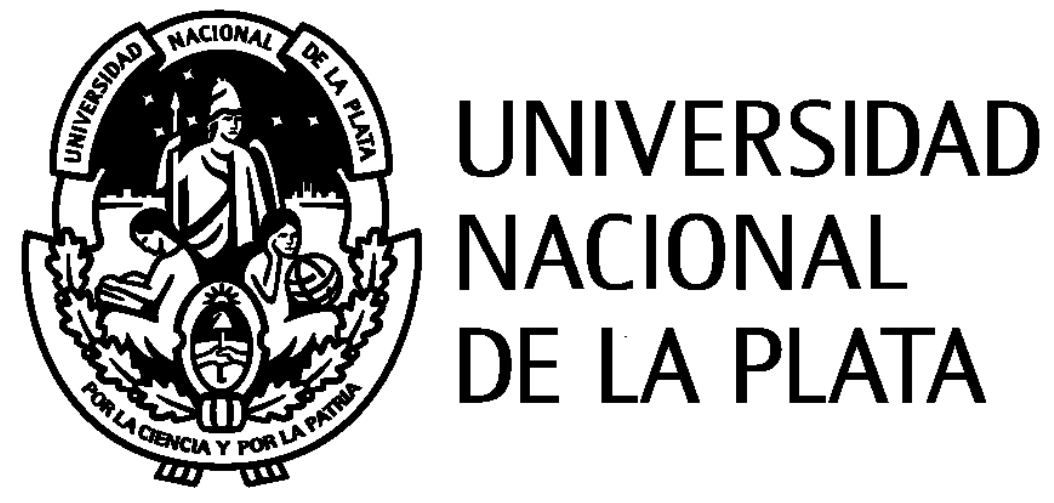

Tesis para obtener el grado académico de

Doctor en Astronomía

\title{
ESTUDIO DEL COMPORTAMIENTO DEL FLUJO ÓPTICO Y DE RAYOS X EN BLAZARES
}

Lic. Lorena C. Zibecchi

Directora: Dra. Ileana Andruchow

Co-Director: Dr. Jorge A. Combi

La Plata, Argentina

- JUNIO DEL 2018 -

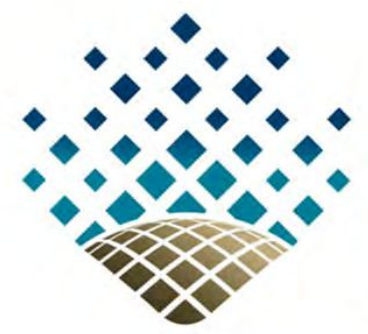

Facultad de Ciencias Astronómicas y Geofísicas 



\section{Prefacio}

Esta Tesis es presentada como parte de los requisitos para obtener el grado académico de Doctor en Astronomía de la Universidad Nacional de La Plata. La misma contiene los resultados de los estudios desarrollados bajo la dirección de la Dra. Ileana Andruchow y el Dr. Jorge A. Combi, investigadores y docentes de la Facultad de Ciencias Astronómicas y Geofísicas de La Plata.

Lorena C. Zibecchi. e-mail: lzibecchi@fcaglp.unlp.edu.ar La Plata, Junio de 2018. 



\title{
ESTUDIO DEL COMPORTAMIENTO DEL FLUJO ÓPTICO Y DE RAYOS X EN BLAZARES
}

\author{
Lic. Lorena C. Zibecchi
}

\section{Directores:}

- Directora: Dra. Ileana Andruchow

- Co-director: Dr. Jorge A. Combi

Jurado de la Tesis:

- Dra. Analía Smith Castelli - FCAGLP (Presidenta)

- Dra. Ana Pichel - IAFE, Buenos Aires

- Dra. Gerogina Coldwell - DGyA - FCEFyN, San Juan

Fecha de defensa: 6 de julio de 2018 



\section{Resumen}

Los blazares son una sub-clase de núcleos de galaxias activos cuyo jet apunta al observador. Su radiación, que se extiende a lo largo de todo el espectro electromagnético, está dominada por procesos no térmicos, y su distribución espectral de energía (SED) es bimodal. Se los divide en dos grandes grupos: BL Lacs y los FSRQs (Flat Spectrum Radio Quasars), a partir de las características de sus espectros ópticos. Sin embargo, aún hay objetos que son clasificados como BZU (blazar uncertain type), los cuales presentan actividad tipo blazar pero con características peculiares, dificultando su clasificación. Dentro de las características principales, se encuentra la evidencia de variabilidad muy rápida, desde algunos pocos minutos hasta horas (escalas menores a un día), conocida como variabilidad intra-día (IDV, Intra-day Variability) o microvariabilidad. Su estudio provee información sobre las estructuras más internas del núcleo activo y permite entender mejor los distintos mecanismos de radiación de la región de emisión. El propósito principal del presente trabajo de Tesis es aportar información basada en el análisis de datos observacionales sobre estas fuentes, en particular aquellas clasificadas como inciertas. Los resultados de este tipo de análisis son de gran importancia para estudiar la física de los procesos así como la estructura de las regiones de emisión. Por lo tanto, los análisis de variabilidad realizados deben tener tanto rigurosidad como robustez. Para esto debemos tener datos observacionales de buena calidad y es fundamental utilizar herramientas estadísticas adecuadas para el análisis del comportamiento de las curvas de luz. En este sentido, cabe destacar que la elección de la estadística aplicada a las curvas arroja resultados contradictorios, donde la determinación del estado de variabilidad de la fuente no estaría relacionada con la física involucrada.

Para analizar adecuadamente la confiabilidad de las distintas herramientas estadísticas bajo diferentes situaciones, hemos llevado a cabo una serie de simulaciones para la construcción de imágenes astronómicas, teniendo en cuenta características instrumentales, condiciones climáticas variables, tales como extinción en la magnitud del objeto por presencia de nubes, variaciones en el comportamiento de la turbulencia atmosférica, etc, obteniéndose un total de 5000 imágenes. Esto permitió confeccionar distintas situaciones observacionales, en semejanza a aquellas que puedan presentarse en la realidad y basadas en la experiencia observacional adquirida. A partir del procesamiento de las imágenes y la aplicación de la fotometría diferencial, se generaron 5.6 $\times 10^{7}$ curvas de luz diferenciales sintéticas, donde se contemplan tanto AGNs variables como no variables, con diferentes magnitudes. Estas curvas fueron estadísticamente analizadas aplicando los dos tests usualmente utilizados $(F$ y el criterio $C$, definido como el cociente de las desviaciones estándares de dos distribuciones) que actualmente presentan controversia en la literatura. Uno de los principales resultados obtenidos es 
la necesidad del uso, en la estadística elegida, de un factor de peso que tenga en cuenta las diferencias en magnitud de los objetos involucrados en la fotometría diferencial. Encontramos también que las variaciones en las condiciones observacionales modifican sensiblemente el comportamiento de las curvas obtenidas. Esto lleva a obtener casos en la clasificación del estado de variabilidad del AGN que son espúreos. Además, dependendiendo del test estadístico aplicado, hay una mayor o menor sensibilidad a la influencia de los cambios en las condiciones observacionales.

Por otro lado se seleccionó una muestra de 19 blazares tomados del Roma BZCAT, constituida por 9 BL Lacs, 7 FSRQs y 3 BZUs. Utilizando el telescopio de 2.15m Jorge Sahade de CASLEO, se realizaron seguimientos de esta muestra en los filtros V y R. Los datos fueron calibrados al sistema estándar. Analizando las curvas de luz obtenidas se detectó tanto variabilidad intra-noche como variaciones inter-noche (en escalas temporales que van desde varios días hasta algunos meses) en 8 objetos. Dado que tuvimos la posibilidad de calibrar los datos al sistema estándar, se complementó este análisis con el estudio del comportamiento de los índices espectrales y los índices de colores y magnitudes en los filtros $\mathrm{V}$ y $\mathrm{R}$, permitiendo relacionar la presencia de las variaciones detectadas con los modelos físicos que explican el origen de estas variabilidades. Para nuestras observaciones, encontramos que el modelo que mejor explica la microvariabilidad en blazares es el modelo de shock-in-jet, el cual explica la variabilidades temporales a partir de la presencia de inhomogeneidades dentro del jet relativista del blazar.

De una sub-muestra conformada por 13 de estos 19 blazares se pudieron obtener observaciones en rayos X (tomadas de la base de datos del satélite Chandra). Estos datos fueron procesados y analizados utilizando el software HEASOFT y CIAO. De las curvas de luz obtenidas, 5 fuentes presentaron evidencia de variabilidad a corto plazo (intra-noche).

De los estudios de variabilidad a cortas escalas, encontramos que sólo a 4 fuentes de la muestra se les detectó variabilidad en el óptico y en rayos- X (datos no simultáneos). Estas fuentes resultaron ser 2 FSRQs (LSP, Low Synchrotron Peak) y 2 BL Lac (HSP, High Synchrotron Peak). Es esperable que los blazares HSP tengan una probabilidad más baja de variar a corto plazo que los LSP. Realizamos un análisis multifrecuencia para una de estas 4 fuentes, con el objetivo de estudiar la variabilidad en estas bandas. Complementando las observaciones del óptico y rayos $\mathrm{X}$ con los datos obtenidos por el satélite Fermi-LAT en la banda de rayos- $\gamma$, encontramos que que no hay variabilidad correlacionada entre las bandas óptico y rayos- $\gamma$, ni tampoco entre rayos X y rayos- $\gamma$, para este conjunto de datos en particular. 


\section{Agradecimientos}

En primer lugar quiero agradecer a Dios por todo lo que Él me ha dado y por haberme permitido llegar hasta donde llegué. Gracias a Dios, a Jesús y al Espíritu Santo por su incondicional amor y por darme la fortaleza y la paz en los momentos que más lo necesitaba.

Gracias a mi esposo Mauro, porque me ha acompañado en cada momento transitado, en los momentos de felicidad así como también en aquellos momentos de frustración, brindándome todo su apoyo y su amor. Sin él, las cosas hubiesen sido más difíciles y mucho menos amenas. También quiero agradecer a mi hijo Ezequiel. Con él, no sólo obtuve uno de los títulos más importantes de mi vida, el de mamá, sino que en todo este proceso de la Tesis Doctoral me ha enseñado a que todo lo que uno sueña, llega si se lo propone. Además, también le agradezco a Javier, mi hijo del corazón, que me ha brindado su apoyo siempre. Gracias a mis padres, Jorge y Carmen, que desde el primer momento me han apoyado en esta carrera tan particular. Gracias por su apoyo, por su amor y por las palabras de aliento cada vez que las necesitaba.

Quiero agradecer especialmente a la Dra. Ileana Andruchow, quien ha sido más que mi directora a lo largo de todos estos años. Le agradezco por todo el tiempo que se ha tomado para ayudarme, incluso en los momentos en que no correspondía. Le agradezco por la infinita paciencia que me ha tenido y por ser siempre tan solícita en todo lo que necesitaba. Gracias porque ha estado a la par mía, me ha enseñado y me sigue enseñando muchas cosas, no sólo en lo laboral. Desde el primer día en que firmó para ser mi profesora consejera hasta el día de hoy, siempre ha estado y se lo agradezco muchísimo.

Agradezco también al Dr. Jorge Combi, quien ha sido mi co-director en todo este proceso. Gracias por toda la ayuda que me ha brindado y por todo lo que ha colaborado en este trabajo. Le doy gracias también al Dr. Sergio Cellone y al Dr. Daniel Carpintero, quienes sin obligación alguna, han colaborado y trabajado arduamente conmigo, y me han ayudado muchísimo con la realización de esta Tesis Doctoral. A todos ellos, muchas gracias por todo lo que me han brindado.

Finalmente, quiero agradecer a la Facultad de Ciencias Astronómicas y Geofísicas de la Universidad Nacional de La Plata, a todo su plantel docente por la formación pública, gratuita y de excelencia que me han permitido capacitarme como profesional. También al Instituto de Astrofísica de La Plata (IALP) y a CONICET quien me ha otorgado una beca para el desarrollo de la presente Tesis. Y a todos mis compañeros/amigos 
que han estado junto a mí, en este largo caminar. Gracias a cada uno de ellos por la palabra y el aliento en el momento justo, y por los mates también! Sin ellos se hubiese hecho díficil llegar hasta la meta! 
A mi Señor Jesucristo,

a mi esposo Mauro, a mi hijo Ezequiel, a mi hijo del corazón Javier y a mi familia. 



\section{Índice general}

$\begin{array}{lll}\text { Prefacio } & \text { iii }\end{array}$

Resumen vii

Agradecimientos $\quad$ ix

Lista de acrónimos $\quad$ xxiii

1. Introducción 1

1.1. Núcleos Galácticos Activos . . . . . . . . . . . . . . . . . . . . 1

1.1.1. El esquema de unificación: fenomenología y zoo de AGNs . . . . 2

1.2. Blazares . . . . . . . . . . . . . . . . . . . 8

1.2.1. Las SEDs en los blazares . . . . . . . . . . . . . . . 99 9

1.3. Variabilidad . . . . . . . . . . . . . . . . . . 12

1.3.1. Microvariabilidad: historia, importancia y modelos . . . . . . . . 13

1.3.2. Variabilidad en altas energías . . . . . . . . . . . . . 16

1.4. Esquema de la presente Tesis Doctoral . . . . . . . . . . . . . . . . . 20

2. Herramientas estadísticas $\quad 21$

2.1. Introducción y presentación de la problemática . . . . . . . . . . . . 21

2.2. Los tests de hipótesis . . . . . . . . . . . . . . . . . . . 22

2.2.1. Estadística $C \ldots \ldots \ldots \ldots$

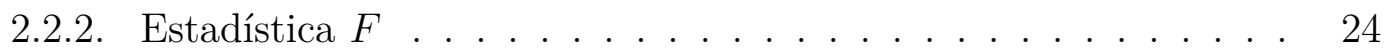

2.3. Resultados sobre datos observacionales . . . . . . . . . . . . 25

2.3.1. Estudio detallado del "test" C . . . . . . . . . . . . . 26

2.3.2. Análisis de $\mathrm{C} \mathrm{y} \mathrm{F}$ en estrellas de campo . . . . . . . . . . . . . 27

2.4. Simulaciones . . . . . . . . . . . . . . . . . . 30

2.4.1. Descripción e implementación . . . . . . . . . . . . . . 30

2.4.2. Situaciones simuladas . . . . . . . . . . . . . . . 35

2.4.3. Factor de peso en las estadísticas . . . . . . . . . . . . . . 42

2.5. Resultados y análisis . . . . . . . . . . . . . . . . 45

2.5.1. Comparación entre casos testigos . . . . . . . . . . . . 47

2.5.2. Comparación entre casos de seeing variable . . . . . . . . . . . . . 6 60

2.5.3. Análisis de los resultados generales . . . . . . . . . . . . . . 71 
2.6. Discusión . . . . . . . . . . . . . . . . . . . . 85

3. Análisis del flujo de la muestra de Blazares $\quad 91$

3.1. Selección de la muestra . . . . . . . . . . . . . . . . . . . . . . . 91

3.2. Análisis en el Óptico . . . . . . . . . . . . . . . . . . . . . . . 92

3.2.1. Observaciones en CASLEO y procesamiento de datos . . . . . . 92

3.2.2. Análisis de los resultados: Variabilidad . . . . . . . . . . . . . 95

3.2.3. Análisis de los colores y magnitudes de la muestra de blazares . 99

3.2.4. Análisis global y discusión sobre cada fuente . . . . . . . . . . . 103

3.2.5. Los blazares BZU . . . . . . . . . . . . . . . . . . . . 164

3.3. Discusión de los resultados obtenidos en el óptico de la muestra seleccionada ......................... 167

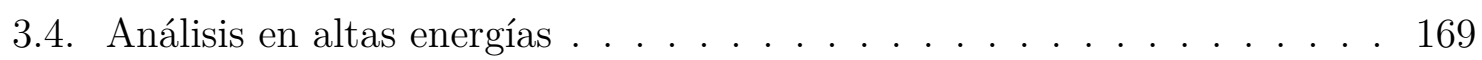

3.4.1. Rayos X . . . . . . . . . . . . . . . . . 169

3.4.2. Análisis multifrecuencia para 3FGL J1958.2-3011 . . . . . . . . 183

4. Conclusiones 191

A. Calibración de los objetos de la muestra al sistema estándar $\quad 195$

A.1. Campos de estándares usados en la calibración . . . . . . . . . . . . . 195

A.2. Resultados . . . . . . . . . . . . . . . . . . . . . 198 


\section{Índice de figuras}

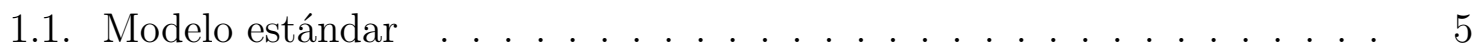

1.2. SED de AGN ...................... 6

1.3. Unificación de AGNs . . . . . . . . . . . . . . . . . . . 7

1.4. Mecanismos de radiación en la SED . . . . . . . . . . . . . . 10

1.5. Espectros sincrotrón e IC . . . . . . . . . . . . . . . . . . . . . . 11

1.6. SED de un blazar . . . . . . . . . . . . . . . . . . . . 12

1.7. Ejemplo de variabilidad LTV . . . . . . . . . . . . . . . 13

1.8. Ejemplo de variabilidad STV y microvariabilidad . . . . . . . . . . 14

1.9. Modelo de variabilidad en rayos X . . . . . . . . . . . . . . . 16

1.10. Correlación entre curvas en rayos X y óptico . . . . . . . . . . . 17

1.11. Correlación entre curvas en rayos- $\gamma$ y óptico . . . . . . . . . . 18

1.12. Análisis multifreciencia . . . . . . . . . . . . . . . . . . . . 19

2.1. Ejemplo de una densidad de distribución de Fisher $F \ldots \ldots \ldots$

2.2. Comparación entre las estadísticas $\mathrm{C} \mathrm{y} \mathrm{F} \mathrm{\ldots} \mathrm{.} \mathrm{.} \mathrm{.} \mathrm{.} \mathrm{.} \mathrm{.} \mathrm{.} \mathrm{.} 26$

2.3. Distribución de los porcentajes de estrellas por campo . . . . . . . . . 29

2.4. Imagen simulada. . . . . . . . . . . . . . . . . . . 31

2.5. Tipos de variabilidades en las simulaciones . . . . . . . . . . . 34

2.6. Comportamiento del seeing . . . . . . . . . . . . . . . . . . . . 36

2.7. Comportamiento de la cobertura de nubes . . . . . . . . . 37

2.8. Ejemplos de curvas de luz diferenciales sintéticas . . . . . . . . . . 40

2.9. Diagrama de flujo de las simulaciones. . . . . . . . . . . . . . 41

2.10. Ejemplo de curva de luz de AGN no variable . . . . . . . . . . . 43

2.11. Histograma del factor de peso de Howell para AGNs NV . . . . . . 46

2.12. Comportamiento de la dispersión de la curva de control frente al factor de peso para los CT para AGNs NV . . . . . . . . . . . . 48

2.13. Comportamiento de la dispersión del AGN frente a la de la curva de

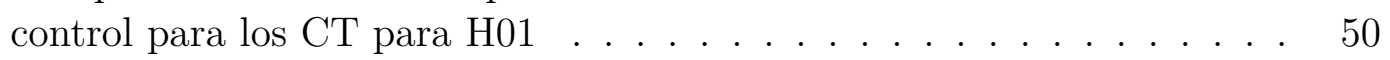

2.14. Comportamiento de la dispersión del AGN frente a la de la curva de control para los CT para $\mathrm{H} 03 \ldots \ldots$. . . . . . . . . . . .

2.15. Comportamiento de la dispersión del AGN frente a la de la curva de control para los CT para $\mathrm{H} 05 \ldots \ldots \ldots$

2.16. Comportamiento de la dispersión de la curva de control frente al factor de peso para los CT para AGNs $\mathrm{V} \ldots \ldots \ldots \ldots$ 
2.17. Histograma de la dispersión de la curva del AGN variables en CT I . . 57

2.18. Histograma de la dispersión de la curva del AGN variables en CT II . . 58

2.19. Comportamiento de la dispersión del AGN frente a la de la curva de control para los CT para AGNs V . . . . . . . . . . . .

2.20. Comportamiento de la dispersión de la curva de control frente al factor de peso para los IQ para AGNs NV . . . . . . . . . . . . .

2.21. Histograma de la dispersión de la curva del AGN para H01, H03 y H05, en los casos CT1 y IQ1. . . . . . . . . . . . . . . . . .

2.22. Comportamiento de la dispersión del AGN frente a la de la curva de control para los IQ para $\mathrm{H} 01 \ldots \ldots . \ldots . . . \ldots$

2.23. Comportamiento de la dispersión del AGN frente a la de la curva de control para los IQ para H03 . . . . . . . . . . . . .

2.24. Comportamiento de la dispersión del AGN frente a la de la curva de control para los IQ para H05 . . . . . . . . . . . . . . . 65

2.25. Histograma de la dispersión de la curva de AGN variables en IQ I . . . 68

2.26. Histograma de la dispersión de la curva de AGN variables en IQ II . . . 69

2.27. Comportamiento de la dispersión del AGN frente a la de la curva de control para los IQ para AGNs V . . . . . . . . . . . .

2.28. Comportamiento de la dispersión de la curva de control frente al factor de peso para CT2, IQ1, IQ1-SB1, IQ1-CC1 y IQ1-SB1-CC1 para AGNs

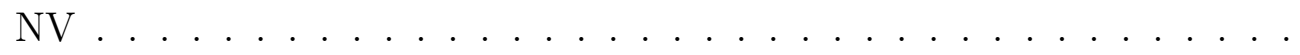

2.29. Comportamiento de la dispersión de la curva de control frente al factor de peso para CT2, IQ1, IQ1-SB1, IQ1-CC1 y IQ1-SB1-CC1 para AGNs $\mathrm{NV}$ para $\mathrm{H} 01 \ldots \ldots \ldots \ldots$

2.30. Comportamiento de la dispersión de la curva de control frente al factor de peso para CT2, IQ1, IQ1-SB1, IQ1-CC1 y IQ1-SB1-CC1 para AGNs NV para $\mathrm{H} 03 \ldots \ldots \ldots \ldots \ldots . \ldots \ldots$

2.31. Comportamiento de la dispersión de la curva de control frente al factor de peso para CT2, IQ1, IQ1-SB1, IQ1-CC1 y IQ1-SB1-CC1 para AGNs

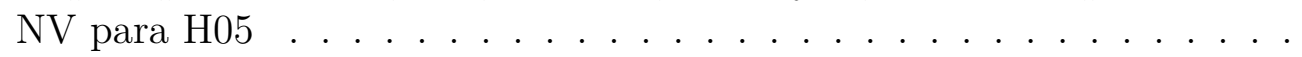

2.32. Comportamiento de la dispersión de la curva de control frente al factor de peso para CT2, IQ1, IQ1-SB1, IQ1-CC1 y IQ1-SB1-CC1 para AGNs V 78

2.33. Comportamiento de la dispersión de la curva de control frente a la de la curva de control para CT2, IQ1, IQ1-SB1, IQ1-CC1 y IQ1-SB1-CC1

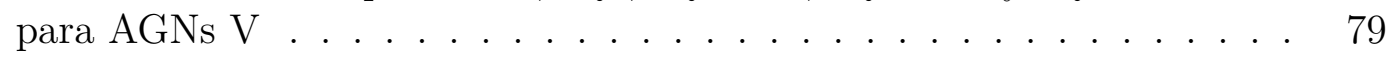

3.1. Catálogo Roma-BZCAT . . . . . . . . . . . . . . . . . . . 92

3.2. Variación de la mag. estándar para 0208-512 . . . . . . . . . . . . . . . 104

3.3. Valores comparativos de la magnitud V para 0208-512 . . . . . . . . . . 105

3.4. Valores comparativos de la magnitud R para 0208-512 . . . . . . . . . . 106

3.5. Índice espectral para 0208-512 . . . . . . . . . . . . . . . . . . . . . . . . 106

3.6. Diagrama V vs. R y DCF para 0208-512 . . . . . . . . . . . . . . 107

3.7. Diagrama V vs. V-R para 0208-512 . . . . . . . . . . . . . . . . . 108

3.8. Variación de la mag. estándar para $0414+009$. . . . . . . . . . . . . . 109

3.9. Valores comparativos entre las mag. V y R para 0414+099 . . . . . . . 110

3.10. Diagrama V vs. R y DCF para 0414+009 . . . . . . . . . . . . . 111 
3.11. Índice espectral para $0414+009$. . . . . . . . . . . . . . . . . . 111

3.12. Comparación del índice espectral para 0414+009 . . . . . . . . . . . . . 112

3.13. Diagrama V vs. V-R para $0414+009$. . . . . . . . . . . . . . . 112

3.14. Variación de la mag. estándar para 0521-365 . . . . . . . . . . . . . . 113

3.15. Valores comparativos entre las mag. V y R para 0521-365 . . . . . . . . 114

3.16. Diagrama V vs. R y DCF para 0521-365 . . . . . . . . . . . . . 115

3.17. Diagrama V vs. V-R para 0521-365 . . . . . . . . . . . . . . . . 115

3.18. Variación de la mag. estándar para 0847-2337 . . . . . . . . . . . . . . 116

3.19. Diagrama V vs. R y DCF para 0847-2337 . . . . . . . . . . . . . . 117

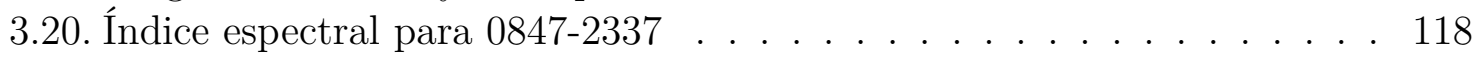

3.21. Diagrama V vs. V-R para 0847-2337 . . . . . . . . . . . . . . . 118

3.22. Variación de la mag. estándar para 1116-462 . . . . . . . . . . . . . . . 119

3.23. Valores comparativos entre las mag. V para 1116-462 . . . . . . . . . . 119

3.24. Diagrama V vs. R y DCF para 1116-462 . . . . . . . . . . . . . . . . 120

3.25. Índice espectral para 1116-462 . . . . . . . . . . . . . . . . . . . . . . . . . . . 121

3.26. Diagrama V vs. V-R para 1116-462 . . . . . . . . . . . . . . . . . 121

3.27. Variación de la mag. estándar para 1127-145 . . . . . . . . . . . . . . . 122

3.28. Diagrama V vs. R y DCF para 1127-145 . . . . . . . . . . . . . . . 122

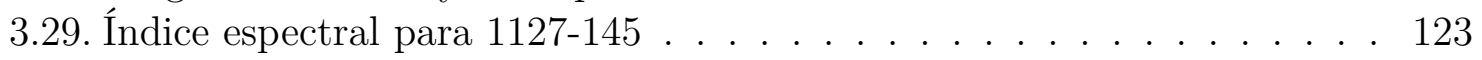

3.30. Diagrama V vs. V-R para 1127-145 . . . . . . . . . . . . . . . . 123

3.31. Variación de la mag. estándar para 1229-02 . . . . . . . . . . . . . . . . 124

3.32. Diagrama V vs. R y DCF para 1229-02 . . . . . . . . . . . . . . 125

3.33. Diagrama V vs. V-R para 1229-02 . . . . . . . . . . . . . . 126

3.34. Variación de la mag. estándar para 1256-1146 . . . . . . . . . . . 126

3.35. Diagrama V vs. R y DCF para 1256-1145 . . . . . . . . . . . . . . . . 127

3.36. Diagrama V vs. V-R para 1256-1145 . . . . . . . . . . . . . . 127

3.37. Variación de la mag. estándar para 1424-418 . . . . . . . . . . . . . . 128

3.38. Valores comparativos de la magnitud V para 1424-418 . . . . . . . . . . 129

3.39. Diagrama V vs. R y DCF para 1424-418 . . . . . . . . . . . . . . . . 129

3.40. Índice espectral para 1424-418 . . . . . . . . . . . . . . . . . . . . 130

3.41. Diagrama V vs. V-R para 1424-418 . . . . . . . . . . . . . . . . . 130

3.42. Variación de la mag. estándar para 1443-3908 . . . . . . . . . . . . . . 131

3.43. Diagrama V vs. R y DCF para 1443-3908 . . . . . . . . . . . . . . . . . 132

3.44. Índice espectral para 1443-3908 . . . . . . . . . . . . . . . . . . . 132

3.45. Diagrama V vs. V-R para 1443-3908 . . . . . . . . . . . . . . . 133

3.46. Variación de la mag. estándar para 1510-089 . . . . . . . . . . . . . . . 134

3.47. Valores comparativos de las magnitudes V y R para 1510-089 . . . . . . 135

3.48. Índice espectral para 1510-089 . . . . . . . . . . . . . . . 135

3.49. Valores comparativos del índice espectral para 1510-089 . . . . . . . . 137

3.50. Diagrama V vs. R y DCF para 1510-089 . . . . . . . . . . . . . . . . . . . 138

3.51. Diagrama V vs V-R para 1510-089 . . . . . . . . . . . . . . . . 138

3.52. Variación de la mag. estándar para 1517-2422 . . . . . . . . . . . . . . 140

3.53. Valores comparativos de la magnitud V para 1517-2422 . . . . . . . . . 141

3.54. Diagrama V vs. R y DCF para 1517-2422 . . . . . . . . . . . . . . . . 142

3.55. Índice espectral para $1517-2422$. . . . . . . . . . . . . . . . . 143

3.56. Valores comparativos del índice espectral para 1517-2422 . . . . . . . . 143 
3.57. Diagrama V vs. V-R para 1517-2422 . . . . . . . . . . . . . . . . . 144

3.58. Variación de la mag. estándar para 1917-1921 . . . . . . . . . . . . . 145

3.59. Diagrama V vs. R y DCF para 1917-1921 . . . . . . . . . . . . . 146

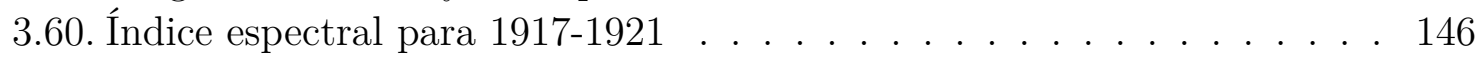

3.61. Diagrama V vs. V-R para 1917-1921 . . . . . . . . . . . . . . . . 146

3.62. Variación de la mag. estándar para 1958-3012 . . . . . . . . . . . . . 147

3.63. Diagrama V vs. R y DCF para 1958-3012 . . . . . . . . . . . . . . 148

3.64. Índice espectral para 1958-3012 . . . . . . . . . . . . . . . . . . . . . . . . . . . . . . . . 148

3.65. Diagrama V vs. V-R para 1958-3012 . . . . . . . . . . . . . . . . 148

3.66. Variación de la mag. estándar para 2005-489 . . . . . . . . . . . . . . . 149

3.67. Valores comparativos de la magnitud V para 2005-489 . . . . . . . . . . 150

3.68. Diagrama V vs. R y DCF para 2005-489 . . . . . . . . . . . . . . 151

3.69. Diagrama V vs. V-R para 2005-489 . . . . . . . . . . . . . . . 151

3.70. Variación de la mag. estándar para 2126-458 . . . . . . . . . . . . . 152

3.71. Valores comparativos de la magnitud V para 2126-158 . . . . . . . . 153

3.72. Diagrama V vs. R y DCF para 2126-158 . . . . . . . . . . . . . . . . 154

3.73. Índice espectral para 2126-158 . . . . . . . . . . . . . . . . 154

3.74. Diagrama V vs. V-R para 2126-158 . . . . . . . . . . . . . . . 155

3.75. Variación de la mag. estándar para 2149-306 . . . . . . . . . . . . . . 155

3.76. Diagrama V vs. R y DCF para 2149-306 . . . . . . . . . . . . 156

3.77. Índice espectral para $2149-306 \ldots$. . . . . . . . . . . . . . 156

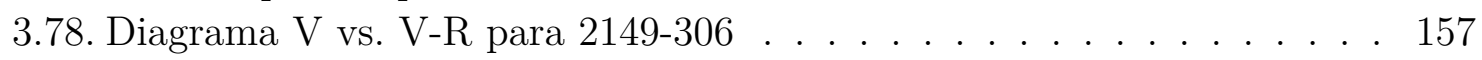

3.79. Variación de la mag. estándar para 2155-304 . . . . . . . . . . . . . 158

3.80. Valores comparativos de la magnitud V para 2155-304 . . . . . . . . . . 159

3.81. Diagrama V vs. R y DCF para 2155-304 . . . . . . . . . . . . . 160

3.82. Comparación del índice espectral para 2155-304 . . . . . . . . . . . . 160

3.83. Diagrama V vs. V-R para 2155-304 . . . . . . . . . . . . . . . . 161

3.84. Variación de la mag. estándar para 2310-437 . . . . . . . . . . . . . 162

3.85. Diagrama V vs. R y DCF para 2310-437 . . . . . . . . . . . . . . . 163

3.86. Diagrama V vs. V-R para 2310-437 . . . . . . . . . . . . . . 163

3.87. Índice de mod. int. vs. Frec. del pico sincrotrón . . . . . . . . . . . . . 165

3.88. Índice de mod. int. vs. Redshift . . . . . . . . . . . . . . . . . . 166

3.89. Satélite Chandra . . . . . . . . . . . . . . . . . . 170

3.90. Ejemplos de curvas de luz de la herramienta glvary . . . . . . . . . . . 174

3.91. Curvas de luz en rayos X de 1127-145 . . . . . . . . . . . . . . 178

3.92. Curvas de luz en rayos X de 1958.2-3011 . . . . . . . . . . . . . . . 179

3.93. Curvas de luz en rayos X de 2005-489 . . . . . . . . . . . . . . . 180

3.94. Curvas de luz en rayos X de 2126-158 . . . . . . . . . . . . . . 181

3.95. Curvas de luz general en rayos X de 2155-304 . . . . . . . . . . . . . 181

3.96. Curvas de luz en rayos X de 2155-304 . . . . . . . . . . . . . . . . 182

3.97. Satélite Fermi-LAT . . . . . . . . . . . . . . . . . . . 184

3.98. Imágenes multifrecuencia de 1958.3011 . . . . . . . . . . . . . . . . 186

3.99. Curvas de luz en rayos- $\gamma$ para $1958.3011 \ldots$. . . . . . . . . . . 187

3.100.Curvas de luz en óptico y rayos- $\gamma$ para $1958.3011 \ldots$. . . . . . . . . . 188

3.101.Curvas de luz en rayos X y rayos- $\gamma$ para 1958.3011 . . . . . . . . . . 189 
A.1. Campos de estándares de Landolt usados . . . . . . . . . . . . . . . . 196

A.2. Campos de blazares I . . . . . . . . . . . . . . . . . . . . . . . 198

A.3. Campos de blazares II . . . . . . . . . . . . . . . . . . . . . . . . . . 199

A.4. Campos de blazares III . . . . . . . . . . . . . . . . . . . . . 200

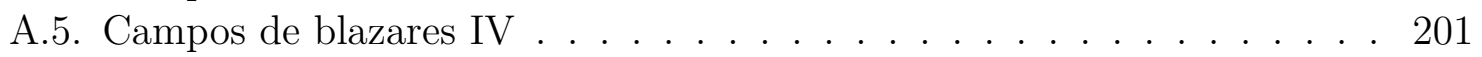

A.6. Campos de blazares V . . . . . . . . . . . . . . . . . . . . . . . 202

A.7. Campos de blazares VI . . . . . . . . . . . . . . . . . . 203 



\section{Índice de tablas}

2.1. Valores de las condiciones instrumentales y atmosféricas. . . . . . . . . 32

2.2. Situaciones básicas simuladas. . . . . . . . . . . . . . . . . . 38

2.3. Cantidad de DLCs variables y no variables sin restricción de Howell. . . 44

2.4. Porcentajes de casos variables y no variables en CT. . . . . . . . . . 53

2.5. Porcentajes de casos variables y no variables en IQ. . . . . . . . . . . . 66

2.6. Porcentajes de casos variables y no variables en la comparación entre situaciones. . . . . . . . . . . . . . . . 76

2.7. Porcentajes de casos variables y no variables en la comparación entre situaciones. . . . . . . . . . . . . . . . . 80

2.8. Porcentajes de casos variables y no variables en la comparación entre situaciones. . . . . . . . . . . . . . . . . 83

2.7. Porcentajes de casos variables y no variables en la comparación entre situaciones. . . . . . . . . . . . . . . . . . 84

2.8. Porcentajes de casos variables y no variables en la comparación entre situaciones. . . . . . . . . . . . . . . 86

2.8. Porcentajes de casos variables y no variables en la comparación entre situaciones. . . . . . . . . . . . . . . . . 87

3.1. Datos de los objetos . . . . . . . . . . . . . . . . . 93

3.2. Datos en el óptico . . . . . . . . . . . . . . . . . . . . . . . . . . . . 94

3.3. Resultados de la variabilidad . . . . . . . . . . . . . . . . . 96

3.3. Cont. . . . . . . . . . . . . . . . . . 97

3.3. Cont. . . . . . . . . . . . . . . . . . . . 98

3.4. Función de correlación . . . . . . . . . . . . . . . . . . 100

3.5. Valores de los índices espectrales . . . . . . . . . . . . . . . . . . . . . . . . . . . . . . . 102

3.6. Datos en la banda de rayos X . . . . . . . . . . . . . . . . . . . . . . . . . . . . . . . 172

3.6. Cont. . . . . . . . . . . . . . . . . . . . 173

3.7. Valores del algoritmo de glvary . . . . . . . . . . . . . . . . . . 174

3.8. Resultados de la variabilidad en rayos X . . . . . . . . . . . . 176

3.8. Cont. . . . . . . . . . . . . . . . . 177

A.1. Información de los campos de estándares . . . . . . . . . . . . . . . 197

A.2. Magnitudes estándares de las estrellas de campo . . . . . . . . . . . . . 204

A.2. Cont. . . . . . . . . . . . . . . . . . 205

A.2. Cont. . . . . . . . . . . . . . . . 206 
A.2. Cont. . . . . . . . . . . . . . . . . . 207

A.2. Cont. . . . . . . . . . . . . . . . . . 208

A.2. Cont. . . . . . . . . . . . . . . . . . . 209

A.2. Cont. . . . . . . . . . . . . . . . . . 210

A.2. Cont. . . . . . . . . . . . . . . . . . 211

A.2. Cont. . . . . . . . . . . . . . . . . . . . 212

A.2. Cont. . . . . . . . . . . . . . . . . . . 213

A.2. Cont. . . . . . . . . . . . . . . . . . . 214

A.2. Cont. . . . . . . . . . . . . . . . . . 215 


\section{Lista de acrónimos}

AGN: Núcleo Galáctico Activo (Active Galactic Nuclei)

BL Lac: objeto tipo BL Lacertae

BLR: región de líneas anchas (Broad line region)

BZU: blazar de tipo incierto (Blazar uncertain type)

CASLEO: Complejo Astronómico El Leoncito

CL: nivel de confiabilidad (Confidence level)

DLC: curva de luz diferencial (Diferential light curve)

FRI: galaxias Fanaroff-Riley I

FRII: galaxias Fanaroff-Riley II

FSRQ: radio-quasar de espectro chato (Flat Spectrum Radio Quasar)

HBL: blazar con pico sincrotrón a altas energías (High energy blazar)

HSP: blazar con pico sincrotrón a altas frecuencias (High Synchrotron Peaked blazar)

IC: proceso de dispersión Compton inverso (Inverse Compton)

IR: Infrarrojo

ISP: blazar con pico sincrotrón a frecuencias intermedias (Intermediate Synchrotron Peaked blazar)

LBL: blazar con pico sincrotrón a bajas energías (Low energy blazar)

LSP: blazar con pico sincrotrón a bajas frecuencias (Low Synchrotron Peaked blazar)

LTV: Variabilidad a escalas largas (Long term variability)

NIR: Infrarrojo cercano (Near Infrared)

NLR: región de líneas angostas (Narrow line region)

PKS: catálogo de Parkes de radio

SED: distribución espectral de energía (Spectral Energy Distribution)

SMBH: Agujero Negro Super Masivo (Super Masive Black Hole)

STV: Variabilidad a escalas cortas (Short term variability)

UV: Ultravioleta

VHE: Muy altas energías (Very high energies) 



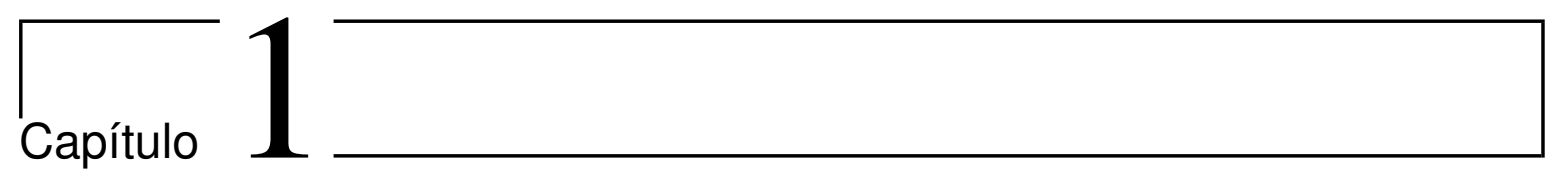

\section{Introducción}

\subsection{Núcleos Galácticos Activos}

Entre las fuentes extragalácticas existentes en el Universo, se encuentran los núcleos galácticos activos (AGNs, de sus siglas en inglés Active Galactic Nuclei). Se los definen como las regiones centrales de galaxias que presentan una gran emisión de energía, la cual no puede explicarse por la emisión estelar ni por las interacciones entre estrellas y el medio interestelar. Las emisiones de energía originadas en estos objetos van desde los $10^{40} \mathrm{erg} / \mathrm{s}$ hasta los $10^{47} \mathrm{erg} / \mathrm{s}$, y se expanden a lo largo de todo el espectro electromagnético, desde radio hasta rayos- $\gamma$. Entre las características más importantes de estos objetos se puede mencionar: luminosidades altas, presencia de jets (flujo colimado de partículas relativistas), variabilidad rápida de su flujo y emisión no térmica en su región central.

\section{Breve contexto histórico}

Cuando se comenzaron a observar las galaxias con telescopios ópticos, se encontró que una fracción de éstas poseía regiones centrales mucho más luminosas que la galaxia en su totalidad. En ese tiempo, una galaxia era considerada como una nebulosa. A comienzos del siglo XX, se tomó el primer espectro de una galaxia activa, NGC 1068 (Fath 1908), donde se observaron líneas de emisión muy fuertes. Años más tarde, Slipher (1913) obtuvo un espectro con una resolución mucho mejor, en el cual se observaron líneas de emisión similares a las presentes en nebulosas planetarias, pero con anchos de varios km/s. Ya en la década del 20, Curtis (1920) propuso que la nebulosa Andrómeda (M31) era una galaxia en sí misma. Todo esto dio lugar a que se consideraran las nebulosas observadas como fuentes extragalácticas.

A partir del trabajo de Seyfert en el año 1943, las galaxias activas se comenzaron a clasificar como un nuevo tipo de fuente. Seyfert observó que varios de los objetos en su muestra de estudio tenían líneas de emisión muy anchas, y que las líneas de Balmer del hidrógeno, en algunos casos, eran más anchas que otras líneas.

Después de la Segunda Guerra Mundial, surge la radioastronomía, la cual permitió, entre otras cosas, descubrir que las galaxias que Seyfert había estudiado, presentaban emisión en radio. Por otra parte, Woltjer (1959) relacionó que el ancho que poseían las 
líneas requería una velocidad muy alta, asociando esto último a una región compacta $(\sim 100 \mathrm{pc})$ y con una gran concentración de masa $\left(10^{8} M_{\odot}\right)$.

Luego, se propuso que en el centro de estas galaxias debía existir un objeto tipo estelar masivo, en el cual los procesos de acreción asociados al disco eran responsables de la emisión (Hoyle \& Fowler, 1963). Un año más tarde se asoció dicho objeto con un agujero negro (Salpeter 1964; Zel'Dovich \& Novikov 1964). Así, la idea de un agujero negro en el centro de estas galaxias permite explicar no sólo las grandes cantidades de energía liberada sino también lo pequeño de las regiones de emisión asociado a la variabilidad con escalas temporales cortas en los AGNs. En 1964, Greenstein y Schmidt propusieron que el brillo extraordinario de estas fuentes $\left(\sim 10^{45} \mathrm{erg} / \mathrm{s}\right)$ era fácil de explicar si existe una gran cantidad de masa $\left(10^{8} M_{\odot}\right)$ en un volumen pequeño de espacio $\left(1 p c^{3}\right)$. Todos estos avances dieron origen al estudio de las galaxias activas, con el objetivo de comprender la física involucrada en el fenómeno de acreción, para ser capaces de explicar la emisión observada en los AGNs a través de todo el espectro electromagnético y así tener una idea cada vez más certera de lo que sucede en estas fuentes.

\subsubsection{El esquema de unificación: fenomenología y zoo de AGNs}

Al observar a los AGNs, se pueden distinguir diversas apariencias y características de los mismos. Esto lleva a pensar que pueden tratarse de objetos distintos intrínsecamente o también puede considerarse que se trata del mismo objeto visto desde diferentes ángulos por los observadores. Esta idea de un escenario en común fue presentada por Blandford \& Rees (1978). Más tarde, Antonucci (1993) sugirió que los AGNs se pueden dividir en dos grandes categorías: los AGNs radio-débiles (radio-quiet) y los AGNs radio-intensos (radio-loud). Además propuso que alrededor de la región central del AGN había un toro ópticamente grueso. Urry \& Padovani (1995) explicaron la unificación de los AGNs radio-intensos y propusieron un comportamiento evolutivo distinto entre los BL Lacs y los FSRQs (subclases de blazares, ver más adelante). Todos estos trabajos permitieron dar el puntapié inicial para poder explicar la subclasificación de las distintas clases de AGNs a través de un escenario en común.

\section{Fenomenología}

Como ya mencionamos, los AGNs radian a lo largo de todo el espectro electromagnético. A continuación, se presenta un breve detalle de lo observable en cada banda del espectro:

- Radio: esta emisión posee una componente extendida, la cual surge de la interacción entre los jets presentes en el AGN y el medio que lo rodea. En cuanto a la región compacta del AGN (correspondiente al núcleo), la cual no puede resolverse a escalas menores a algunos segundos de arco, coincidiría con una posición cercana al agujero negro central, donde el jet se vuelve ópticamente delgado. La radiación en esta banda está asociada al mecanismo de sincrotrón de electrones con velocidades relativistas que se mueven en regiones con campos magnéticos intensos. 
- IR: esta emisión infrarroja (IR) se expande desde $1 \mu m$ hasta $300 \mu m$ y puede originarse a partir de un proceso térmico como así también no-térmico. Para el caso de los AGNs radio-intensos y en particular para los blazares, la radiación IR proviene del proceso sincrotrón. Para las galaxias Seyferts y otros AGNs de luminosidad baja, la radiación proviene de varias componentes térmicas, como la radiación del polvo que se encuentra cerca del agujero negro super masivo y el continuo térmico asociado a la formación estelar de la galaxia que alberga al AGN.

- Óptico: la emisión en esta banda puede explicarse por procesos térmicos y notérmicos. Respecto a la emisión térmica, se suele observar un exceso en la radiación, el cual se extiende hasta el ultravioleta (UV): la Big Blue Bump (del inglés, la Gran Loma Azul). Su origen está asociado a la emisión viscosa del disco de acreción. Existe también una componente térmica correspondiente a la radiación de la galaxia anfitriona del AGN. Por otra parte, en lo que respecta a la emisión no-térmica, cuando los jets presentes en el AGN están orientados hacia el observador, se tiene contribución a la emisión óptica, relacionada al proceso sinctrotrón y al proceso de Compton inverso (IC, de sus siglas en inglés Inverse Compton). En general, esta componente puede describirse por una ley de potencias. En cuanto a su espectro, se caracteriza por la presencia de líneas de emisión anchas, resultado de la interacción entre el agujero negro y su material circundante. En varios objetos también se observan líneas de emisión angostas, las cuales se originan de un material más frío en comparación a aquel que produce las líneas anchas.

- UV: la emisión en el UV es de carácter térmico y proviene del disco de acreción que se encuentra alrededor del agujero negro central. Algunos de los fotones UV son dispersados a través del IC hasta llegar a energías asociadas a la banda de rayos $\mathrm{X}$. Una parte de estos son emitidos nuevamente hacia el disco, produciendo un calentamiento del mismo e incrementando la emisión en el UV.

- Rayos X: esta emisión se localiza entre los 120 eV y los 120 keV. La parte térmica de su espectro puede producirse por el disco de acreción en torno al agujero negro super masivo. Los fotones de bajas energías (originados en el disco de acreción), que son responsables del espectro son dispersados a energías más altas a través del IC. Dado que tanto la temperatura del disco como la distribución de energía de los electrones relativistas son limitados, el espectro resultante del IC tiene un corte (cutoff) en altas energías. El resultado es un espectro tipo ley de potencias, que se extiende hasta unos cientos de keV, caracterizado por absorción fotoeléctrica y exceso de rayos $\mathrm{X}$ blandos (el cual se explicaría por una componente adicional de Comptonización, una reflección ionizada o una absorción ionizada). Este espectro es característico de los AGNs no colimados. Para el caso de AGNs colimados (como los blazares) el espectro es una ley de potencias simple, modificada por la absorción propia de nuestra Galaxia. Su índice espectral típico ronda $\alpha \sim 2$.

- Rayos- $\gamma$ : la radiación $\gamma$ comienza a partir de los $120 \mathrm{keV}$. En líneas generales, aquellos AGNs que no poseen componentes colimadas, como las galaxias Seyferts, no se los puede detectar por encima de los $100 \mathrm{MeV}$. En cambio, los blazares son capaces de generar fotones mucho más energéticos. Esto es debido a que 
las partículas que se encuentran en el jet (los cuales están involucrados en el proceso IC) tienen energías mucho más grandes que los electrones térmicos que se encuentran cerca del disco de acreción.

- VHE: la detección de emisión en esta banda de energía es dificultosa debido al escaso número de fotones provenientes del AGN por unidad de área y de tiempo. Esta detección se realiza a través de las cascadas de pares (que se generan cuando se atraviesa la atmósfera terrestre), las cuales producen fotones $\gamma$ con energías entre $\mathrm{GeV}$ y TeV. Por encima de los $100 \mathrm{GeV}$, la mayor parte de las fuentes que se detectan son AGNs y en particular, blazares dentro de los cuales, en su mayoría son del tipo BL Lac (HSP, High Peaked Syncrhotron blazars, Sect. 1.2.1).

\section{Modelo estándar}

Los AGNs presentan variaciones en sus flujos en todas las longitudes de onda y para distintas escalas temporales que pueden ir desde minutos, horas o incluso años. En particular, si un AGN varía rápidamente (en un tiempo t), el tamaño del núcleo (R) debe ser menor que el tiempo-luz de cruce del objeto (ct) a partir del principio de causalidad $(R<c t)$. Por otra parte, como ya se mencionó, los AGNs se caracterizan por tener altas luminosidades, las cuales están asociadas a grandes volúmenes de masa. Combinando ambos conceptos, se da lugar a objetos compactos muy densos, lo que sugiere que en el núcleo del AGN se encuentra un agujero negro super masivo (SMBH, del inglés Super Masive Black Hole), con una masa de $M_{\bullet} \geq 10^{8} M_{\odot}$. Este SMBH acreta el material del centro de la galaxia, el cual consiste básicamente en gas y polvo (disco de acreción). El mecanismo de acreción se da, por un lado, gracias al momento angular que tiene el SMBH (debido a que se lo considera como un agujero negro de Kerr, esto es, un agujero negro rotante), y por el otro, debido a que este material está circundante al agujero. Dado que el material que está siendo acretado es acelerado, éste emite radiación térmica, la cual se expande desde el óptico-NIR (IR cercano, del inglés Near Infrared) hasta el UV y rayos X.

Por otra parte, Antonucci (1993) propuso que alrededor de la región central de los AGNs, se encuentra un toro ópticamente grueso el cual tiene influencia en cómo se observan las distintas clases de galaxias Seyfert. Este anillo es el encargado del reprocesamiento de la radiación térmica del disco. Su emisión abarca las longitudes de onda milimétricas y sub-milimétricas. Con respecto a las líneas de emisión anchas y angostas que se observan en el óptico, pueden ser explicadas gracias a la presencia de nubes de gas (cercanas al SMBH). Aquellas que poseen altas densidades y que se mueven con velocidades altas, son las responsables de las líneas anchas, mientras que aquellas que están un poco más alejadas del SMBH con bajas densidades y velocidades, producen las líneas angostas. Las regiones donde se encuentran estas nubes se las denomina región de líneas anchas (BLR, del inglés Broad line region) y región de líneas angostas (NLR, del inglés Narrow line region). Finalmente, parte de la emisión no-térmica que se recibe de los AGNs es producida por flujos colimados de partículas relativistas, conocidos como jets (la primer evidencia observacional fue hecha por Curtis (1917) en la galaxia M87). Esta emisión no-térmica se debe al proceso sincrotrón, responsable de la radiación emitida en la banda de radio, así como también (en parte) de la emisión en óptico y rayos X; y del IC que explica la emisión en altas energías (incluyendo rayos- $\gamma$ ). 
De esta manera, a partir de la fenomenología observada en los AGNs en cada banda y de los distintos procesos radiativos que permiten explicar los diferentes tipos de emisión que se observan, surge lo que se conoce como el modelo estándar de acreción a un $S M B H$, como se muestra en la Fig. 1.1.



Figura 1.1. Componentes del modelo estándar (Brooks/Cole Thomson, AstronomyOnline.org, versión original en inglés).

Cada una de las distintas componentes de emisión que provienen de los AGNs se puede ver reflejada en el espectro electromagnético, a través de su distribución espectral de energía (SED, del inglés Spectral Energy Distribution), como se muestra en la Fig. 1.2. Como se puede ver, hay una diferencia entre la forma de la SED que presentan los AGNs colimados y los no-colimados (esto es, presencia o no de jets). En cuanto a los primeros, como los blazares, la forma de la SED es bimodal con un pico en la región entre radio y óptico, y el otro en la región de rayos X y rayos- $\gamma$. Su espectro se caracteriza por procesos no-térmicos y puede ajustarse por una ley de potencias. Con respecto a los AGNs no-colimados, como las galaxias Seyfert, es más complejo explicar la forma de la SED pues están presentes varias componentes emisoras. En las Seyfert, la SED presenta una estructura con varios picos máximos en el IR, óptico/UV y rayos $\mathrm{X}$.

\section{Modelo de unificación geométrica}

A partir del planteo del modelo estándar, es posible explicar la amplia fenomenología observada (descripta previamente) a través de un único escenario en donde las propiedades observacionales de los distintos tipos de AGNs puedan explicarse simplemente por la orientación que tenga la estructura presentada en el modelo estándar (ver Fig. 1.1). De esta manera, surge lo que se conoce como el modelo de unificación geométrica, Fig. 1.3. Este concepto fue propuesto primeramente por Blandford 


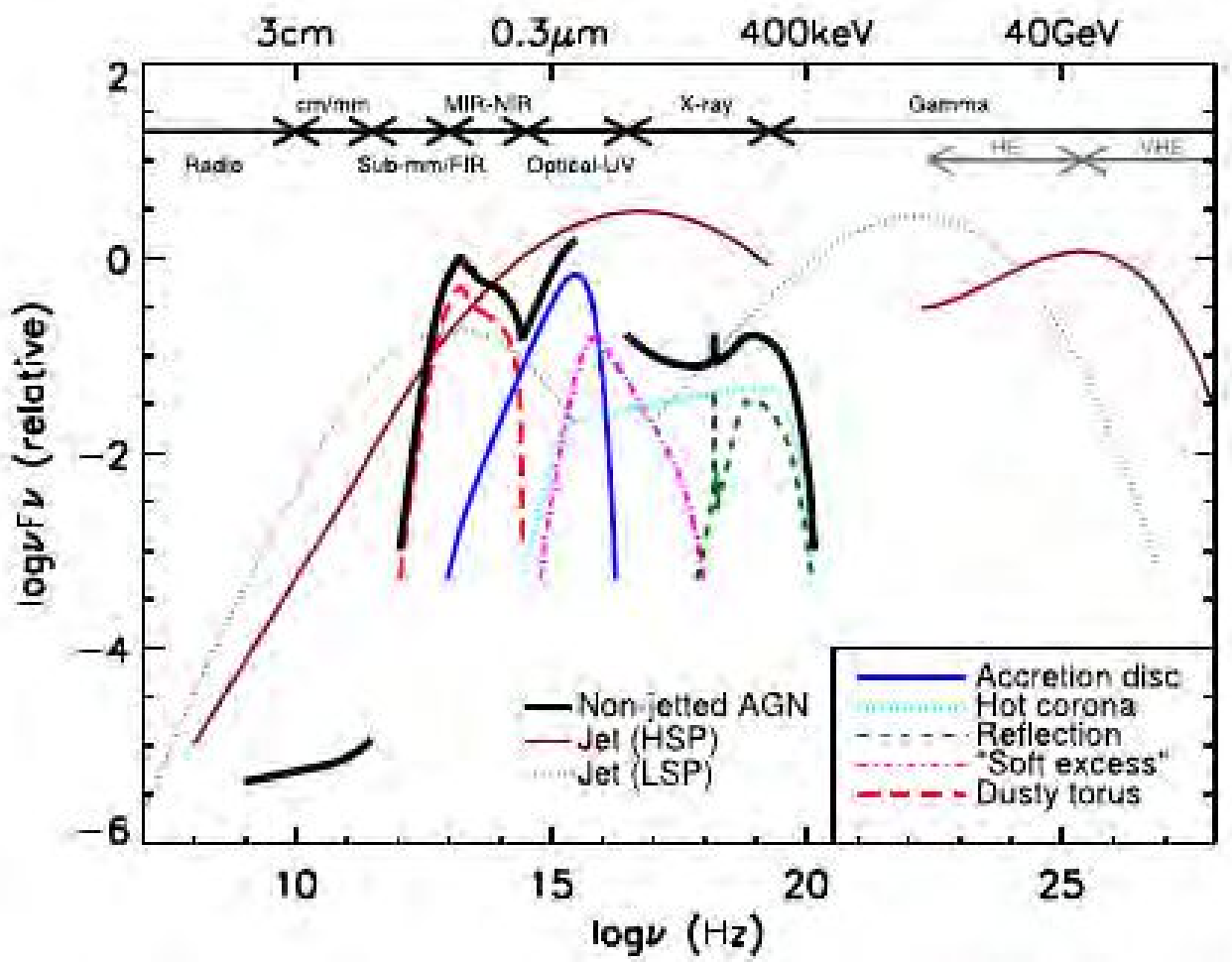

Figura 1.2. Representación esquemática de la distribución espectral de energía de un AGN (Padovani et al., 2017).

\& Rees (1978); luego Antonucci (1993), quien propone la existencia de dos tipos de AGNs (radio-intensos y radio-débiles); y Urry \& Padovani (1995), quienes presentan un esquema de unificación para los AGNs radio-intensos.

Debido a la gran asimetría del sistema, dependiendo del ángulo visual con el que se observe al núcleo activo, se tienen las distintas subclases de AGNs:

- Blazares: en esta clase de AGN, el jet relativista está apuntando al observador o se encuentra muy cerca de la línea de la visual. Son objetos altamente variables y emiten a lo largo de todo el espectro, desde radio hasta altas energías (VHE, Very high energies, por encima de $1 \mathrm{TeV}$ ). Se los divide, principalmente, en BL Lacs y FSRQ (Flat Spectrum Radio Quasars). Los blazares muestran variaciones que pueden alcanzar intensidades importantes en todo el espectro electromagnético, con escalas temporales muy cortas (minutos) hasta largas (años). En la Sec. 1.2 se encuentra una descripción más completa y detallada de este tipo de AGN.

- Galaxias Seyferts 1 y 2: son la clase más común de AGN que se observa en el Universo local. Dada su proximidad, se tienen espectros e imágenes de muy buena resolución, permitiendo el estudio en detalle de su geometría y procesos físicos asociados. Se dividen en dos clases de acuerdo a sus anchos relativos de líneas angostas (prohibidas) y líneas de Balmer. Las Seyfert 1 (Sy 1) presentan líneas anchas y angostas muy intensas, siendo las líneas de Balmer mucho más anchas que las líneas prohibidas. En las Seyfert 2 (Sy 2), tanto las líneas prohibidas como las de Balmer tienen el mismo ancho angosto. Por otra parte, en lo que respecta al continuo, las Sy 1 exhiben un continuo potente sin líneas de absorción, 


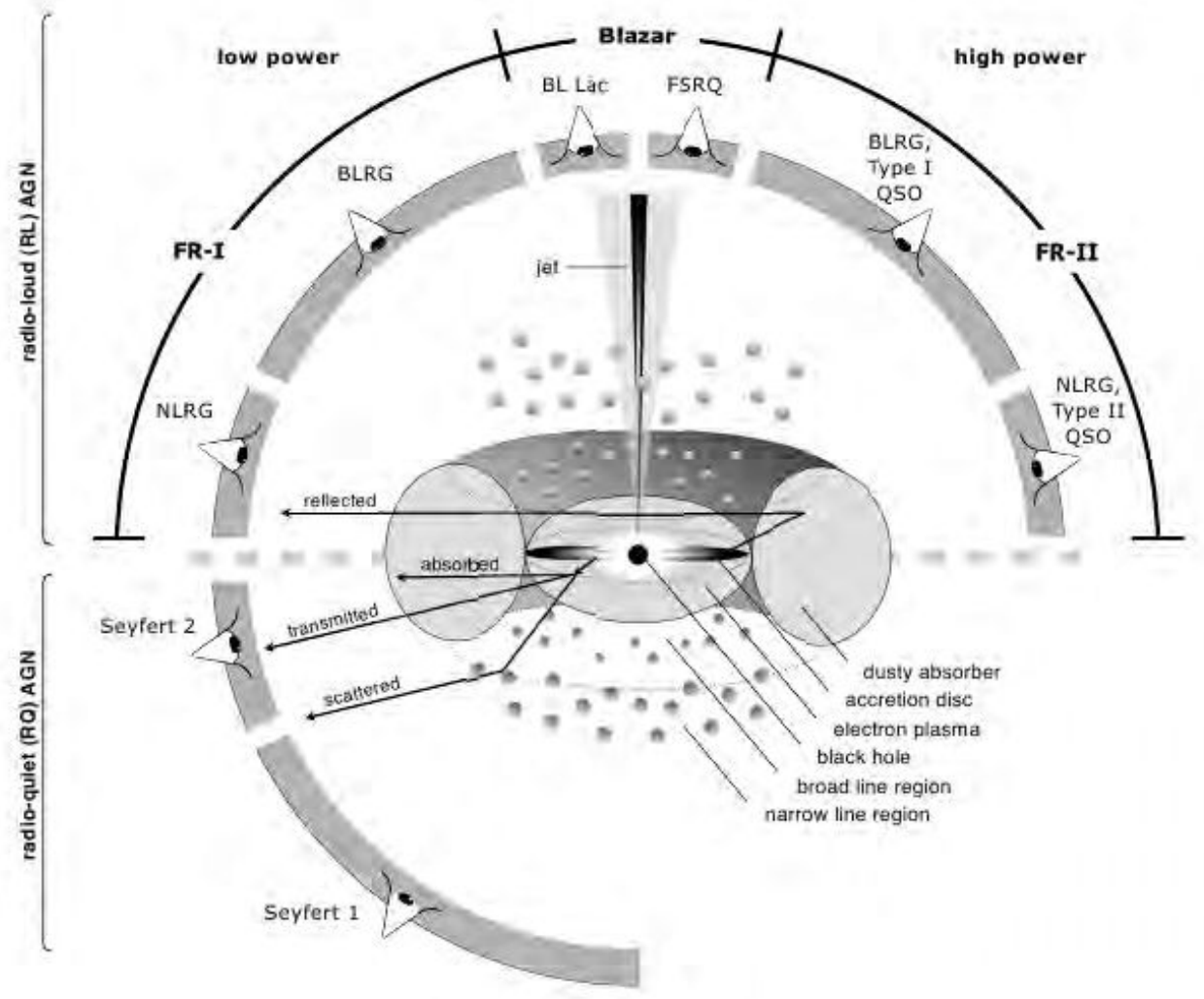

Figura 1.3. Esquema ilustrativo del modelo de unificación de AGNs. El tipo de objeto depende del ángulo visual, si el AGN produce o no emisión significativa asociada al jet y cuán potente es su motor central. (Marie-Luise Menzel, Active Galactic Nuclei, Beckmann \& Shrader.)

dominado por el objeto central y no por la contribución de las estrellas de la galaxia anfitriona. En las Sy 2 el continuo es más débil y pasa a ser dominado por la emisión estelar de la galaxia.

- Radio galaxias: cuando la región central de un quasar está oculta pero el objeto produce radio jets orientados a 90 grados respecto a un observador y luminosidades en radio muy grandes, se habla de radio galaxias. Éstas se subdividen en las galaxias Fanaroff-Riley I (FRI), con luminosidades bajas y una fuerte emisión hacia la zona más compacta y cercana a la región central; y las galaxias Fanaroff-Riley II (FRII), con luminosidades más altas y cuya emisión está más alejada del centro, hacia sus lóbulos (estructura dominante en las FRII) (Fanaroff \& Riley, 1974). El quiebre en dichas luminosidades se estableció en $L_{R}=10^{32} \mathrm{ergs}^{-1} \mathrm{~Hz}^{-1} \mathrm{sr}^{-1}$ a $175 \mathrm{MHz}$.

- Quasars: son los AGNs más luminosos y se los puede observar hasta muy altos redshifts (llegando a $z=7.085$, Mortlock et al., 2011). Son fuertes emisores en IR $\mathrm{y}$ en rayos $\mathrm{X}$, y se dividen de acuerdo a su emisión en radio: radio-loud y radio-quiet. Éstos últimos representan el $90 \%$ de la totalidad de quasars conocidos. Que sean radio-quiet implica que no presentan fuerte emisión en radio y 
además no producen las estructuras de jets. Por otra parte, los radio-loud, al ser fuertes emisores en radio, fueron los primeros en ser descubiertos. Sus galaxias anfitrionas tienden a ser más masivas que las de los radio-quiet (Dunlop et al., 2003). Ambos tipos de quasars comparten características tales como un flujo del continuo variable, una componente UV fuerte y líneas de emisión anchas.

\subsection{Blazares}

Los blazares son la clase que muestra el comportamiento más extremo de los AGNs, caracterizados por su emisión extendida desde radio hasta las bandas de los $\mathrm{GeV}$ y TeV, además de su alta polarización. Esta emisión es de carácter no-térmica y variable. Se dividen básicamente en dos clases: los BL Lac y los FSRQs (del inglés, Flat spectrum radio quasars). La principal diferencia entre ambos se encuentra en las propiedades ópticas de sus espectros: los FSRQs muestran líneas de emisión anchas y fuertes, mientras que los BL Lacs carecen de líneas de emisión, o éstas son muy débiles. Esta división histórica entre ambas clases se da a partir de considerar, como límite de separación, al ancho equivalente (en el marco en reposo) de la línea (EW, del inglés equivalent width), con $\mathrm{EW}=5 \AA$ (Stocke et al., 1991). Sin embargo, no hay evidencias sobre esta modalidad en los valores de EW. Scarpa \& Falomo (1997) plantearon que los blazares se pueden agrupar de la siguiente manera: por un lado, los FSRQs junto con los BL Lacs seleccionados en radio, dado que tienen propiedades en las líneas de emisión muy similares (aunque estos BL Lacs poseen un continuo más fuerte), y por el otro, los BL Lacs seleccionados en rayos X, quienes tienen líneas de emisión muy débiles. Se entiende por BL Lacs seleccionados en radio (RBL, del inglés Radio-selected Blazars) a aquellos que han sido identificados con emisión en radio muy fuerte y polarizada. Los $B L$ Lacs seleccionados en rayos $X$ (XBL, del inglés $X$-ray-selected Blazars) son fuentes seleccionadas debido a que su flujo en la banda de rayos $\mathrm{X}$ es alto en comparación a su emisión en el óptico.

Otras diferencias que pueden encontrarse entre los FSRQs y los BL Lacs (Giommi et al., 2011) son las siguientes: (i) en cuanto a su potencia extendida en radio, la mayoría de los BL Lacs tienen tanto su potencia como su morfología extendidas similares a las que se encuentran en las galaxias FR I, mientras que los FSRQs son del tipo FR II (Urry \& Padovani 1995); (ii) presentan distribuciones en sus corrimientos al rojo (z, redshift) distintas, dado que los BL Lacs se mantienen por debajo de $z<0.6$ y los FSRQs poseen valores desde $z=1-2$ hasta $z=5.5$ (aunque Giommi et al. 2011 argumentan que esta distinción está relacionada fuertemente con efectos de selección, debido a que muchos BL Lacs no poseen sus redshifts medidos por carecer de líneas de emisión en sus espectros ópticos); (iii) respecto a la distribución de la energía del pico sincrotrón $\left(\nu_{\text {peak }}^{S}\right)$, para el caso de los FSRQs ésta posee un pico a bajas energías $\left(\sim 10^{13.1 \pm 0.1} \mathrm{~Hz}\right)$ y no llegan a alcanzar valores muy altos, mientras que para los BL Lacs la distribución tiene su pico a valores más altos, llegando a $\nu_{\text {peak }}^{S}>10^{18} \mathrm{~Hz}$; etc.

Más allá de sus diferencias en la clasificación y de las ambigüedades que se presentan en algunos casos, se sigue adoptando esta división entre FSRQs y BL Lacs. Sin embargo, aún existen blazares que no han podido clasificarse, los BZU (del inglés, blazar of uncertain type). Éstos presentan actividad tipo blazar pero con características peculiares, tales como presencia/ausencia ocasional de líneas de emisión anchas; 
objetos en transición entre radio galaxias y BL Lacs; etc. (Massaro et al. 2009), lo que dificulta su clasificación.

Como se mencionó anteriormente, dentro del esquema de unificación, los blazares son aquellos AGNs cuyo jet está en dirección al observador, o muy cercano a la línea de la visual. La emisión de estos jets puede modelarse a partir de dos parámetros esenciales: el factor de Lorentz $(\Gamma)$ el cual describe la velocidad que se tiene en el jet, y el ángulo de visión $(\theta)$, que se lo define como el ángulo entre el jet y la línea de la visual. Ambas cantidades pueden calcularse si se conoce el factor boosting de Doppler ( $\delta$, expresión que se utiliza para medir cuán fuertes o débiles son los efectos relativistas en estas fuentes y se basa tanto en la velocidad que tiene el jet como el ángulo con la visual del mismo) y la velocidad aparente $\left(\beta_{a p p}=v / c\right.$, con $c$ la velocidad de la luz). Dado que el jet está apuntando al observador, se puede evidenciar lo que se conoce como movimientos superlumínicos. Sólo por efectos geométricos, estos movimientos dan lugar a velocidades superlumínicas, es decir, mayores a la velocidad de la luz. Además, todas las cantidades medidas se verán afectadas. Debido al movimiento relativista del material del jet con una velocidad $v_{j e t}=\beta_{\Gamma} c$ con $\beta_{\Gamma} \sim 1$, el flujo $v F_{v}$ de la emisión del continuo del jet es amplificado a la cuarta potencia del factor Doppler relativista $\delta$. La amplificación relativista (boosting) a partir de factores de $\delta^{4}$ explica por qué los blazares se encuentran entre los objetos más brillantes en el Universo. Por otro lado, las frecuencias medidas en el marco del observador son más azules que las frecuencias asociadas al marco del objeto $\left(\nu^{\prime}=\delta \nu\right)$; y el tiempo medido en el marco del observador se acorta respecto al objeto $\left(t^{\prime}=\delta^{-1} t\right)$.

\subsubsection{Las SEDs en los blazares}

Las distribuciones espectrales de energía (SEDs) resultan ser una herramienta muy útil para poder distinguir y clasificar a los distintos tipos de blazares. La característica principal de sus SEDs es la estructura bimodal (de doble pico) que presentan. El primer pico se encuentra en la región de radio/UV, y el otro pico en el rango entre rayos $\mathrm{X}$ y rayos- $\gamma$. El continuo que caracteriza a estas SEDs es no-térmico. Respecto a la emisión correspondiente a las bajas frecuencias, se relaciona con el proceso sincrotrón de los electrones relativistas que están en el jet. A altas frecuencias, se hace dificultoso explicar y modelar los procesos radiativos de la parte de rayos- $\gamma$ (Fig. 1.4), con lo que se proponen dos tipos de modelos: leptónicos y hadrónicos.

- Leptónicos: las partículas involucradas son electrones y positrones. Para la parte del espectro que va desde radio hasta el UV se puede modelar usando el proceso de radiación sincrotrón de electrones que tienen energías altas dentro del jet relativista (Blandford \& Königl, 1979). Para la región de los MeV y GeV, se puede explicar a través de la dispersión Compton inversa de electrones y positrones en el jet con los fotones sincrotrón del mismo jet (SSC, del inglés Synchrotron Self-Compton) o con un campo de fotones externo (EIC, de sus siglas en inglés External Inverse Compton). Éste último puede ser: la emisión directa del disco de acreción (ECD, del inglés External Compton of disk emission), la emisión de la BLR (ECL, del inglés External Compton of line emission), emisión del toro de polvo (ECT, de sus siglas en inglés External Comptonization of torus photons). En la Fig. 1.5 se puede ver el esquema gráfico del modelo leptónico junto con 


\section{Introducción}

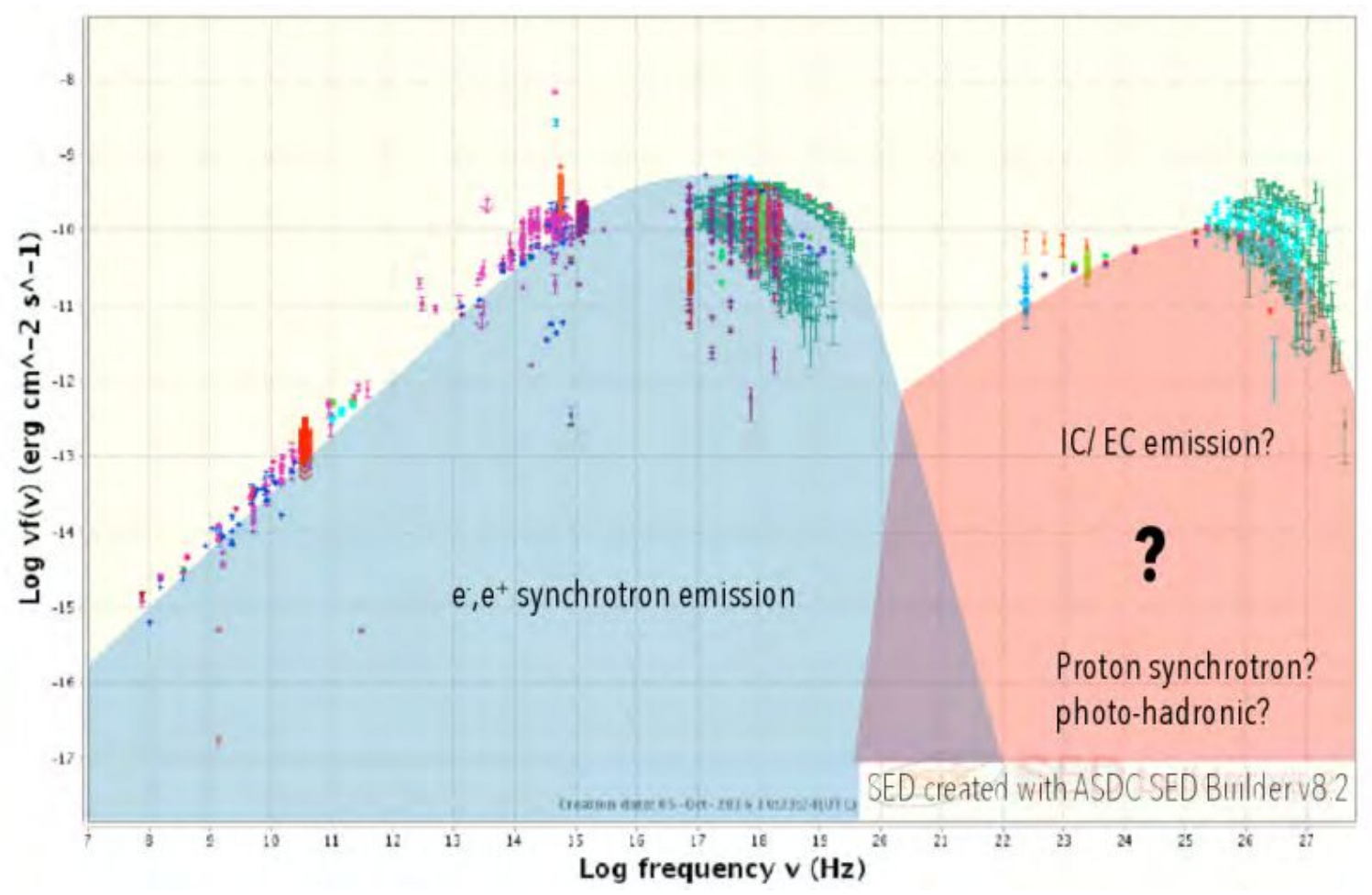

Figura 1.4. Mecanismos de radiación presentes en la SED del blazar Mrk 421. Se puede observar que cada parte de su estructura bimodal es descripta por un proceso distinto (Kreikenbohm et al. 2017).

la distribución espectral para un blazar, en donde se ven reflejadas las distintas componentes.

- Hadrónicos: en estos modelos, la emisión está asociada con protones, ya sea a través del proceso sincrotrón de estos protones como así también de la interacción electromagnética que produce partículas secundarias, tales como pares electrónpositrón, fotones y neutrinos. Esta interacción se da en forma de cascada de pares dentro de la estructura del jet.

A principios de la década del 90, con el advenimiento de la nueva generación de satélites orbitales de rayos X, se comenzó a clasificar a los blazares de acuerdo a si eran detectados en radio o en rayos X. Así, surgen los RBL y los XBL, mencionados previamente en este capítulo. Años más tarde, se propuso una división para los objetos BL Lac, la cual depende de las características presentes en las SEDs. Los primeros en proponer dicha división fueron Padovani \& Giommi (1995), los cuales utilizaron el pico en la energía asociada a la emisión sincrotrón. Este pico refleja la energía máxima a la cual las partículas pueden acelerarse en el jet. Basados en esto, clasificaron a los BL Lacs entre objetos con pico sincrotrónico a bajas energías (LBL, del inglés Low Synchrotron Peaked BL Lacs) y a altas energías (HBL, del inglés High Synchrotron Peaked BL Lacs). Posteriormente, Abdo et al. (2010) extiende esta definición para todos los tipos de AGNs dominados por procesos no térmicos. Por un lado tenemos a los blazares LSP (Low synchrotron peaked blazars), en los cuales el pico cae en la región de bajas energías (en la banda del IR o IR lejano), con la frecuencia del pico 

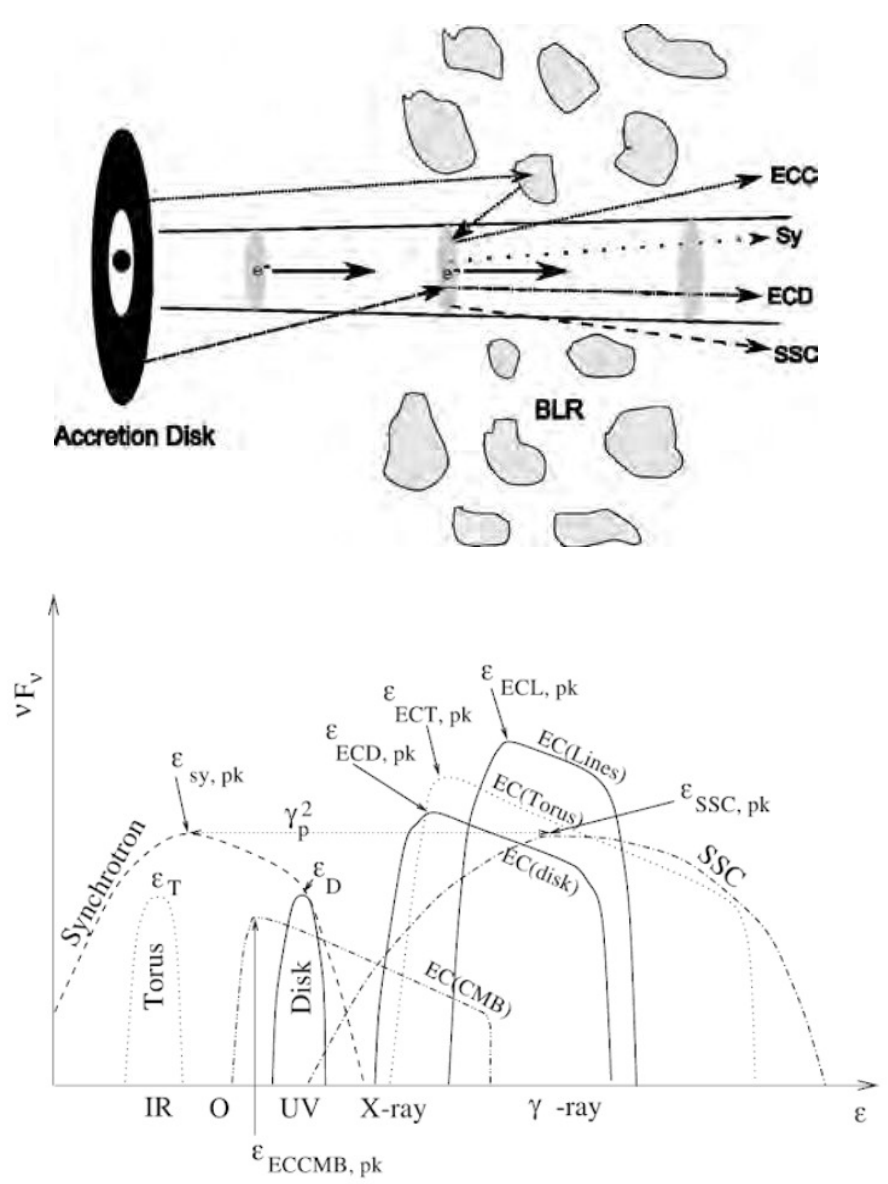

Figura 1.5. Esquema del modelo leptónico que se adopta para los blazares (arriba). Distribución espectral de un blazar en donde se muestran las distintas contribuciones de los procesos involucrados en el modelo leptónico (abajo, Relativistic Jets in Active Galactic Nuclei, Cap.8, Fig. 8.5).

$\nu_{\text {peak }}<10^{14} \mathrm{~Hz}$, y su emisión en rayos $\mathrm{X}$ es chata (siendo el índice espectral en la banda de rayos $\left.\mathrm{X} \alpha_{X} \sim 0.4-0.7\right)$ debido a la parte creciente de la componente de IC. La dispersión del IC, en estas bajas energías, ocurre dentro del régimen de Thompson. Por otro lado, se encuentran los objetos ISP (Intermediate synchrotron peaked blazars), donde el pico sincrotrónico cae a energías intermedias $\left(10^{14}<\nu_{\text {peak }}<10^{15} \mathrm{~Hz}\right)$. En este caso, la región de rayos $\mathrm{X}$ comprende tanto la cola de la emisión sincrotrón como la parte donde comienza a crecer la componente del IC. Finalmente, se tienen los HSP (High synchrotron peaked blazars), en los cuales las partículas emisoras son aceleradas a energías mucho más altas que en los LSPs, de modo que el pico sincrotrón alcanza la región del UV o incluso energías más altas $\left(\nu_{\text {peak }}>10^{15} \mathrm{~Hz}\right)$. En este escenario, la emisión sincrotrón domina el flujo observado en rayos X y la dispersión del IC ocurre en el régimen de Klein-Nishina. En la Fig. 1.6 se muestran las SEDs de estas 3 clases de BL Lacs. 


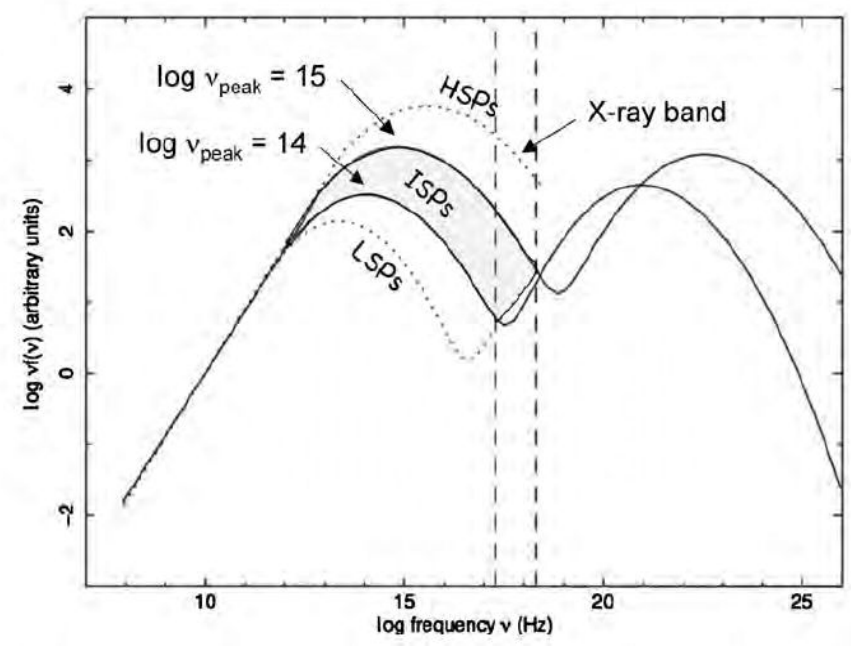

Figura 1.6. Tipos de blazares de acuerdo a la posición del pico sincrotrón en la SED. De abajo para arriba, se tienen: blazares LSP (Low synchrotron peaked blazars); ISP (Intermediate synchrotron peaked blazars), y HSP (High synchrotron peaked blazars) (Abdo et al. 2010).

\subsection{Variabilidad}

El fenómeno de variabilidad (variaciones temporales en el flujo observado) en los blazares se caracteriza por encontrarse en todas las longitudes de onda del espectro electromagnético (Falomo et al. 2014), y es una herramienta fundamental para entender los mecanismos de emisión de los AGNs y para la caracterización de los modelos físicos de estas fuentes, dado que provee información del tamaño de la región emisora.

Las escalas temporales involucradas pueden abarcar desde meses hasta años y la variabilidad asociada a esta escala se la conoce como variabilidad a largo plazo (LTV, Long term variability, Sillanpää et al., 1988; Kidger et al. 1992; Raitieri et al., 2001; Valtonen et al., 2006; Rani et al., 2009; Chatterjee et al., 2012). Su estudio sirve para poder estimar la estructura del jet relativista la cual, en algunos casos, puede presentar un patrón helicoidal global y una posible estratificación en la dirección perpendicular al jet axial (Marscher et al. 2008). A este tipo de variabilidad no se le puede asociar algún período y en determinadas ocasiones, puede describir mecanismos turbulentos provocados por cierta inestabilidad en el proceso de acreción (e.g. Ulrich et al. 1997). Como ejemplo, en la Fig.1.7 se muestra la variabilidad en las bandas del NIR y óptico para 3C279 (Sandrinelli et al. 2016).

Por otro lado, se observan variaciones en el flujo en escalas de días a semanas, lo que se denomina como variabilidad a escala corta (STV, Short term variability), o también variabilidad inter-noche (Romero et al., 2000; Joshi et al., 2007; Bonning et al., 2009; Marchesini et al., 2016). Finalmente se pueden encontrar escalas temporales muy cortas, que van desde minutos hasta horas, las cuales pueden aportar mayor información sobre tamaños comparables con el radio gravitacional del agujero negro (Urry \& Padovani 1991). Este tipo de variabilidad se la conoce como variabilidad intra-noche o más comúnmente llamada, microvariabilidad (Miller \& Noble 1996; Romero et al. 1999, 2002; Andruchow et al. 2003, 2008; Gupta et al. 2008). Un ejemplo de esto puede verse en la Fig.1.8, donde Romero et al. (2000) reportan variabilidades extremas 


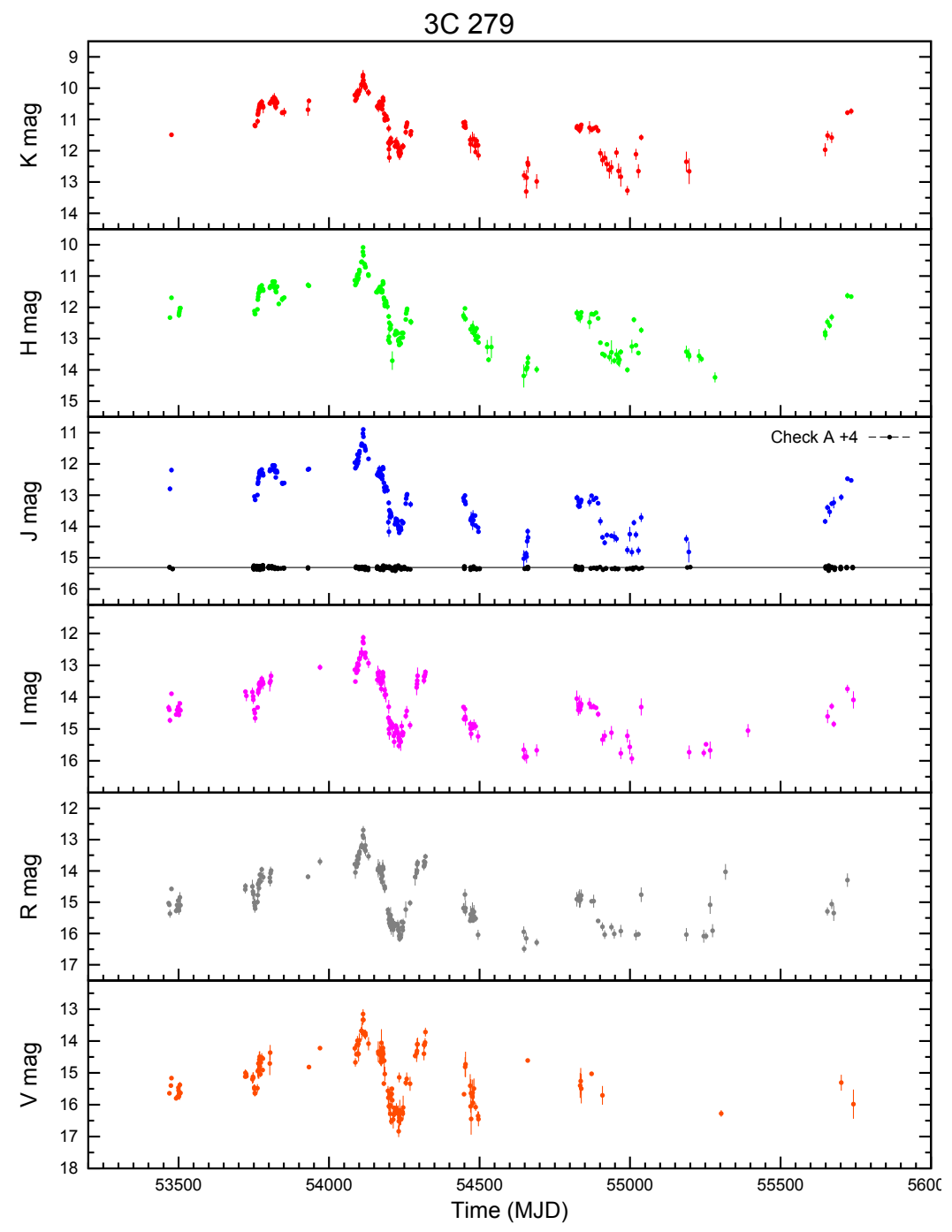

Figura 1.7. Curvas de luz de 3C279 en las bandas del NIR y óptico (Sandrinelli et al. 2016).

tanto intra-noche como inter-noche para el BL Lac AO $0235+164$, observado por 6 noches consecutivas en Noviembre 1999. En esta Figura se muestra la curva de variabilidad inter-noche, compuesta a su vez por las curvas que muestran microvariabilidad correspondientes a cada una de las noches.

\subsubsection{Microvariabilidad: historia, importancia y modelos}

El origen del concepto de microvariabilidad data de finales de la década del 80 y comienzos de los 90, donde comenzaron a reportarse los primeros estudios sobre estas variaciones en el flujo óptico en escalas temporales muy cortas, que van desde minutos hasta horas (Miller et al. 1989, Carini et al. 1990, 1991, 1992; Noble et al. 1997). En estos trabajos se comenzó a usar la nomenclatura de microvariabilidad. Este 


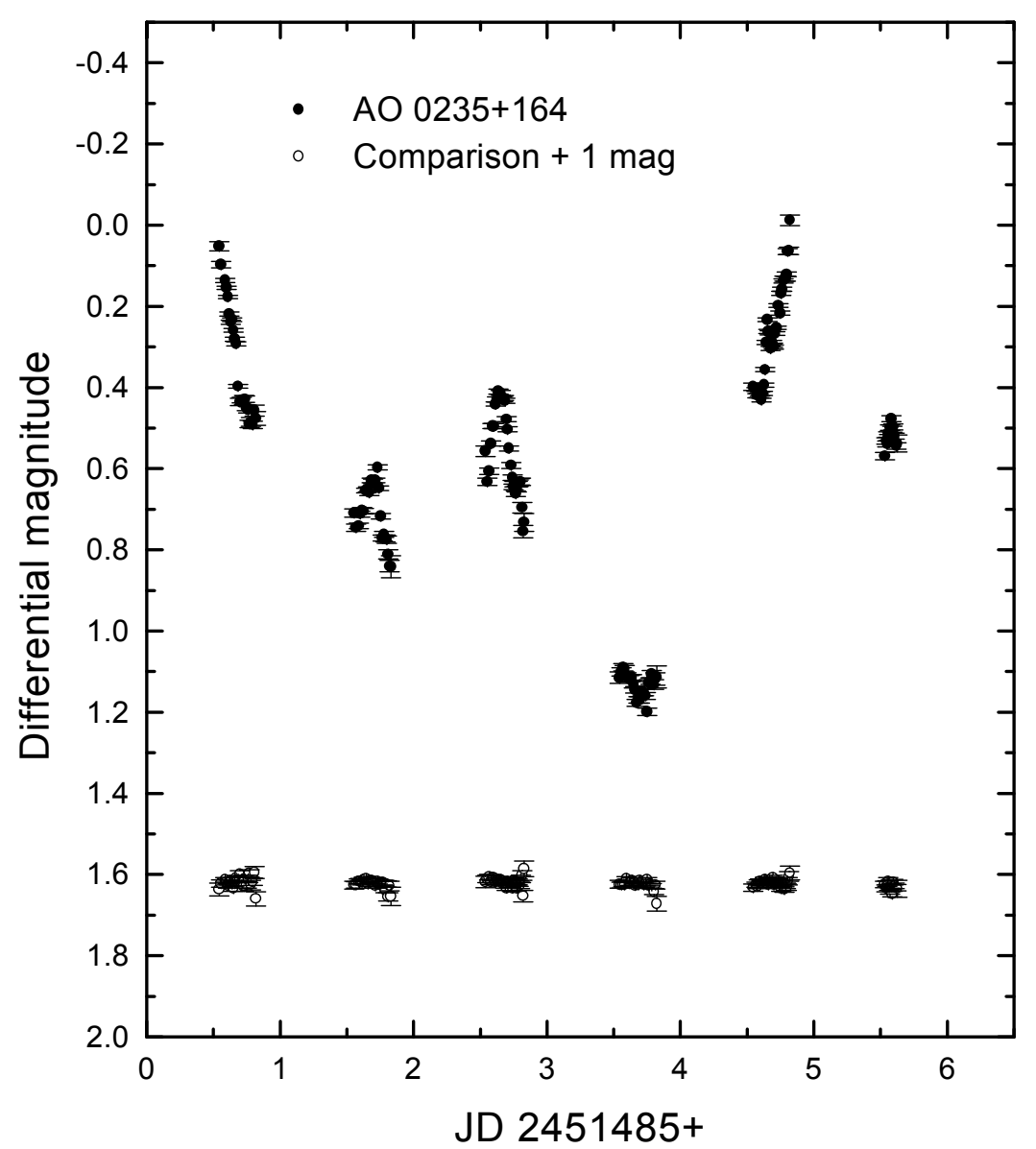

Figura 1.8. Curva de luz diferencial de AO 0235-365 (con su curva de control) en el óptico, en el filtro V (Romero et al. 2000).

tipo de análisis fue posible por el desarrollo y utilización de los detectores CCD (del inglés, Charge-coupled device, dispositivo de carga acoplada) usados para la fotometría. Aunque se conoce que los primeros estudios se sitúan en la década del 70 (Racine 1970).

Para comienzos del presente siglo, son numerosos los trabajos acerca de este tipo de variabilidad en blazares (Romero et al. 1999, 2002; Cellone et al. 2000; Andruchow et al. 2003, 2008; Sagar et al. 2004; Gupta et al. 2008; Joshi et al. 2011; Marchesini et al. 2016). Y como se mencionó anteriormente, el estudio y análisis de la microvariabilidad es de suma importancia para poder inferir y conocer más en detalle cómo es la física en la región emisora presente cerca al núcleo, ya que (a excepción de las estructuras en radio de los jets y lóbulos) las componentes físicas del núcleo activo no pueden resolverse espacialmente.

Este tipo de variabilidad a escala tan corta está asociada con los procesos que se dan lugar en el jet relativista (Wagner \& Witzel, 1995). Existen diversos modelos que tratan de explicar el origen de estos procesos que dan lugar a la microvariabilidad. Básicamente, se los puede dividir en dos grandes grupos: los modelos intrínsecos (que a su vez, pueden dividirse en físicos, asociados a la emisión de la fuente y geométricos)y los modelos extrínsecos. El modelo más utilizado para este último grupo es el modelo de microlensing gravitacional, el cual fue propuesto por Gopal-Krishna \& Sumbramanian 
(1991). El modelo intenta explicar que las variaciones ultra-rápidas (detectadas en algunos blazares) pueden deberse a la presencia, en la línea de la visual, de estrellas que actúa como microlentes gravitacionales. Como resultado, se tiene movimientos superlumínicos que causan estas variaciones, inlcuso si no se tienen variaciones intrínsecas al objeto. A continuación, explicamos brevemente los distintos modelos intrínsecos:

- Modelos físicos:

1. Shock-in-jet: este modelo fue propuesto por Blandford \& Königl (1979). Básicamente consiste en la formación de un frente de choque dentro del jet relativista debido a la presencia de nubes densas (las cuales son aceleradas) o debido a un campo de velocidades inestable dentro del jet. Esto hace que se tengan variabilidades temporales y espaciales dado el movimiento de estas inhomogeneidades dentro de las partes más externas del jet (Marscher 2016).

2. Hot-spot: este modelo asocia las variaciones observadas con inestabilidades en el disco de acreción. Fue desarrollado por Mangalam \& Wiita (1993) y pudieron explicar las microvariaciones de baja amplitud en las bandas del UV y óptico. Las inestabilidades en el disco pueden ser de carácter magnético o gravitacional, entre otros.

3. Inestabilidades de Kelvin-Helmholtz: propuesto por Romero (1995). Consiste en la presencia de inestabilidades surgidas de la interfase entre dos fluidos con velocidades distintas dentro del jet. Estas inestabilidades son capaces de formar grumos dentro del jet, que originarían las variaciones observadas (escenario que se relaciona con los modelos geométricos como el del swinging jets).

4. Jet desacelerado: este modelo fue propuesto por Böttcher \& Principe (2009), para poder explicar el decaimiento exponencial en las curvas de luz de 3C279, en los filtros V, R e I, en un lapso de dos semanas. Ellos desarrollaron un modelo en el cual se contempla la desaceleración de un plasmoide en el jet de esta fuente. A partir de tener en cuenta la dinámica de partículas junto con procesos de radiación, al desacelerarse este plasmoide, este evento se refleja en el decaimiento del flujo en las curvas de luz.

- Modelos geométricos:

1. Swinging jets: propuesto por Gopal-Krishna \& Wiita (1992). Este modelo contempla el patrón de grumos que se mueven relativísticamente a lo largo del jet. Este patrón se desvía de la línea de la visual en pequeños ángulos, haciendo que el factor Doppler sea variable, al igual que la emisión de este grumo, lo que explicaría las variaciones rápidas detectadas.

2. Movimiento helicoidal del jet: Jiménez-Andrade et al. (2017) detectaron un movimiento helicoidal en el jet óptico del blazar PKS 0521-365. Dicho patrón lo asocian a campos magnéticos helicoidales y a la precesión del jet relativista. Además de poder explicar lo que se observa a escalas de pc, también sería una alternativa (aunque poco usada) para explicar la variabilidad que se detecta. 


\subsubsection{Variabilidad en altas energías}

\section{Rayos X}

La emisión en rayos X se caracteriza por variar muy rápidamente y por no mostrar periodicidad, que proviene del jet. En cuanto al origen de la variabilidad en rayos X, se la suele asociar con eventos del tipo estallidos o flares (Wehrle et al., 1998), donde éstos últimos estarían relacionados con choques en el jet (Marscher, 1985). Otro modelo plausible para explicar la variabilidad se apoya en la existencia de fluctuaciones en el flujo de acreción proveniente del disco (Fig. 1.9). Estas fluctuaciones serían las que modulan las emisiones en X sobre el disco o alrededor del SMBH. De esta manera, el flujo de acreción que fluctúa, se propagaría hacia el jet, produciendo la emisión en rayos X variable (Marscher, 1985).

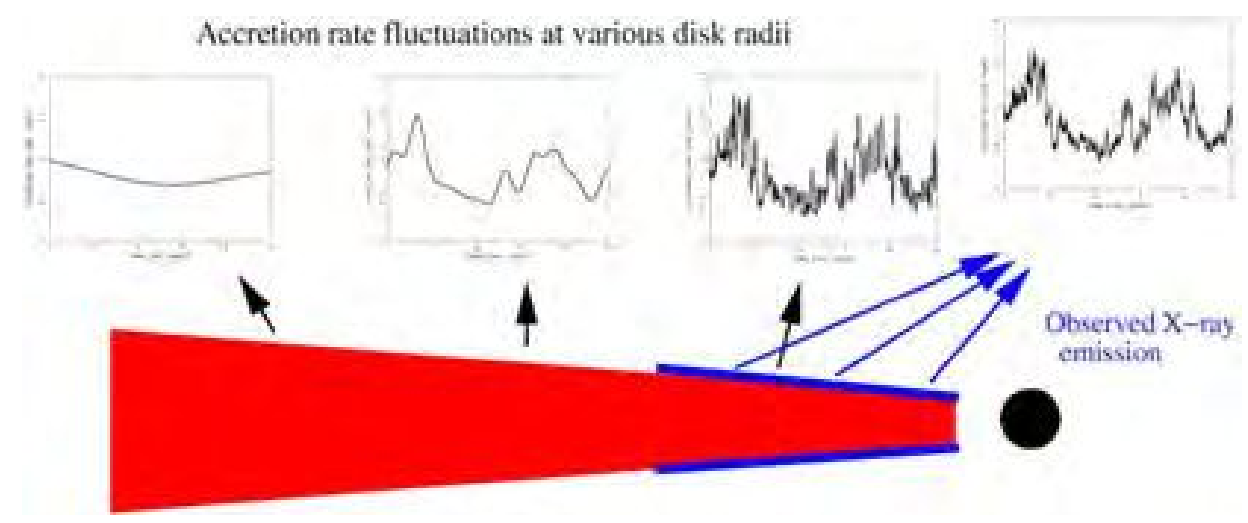

Figura 1.9. Modelo para la variabilidad en rayos X (Mc.Hardy 2008).

Por otra parte, la variabilidad en rayos X está relacionada con la del óptico. Un ejemplo de esto se puede ver en la Fig. 1.10, en las curvas de luz presentadas para 3C279. Chatterjee et al. (2008) encontraron que las variaciones presentes en esta fuente en la banda de rayos $\mathrm{X}$ correlacionan con aquellas en el óptico si los rayos $\mathrm{X}$ son producidos en el jet. Además, los flares en rayos $\mathrm{X}$ estarían asociados con grumos superlumínicos $\mathrm{y}$, del análisis en particular de las curvas de luz en rayos X y óptico, encontraron que los flares en rayos X son producidos por la dispersión SSC, mientras que los flares en el óptico por el proceso sincrotrón. Finalmente, aquellos flares simultáneos en rayos $\mathrm{X}$ y óptico con energías radiativas similares son originados cerca de la base del jet relativista.

\section{Rayos- $\gamma$}

El estudio de la variabilidad en la banda de rayos- $\gamma$ es importante dado que permite indagar sobre los procesos físicos en la parte más interna del jet, la cual está próxima al SMBH. En general, las curvas de luz de los blazares a estas energías están dominadas por flares muy fuertes, cuyo origen puede deberse a la inyección de partículas nuevas en el jet o shocks internos intensos. En la Fig. 1.11 se muestra que también existe la correlación entre las emisiones en rayos- $\gamma$ y la banda del óptico, a través de las curvas de luz del blazar PKS 0537-441. Larsson et al. (2012) estudiaron la variabilidad en rayos- $\gamma$ de una muestra de blazares con datos del satélite Fermi-LAT. Encontraron que más 


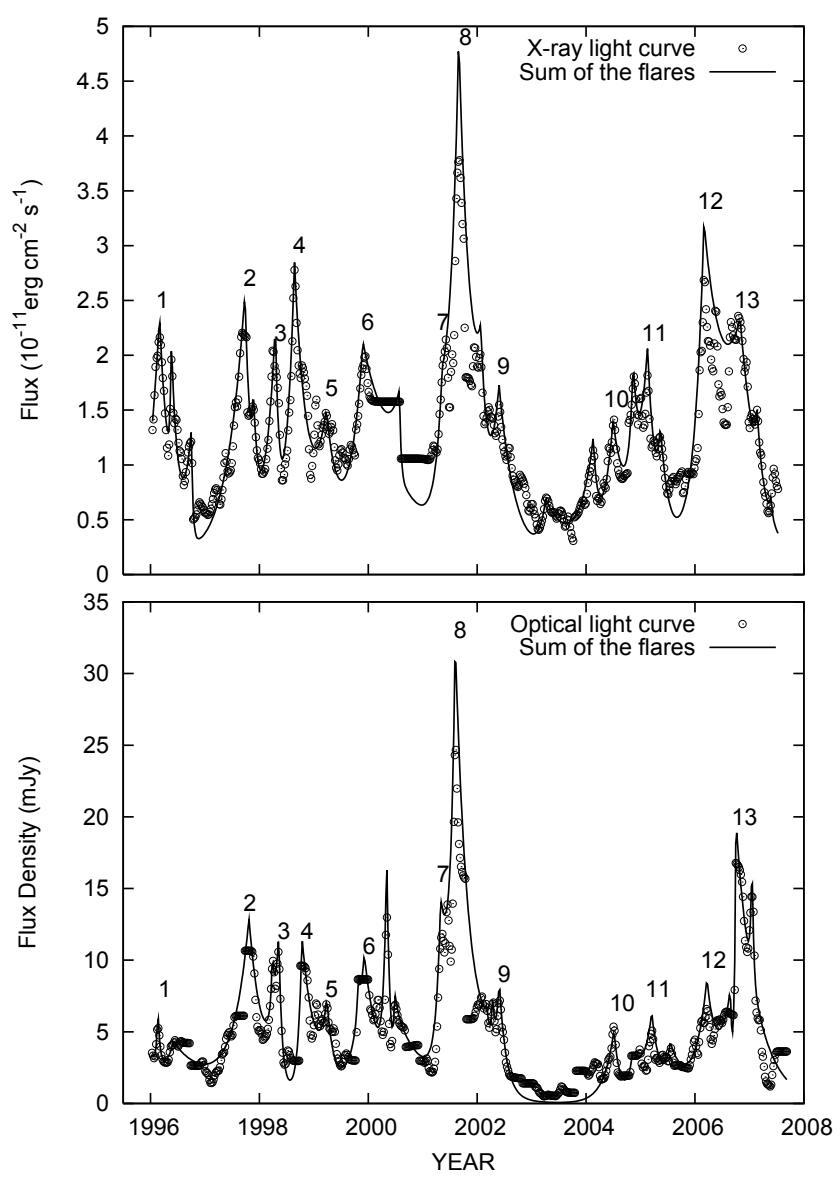

Figura 1.10. Curvas de luz en las bandas de rayos X y óptico para $3 \mathrm{C} 279$, desde 1996 hasta el 2007, en donde se puede ver una correlación entre los flares en rayos $\mathrm{X}$ y óptico (Chatterjee et al. 2008).

del $60 \%$ de las fuentes resultan variables; que del total del tiempo de observación, en el $25 \%$ del mismo la fuente está en su estado high; y que el perfil de los flares detectados suele ser simétrico. Por otra parte, concluyen que el estudio de la variabilidad de la emisión en rayos- $\gamma$ está limitado por el lapso de las observaciones disponibles. Además las propiedades de variabilidad a corto plazo podrían diferir de aquellas asociadas a las de largo plazo, debido a la naturaleza estocástica de la variabilidad.

Chatterjee et al. (2012) también estudiaron la correlación entre las emisiones de rayos- $\gamma$ y óptico para PKS 0208-512. En la Fig. 1.12, se muestran conjuntamente las curvas de luz simultáneas de las emisiones en rayos- $\gamma$ (entre $0.1-300 \mathrm{GeV}$ ), en rayos $\mathrm{X}$ (entre $0.3-10 \mathrm{keV}$ ) y las bandas ópticas $B, R$ y $J$. De la comparación en estas bandas, los autores encontraron diversas correlaciones. Por un lado, de la Fig. 1.12 se muestran marcados los intervalos 1 y 3 , en donde se detectó una fuerte correlación entre rayos- $\gamma$ y la banda R, sin desfasaje de tiempo. Esto lo pueden explicar a partir del modelo leptónico, en donde las emisiones en rayos- $\gamma$ y óptico/IR (OIR) son generadas por los mismos electrones relativistas en el jet a través de los procesos IC y sincrotrón, respectivamente. Los fotones semillas que son dispersados pueden provenir del proceso SSC o del EC. Por otra parte, en el intervalo 2 no detectaron incremento alguno en el flujo de 


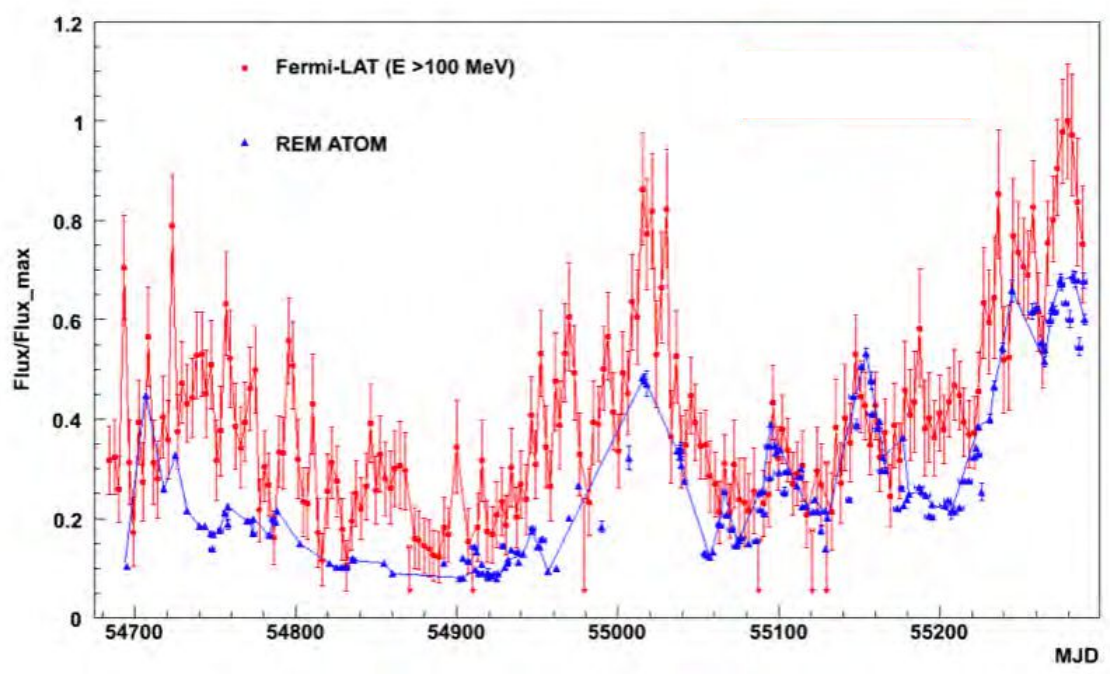

Figura 1.11. Curvas de luz en las bandas de rayos- $\gamma$ y óptico para PKS 0537-441, en donde se puede ver una correlación entre los flares de ambas bandas (Larsson et al., 2012).

rayos- $\gamma$. Para poder explicar este comportamiento, los autores plantearon dos posibles escenarios: en el primero, el estallido detectado en óptico/IR y no en rayos- $\gamma$ podría ser debido sólo a un cambio en el campo magnético de la región emisora, afectando la emisión sincrotrón en el óptico, pero dejando invariante la emisión en rayos- $\gamma$ (generada por IC). En el segundo escenario, la gran diferencia entre los cocientes GeV/OIR entre los intervalos 1 y 3 versus el 2 sería por la posición en donde se genera el estallido. Si éste se forma cerca del $\mathrm{BH}$, posee un factor de Lorentz $(\Gamma)$ bajo, pero el campo magnético y la densidad de partículas es mayor debido a lo compacto de la región. En este contexto, si el estallido del intervalo 2 se genera cerca del BH pero los estallidos de los intervalos 1 y 3 se producen más lejos, la emisión óptico/IR es mucho más dominante que en la banda de rayos- $\gamma$ durante el intervalo 2 .

De todo lo planteado, vemos que los estudios multifrecuencia son de gran relevancia para identificar los procesos físicos involucrados y tratar de inferir cómo se conectan unos con otros. 


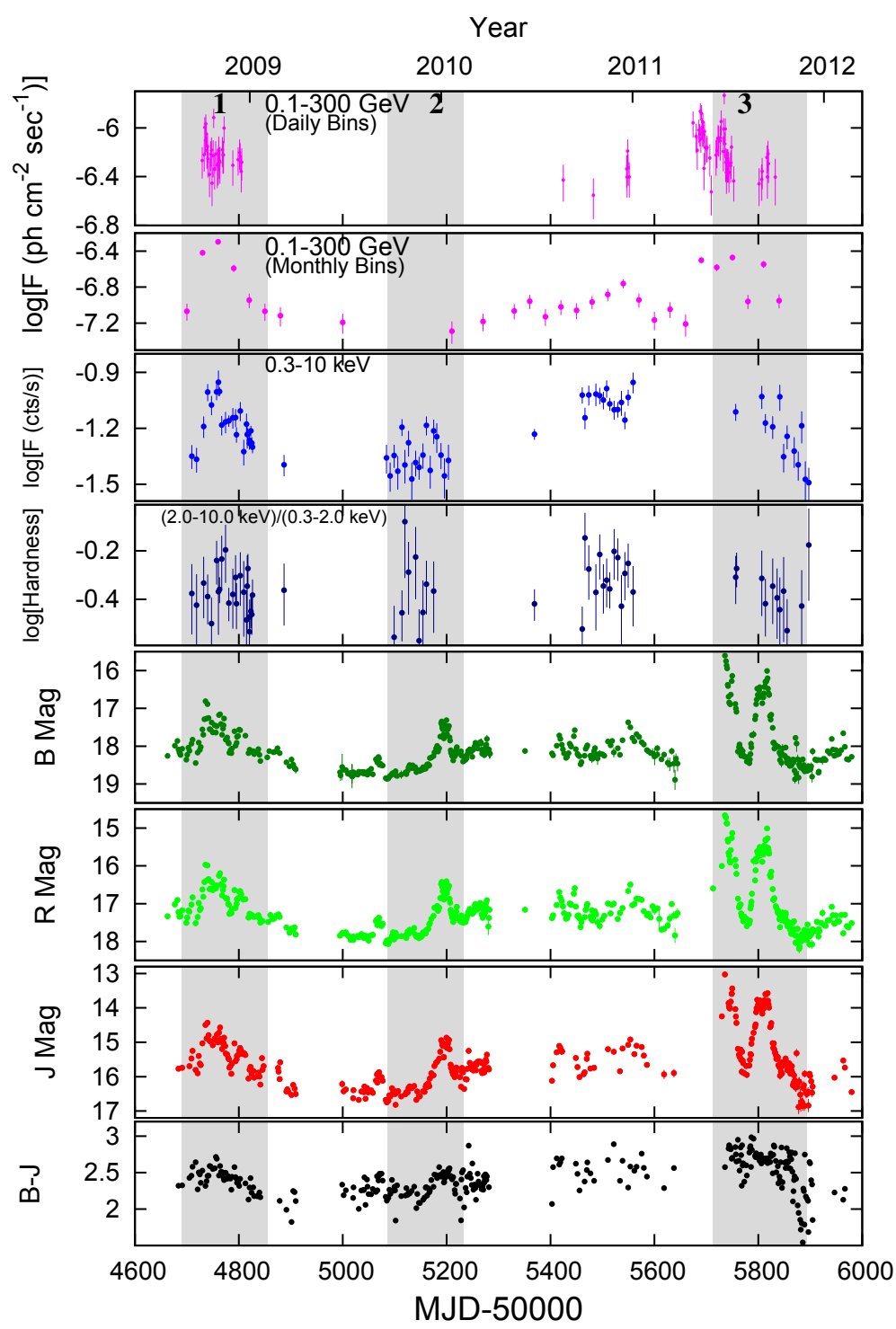

Figura 1.12. Análisis multifrecuencia del blazar PKS 0208-512 (Chatterjee et al. 2012). 


\subsection{Esquema de la presente Tesis Doctoral}

En esta Tesis Doctoral nos focalizamos en el estudio de la variabilidad y en particular de la microvariabilidad, de blazares, en el óptico. Para ello, recurrimos al uso de herramientas estadísticas con el objetivo de analizar las curvas de luz diferenciales obtenidas para los objetos de la muestra. Estas herramientas deben ser lo más confiables posible, con lo cual estudiamos su robustez y estabilidad a partir de una serie de simulaciones de curvas de luz sintéticas. En cuanto al estudio del comportamiento de las fuentes, se observó y analizó el flujo óptico de una muestra de 19 blazares, complementadas con su comportamiento a mayores energías de manera de contar con un escenario astrofísico más realista, capaz de explicar las observaciones analizadas. Se presenta a continuación el esquema general de la Tesis:

En el Capítulo 2 se muestran los resultados del estudio de las herramientas estadísticas implementadas en este trabajo, a partir de simulaciones que han sido llevadas a cabo bajo diferentes situaciones observacionales de AGNs tanto variables como no variables, afectadas por diversas condiciones atmosféricas e instrumentales.

En el Capítulo 3 se presentan los resultados de las observaciones realizadas sobre una muestra de blazares seleccionada del Roma BZCAT. Dicha muestra está constituida por 19 blazares divididos en BL Lacs, FSRQs y BZUs. Estas fuentes fueron analizadas tanto en la banda óptica, a través del estudio de las curvas de luz bajo las herramientas estadísticas mencionadas, como también en rayos X. Los datos en el óptico fueron obtenidos con el telescopio 2.15m "Jorge Sahade", en CASLEO. Las observaciones en rayos $\mathrm{X}$ fueron obtenidas de la base de datos del satélite Chandra. Como complemento, se realizó un análisis multifrecuencia para 3FGL J1958.2-3011, en el que se incorporaron datos en la banda de rayos- $\gamma$, obtenidos de la base de datos del satélite Fermi. Además, se puede encontrar la discusión de los resultados obtenidos.

En el Capítulo 4, se presentan las conclusiones finales. 


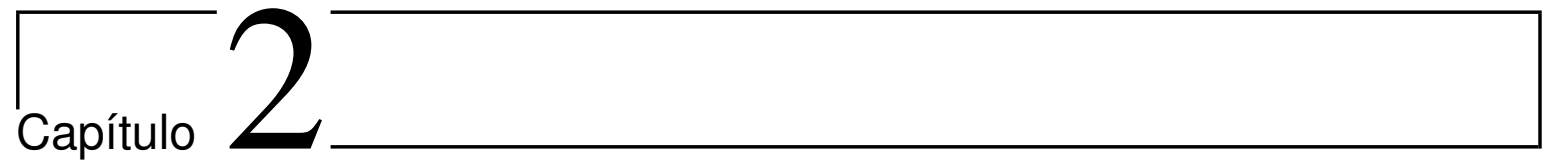

\section{Herramientas estadísticas}

\subsection{Introducción y presentación de la problemática}

Como vimos en el capítulo anterior, los estudios acerca de microvariabilidad brindan información importante sobre las características físicas de la región de emisión y también sobre los parámetros involucrados en los diferentes modelos de la emisión electromagnética. Dada la relevancia que tiene poder detectar estas variaciones, es importante conocer cuáles son las herramientas más confiables, con las cuales podamos analizar las curvas de luz obtenidas para las fuentes bajo estudio, de manera de poder detectar tales variaciones de la forma más robusta. Las herramientas naturales para detectar variaciones son los tests estadísticos. Esta detección no es sencilla, ya que se pueden llegar a obtener resultados falsos a partir de la detección de variaciones espúreas. Esto puede deberse a errores sistemáticos introducidos por contaminación de la luz de la galaxia anfitriona (Cellone, Romero \& Combi 2000), metodologías observacionales/fotométricas inapropiadas (Cellone, Romero \& Araudo 2007), o también un uso inadecuado de métodos estadísticos para la detección de variabilidad (de Diego 2010; Joshi et al. 2011).

Dentro de la literatura se pueden encontrar diversos trabajos dedicados al estudio y uso de estas herramientas para la detección de microvariabilidad en las curvas de luz en AGNs y en particular, blazares. Las herramientas estadísticas más comúnmente utilizadas son: el test $\chi^{2}$, el cual compara la varianza de la curva del posible objeto variable con una varianza teórica calculada para un objeto no variable, propuesto por Kesteven, Bridle \& Brandie (1976) y usado para las series temporales fotométricas y polarimétricas (Romero, Combi \& Colomb 1994; Andruchow et al. 2003, 2005; de Diego 2010); el One Way ANOVA (Analysis of Variances), el cual consta de una familia de tests que comparan las medias de un número de muestras (de Diego et al. 1998; Ramírez et al. 2004, 2009; de Diego 2010); el criterio $C$, el cual involucra el cociente de las desviaciones estándares de dos distribuciones (Howell, Mitchell \& Warnock III 1988; Romero et al. 1999, 2002; Andruchow, Romero \& Cellone 2005; de Diego 2010; Joshi et al. 2011); y el test $F$, el cual tiene en cuenta el cociente entre las varianzas de dos distribuciones (de Diego 2010; Joshi et al. 2011). Sin embargo, pese a la diversidad de herramientas que se tiene y a su amplia aplicación, se suelen hallar resultados contradictorios entre sí cuando se las aplica. Por lo tanto, se busca que la clasificación 
del estado de variabilidad de una determinada fuente sea independiente del método estadístico utilizado, y por ende, es fundamental buscar tal herramienta que garantice dicha independencia.

\section{Fotometría Diferencial}

Para poder analizar la variabilidad de los blazares a través de las herramientas estadísticas mencionadas, necesitamos poder visualizar de alguna manera las posibles variaciones que se tengan en el flujo observado. Para ello, recurrimos a las curvas de luz y en particular, nos centramos en las curvas de luz diferenciales (DLC, de sus siglas en inglés Differential light curves), producto de la aplicación de la fotometría diferencial. La fotometría diferencial fue propuesta por Howell \& Jacobi (1986). Consiste en tomar exposiciones pequeñas, de manera reiterada, de un determinado campo estelar, el cual contenga a la fuente que se está analizando. Además de la fuente, el resto de las estrellas que estén en el campo son utilizadas como comparaciones durante el proceso de reducción, para obtener las magnitudes instrumentales de todos los objetos de interés. A partir de estas magnitudes, se construyen las magnitudes diferenciales usando las estrellas que fueron elegidas como comparación y control en la fotometría diferencial. Basándonos en la nomenclatura usada por Howell \& Jacobi (1986), si la magnitud instrumental $V$ representa al objeto y la de las estrellas de comparación y control son $C$ y $K$, respectivamente, se determinan las diferencias $V-C$ y $K-C$. Ésta última sirve como estimación de la precisión del sistema instrumental, y principalmente, provee una comparación para poder determinar si la curva de luz de la fuente es variable o no.

Es de suma importancia la elección correcta de estas estrellas de comparación y control. Para esto nos basamos en lo propuesto por Howell et al. (1988), en donde se aconseja tomar como estrella de control a aquella cuyo valor de la magnitud sea lo más parecido a la magnitud de la fuente, mientras que la estrella de comparación debe ser un poco más brillante. Esto es lo que llamaremos el "criterio de Howell".

\subsection{Los tests de hipótesis}

Los tests de hipótesis se basan en estudiar si una proposición o un valor predeterminado es aceptable o no de acuerdo a los datos que se tengan. Esta proposición se la conoce como hipótesis estadística, y está basada en la distribución de probabilidad de una variable que es aleatoria. Ésta es establecida a partir de la distribución bajo estudio y no de los datos. Por otra parte, se define un estadístico, cuya distribución de probabilidad está relacionada con la hipótesis. Básicamente se tienen dos tipos de hipótesis:

- Hipótesis nula $H_{0}$ : es la afirmación que se compara con los resultados obtenidos. Esta hipótesis se mantiene, a menos que los datos señalen que ésta es falsa.

- Hipótesis alternativa $H_{1}$ : es la que establece una afirmación opuesta/distinta a la hipótesis nula.

Por otra parte, se define la existencia de un punto crítico el cual determina los valores que serán aceptados o rechazados. A partir de la comparación de este valor 
con el estadístico, se decide si se rechaza o no la hipótesis planteada. Sin embargo, al comparar estos valores, se pueden cometer dos tipos de errores: rechazar la hipótesis nula cuando en realidad es verdadera (error de tipo I), o aceptar la hipótesis nula cuando ésta es falsa (error de tipo II, falsos positivos). Y al estar involucradas variables aleatorias, se pueden asociar probabilidades a estos errores. En particular, la probabilidad de cometer un error del tipo I se la conoce como el nivel de significancia del test, $\alpha$. Por otro lado, se define la potencia del test como la probabilidad de rechazar la hipótesis nula cuando ésta es falsa (o dicho de otra manera, la probabilidad de aceptar la hipótesis alternativa). La potencia es una medida de la sensibilidad del test. En lugar de trabajar con un valor específico de $\alpha$ para rechazar o no la hipótesis nula, se puede usar el p-valor, el cual se define como la probabilidad de que el estadístico tome un valor que sea tan extremo como el valor observado del estadístico cuando la hipótesis nula es verdadera. Es decir, es el nivel de significancia más pequeño que conduce al rechazo de la hipótesis nula. Si se obtiene un p-valor muy chico se puede rechazar, de manera robusta, la hipótesis nula; caso contrario (un p-valor alto), no se puede rechazar la hipótesis nula.

\subsubsection{Estadística $C$}

Con sus orígenes en los trabajos de Carini et al. (1990) y Jang \& Muller (1995), la estadística $C$ es una herramienta basada en el cociente de las desviaciones estándares o dispersiones de las curvas involucradas. En nuestro caso, son los correspondientes a las curvas de luz diferenciales de objeto-comparación $(V-C), \sigma_{1}$, y de control-comparación $(K-C), \sigma_{2}$ (producto de la fotometría diferencial):

$$
C=\frac{\sigma_{1}}{\sigma_{2}}
$$

Tomando un nivel de significancia $\alpha=0.995$, se puede establecer el valor crítico para el cual se acepta o se rechaza la hipótesis nula (i.e. igualdad estadística de las desviaciones). Se considera a una curva de luz como variable si $C \geq 2.576$, con un $99.5 \%$ de nivel de confiabilidad, dado que la hipótesis nula es rechazada (para el 99\%, el valor crítico corresponde a 2.367 y para el $95 \%$, corresponde a 1.671). Entonces la clasificación de la curva de luz resulta variable. Este estadístico está basado en suponer que la distribución de valores es una gaussiana.

Howell et al. (1988) definen un factor de peso, $\Gamma$, el cual tiene en cuenta la diferencia en magnitud de las estrellas de comparación, de control y del objeto cuando se aplica la fotometría diferencial. El factor de peso $\Gamma$ se define:

$$
\Gamma^{2}=\left(\frac{N_{K}}{N_{V}}\right)^{2}\left\lfloor\frac{N_{C}^{2}\left(N_{V}+P\right)+N_{V}^{2}\left(N_{C}+P\right)}{N_{K}^{2}\left(N_{C}+P\right)+N_{C}^{2}\left(N_{K}+P\right)}\right\rfloor
$$

donde $N_{V}$ son las cuentas sobre la imagen que tiene el objeto, $N_{C}$ y $N_{K}$ las cuentas que tienen las estrellas de comparación y control, respectivamente, y $P$ es un parámetro que involucra las cuentas en el cielo, $N_{S}$. 
De esta manera, es posible tener en cuenta las diferencias en magnitud de los objetos usados en la fotometría diferencial y usar una estadística pesada. Se considera como parámetro de la variación al mismo estadístico $C$, definido previamente, pero dividido por ese factor de peso $\Gamma$, que tiene en cuenta la diferencia de magnitudes y errores relativos, entre las estrellas de comparación y el objeto:

$$
C=\frac{\sigma_{1}}{\Gamma \sigma_{2}}
$$

El criterio de variabilidad es el mismo dado para el test sin el peso, esto es, la fuente es variable si $C \geq 2.576$.

\subsection{2. $\quad$ Estadística $F$}

El test estadístico $F$ se utiliza para comparar las varianzas de dos muestras determinadas. En este caso, la hipótesis nula es que las varianzas son estadísticamente las mismas. A su vez, existen otras suposiciones que deben asumirse al aplicar este test. La población bajo estudio debe estar aproximadamente distribuida en forma normal. Además, los datos considerados tienen que ser independientes entre sí. El procedimiento para trabajar con el test $F$ es el siguiente: en primer lugar, hay que elegir un nivel de confiabilidad $\alpha$. Segundo, los grados de libertad, $\nu_{i}=n_{i}-1, i=1,2$ son computados, donde $n_{i}, i=1,2$ son los número de las mediciones de cada muestra. Tercero, usando la distribución de densidad de probabilidad de la variable estadística $F$ con $\nu_{1}$ y $\nu_{2}$ grados de libertad, se calcula un valor $F_{\alpha}$, tal que el área bajo la distribución mencionada antes hacia la izquierda de $F_{\alpha}$ sea $\alpha$ (Fig. 2.1). Cuarto, se calcula un valor $F_{\text {obs }}=\sigma_{1}^{2} / \sigma_{2}^{2}$ con los datos, a partir de usar para cada muestra la fórmula:

$$
\sigma^{2}=\frac{1}{n-1} \sum_{i=1}^{n}\left(x_{i}-\mu\right)^{2},
$$

donde $n$ es el tamaño de la muestra, $x_{i}$ son las mediciones, y $\mu$ es el valor medio de la muestra. Finalmente, se comparan $F_{\text {obs }}$ contra $F_{\alpha}$. Si $F_{\text {obs }}>F_{\alpha}$, entonces la hipótesis nula es rechazada; de lo contrario, la hipótesis nula no es rechazada.

Aplicando todo esto a nuestro análisis y suponiendo que los errores de las curvas a comparar están distribuidos normalmente, se tiene:

$$
F=\frac{\sigma_{1}^{2}}{\sigma_{2}^{2}}
$$

donde $\sigma_{1}^{2}$ corresponde a la varianza de la curva de luz diferencial de objeto-comparación $(V-C)$ y $\sigma_{2}^{2}$, la de control-comparación $(K-C)$. Tomando en cuenta la cantidad de puntos en las curvas V-C, $N_{o c}$, y en la K-C, $N_{c c}$ (los cuales cumplen que $N_{o c}=N_{c c}=$ $N$ ), los grados de libertad en nuestro caso resultan ser $N-1$. A partir de lo mencionado antes, si nuestro valor $F$ es mayor que los valores críticos para los grados de libertad de cada curva, entonces la hipótesis nula del test es rechazada. Esto se traduce en que la curva bajo estudio se la puede clasificar como variable.

De la misma manera, siguiendo el lineamiento presentado para el criterio $\mathrm{C}$, se utiliza el mismo factor de peso $\Gamma$. De esta manera, el estadístico $\mathrm{F}$ pesado resulta: 


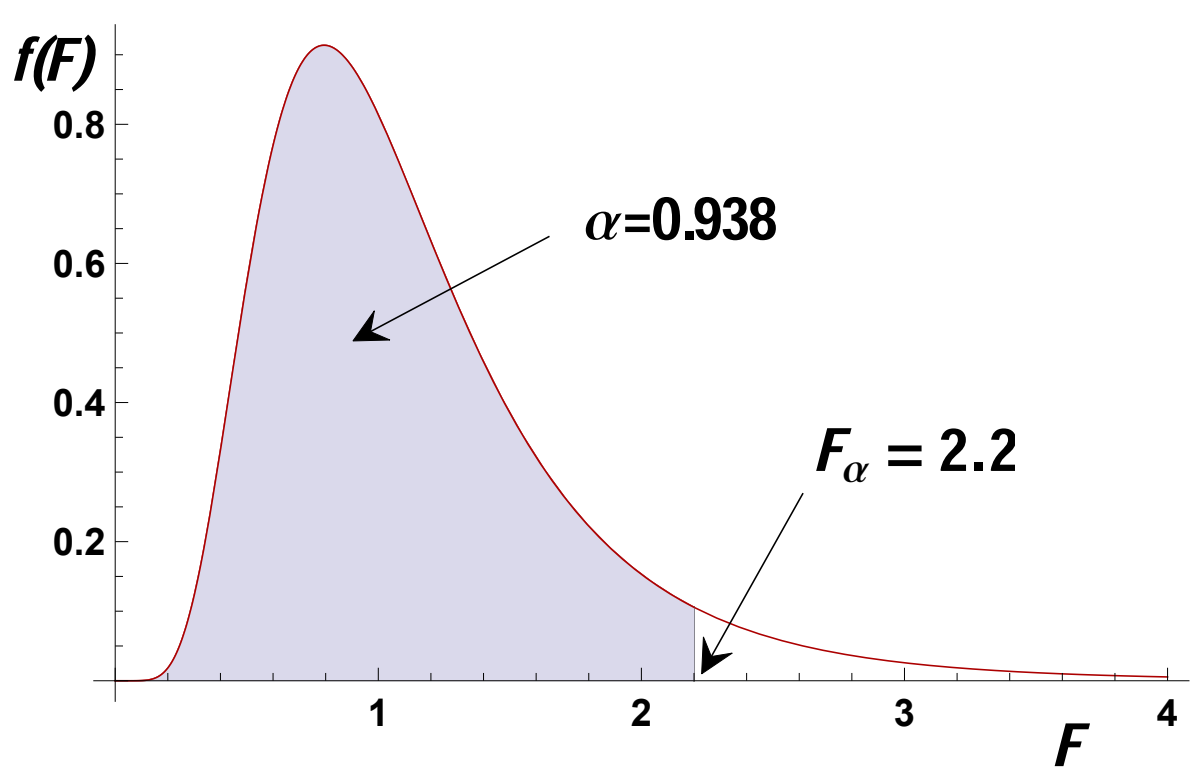

Figura 2.1. Ejemplo de una densidad de distribución de Fisher $F$, aquí con $\nu_{1}=20$, $\nu_{2}=15$, i.e. la muestra con la mayor dispersión tiene 21 mediciones, y la otra 16 . El nivel de confiabilidad es elegido como $\alpha=0.938$, el cual da un valor de $F_{\alpha}=2.2$. Si resulta que $F_{\text {obs }}>F_{\alpha}$, la hipótesis nula es rechazada; de lo contrario, la hipótesis nula es la correcta.

$$
F=\frac{\sigma_{1}^{2}}{\sigma_{2}^{2} \Gamma^{2}}
$$

\subsection{Resultados sobre datos observacionales}

En Zibecchi (2013), estudiamos las estadísticas C y F a partir de una muestra grande y homogénea de datos observacionales reales. Se trabajó con 39 AGNs del sur (reportados en Romero et al. 1999, 2002), los cuales fueron observados con el telescopio 2.15 m "Jorge Sahade", San Juan, Argentina, obteniendo 78 curvas de luz diferenciales, a las que se les aplicó las herramientas estadísticas. Las observaciones estaban caracterizadas por valores de seeing entre 2.0 y 4.0 arcsec, tiempos de exposición entre 2 y $15 \mathrm{~min}$, y valores de la masa de aire entre 1.00 y 2.40. Se analizó el comportamiento de ambas estadísticas con 3 niveles de confiabilidad distintos: $95 \%$ y 99 \% y $99.5 \%$. Como ejemplo, en la Fig. 2.2 presentamos una comparación entre los valores obtenidos para los parámetros pesados de $\mathrm{C}$ y $\mathrm{F}$ al $99.5 \%$ del nivel de confiabilidad. Estos valores están referidos a los valores límites correspondientes en cada caso particular, para comparar de mejor manera a cada uno. Las líneas sólidas indican el límite de los valores críticos para ambas estadísticas, marcando la división para los cuatro posibles casos. Es posible apreciar que el cuadrante, en el cual el criterio C resultaría variable y el test $F$ no lo sería, está vacío, en contraste con su cuadrante opuesto (no variable con $\mathrm{C}$ y variable con $\mathrm{F}$ ).

A partir de los resultados de aplicar los tests $\mathrm{C}$ y $\mathrm{F}$ a la muestra, encontramos que, con respecto a la amplitud de DLC $(\Delta m)$, los valores calculados para $F$ tienden 


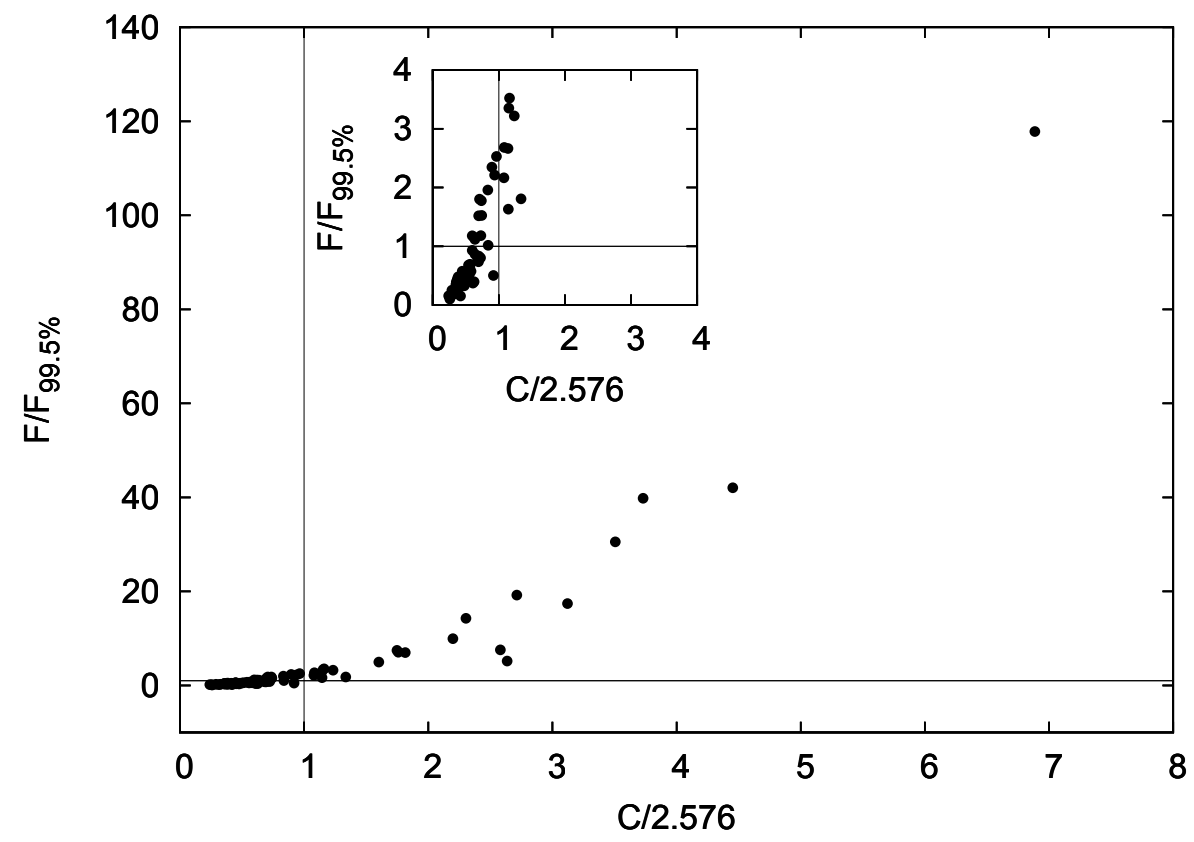

Figura 2.2. Comparación entre las estadísticas $C$ y $F(99.5 \%$ nivel de significancia). Un ampliamiento de la zona cercana a $(1,1)$ se muestra. Las líneas sólidas indican el límite de los valores críticos para ambas estadísticas.

a clasificar como variables las DLCs con $\Delta m$ cerca del error rms (esto es, con valores de $\Delta m \sim 0.03 \mathrm{mag}$ ), mientras que para DLCs con una amplitud más alta a este valor (DLCs con $\Delta m>0.1-0.2 \mathrm{mag}$ ), ambas estadísticas tienden a detectar variabilidad. Para el intervalo de tiempo en el cual se registran el máximo y el mínimo valor de la diferencia en magnitud $(\Delta t)$, las DLCs con valores de $\Delta t>0.1-0.2 \mathrm{hr}$ se clasifican como variables, de acuerdo con el hecho de que este alto valor aparece generalmente en curvas de luz donde se observa variabilidad sistemática. Ambas estadísticas parecen ser robustas en la detección (o no detección) de la variabilidad cuando las DLCs presentan una dispersión instrumental baja $\left(\sigma_{2} \sim 0.01\right)$, pero si esta dispersión alcanza valores más altos (esto es, $\sigma_{2}>0.03$ ), una variación de baja amplitud en la fuente podría ser enmascarada dada la baja relación $\mathrm{S} / \mathrm{N}$ en la DLC.

\subsubsection{Estudio detallado del "test" C}

En esta sección vamos a inspeccionar más de cerca al "estadístico" C. Como vimos en la Sec. 2.2.1, el origen del test C proviene del trabajo de Carini et al. (1990). Los autores propusieron que las dispersiones de las magnitudes diferenciales de la curva de luz de control podían considerarse como estimadores de cuán estables eran las estrellas que se usan en la fotometría, tomándose como una medida de la incerteza observacional. Luego, Jang \& Miller (1995) ajustaron rectas a las curvas de luz diferenciales a modo de poder calcular las desviaciones estándares, donde la dispersión más grande era considerada como una estimación del error observacional. Ya en el trabajo de Romero et al. (1999), el parámetro C queda establecido donde utilizaron la dispersión de la curva del AGN en lugar de la amplitud de la curva (Jang \& Miller 1997). Todo este 
desarrollo fue hecho con el propósito de mejorar la estimación en los errores observacionales, a la vez que se conforma como criterio de variabilidad robusto ante los falsos positivos que se obtienen de los errores sistemáticos.

Al compararlo con el test $\mathrm{F}$, se observa que ambos parámetros dan resultados diferentes. Recordemos que tanto el criterio $\mathrm{C}$ como el test $\mathrm{F}$ se construyen a partir del cociente de las desviaciones estándares o de las varianzas, respectivamente, de dos poblaciones en particular. Cada cociente (i.e. cada estadístico) es comparado con un valor crítico a partir de decidir cuál va a ser el nivel de significancia del test. La diferencia principal entre ambos parámetros es que, mientras que el valor crítico del test $\mathrm{F}$ no es fijo (es decir, se construye no sólo a partir del nivel de significancia, sino también de los grados de libertad de cada grupo de datos), no es el caso del criterio C. Para este último fijando, por ejemplo, el nivel de significancia $\alpha=0.995$, el valor crítico resulta ser 2.576, es decir, un valor fijo independientemente de los grados de libertad que tengan las dos poblaciones bajo estudio.

Así, el criterio $\mathrm{C}$ no es un test estricta y teóricamente estadístico. En principio, el C proviene de lo que se conoce como el proceso de rechazo de una mala medición: se compara una medición particular con el valor medio de la densidad de distribución de las mediciones, y la distancia (i.e. cuánto se aleja dicha medición de la media) no es otra cosa que la dispersión de la densidad de distribución de las mediciones. Pero, analizando más en detalle cómo está construido el $\mathrm{C}$, se está queriendo comparar la dispersión $\sigma_{1}$ con una dispersión de referencia $\sigma_{2}$, la cual debería ser el valor medio de la densidad de distribución de las dispersiones y a la vez, debería ser la dispersión de dicha densidad de distribución (pues $\sigma_{1} / \sigma_{2}$ vendría a ser la distancia). Resumiendo, $\sigma_{2}$ tiene un doble rol: valor medio y dispersión de una distribución de dispersiones desconocida.

Por lo tanto, no podemos tomar al criterio C como un test estadístico propiamente dicho, aunque sí sigue siendo un parámetro útil y robusto para el análisis de la variabilidad en las curvas de luz, siempre que se elija el nivel de significancia adecuado.

\subsubsection{Análisis de $\mathrm{C} \mathrm{y} \mathrm{F}$ en estrellas de campo}

Para poder analizar un poco más profundamente la estabilidad de ambos parámetros $\mathrm{C}$ y F, se generaron (a partir de la fotometría diferencial) las curvas de luz diferenciales quitando al AGN y poniendo en su lugar alguna de las estrellas de campo que se encuentran en el frame observado (que no sea ni la estrella de comparación ni la de control, pues éstas siguen estando en su rol). Las curvas de luz obtenidas fueron usadas para el cálculo de ambos parámetros C y F. Del total de curvas de luz generadas, se descartaron aquellas que fueron afectadas por saturación, por rayos cósmicos, por estrellas que estén cerca del borde de la imagen; aquellas que presentaban comportamientos con amplitudes en la magnitud $>0.4$ mag y también aquellas curvas que involucraron estrellas variables conocidas. Así, del total de 1039 curvas de luz diferenciales, trabajamos con 981. En principio, habiendo considerado lo mencionado antes, no debería ser alto el porcentaje de curvas de luz variables, siendo las estrellas de campo no variables. Del trabajo de Howell (2008) se espera que el $7 \%$ de las estrellas de campo resulten ser variables (con una precisión de $0.01 \mathrm{mag}$ ). De esta manera, esperamos que 
la fracción de estrellas cuyas curvas de luz resulten variables en los campos de nuestra muestra observacional (que varían a un nivel $>0.01$ mag en algunas horas) sea como máximo $10 \%$.

De las 981 DLCs, el $16.9 \%$ resultó ser clasificado como variable con el parámetro F, mientras que el criterio C clasificó variable el 9.5\% de las DLCs. Es decir, se está obteniendo un porcentaje de variabilidad mayor al esperado (5\%) en ambos parámetros, siendo nuevamente el test $\mathrm{F}$ el más sensible. Analizando más en detalle los parámetros que caracterizan a las DLCs, encontramos que existe una dependencia entre el valor de la amplitud $(\Delta m)$ y la dispersión de la curva de control escaleada $\left(\Gamma \sigma_{2}\right)$ con las distintas distribuciones de casos (i.e. la correspondiente a la clasificación como no variable con los parámetros C y F; la que corresponde a la clasificación de variable con ambos; y aquella que involucra la no variabilidad con el criterio $\mathrm{C}$ pero sí con el test F). Si ambos parámetros no clasifican como variable a la curva de luz, el cociente $\Delta m / \Gamma \sigma_{2}$ agrupa a las curvas por debajo de 0.9. Si en cambio, ambos parámetros clasifican como variables a las curvas, este cociente pasa a tomar valores mayores a 0.9 . Y si el criterio $\mathrm{C}$ no clasifica como variable a una curva pero sí lo hace el test $\mathrm{F}$, este cociente ronda el valor de 0.9. Resultados similares a éstos los obtuvimos al reemplazar de nuevo al AGN en su lugar. Todos estos resultados tienen en cuenta el uso del factor de peso $\Gamma$. Si no se lo considera, el porcentaje de curvas de luz variables se incrementa a $20.7 \%$ y $33.4 \%$ para los parámetros $\mathrm{C}$ y $\mathrm{F}$, respectivamente. Por último, también calculamos el porcentaje de variabilidad no sólo para el nivel de confiabilidad de $99.5 \%$, sino también para el $99 \%$ y $95 \%$, encontrando que sus distribuciones tienen un claro pico alrededor del $10 \%$. Dentro de un nivel de confiabilidad específico, la distribución de F se extiende a mayores porcentajes de variabilidad. En la Fig. 2.3, comparamos los casos del F al $99.5 \%$ y del C al $95 \%$, obteniendo distribuciones casi idénticas (ésto último respaldado por un test de KS), lo que nos da a entender que, para nuestros datos, hay que relajar el nivel de confiabilidad del $\mathrm{C}$ al $95 \%$ para obtener los mismos resultados que el $\mathrm{F}$ al $99.5 \%$.

En líneas generales, vemos que el $\mathrm{F}$ sigue siendo el que más variabilidad detecta y es debido a que el $\mathrm{F}$ es particularmente sensible a los errores no gaussianos (Well \& Jenkins 2012). 


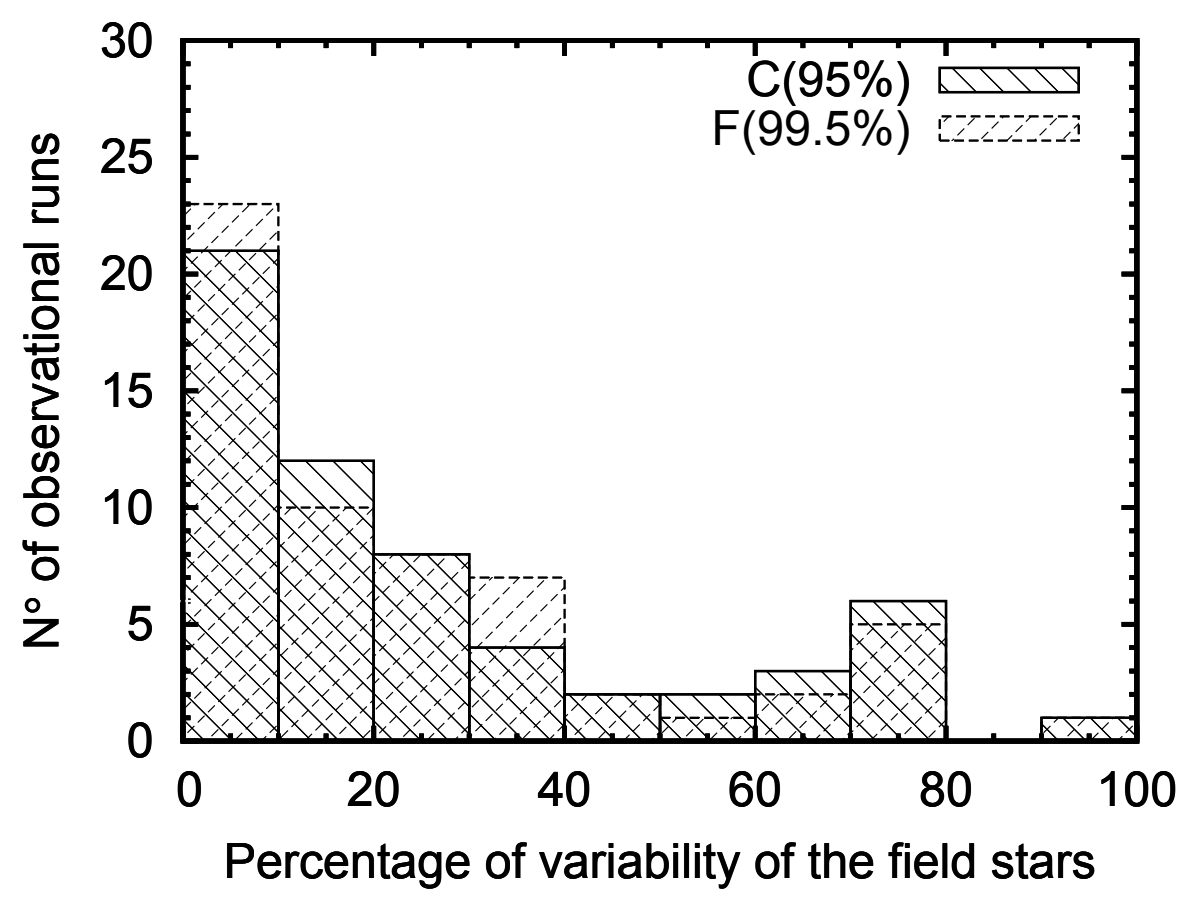

Figura 2.3. Distribución de los porcentajes de estrellas por campo que resultó variable usando $C$ al $95 \%$-CL y $F$ al $99.5 \%$-CL (Zibecchi et al. 2017). 


\subsection{Simulaciones}

\subsubsection{Descripción e implementación}

Uno de los objetivos principales de la presente Tesis es estudiar y analizar las herramientas estadísticas con las que se analiza la variabilidad. Para ello, planteamos realizar un estudio utilizando la generación de curvas de luz diferenciales sintéticas a las cuales aplicar las dos herramientas bajo estudio. Una forma de obtener dichas curvas es simplemente generándolas a partir de algoritmos numéricos y simulaciones que generen distribuciones de puntos al azar, con determinados efectos adicionales (de Diego 2010, 2014; de Diego et al. 2015; Emmanoulopoulos et al. 2013; Wang \& Cao 2014). En nuestro caso, buscamos obtener las curvas de luz a través de la generación de imágenes astronómicas, en las cuales estén presentes las características más comunes que uno se puede encontrar a la hora de observar, tales como condiciones climáticas adversas.

A partir de la aplicación de diversas tareas de IRAF, desarrollamos un script que genera las imágenes astronómicas sintéticas. Este script contempla tareas tales como: mkobjects, tarea principal encargada de la generación/modificación de imágenes; hedit, para la modificación de los encabezados de las imágenes; setjd, que establece la información de la fecha de observación a partir los valores de ascensión recta y declinación del objeto, tiempo de exposición de la imagen, el lugar del observatorio; etc. Dado que requerimos curvas de luz diferenciales, en cada una de las imágenes se muestra un conjunto de AGNs no variables y otro conjunto de estrellas de campo, las cuales son utilizadas como comparación y control en la fotometría diferencial. Como resultado, obtenemos imágenes como la Fig. 2.4. En la parte superior de la imagen, se encuentran 200 AGNs no variables con magnitudes entre 16 y 17 mag, valores característicos de las magnitudes aparentes de estos objetos. En la parte inferior, se tienen 63 estrellas de campo, las cuales poseen magnitudes que cubren un rango desde 15 a 17 mag. Este rango de valores permite aplicar el criterio de Howell con diversas combinaciones entre el AGN y las estrellas de comparación y control.

En el script, se especifican las características instrumentales del CCD y del telescopio, así como también las condiciones atmosféricas variables, las coordenadas del objeto y del observatorio, y además, tomamos en cuenta el overhead (tiempo muerto entre exposiciones), el cual se utiliza para el cálculo del tiempo universal (UT) que se asigna luego a cada una de las imágenes y una componente adicional de ruido aleatorio. La salida resulta en un conjunto de imágenes, cada una con un set de parámetros observacionales y atmosféricos, que se detallan a continuación:

- Ruido instrumental: está relacionado con las propiedades del CCD. Contemplamos valores del ruido de lectura (readout noise) y la ganancia (gain) sustraídos de CCD TeK1024 en CASLEO ${ }^{1}$.

- Seeing (IQ Image quality): está asociado a la calidad de la imagen que se tiene al momento de observar. Contempla las variaciones que se introducen debido a la turbulencia atmosférica.

\footnotetext{
${ }^{1}$ Este telescopio es tomado como ejemplo ya que posee el tamaño de espejo $(2.15 \mathrm{~m})$ que se utiliza comúnmente para estos seguimientos de variabilidad.
} 


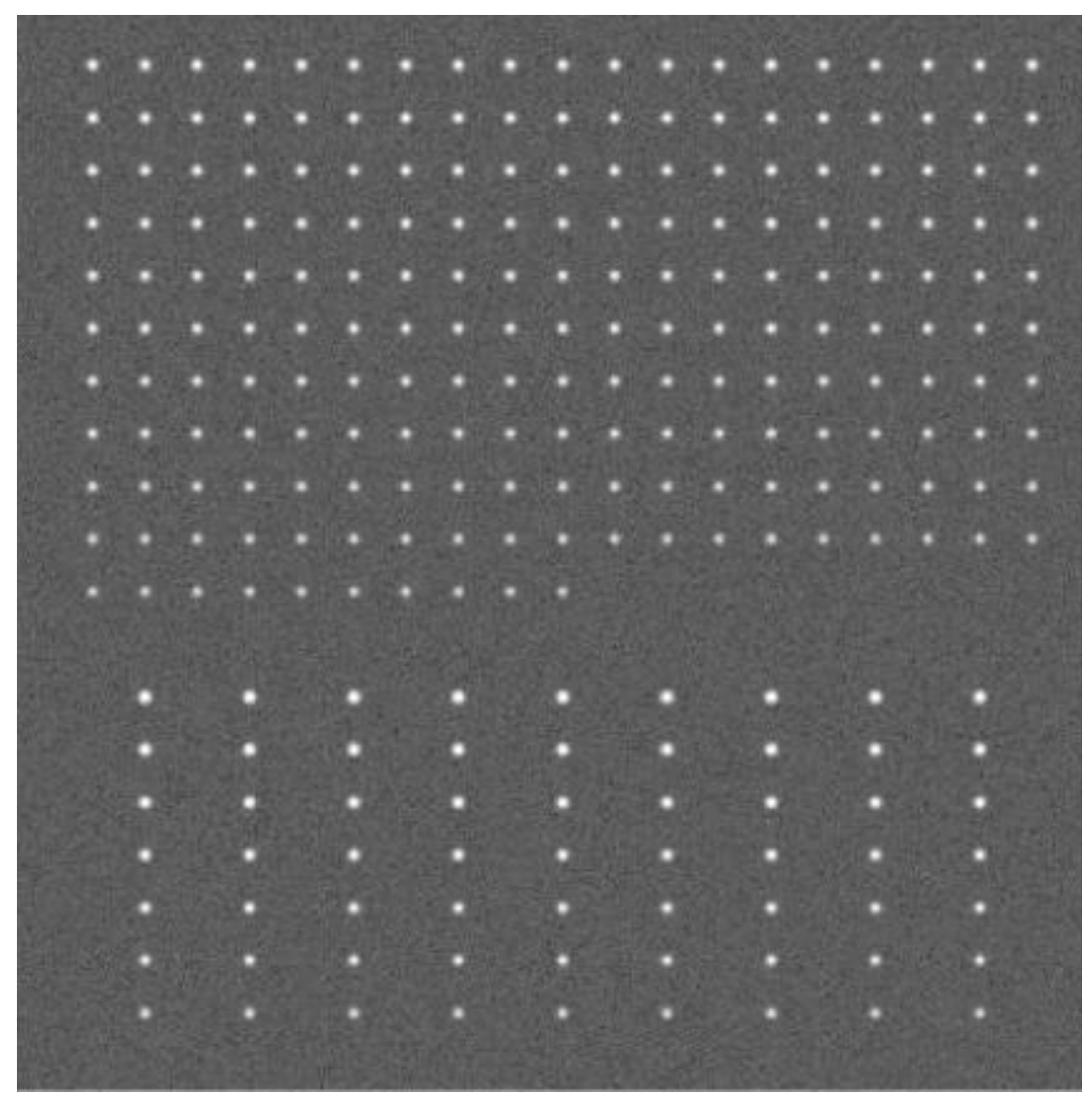

Figura 2.4. Ejemplo de una de las imágenes simuladas. Arriba: AGNs con diferentes magnitudes. Abajo: candidatos a las estrellas de control y comparación.

- Cobertura de nubes (CC Cloud cover): este efecto simula la extinción en magnitud para todos los objetos de la imagen causada por nubes, y también los casos en los que está presente velo (el valor de extinción representa el promedio en el tiempo de exposición).

- Brillo del cielo (SB Sky brightness): tiene en cuenta la presencia de la Luna, y cómo esto afecta al brillo del cielo, y así, al número de cuentas en la imagen.

- Masa de aire: relacionado con la posición de la fuente en el cielo. Dependiendo de la posición del objeto, la atenuación de la luz es mayor o menor.

Finalmente, con la colaboración del Dr. Sergio Cellone, se transformó el script en una tarea de IRAF, utilizada por el grupo de trabajo.

En la Tabla 2.1 mostramos los valores considerados para cada uno de estos parámetros en la generación de imágenes. En las columnas 1 y 2 se muestran los valores de ruido de lectura y ganancia del CCD, en la columna 3 se presentan los valores del seeing, en la columna 4 se tiene la extinción provocada por la presencia de velo y nubes. En la columna 5 se muestra el brillo del cielo debido a la ausencia o presencia total o parcial de la Luna. Para el caso de la masa de aire, usamos un total de 40 valores esparcidos desde 1.2 hasta 2.0. De esta manera, teniendo en cuenta la cantidad de combinaciones de los efectos que puede tener una imagen, se generó un total de 5000 imágenes. 
Tabla 2.1. Valores de las condiciones instrumentales y atmosféricas usadas en las simulaciones. En las columnas 1 y 2 se muestran los valores de ruido de lectura y ganancia del CCD, en la columna 3 se presentan los valores del seeing, en la columna 4 se tienen la extinción provocada por la presencia de velo y nubes. En la columna 5 se muestra el brillo del cielo debido a la ausencia o presencia total o parcial de la Luna.

\begin{tabular}{ccccc}
\hline \hline $\begin{array}{c}\text { Ruido de lectura } \\
e^{-}\end{array}$ & $\begin{array}{c}\text { Ganancia } \\
e^{-} / a d u\end{array}$ & $\begin{array}{c}\text { Seeing } \\
\text { " }\end{array}$ & $\begin{array}{c}\mathrm{CC} \\
\mathrm{mag}\end{array}$ & $\begin{array}{c}\mathrm{SB} \\
\mathrm{mag}^{\operatorname{arcsec}^{-2}}\end{array}$ \\
\hline 9.60 & 1.98 & 0.6 & 0.00 & 22.2 \\
& & 1.5 & 0.25 & 21.2 \\
& & 2.0 & 0.50 & 20.7 \\
& & 3.0 & 0.75 & 20.2 \\
& & 4.0 & 1.00 & 19.7 \\
\hline
\end{tabular}

Una vez que todas las imágenes fueron generadas, realizamos el proceso de reducción usual con las tareas de IRAF. Las tareas del paquete APPHOT fueron usadas para la fotometría de apertura. Seleccionamos un radio de apertura de 8 pixels (equivalente a 6.5 arcsec), resultando este el valor óptimo para la fotometría diferencial. Como salida, se obtuvieron los archivos de fotometría, los cuales contienen información sobre la magnitud de los objetos, el flujo, el radio de apertura usado, entre otras cosas. Estos archivos son la base del script desarrollado en IRAF para el armado de las distintas situaciones observacionales y de la aplicación de la fotometría diferencial. Por un lado, el script comienza generando la situación observacional a simular. Cada una de estas situaciones están identificadas en un archivo de entrada, en el cual se especifica cómo es la variación de cada uno de los efectos considerados. Utilizando ese archivo y los de fotometría, se arma la situación y la correspondiente tabla de fotometría. Por otro lado, el programa toma estas tablas generadas y, a partir de combinar las magnitudes de los AGNs no variables, arma las variaciones de los AGNs variables. Respecto a las formas en que varían estos AGNs, son el resultado de una combinación entre casos reales observados y variaciones que buscan replicar curvas de luz ruidosas. A continuación, se presentan cada una de ellas:

1. sistemática: curvas que corresponden a un incremento (o decrecimiento) continuo a lo largo de toda la observación. La variabilidad sistemática decreciente posee una amplitud de $0.2 \mathrm{mag}$, mientras que la creciente presenta una amplitud de 0.3 mag.

2. flickering: este tipo de variación usualmente es confundida con el ruido presente en la curva de control. Está relacionado con una variación aleatoria. En total se consideraron 5 tipos de flickering, con amplitud variada desde 0.3 mag para el primero, hasta 0.05 para el último, pasando por valores de $0.2,0.15$ y 0.10 mag.

3. burst suave: relacionada con un incremento intermedio en el flujo seguido de un decaimiento suave. Presenta una amplitud de 0.15 mag. 
4. serrucho: similar al flickering, pero con variaciones en amplitud mayores. Posee una amplitud de 0.15 mag.

En total, tenemos 9 tipos de variabilidad, como puede verse en la Fig. 2.5 (para el caso del flickering sólo se muestra uno de los 5 tipos considerados).

Finalmente, en lo que respecta a las curvas de luz correspondientes a los AGNs no variables, se tomaron 3 restricciones al criterio de Howell. Cada uno de ellos contempla un determinado rango en la diferencia entre las magnitudes involucradas. Lo que se busca testear es cómo influye la elección de las estrellas de comparación y de control, sobretodo en los casos en que no se disponen de muchas estrellas en el campo, impidiendo aplicar el criterio de manera precisa. Estas 3 restricciones se las designó como:

- H01: diferencia en las magnitudes menores a 0.1 y mayores a 0.001.

- H03: diferencia en las magnitudes entre 0.1 y 0.3

- H05: diferencia en las magnitudes entre 0.3 y 0.5

Nótese que, desde H01 hasta H05, los criterios se van relajando, son cada vez menos estrictos. 


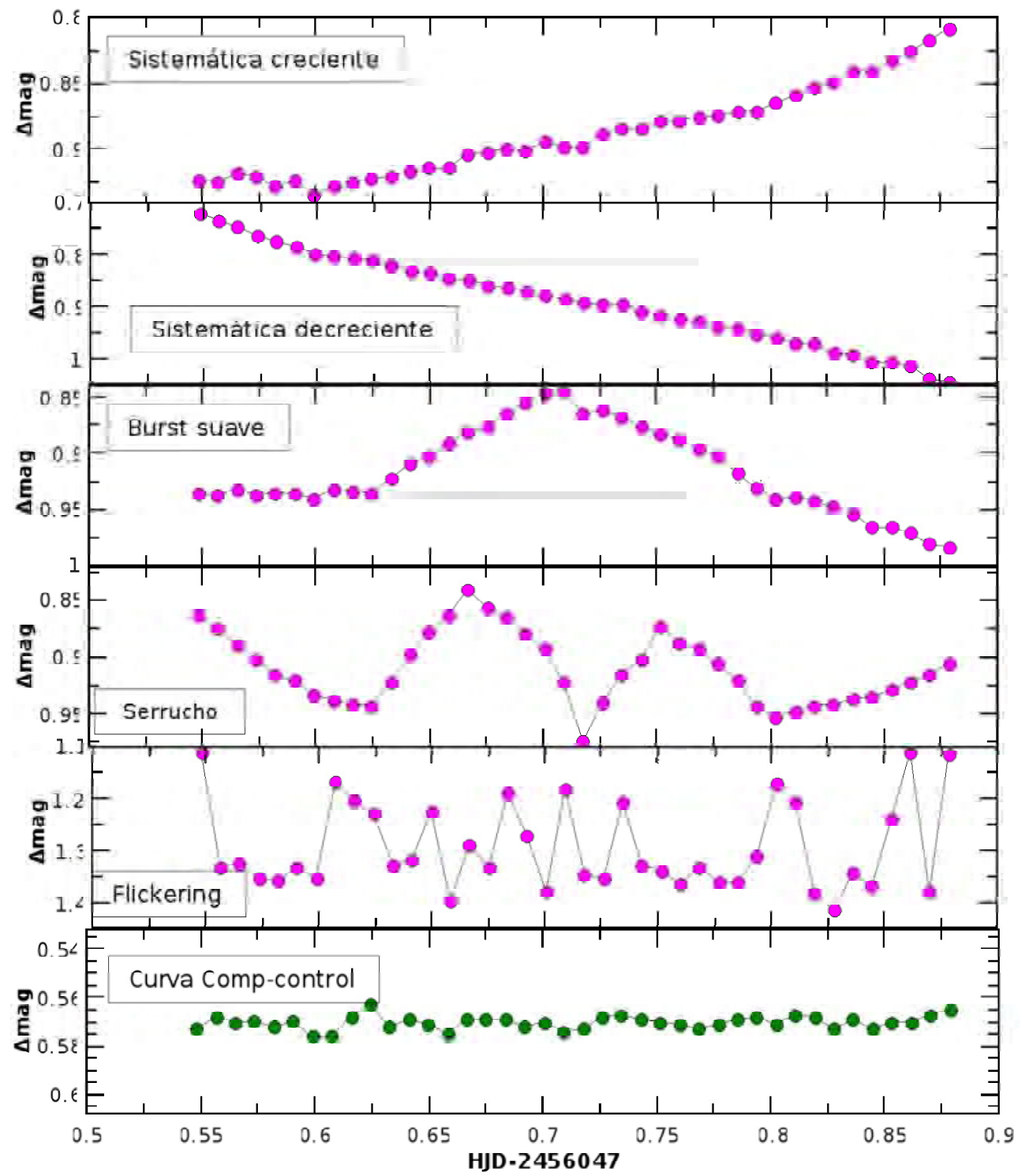

Figura 2.5. Distintos tipos de variaciones, junto con la curva de control. Desde arriba hacia abajo: variabilidad sistemática creciente, variabilidad sistemática decreciente, variabilidad tipo burst suave, variabilidad tipo serrucho y variabilidad tipo flickering. 


\subsubsection{Situaciones simuladas}

Como mencionamos anteriormente, en los scripts se contemplan diversas situaciones basadas en los cambios que ocurren comúnmente a la hora de observar. Cada curva de luz posee 40 puntos (40 observaciones correspondientes a las 40 masas de aire). A continuación las detallamos, donde el número máximo de casos en cada una de las situaciones contempladas tiene relación con la cantidad de tiempo de cómputo que lleva correr cada una de las simulaciones:

- Casos testigo (CT): son aquellas situaciones en donde el valor del brillo del cielo es fijo y sin la presencia de la Luna (22.2 mag $\operatorname{arcsec}^{-2}$ ). Además tampoco se tienen nubes (sin extinción), y el valor del seeing permanece fijo durante toda la noche, pero tomando cada uno de los valores elegidos. Entonces tenemos Caso testigo 1 (CT1) al 5 (CT5), con el seeing de 0.6 arcsec a 4.0 arcsec, respectivamente.

- Seeing variable (IQ): situaciones en donde el valor del brillo del cielo es fijo y sin la presencia de la Luna y sin nubes, pero ahora el valor del seeing varía durante toda la noche. Se consideraron 5 casos cuyas variaciones son construidas a partir de la experiencia observacional:

1. Seeing variable 1 (IQ1): en los primeros 8 puntos el valor del seeing es de 1.5 arcsec. En los segundos 8 puntos, decae a 0.6 arcsec. En los siguientes 8 puntos, vuelve a crecer hasta 1.5 arcsec. Y en los últimos 16 puntos, crece a 2.0 arcsec.

2. Seeing variable 2 (IQ2): en los primeros 8 puntos el valor del seeing es de 1.5 arcsec. En los segundos 8 puntos, crece a 2.0 arcsec. En los siguientes 8 puntos, sigue en 2.0 arcsec. Y en los últimos 16 puntos, crece a 3.0 arcsec.

3. Seeing variable 3 (IQ3): en los primeros 16 puntos el valor del seeing es de 2.0 arcsec. En los siguientes 8 puntos, crece a 3.0 arcsec. En los siguientes 8 puntos, vuelve a crecer hasta 4.0 arcsec. Y en los últimos 8 puntos, decrece a 3.0 arcsec.

4. Seeing variable 4 (IQ4): en los primeros 16 puntos el valor del seeing es de 3.0 arcsec. En los siguientes 16 puntos, decrece a 2.0 arcsec. Y en los últimos 8 puntos, crece a 3.0 arcsec.

5. Seeing variable 5 (IQ5): en los primeros 16 puntos el valor del seeing es de 0.6 arcsec. En los siguientes 16 puntos, crece a 1.5 arcsec. Y en los últimos 8 puntos, crece a 2.0 arcsec. En la Fig. 2.6 puede verse un ejemplo.

- Cobertura de nubes variable $(\mathbf{C C})^{2}$ : situaciones en las que hay cambios en la cobertura de nubes. Se consideraron 5 casos, reflejados en la Fig. 2.7. Y para cada uno de ellos se consideraron los 5 casos de seeing fijo.

\footnotetext{
${ }^{2}$ Los valores de las extinciones planteadas son el promedio de disminución del flujo que ocurre cuando se observa con presencia de nubes.
} 


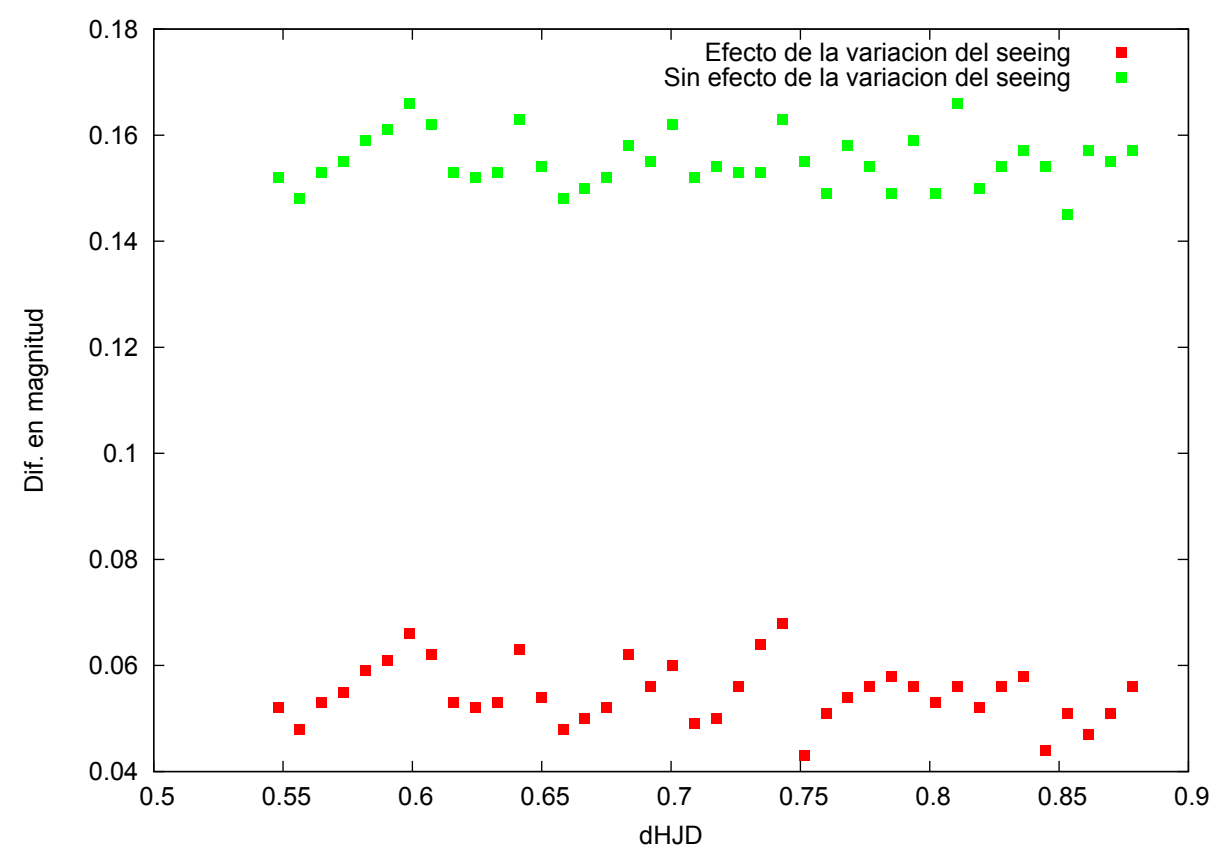

Figura 2.6. Comportamiento de la variación del seeing para IQ5, sin considerar y considerando su efecto.

1. Cobertura de nubes variable 1 (CC1): situación de nubosidad en aumento. En los primeros 8 puntos no hay extinción. En los segundos 8 puntos, comienza a aparecer un velo, que extingue las magnitudes en 0.25 mag. En los siguientes 8 puntos, aparecen más nubes, extinguiendo en 0.5 mag. Y en los últimos 16 puntos, las nubes extinguen las magnitudes en 0.75 mag.

2. Cobertura de nubes variable 2 (CC2): situación de pasaje de nubes. En los primeros 8 puntos no hay presencia de velo ni nubes. En los segundos 8 puntos, aparecen nubes que extinguen las magnitudes en 1.0 mag. En los siguientes 8 puntos, vuelve a estar despejado. En los siguientes 8 puntos, vuelve a nublarse con una extinción de 0.75 mag. Y en los últimos 8 puntos, se despeja otra vez.

3. Cobertura de nubes variable 3 (CC3): situación de velo. En los primero 16 puntos hay extinción de 0.25 mag. En los siguientes 8 puntos, empiezan a aparecer algunas nubes, que extinguen las magnitudes en 0.5 mag. En los últimos 16 puntos, se vuelve a despejar dejando un velo con extinción de 0.25 mag.

4. Cobertura de nubes variable 4 (CC4): situación de nubosidad en descenso. En los primeros 8 puntos las nubes extinguen las magnitudes en 0.75 mag. En los segundos 8 puntos, comienza a desaparecer algunas nubes, extinguiendo en $0.5 \mathrm{mag}$ las magnitudes. En los siguientes 8 puntos, aparece un velo, extinguiendo en $0.25 \mathrm{mag}$. Y en los últimos 16 puntos, no hay presencia de velo ni nubes.

5. Cobertura de nubes variable 5 (CC5): situación de velo intermedio. En los primero 16 puntos no hay extinción. En los siguientes 8 puntos, empieza a 
aparecer un velo, que extingue las magnitudes en 0.25 mag. En los siguientes 8 puntos, se nubla llegando la extinción a 0.5 mag. En los últimos 16 puntos, se vuelve a despejar, sin extinción.
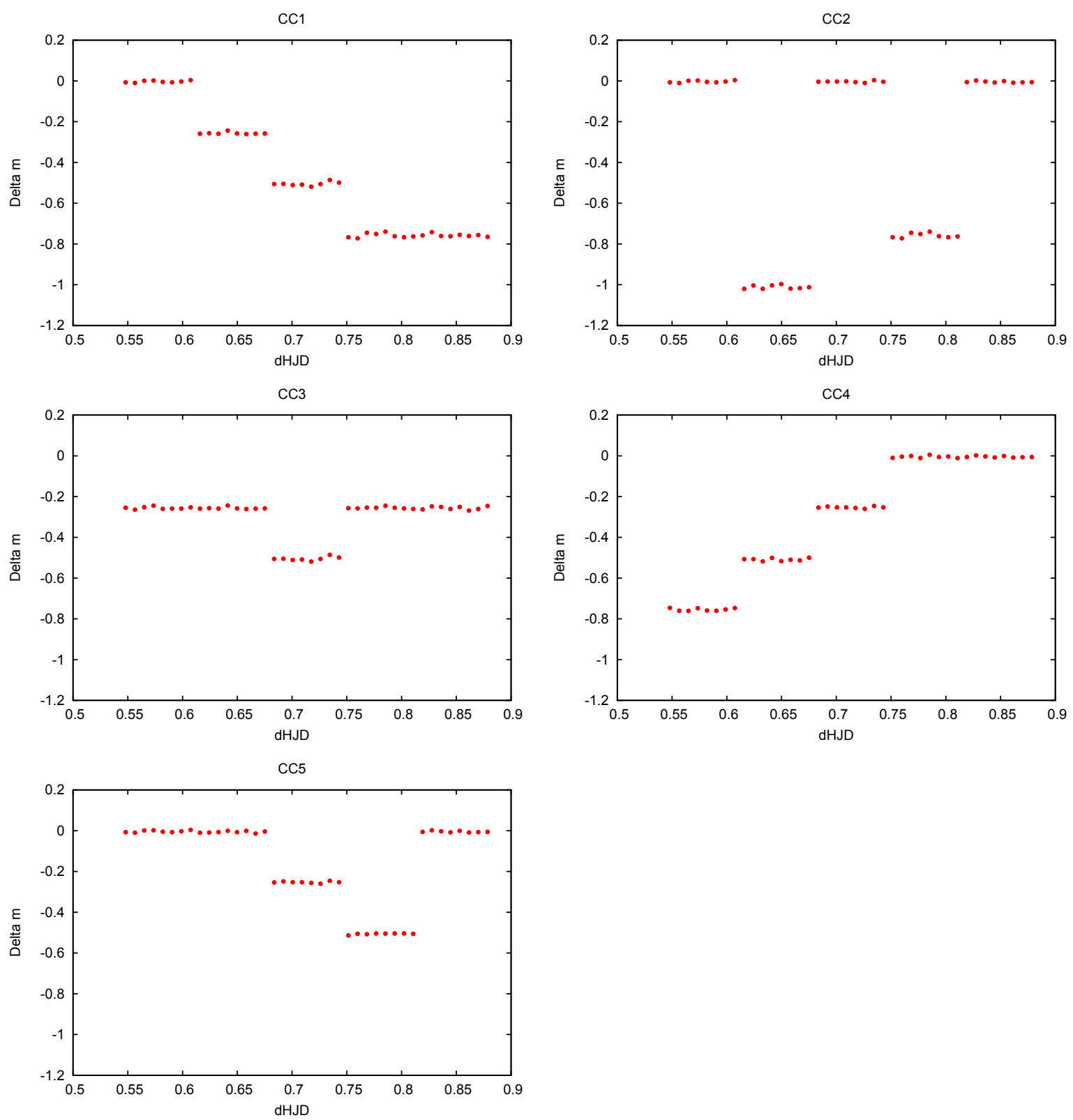

Figura 2.7. Comportamiento de los distintos casos de variaciones de la cobertura de nubes, desde CC1 hasta CC5. 
Tabla 2.2. Detalle de las situaciones IQ, CC y SB para los 40 puntos de las DLCs.

\begin{tabular}{|c|c|c|c|c|c|c|c|c|c|c|c|c|c|c|c|}
\hline Casos & IQ1 & IQ2 & IQ3 & IQ4 & IQ5 & $\mathrm{CC} 1$ & $\mathrm{CC} 2$ & $\mathrm{CC} 3$ & $\mathrm{CC} 4$ & $\mathrm{CC5}$ & SB1 & SB2 & SB3 & SB4 & SB5 \\
\hline & $\%$ & $\%$ & $"$ & " & " & mag & mag & mag & mag & mag & $\mathrm{mag} / " 2$ & $\mathrm{mag} / " 2$ & $\mathrm{mag} / ", 2$ & $\operatorname{mag} / "{ }^{2}$ & $\mathrm{mag} / " 2$ \\
\hline $1-8$ & 1.5 & 1.5 & 2.0 & 3.0 & 0.6 & 0.0 & 0.0 & 0.25 & 0.75 & 0.0 & 19.7 & 20.5 & 22.2 & 21.1 & 22.2 \\
\hline $9-16$ & 0.6 & 2.0 & 2.0 & 3.0 & 0.6 & 0.25 & 1.0 & 0.25 & 0.50 & 0.0 & 19.7 & 21.1 & 21.1 & 22.2 & 21.1 \\
\hline $17-24$ & 1.5 & 2.0 & 3.0 & 2.0 & 1.5 & 0.50 & 0.0 & 0.50 & 0.25 & 0.25 & 19.7 & 22.2 & 20.5 & 22.2 & 21.1 \\
\hline $25-32$ & 2.0 & 3.0 & 4.0 & 2.0 & 1.5 & 0.75 & 0.75 & 0.25 & 0.0 & 0.50 & 19.7 & 22.2 & 20.5 & 22.2 & 21.1 \\
\hline $33-40$ & 2.0 & 3.0 & 3.0 & 3.0 & 2.0 & 0.75 & 0.0 & 0.25 & 0.0 & 0.0 & 19.7 & 22.2 & 20.5 & 22.2 & 21.1 \\
\hline
\end{tabular}

- Brillo del cielo variable (SB): Se consideraron 5 situaciones. Y para cada una de ellas se consideraron los 5 casos de seeing fijo, sin considerar la presencia de nubes.

1. Brillo del cielo variable 1 (SB1): a lo largo de toda la noche se tiene la presencia de la Luna Llena, con un brillo de 19.7 mag $\operatorname{arcsec}^{-2}$.

2. Brillo del cielo variable 2 (SB2): presencia de la Luna en los primeros 16 puntos (20.5-21.1 mag $\operatorname{arcsec}^{-2}$, Luna en cuarto creciente).

3. Brillo del cielo variable 3 (SB3): se tiene la presencia de la Luna en los últimos 16 puntos (20.5-21.1 mag $\operatorname{arcsec}^{-2}$, Luna en cuarto creciente).

4. Brillo del cielo variable 4 (SB4): se tiene la presencia de la Luna en los primeros 8 puntos (21.1 mag $\operatorname{arcsec}^{-2}$, Luna en cuarto creciente).

5. Brillo del cielo variable 5 (SB5): presencia de la Luna en los últimos 8 puntos (21.1 mag $\operatorname{arcsec}^{-2}$, Luna en cuarto creciente).

Estas situaciones están resumidas en la Tabla 2.2. Las columnas de IQ están en arcsec, las de CC corresponden a mag y las de IQ a mag/ $\operatorname{arcsec}^{2}$.

Con estas situaciones planteadas, comenzamos a realizar las posibles combinaciones entre estas variaciones. En este sentido, obtenemos:

- Variaciones en el seeing y en la cobertura de nubes (IQ-CC): se combinan las distintas situaciones presentadas de variación en el seeing junto con los distintos casos de cobertura de nube. En todos estos casos no se tiene la presencia de la Luna llena (22.2 mag $\operatorname{arcsec}^{-2}$ ). En total, son 25 casos. A modo de ejemplo, presentamos en detalle una de las combinaciones obtenidas:

1. IQ2-CC4: se comienza la noche con un valor del seeing de 1.5 arcsec y una cobertura de nubes que extingue en 0.75 mag las magnitudes de los objetos. Luego, el valor del seeing crece a 2.0 arcsec y comienza a despejarse un poco, con extinción de 0.5 mag. Más tarde en la noche, el seeing se mantiene en 2.0 arcsec, mientras que la nubosidad sigue en descenso, hasta extinguir la 
magnitud en 0.25 mag. Y para el final de la noche, el seeing crece a 3.0 arcsec y ya no se tienen nubes.

- Variaciones en el seeing y en el brillo del cielo (IQ-SB): se combinan distintas situaciones presentadas de variación en el seeing junto con los distintos casos de brillo de cielo. En todos estos casos no se tiene la presencia de nubes (0.0 mag, sin extinción). Nuevamente, obtenemos 25 casos. Como ejemplo:

1. IQ5-SB3: Se comienza con un valor del seeing de 0.6 arcsec y sin la presencia de la Luna. Pasado un tiempo, el valor del seeing se mantiene en 0.6 arcsec y sale la Luna (21.1 mag $\left.\operatorname{arcsec}^{-2}\right)$. Luego, el seeing crece a 1.5 arcsec, y ya se tiene la Luna en cuarto creciente presente en el cielo (20.5 mag $\operatorname{arcsec}^{-2}$ ). Más tarde, el seeing sigue en 1.5 arcsec, al igual que la Luna. $\mathrm{Y}$ en el final de la noche, el seeing aumenta a 2.0 arcsec con la Luna presente.

Por último, llegamos a considerar las situaciones más realistas posibles: cambios de seeing, de brillo del cielo y de la cobertura de nubes.

- Variaciones en el seeing, en el brillo del cielo y en la cobertura de nubes (IQ-SB-CC): son aquellas situaciones en donde se mezclan todas las situaciones presentadas de variación en el seeing junto con los distintos casos de brillo de cielo más la variación en la cobertura de nubes. Se obtienen 125 casos. Como ejemplo:

1. IQ1-SB1-CC1: a lo largo de toda la noche se tiene la presencia de la Luna Llena, con un brillo de $19.7 \mathrm{mag} \operatorname{arcsec}^{-2}$. Al comienzo de la misma, se tiene un valor del seeing de 1.5 arcsec y sin cobertura de nubes. Más tarde, el valor del seeing decae a 0.6 arcsec y comienza a aparecer un velo, con extinción de 0.25 mag. Luego, el seeing vuelve a aumentar a 1.5 arcsec, mientras que la nubosidad sigue creciendo, hasta extinguir la magnitud en 0.5 mag. En el final de la noche, el seeing crece a 2.0 arcsec y la nubosidad vuelve a aumentar, extinguiendo todo en 0.75 mag.

De esta manera, teniendo en cuenta el set completo de 235 situaciones simuladas, junto con los 3 criterios de Howell para los AGNs no variables más los AGNs variables (sin restricción de Howell), se llega a un total de $5.6 \times 10^{7}$ curvas de luz diferenciales sintéticas. Como ejemplo, presentamos en la Fig. 2.8 un caso de AGN variable (variabilidad sistemática decreciente) para 5 situaciones en particular.

Como resumen de todo el procedimiento involucrado en la generación de las curvas de luz sintéticas, mostramos en la Fig. 2.9 un diagrama de flujo con los pasos más importantes de las simulaciones. 

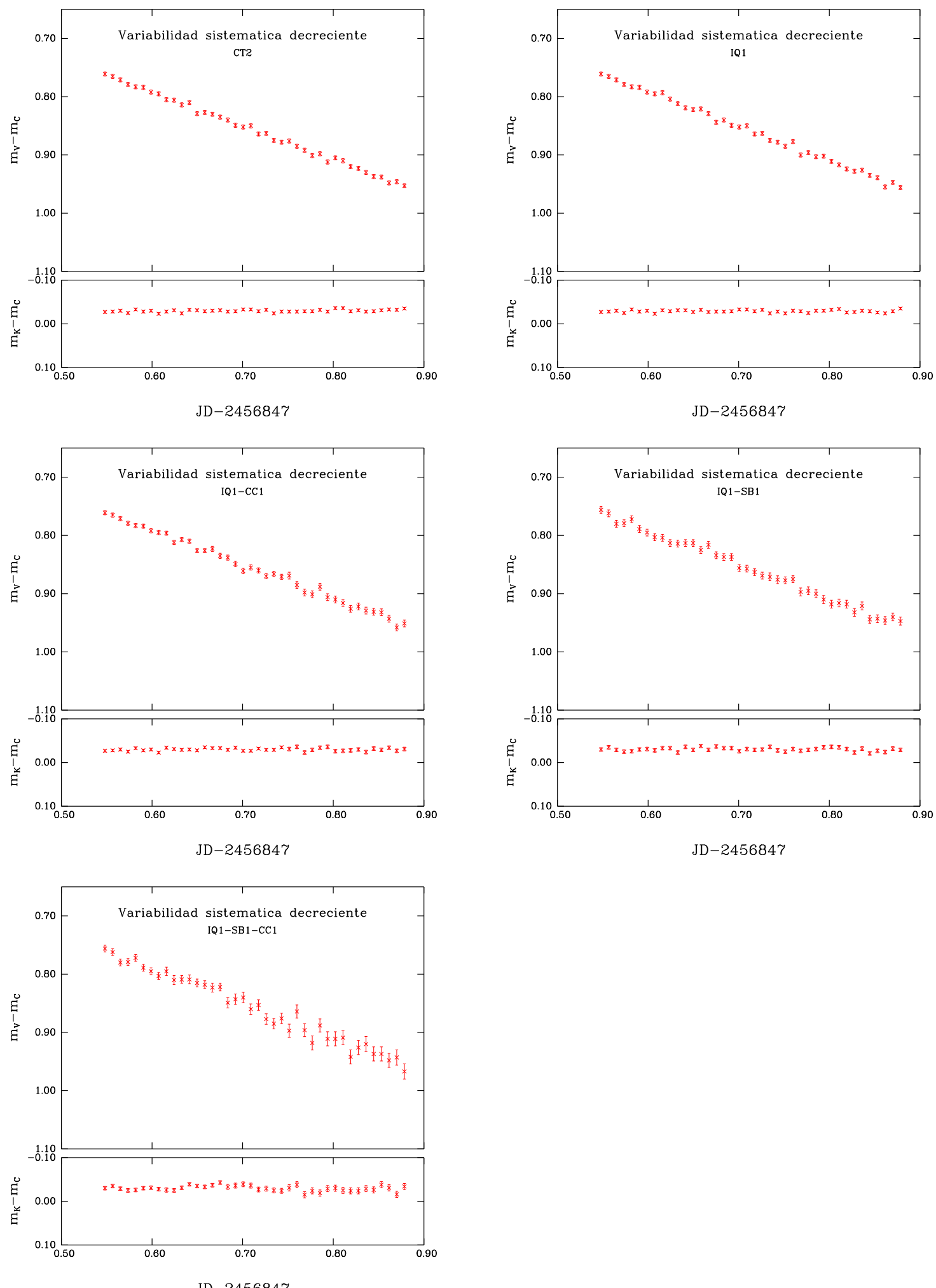

Figura 2.8. Ejemplos de curvas de luz diferenciales sintéticas para las situaciones CT2, IQ1, IQ1-CC1, IQ1-SB1 y IQ1-SB1-CC1 


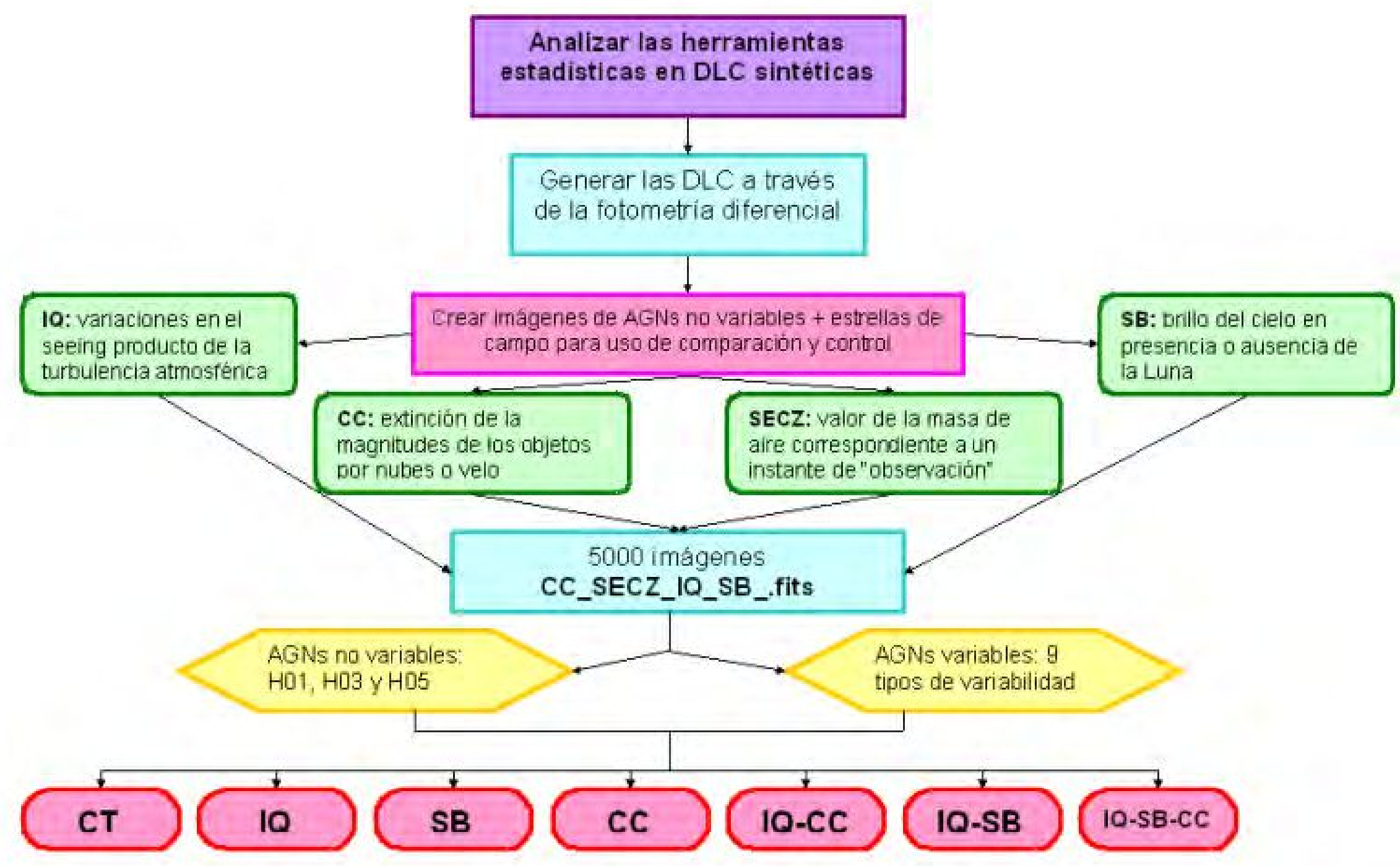

Figura 2.9. Diagrama de flujo de las simulaciones. 


\subsubsection{Factor de peso en las estadísticas}

En la Sec. 2.2.1, se introdujo el factor de peso estadístico $\Gamma$. Al trabajar con datos observacionales, lo más común es no tener estrellas en el campo que cumplan con el criterio de Howell. Este factor se calcula para cada curva de luz involucrando la combinación de AGN, estrellas de comparación y estrella de control. Por cada grupo de combinaciones tomadas en la fotometría, se calcula un valor de $\Gamma$, el cual es utilizado en el cálculo de los parámetros $\mathrm{C}$ y F, al escalear a la dispersión de la curva de control (como se explicó en las Sec. 2.2.1 y 2.2.2). Esto implica que el factor $\Gamma$ no es único para todas las curvas de luz ni para todos los objetos de la muestra de estudio, sino que es un valor individual por cada curva. Existen otros factores de peso planteados en la literatura. Se tiene el factor de peso $\kappa$, propuesto por Joshi et al. (2011). Este factor involucra el cociente del ruido en las curvas de luz del objeto y de control a través del promedio del error cuadrático en las curvas. Se construye a partir de los errores presentes en las curvas (asociados a las magnitudes). Este valor $\kappa$ es un valor único por objeto, independientemente de la cantidad de curvas de luz que tenga cada objeto. Por otro lado, varios autores utilizan un factor de peso $\eta$, el cual proviene de una subestimación de los errores fotométricos introducidos por los paquetes de fotometría de IRAF. Gopal-Krishna et al. (1995) calculan un valor de $\eta=1.75$, mientras que García et al. (1999) obtienen un valor similar, $\eta=1.73$. Stalin et al. (2004), a partir de calcular este factor para cada curva y promediar, utilizan un valor de $\eta=1.5$, al igual que lo hacen Goyal et al. (2012). Como el origen de este factor de peso está en los errores de la fotometría, este valor es único para las curvas de luz de los diferentes objetos.

Dado que el factor de peso $\Gamma$ tiene en cuenta el número de cuentas de cada objeto involucrado, estudiamos la relación entre la cantidad de cuentas que se tiene y el valor que toma $\Gamma$. Esto es, cómo se comporta $\Gamma$ respecto a las diferencias de magnitud entre los objetos involucrados. Cuando el AGN resulta ser el objeto más brillante respecto de las estrellas de comparación y control, el valor de $\Gamma<1$, al igual que cuando la magnitud del AGN se encuentra en medio entre la magnitud de la estrella de comparación (siendo más brillante) y la de control (siendo la más débil). En cambio, si el AGN es el más débil de los objetos, o si la magnitud del AGN vuelve a quedar en el medio pero ahora con la estrella de control siendo la más brillante y la de comparación más débil, el valor de $\Gamma>1$. Luego, que $\Gamma>1$ o $\Gamma<1$ depende de la relación entre las magnitudes del AGN y de la estrella de control: si el AGN es más brillante que la estrella de control, $\Gamma<1$; caso contrario, $\Gamma>1$.

Como ejemplo, presentamos una curva de luz sintética en donde se refleja la importancia del uso de este factor. Tomamos uno de los AGNs no variable, de magnitud 17.255, junto a dos estrellas de comparación y control, de magnitudes 15.429 y 15.398, respectivamente. La curva corresponde a una situación de IQ1, SB5 y CC5 (ver Sec. 2.4.2 para el detalle de cada una de las situaciones). Tomando los parámetros C y F sin tener en cuenta el factor de peso $\Gamma$, ambos detectan variabilidad en la curva, siendo sus valores $\mathrm{C}=2.721$ y areaF $=1.000$. En este caso, $\Gamma=3.526$. Cuando se incluye el factor de peso, ambos parámetros devuelven el mismo estado de variabilidad original del AGN, es decir, el criterio $\mathrm{C}$ y el test $\mathrm{F}$ no detectan variabilidad en la curva. Sus valores pasan a ser $\mathrm{C}=0.772$ y areaF $=0.890$. En la Fig. 2.10, se muestra cómo son las curvas del AGN y de control para este caso.

Por otra parte, de los resultados de los casos testigo, en particular para los AGNs 


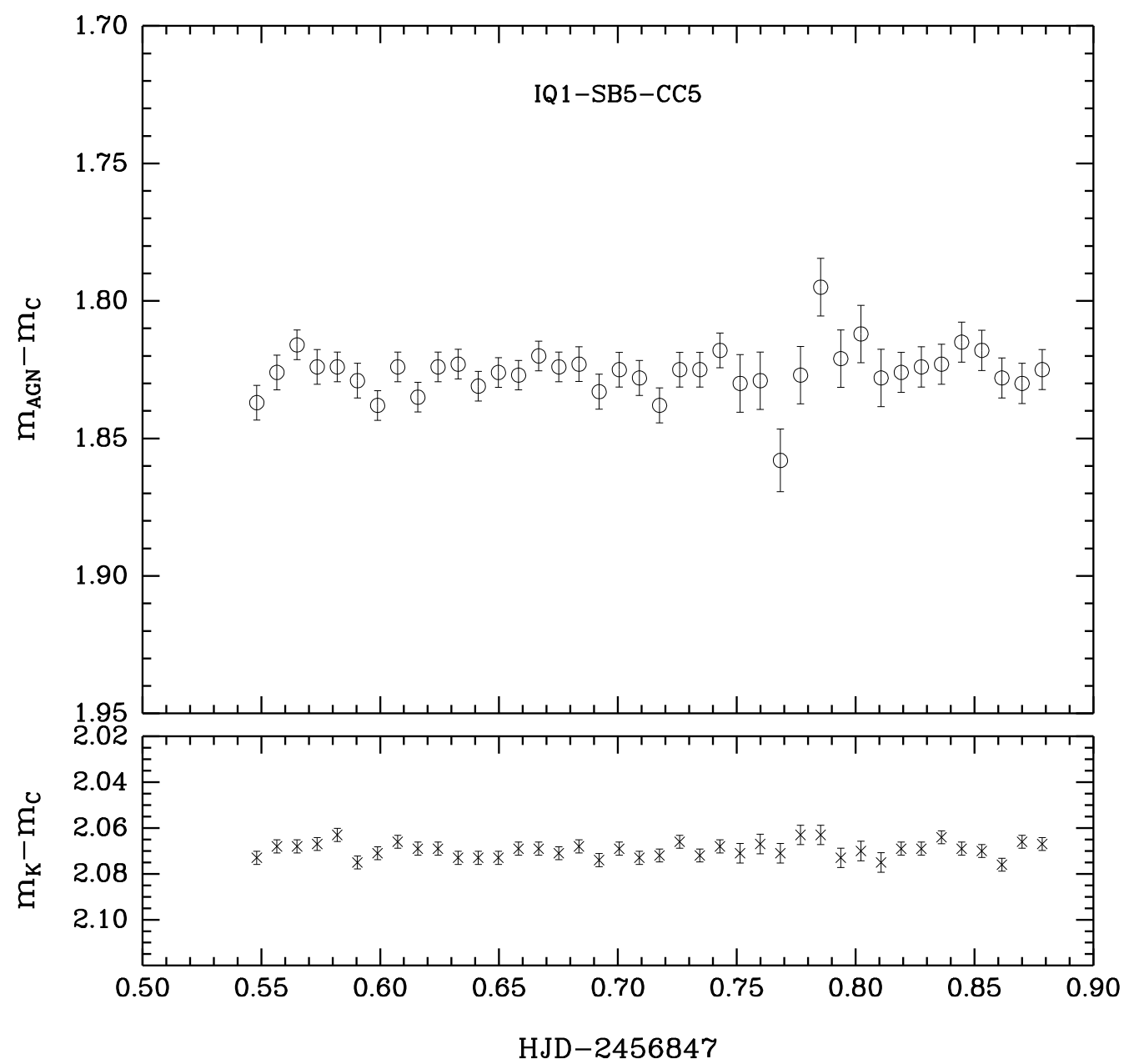

Figura 2.10. Ejemplo de una curva de luz diferencial para un AGN no variable (arriba), junto a la curva de control (abajo). El despliegue tiene un offset para poder visualizar mejor ambas curvas.

no variables, obtuvimos los porcentajes de casos variables y no variables que detectan ambos parámetros, teniendo o no en cuenta el factor de peso. Recordemos que, dentro de las restricciones al criterio de Howell, estamos considerando como máximo una diferencia en magnitud entre los objetos de $\Delta m=0.5$ mag. De este análisis, observamos que el parámetro C recupera el estado de no variabilidad de los AGNs en un $100 \%$, mientras que el test F detecta DLCs variables. En estos casos, la diferencia numérica entre no considerar y considerar el peso en la estadística cae en un orden de magnitud. A su vez, este mismo comportamiento ocurre cuando vamos de los casos de H01 a H05. Por ejemplo, considerando el caso testigo CT5 con el criterio H05, sobre un total de 21193 DLCs, se detectan 309 DLCs variables aplicando el test F sin el peso (1.46\%), cayendo este número a 21 DLCs si se considera el peso $(0.1 \%)$. Estos valores crecen cuando consideramos las situaciones en donde se ven involucradas las distintas condiciones atmosféricas. Como ejemplo, si tomamos el caso IQ4-SB1-CC3, con el criterio H03 (siempre considerando AGNs no variables), de un total de 27833 DLCs, 3224 son 
clasificadas como variables con el test $\mathrm{F}$ sin el peso (11.6\%), mientras que este número disminuye a 722 DLCs cuando se considera el peso $(2.6 \%)$.

Como complemento, estudiamos estos porcentajes en el caso de no contemplar restricción alguna en el criterio de Howell. Esto es, la diferencia en magnitud de los objetos no tiene ni cota mayor ni cota menor, se toman todas las posibles combinaciones. El número total de DLCs obtenidas por cada AGN es de 3906. Los resultados los mostramos en la Tabla 2.3. Tomamos 3 AGNs diferentes, mostrados en la columna 1, los cuales resultan ser el más brillante, el más débil y uno intermedio. En las columnas 2 a la 5 están los resultados del parámetro $\mathrm{C}$ sin pesar, tanto no variable (NV) como variable $(\mathrm{V})$ y el parámetro con el peso $(\mathrm{CW})$, tanto no variable como variable. En el mismo sentido, de las columnas 6 a la 9 mostramos los valores para la estadística F sin pesar, considerando los no variables y los variables, y la estadística pesada (FW) tanto variable como no variable.

Tabla 2.3. Cantidad de DLCs que resultaron variables y no variables con los parámetros $\mathrm{C}$ y $\mathrm{F}$ en el $\mathrm{CT} 1$, sin considerar ninguna restricción sobre el criterio de Howell. Se presenta en la columna 1 el AGN involucrado con su respectiva magnitud, en las columnas 2, 3, 4 y 5 la información del total de DLCs que resultaron no variables y variables con el parámetro $\mathrm{C}$ sin pesar y con el peso $(\mathrm{CW})$, en las columnas $6,7,8$ y 9 las DLCs que resultaron no variables y variables con la estadística F, tanto sin el peso como con el peso (FW). Para cada AGN, se obtuvo un total de 3906 DLCs.

\begin{tabular}{|c|c|c|c|c|c|c|c|c|}
\hline \hline AGN & C NV & C V & CW NV & CW V & F NV & F V & FW NV & FW V \\
\hline \hline$\# 1(16.0 \mathrm{mag})$ & 3906 & 0 & 3906 & 0 & 3848 & 58 & 3897 & 9 \\
\hline$\# 100(16.5 \mathrm{mag})$ & 3906 & 0 & 3906 & 0 & 2645 & 1261 & 3878 & 28 \\
\hline$\# 200(17.0 \mathrm{mag})$ & 3885 & 21 & 3906 & 0 & 1236 & 2670 & 3904 & 2 \\
\hline
\end{tabular}

En primer lugar observamos que, para el parámetro C sin tener en cuenta el factor de peso, aparecen DLCs clasificadas como variables sólo en el caso en que el AGN es el objeto más débil de todos, mientras que en los restantes dos, más todos los casos donde se involucra el peso, se recupera el $100 \%$ de la no-variabilidad presente en el AGN. En cambio, para el test F, se puede ver que, más allá de tener involucrado o no al factor de peso en la estadística, siempre se tienen DLCs clasificadas como variables. El test F resulta más sensible en la clasificación de las DLCs como variables. Además, a medida que pasamos de considerar del AGN más brillante al más débil, es mayor la cantidad de DLCs que se clasifican como variables si no se tiene presente al factor de peso de Howell. En particular, cuando el AGN es el objeto más débil, sobre un total de 3906 DLCs, 2670 resultan variables (68.35\%) sin el peso, mientras que este número desciende a $2(0.05 \%)$ cuando se involucra al factor de peso. Por otro lado, respecto al comportamiento de los valores de los parámetros con el peso, lo podemos asociar con lo que analizamos respecto al valor de $\Gamma$ y cómo es la relación entre las magnitudes del AGN, estrella de comparación y estrella de control. Para el primer caso, donde el AGN es el más brillante, vimos que siempre el valor de $\Gamma<1$. Con lo cual, dado que $\Gamma$ escalea al valor de $\sigma_{2}$ en el denominador de ambos parámetros, es lógico que se tengan valores 
mayores para los parámetros $\mathrm{C}$ y $\mathrm{F}$, lo que se traduce en un número mayor de DLCs variables. Si en cambio, vamos al caso contrario, en donde el AGN es el objeto más débil, obtuvimos que $\Gamma>1$ siempre. Esto implica que se obtienen valores de los parámetros C y $\mathrm{F}$ más chicos y la cantidad de DLCs clasificadas como variables disminuye. Por último, en el caso en el que AGN tiene un valor intermedio en su magnitud, se van a tener casos en donde el AGN es más brillante que las estrellas, o más débil, o estará en el medio. En esos casos podemos llegar a tener tanto $\Gamma<1$ como $\Gamma>1$ y es por eso que el número de casos de DLCs variables es mayor. Esto se refleja en el comportamiento del test F con el peso (columna 9 de la Tabla 2.3).

En la Fig. 2.11 muestran las distribuciones del factor de peso de Howell para los AGNs no variables y variables, respectivamente, para el caso testigo 1 (CT1). Las distribuciones presentan la misma forma sin importar en qué caso testigo sea el considerado, ya sea para los AGNs no variable como para los variables. Estos resultados fueron corroborados por los resultados obtenidos en los tests de KS aplicados. Es decir, resultan invariantes ante un cambio en el valor del seeing. En el caso de la Fig. 2.11 (panel superior), se obtiene lo esperado para cada restricción del criterio, en donde la distribución del criterio más estricto (H01) tiene un pico alrededor de $\Gamma=1$ y se concentra en un rango de valores $\Gamma=1 \pm 0.1$. A medida que el criterio es menos estricto, las distribuciones se alejan de $\Gamma=1$ y se dispersan más. Con respecto a los AGNs variables (Fig. 2.11, panel inferior) la forma de la distribución es similar para las distintas variabilidades contempladas, teniendo el pico en un valor de $\Gamma \sim 1.1$. Sin embargo, para los casos particulares de flickering, la distribución se extiende a valores mucho más altos que las otras variabilidades, llegando a $\Gamma \sim 2.6$. En el caso de las variabilidades no se ha aplicado restricciones al criterio de Howell.

\subsection{Resultados y análisis}

Para poder analizar el gran volumen de resultados que arrojan las simulaciones, generamos una serie de gráficos que nos sean útiles para poder realizar comparaciones entre las distintas situaciones planteadas.

1. $\sigma_{2}$ vs. $\Gamma$ : muestra cómo es el comportamiento de la dispersión de la curva de control ( $\sigma_{2}$, la cual es, a su vez, una medida del error) frente a los valores que toma el factor de peso de Howell $(\Gamma)$.

- AGNs no variables: se grafica dicho comportamiento para cada una de las 3 restricciones impuestas al criterio de Howell (H01, H03 y H05).

- AGNs variables: se grafican cada una de las variabilidades consideradas.

2. $\sigma_{1}$ vs. $\sigma_{2}$ : relación entre las dispersiones de las curvas del objeto y de control. Estas son las cantidades involucradas en la construcción de los parámetros estadísticos $\left(\sigma^{2}\right.$ en el test $\left.\mathrm{F}\right)$.

- AGNs no variables: se muestra el comportamiento de ambas dispersiones para cada una de las restricciones. Se grafica teniendo en cuenta el valor 

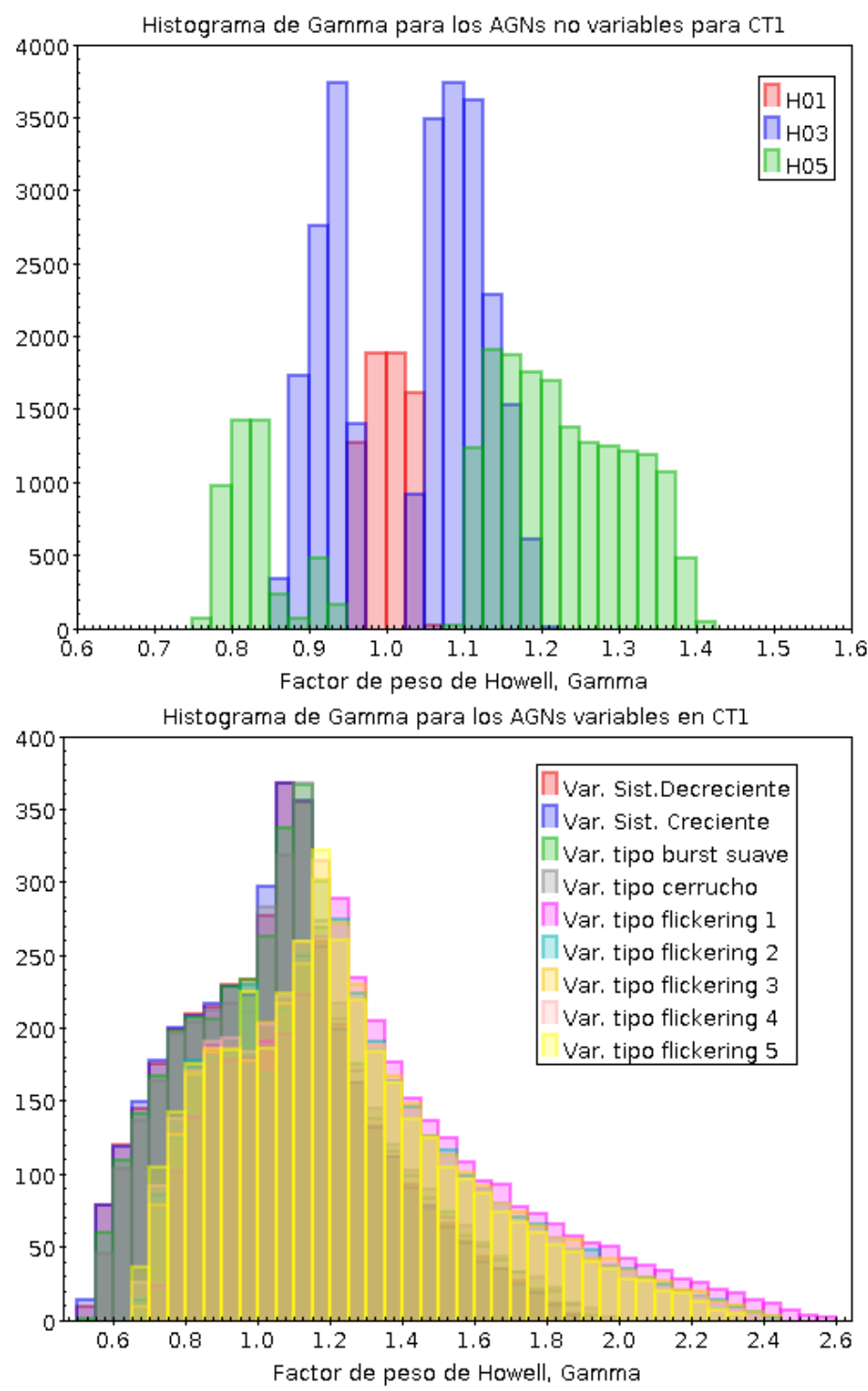

Figura 2.11. Histograma del factor de peso de Howell, $\Gamma$ para los AGNs no variables (panel superior) y para los AGNs variables (panel inferior), en el caso CT1.

de la magnitud que toma el AGN, para poder ver cómo se distribuyen las magnitudes en esta relación.

- AGNs variables: se muestran cada una de las distintas variabilidades.

3. Distribución de $\sigma_{1}$ : histograma para los diferentes estados planteados para los AGNs (tanto variables como no variables) de los valores de $\sigma_{1}$.

4. Distribución de $\sigma_{2}$ : histograma para los diferentes estados planteados para los AGNs (tanto variables como no variables) de los valores de $\sigma_{2}$.

5. Distribución de $\Gamma$ : histograma para los diferentes estados planteados para los AGNs (tanto variables como no variables) de los valores de $\Gamma$. 


\subsubsection{Comparación entre casos testigos}

En la Fig. 2.12 se muestran los valores del factor de peso $\Gamma$ en función de la dispersión de la curva de control, $\sigma_{2}$, para el caso de los AGNs no variables. Para los 5 casos testigo, no se encuentran grandes diferencias en la distribución de valores de $\Gamma$, donde éstos se esparcen desde 0.7 hasta 1.4. Además, dentro de cada criterio de Howell, los valores que toma el factor de peso se encuentran en el mismo rango. Es decir, el criterio más estricto (H01) se mantiene dentro del rango [0.9:1.1], en cualquiera de los 5 casos testigo. Lo mismo sucede con el criterio H03 y H05. Lo que sí cambia es la distribución de valores de la dispersión. Con los distintos casos testigo, el valor del seeing es fijo pero va en aumento, esto es, a medida que va empeorando el seeing de un caso al otro, $\sigma_{2}$ toma valores cada vez más grandes: como es esperable, las curvas son más ruidosas. Esto se debe al hecho de que, cuando el seeing aumenta, la cantidad de flujo que cae dentro del radio de apertura tomado para el objeto es menor (ya que el seeing tiende a desparramar la luz en el frame).

En cuanto a la distribución del valor de la dispersión de la curva de control, vemos que estos valores toman el mismo rango que en el caso del gráfico $\sigma_{2}$ vs. $\Gamma$. Es decir, a medida que se consideran valores mayores del seeing, la distribución en $\sigma_{2}$ se expande a valores mayores. La diferencia que se destaca entre los 5 histogramas es el pico de la distribución del criterio de Howell H03, destacándose principalmente en el CT3. Que haya una mayor cantidad de DLCs en el criterio H03 respecto a H01 y H05 puede explicarse por cómo se van poblando estos 3 grupos de acuerdo a los valores que va tomando $\sigma_{2}$. Dado que el valor del seeing aumenta, es menos la cantidad de fotones que ingresan en el radio de apertura tomado para la fotometría. Esto lleva a que, dentro del radio, se tenga menos flujo y por ende una magnitud instrumental más débil. Al verse afectadas las magnitudes, se verá afectado el $\Gamma$ y aquellos objetos que antes podían cumplir con el criterio más estricto de Howell (H01), ahora poseen magnitudes más débiles (lo que se traduce a valores más grandes de la magnitud), haciendo que caigan en el grupo siguiente. Aquellos que quedan afuera del H01, entrarán al H03, y algunos de los que pertenecen al H03 se moverán al H05, pero este último grupo no va a estar más poblado que el H03, dado que por la misma razón explicada antes, las magnitudes se debilitan y habrá casos que queden fuera del H05, los cuales no fueron considerados.

Para visualizar las distribuciones de las dispersiones, tanto $\sigma_{1}$ como $\sigma_{2}$, se tienen los gráficos de las Fig. 2.13, 2.14 y 2.15. En estos gráficos se muestra la relación entre las dispersiones de las curvas del AGN y de control, en función de la magnitud que posee el AGN. El valor de la magnitud del AGN está representada por la paleta de colores, en donde el color verde indica al AGN más brillante (con un valor de $16.1 \mathrm{mag}$ ) y el color amarillo indica el AGN más débil (con magnitud $17.2 \mathrm{mag}$ ). Para los casos testigos, estos gráficos están confinados dentro de los rangos [0.001:0.01] para $\sigma_{1}$ y [0.002:0.009] para $\sigma_{2}$. Comparando entre los 3 criterios de Howell, vemos que a medida que se va relajando el criterio, se encuentran casos que involucran AGNs más débiles. Por otro lado, dentro de cada criterio de Howell, se puede ver que cuanto más alto es el valor del seeing, aparecen más objetos débiles. Y a su vez estos objetos se van expandiendo mucho más a lo largo del gráfico. Es decir, el rango de valores tanto de $\sigma_{1}$ como de $\sigma_{2}$ se extiende. Este resultado es esperable debido a que las curvas se vuelven más ruidosas a medida que se consideran valores mayores de seeing.

Una manera de ver todos los efectos juntos (esto es, aumento de seeing y relajación 

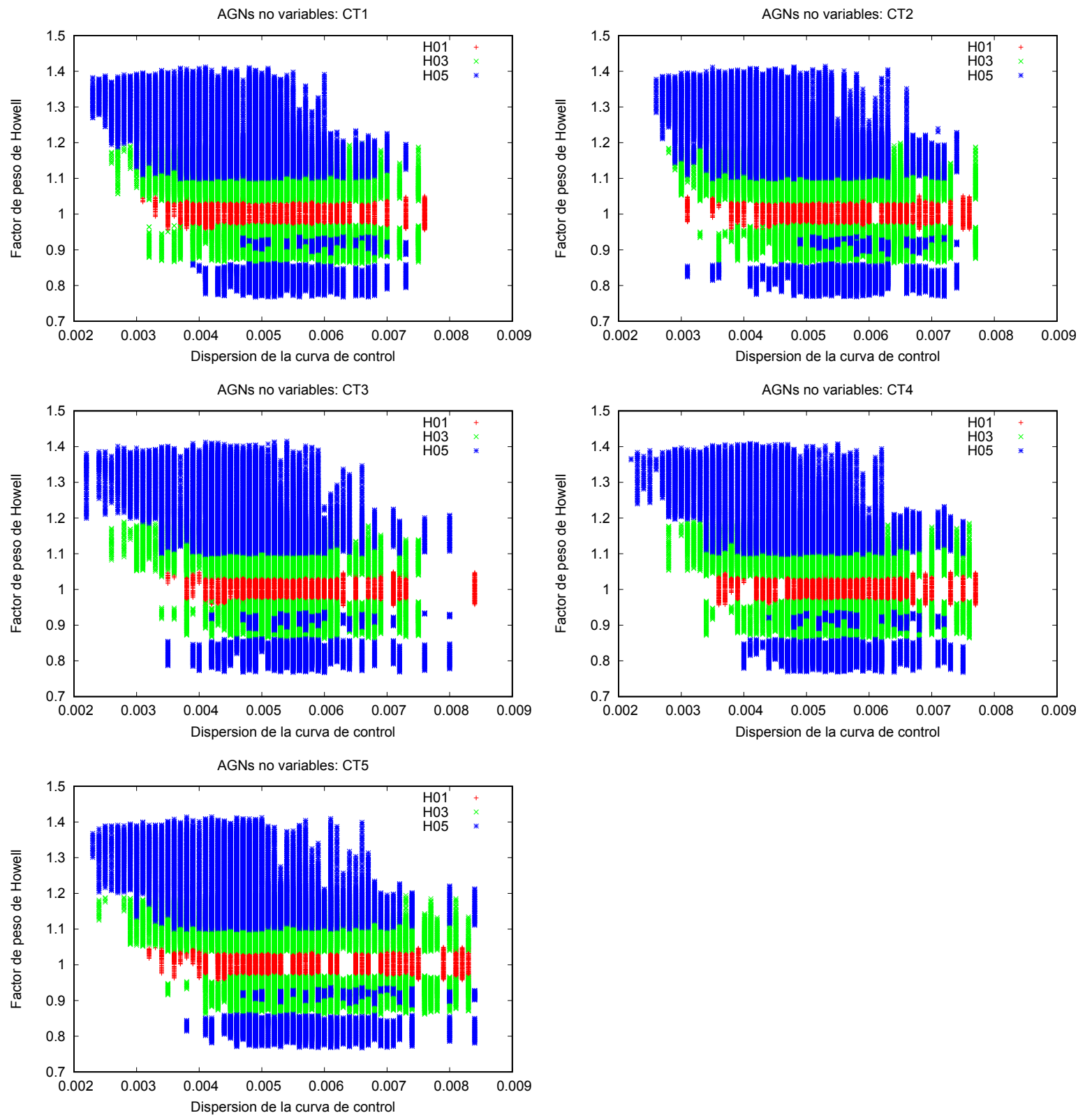

Figura 2.12. Comportamiento de la dispersión de la curva de control frente al valor del factor de peso de Howell, $\Gamma$, para las distintas restricciones del criterio de Howell, en los casos CT1 (panel superior izquierdo), CT2 (panel superior derecho), CT3 (panel del medio izquierdo), CT4 (panel del medio derecho) y CT5 (panel inferior).

del criterio de Howell) es comparar el gráfico de la Fig. 2.13 (panel superior izquierdo, H01 para CT1) con el gráfico de la Fig. 2.15 (panel inferior, H05 para CT5). Éstos representan los extremos de las situaciones simuladas. Comparando ambos gráficos, vemos primero que el rango de valores de ambas dispersiones aumenta en el caso de H05 para CT5. Segundo, la distribución de colores es diferente, teniendo no sólo una mayor cantidad de objetos débiles en el caso menos estricto, sino que además los colores presentan mayores dispersiones. Esto se traduce en que, dentro de un rango acotado 
de valores de $\sigma_{1}$ y $\sigma_{2}$ (sobretodo en la región central del gráfico), se pueden tener tanto AGNs brillantes como débiles. Estos AGNs con diferentes magnitudes tienen valores muy similares en sus dispersiones y en las dispersiones de sus curvas de control, haciendo que los parámetros $\mathrm{C}$ y $\mathrm{F}$ resulten similares en ambos AGNs (mismos resultados del estado de variabilidad), si no se considerara el factor de peso de Howell.

Las variaciones presentes en los valores de las dispersiones y de las magnitudes de los objetos se verán reflejados en la aplicación de las herramientas estadísticas (esto es, los valores que tomen los parámetros $\mathrm{C}$ y $\mathrm{F}$ en cada caso). En cuanto al H01, se puede ver que en los casos testigo con los menores valores del seeing (CT1 y CT2) todas las DLCs son clasificadas como no variables con ambos parámetros. Cuando se sigue aumentando el valor del seeing, a partir del CT3 (2.0 arcsec) en adelante (CT4 y CT5), empiezan a aparecer curvas que son clasificadas como variables, pero sólo con la estadística F.

Para el criterio de Howell de H03, se tiene que en todos los casos testigo se registran DLCs variables. Cabe destacar, nuevamente, que en el caso del parámetro C, se recupera el $100 \%$ del estado de no variabilidad para las curvas de los AGNs no variables, sin importar el valor del seeing que se tome. En cuanto al estadístico F, ya desde el CT1 aparece un número de DLCs variables. Lo mismo sucede con el criterio más relajado de Howell (H05). En la Tabla 2.4 se muestran para cada caso testigo y cada criterio de Howell los porcentajes de variabilidad y la cantidad de DLCs sintéticas obtenidas en cada situación. Los porcentajes de DLCs variables obtenidos en los casos en los cuales se aplica el test $F$ pueden estar asociados con fluctuaciones estadísticas. Sin embargo, es notable que aún en los casos más favorables (con el menor ruido posible), el test $\mathrm{F}$ encuentra DLCs variables. Este no es el caso cuando se aplica el parámetro C. En esta caso se recupera el $100 \%$ de DLCs no variables que fue supuesto al comienzo. 

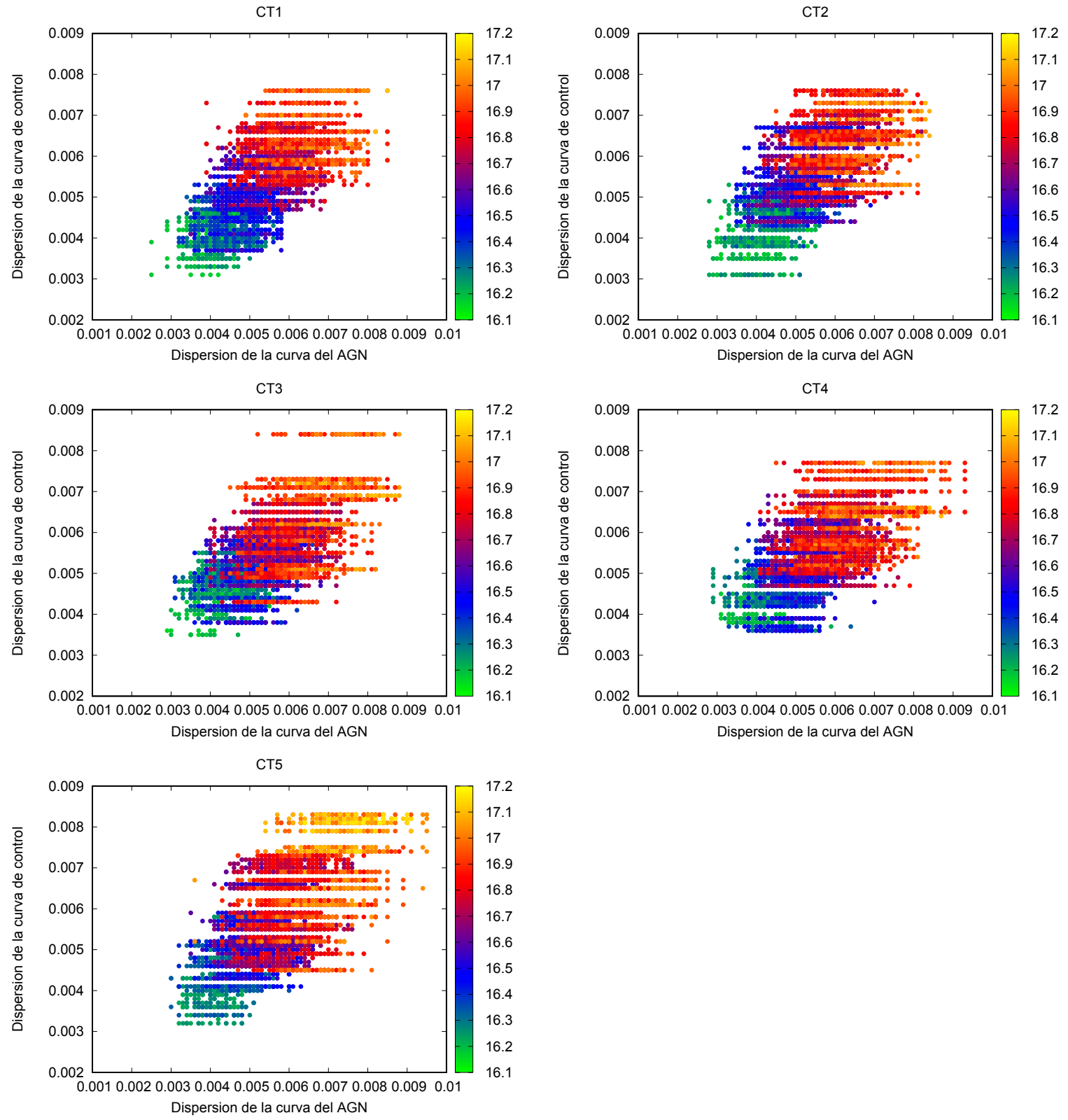

Figura 2.13. Comportamiento de la dispersión de la curva del AGN frente a la dispersión de la curva de control para H01, en los casos CT1 (panel superior izquierdo), CT2 (panel superior derecho), CT3 (panel del medio izquierdo), CT4 (panel del medio derecho) y CT5 (panel inferior). 

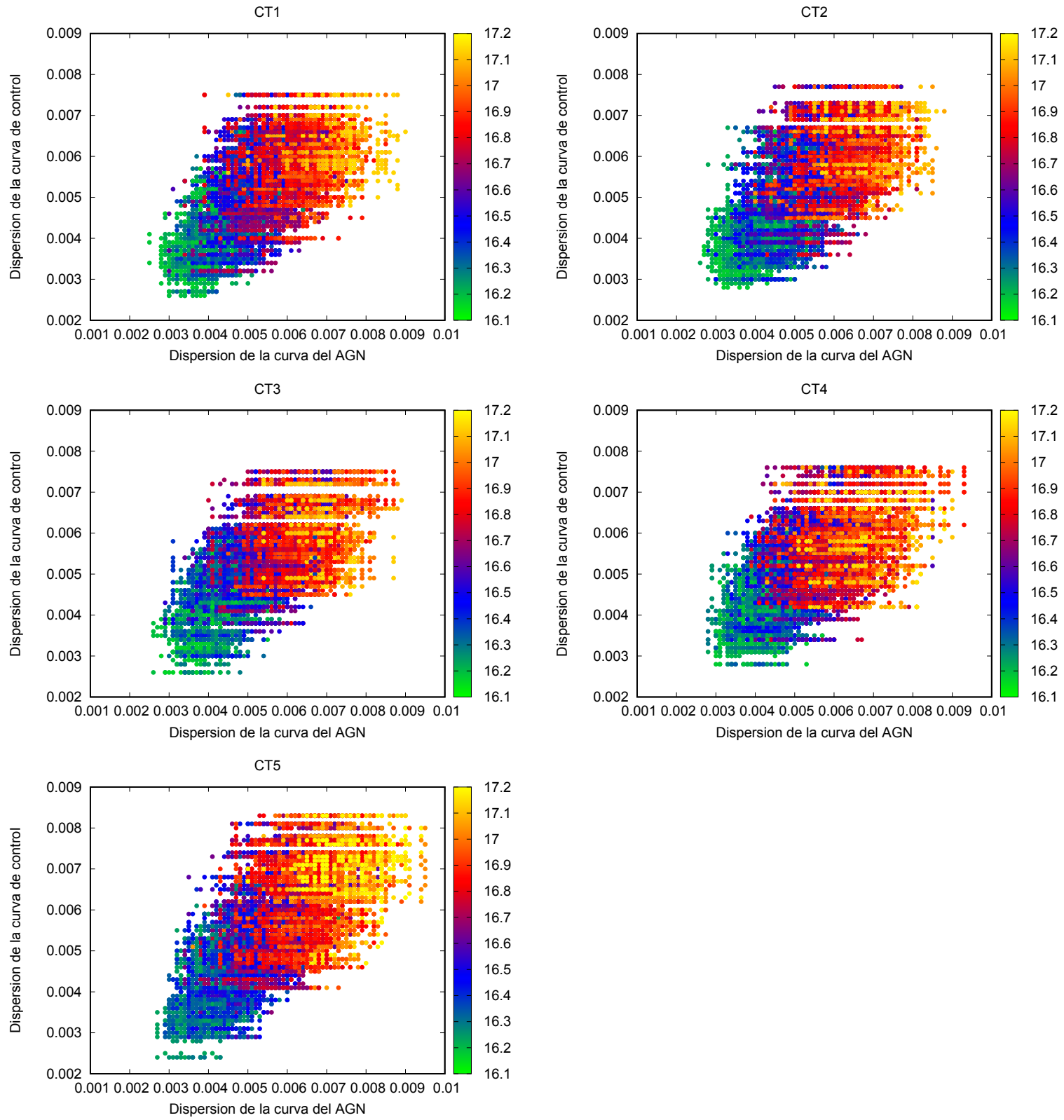

Figura 2.14. Comportamiento de la dispersión de la curva del AGN frente a la dispersión de la curva de control para H03, en los casos CT1 (panel superior izquierdo), CT2 (panel superior derecho), CT3 (panel del medio izquierdo), CT4 (panel del medio derecho) y CT5 (panel inferior). 

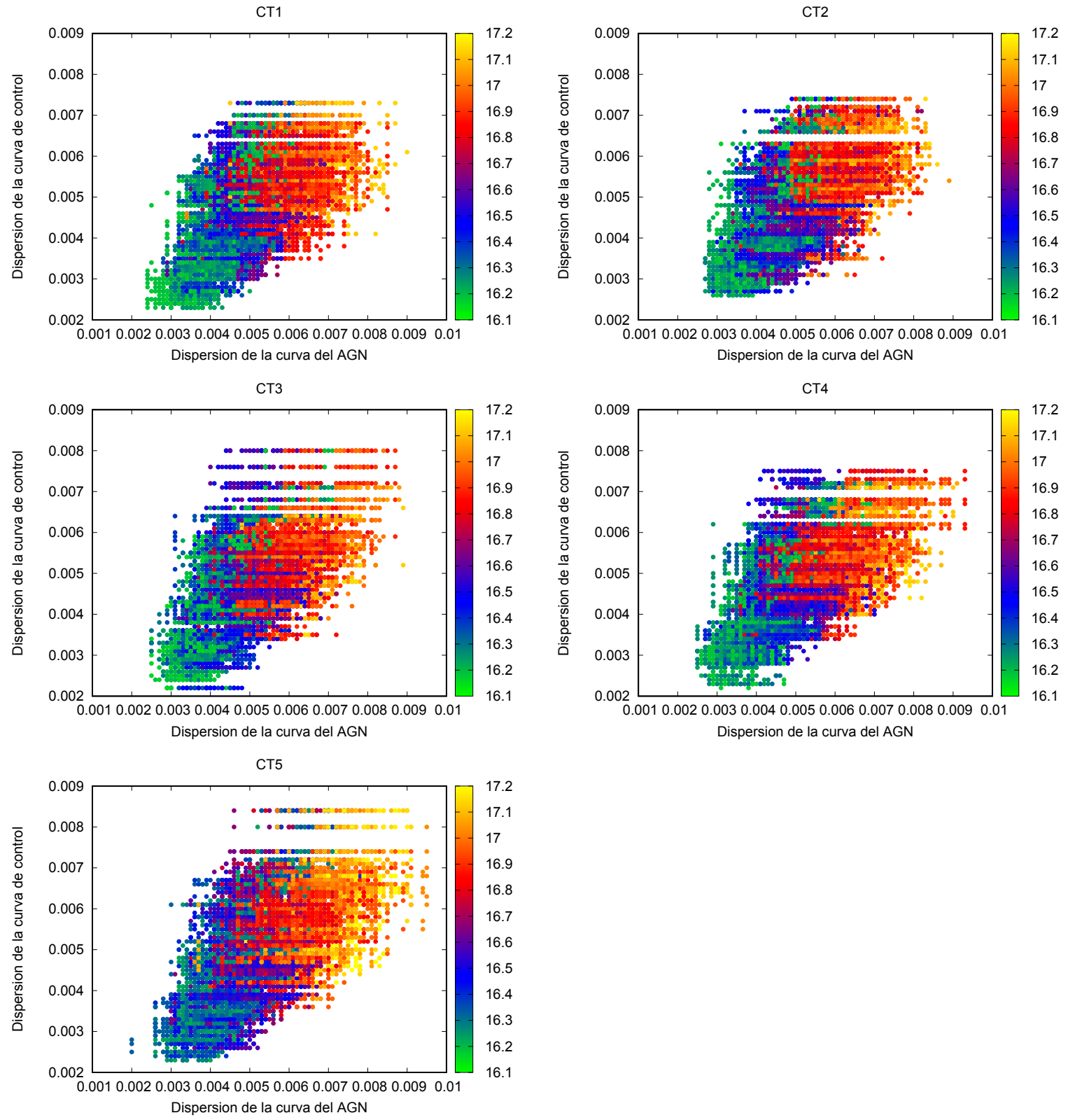

Figura 2.15. Comportamiento de la dispersión de la curva del AGN frente a la dispersión de la curva de control para H05, en los casos CT1 (panel superior izquierdo), CT2 (panel superior derecho), CT3 (panel del medio izquierdo), CT4 (panel del medio derecho) y CT5 (panel inferior). 
Tabla 2.4. Porcentajes de los casos de DLCs que resultaron variables y no variables con los parámetros $\mathrm{C}$ y $\mathrm{F}$ en cada caso testigo. Se presenta en la columna 1 el caso testigo, en las columnas 2, 3 y 4 la información del total de DLCs y los porcentajes de variabilidad para $\mathrm{C}$ y F, para el criterio de Howell H01; en las columnas 5, 6 y 7 ídem anterior pero para H03; y en las columnas 8, 9 y 10 ídem para H05.

\begin{tabular}{|c|c|c|c|c|c|c|c|c|c|}
\hline \multirow[t]{2}{*}{ Caso testigo } & \multicolumn{3}{|c|}{ H01 } & \multicolumn{3}{|c|}{ H03 } & \multicolumn{3}{|c|}{$\mathrm{H} 05$} \\
\hline & Total DLCs & $\mathrm{C}$ & $\mathrm{F}$ & Total DLCs & $\mathrm{C}$ & $\mathrm{F}$ & Total DLCs & $\mathrm{C}$ & $\mathrm{F}$ \\
\hline CT1 & 66663 & $\begin{array}{c}100 \% \mathrm{NV} \\
0 \% \mathrm{~V}\end{array}$ & $\begin{array}{c}100 \% \mathrm{NV} \\
0 \% \mathrm{~V}\end{array}$ & 26108 & $\begin{array}{c}100 \% \mathrm{NV} \\
0 \% \mathrm{~V}\end{array}$ & $\begin{array}{c}99.96 \% \mathrm{NV} \\
0.04 \% \mathrm{~V}\end{array}$ & 21161 & $\begin{array}{c}100 \% \mathrm{NV} \\
0 \% \mathrm{~V}\end{array}$ & $\begin{array}{c}99.92 \% \mathrm{NV} \\
0.08 \% \mathrm{~V}\end{array}$ \\
\hline CT2 & 6685 & $\begin{array}{c}100 \% \mathrm{NV} \\
0 \% \mathrm{~V}\end{array}$ & $\begin{array}{c}100 \% \mathrm{NV} \\
0 \% \mathrm{~V}\end{array}$ & 25949 & $\begin{array}{c}100 \% \mathrm{NV} \\
0 \% \mathrm{~V}\end{array}$ & $\begin{array}{c}99.98 \% \mathrm{NV} \\
0.02 \% \mathrm{~V}\end{array}$ & 21368 & $\begin{array}{c}100 \% \mathrm{NV} \\
0 \% \mathrm{~V}\end{array}$ & $\begin{array}{c}99.93 \% \mathrm{NV} \\
0.07 \% \mathrm{~V}\end{array}$ \\
\hline CT3 & 6634 & $\begin{array}{c}100 \% \mathrm{NV} \\
0 \% \mathrm{~V}\end{array}$ & $\begin{array}{c}99.93 \% \mathrm{NV} \\
0.07 \% \mathrm{~V}\end{array}$ & 25947 & $\begin{array}{c}100 \% \mathrm{NV} \\
0 \% \mathrm{~V}\end{array}$ & $\begin{array}{c}99.97 \% \mathrm{NV} \\
0.03 \% \mathrm{~V}\end{array}$ & 21453 & $\begin{array}{c}100 \% \mathrm{NV} \\
0 \% \mathrm{~V}\end{array}$ & $\begin{array}{c}99.56 \% \mathrm{NV} \\
0.44 \% \mathrm{~V}\end{array}$ \\
\hline CT4 & 6706 & $\begin{array}{c}100 \% \mathrm{NV} \\
0 \% \mathrm{~V}\end{array}$ & $\begin{array}{c}99.94 \% \mathrm{NV} \\
0.06 \% \mathrm{~V}\end{array}$ & 25369 & $\begin{array}{c}100 \% \mathrm{NV} \\
0 \% \mathrm{~V}\end{array}$ & $\begin{array}{c}99.81 \% \mathrm{NV} \\
0.19 \% \mathrm{~V}\end{array}$ & 21586 & $\begin{array}{c}100 \% \mathrm{NV} \\
0 \% \mathrm{~V}\end{array}$ & $\begin{array}{c}99.95 \% \mathrm{NV} \\
0.05 \% \mathrm{~V}\end{array}$ \\
\hline CT5 & 6659 & $\begin{array}{c}100 \% \mathrm{NV} \\
0 \% \mathrm{~V}\end{array}$ & $\begin{array}{c}99.87 \% \mathrm{NV} \\
0.13 \% \mathrm{~V}\end{array}$ & 26197 & $\begin{array}{c}100 \% \mathrm{NV} \\
0 \% \mathrm{~V}\end{array}$ & $\begin{array}{c}99.96 \% \mathrm{NV} \\
0.04 \% \mathrm{~V}\end{array}$ & 21193 & $\begin{array}{c}100 \% \mathrm{NV} \\
0 \% \mathrm{~V}\end{array}$ & $\begin{array}{c}99.01 \% \mathrm{NV} \\
0.09 \% \mathrm{~V}\end{array}$ \\
\hline
\end{tabular}


En cuanto al comportamiento en los casos de AGNs variables, se ha procedido a realizar el mismo tipo de análisis descripto anteriormente. Recordemos que para los AGNs variables se consideraron 9 tipos distintos de variabilidad (ver Sec. 2.4.1). Por un lado, considerando el comportamiento de los valores de la dispersión de la curva de control $\left(\sigma_{2}\right)$ frente al factor de peso de Howell $(\Gamma)$, vemos en la Fig. 2.16 que para los 5 casos testigo la forma del gráfico es similar: para valores del $\sigma_{2}$ bajos, los valores de $\Gamma$ se expanden en un amplio rango, desde 1.0 hasta 2.8 y a medida que va aumentando $\sigma_{2}$, los valores del factor de peso tienden a estabilizarse alrededor de $\Gamma=1.0$, entre 0.8 y 1.2. Comparando entre los distintos casos testigo, vemos que una de las principales diferencias radica en los valores que toma la dispersión de la curva de control. Conforme vamos aumentando el valor del seeing, $\sigma_{2}$ alcanza valores más grandes: en CT1, llega hasta 0.075 mientras que en CT5 se expande hasta casi 0.009. Estos valores son similares a los hallados para los AGNs no variables, dado que las estrellas de comparación y control son las mismas. Otra diferencia (más sutil) está en cómo se distribuyen los valores para cada variabilidad, de las cuales la que alcanza los mayores valores de $\Gamma$ es el flickering 1, en todos los casos. Recordemos que para estos casos de AGNs variables, no se han tomado restricciones al criterio de Howell, entonces tenemos todas las combinaciones posibles entre las estrellas de control y comparación.

En las Fig. 2.17 y 2.18 se muestran las distribuciones del valor de la dispersión de la curva del AGN para las distintas variabilidades simuladas. Para el caso de la variabilidad sistemática decreciente (Fig. 2.17, panel superior izquierdo), es posible determinar dos grupos de acuerdo a las distribuciones del valor de $\sigma_{1}$. La amplitud planteada originalmente para esta variabilidad es de 0.3 mag. Por un lado, para el CT1 la distribución se sitúa por encima de $\sigma_{1}=0.059$, con un pico en su distribución alrededor de $\sigma_{1}=0.0605$, mientras que el resto de los casos testigo se sitúan por debajo de este valor de $\sigma_{1}=0.059$. Para estas 4 distribuciones (CT2, CT3, CT4 y CT5), el pico de la distribución se encuentra entre los valores $\sigma_{1}=0.0582-0.0585$. Vemos que, para el caso testigo cuyo valor del seeing es el más bajo ( 0.8 arcsec) las curvas de luz tienen valores más altos de $\sigma_{1}$, pero a medida que el seeing crece, esta dispersión de la curva del AGN se hace más chica. Esto es esperable ya que el ruido en las curvas es más bajo cuando menor es el valor del seeing.

Con respecto a la variabilidad sistemática creciente, ésta posee una amplitud original de 0.2 mag. Los valores obtenidos de la dispersión de la curva del AGN caen en los mismos rangos que para la variabilidad sistemática decreciente, es decir, en el rango $\sigma_{1}=0.056-0.062$. Lo que se encuentra en este caso es que se tiene otro comportamiento para las distribuciones. Para los CT1, CT2 y CT5, la distribución se encuentra a valores más altos que donde se encuentran para los CT3 y CT4, ubicándose a partir de $\sigma_{1}=0.058$. Estas 3 distribuciones poseen un pico alrededor de $\sigma_{1}=0.0594$. Con respecto a CT3 y CT4, ambas distribuciones se sitúan por debajo de $\sigma_{1}=0.058$, y el pico de sus distribuciones cae en $\sigma_{1}=0.0582$ para CT3 y $\sigma_{1}=0.0578$ para CT4.

Para la variabilidad tipo burst suave, el rango de valores que toma la dispersión es un poco más chico respecto al de las variabilidades sistemáticas decreciente y creciente, situándose entre $\sigma_{1}=0.045$ y 0.050 . Esto tiene relación con la amplitud original que tiene esta variabilidad (0.15 mag), la cual es menor a las amplitudes de las otras dos variaciones (0.2 mag y $0.3 \mathrm{mag}$ ). En cuanto al comportamiento de las distintas distribuciones, podemos ver que se mantienen dentro de rangos similares de $\sigma_{1}$. Lo que 

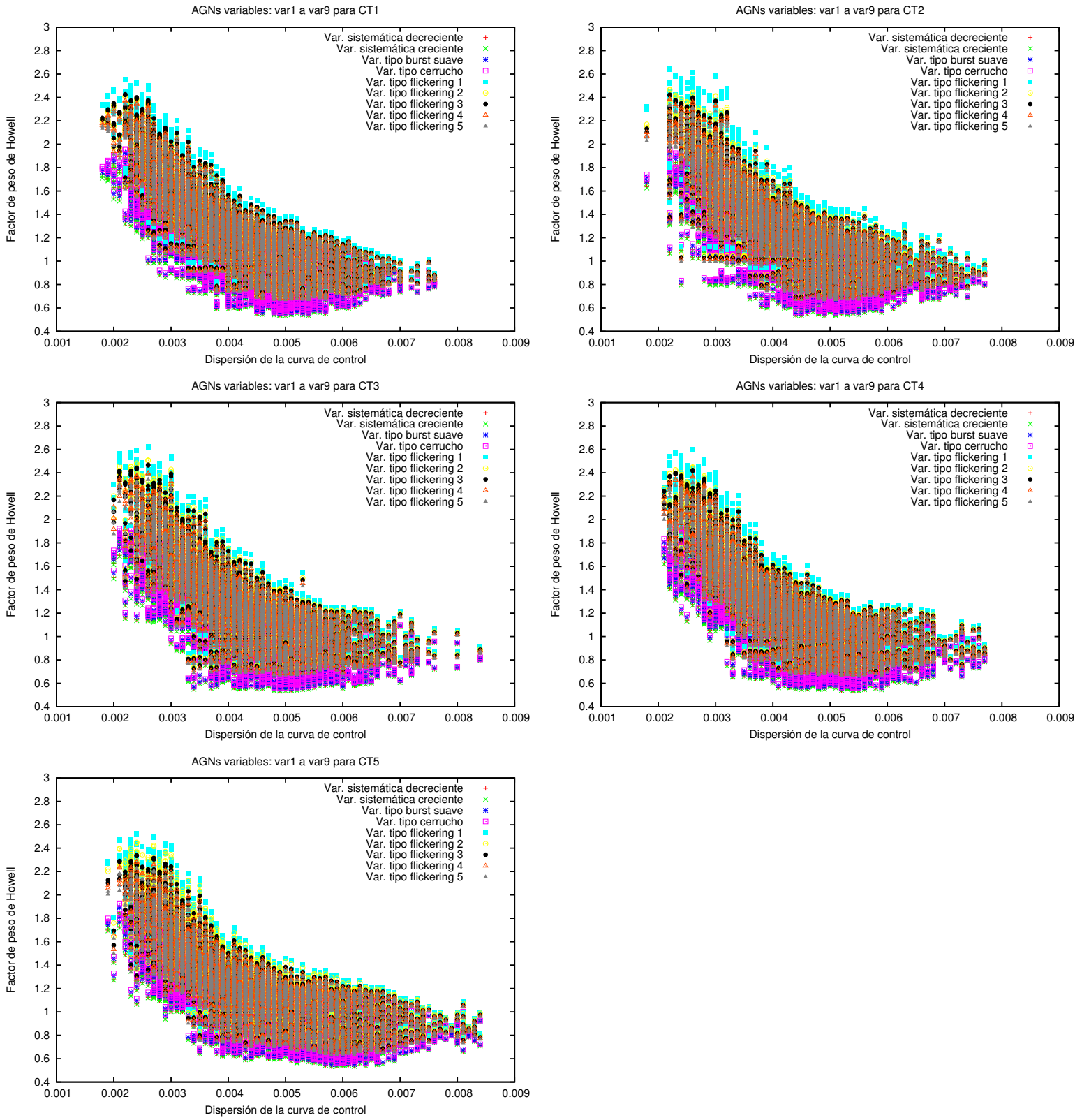

Figura 2.16. Comportamiento de la dispersión de la curva de control frente al valor del factor de peso de Howell, $\Gamma$, para las distintas variaciones de los AGNs, en los casos CT1 (panel superior izquierdo), CT2 (panel superior derecho), CT3 (panel del medio izquierdo), CT4 (panel del medio derecho) y CT5 (panel inferior). 
sí cambia es la posición del pico de su distribución. A medida que el valor del seeing crece, el pico de la distribución se va desplazando hacia valores levemente mayores de $\sigma_{1}$.

Consideramos ahora la variabilidad tipo serrucho, cuya amplitud original es de 0.15 mag. Las dispersiones toman valores aún menores respecto de las otra variabilidades mencionadas, en un rango de $\sigma_{1}=0.029$ - 0.034 . Como explicamos antes, estos valores obtenidos también se relacionan con el hecho que la amplitud original de esta variabilidad es menor y por ende se obtienen valores más bajos de $\sigma_{1}$. Respecto a la posición de las distintas distribuciones, los picos oscilan alrededor de $\sigma_{1}=0.031$.

Para el caso de los flickerings, tenemos comportamientos más extremos. Comenzando con el flickering 1 (con una amplitud original en la variación es de $0.3 \mathrm{mag}$ ), esta variabilidad tiene sus distribuciones ubicadas a los valores más altos entre todas las variabilidades, en el rango de $\sigma_{1}=0.082$ - 0.092. Se tienen diferentes comportamientos. Podríamos separarlos en tres grupos: para los casos testigo CT1 y CT2 (los casos de menor valor del seeing) tienen distribuciones correspondientes a los valores más altos de $\sigma_{1}$, con pico en 0.091; por su parte, el CT3 tiene el comportamiento opuesto, con su distribución ubicada a valores más bajos de la dispersión, con pico alrededor de 0.083 ; y finalmente los casos CT4 y CT5 (los de mayor seeing) tienen distribuciones ubicadas en valores intermedios, con picos en $\sigma_{1}=0.088$. A medida que el valor de la amplitud del flickering se achica, vemos que los valores de $\sigma_{1}$ también lo hacen. Se pueden apreciar algunas diferencias en las distribuciones de los valores de $\sigma_{1}$ para cada caso testigo dentro de cada amplitud de flickering. Las mismas están relacionadas con la amplitud de la variación original planteada y el valor de seeing en cada caso testigo. Este último es influencia directa de los errores en las curva de luz, esto es, de lo ruidosas que éstas sean.

Para los valores de la dispersión de la curva de control en los casos de AGNs variables, los valores van desde $\sigma_{2}=0.002$ hasta 0.0085 . Todas las distribuciones presentan un pico alrededor de $\sigma_{2}=0.004-0.0045$. Esto sucede debido a que se están considerando todas las combinaciones posibles entre las estrellas candidatas a control y comparación. Y esto es así para todos los casos de AGN variables por igual.

La Fig. 2.19 resume lo visto anteriormente en los histogramas presentados. Se muestra cómo es la relación entre las dispersiones de la curva del AGN y de control para cada caso testigo. Lo que es interesante de ver es la posición de cada variabilidad según el valor de seeing que se considere. En algunos casos, el valor de la dispersión de la curva del AGN permanece invariante frente a un cambio en el valor del seeing, como por ejemplo la variabilidad tipo serrucho (color rosa en los gráficos), el flickering 1 (color celeste), el flickering 4 (color naranja), la variabilidad tipo burst suave (color azul), las variabilidades sistemáticas decreciente y creciente (colores rojo y verde, respectivamente). Cuando consideramos variabilidades con amplitudes menores (como es el caso de los flickering del 2 al 5), vemos que las dispersiones de la curva de luz del AGN ya no se mantienen invariantes frente a los cambios del valor del seeing, si bien, el cambio que se observa no modifica su estado de variabilidad.

Por último, analizando los resultados de aplicar los parámetros $\mathrm{C}$ y $\mathrm{F}$ a los AGNs variables, obtenemos que para cada caso testigo y cada tipo de variabilidad, se recupera el $100 \%$ del estado de variabilidad de las DLCs. Es decir, sin importar qué valor de seeing fijo se tenga, ni qué tipo de variabilidad, ambos parámetros detectan como 

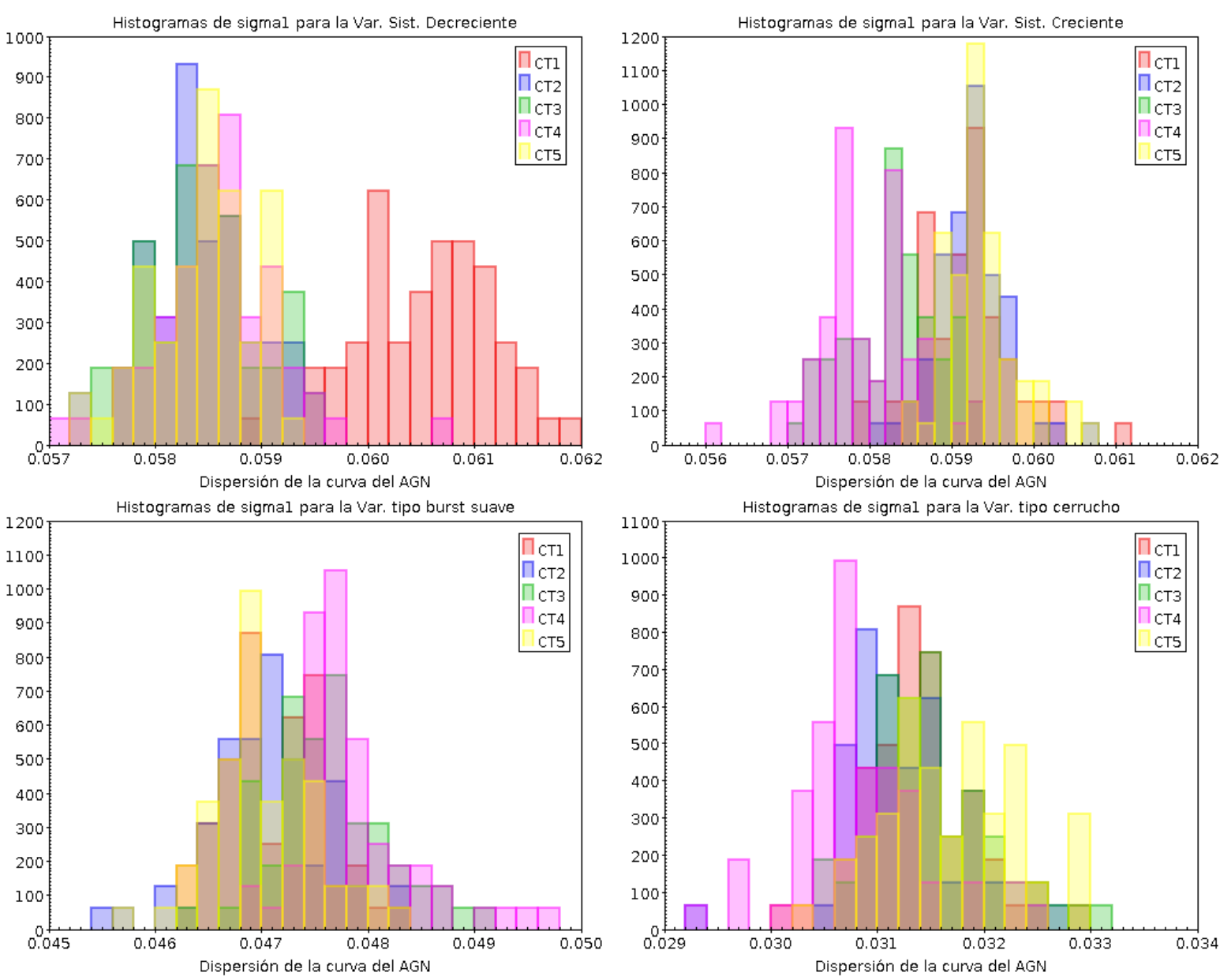

Figura 2.17. Histograma de la dispersión de la curva del AGN para la variabilidad sistemática decreciente (panel superior izquierdo), creciente (panel superior derecho), burst suave (panel inferior izquierdo) y serrucho (panel inferior derecho) en los casos CT1, CT2, CT3, CT4 y CT5.

variables el $100 \%$ de la DLCs de los AGNs variables, como es esperable. Un valor del seeing fijo a lo largo de toda la noche no afecta al estado de variabilidad del AGN, por más que ese valor del seeing sea cada vez más alto. Y además, dentro de estas situaciones ideales, ambos parámetros son robustos en sus resultados. 

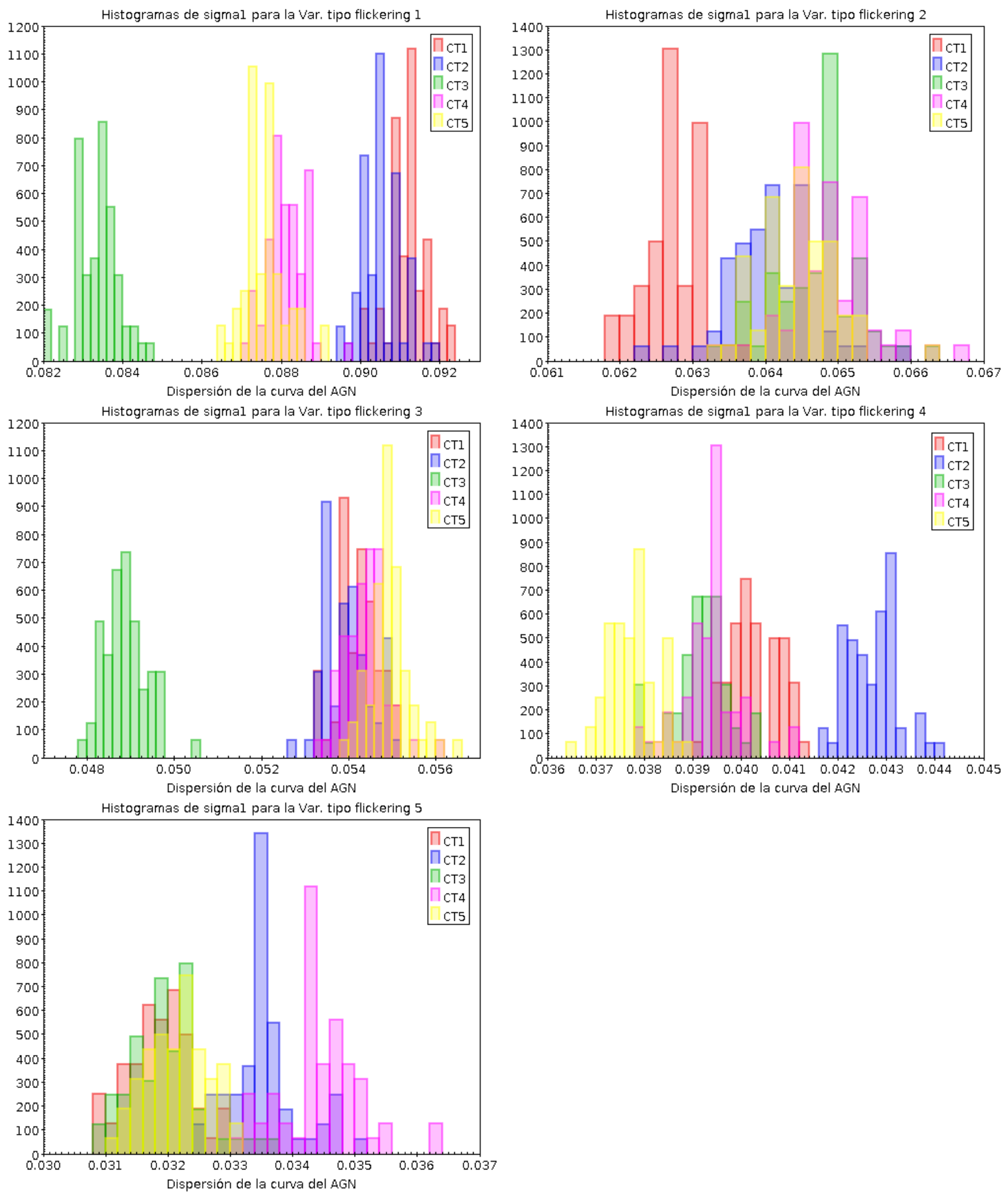

Figura 2.18. Histograma de la dispersión de la curva del AGN para las variabilidades relacionadas con los 5 flickerings en los casos CT1, CT2, CT3, CT4 y CT5. 

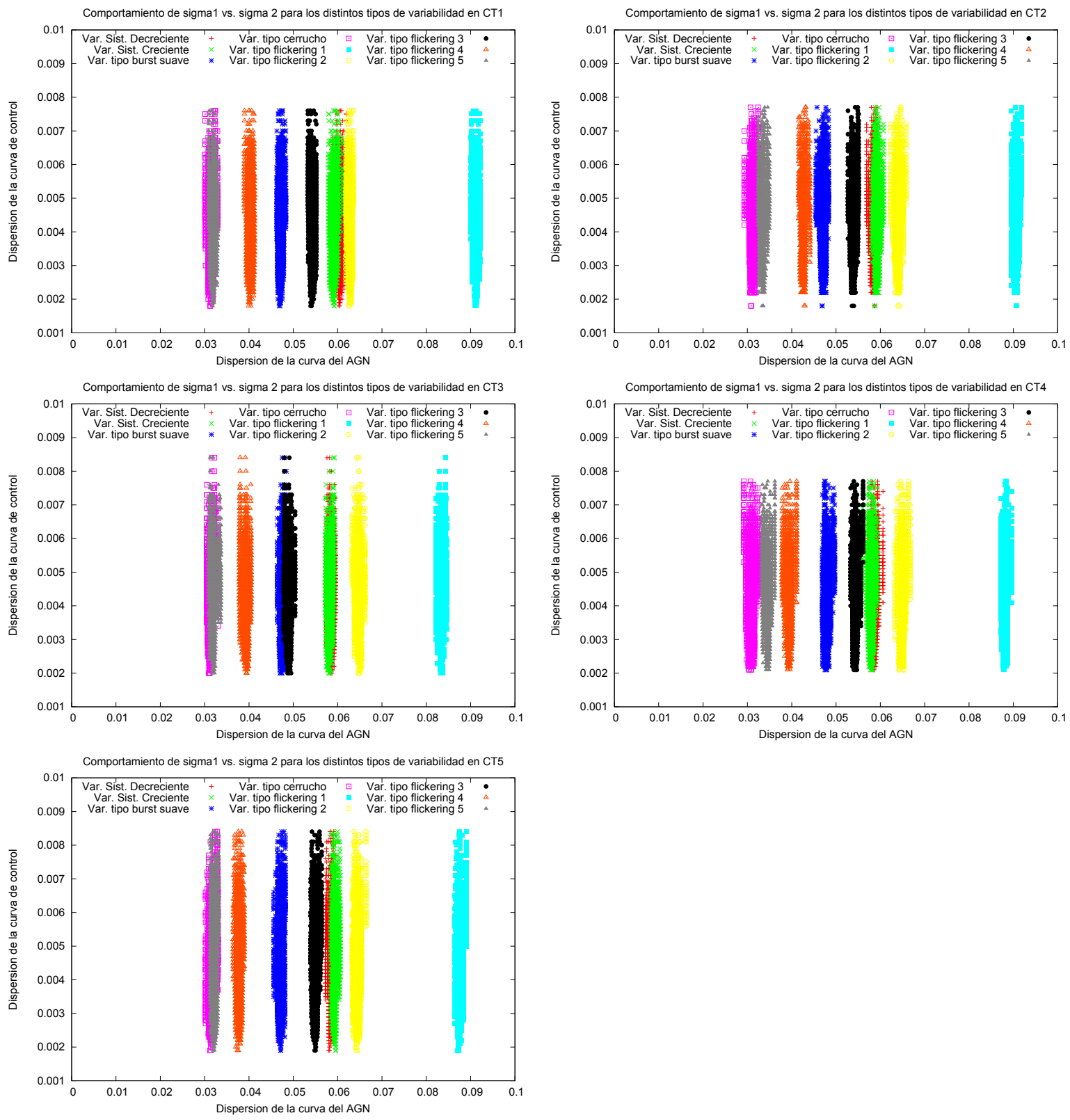

Figura 2.19. Comportamiento de la dispersión de la curva del AGN frente a la dispersión de la curva de control para las distintas variabilidades, en los casos CT1 (panel superior izquierdo), CT2 (panel superior derecho), CT3 (panel del medio izquierdo), CT4 (panel del medio derecho) y CT5 (panel inferior). 


\subsubsection{Comparación entre casos de seeing variable}

Analizamos ahora los resultados obtenidos sobre los AGNs no variables bajo cambios del valor del seeing en la noche. Al igual que para los CT presentamos los mismos tipos de gráficos, comenzando con la relación entre la dispersión de la curva de control en función del factor de peso de Howell, Fig. 2.20. Para las 5 situaciones de variación en seeing, los valores de $\Gamma$ se esparcen desde 0.60 hasta 1.55 . Comparando con los casos testigo, donde estos valores se encontraban entre 0.80 y 1.40 , para los IQ se obtiene un rango un poco más extenso. Mientras que H01 se sigue manteniendo dentro de los mismos valores para $\Gamma$ que se tenían en los $\mathrm{CT}$, los valores de $\Gamma$ para las restricciones H03 y H05 cambian respecto de los obtenidos anteriormente. Esto puede deberse al hecho de que, al tener un seeing variable, la cantidad de cuentas que caen dentro del radio de apertura tomado para la fotometría varía. Esto hace que se obtengan magnitudes más débiles o más brillantes, tanto para el AGN como para las estrellas de campo. Y estas diferencias en magnitud se reflejan en el valor del $\Gamma$. La variación del seeing a lo largo de la noche modifica las magnitudes, la relación entre ellas y por ende, se tienen valores de $\Gamma$ más chicos o más grandes, dependiendo en dónde quede el AGN en estas relaciones. Respecto a los valores de la dispersión de la curva de control, $\sigma_{2}$, se mantienen en un rango similar al encontrado para los CT, entre 0.002 hasta 0.008 , aunque para los últimos 3 casos de IQ (IQ3, IQ4, IQ5), se tienen algunos casos aislados con valores de $\sigma_{2}$ mayores. En particular, IQ3 y IQ4 pertenecen a los casos de variación de seeing con los valores más altos del mismo y representan aquellas situaciones con las peores condiciones en los valores y variación del seeing.

Las distribuciones de los valores de la dispersión de la curva del AGN, entre los 5 IQ no presentan diferencias significativas (sostenido por el test de KS). Y dentro de cada IQ, la forma de la distribución de cada criterio de Howell es similar a una distribución normal, donde H01 se expande desde $\sigma_{1}=0.003$ hasta 0.008 y manteniéndose por debajo de las 1000 DLCs; H03 tiene valores de la dispersión entre 0.003 hasta 0.009, y la cantidad de curvas llega aproximadamente a 3500; y H05, cuyos valores de $\sigma_{1}$ van desde 0.002 hasta 0.01 y llegando a las 4000 DLCs. Para el caso de las distribuciones de los valores de la dispersión de la curva de control $\left(\sigma_{2}\right)$, para todas las situaciones de IQ, los valores se encuentran en el rango que va desde 0.002 hasta 0.008 , y son distribuciones estadísticamente similares, donde nuevamente un test de KS corrobora esto. Con la excepción de IQ3 (cuya distribución tiene un pico por encima de las 4000 DLCs), el resto de las situaciones tienen sus distribuciones por debajo de las 2000 DLCs. Además, el pico de la distribución cae alrededor de $\sigma_{2}=0.005$ para todos los IQ.

Por otra parte, en cuanto a las distribuciones del factor de peso $\Gamma$, se obtiene un comportamiento esperado para cada restricción de Howell, teniendo los valores de $\Gamma$ para $\mathrm{H} 01$ distribuidos alrededor de 1.00, mientras que para el resto de las restricciones (H03 y H05) toman valores más grandes, esparciéndose entre 0.60 y 1.55. Estos rangos de valores para cada restricción de Howell es muy similar a la obtenida para los CT. Lo que se observa de diferente es la forma en la distribución respecto de las obtenidas para los CT. Mientras que en los casos testigo, la forma se asemeja a una distribución normal, para los IQ, la distribución presenta una forma más plana. Para visualizar mejor esto, presentamos en la Fig. 2.21 los histogramas de $\Gamma$ para el CT2 y para IQ1. La principal diferencia se encuentra en la distribución de valores de $\Gamma$ en la restricción de Howell H03. Elegimos estos dos casos en la comparación ya que el valor promedio 
del seeing para el caso de IQ1 es de 1.52 arcsec, lo cual es representado por el caso CT2 (1.50 arcsec).
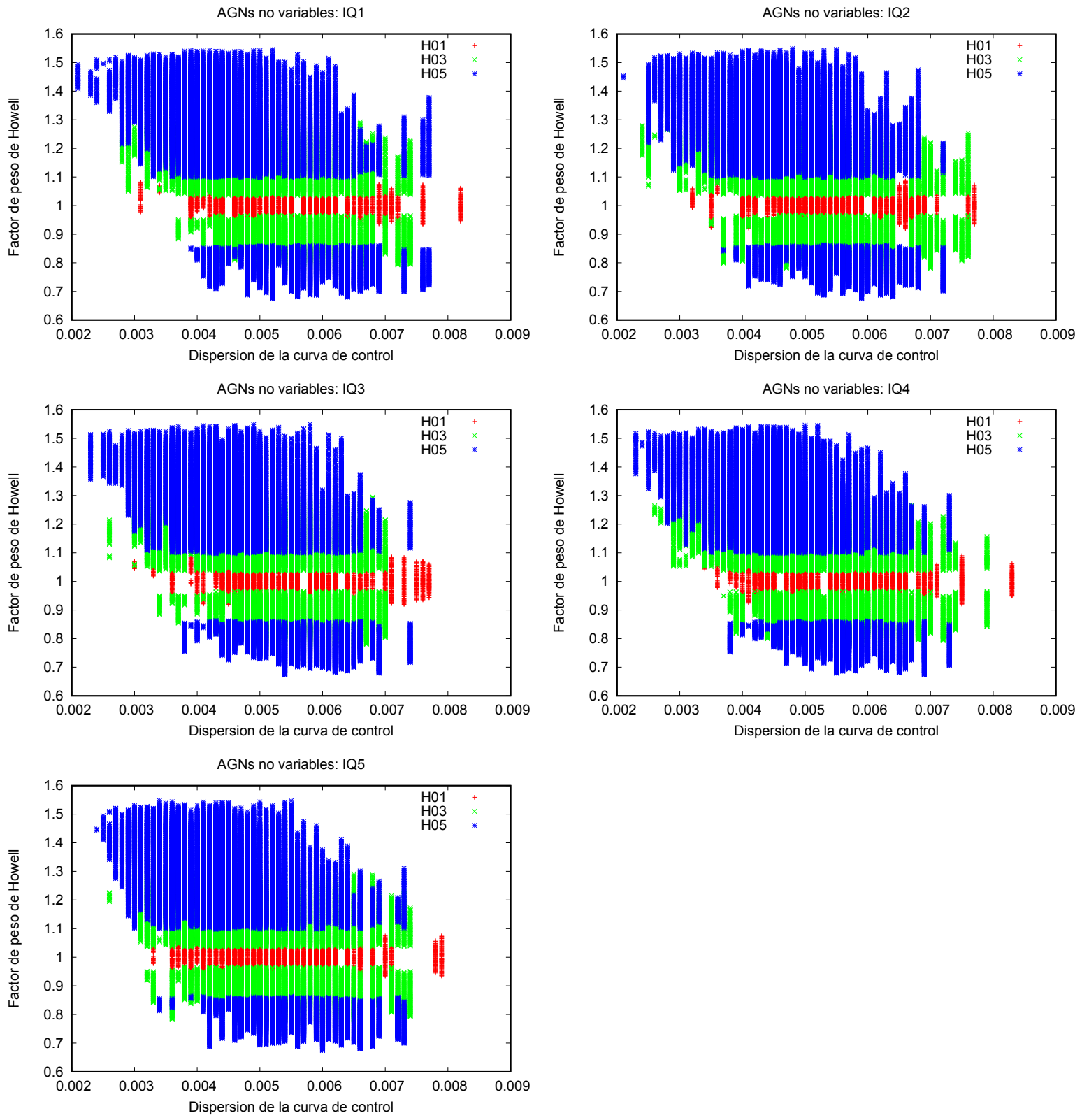

Figura 2.20. Comportamiento de la dispersión de la curva de control frente al valor del factor de peso de Howell, $\Gamma$, para las distintas restricciones del criterio de Howell, en los casos IQ1 (panel superior izquierdo), IQ2 (panel superior derecho), IQ3 (panel del medio izquierdo), IQ4 (panel del medio derecho) y IQ5 (panel inferior).

En las Fig. 2.22, 2.23 y 2.24 se muestran cómo se comportan las dispersiones de las curvas del AGN y de control frente a la magnitud del AGN, para cada criterio de Howell y para cada situación IQ. Para los distintos criterios H01, H03 y H05, obtenemos los mismos valores de las dispersiones que para los CT: cuando $\Gamma$ es cercano a uno, frente a las diferentes variaciones del seeing, aparecen más AGNs con valores intermedios de 

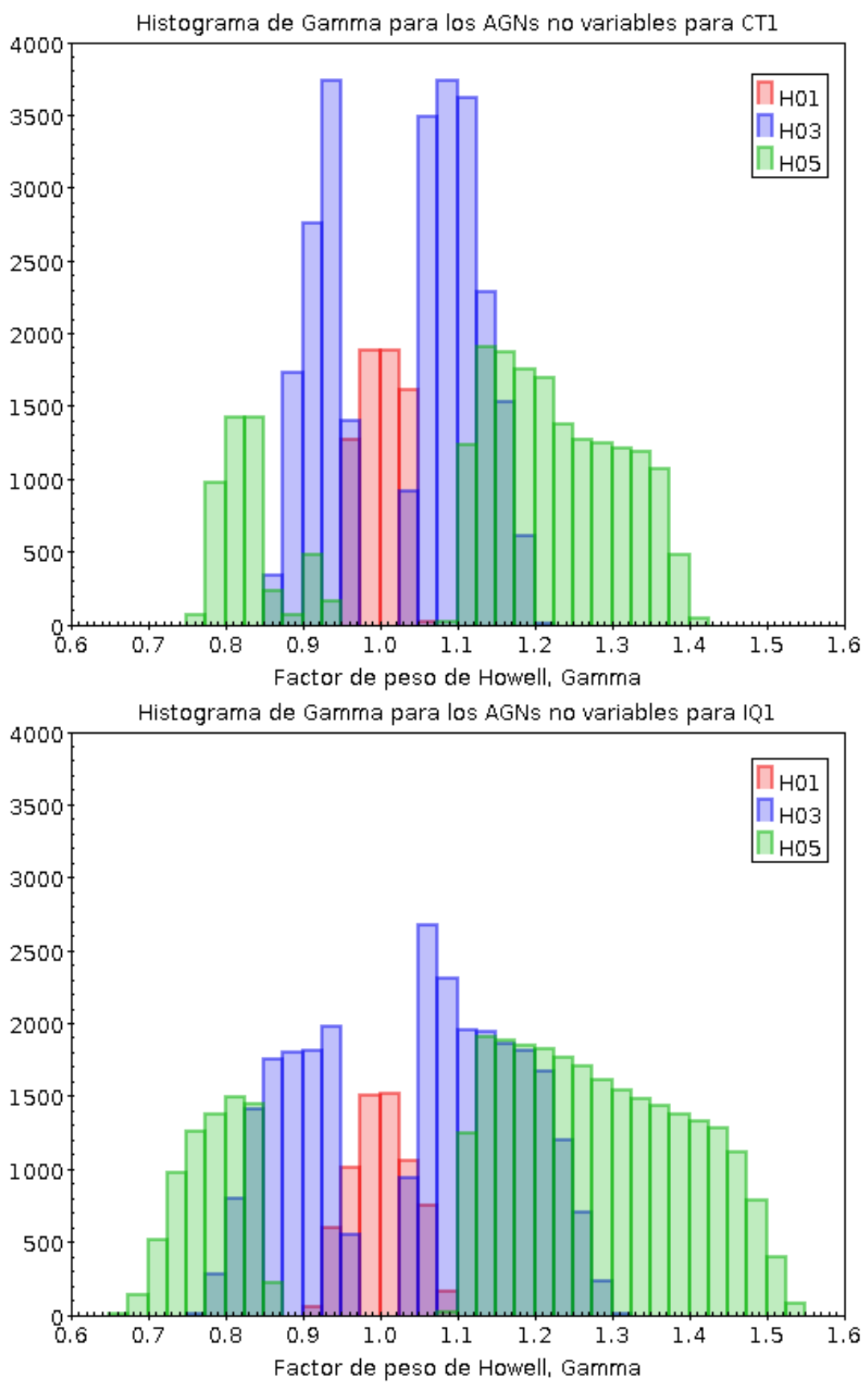

Figura 2.21. Histograma de la dispersión de la curva del AGN para H01, H03 y H05, en los casos CT1 y IQ1.

magnitud y, a medida que nos alejamos de $\Gamma=1$, se comienzan a poblar los gráficos con AGNs más débiles. Este comportamiento se acentúa aún más que en los CT cuando se estudia qué sucede en los casos de H05. Éste se debe al hecho de que el seeing es variable en todos los casos. Esto se suma al hecho de tener curvas más ruidosas por los valores de seeing en sí mismos, una componente adicional de ruido en la curva originada en la variación del valor del seeing a lo largo de la DLC.

Al analizar los resultados en la clasificación de las curvas de luz para los IQ, se observa que el parámetro $\mathrm{C}$ sigue siendo el más conservador, dado que se recupera el 

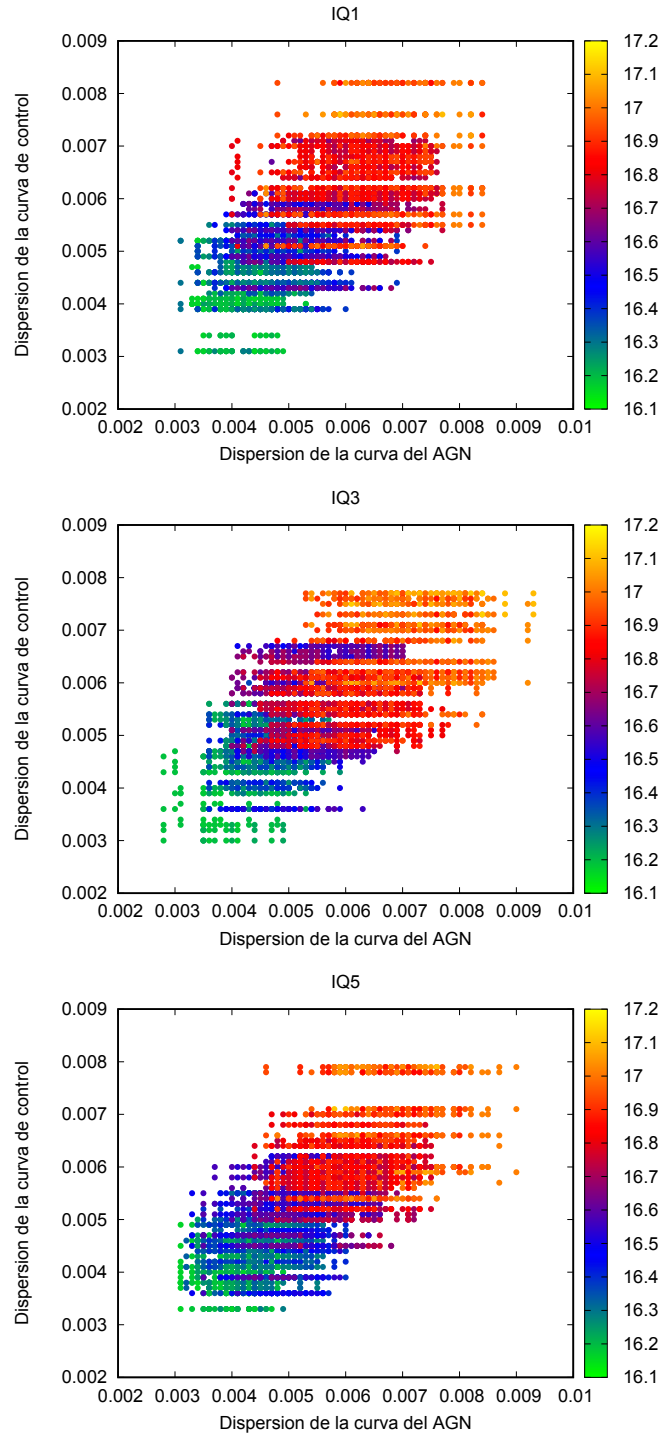
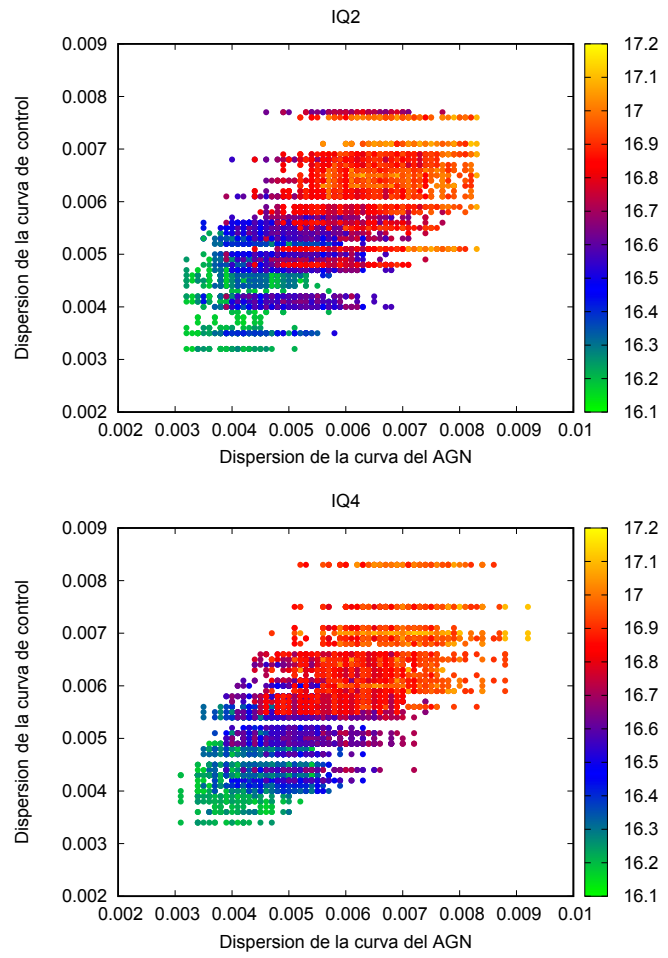

Figura 2.22. Comportamiento de la dispersión de la curva del AGN frente a la dispersión de la curva de control para H01, en los casos IQ1 (panel superior izquierdo), IQ2 (panel superior derecho), IQ3 (panel del medio izquierdo), IQ4 (panel del medio derecho) y IQ5 (panel inferior).

$100 \%$ del estado de no variabilidad en las curvas de los AGNs no variables, mientras que el test $\mathrm{F}$ detecta casos como variables (son porcentajes bajos, alrededor del $0.2 \%$ en promedio, ver Tabla 2.5). En segundo lugar, a medida que nos movemos de un criterio al otro, es mayor la cantidad de DLCs que aparecen como variables con el test F. En la Tabla 2.5 se detallan los porcentajes encontrados en cada IQ. Se presenta en la columna 1 la variación del seeing correspondiente, en las columnas 2, 3 y 4 la información del total de DLCs y los porcentajes de variabilidad para $\mathrm{C}$ y F, para el criterio de Howell H01; en las columnas 5, 6 y 7 ídem anterior pero para H03; y en las columnas 8, 9 y 10 ídem para H05. Si comparamos estos valores en relación a los obtenidos en los CT (ver Tabla 2.3), vemos que al incluir variabilidades en el seeing obtenemos porcentajes levemente mayores en las DLCs que detecta el test F. 

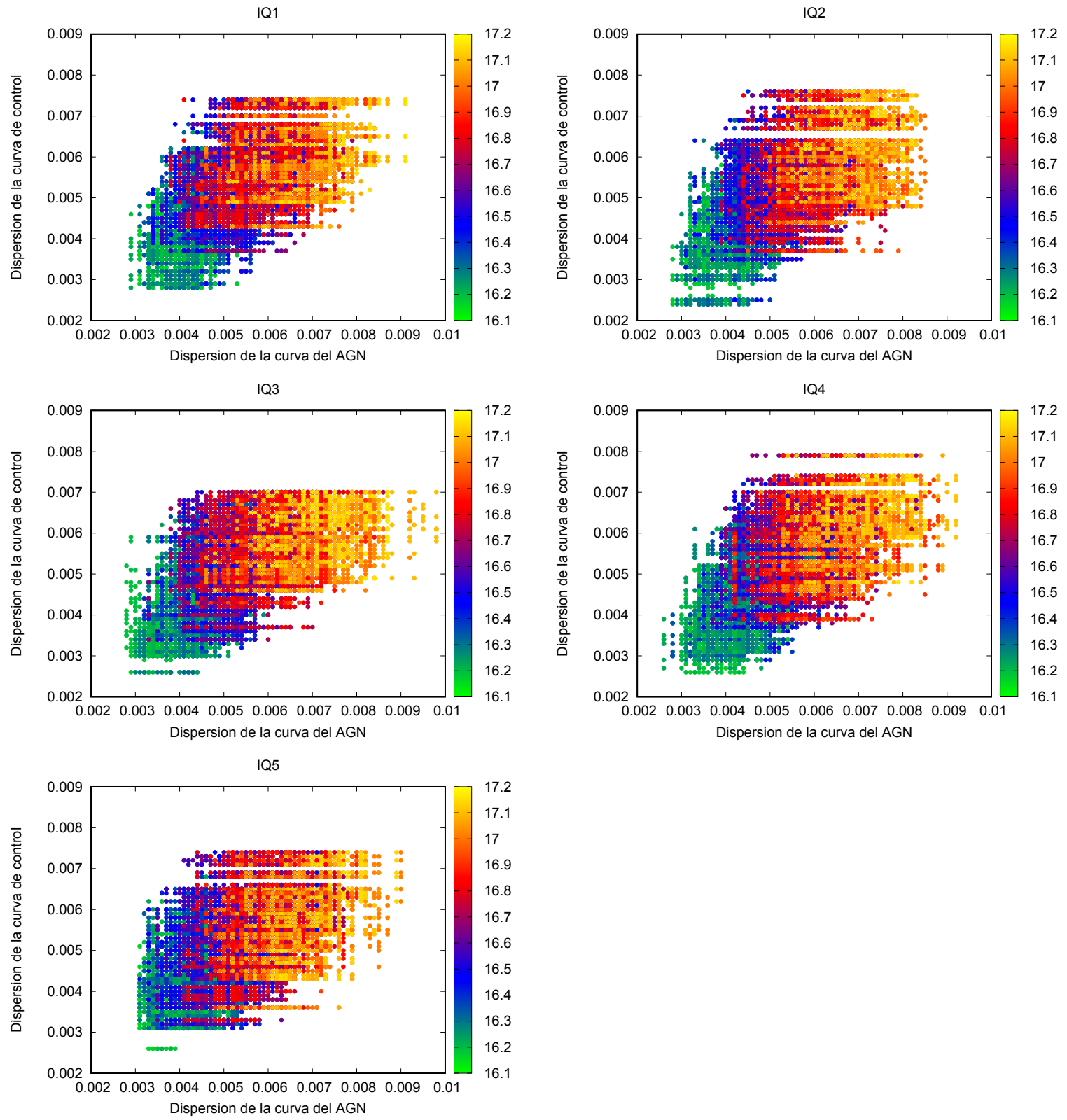

Figura 2.23. Comportamiento de la dispersión de la curva del AGN frente a la dispersión de la curva de control para H03, en los casos IQ1 (panel superior izquierdo), IQ2 (panel superior derecho), IQ3 (panel del medio izquierdo), IQ4 (panel del medio derecho) y IQ5 (panel inferior). 

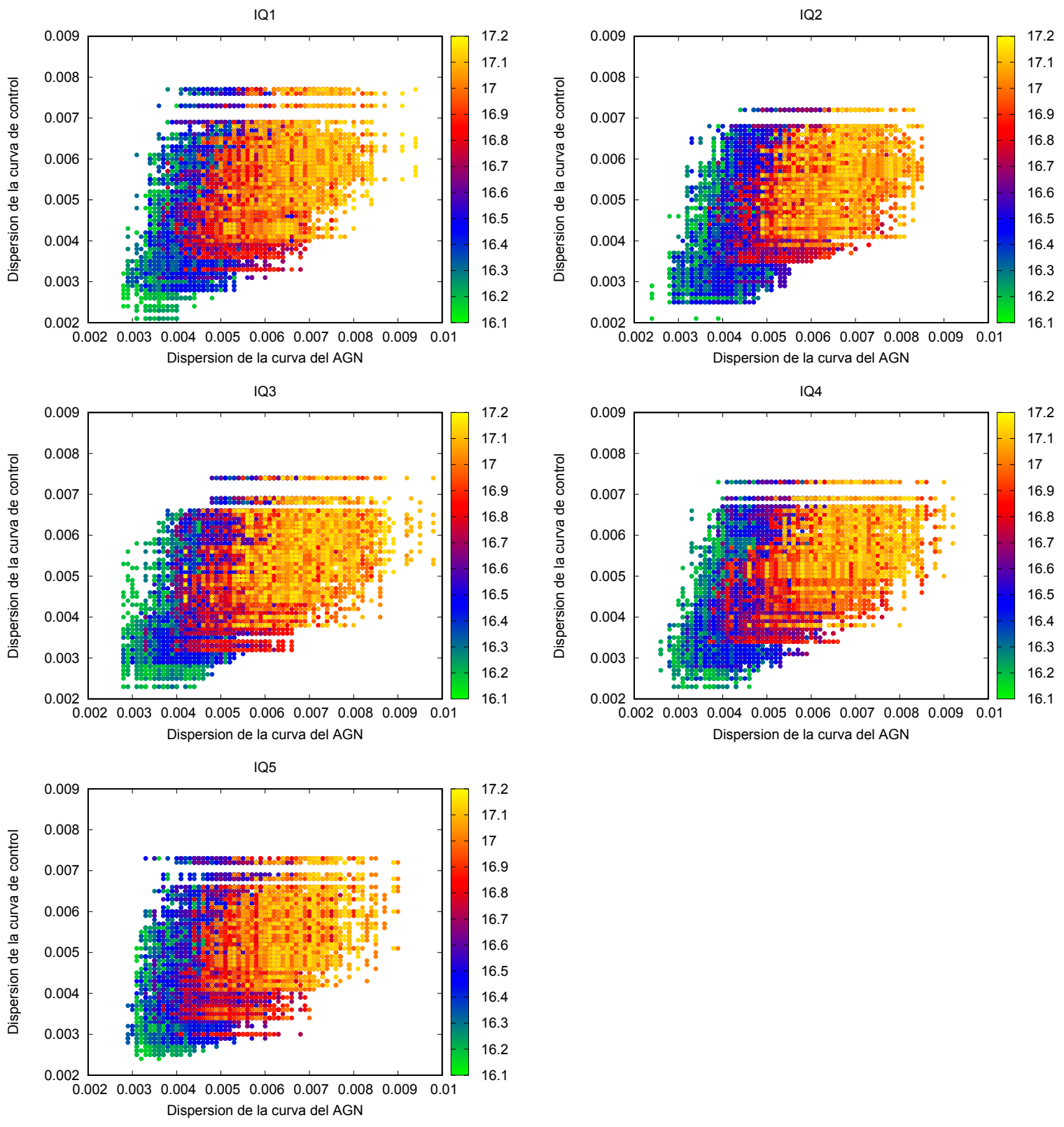

Figura 2.24. Comportamiento de la dispersión de la curva del AGN frente a la dispersión de la curva de control para H05, en los casos IQ1 (panel superior izquierdo), IQ2 (panel superior derecho), IQ3 (panel del medio izquierdo), IQ4 (panel del medio derecho) y IQ5 (panel inferior). 


\begin{tabular}{|c|c|c|c|c|c|c|c|c|c|}
\hline \multirow[t]{2}{*}{ Var. seeing } & \multicolumn{3}{|c|}{ H01 } & \multicolumn{3}{|c|}{ H03 } & \multicolumn{3}{|c|}{ H05 } \\
\hline & Total DLCs & $\mathrm{C}$ & $\mathrm{F}$ & Total DLCs & $\mathrm{C}$ & $\mathrm{F}$ & Total DLCs & $\mathrm{C}$ & $\mathrm{F}$ \\
\hline IQ1 & 6639 & $\begin{array}{c}100 \% \mathrm{NV} \\
0 \% \mathrm{~V}\end{array}$ & $\begin{array}{c}100 \% \mathrm{NV} \\
0 \% \mathrm{~V}\end{array}$ & 27698 & $\begin{array}{c}100 \% \mathrm{NV} \\
0 \% \mathrm{~V}\end{array}$ & $\begin{array}{c}99.97 \% \mathrm{NV} \\
0.03 \% \mathrm{~V}\end{array}$ & 21665 & $\begin{array}{c}100 \% \mathrm{NV} \\
0 \% \mathrm{~V}\end{array}$ & $\begin{array}{c}99.84 \% \mathrm{NV} \\
0.16 \% \mathrm{~V}\end{array}$ \\
\hline IQ2 & 6601 & $\begin{array}{c}100 \% \mathrm{NV} \\
0 \% \mathrm{~V}\end{array}$ & $\begin{array}{c}99.89 \% \mathrm{NV} \\
0.11 \% \mathrm{~V}\end{array}$ & 27598 & $\begin{array}{c}100 \% \mathrm{NV} \\
0 \% \mathrm{~V}\end{array}$ & $\begin{array}{c}99.77 \% \mathrm{NV} \\
0.23 \% \mathrm{~V}\end{array}$ & 21487 & $\begin{array}{c}100 \% \mathrm{NV} \\
0 \% \mathrm{~V}\end{array}$ & $\begin{array}{c}99.83 \% \mathrm{NV} \\
0.17 \% \mathrm{~V}\end{array}$ \\
\hline IQ3 & 6609 & $\begin{array}{c}100 \% \mathrm{NV} \\
0 \% \mathrm{~V}\end{array}$ & $\begin{array}{c}99.95 \% \mathrm{NV} \\
0.05 \% \mathrm{~V}\end{array}$ & 27910 & $\begin{array}{c}100 \% \mathrm{NV} \\
0 \% \mathrm{~V}\end{array}$ & $\begin{array}{c}99.91 \% \mathrm{NV} \\
0.09 \% \mathrm{~V}\end{array}$ & 21059 & $\begin{array}{c}100 \% \mathrm{NV} \\
0 \% \mathrm{~V}\end{array}$ & $\begin{array}{c}99.92 \% \mathrm{NV} \\
0.08 \% \mathrm{~V}\end{array}$ \\
\hline IQ4 & 6651 & $\begin{array}{c}100 \% \mathrm{NV} \\
0 \% \mathrm{~V}\end{array}$ & $\begin{array}{c}100 \% \mathrm{NV} \\
0 \% \mathrm{~V}\end{array}$ & 27341 & $\begin{array}{c}100 \% \mathrm{NV} \\
0 \% \mathrm{~V}\end{array}$ & $\begin{array}{c}99.95 \% \mathrm{NV} \\
0.05 \% \mathrm{~V}\end{array}$ & 21487 & $\begin{array}{c}100 \% \mathrm{NV} \\
0 \% \mathrm{~V}\end{array}$ & $\begin{array}{c}99.94 \% \mathrm{NV} \\
0.06 \% \mathrm{~V}\end{array}$ \\
\hline IQ5 & 6631 & $\begin{array}{c}100 \% \mathrm{NV} \\
0 \% \mathrm{~V}\end{array}$ & $\begin{array}{c}99.98 \% \mathrm{NV} \\
0.02 \% \mathrm{~V}\end{array}$ & 28162 & $\begin{array}{c}100 \% \mathrm{NV} \\
0 \% \mathrm{~V}\end{array}$ & $\begin{array}{c}99.70 \% \mathrm{NV} \\
0.30 \% \mathrm{~V}\end{array}$ & 21668 & $\begin{array}{c}100 \% \mathrm{NV} \\
0 \% \mathrm{~V}\end{array}$ & $\begin{array}{c}99.81 \% \mathrm{NV} \\
0.19 \% \mathrm{~V}\end{array}$ \\
\hline
\end{tabular}

2.5. Porcentajes de los casos de DLC que resultaron variables y no variables con los parámetros $\mathrm{C}$ y $\mathrm{F}$ en cada IQ. Se presenta en la columna 1 la variación del seeing, en las columnas 2, 3 y 4 la información del total de DLCs y los porcentajes de variabilidad para $\mathrm{C}$ y F, para el criterio de Howell H01; en las columnas 5, 6 y 7 ídem anterior pero para H03; y en las columnas 8, 9 y 10 ídem para H05. 
En cuanto al comportamiento de los valores de la dispersión de la curva de control $\left(\sigma_{2}\right)$ frente al factor de peso de Howell $(\Gamma)$ para los distintos IQ en los AGNs variables, obtuvimos resultados muy similares respecto a los CT (Fig. 2.16). Para valores de $\sigma_{2}$ bajos, los valores de $\Gamma$ se expanden en un amplio rango, desde 1.00 hasta 2.80. A medida que va aumentando $\sigma_{2}$, los valores del factor de peso tienden a estabilizarse alrededor de $\Gamma=1.00$, entre 0.80 y 1.20 (siendo $\Gamma=1.00$ el valor óptimo alcanzado cuando se cumple estrictamente con el criterio propuesto por Howell). Y esta forma es similar entre los IQ. Este mismo tipo de comportamiento se observa cuando se considera la variabilidad sistemática decreciente, con leves cambios en alguno de los valores. En la variabilidad tipo burst suave, el rango de las dispersiones es similar al obtenido en los dos casos anteriores y, al igual que en los CT, se observa que el pico de las distribuciones cae en posiciones diferentes de acuerdo al caso de IQ que se considere. Para el caso de la variabilidad tipo serrucho, las dispersiones toman valores menores respecto de los casos anteriores y también de las situaciones de los CT. Sin embargo, su comportamiento general es similar al encontrado en estos últimos.

Para el caso de los flickerings, éstos también presentan comportamientos similares a los CT. Respecto del flickering 1, tiene sus distribuciones ubicadas en los valores más altos entre todas las variabilidades. Este rango de valores es más extendido que el que se obtuvo para los CT. Podemos distinguir dos grupos (Fig. 2.26, panel superior izquierdo). Por un lado, se tienen los casos de IQ1, IQ2 y IQ3, y por el otro a IQ4 y IQ5. Además, las primeras tres distribuciones son las que muestran los valores más altos en sus picos, llegando hasta las 1100 DLCs. Para el otro grupo, se observa que las distribuciones se encuentran en un rango de valores de $\sigma_{1}$ mayor al del grupo anterior. En lo que respecta al flickering 2, los valores de la dispersión de la curva del AGN están esparcidos en valores más bajos que en el caso anterior. Para la variabilidad tipo flickering 3, notamos una diferencia respecto a lo obtenido para los CT: se distinguen tres picos en las distribuciones con leves diferencias entre los valores donde se ubican sus máximos. Y continuando con los otros casos, a medida que la amplitud del flickering decae, encontramos que las dispersiones se distribuyen hacia valores más bajos, como es razonable de obtener. Las diferencias que se presentan están en las ubicaciones de los máximos de las distribuciones, pero sin cambios significativos en sus valores.

En la Fig. 2.27 se muestra cómo es la relación entre las dispersiones de la curva del AGN y de control para cada IQ. En lo que respecta a los valores de $\sigma_{2}$ todos los IQ toman el mismo rango de valores. Una diferencia entre cada variación del seeing es hasta dónde llega la distribución de valores de $\sigma_{1}$, ya que, nuevamente, en los casos de AGNs variables se dejó libre la elección de las estrellas de comparación y de control y están tomados todos los casos posibles de combinaciones.

Por otro lado, en lo que respecta a la posición de cada variabilidad en el gráfico, uno de los casos que más sobresale es el del flickering 1 (color celeste). Para las primeras 3 situaciones de IQ, se ubica en un valor de $\sigma_{2}=0.008$, mientras que para las restantes dos, se corre a $\sigma_{2}=0.0095$. Este resultado es opuesto a lo que se encontró para los CT (en los cuales el flickering 1, entre otras variabilidades, permanecía invariante frente a un cambio en el valor fijo del seeing). Las distribuciones de las amplitudes de cada caso de variabilidad cambian en algunas situaciones y en otras no. Esto se debe a que la amplitud de la variabilidad que se plantea sumado al tipo de variabilidad a lo largo de la curva hacen se compensan en algunos casos y en otros es una suma de efectos. 

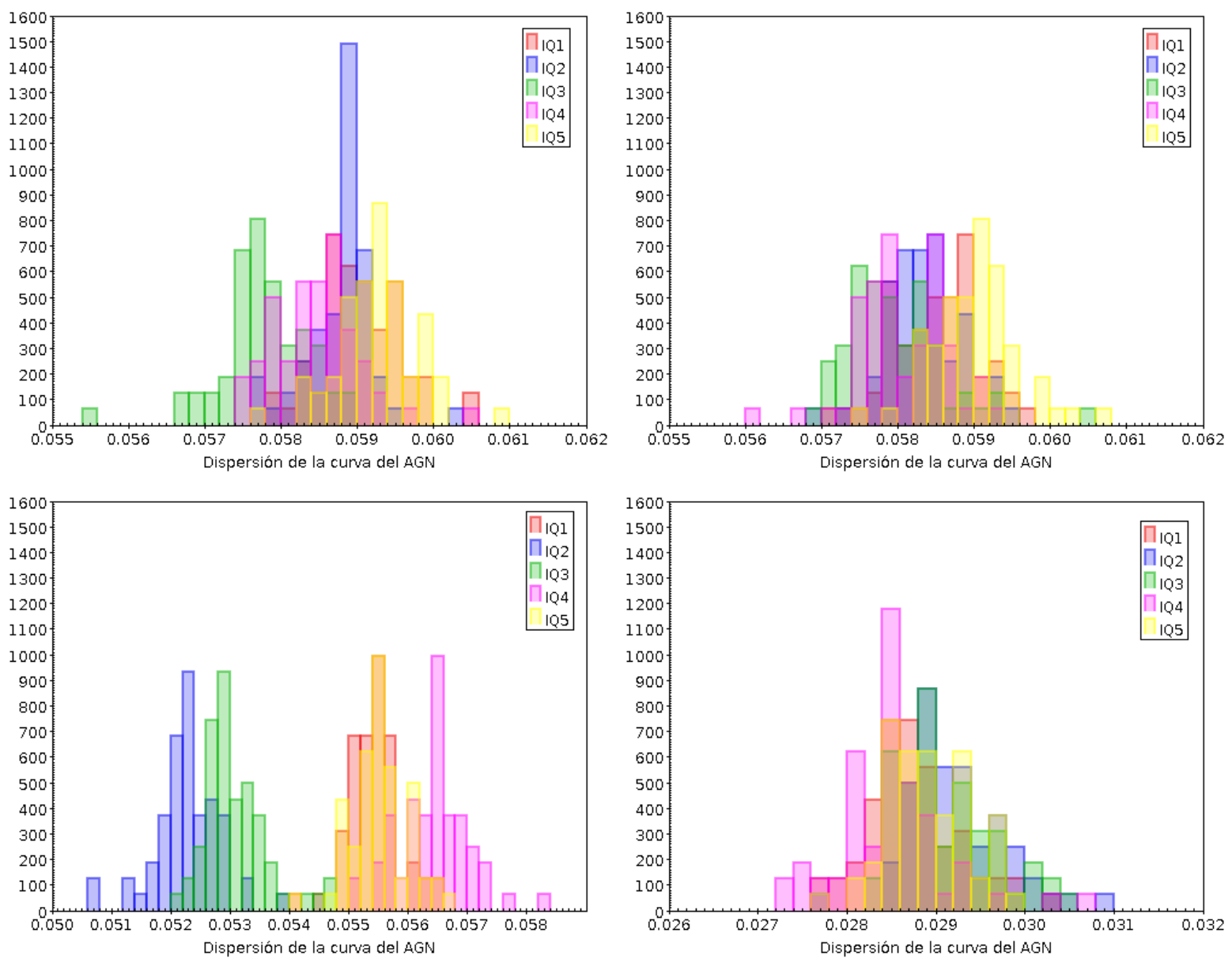

Figura 2.25. Histograma de la dispersión de la curva del AGN para la variabilidad sistemática decreciente (panel superior izquierdo), creciente (panel superior derecho), burst suave (panel inferior izquierdo) y serrucho (panel inferior derecho) en los casos IQ1, IQ2, IQ3, IQ4 y IQ5.

Finalmente, a partir de los resultados de aplicar los parámetros $\mathrm{C}$ y $\mathrm{F}$ a los AGNs variables, obtenemos que para cada IQ y cada tipo de variabilidad, se recupera el $100 \%$ del estado de variabilidad de las DLCs para ambos parámetros. Luego, lo que estamos encontrando es que, aún frente a una situación realista de variabilidad del seeing a lo largo de la noche, si el objeto observado presenta una variabilidad intrínseca, es posible recuperar ese estado de variabilidad. Si en las curvas de IQ tomamos un valor promedio representativo de los valores en toda la curva, y comparando con los CT, donde el valor del seeing es fijo, recuperamos (en un 99.6\%) las mismas situaciones. Esto cobra importancia cuando vamos a los casos reales de las observaciones, donde se suele tomar un valor medio del seeing en la noche. 

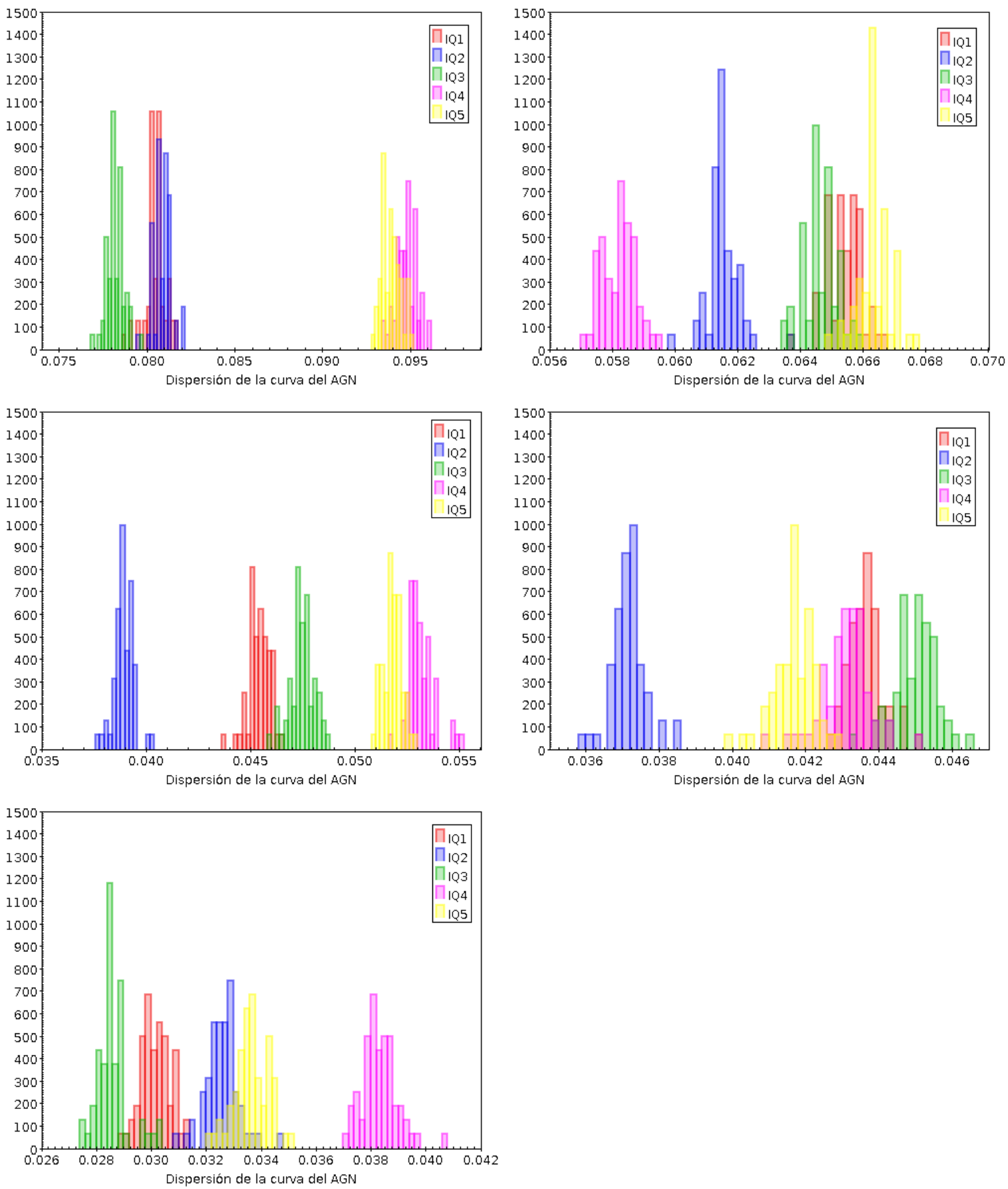

Figura 2.26. Histograma de la dispersión de la curva del AGN para las variabilidades relacionadas con los 5 flickerings en los casos IQ1, IQ2, IQ3, IQ4 y IQ5. 

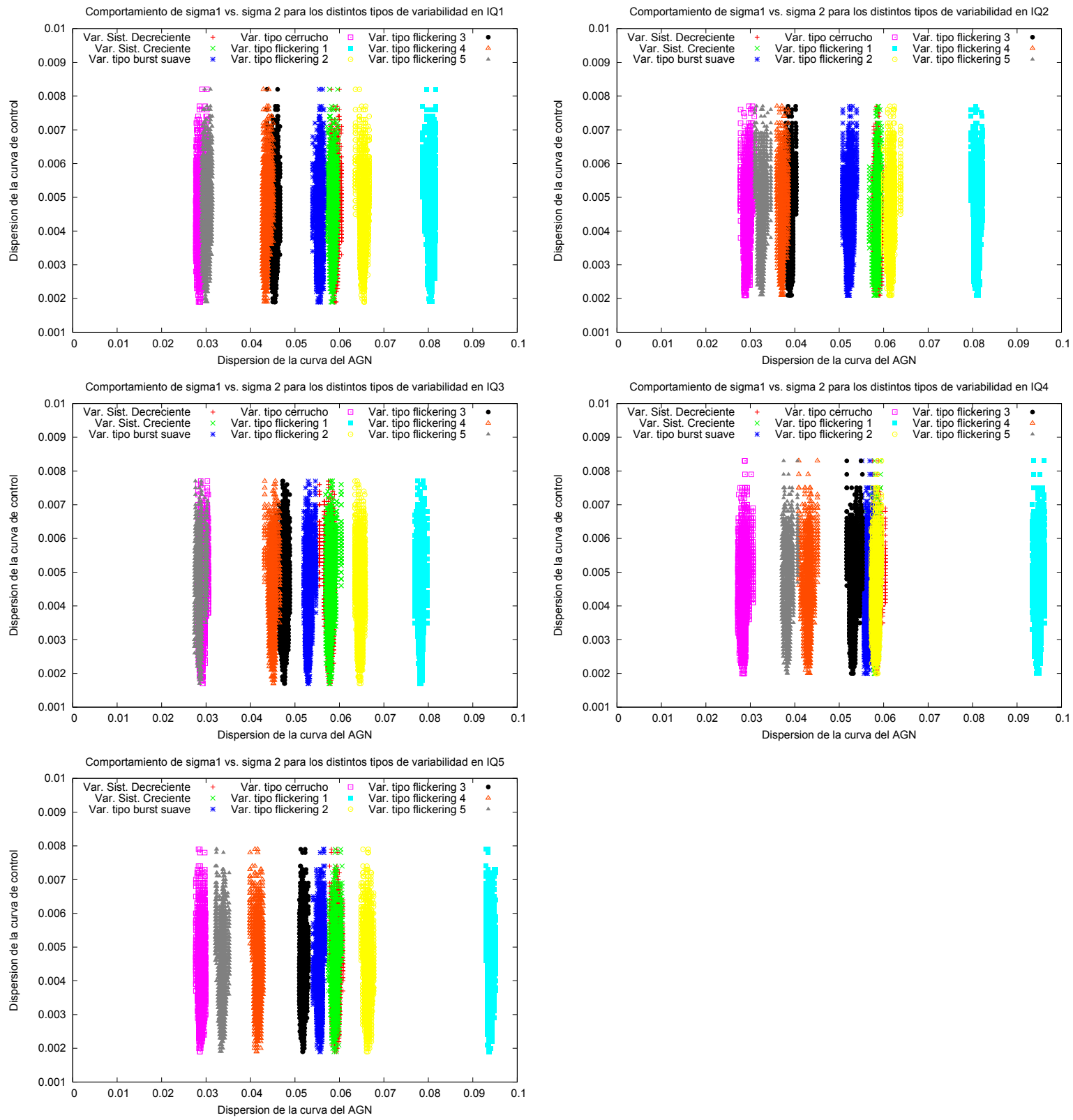

Figura 2.27. Comportamiento de la dispersión de la curva del AGN frente a la dispersión de la curva de control para las distintas variabilidades, en los casos IQ1 (panel superior izquierdo), IQ2 (panel superior derecho), IQ3 (panel del medio izquierdo), IQ4 (panel del medio derecho) y IQ5 (panel inferior). 


\subsubsection{Análisis de los resultados generales}

A continuación, proponemos realizar el análisis de los resultados obtenidos en general a través de una comparación entre diferentes situaciones. Esto lo hacemos partiendo de las situaciones ideales (no existentes en la realidad) y viendo cuales son los efectos sobre las mismas de agregar diferentes situaciones.

\section{CT2 vs. IQ1 vs. IQ1-SB1 vs. IQ1-CC1 vs. IQ1-SB1-CC1}

Comenzamos con el CT2, cuyo valor fijo del seeing es de 1.50 arcsec; luego contemplamos la situación IQ1, la cual posee una variación del seeing cuyo valor promedio en toda la noche es de 1.52 arcsec; a esta situación le agregamos dos efectos por separado: por un lado sumamos la presencia de la Luna (IQ1-SB1) y por otro agregamos los cambios en la cobertura de nubes (IQ1-CC1). Finalmente IQ1-SB1-CC1, en donde se suman los 3 efectos, esto es, variación de seeing, de nubes y Luna Llena.

En cuanto a la relación entre la dispersión $\sigma_{2}$ en función del factor de peso de Howell (Fig.2.28) se observa que, conforme se van sumando los efectos, se amplía el rango de valores que toma $\Gamma$. En particular, al sumar la Luna, vemos que para todas las restricciones de Howell se tienen DLCs donde se obtienen valores de $\Gamma$ cercanos a 1 . Con respecto a los valores de $\sigma_{2}$, también se puede ver que a medida que se incrementan los efectos, se obtienen valores cada vez más altos de la dispersión, pasando de $\sigma_{2} \sim 0.008$ (CT2) a $\sigma_{2} \sim 0.026$ (IQ1-SB1-CC1). La explicación de este comportamiento es que, dado que estamos teniendo en cuenta todos los efectos al mismo tiempo, es mayor el error introducido en las imágenes de los AGNs y estrellas de campo, lo que termina dejando curvas de luz muy ruidosas.

Respecto al comportamiento entre las dispersiones de las curvas del AGN y de control en función de la magnitud que posee el AGN en cada situación, vemos que el comportamiento difiere a medida que introducimos los distintos efectos, como lo muestran las Fig. 2.29, 2.30 y 2.31. Considerando la primer restricción en el criterio de Howell (H01), no hay diferencias significativas cuando pasamos de tener un valor del seeing fijo a la situación de que éste varíe (Fig. 2.29, panel superior izquierdo y panel superior derecho). Si a la variación del seeing se le agrega la presencia de la Luna, los puntos pasan a tener una distribución más ancha, donde los valores de $\sigma_{1}$ se expanden de forma similar que los valores de $\sigma_{2}$. Si en cambio, tomamos una de las situaciones de cobertura de nubes (CC1), los valores no se dispersan tanto como con la presencia de la luna y los AGNs involucrados tienen magnitudes más débiles. Al introducir la situación en donde la nubosidad va en aumento, es esperable que las magnitudes de los AGNs sean más débiles (Fig. 2.29, panel central derecho). Finalmente, tomando todos los efectos juntos, observamos que las dispersiones de la curva del AGN, $\sigma_{1}$, y de la curva de control, $\sigma_{2}$, se dispersan mucho más en el gráfico (Fig. 2.29, panel inferior), alcanzando valores que triplican lo obtenido para el caso ideal. Lo que está sucediendo es que se suma el hecho de llegar a magnitudes menores por la presencia de las nubes a la mayor dispersión obtenida por el hecho de contar con la iluminación de la luna llena en toda la curva luz.

En cuanto a la siguiente restricción contemplada (H03), el comportamiento encontrado es similar al caso de H01, en donde a medida que la situación involucra más efectos, los valores de la dispersión de las curvas se hacen cada vez más grandes, es 

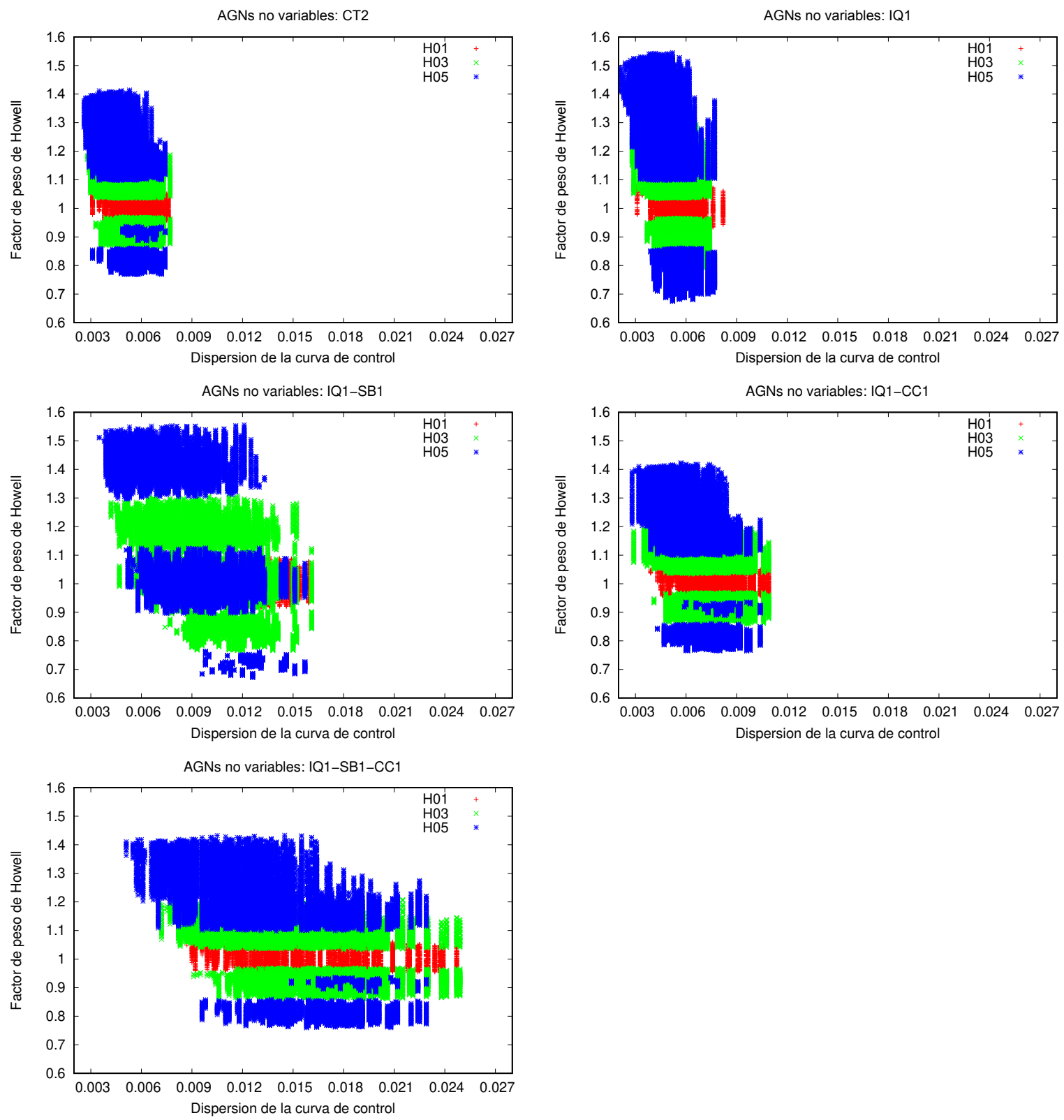

Figura 2.28. Comportamiento de la dispersión de la curva de control frente al valor del factor de peso de Howell, $\Gamma$, para las distintas restricciones del criterio de Howell, en los casos CT2 (panel superior izquierdo), IQ1 (panel superior derecho), IQ1-SB1 (panel del medio izquierdo), IQ1-CC1 (panel del medio derecho) y IQ1-SB1-CC1 (panel inferior).

decir, se introduce más error en la curvas, dado los efectos climáticos y observacionales que se están incluyendo (ver Fig. 2.30). Lo que resalta respecto a la comparación con H01 es análogo a lo que hallamos en los CT. Cuando pasamos de un criterio estricto como H01 a H03, que es más relajado, en cada uno de los gráficos aparecen más objetos débiles. Esto es razonable y esperable, ya que H03 tiene en cuenta diferencias en las magnitudes de los objetos mayores a las que contempla H01. Esto se refleja en la distribución de colores, principalmente es más notorio en los casos menos afectados 

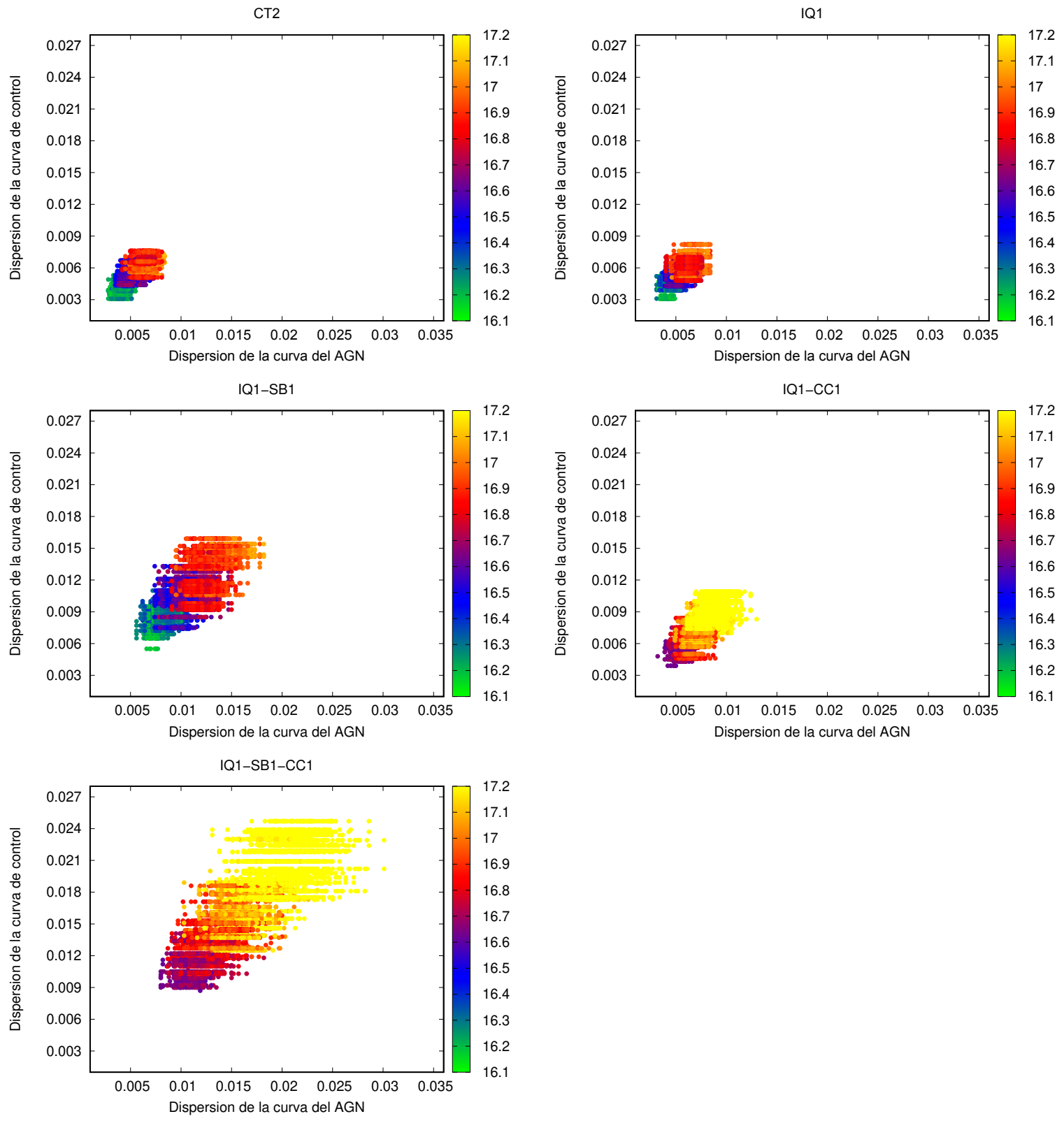

Figura 2.29. Comportamiento de la dispersión de la curva del AGN frente a la dispersión de la curva de control para H01, en los casos CT2 (panel superior izquierdo), IQ1 (panel superior derecho), IQ1-SB1 (panel del medio izquierdo), IQ1-CC1 (panel del medio derecho) y IQ1-SB1-CC1 (panel inferior).

(CT2, IQ1 y IQ-SB1). Notamos que, comparando con los casos H01, para un mismo rango de valores de $\sigma_{1}$ y $\sigma_{2}$, se tiene una mayor variedad de AGNs con magnitudes bien distintas entre sí.

Por último, para el criterio de Howell menos estricto (H05), también volvemos a encontrar que, conforme se van sumando los distintos efectos climáticos, los valores de $\sigma_{1}$ y $\sigma_{2}$ se esparcen a lo largo del diagrama (ver Fig. 2.31). Dado que este criterio tiene en cuenta las diferencias en magnitud más grandes entre AGNs y estrellas, se obtienen 

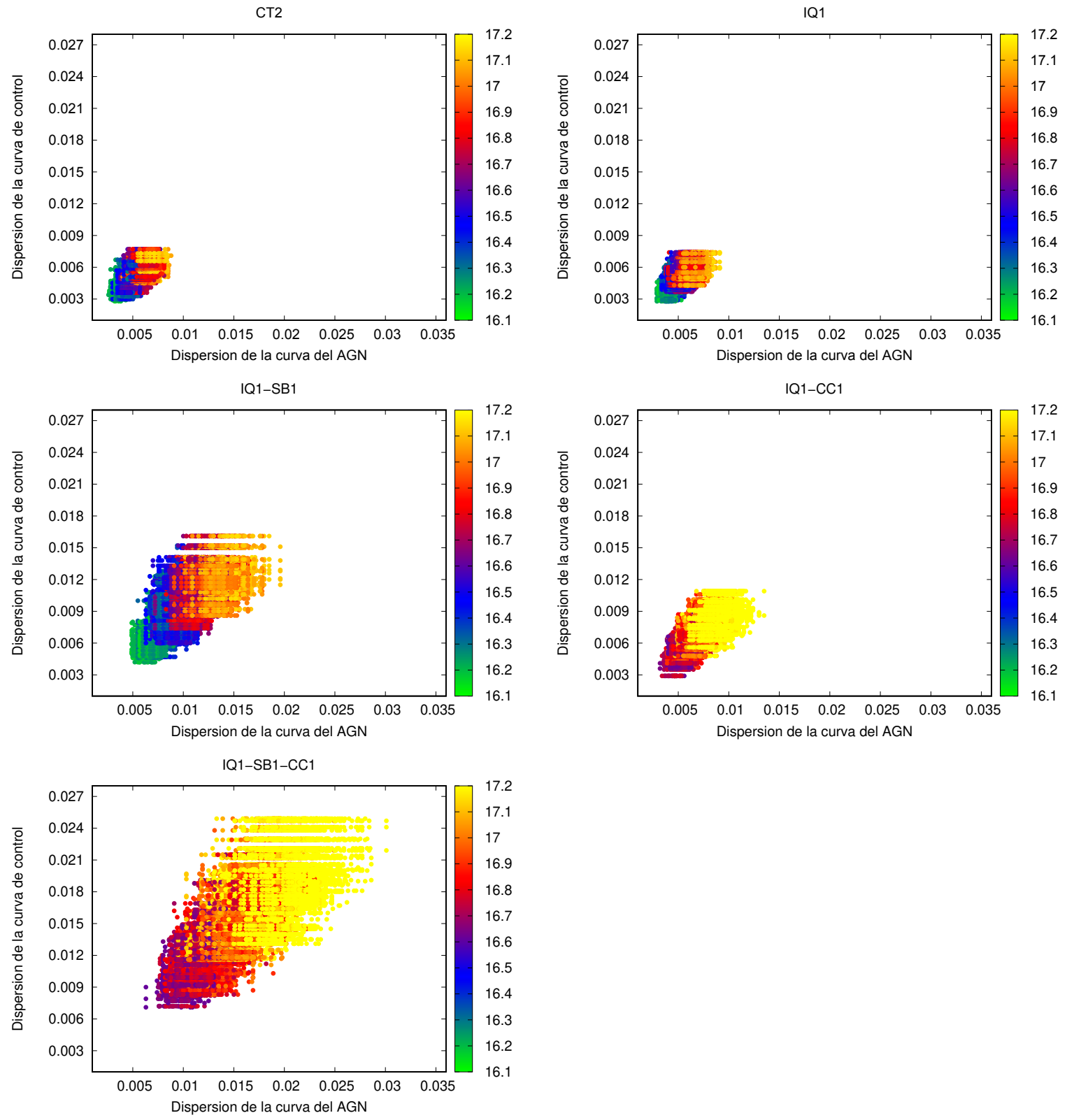

Figura 2.30. Comportamiento de la dispersión de la curva del AGN frente a la dispersión de la curva de control para H03, en los casos CT2 (panel superior izquierdo), IQ1 (panel superior derecho), IQ1-SB1 (panel del medio izquierdo), IQ1-CC1 (panel del medio derecho) y IQ1-SB1-CC1 (panel inferior).

valores en la magnitud más débiles que en los casos anteriores, comportamiento también esperable. Se acentúa la contribución de objetos con distintas magnitudes a cada par de valores $\sigma_{1}-\sigma_{2}$ (por ejemplo, IQ1-SB1).

Los cambios en las cantidades estudiadas $\left(\sigma_{1}, \sigma_{2}\right.$ y $\left.\Gamma\right)$ debido a los distintos efectos observacionales y climáticos, se verán reflejados en los resultados de la aplicación de las herramientas C y F. En la Tabla 2.6 se presentan los resultados de la clasificación de las DLCs para los AGNs no variables, en las distintas situaciones estudiadas. La 

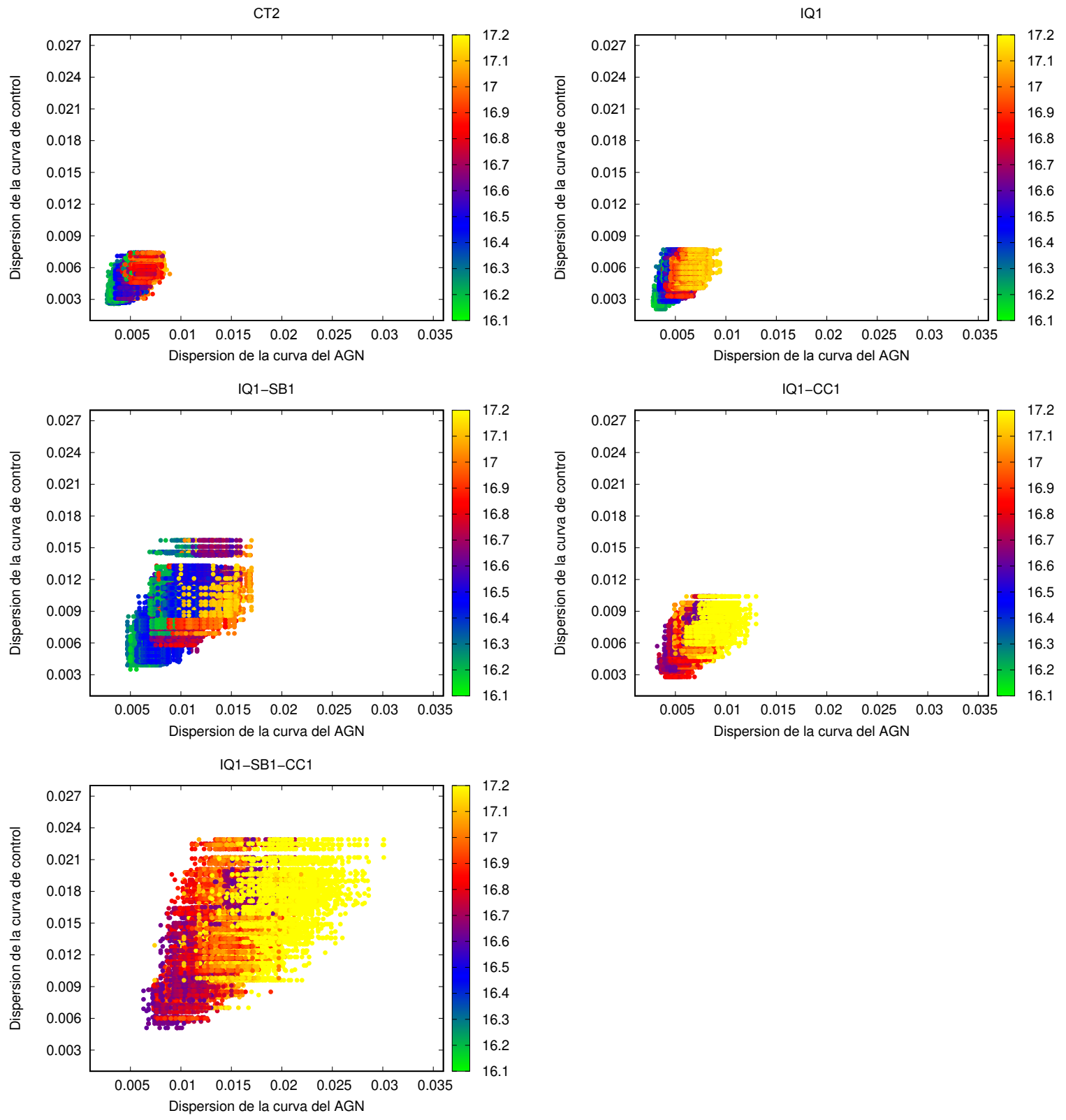

Figura 2.31. Comportamiento de la dispersión de la curva del AGN frente a la dispersión de la curva de control para H05, en los casos CT2 (panel superior izquierdo), IQ1 (panel superior derecho), IQ1-SB1 (panel del medio izquierdo), IQ1-CC1 (panel del medio derecho) y IQ1-SB1-CC1 (panel inferior).

Tabla comprende en la columna 1 la situación observacional, en las columnas 2, 3 y 4 la información del total de DLCs y los porcentajes de variabilidad para C y F, para el criterio de Howell H01; en las columnas 5, 6 y 7 ídem anterior pero para H03; y en las columnas 8, 9 y 10 ídem para H05. 


\begin{tabular}{|c|c|c|c|c|c|c|c|c|c|}
\hline \multirow[t]{2}{*}{ Situación } & \multicolumn{3}{|c|}{ H01 } & \multicolumn{3}{|c|}{ H03 } & \multicolumn{3}{|c|}{ H05 } \\
\hline & Total DLCs & $\mathrm{C}$ & $\mathrm{F}$ & Total DLCs & $\mathrm{C}$ & $\mathrm{F}$ & Total DLCs & $\mathrm{C}$ & $\mathrm{F}$ \\
\hline $\mathrm{CT} 2$ & 6685 & $\begin{array}{c}100 \% \mathrm{NV} \\
0 \% \mathrm{~V}\end{array}$ & $\begin{array}{c}100 \% \mathrm{NV} \\
0 \% \mathrm{~V}\end{array}$ & 25949 & $\begin{array}{c}100 \% \mathrm{NV} \\
0 \% \mathrm{~V}\end{array}$ & $\begin{array}{c}99.98 \% \mathrm{NV} \\
0.02 \% \mathrm{~V}\end{array}$ & 21368 & $\begin{array}{c}100 \% \mathrm{NV} \\
0 \% \mathrm{~V}\end{array}$ & $\begin{array}{c}99.93 \% \mathrm{NV} \\
0.07 \% \mathrm{~V}\end{array}$ \\
\hline IQ1 & 6639 & $\begin{array}{c}100 \% \mathrm{NV} \\
0 \% \mathrm{~V}\end{array}$ & $\begin{array}{c}100 \% \mathrm{NV} \\
0 \% \mathrm{~V}\end{array}$ & 27698 & $\begin{array}{c}100 \% \mathrm{NV} \\
0 \% \mathrm{~V}\end{array}$ & $\begin{array}{c}99.97 \% \mathrm{NV} \\
0.03 \% \mathrm{~V}\end{array}$ & 21665 & $\begin{array}{c}100 \% \mathrm{NV} \\
0 \% \mathrm{~V}\end{array}$ & $\begin{array}{c}99.84 \% \mathrm{NV} \\
0.16 \% \mathrm{~V}\end{array}$ \\
\hline IQ1-SB1 & 6671 & $\begin{array}{c}100 \% \mathrm{NV} \\
0 \% \mathrm{~V}\end{array}$ & $\begin{array}{c}99.95 \% \mathrm{NV} \\
0.05 \% \mathrm{~V}\end{array}$ & 26499 & $\begin{array}{c}100 \% \mathrm{NV} \\
0 \% \mathrm{~V}\end{array}$ & $\begin{array}{c}99.95 \% \mathrm{NV} \\
0.05 \% \mathrm{~V}\end{array}$ & 21160 & $\begin{array}{c}100 \% \mathrm{NV} \\
0 \% \mathrm{~V}\end{array}$ & $\begin{array}{c}99.98 \% \mathrm{NV} \\
0.02 \% \mathrm{~V}\end{array}$ \\
\hline IQ1-CC1 & 6663 & $\begin{array}{c}100 \% \mathrm{NV} \\
0 \% \mathrm{~V}\end{array}$ & $\begin{array}{c}99.80 \% \mathrm{NV} \\
0.20 \% \mathrm{~V}\end{array}$ & 26404 & $\begin{array}{c}100 \% \mathrm{NV} \\
0 \% \mathrm{~V}\end{array}$ & $\begin{array}{c}99.89 \% \mathrm{NV} \\
0.11 \% \mathrm{~V}\end{array}$ & 21097 & $\begin{array}{c}100 \% \mathrm{NV} \\
0 \% \mathrm{~V}\end{array}$ & $\begin{array}{c}99.80 \% \mathrm{NV} \\
0.20 \% \mathrm{~V}\end{array}$ \\
\hline IQ1-SB1-CC1 & 6692 & $\begin{array}{c}100 \% \mathrm{NV} \\
0 \% \mathrm{~V}\end{array}$ & $\begin{array}{c}100 \% \mathrm{NV} \\
0 \% \mathrm{~V}\end{array}$ & 25880 & $\begin{array}{c}100 \% \mathrm{NV} \\
0 \% \mathrm{~V}\end{array}$ & $\begin{array}{c}99.95 \% \mathrm{NV} \\
0.05 \% \mathrm{~V}\end{array}$ & 21479 & $\begin{array}{c}100 \% \mathrm{NV} \\
0 \% \mathrm{~V}\end{array}$ & $\begin{array}{c}99.89 \% \mathrm{NV} \\
0.11 \% \mathrm{~V}\end{array}$ \\
\hline
\end{tabular}

Tabla 2.6. Porcentajes de los casos de DLC que resultaron variables y no variables con los parámetros C y F en cada situación considerada: CT2, IQ1, IQ1-SB1, IQ1CC1 y IQ1-SB1-CC1. Se presenta en la columna 1 la vsituación contemplada, en las columnas 2, 3 y 4 la información del total de DLCs y los porcentajes de variabilidad para C y F, para el criterio de Howell H01; en las columnas 5, 6 y 7 ídem anterior pero para H03; y en las columnas 8, 9 y 10 ídem para H05. 
Lo primero que se desprende de los resultados obtenidos para esta primera comparación de situaciones es que, independientemente de qué efecto se esté involucrando en las simulaciones, el parámetro C siempre recupera el $100 \%$ de las DLCs clasificadas como no variables, para los AGNs no variables. En cambio, el parámetro F detecta casos de DLCs como variables de AGNs no varían, aunque los porcentajes son muy bajos. Por otra parte, la restricción del criterio de Howell H05 es la que registra el mayor porcentaje de DLCs clasificadas como variables con el parámetro F. De los gráficos analizados anteriormente, vimos que para H05 se tienen los mayores valores de las dispersiones de las curvas, además de que aparecen más cantidad de AGNs débiles. Todo esto introduce errores en las curvas, haciendo que el test $\mathrm{F}$ detecte DLC no variables como si lo fueran. En este sentido, el test $\mathrm{F}$ resultaría ser más sensible a las distintas variaciones observacionales, no siendo así para el parámetro C.

Para el caso de los AGNs variables, en la Fig. 2.32 mostramos la relación entre $\sigma_{2}$ y el factor de peso $\Gamma$. En lo que respecta al rango de valores de $\Gamma$ que toman las diferentes situaciones, se mantiene entre 0.40 y 2.60, en la mayoría de los casos, salvo cuando se incorporan las nubes. El efecto de la cobertura de nubes es que los valores de $\Gamma$ se incrementan, llegando hasta 3.10, como es el caso de IQ1-CC1 y IQ1-SB1CC1. El comportamiento de los valores de $\sigma_{2}$ es similar al de los AGNs no variables, donde para el caso más ideal de seeing fijo (CT2), el rango de $\sigma_{2}$ llega hasta 0.008, mientras que para el caso con todos los efectos juntos (IQ1-SB1-CC1), se tienen $\sigma_{2}$ de hasta 0.026. Por otro lado, se puede ver que las distintas distribuciones de puntos correspondientes a las diferentes variabilidades, se pueden dividir en dos grupos: por un lado, se encuentran las variabilidades sistemáticas decreciente, creciente, burst suave y serrucho, y por el otro, los 5 tipos de flickering. Y a medida que se van sumando los efectos, estos grupos se separan uno respecto del otro dentro del diagrama. Es el caso de los flickering, donde pasan a tener valores mayores de $\Gamma$ conforme la situación va incorporando los efectos de la Luna y de la cobertura de nubes.

En la Fig. 2.33, se presenta la relación entre las dispersiones de las curvas del AGN y de control. Los distintos efectos que vamos involucrando repercuten en los valores de $\sigma_{1}$ y $\sigma_{2}$. Por un lado, de la Fig. 2.33 (panel del medio izquierdo) (IQ1-SB1), vemos que la presencia de la Luna afecta más a la dispersión de la curva de control $\left(\sigma_{2}\right)$ que a la dispersión de la curva del AGN variable $\left(\sigma_{1}\right)$, pues comparando con los casos CT2 y IQ1, los valores de $\sigma_{2}$ se extienden a rangos mayores. Para CT2 y IQ1, $\sigma_{2}$ llega hasta 0.008, mientras que para IQ1-SB1 llega a duplicarse. Por otro lado, si se considera la cobertura de nubes (Fig. 2.33, panel del medio derecho, IQ1-SB1), se da el efecto contrario. Las nubes afectan más a $\sigma_{1}$ que a $\sigma_{2}$, donde para CT2 y IQ1 $\sigma_{1}$ llega a 0.08, mientras que para IQ1-CC1 llega a 0.09. Y al considerar todos los efectos juntos, la variación de la presencia de las nubes propuesta afecta algo más a la dispersión de la curva del AGNs que la curva de control, siendo $\sigma_{2}$ la que se ve más afectada que $\sigma_{1}$.

De la misma manera que hicimos con los AGNs no variables, veamos cómo impactan los distintos efectos atmosféricos en los resultados de la clasificación de las DLC con los parámetros C y F. Para la mayor parte de los casos contemplados junto con las diferentes variabilidades, se tiene que con ambos parámetros C y F se recupera el $100 \%$ del estado de variabilidad de los AGNs variables. Sin embargo, existen varios casos en donde, pese a tener AGNs variables, se tienen DLCs clasificadas como no variables, 

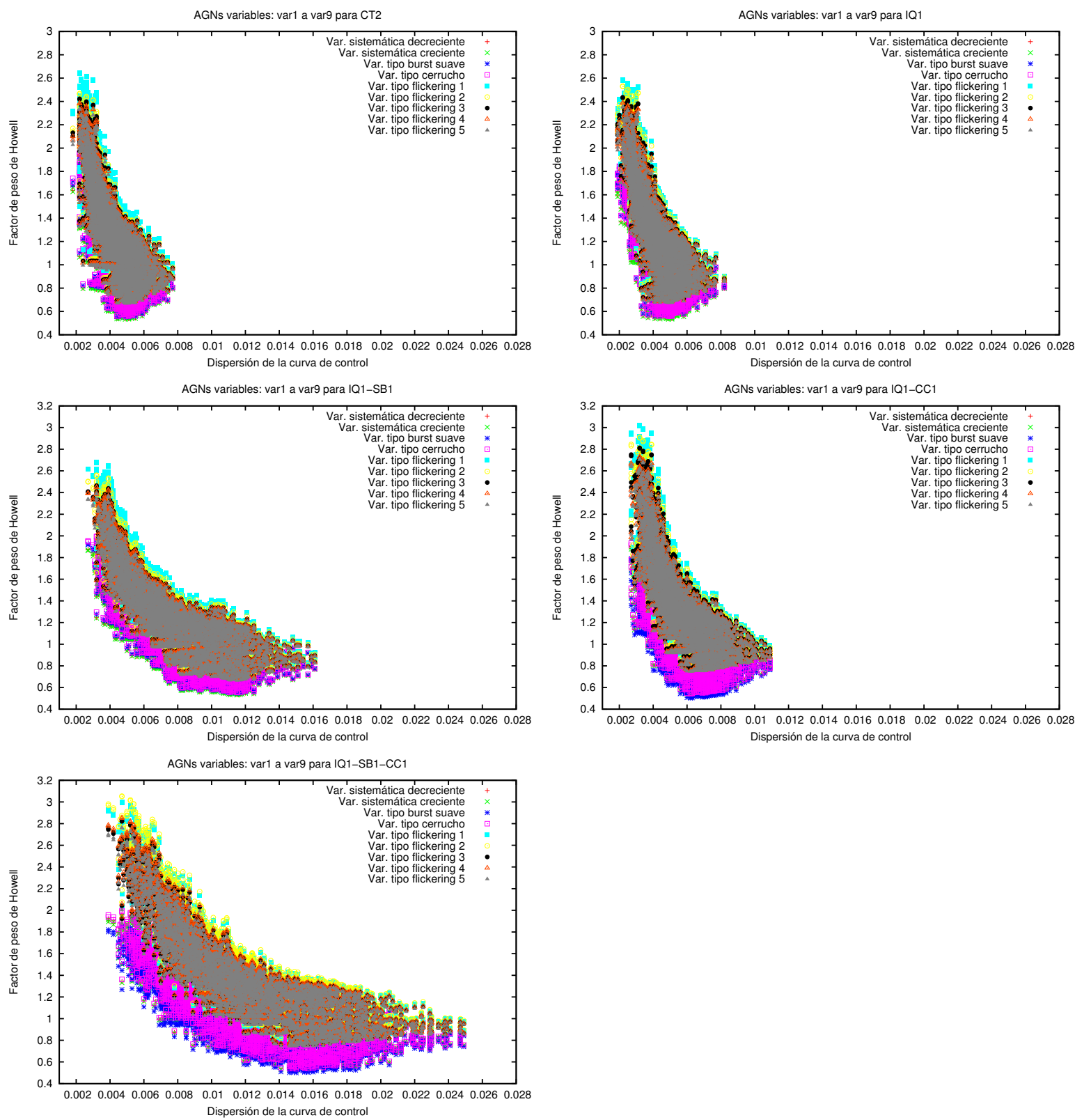

Figura 2.32. Comportamiento de la dispersión de la curva de control frente al valor del factor de peso de Howell, $\Gamma$, para las distintas variabilidades, en los casos CT2 (panel superior izquierdo), IQ1 (panel superior derecho), IQ1-SB1 (panel del medio izquierdo), IQ1-CC1 (panel del medio derecho) y IQ1-SB1-CC1 (panel inferior).

tanto con el criterio $\mathrm{C}$ como con el test F. Y la mayor cantidad de casos se da cuando se tienen involucrados todos los efectos juntos. En la Tabla 2.7 se presentan todos los casos, con sus respectivos porcentajes, en que las DLCs han sido clasificadas como no variables. Se presenta en la columna 1 la situación contemplada, en las columnas 2 y 3 los porcentajes de variabilidad para los parámetros $\mathrm{C}$ y $\mathrm{F}$ de la variabilidad burst suave; en las columnas 4 y 5 ídem para la variabilidad serrucho; en las columnas 6 y 7 ídem para la variabilidad flickering 3; en las columnas 8 y 9 ídem para la variabilidad 

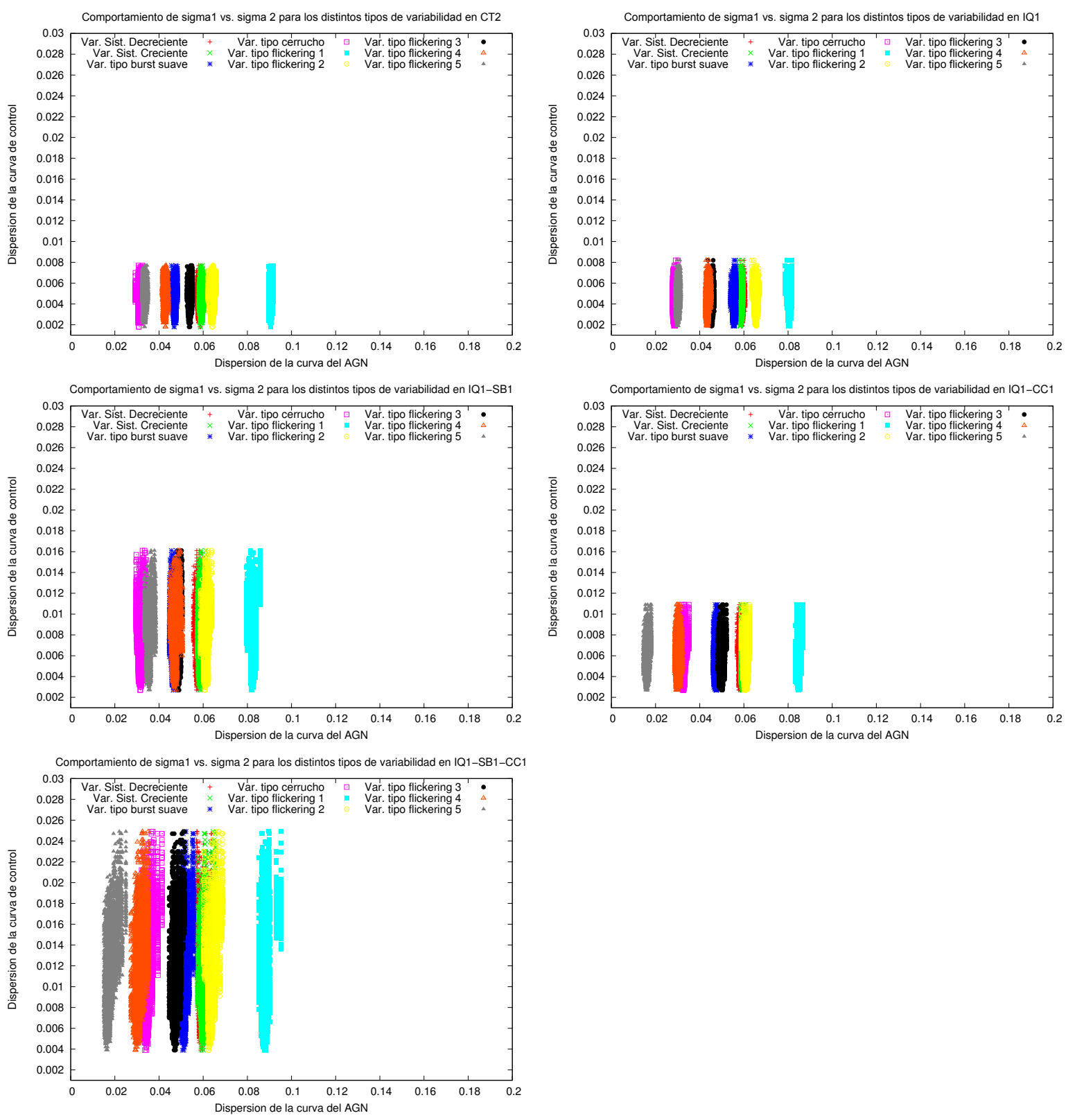

Figura 2.33. Comportamiento de la dispersión de la curva del AGN frente a la dispersión de la curva de control para las distintas variabilidades, en los casos CT2 (panel superior izquierdo), IQ1 (panel superior derecho), IQ1-SB1 (panel del medio izquierdo), IQ1-CC1 (panel del medio derecho) y IQ1-SB1-CC1 (panel inferior).

flickering 4; y en las columnas 10 y 11 ídem para la variabilidad flickering 5. Los porcentajes son sobre un total del 3906 DLCs. 
Tabla 2.7. Porcentajes de los casos de DLC que resultaron variables y no variables con los parámetros C y F en cada situación considerada: IQ1-SB1, IQ1-CC1 y IQ1SB1-CC1. Se presenta en la columna 1 la situación contemplada, en las columnas 2 y 3 los porcentajes de variabilidad para los parámetros $\mathrm{C}$ y $\mathrm{F}$ de la variabilidad burst suave; en las columnas 4 y 5 ídem para la variabilidad serrucho; en las columnas 6 y 7 ídem para la variabilidad flickering 3; en las columnas 8 y 9 ídem para la variabilidad flickering 4; y en las columnas 10 y 11 ídem para la variabilidad flickering 5 . Los porcentajes son sobre un total del 3906 DLCs.

\begin{tabular}{|c|cc|cc|cc|cc|c|c|}
\hline \hline Situación & \multicolumn{2}{|c}{ Var. burst suave } & \multicolumn{2}{c}{ Var. serrucho } & \multicolumn{2}{c}{ Var. flickering 3 } & \multicolumn{2}{c}{ Var. flickering 4} & \multicolumn{2}{c}{ Var. flickering 5 } \\
\hline & $\mathrm{C}$ & $\mathrm{F}$ & $\mathrm{C}$ & $\mathrm{F}$ & $\mathrm{C}$ & $\mathrm{F}$ & $\mathrm{C}$ & $\mathrm{F}$ & $\mathrm{C}$ \\
\hline \hline \multirow{2}{*}{ IQ1-CC1 } & $0 \% \mathrm{NV}$ & $0 \% \mathrm{NV}$ & $0 \% \mathrm{NV}$ & $0 \% \mathrm{NV}$ & $0 \% \mathrm{NV}$ & $0 \% \mathrm{NV}$ & $0 \% \mathrm{NV}$ & $0 \% \mathrm{NV}$ & $81.36 \% \mathrm{NV}$ & $0.38 \% \mathrm{NV}$ \\
& $100 \% \mathrm{~V}$ & $100 \% \mathrm{~V}$ & $100 \% \mathrm{~V}$ & $100 \% \mathrm{~V}$ & $100 \% \mathrm{~V}$ & $100 \% \mathrm{~V}$ & $100 \% \mathrm{~V}$ & $100 \% \mathrm{~V}$ & $18.64 \% \mathrm{~V}$ & $99.62 \% \mathrm{~V}$ \\
\hline \multirow{2}{*}{ IQ1-SB1 } & $0 \% \mathrm{NV}$ & $0 \% \mathrm{NV}$ & $2.18 \% \mathrm{NV}$ & $0 \% \mathrm{NV}$ & $0 \% \mathrm{NV}$ & $0 \% \mathrm{NV}$ & $0 \% \mathrm{NV}$ & $0 \% \mathrm{NV}$ & $0.28 \% \mathrm{NV}$ & $0 \% \mathrm{NV}$ \\
& $100 \% \mathrm{~V}$ & $100 \% \mathrm{~V}$ & $97.82 \% \mathrm{~V}$ & $100 \% \mathrm{~V}$ & $100 \% \mathrm{~V}$ & $100 \% \mathrm{~V}$ & $100 \% \mathrm{~V}$ & $100 \% \mathrm{~V}$ & $99.72 \% \mathrm{~V}$ & $100 \% \mathrm{~V}$ \\
\hline \multirow{2}{*}{ IQ1-SB1-CC1 } & $0.28 \% \mathrm{NV}$ & $0 \% \mathrm{NV}$ & $72.12 \% \mathrm{NV}$ & $0 \% \mathrm{NV}$ & $86.79 \% \mathrm{NV}$ & $0 \% \mathrm{NV}$ & $95.06 \% \mathrm{NV}$ & $6.02 \% \mathrm{NV}$ & $100 \% \mathrm{NV}$ & $98.28 \% \mathrm{NV}$ \\
& $99.72 \% \mathrm{~V}$ & $100 \% \mathrm{~V}$ & $27.88 \% \mathrm{~V}$ & $100 \% \mathrm{~V}$ & $13.21 \% \mathrm{~V}$ & $100 \% \mathrm{~V}$ & $4.94 \% \mathrm{~V}$ & $93.98 \% \mathrm{~V}$ & $0 \% \mathrm{~V}$ & $1.72 \% \mathrm{~V}$ \\
\hline
\end{tabular}


Analizando los porcentajes obtenidos para cada situación y cada variabilidad, observamos que, en primer lugar, si el valor del seeing es fijo (1.50 arcsec) o si varía pero se mantiene con un valor promedio de 1.52 arcsec, recuperamos el $100 \%$ del estado de variabilidad de los AGNs variables, tanto con el criterio C como con el test F. Segundo, al incorporar la cobertura de nubes a la variación del seeing, la única variabilidad afectada es la del flickering 5, cuya amplitud original es de 0.05 mag (comparable en orden de magnitud al ruido esperado en las curvas de control). Y en este caso, tanto el índice C como el parámetro F registran DLCs no variables, aunque la cantidad de DLCs es mucho mayor con el criterio C (3178 DLCs) que con el parámetro F (15 DLCs). Tercero, si ahora a la variación de seeing sólo se le agrega la presencia de la Luna Llena, además de verse afectada la variabilidad tipo flickering 5 , también lo es la variabilidad tipo serrucho, cuya amplitud original es de 0.15. Para ambas variabilidades, el parámetro F recupera el $100 \%$ del estado de variabilidad de las DLCs, mientras que el criterio C detecta en ambas, un porcentaje bajo de DLCs no variables. En el caso de la variabilidad tipo serrucho, son 85 de 3906 DLCs las clasificadas como no variables, mientras que para la variabilidad tipo flickering 5 son 11 de 3906 DLCs. Finalmente, al contemplar todos los efectos juntos, encontramos que son más las variabilidades afectadas en sus resultados. Obtuvimos que 5 de las 9 variabilidades que contemplamos presentan DLCs clasificadas como no variables. Y lo interesante es que estas 5 variabilidades tienen amplitudes originales $\Delta m<0.15 \mathrm{mag}$, mientras que aquellas variabilidades que no se vieron afectadas poseen amplitudes originales $\Delta m>0.2$ mag. La estadística $\mathrm{F}$, en particular, detecta DLCs como no variables en los últimos dos casos de variabilidad tipo flickering, donde se tienen $\Delta m<0.1$ mag. Y en particular, para la variabilidad tipo flickering $5(\Delta m<0.05 \mathrm{mag})$, la cantidad de DLCs clasificadas como no variables es casi $100 \%$ (3839 de 3906 DLCs). Por otro lado, el parámetro C encuentra DLCs no variables en estas 5 variabilidades. Y a medida que la amplitud de la variabilidad se hace menor, son más los casos detectados como no variables. Como ejemplo, si tomamos la variabilidad burst suave $(\Delta m<0.15 \mathrm{mag})$, el criterio C clasifica $0.28 \%$ de las DLCs como no variables. Moviéndonos hacia las variaciones con amplitudes menores, este número tiene un importante incremento llegando a detectar el $100 \%$ de las DLCs como no variables en el caso de la variabilidad tipo flickering de menor amplitud.

\section{Comparación con otras situaciones de cobertura de nubes}

Analizamos los resultados obtenidos de las herramientas C y F si a la situación anterior (con una determinada variación del seeing y la presencia de la Luna, IQ1SB1), le aplicamos el resto de las coberturas de nubes simuladas.

En lo que respecta a los AGNs no variables, se obtienen porcentajes similares en las estadísticas respecto de lo que se obtuvo al considerar la cobertura de nubes CC1. En cada uno de los distintos criterios de Howell, tanto el C como el F recuperan $99.70 \%$ la clasificación de no variable de las DLCs. Los valores que se obtuvieron en cada una de las situaciones mencionadas en el párrafo anterior son muy similares a los resultados mostrados en la Tabla 2.6 (filas 4 y 5).

Por otra parte, en cuanto a los AGNs variables, si consideramos primero la variación del seeing junto con la cobertura de nubes, resulta nuevamente que la única variabilidad afectada es la variabilidad tipo flickering 5, cuya amplitud original es de $\Delta m=0.05$ mag. Lo que sí es diferente es el valor del porcentaje de las DLCs que han resultado ser 
variable y no variables en cada cobertura. Para el caso de IQ1-CC2, los porcentajes de DLCs variables y no variables con el test $\mathrm{F}$ es el mismo que para IQ1-CC1, mientras que el porcentaje del parámetro $\mathrm{C}$ de DLCs variables es más alto, con un valor de $\sim 24 \%$ (respecto de un 18.60\%). Para los restantes casos de IQ1-CC3, IQ1-CC4 y IQ1-CC5, el parámetro F recupera el $100 \%$ del estado de variabilidad de la DLCs (salvo IQ1-CC4, con un valor de 99.54\%). En cambio, los porcentajes correspondientes al criterio C varían de una situación a otra. La cantidad de DLCs que recuperan su clasificación como variables con el C es del $32.75 \%$ para IQ1-CC3, $19.3 \%$ para IQ1-CC4 y $59.14 \%$ para IQ1-CC5. En relación con el tipo de cobertura de nubes, se tiene que el caso en donde el parámetro C recupera el mayor porcentaje de DLCs variables corresponde a la situación CC5, donde recordemos que ésta involucra un velo muy tenue en una parte de la noche teniendo el resto de la misma sin nubes. En cambio, el porcentaje más bajo que clasifica como variables a las DLCs está asociado a CC1 y CC4, donde se tiene la situación de nubosidad en aumento en el primero y un cielo nublado que luego se despeja en el segundo. Esto es, así como el test F tiende a clasificar como variables curvas sólo ruidosas (en los casos NV), el parámetro C, cuando se obtienen curvas con variaciones de baja amplitud, no detecta la variabilidad. Estas variaciones de baja amplitud se ven más afectadas por los cambios en las condiciones de observación.

Finalmente le sumamos la presencia de la Luna Llena a las situaciones descriptas anteriormente. Nuevamente, obtenemos que el número de variabilidades afectadas al sumar todos los efectos observacionales es mayor respecto de considerar los efectos individualmente. Se sigue observando que las variabilidades más afectadas son aquellas cuya amplitud es de $\Delta m<0.15$ mag. Sin embargo para una situación particular, la IQ1-SB1-CC2 (cobertura de nubes donde a lo largo de la noche se nubla y se despeja alternativamente), casi todas las variabilidades se vieron afectadas. En la Tabla 2.8 se resumen los resultados obtenidos para esta situaciones.

Como se puede ver en la Tabla, para el caso IQ1-SB1-CC2, se sigue manteniendo la tendencia que, al incorporar todos los efectos, los resultados en la clasificación de las DLCs asociadas a las variabilidades cuya amplitud original sea $\Delta m<0.15$ mag serán las más afectadas. 
Tabla 2.8. Porcentajes de los casos de DLC que resultaron variables y no variables con los parámetros $\mathrm{C}$ y $\mathrm{F}$ en cada situación considerada: IQ1-SB1-CC2, IQ1-SB1CC3, IQ1-SB1-CC4 y IQ1-SB1-CC5. Se presenta en la columna 1 la situación contemplada, en las columnas 2 y 3 los porcentajes de variabilidad para $\mathrm{C}$ y $\mathrm{F}$ de la var. sist. decreciente; en las columnas 4 y 5 ídem para la var. sist. creciente; en las columnas 6 y 7 ídem para la var. burst suave y en las columnas 8 y 9 ídem para la var. serrucho. Los porcentajes son sobre un total del 3906 DLCs.

\begin{tabular}{|c|cc|cc|cc|cc|}
\hline \hline Situación & \multicolumn{2}{|c|}{ Var. sist. decreciente } & \multicolumn{2}{c}{ Var. sist. creciente } & \multicolumn{2}{c|}{ Var. burst suave } & \multicolumn{2}{c|}{ Var. serrucho } \\
\hline & $\mathrm{C}$ & $\mathrm{F}$ & $\mathrm{C}$ & $\mathrm{F}$ & $\mathrm{C}$ & $\mathrm{F}$ & $\mathrm{C}$ & $\mathrm{F}$ \\
\hline \hline IQ1-SB1-CC2 & $0.05 \% \mathrm{NV}$ & $0 \% \mathrm{NV}$ & $0.03 \% \mathrm{NV}$ & $0 \% \mathrm{NV}$ & $2.82 \% \mathrm{NV}$ & $0 \% \mathrm{NV}$ & $32.24 \% \mathrm{NV}$ & $0.03 \% \mathrm{NV}$ \\
& $99.95 \% \mathrm{~V}$ & $100 \% \mathrm{~V}$ & $99.97 \% \mathrm{~V}$ & $100 \% \mathrm{~V}$ & $97.18 \% \mathrm{~V}$ & $100 \% \mathrm{~V}$ & $67.76 \% \mathrm{~V}$ & $99.97 \% \mathrm{~V}$ \\
\hline IQ1-SB1-CC3 & $0 \% \mathrm{NV}$ & $0 \% \mathrm{NV}$ & $0 \% \mathrm{NV}$ & $0 \% \mathrm{NV}$ & $0 \% \mathrm{NV}$ & $0 \% \mathrm{NV}$ & $23.66 \% \mathrm{NV}$ & $0 \% \mathrm{NV}$ \\
& $100 \% \mathrm{~V}$ & $100 \% \mathrm{~V}$ & $100 \% \mathrm{~V}$ & $100 \% \mathrm{~V}$ & $100 \% \mathrm{~V}$ & $100 \% \mathrm{~V}$ & $76.34 \% \mathrm{~V}$ & $100 \% \mathrm{~V}$ \\
\hline IQ1-SB1-CC4 & $0 \% \mathrm{NV}$ & $0 \% \mathrm{NV}$ & $0 \% \mathrm{NV}$ & $0 \% \mathrm{NV}$ & $0.62 \% \mathrm{NV}$ & $0 \% \mathrm{NV}$ & $22.02 \% \mathrm{NV}$ & $0 \% \mathrm{NV}$ \\
& $100 \% \mathrm{~V}$ & $100 \% \mathrm{~V}$ & $100 \% \mathrm{~V}$ & $100 \% \mathrm{~V}$ & $99.38 \% \mathrm{~V}$ & $100 \% \mathrm{~V}$ & $77.98 \% \mathrm{~V}$ & $100 \% \mathrm{~V}$ \\
\hline IQ1-SB1-CC5 & $0 \% \mathrm{NV}$ & $0 \% \mathrm{NV}$ & $0 \% \mathrm{NV}$ & $0 \% \mathrm{NV}$ & $0 \% \mathrm{NV}$ & $0 \% \mathrm{NV}$ & $6.96 \% \mathrm{NV}$ & $0 \% \mathrm{NV}$ \\
& $100 \% \mathrm{~V}$ & $100 \% \mathrm{~V}$ & $100 \% \mathrm{~V}$ & $100 \% \mathrm{~V}$ & $100 \% \mathrm{~V}$ & $100 \% \mathrm{~V}$ & $93.04 \% \mathrm{~V}$ & $100 \% \mathrm{~V}$ \\
\hline
\end{tabular}




\begin{tabular}{|c|cc|cc|cc|cc|}
\hline \hline Situación & \multicolumn{2}{|c}{ Var. flickering 2} & \multicolumn{2}{c}{ Var. flickering 3 } & \multicolumn{2}{c|}{ Var. flickering 4} & \multicolumn{2}{c|}{ Var. flickering 5} \\
\hline & $\mathrm{C}$ & $\mathrm{F}$ & $\mathrm{C}$ & $\mathrm{F}$ & $\mathrm{C}$ & $\mathrm{F}$ & $\mathrm{C}$ & $\mathrm{F}$ \\
\hline \hline IQ1-SB1-CC2 & $9.5 \% \mathrm{NV}$ & $0 \% \mathrm{NV}$ & $8.37 \% \mathrm{NV}$ & $0 \% \mathrm{NV}$ & $74.83 \% \mathrm{NV}$ & $2.28 \% \mathrm{NV}$ & $99.97 \% \mathrm{NV}$ & $54.63 \% \mathrm{NV}$ \\
& $90.5 \% \mathrm{~V}$ & $100 \% \mathrm{~V}$ & $91.63 \% \mathrm{~V}$ & $100 \% \mathrm{~V}$ & $25.17 \% \mathrm{~V}$ & $97.72 \% \mathrm{~V}$ & $0.03 \% \mathrm{~V}$ & $45.37 \% \mathrm{~V}$ \\
\hline IQ1-SB1-CC3 & $0 \% \mathrm{NV}$ & $0 \% \mathrm{NV}$ & $6.58 \% \mathrm{NV}$ & $0 \% \mathrm{NV}$ & $60.91 \% \mathrm{NV}$ & $0.05 \% \mathrm{NV}$ & $100 \% \mathrm{NV}$ & $43.75 \% \mathrm{NV}$ \\
& $100 \% \mathrm{~V}$ & $100 \% \mathrm{~V}$ & $93.42 \% \mathrm{~V}$ & $100 \% \mathrm{~V}$ & $39.09 \% \mathrm{~V}$ & $99.95 \% \mathrm{~V}$ & $0 \% \mathrm{~V}$ & $56.25 \% \mathrm{~V}$ \\
\hline IQ1-SB1-CC4 & $0.05 \% \mathrm{NV}$ & $0 \% \mathrm{NV}$ & $14.67 \% \mathrm{NV}$ & $0 \% \mathrm{NV}$ & $70.58 \% \mathrm{NV}$ & $0.87 \% \mathrm{NV}$ & $99.97 \% \mathrm{NV}$ & $68.9 \% \mathrm{NV}$ \\
& $99.95 \% \mathrm{~V}$ & $100 \% \mathrm{~V}$ & $85.33 \% \mathrm{~V}$ & $100 \% \mathrm{~V}$ & $29.42 \% \mathrm{~V}$ & $99.13 \% \mathrm{~V}$ & $0.03 \% \mathrm{~V}$ & $31.1 \% \mathrm{~V}$ \\
\hline IQ1-SB1-CC5 & $0 \% \mathrm{NV}$ & $0 \% \mathrm{NV}$ & $0.03 \% \mathrm{NV}$ & $0 \% \mathrm{NV}$ & $33.05 \% \mathrm{NV}$ & $0 \% \mathrm{NV}$ & $100 \% \mathrm{NV}$ & $60.47 \% \mathrm{NV}$ \\
& $100 \% \mathrm{~V}$ & $100 \% \mathrm{~V}$ & $99.97 \% \mathrm{~V}$ & $100 \% \mathrm{~V}$ & $66.95 \% \mathrm{~V}$ & $100 \% \mathrm{~V}$ & $0 \% \mathrm{~V}$ & $39.53 \% \mathrm{~V}$ \\
\hline
\end{tabular}




\section{Comparación con otras situaciones de variación de seeing}

Por último, analizamos los resultados de las herramientas $\mathrm{C}$ y $\mathrm{F}$, agregando a las situaciones de la sección anterior, el resto de las variaciones en el seeing.

Nuevamente, para los AGNs no variables, los resultados de variabilidad de las DLCs con los parámetros $\mathrm{C}$ y $\mathrm{F}$ son similares a los que se obtuvieron para el resto de las situaciones ya analizadas (por ej., ver Tabla 2.5).

Para los AGNs variables, el comportamiento en los resultados de la clasificación de las DLCs con los parámetros C y F también resulta similar a lo obtenido anteriormente (ver Tabla 2.8). Las variabilidades que más resultan ser afectadas siguen siendo aquellas que presentan una amplitud original $\Delta m<0.15 \mathrm{mag}$. Pero en particular, la variabilidad que siempre se ve afectada y sobretodo, en los resultados del parámetro $\mathrm{C}$ cuando se consideran todos los efectos juntos es la variabilidad tipo serrucho. Esta variabilidad es una de las que posee una amplitud $\Delta m<0.15$ mag. En la Tabla 2.8 mostramos todas las combinaciones entre las distintas variaciones del seeing, presencia de la Luna y cobertura de nubes y cómo son los porcentajes obtenidos para esta variabilidad en particular.

\subsection{Discusión}

Con respecto a los casos planteados como no variables, encontramos que, siempre que usemos el factor de peso, ambos índices de variabilidad ( $\mathrm{C}$ y F) son confiables para clasificar las DLCs. Sin embargo, encontramos que el F es más sensible a los ruidos presentes en las observaciones, lo que deriva en porcentajes de DLCs clasificadas como variables. Si bien estos valores son bajos $(<1 \%)$, se puede decir que con el test F encontramos resultados de falsos positivos.

En el análisis de los AGNs variables, considerar diferentes situaciones arroja distintos resultados. Por un lado, la sola presencia del seeing, tanto fijo como variable a lo largo de la noche, no influye en la robustez de la herramienta estadística utilizada para la clasificación de las DLCs. En todos los casos (CT y IQ) se recupera el $100 \%$ de la variabilidad para todos los casos considerados, involucrando distintos tipos de variabilidad y diferentes amplitudes de la misma. Dentro de los valores de seeing planteados para las simulaciones, encontramos que no hay diferencias entre considerar los valores del seeing punto a punto y analizar el comportamiento de las DLCs tomando un valor promedio representativo del mismo. Dado que las condiciones de seeing fijo y variable nos devuelven los mismos resultados, la incorporación de los demás efectos se realizó sobre los casos que contemplan la variación del seeing, debido a que en los casos reales de observación siempre se tiene variación en los valores del seeing (pues éste depende de la turbulencia que tiene la atmósfera terrestre).

Considerando las distintas combinaciones de IQ variables con las distintos valores del brillo del cielo debido a la mayor o menor presencia de la Luna, encontramos que la mayor afectación de la presencia del brillo de la Luna se da sobre los flickering de menor amplitud (0.10 y $0.05 \mathrm{mag}$ ). Estos porcentajes son mayores cuando se considera la Luna Llena, siendo el índice C el más afectado. Esto es, detecta un número mayor de casos no variables que el test $\mathrm{F}$. El efecto combinado de un seeing variable con la 
Tabla 2.8. Porcentajes de los casos de DLC que resultaron variables y no variables con los parámetros C y F en cada situación considerada. Se presenta en la columna 1 la situación contemplada, en las columnas 2 y 3 los porcentajes de variabilidad para $\mathrm{C}$ y $\mathrm{F}$ de la var. sist. serrucho y en las columnas 4 y 5 ídem para la var. flickering 5. Los porcentajes son sobre un total del 3906 DLCs.

\begin{tabular}{|c|c|c|c|c|}
\hline \multirow[t]{2}{*}{ Situación } & \multicolumn{2}{|c|}{ Var. serrucho } & \multicolumn{2}{|c|}{ Var. flickering 5} \\
\hline & $\mathrm{C}$ & $\mathrm{F}$ & $\mathrm{C}$ & $\mathrm{F}$ \\
\hline IQ1-SB1-CC1 & $\begin{array}{c}72.12 \% \mathrm{NV} \\
27.88 \% \mathrm{~V}\end{array}$ & $\begin{array}{l}0 \% \mathrm{NV} \\
100 \% \mathrm{~V}\end{array}$ & $\begin{array}{c}100 \% \mathrm{NV} \\
0 \% \mathrm{~V}\end{array}$ & $\begin{array}{c}98.28 \% \mathrm{NV} \\
1.72 \% \mathrm{~V}\end{array}$ \\
\hline IQ1-SB1-CC2 & $\begin{array}{c}32.24 \% \mathrm{NV} \\
67.76 \% \mathrm{~V}\end{array}$ & $\begin{array}{l}0.03 \% \mathrm{NV} \\
99.97 \% \mathrm{~V}\end{array}$ & $\begin{array}{c}99.97 \% \mathrm{NV} \\
0.03 \% \mathrm{~V}\end{array}$ & $\begin{array}{c}54.63 \% \mathrm{NV} \\
45.37 \% \mathrm{~V}\end{array}$ \\
\hline IQ1-SB1-CC3 & $\begin{array}{c}23.66 \% \mathrm{NV} \\
76.34 \% \mathrm{~V}\end{array}$ & $\begin{array}{l}0 \% \mathrm{NV} \\
100 \% \mathrm{~V}\end{array}$ & $\begin{array}{c}100 \% \mathrm{NV} \\
0 \% \mathrm{~V}\end{array}$ & $\begin{array}{c}43.75 \% \mathrm{NV} \\
56.25 \% \mathrm{~V}\end{array}$ \\
\hline IQ1-SB1-CC4 & $\begin{array}{c}22.02 \% \mathrm{NV} \\
77.98 \% \mathrm{~V}\end{array}$ & $\begin{array}{l}0 \% \mathrm{NV} \\
100 \% \mathrm{~V}\end{array}$ & $\begin{array}{c}99.97 \% \mathrm{NV} \\
0.03 \% \mathrm{~V}\end{array}$ & $\begin{array}{c}68.9 \% \mathrm{NV} \\
31.1 \% \mathrm{~V}\end{array}$ \\
\hline IQ1-SB1-CC5 & $\begin{array}{l}6.96 \% \mathrm{NV} \\
93.04 \% \mathrm{~V}\end{array}$ & $\begin{array}{l}0 \% \mathrm{NV} \\
100 \% \mathrm{~V}\end{array}$ & $\begin{array}{c}100 \% \mathrm{NV} \\
0 \% \mathrm{~V}\end{array}$ & $\begin{array}{c}60.47 \% \mathrm{NV} \\
39.53 \% \mathrm{~V}\end{array}$ \\
\hline IQ2-SB1-CC1 & $\begin{array}{c}37.92 \% \mathrm{NV} \\
62.08 \% \mathrm{~V}\end{array}$ & $\begin{array}{l}0.4 \% \mathrm{NV} \\
99.6 \% \mathrm{~V}\end{array}$ & $\begin{array}{l}0 \% \mathrm{NV} \\
100 \% \mathrm{~V}\end{array}$ & $\begin{array}{l}0 \% \mathrm{NV} \\
100 \% \mathrm{~V}\end{array}$ \\
\hline IQ2-SB1-CC2 & $\begin{array}{c}44.19 \% \mathrm{NV} \\
55.81 \% \mathrm{~V}\end{array}$ & $\begin{array}{l}0.3 \% \mathrm{NV} \\
99.7 \% \mathrm{~V}\end{array}$ & $\begin{array}{l}0 \% \mathrm{NV} \\
100 \% \mathrm{~V}\end{array}$ & $\begin{array}{l}0 \% \mathrm{NV} \\
100 \% \mathrm{~V}\end{array}$ \\
\hline IQ2-SB1-CC3 & $\begin{array}{c}18.25 \% \mathrm{NV} \\
81.75 \% \mathrm{~V}\end{array}$ & $\begin{array}{l}0 \% \mathrm{NV} \\
100 \% \mathrm{~V}\end{array}$ & $\begin{array}{l}0 \% \mathrm{NV} \\
100 \% \mathrm{~V}\end{array}$ & $\begin{array}{l}0 \% \mathrm{NV} \\
100 \% \mathrm{~V}\end{array}$ \\
\hline IQ2-SB1-CC4 & $\begin{array}{c}23.1 \% \mathrm{NV} \\
76.9 \% \mathrm{~V}\end{array}$ & $\begin{array}{l}0 \% \mathrm{NV} \\
100 \% \mathrm{~V}\end{array}$ & $\begin{array}{l}0 \% \mathrm{NV} \\
100 \% \mathrm{~V}\end{array}$ & $\begin{array}{l}0 \% \mathrm{NV} \\
100 \% \mathrm{~V}\end{array}$ \\
\hline IQ2-SB1-CC5 & $\begin{array}{l}6.89 \% \mathrm{NV} \\
93.11 \% \mathrm{~V}\end{array}$ & $\begin{array}{l}0 \% \mathrm{NV} \\
100 \% \mathrm{~V}\end{array}$ & $\begin{array}{l}0 \% \mathrm{NV} \\
100 \% \mathrm{~V}\end{array}$ & $\begin{array}{l}0 \% \mathrm{NV} \\
100 \% \mathrm{~V}\end{array}$ \\
\hline IQ3-SB1-CC1 & $\begin{array}{c}28.93 \% \mathrm{NV} \\
71.07 \% \mathrm{~V}\end{array}$ & $\begin{array}{l}0.13 \% \mathrm{NV} \\
99.87 \% \mathrm{~V}\end{array}$ & $\begin{array}{l}0 \% \mathrm{NV} \\
100 \% \mathrm{~V}\end{array}$ & $\begin{array}{l}0 \% \mathrm{NV} \\
100 \% \mathrm{~V}\end{array}$ \\
\hline IQ3-SB1-CC2 & $\begin{array}{c}36.12 \% \mathrm{NV} \\
63.88 \% \mathrm{~V}\end{array}$ & $\begin{array}{l}0.44 \% \mathrm{NV} \\
99.56 \% \mathrm{~V}\end{array}$ & $\begin{array}{l}0 \% \mathrm{NV} \\
100 \% \mathrm{~V}\end{array}$ & $\begin{array}{l}0 \% \mathrm{NV} \\
100 \% \mathrm{~V}\end{array}$ \\
\hline IQ3-SB1-CC3 & $\begin{array}{l}5.15 \% \mathrm{NV} \\
94.85 \% \mathrm{~V}\end{array}$ & $\begin{array}{l}0 \% \mathrm{NV} \\
100 \% \mathrm{~V}\end{array}$ & $\begin{array}{l}0 \% \mathrm{NV} \\
100 \% \mathrm{~V}\end{array}$ & $\begin{array}{l}0 \% \mathrm{NV} \\
100 \% \mathrm{~V}\end{array}$ \\
\hline IQ3-SB1-CC4 & $\begin{array}{c}23.8 \% \mathrm{NV} \\
76.2 \% \mathrm{~V}\end{array}$ & $\begin{array}{l}0 \% \mathrm{NV} \\
100 \% \mathrm{~V}\end{array}$ & $\begin{array}{l}0 \% \mathrm{NV} \\
100 \% \mathrm{~V}\end{array}$ & $\begin{array}{l}0 \% \mathrm{NV} \\
100 \% \mathrm{~V}\end{array}$ \\
\hline IQ3-SB1-CC5 & $\begin{array}{l}9.32 \% \mathrm{NV} \\
90.68 \% \mathrm{~V}\end{array}$ & $\begin{array}{l}0 \% \mathrm{NV} \\
100 \% \mathrm{~V}\end{array}$ & $\begin{array}{l}0 \% \mathrm{NV} \\
100 \% \mathrm{~V}\end{array}$ & $\begin{array}{l}0 \% \mathrm{NV} \\
100 \% \mathrm{~V}\end{array}$ \\
\hline
\end{tabular}


Tabla 2.8. Cont.

\begin{tabular}{|c|cc|cc|}
\hline \hline Situación & \multicolumn{2}{|c}{ Var. serrucho } & \multicolumn{2}{c}{ Var. flickering 5} \\
\hline & $\mathrm{C}$ & $\mathrm{F}$ & $\mathrm{C}$ & $\mathrm{F}$ \\
\hline \hline IQ4-SB1-CC1 & $32.87 \% \mathrm{NV}$ & $0.15 \% \mathrm{NV}$ & $0 \% \mathrm{NV}$ & $0 \% \mathrm{NV}$ \\
& $67.13 \% \mathrm{~V}$ & $99.85 \% \mathrm{~V}$ & $100 \% \mathrm{~V}$ & $100 \% \mathrm{~V}$ \\
\hline IQ4-SB1-CC2 & $36.36 \% \mathrm{NV}$ & $0.1 \% \mathrm{NV}$ & $0 \% \mathrm{NV}$ & $0 \% \mathrm{NV}$ \\
& $63.64 \% \mathrm{~V}$ & $99.9 \% \mathrm{~V}$ & $100 \% \mathrm{~V}$ & $100 \% \mathrm{~V}$ \\
\hline IQ4-SB1-CC3 & $29.99 \% \mathrm{NV}$ & $0 \% \mathrm{NV}$ & $0 \% \mathrm{NV}$ & $0 \% \mathrm{NV}$ \\
& $70.01 \% \mathrm{~V}$ & $100 \% \mathrm{~V}$ & $100 \% \mathrm{~V}$ & $100 \% \mathrm{~V}$ \\
\hline IQ4-SB1-CC4 & $15.36 \% \mathrm{NV}$ & $0 \% \mathrm{NV}$ & $0 \% \mathrm{NV}$ & $0 \% \mathrm{NV}$ \\
& $84.64 \% \mathrm{~V}$ & $100 \% \mathrm{~V}$ & $100 \% \mathrm{~V}$ & $100 \% \mathrm{~V}$ \\
\hline IQ4-SB1-CC5 & $11.14 \% \mathrm{NV}$ & $0 \% \mathrm{NV}$ & $0 \% \mathrm{NV}$ & $0 \% \mathrm{NV}$ \\
& $88.86 \% \mathrm{~V}$ & $100 \% \mathrm{~V}$ & $100 \% \mathrm{~V}$ & $100 \% \mathrm{~V}$ \\
\hline IQ5-SB1-CC1 & $40.27 \% \mathrm{NV}$ & $0.2 \% \mathrm{NV}$ & $0 \% \mathrm{NV}$ & $0 \% \mathrm{NV}$ \\
& $59.73 \% \mathrm{~V}$ & $99.8 \% \mathrm{~V}$ & $100 \% \mathrm{~V}$ & $100 \% \mathrm{~V}$ \\
\hline IQ5-SB1-CC2 & $48.18 \% \mathrm{NV}$ & $0.23 \% \mathrm{NV}$ & $0 \% \mathrm{NV}$ & $0 \% \mathrm{NV}$ \\
& $51.82 \% \mathrm{~V}$ & $99.77 \% \mathrm{~V}$ & $100 \% \mathrm{~V}$ & $100 \% \mathrm{~V}$ \\
\hline IQ5-SB1-CC3 & $20.43 \% \mathrm{NV}$ & $0 \% \mathrm{NV}$ & $0 \% \mathrm{NV}$ & $0 \% \mathrm{NV}$ \\
& $79.57 \% \mathrm{~V}$ & $100 \% \mathrm{~V}$ & $100 \% \mathrm{~V}$ & $100 \% \mathrm{~V}$ \\
\hline IQ5-SB1-CC4 & $24.27 \% \mathrm{NV}$ & $0 \% \mathrm{NV}$ & $0 \% \mathrm{NV}$ & $0 \% \mathrm{NV}$ \\
& $75.73 \% \mathrm{~V}$ & $100 \% \mathrm{~V}$ & $100 \% \mathrm{~V}$ & $100 \% \mathrm{~V}$ \\
\hline IQ5-SB1-CC5 & $9.9 \% \mathrm{NV}$ & $0 \% \mathrm{NV}$ & $0 \% \mathrm{NV}$ & $0 \% \mathrm{NV}$ \\
& $90.1 \% \mathrm{~V}$ & $100 \% \mathrm{~V}$ & $100 \% \mathrm{~V}$ & $100 \% \mathrm{~V}$ \\
\hline
\end{tabular}


presencia de la Luna aumenta el ruido presente en las curvas y éste tiene una influencia mayor para las variaciones de baja amplitud.

El siguiente efecto considerado sumado a las variaciones del seeing es la cobertura de nubes. Nuevamente los casos que se ven afectados son aquellos en los cuales la variación es del orden de $0.05 \mathrm{mag}$. Volvemos a encontrar que el criterio $\mathrm{C}$ detecta falsos negativos (llegando a valores de más del $90 \%$ en los casos en los cuales se tiene el mayor valor del seeing). En el caso del test F existen combinaciones de IQ y CC con los cuales se obtienen falsos negativos en un bajo porcentaje.

Lo que podemos observar de este comportamiento es que la combinación de un cambio en la calidad de la imagen sumado a un efecto que modifica el número de cuentas que se miden sobre la imagen, ya sea incrementándolo (presencia de la Luna) o disminuyéndolo (presencia de nubes), aumenta la componente del ruido en las DLCs de manera tal que se enmascaran las variaciones de baja amplitud. Esto se ve reflejado mayormente sobre el índice $\mathrm{C}$ ya que, como se vio en los casos no variables, nunca detecta al ruido como variabilidad. Esto no ocurre con el parámetro F ya que el mismo es sensible a reportar falsos positivos, reconociendo ruido como variabilidad.

Otro resultado importante que encontramos es con respecto a los resultados obtenidos para la variabilidad tipo serrucho. Esta variabilidad fue planteada originalmente considerando grupos de aproximadamente 8 puntos, de la misma manera que se consideraron las variaciones de los distintos parámetros atmosféricos. Esto nos permitió estudiar si las variaciones de la fuente y de las situaciones atmosféricas podían correlacionarse o no, influyendo en la clasificación de las DLCs. Esto se puede ver en los resultados mostrados en la Tabla 2.8. En todos los casos obtenemos falsos negativos aplicando el índice C, en distinta medida para cada caso en particular. Así vemos cómo en los casos en donde al tiempo que la fuente está aumentando su brillo y a la vez se incrementa la cobertura de nubes, la dispersión de la curva de control aumenta al mismo tiempo que disminuye la dispersión de la curva del AGN. A esto se le suma el ruido introducido por la presencia de la Luna y la variación en el seeing. Lo que estamos obteniendo es que existen casos en los cuales las variaciones en la fuente son contrarrestadas por los efectos variables en las condiciones atmosféricas. Si bien estamos trabajando con fotometría diferencial, los efectos introducidos afectan en distinta medida a objetos en un mismo campo de distinta magnitud (tanto las estrellas como a $\operatorname{los} \mathrm{AGNs}$ ).

Una dificultad importante para detectar variaciones, sobretodo las de baja amplitud utilizando fotometría, es la influencia de los distintos errores presentes. Esto lo vemos con los resultados obtenidos. En cualquiera de los dos índices estudiados, obtenemos resultados opuestos al estado de variabilidad inicial. En los casos de los AGNs no variables, el índice $\mathrm{C}$ resulta totalmente robusto. Con el test $\mathrm{F}$, encontramos algunos casos de falsos positivos. Para los casos de AGNs variables, encontramos, dependiendo del tipo de variación y la amplitud de la misma, falsos negativos. Esto ocurre en mayor medida cuando aplicamos el índice $\mathrm{C}$, lo cual es esperable, dado que el índice $\mathrm{C}$ clasifica como no variables a las curvas ruidosas. Obtuvimos que este nivel de ruido en las DLCs enmascara la presencia de variaciones intrínsecas, sobretodo las de baja amplitud. Esto no se obtienen al aplicar el test $\mathrm{F}$ ya que el mismo tiende a clasificar como variables curvas ruidosas. 
Encontramos que, tanto el criterio $\mathrm{C}$ como el test $\mathrm{F}$ son buenos indicadores de la no variabilidad de las DLCs, siendo el $\mathrm{C}$ un poco más robusto que el $\mathrm{F}$ (siempre utilizando el factor de peso). Por otra parte, hallamos que ambos índices no distinguen errores en las mediciones respecto a variaciones reales, y esto se debe a que los errores incrementan la dispersión presente en las DLCs, y al hecho de que los diferentes orígenes de estos errores pueden correlacionar con la variabilidad. Si nos fuera posible identificar cada una de estas fuentes de error con sus amplitudes y sus variaciones, y las pudiéramos extraer de las observaciones, sería posible obtener el estado de variabilidad del objeto de manera certera. En un estudio recientemente publicado sobre la eficiencia de distintos índices de variabilidad para detectar estrellas variables, Sokolovsky et al. (2017) encuentran resultados similares. En este trabajo no se estudian ni el índice $\mathrm{C}$ ni el test $\mathrm{F}$, sino que trabajan sobre una serie de índices comúnmente utilizados para la detección de estrellas variables. 



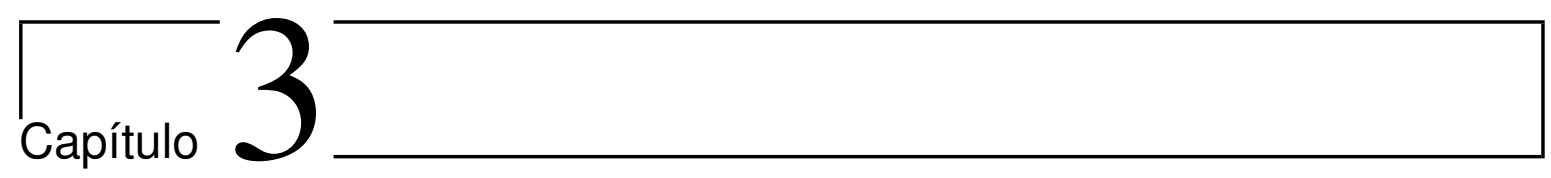

\section{Análisis del flujo de la muestra de Blazares}

\subsection{Selección de la muestra}

La muestra seleccionada para esta Tesis está basada en el catálogo del RomaBZCAT (Massaro et al., 2014, 5ta edición ${ }^{1}$ ). Este catálogo fue diseñado para tener la lista más completa y extensa de blazares publicados, con el objetivo de poder estudiar las contrapartes en altas energías de estas fuentes y, por otro lado, permite tener la información de la mayor base de datos de blazares. Esta información incluye la SED de cada objeto, lo que es de suma utilidad para el estudio y análisis de los mecanismos de radiación presentes en estas fuentes. El Roma-BZCAT fue construido a partir de la recopilación de información disponible en numerosos catálogos conocidos, tales como 1 Jy NVSS-RASS (Taker 2000, Giommi et al. 2002), CLASS (Cosmic Lens All-Sky Survey, Myers et al. 2003), CGRaBS (Candidate Gamma Ray Blazar Survey, Healey et al. 2008), etc. En la actualidad, se encuentra disponible la 5 ta edición, con un total de 3561 fuentes (Massaro et al. 2014), distribuidos como se muestra en la Fig. 3.1.

Este catálogo comprende básicamente 4 tipo de objetos: los BZB, que corresponden a los BL Lacs; los BZQ, quienes comprenden a los FSRQs; los BZG, los cuales son objetos tipo BL Lacs con una SED dominada principalmente por la emisión de la galaxia; y los BZU, que son los blazares de tipo incierto. El criterio de selección para nuestra muestra fue el siguiente: buscamos aquellas fuentes cuyas características en la banda óptica (posición en el cielo, magnitudes, etc.) permitieran su observación desde el Hemisferio Sur. Además, estos objetos debían tener observaciones con el satélite de rayos- $\gamma$, Fermi-LAT. Por otra parte, era necesario que nuestra muestra contemple tanto fuentes conocidas y bien estudiadas, como también fuentes que no hayan sido estudiadas, sobretodo en cuanto al análisis de la variabilidad y a su clasificación. En nuestro caso, seleccionamos 19 blazares de este catálogo, los cuales se dividen en BZB, BZQ y BZU. De aquí en adelante a los BZB lo llamaremos como BL Lacs y los BZQ como FSRQs.

Los datos de la muestra se presentan en la Tabla 3.1, teniendo en la columna 1 el nombre de la fuente, en la columna 2 su clasificación (referida al catálogo Roma BZCAT) junto con el tipo de SED que tiene, en las columnas 3 y 4 las coordenadas referidas al J2000.0, en la columna 5 el valor de su redshift y en la columna 6 la mag-

\footnotetext{
${ }^{1}$ http://www.asdc.asi.it/bzcat/
} 


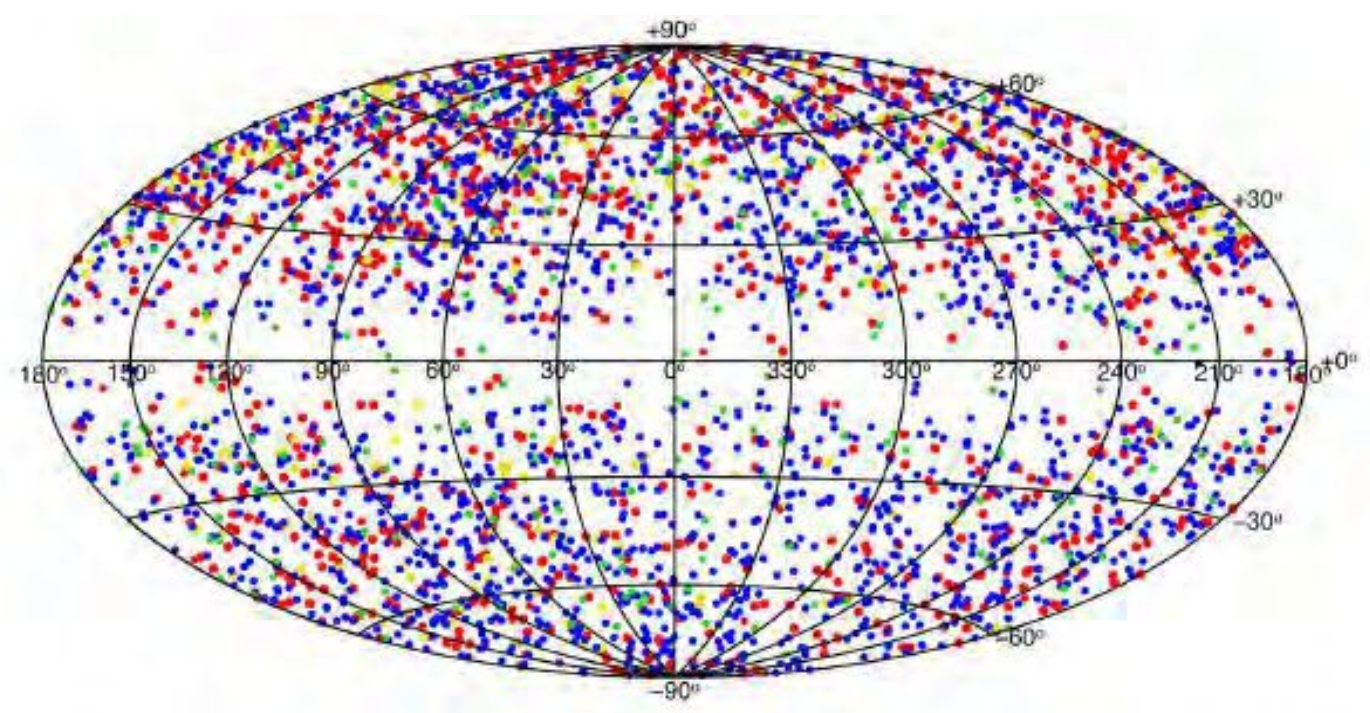

Figura 3.1. Distribución de todos los blazares comprendidos en la 5ta Edición del Catálogo de Roma-BZCAT. En rojo se muestran los BL Lacs, en azul los FSRQs, en amarillo los BZG y en verde los BZU (Massaro et al. 2014).

nitud visual publicada en la base de datos NED (de sus siglas en inglés, NASA/IPAC Extragalactic Databse).

\subsection{Análisis en el Óptico}

\subsubsection{Observaciones en CASLEO y procesamiento de datos}

Para estudiar el comportamiento del flujo óptico de la muestra seleccionada, se obtuvieron 4 pedidos de turno de observación con el Telescopio de 2.15m "Jorge Sahade" en CASLEO y se utilizó la cámara directa (ubicada en el foco Cassegrain) Roper Scientific. Este CCD tiene un área de $2048 \times 2048$ pixels de $13.5 \mu \mathrm{m}$ de lado c/u, instalado en un dewar enfriado con nitrógeno líquido. La escala en este CCD es de 0.15 $\operatorname{arcsec} / \mathrm{px}$, con un campo útil cuadrado de $5.2 \times 5.2$ arcmin. Si se utiliza el reductor focal, la escala resultante es de $0.45 \mathrm{arcsec} / \mathrm{px}$, con un campo útil circular de 9 arcmin de diámetro. Estos turnos comprendieron los siguientes períodos:

- Semestre 2014A: del 22 al 27 de abril del 2014 (6 noches de observación).

- Semestre 2015A: del 10 al 14 de abril del 2015 (5 noches de observación).

- Semestre 2015B: del 11 al 13 de agosto; del 15 al 17 de septiembre y del 11 al 12 de diciembre del 2015 (8 noches de observación).

- Semestre 2016B: del 27 al 28 de noviembre del 2016 (2 noches de observación).

Para cada objeto, se tomaron imágenes en los filtros V y R del sistema de JohnsonCousins. El número de imágenes obtenido para cada objeto depende de las condiciones climáticas que se tuvieron en cada turno, de la magnitud del objeto y del momento de 
Tabla 3.1. Datos de los objetos, donde se muestra en la columna 1 el nombre de la fuente, en la columna 2 su clasificación junto con el tipo de SED que tiene, en las columnas 3 y 4 las coordenadas referidas al J2000.0, en la columna 5 el valor de su redshift y en la columna 6 la magnitud visual publicada en la base de datos NED.

+ blazar tipo incierto.

\begin{tabular}{|c|c|c|c|c|c|}
\hline Objeto & Clasificación & $\begin{array}{c}\alpha(\mathrm{J} 2000.0) \\
\mathrm{h} \mathrm{m} \mathrm{s}\end{array}$ & $\begin{array}{c}\delta(\mathrm{J} 2000.0) \\
\circ,,\end{array}$ & $z$ & $m_{\text {visual }}$ \\
\hline PKS 0208-512 & $\mathrm{BZU}^{+}$ & 021046.2 & -510101.8 & 1.003 & $14.82(\mathrm{R})$ \\
\hline $1 \mathrm{H} 0414+009$ & BL Lac (HSP) & 041652.5 & +010523.9 & 0.287 & - \\
\hline [HB89] 0521-365 & $\mathrm{BZU}^{+}$ & 052257.9 & -362730.8 & 0.055 & $14.48(\mathrm{R})$ \\
\hline PMN J0847-2337 & $\mathrm{BZU}^{+}$ & 084701.5 & -233701.6 & 0.059 & - \\
\hline [HB89] 1116-462 & FSRQ (LSP) & 111826.9 & -463415.0 & 0.713 & $17.02(\mathrm{~V})$ \\
\hline [HB89] 1127-145 & FSRQ (LSP) & 113007.1 & -144927.4 & 1.184 & $16.70(\mathrm{~V})$ \\
\hline PKS 1229-02 & FSRQ (LSP) & 123200.0 & -022405.3 & 1.045 & - \\
\hline PMN J1256-1146 & BL Lac (HSP) & 125615.9 & -114637.4 & 0.057 & - \\
\hline [HB89] 1424-418 & FSRQ (LSP) & 142756.3 & $-4206 \quad 19.4$ & 1.522 & $16.30(\mathrm{R})$ \\
\hline PKS J1443-3908 & BL Lac (HSP) & 144357.2 & -390839.7 & 0.065 & - \\
\hline PKS 1510-089 & FSRQ (LSP) & 151250.5 & -0905 59.8 & 0.360 & - \\
\hline PKS J1517-242 & BL Lac (ISP) & 151741.8 & -242219.5 & 0.048 & $10.78(\mathrm{R})$ \\
\hline BZB J1917-192 & BL Lac (HSP) & 191744.8 & -192131.6 & 0.137 & $15.24(\mathrm{R})$ \\
\hline 3FGL J1958.2-3011 & BL Lac (HSP) & 195814.9 & -301111.8 & 0.119 & - \\
\hline PKS 2005-489 & BL Lac (HSP) & 200925.3 & -484953.7 & 0.071 & $11.41(\mathrm{R})$ \\
\hline [HB89] 2126-158 & FSRQ (LSP) & 212912.1 & -153841.0 & 3.268 & $16.43(\mathrm{R})$ \\
\hline PKS 2149-306 & FSRQ (LSP) & 215155.5 & -302753.6 & 2.345 & - \\
\hline PKS 2155-304 & BL Lac (HSP) & 215852.0 & -301332.1 & 0.116 & - \\
\hline PMN J2310-4347 & BL Lac & 231041.7 & -434734.1 & 0.088 & - \\
\hline
\end{tabular}

la noche en que se lo observa (i.e. si el objeto puede seguirse a lo largo de toda la noche o sólo al comienzo/final). Los tiempos de integración en las imágenes fueron de 60 seg hasta 360 seg.

La reducción de las observaciones se hizo utilizando el amplio rango de tareas y subrutinas que ofrece el software de reducción de imágenes IRAF ${ }^{2}$. Las imágenes de ciencia de los blazares fueron calibrados y procesados por bias y flats de cúpula. Y también fueron corregidas por overscan y trimming. En la Tabla 3.2, se muestra la información de los datos en el óptico de la muestra de blazares. En la columna 1 está el nombre de la fuente, en la columna 2, la fecha de observación. En la columna 3, se especifica las dimensiones del campo de visión que tienen las imágenes, donde un valor de $5.2 \times 5.2$ corresponden a imágenes sin reductor focal, mientras que si se utiliza el reductor, se obtienen campos de $9.0 \times 9.0$. Finalmente en la última columna, se muestra el radio de apertura usado en el proceso de reducción fotométrico.

\footnotetext{
${ }^{2}$ IRAF es distribuido por el National Optical Astronomy Observatories, los cuales son operados por la Association of Universities for Research in Astronomy, Inc., bajo acuerdo cooperativo con la National Science Foundation.
} 
Tabla 3.2. Información de los datos ópticos de la muestra. Las columnas son: nombre de la fuente, turno en CASLEO, FOV y el radio de apertura usado en el proceso de reducción fotométrico.

\begin{tabular}{|c|c|c|c|}
\hline Objeto & Turno CASLEO & FOV & Radio de apertura \\
\hline \multirow[t]{2}{*}{ PKS 0208-512 } & 13 agosto 2015 & $9.0^{\prime} \times 9.0^{\prime}$ & 16.00 arcsec \\
\hline & 15 y 17 septiembre 2015 & $9.0^{\prime} \times 9.0^{\prime}$ & 16.00 arcsec \\
\hline$[H B 89] 0414+009$ & 27 y 28 noviembre 2016 & $9.0^{\prime} \times 9.0^{\prime}$ & 10.00 arcsec \\
\hline$[H B 89] 0521-365$ & 11 diciembre 2015 & $9.0^{\prime} \times 9.0^{\prime}$ & $14.00 \operatorname{arcsec}$ \\
\hline PMN J0847-2337 & 22 al 27 abril 2014 & $5.2^{\prime} \times 5.2^{\prime}$ & 18.00 arcsec \\
\hline$[H B 89] 1116-462$ & 13 y 14 abril 2015 & $9.0^{\prime} \times 9.0^{\prime}$ & $8.00 \operatorname{arcsec}$ \\
\hline$[$ HB B 89]1127 - 145 & 10 y 12 abril 2015 & $9.0^{\prime} \times 9.0^{\prime}$ & $8.00 \operatorname{arcsec}$ \\
\hline PKS 1229-02 & 12 abril 2015 & $9.0^{\prime} \times 9.0^{\prime}$ & 10.00 arcsec \\
\hline PMN J1256-1146 & 23 abril 2014 & $5.2^{\prime} \times 5.2^{\prime}$ & 18.00 arcsec \\
\hline$[H B 89] 1424-418$ & 14 abril 2015 & $9.0^{\prime} \times 9.0^{\prime}$ & $10.00 \operatorname{arcsec}$ \\
\hline PKS J1443-3908 & 24,25 y 27 abril 2014 & $5.2^{\prime} \times 5.2^{\prime}$ & 12.00 arcsec \\
\hline PKS 1510-089 & 12 al 14 abril 2015 & $9.0^{\prime} \times 9.0^{\prime}$ & 10.00 arcsec \\
\hline \multirow[t]{2}{*}{ PKS J1517-242 } & 22,23 y 26 abril 2014 & $5.2^{\prime} \times 5.2^{\prime}$ & 18.00 arcsec \\
\hline & 10 y 11 abril 2015 & $9.0^{\prime} \times 9.0^{\prime}$ & 18.00 arcsec \\
\hline BZB J1917-192 & 25 y 26 abril 2014 & $5.2^{\prime} \times 5.2^{\prime}$ & 18.00 arcsec \\
\hline 3FGL J1958.2-3011 & 23 y 24 abril 2014 & $5.2^{\prime} \times 5.2^{\prime}$ & $12.00 \operatorname{arcsec}$ \\
\hline PKS 2005-489 & 12 agosto 2015 & $9.0^{\prime} \times 9.0^{\prime}$ & $8.00 \operatorname{arcsec}$ \\
\hline$[H B 89] 2126-158$ & 15 y 17 septiembre 2015 & $9.0^{\prime} \times 9.0^{\prime}$ & $8.00 \operatorname{arcsec}$ \\
\hline \multirow[t]{2}{*}{ PKS 2149-306 } & 12 agosto 2015 & $9.0^{\prime} \times 9.0^{\prime}$ & $10.43 \operatorname{arcsec}$ \\
\hline & 17 septiembre 2015 & $9.0^{\prime} \times 9.0^{\prime}$ & $10.43 \operatorname{arcsec}$ \\
\hline PKS 2155-304 & 13 agosto 2015 & $9.0^{\prime} \times 9.0^{\prime}$ & 18.00 arcsec \\
\hline PMN J2310-4347 & 17 septiembre 2015 & $9.0^{\prime} \times 9.0^{\prime}$ & 16.00 arcsec \\
\hline
\end{tabular}


Por otro lado, se realizó la fotometría diferencial a través de las subrutinas del paquete APPHOT de IRAF. Dependiendo del tamaño aparente de la fuente en la imagen así como también de cuán poblado es el campo del objeto, se seleccionaron distintos radios de apertura (Co. 4, Tab. 3.2). Como se mencionó en el capítulo anterior, para la fotometría diferencial, necesitamos dos estrellas, una de comparación y otra de control. Éstas fueron seleccionadas dentro del campo de cada uno de los objetos, buscando cumplir con el criterio de Howell. De esta manera, con el blazar y las estrellas seleccionadas, generamos las DLCs del blazar y la de control, y se aplicaron los parámetros C y F estudiados en el capítulo anterior para el análisis de la variabilidad.

\subsubsection{Análisis de los resultados: Variabilidad}

Presentamos los resultados del estado de variabilidad de los blazares en la banda óptica. Se tomaron los valores al $99.5 \%$ de nivel de significancia, para ambos parámetros C y F. Se consideraron principalmente dos escalas temporales distintas para analizar la variabilidad: por un lado, se analizó la microvariabilidad (esto es, la variabilidad intranoche en escalas de horas) y por otro lado, se estudiaron las DLCs en escalas de días (lo que se conoce como variabilidad inter-noche). En algunos objetos, también tuvimos la posibilidad de observarlo en escala de meses y de años. El detalle de la escala analizada se encuentra en la segunda columna de la Tabla 3.3. En base a los resultados obtenidos en el análisis de las simulaciones del Cap. 2, si la DLC es clasificada como variable (no variable) por ambos parámetros, se determina que ésta es variable (no variable). En aquellos casos en que los parámetros no coincidieron en el resultado de su clasificación, se analizó detalladamente la DLC, teniendo en cuenta cada uno de los resultados de los parámetros $\mathrm{C}$ y $\mathrm{F}$, así como también cómo han sido las condiciones observacionales en esa noche. Los resultados se encuentran resumidos en la Tabla 3.3, donde se muestra la siguiente información: el nombre del objeto, la fecha de observación, el valor del criterio $\mathrm{C}$ en las bandas $\mathrm{V}$ y $\mathrm{R}$, el valor del areaF en las bandas $\mathrm{V}$ y $\mathrm{R}$, el resultado de si es o no variable la fuente, el número de puntos en la curva de luz, el factor de peso de Howell en los filtros V y R $(\Gamma)$ y el valor de la dispersión de la curva de control $\left(\sigma_{2}\right)$. En el apéndice A, se muestran los campos para cada objeto donde están señaladas las estrellas de campo. 
Tabla 3.3. Resultados del estado de variabilidad de las fuentes en la banda óptica. Se muestran el nombre del objeto, la fecha de observación, el valor del criterio C en las bandas $\mathrm{V}$ y $\mathrm{R}$, el valor del areaF en las bandas $\mathrm{V}$ y $\mathrm{R}$, el resultado de si es o no variable la fuente, el número de puntos en la curva de luz, el factor de peso de Howell en los filtros V y R $(\Gamma)$ y el valor de a dispersión de la curva de control $\left(\sigma_{2}\right)$.

\begin{tabular}{|c|c|c|c|c|c|c|c|c|c|c|c|}
\hline \multirow[t]{2}{*}{ Objeto } & \multirow[t]{2}{*}{ Fecha } & \multicolumn{2}{|c|}{$C$} & \multicolumn{2}{|c|}{ areaF } & \multirow[t]{2}{*}{ Variable? } & \multirow[t]{2}{*}{$\mathrm{N}$} & \multicolumn{2}{|c|}{$\Gamma$} & \multicolumn{2}{|c|}{$\sigma_{2}$} \\
\hline & & $\mathrm{V}$ & $\mathrm{R}$ & $\mathrm{V}$ & $\mathrm{R}$ & & & $\mathrm{V}$ & $\mathrm{R}$ & $\mathrm{V}$ & $\mathrm{R}$ \\
\hline \multirow[t]{5}{*}{ PKS 0208-512 } & $13 / 08 / 2015$ & 2.1118 & 3.6250 & 0.9508 & 0.9985 & Sí (R) & 9 & 1.5270 & 1.5537 & 0.0161 & 0.0104 \\
\hline & $15 / 09 / 2015$ & 2.6002 & 0.5829 & 0.9440 & 0.7388 & No & 6 & 2.3378 & 2.7089 & 0.0221 & 0.0329 \\
\hline & $17 / 09 / 2015$ & 0.5637 & 0.6210 & 0.8466 & 0.7680 & No & 8 & 2.3949 & 2.6770 & 0.0330 & 0.0174 \\
\hline & Todas las noches Sept. & 0.9802 & 0.9490 & 0.0564 & 0.1469 & No & 14 & 2.3832 & 2.7391 & 0.0473 & 0.0343 \\
\hline & Todas las noches & 6.2279 & 11.816 & 1.0000 & 1.0000 & Sí & 23 & 2.2073 & 2.5351 & 0.0450 & 0.0279 \\
\hline \multirow[t]{3}{*}{ [HB89] 0414+009 } & $27 / 11 / 2016$ & 1.7445 & 1.5755 & 0.9347 & 0.8708 & No & 13 & 0.5939 & 0.7018 & 0.0088 & 0.0066 \\
\hline & $28 / 11 / 2016$ & 2.0108 & 2.0572 & 0.9710 & 0.9677 & No & 11 & 0.5981 & 0.6991 & 0.0076 & 0.0052 \\
\hline & Todas las noches & 1.9526 & 1.5793 & 0.9983 & 0.9670 & No & 24 & 0.5916 & 0.6953 & 0.0083 & 0.0065 \\
\hline [HB89] 0521-365 & $11 / 12 / 2015$ & 1.6877 & 1.7143 & 0.9763 & 0.9766 & No & 21 & 0.9894 & 0.9499 & 0.0084 & 0.0085 \\
\hline \multirow[t]{7}{*}{ PMN J0847-2337 } & $22 / 04 / 2014$ & 1.4760 & 1.7280 & 0.7877 & 0.9169 & No & 12 & 0.9205 & 0.7447 & 0.0081 & 0.0069 \\
\hline & $23 / 04 / 2014$ & 2.1700 & 0.6452 & 0.6497 & 0.4122 & No & 3 & 0.9218 & 0.7449 & 0.0010 & 0.0020 \\
\hline & $24 / 04 / 2014$ & 1.0458 & 1.3407 & 0.0670 & 0.4161 & No & 5 & 0.9255 & 0.7395 & 0.0081 & 0.0048 \\
\hline & $25 / 04 / 2014$ & 0.7125 & 0.8782 & 0.4725 & 0.1926 & No & 5 & 0.9125 & 0.7312 & 0.0136 & 0.0101 \\
\hline & $26 / 04 / 2014$ & 1.9676 & 0.2981 & 0.5894 & 0.8368 & No & 4 & 0.9462 & 0.7370 & 0.0103 & 0.0134 \\
\hline & $27 / 04 / 2014$ & 0.7238 & 1.1103 & 0.5488 & 0.1940 & No & 7 & 0.9561 & 0.7668 & 0.0070 & 0.0070 \\
\hline & Todas las noches & 1.6999 & 2.7685 & 0.9973 & 1.0000 & Sí & 36 & 0.9185 & 0.7434 & 0.0165 & 0.0096 \\
\hline \multirow[t]{3}{*}{ [HB89] 1116-462 } & $13 / 04 / 2015$ & 3.9413 & 1.3329 & 1.0000 & 0.7060 & Sí (V) & 15 & 0.9538 & 1.1599 & 0.0085 & 0.0075 \\
\hline & $14 / 04 / 2015$ & 6.5250 & 2.9342 & 1.0000 & 0.9987 & Sí & 12 & 0.9176 & 1.1300 & 0.0067 & 0.0076 \\
\hline & Todas las noches & 6.7333 & 3.2910 & 1.0000 & 1.0000 & Sí & 27 & 0.9324 & 1.1520 & 0.0102 & 0.0081 \\
\hline \multirow[t]{3}{*}{ [HB89] 1127-145 } & $10 / 04 / 2015$ & 1.3390 & 1.2720 & 0.7136 & 0.6206 & No & 15 & 0.8831 & 0.8890 & 0.0042 & 0.0053 \\
\hline & $12 / 04 / 2015$ & 1.7780 & 1.1360 & 0.8741 & 0.2737 & No & 9 & 0.8833 & 0.8935 & 0.0047 & 0.0049 \\
\hline & Todas las noches & 1.4420 & 1.4180 & 0.9140 & 0.8995 & No & 24 & 0.8866 & 0.8890 & 0.0058 & 0.0056 \\
\hline PKS 1229-02 & $12 / 04 / 2015$ & 0.6690 & 0.8070 & 0.8204 & 0.5308 & No & 13 & 0.7419 & 1.0453 & 0.0092 & 0.0067 \\
\hline
\end{tabular}


Tabla 3.3. Cont.

\begin{tabular}{|c|c|c|c|c|c|c|c|c|c|c|c|}
\hline \multirow[t]{2}{*}{ "Objeto } & \multirow[t]{2}{*}{ "Fecha } & \multicolumn{2}{|c|}{ 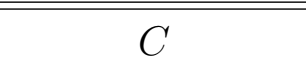 } & \multicolumn{2}{|c|}{ areaF } & \multirow[t]{2}{*}{ Variable? } & \multirow[t]{2}{*}{$\mathrm{N}$} & \multicolumn{2}{|c|}{ 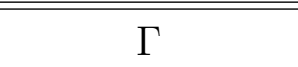 } & \multicolumn{2}{|c|}{$\overline{\sigma_{2}}$} \\
\hline & & $\mathrm{V}$ & $\mathrm{R}$ & $\mathrm{V}$ & $\mathrm{R}$ & & & $\mathrm{V}$ & $\mathrm{R}$ & $\mathrm{V}$ & $\mathrm{R}$ \\
\hline PMN J1256-1146 & $23 / 04 / 2014$ & 1.5443 & 3.3689 & 0.6858 & 0.9906 & No & 7 & 0.4123 & 0.3435 & 0.0033 & 0.0034 \\
\hline [HB89] 1424-418 & $14 / 04 / 2015$ & 2.5330 & 1.5750 & 0.9836 & 0.7799 & No & 9 & 0.9936 & 0.9373 & 0.0157 & 0.0102 \\
\hline \multirow[t]{4}{*}{ PKS J1443-3908 } & $24 / 04 / 2014$ & 0.9020 & 2.9590 & 0.4932 & 1.0000 & Sí (R) & 43 & 0.9038 & 0.9421 & 0.0089 & 0.0041 \\
\hline & $25 / 04 / 2014$ & 1.9430 & 3.4240 & 0.9982 & 1.0000 & Sí & 25 & 0.9137 & 0.9280 & 0.0104 & 0.0103 \\
\hline & $27 / 04 / 2014$ & 4.1530 & 3.6130 & 1.0000 & 1.0000 & Sí & 16 & 0.8630 & 0.8984 & 0.0079 & 0.0066 \\
\hline & Todas las noches & 1.6950 & 2.0850 & 1.0000 & 1.0000 & No & 84 & 0.9088 & 0.9471 & 0.0113 & 0.0115 \\
\hline \multirow[t]{4}{*}{ PKS 1510-089 } & $12 / 04 / 2015$ & 0.9940 & 1.5030 & 0.0164 & 0.8606 & No & 15 & 0.9686 & 0.9490 & 0.0054 & 0.0032 \\
\hline & $13 / 04 / 2015$ & 8.3140 & 3.6240 & 1.0000 & 0.9999 & Sí & 13 & 0.9961 & 0.9713 & 0.0030 & 0.0058 \\
\hline & $14 / 04 / 2015$ & 5.5070 & 4.1660 & 1.0000 & 1.0000 & Sí & 12 & 0.9797 & 0.9661 & 0.0036 & 0.0039 \\
\hline & Todas las noches & 5.1580 & 4.1690 & 1.0000 & 1.0000 & Sí & 40 & 0.9779 & 0.9605 & 0.0056 & 0.0063 \\
\hline \multirow[t]{8}{*}{ PKS J1517-242 } & $22 / 04 / 2014$ & 1.4286 & 1.6276 & 0.9488 & 0.9917 & No & 32 & 0.7599 & 0.7382 & 0.0056 & 0.0050 \\
\hline & $23 / 04 / 2014$ & 1.2526 & 2.4160 & 0.6071 & 0.9985 & No & 16 & 0.7528 & 0.7281 & 0.0052 & 0.0029 \\
\hline & $26 / 04 / 2014$ & 2.3333 & 1.6086 & 0.9996 & 0.9608 & No & 21 & 0.7483 & 0.7241 & 0.0087 & 0.0096 \\
\hline & Todas las noches & 7.6741 & 7.2382 & 1.0000 & 1.0000 & Sí & 69 & 0.7582 & 0.7370 & 0.0080 & 0.0072 \\
\hline & $10 / 04 / 2015$ & 1.3714 & 1.6205 & 0.8864 & 0.9853 & No & 28 & 0.7282 & 0.7107 & 0.0155 & 0.0108 \\
\hline & $11 / 04 / 2015$ & 1.2134 & 0.8006 & 0.4030 & 0.4563 & No & 9 & 0.7243 & 0.7064 & 0.0109 & 0.0042 \\
\hline & Todas las noches & 1.4015 & 1.6526 & 0.9505 & 0.9956 & No & 37 & 0.7265 & 0.7093 & 0.0145 & 0.0090 \\
\hline & Total & 8.6806 & 11.345 & 1.0000 & 1.0000 & Sí & 106 & 0.7536 & 0.7326 & 0.0108 & 0.0091 \\
\hline \multirow[t]{3}{*}{ BZB J1917-192 } & $25 / 04 / 2014$ & 0.7026 & 1.1086 & 0.7643 & 0.2733 & No & 13 & 1.0156 & 0.9954 & 0.0065 & 0.0055 \\
\hline & $26 / 04 / 2014$ & 1.0370 & 0.9820 & 0.0847 & 0.0459 & No & 11 & 0.9971 & 0.9753 & 0.0194 & 0.0129 \\
\hline & Todas las noches & 1.6420 & 2.4220 & 0.9759 & 0.9999 & No & 24 & 1.0075 & 0.9807 & 0.0142 & 0.0094 \\
\hline
\end{tabular}


Tabla 3.3. Cont.

\begin{tabular}{|c|c|c|c|c|c|c|c|c|c|c|c|}
\hline \multirow[t]{2}{*}{ Objeto } & \multirow[t]{2}{*}{ Fecha } & \multicolumn{2}{|c|}{$\bar{C}$} & \multicolumn{2}{|c|}{ areaF } & \multirow[t]{2}{*}{ Variable? } & \multirow[t]{2}{*}{$\mathrm{N}$} & \multicolumn{2}{|c|}{$\bar{\Gamma}$} & \multicolumn{2}{|c|}{$\sigma_{2}$} \\
\hline & & $\mathrm{V}$ & $\mathrm{R}$ & $\mathrm{V}$ & $\mathrm{R}$ & & & $\mathrm{V}$ & $\mathrm{R}$ & $\mathrm{V}$ & $\mathrm{R}$ \\
\hline \multirow[t]{3}{*}{ 3FGL J1958.2-3011 } & $23 / 04 / 2014$ & 2.3823 & 1.0080 & 0.9888 & 0.0197 & No & 11 & 0.8747 & 0.7535 & 0.0039 & 0.0091 \\
\hline & $24 / 04 / 2014$ & 0.8615 & 1.3443 & 0.3358 & 0.6087 & No & 10 & 0.8716 & 0.7536 & 0.0039 & 0.0072 \\
\hline & Todas las noches & 1.6970 & 1.0340 & 0.9777 & 0.1184 & No & 21 & 0.8750 & 0.7555 & 0.0056 & 0.0072 \\
\hline PKS 2005-489 & $12 / 08 / 2015$ & 2.8430 & 2.5110 & 1.0000 & 1.0000 & Sí & 26 & 0.9449 & 0.9556 & 0.0053 & 0.0084 \\
\hline \multirow[t]{3}{*}{ [HB89] 2126-158 } & $15 / 09 / 2015$ & 1.6342 & 1.0515 & 0.9118 & 0.1409 & No & 14 & 1.0156 & 1.0985 & 0.0106 & 0.0160 \\
\hline & $17 / 09 / 2015$ & 0.3609 & 0.5135 & 0.9979 & 0.9634 & No & 12 & 0.9868 & 1.0956 & 0.0167 & 0.0147 \\
\hline & Todas las noches & 0.7665 & 0.7188 & 0.8094 & 0.8948 & No & 26 & 1.0028 & 1.0952 & 0.0179 & 0.0194 \\
\hline \multirow{3}{*}{ PKS 2149-306 } & $12 / 08 / 2015$ & 1.2078 & 0.4268 & 0.2362 & 0.6918 & No & 4 & 1.5750 & 1.8497 & 0.0284 & 0.1031 \\
\hline & $17 / 09 / 2015$ & 1.0250 & 0.7854 & 0.0370 & 0.3488 & No & 5 & 1.5439 & 1.7880 & 0.0130 & 0.0139 \\
\hline & Todas las noches & 1.3797 & 0.5487 & 0.6186 & 0.8641 & No & 9 & 1.5574 & 1.7796 & 0.0257 & 0.0772 \\
\hline \multirow[t]{3}{*}{ PKS 2155-304 } & $13 / 08 / 2015$ & 3.2841 & 2.7842 & 1.0000 & 0.9999 & Sí & 19 & 0.8029 & 0.8185 & 0.0057 & 0.0086 \\
\hline & $15 / 09 / 2015$ & 6.8839 & 2.0377 & 1.0000 & 0.9846 & Sí (V) & 14 & 0.5985 & 0.6075 & 0.0038 & 0.0053 \\
\hline & Todas las noches & 25.105 & 15.889 & 1.0000 & 1.0000 & Sí & 33 & 0.7452 & 0.7721 & 0.0060 & 0.0092 \\
\hline PMN J2310-4347 & $17 / 09 / 2015$ & 2.5547 & 1.1443 & 0.9935 & 0.3220 & No & 11 & 1.0287 & 1.0734 & 0.0044 & 0.0042 \\
\hline
\end{tabular}




\subsubsection{Análisis de los colores y magnitudes de la muestra de blazares}

\section{Diagramas magnitud-magnitud y la función de correlación discreta}

Del análisis anterior de variabilidad, se observa que en los casos en que se detectan variaciones, ésta se refleja en ambas bandas V y R. Para poder comprobar si hay una correlación entre las variaciones en cada banda, utilizamos los diagramas magnitudmagnitud junto con la función de correlación discreta (DCF, del inglés discrete correlation function). Este método fue desarrollado por Edelson \& Krolik (1988), el cual consiste en tomar cada par de datos a correlacionar $\left(x_{i}, y_{j}\right)$, con $0 \leq i, j \leq N$ y $\mathrm{N}$ el número de puntos observados y calcular la función de correlación discreta sin binear (UDCF, de inglés unbined discrete correlation function), dada por:

$$
U D C F_{i j}(\tau)=\frac{\left(x_{i}-\bar{x}\right)\left(y_{j}-\bar{y}\right)}{\sqrt{\left(\sigma_{x}^{2}-e_{x}^{2}\right)\left(\sigma_{y}^{2}-e_{y}^{2}\right)}}
$$

donde $\bar{x}, \bar{y}$ son los valores medios de los conjuntos de datos $x_{i}, y_{j}$, con desviaciones estándares $\sigma_{x}, \sigma_{y} \mathrm{y}$ errores en la medición $e_{x}, e_{y}$.

La función DCF se calcula promediando los $M$ valores de UDCF para cada lapso temporal $\Delta t_{i, j}=\left(t_{y j}-t_{x i}\right)$ (donde $\mathrm{M}$ es la cantidad de pares de datos que caen dentro del intervalo $\Delta t_{i, j}$ y $\left.\tau-\frac{\Delta \tau}{2} \leq \Delta t_{i, j} \leq \tau+\frac{\Delta \tau}{2}\right)$ :

$$
D C F(\tau)=\frac{\sum_{k=1}^{M} U D C F_{k}}{M}
$$

con $\tau$ el centro del bin de tamaño $\Delta \tau$. El error viene dado por:

$$
\sigma_{D C F(\tau)}=\frac{\sqrt{\sum_{k=1}^{M}\left(U D C F_{k}-D C F(\tau)\right)^{2}}}{M-1}
$$

Un valor de DCF mayor a 0 implica que ambos conjuntos de datos están correlacionados; si DCF es menor a 0, están anticorrelacionados; y si DCF es nula, no hay correlación.

Trasladando este análisis a nuestro datos, una tendencia creciente en los diagramas implica que las variaciones en las magnitudes están bien correlacionadas, indicando que el mecanismo de variabilidad sería el mismo en ambas bandas. Además de la DCF, también realizamos un ajuste lineal a los diagramas mediante el método de regresión lineal, corroborado con el test de $\chi^{2}$. Los resultados están resumidos en la Tabla 3.4, donde se tienen: objeto, valores de la pendiente y ordenada del ajuste lineal, el valor del estadístico asociado $\chi^{2}$ reducido, el valor de la función de correlación discreta y el resultado de la correlación. 
Tabla 3.4. Resultados del ajuste lineal y de la función de correlación para los objetos de la muestra. Se presentan: objeto, valores de la pendiente y ordenada del ajuste lineal, el valor del estadístico asociado $\chi^{2}$ reducido, el valor de la función de correlación discreta y cuál es el resultado de la correlación.

\begin{tabular}{|c|c|c|c|c|c|}
\hline \multirow[t]{2}{*}{ Objeto } & \multicolumn{2}{|c|}{ Ajuste lineal } & \multirow[t]{2}{*}{$\chi^{2}$ reducido } & \multirow[t]{2}{*}{ DCF } & \multirow[t]{2}{*}{ Resultado } \\
\hline & Pendiente $\left(\times 10^{-1}\right)$ & Ordenada & & & \\
\hline PKS 0208-512 & $1.177 \pm 0.014$ & $-3.507 \pm 0.243$ & 0.989 & $1.007 \pm 0.145$ & Correlación \\
\hline$[H$ B89]0414 + 009 & $0.229 \pm 0.120$ & $12.056 \pm 1.951$ & 0.127 & $0.213 \pm 0.160$ & Correlación \\
\hline$[H$ B 89]0521 - 365 & $0.215 \pm 0.066$ & $11.09 \pm 1.020$ & 0.016 & $0.081 \pm 0.088$ & Correlación \\
\hline PMN J0847-2337 & $6.61 \pm 0.820$ & $5.160 \pm 1.350$ & 0.704 & $0.801 \pm 0.198$ & Correlación \\
\hline$[H B 89] 1116-462$ & $3.15 \pm 0.815$ & $11.5 \pm 0.780$ & 0.821 & $0.889 \pm 0.108$ & Correlación \\
\hline$[$ H B 89] $1127-145$ & $0.535 \pm 0.159$ & $7.647 \pm 2.904$ & 0.305 & $1.098 \pm 0.280$ & Correlación \\
\hline PKS 1229-02 & $-0.228 \pm 0.291$ & $20.322 \pm 4.888$ & 0.065 & $-0.210 \pm 1.160$ & Anticorrelación \\
\hline PMN J1256-1146 & $0.613 \pm 0.562$ & $4.354 \pm 6.781$ & 0.072 & $0.097 \pm 0.340$ & Correlación \\
\hline [H B89]1424 - 418 & $0.054 \pm 0.211$ & $16.086 \pm 3.721$ & 0.022 & $-0.186 \pm 0.156$ & no coinciden \\
\hline PKS J1443-3908 & $1.107 \pm 0.034$ & $-2.038 \pm 0.527$ & 0.673 & $0.053 \pm 0.030$ & Correlación \\
\hline PKS 1510-089 & $0.868 \pm 0.054$ & $1.507 \pm 0.833$ & 0.907 & $0.728 \pm 0.080$ & Correlación \\
\hline PKS J1517-242 (2014) & $0.832 \pm 0.015$ & $1.956 \pm 0.222$ & 0.962 & $0.996 \pm 0.067$ & Correlación \\
\hline$(2015)$ & $0.192 \pm 0.223$ & $11.489 \pm 3.344$ & 0.076 & $0.035 \pm 0.093$ & Correlación \\
\hline$(2014-2015)$ & $0.767 \pm 0.014$ & $2.934 \pm 0.214$ & 0.880 & $0.636 \pm 0.053$ & Correlación \\
\hline BZB J1917-192 & $0.812 \pm 0.084$ & $2.472 \pm 1.301$ & 0.559 & $0.751 \pm 0.090$ & Correlación \\
\hline 3FGL J1958.2-3011 & $0.361 \pm 0.362$ & $10.284 \pm 6.163$ & 0.216 & $0.076 \pm 0.090$ & Correlación \\
\hline PKS 2005-489 & $1.024 \pm 0.155$ & $-1.008 \pm 2.265$ & 0.542 & $0.285 \pm 0.084$ & Correlación \\
\hline$[H B 89] 2126-158$ & $0.163 \pm 0.402$ & $14.266 \pm 7.109$ & 0.031 & $-0.215 \pm 0.087$ & no coinciden \\
\hline PKS 2149-306 & $1.607 \pm 0.578$ & $14.686 \pm 10.261$ & 0.106 & $-0.182 \pm 0.234$ & no coinciden \\
\hline PKS 2155-304 & $0.984 \pm 0.006$ & $0.069 \pm 0.080$ & 0.990 & $0.915 \pm 0.055$ & Correlación \\
\hline PMN J2310-4347 & $0.302 \pm 0.306$ & $10.732 \pm 5.016$ & 0.520 & $0.304 \pm 0.163$ & Correlación \\
\hline
\end{tabular}




\section{Cálculo del índice espectral y diagramas color-magnitud}

Con la obtención de las magnitudes $\mathrm{V}$ y R, podemos calcular el índice de color (V-R). Y con él, se puede calcular el promedio del índice espectral para cada blazar, como lo hizo Wierzcholska et al. (2015):

$$
\left\langle\alpha_{V R}\right\rangle=\frac{0.4\langle V-R\rangle}{\log \left(\lambda_{R} / \lambda_{V}\right)}
$$

donde $\lambda_{V}$ y $\lambda_{R}$ son las longitudes de onda efectivas de las bandas $\mathrm{V}$ y $\mathrm{R}$, respectivamente (Bessell, Castelli \& Plez 1998). El índice espectral es una cantidad que está relacionada a la densidad de flujo de una fuente $\left(\mathrm{S}_{\nu}\right)$ si se supone que el flujo del objeto sigue una ley de potencias del tipo $\mathrm{S}_{\nu} \sim \nu^{-\alpha}$. Permite conocer cómo es el comportamiento del flujo y determinar cómo es la radiación presente en la fuente y qué mecanismos están involucrados. Cambios en los valores de este índice implican cambios en las pendientes de la distribución espectral, lo que se traduce a variaciones en esta distribución. Son las condiciones del medio las que se ven modificadas haciendo que la pendiente cambie.

Los valores calculados para nuestra muestra observacional se presentan en la Tabla 3.5. Allí se dan: el nombre de la fuente, el promedio del índice de color (V-R) y el promedio del índice espectral $\alpha_{V R}$. Ambos promedios fueron tomados en escalas de días, con excepción de aquellas fuentes que se las observó una sola noche, en donde se utilizó directamente el valor obtenido para dicha noche.

\section{Diagramas color-magnitud y su conexión con los modelos de variabilidad}

Las variaciones detectadas en el flujo óptico de los blazares pueden estar relacionadas con variaciones en su forma espectral. Éstas últimas pueden proveer información sobre los procesos físicos que son responsables de las variaciones. A su vez, los cambios espectrales se relacionan con cambios en el color de la fuente. Por esta razón, analizamos los diagramas de color-magnitud: V vs. (V-R). De acuerdo al comportamiento de estas cantidades, se pueden inferir 5 comportamientos (Zhang et al. 2014):

- BWB: del inglés Bluer when brighter, cuanto más azul, más brillante. Suele darse en todo el conjunto de datos.

- RWB: del inglés Redder when brighter, cuanto más roja, más brillante. Suele darse en todo el conjunto de datos.

- RWB to BWB: se suele tener un comportamiento RWB cuando el blazar está en su estado más bajo de actividad, mientras que se da el BWB en su estado más alto.

- SWB: del inglés Stable when brighter, permanece estable a medida que aumenta su brillo.

- Sin correlación: no se presenta correlación alguna a lo largo de todo el conjunto de datos. 
Tabla 3.5. Valores de los índices espectrales para los blazares de la muestra. Se presenta el nombre de la fuente, el promedio del índice de color $(\mathrm{V}-\mathrm{R})$ y el promedio del índice espectral $\alpha_{V R}$.

\begin{tabular}{ccc}
\hline \hline Objeto & $\langle V-R\rangle$ & $\left\langle\alpha_{V R}\right\rangle$ \\
\hline PKS 0208-512 & $0.410 \pm 0.065$ & $2.33 \pm 0.065$ \\
{$[H B 89] 0414+009$} & $0.423 \pm 0.015$ & $2.40 \pm 0.015$ \\
{$[H B 89] 0521-365$} & $0.917 \pm 0.016$ & $5.20 \pm 0.016$ \\
PMN J0847-2337 & $0.618 \pm 0.011$ & $3.51 \pm 0.011$ \\
{$[H B 89] 1116-462$} & $0.187 \pm 0.045$ & $1.06 \pm 0.045$ \\
{$[H B 89] 1127-145$} & $0.823 \pm 0.007$ & $4.67 \pm 0.007$ \\
PKS 1229-02 & $0.311 \pm 0.009$ & $1.76 \pm 0.009$ \\
PMN J1256-1146 & $0.305 \pm 0.011$ & $1.73 \pm 0.011$ \\
{$[H B 89] 1424-418$} & $0.589 \pm 0.040$ & $3.34 \pm 0.040$ \\
PKS J1443-3908 & $0.403 \pm 0.016$ & $2.29 \pm 0.016$ \\
PKS 1510-089 & $0.529 \pm 0.009$ & $3.00 \pm 0.009$ \\
PKS J1517-242 & $0.565 \pm 0.046$ & $3.21 \pm 0.046$ \\
BZB J1917-192 & $0.446 \pm 0.017$ & $2.53 \pm 0.017$ \\
3FGL J1958.2-3011 & $0.585 \pm 0.009$ & $3.32 \pm 0.009$ \\
PKS 2005-489 & $0.362 \pm 0.014$ & $2.06 \pm 0.014$ \\
{$[H B 89] 2126-158$} & $0.260 \pm 0.016$ & $1.48 \pm 0.016$ \\
PKS 2149-306 & $0.207 \pm 0.021$ & $1.18 \pm 0.021$ \\
PKS 2155-304 & $0.142 \pm 0.023$ & $0.81 \pm 0.023$ \\
PMN J2310-4347 & $0.695 \pm 0.008$ & $3.95 \pm 0.008$ \\
\hline
\end{tabular}


En general, los comportamientos más comúnmente observados en los blazares son el RWB y BWB. En particular, la tendencia BWB está asociada a los BL Lacs, los cuales pueden ser explicados con el modelo de shock-in-jet. Esto implicaría la presencia de una componente variable con un color constante y relativamente azul junto con una componente roja subyacente (Fiorucci et al. 2004, Ikejiri et al. 2011). Con respecto a la tendencia RWB, se la asocia a los FSRQs, lo que puede ser interpretado como una contribución fuerte de emisión térmica azul del disco de acreción, el cual afecta a la región más azul del espectro óptico cuando la emisión del jet es débil (Villata et al. 2006, Rani et al. 2010, Bonning et al. 2012).

\subsubsection{Análisis global y discusión sobre cada fuente}

* [HB89] 0208-512: está clasificado como BZU, según el catálogo de Roma BZCAT. Aunque la gran mayoría de los trabajos publicados lo clasifican como un FSRQ (LSP, Blom et al. 1995; Pohl et al. 1997; Sefako et al. 1999; Romero et al. 2002; Fan et al. 2002; Nolan et al. 2003; Vercellone et al. 2004; Li et al. 2010.), existen algunos autores que lo sitúan dentro del grupo de los BL Lacs (Chen at al. 2005; Healey et al. 2008; Mao et al. 2011; Taris et al. 2013). Posee un redshift de $z=1.003$ (Peterson et al. $1976 ; 1979)$. En la banda de rayos- $\gamma$, fue una de las primeras fuentes extragalácticas en ser detectadas por el satélite EGRET (Bertsch et al. 1993; Fitchel et al. 1993) y una de las pocas en ser candidata a "MeV-blazar", observada por el satélite COMPTEL (Blom et al. 1995) debido a sus inusuales flares en energías de MeV. Por encima de los $100 \mathrm{MeV}$, se encontró que la variabilidad presente en dicha banda se daba en escalas cortas de 8 días, lo que sugería que la región de emisión $\gamma$ tenía varios radios de Schwarzschild para un agujero negro de $10^{10} M_{\odot}$ (von Montigny et al. 1995); mientras que Bhattacharya et al. (2013) estudiaron la variabilidad $\gamma$ en escalas más largas (10 años), encontrando variaciones de un orden de magnitud. En cuanto a la banda de rayos $\mathrm{X}$, los primeros reportes fueron con el satélite ROSAT, con el cual Brinkmann et al. (1994), a partir de las correlaciones cruzadas con el survey Mongolo de $408 \mathrm{MHz}$, lograron identificar más de 500 fuentes. Para [HB89] 0208-512, obtuvieron el flujo en rayos $\mathrm{X}$, siendo su valor $\mathrm{F}=6.15 \times 10^{-12} \pm 8.16 \times 10^{-13} \mathrm{erg} \mathrm{cm}^{-2} \mathrm{~s}^{-1}$. Por otra parte, con el lanzamiento del satélite BeppoSAX, Donato et al. (2005) presentaron un catálogo espectral para blazares, donde para esta fuente ajustaron una ley de potencia simple con un índice de $\Gamma=1.64 \pm 0.10$. Más tarde, comienzan las primeras observaciones en imagen y espectroscopía con el satélite Chandra (Marshall et al. 2005; Schwartz et al. 2006), encontrando un jet de 4 arcsec, con posición similar al jet de miliarcsec en radio. Hacia la banda óptica, la primera medición de su polarización fue hecha por Impey et al. (1988), encontrando un valor alto en el porcentaje de polarización, $11.5 \%$, y corroborando que la fuente se trataba de un blazar. Romero et al. (2002) presentan un estudio fotométrico de la incidencia de la microvariabilidad en la emisión óptica, en el cual este blazar muestra variaciones de amplitud considerable $(\Delta m=0.131 \mathrm{mag})$ en escalas cortas de tiempo (horas). Por otra parte, se han realizado estudios multifrecuencia: Perlman et al. (2011) quienes estudiaron el jet de este objeto con el HST (Hubble Space Telescope), Chandra, VLA (Very Large Array) y ATCA (Australian Telescope Compact Array), encontrando que dicho jet presenta una estructura compleja en radio con evidencia de curvatura de $90^{\circ}$, mientras que en rayos $\mathrm{X}$ el jet se extiende 
varios arcsec. Chatterjee et al. (2013) reportan un flare anómalo y lo estudian en la bandas óptica, NIR, X y $\gamma$, detectando 3 intervalos en los cuales la variación se da en 1 mag durante 3 meses para el óptico y NIR, mientras que para energías de GeV las variaciones se dan en el primer y tercer intervalo durante 1 mes.

Este blazar fue observado durante 3 noches, el 13 agosto, y el 15 y 17 septiembre 2015. Un detalle importante es que el blazar es el objeto más débil dentro del campo, con lo cual las estrellas de comparación y control más cercanas son entre 1 y 2 mag más brillantes. En la Tabla 3.3, se tienen los resultados de la aplicación de las herramientas C y F. En el caso de la variabilidad intra-noche, no se detectan variaciones en los datos de septiembre con ningunos de los dos parámetros, mientras que para la noche de agosto sólo el filtro $\mathrm{R}$ registra variación con ambos parámetros estadísticos. En cuanto a las variaciones inter-noche dentro del mes de septiembre, tampoco se detecta variabilidad alguna (ambos parámetros coinciden en los resultados de la no variabilidad), pero al considerar la escala temporal de meses (entre agosto y septiembre), se detecta una variación de gran amplitud, siendo la variación promedio de $\Delta m \sim 0.7$.

En la Fig. 3.2 se muestran las curvas de luz de las magnitudes estándares del blazar en el filtro $\mathrm{V}$ (puntos verdes) y en el $\mathrm{R}$ (puntos rojos), donde el valor promedio de la variación (en escala de meses) es de $\Delta m=0.702$ en el filtro $\mathrm{V}$ y $\Delta m=0.756$ en el filtro R. Las magnitudes estándares promedio para esta fuente resultaron ser $17.09 \pm 0.01 \mathrm{en}$ $\mathrm{V}$ y $16.62 \pm 0.02$ en $\mathrm{R}$ para los datos de agosto, y $17.64 \pm 0.02$ en $\mathrm{V}$ y $17.27 \pm 0.02$ en $\mathrm{R}$ para los datos de septiembre.

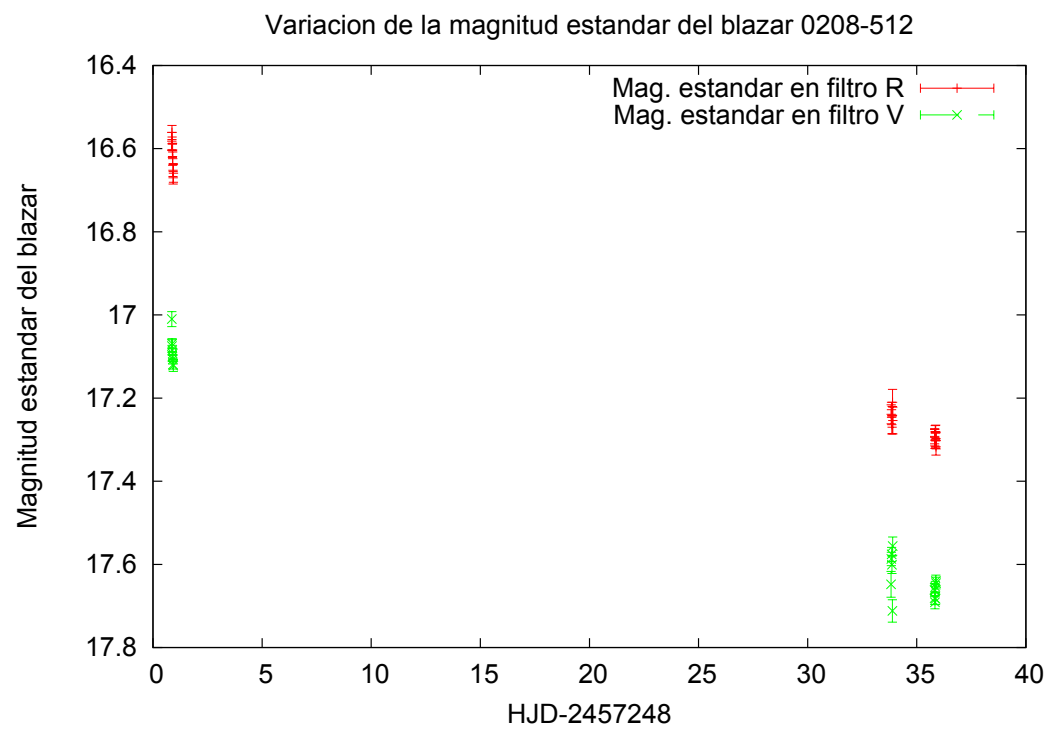

Figura 3.2. Curva de luz para la magnitud estándar de [HB89] 0208-512 en el filtro $\mathrm{R}$ (rojo) y en el V (verde).

Los valores que obtuvimos lo comparamos con los encontrados en la literatura. El primer registro de la magnitud en el filtro V lo obtuvo Adam (1985), con un valor de $\mathrm{V}=16.93$. Luego, Romero et al. (2002) estudiaron la variabilidad a escala corta, encontrando variaciones significativas en un corto lapso de tiempo. Las variaciones eran de $\Delta m_{V}=0.131$ en un lapso de $7.75 \mathrm{hs}$ y obtuvieron una magnitud promedio de $V=15.63 \pm 0.02$. Otros trabajos registraron valores similares a los de Adam (1985), 
siendo V=16.93 en el caso de Chen et al. (2005) y Abdo et al. (2010) con V=16.90. Comparando lo obtenido con estos valores, encontramos que, para nuestras observaciones, el objeto presenta la magnitud más débil registrada hasta el momento. Analizando de manera global, la fuente pasa de tener una magnitud de 16.93 a 15.63 en 17 años, esto es, se vuelve más brillante en más de 1mag. Luego su brillo vuelve a decaer a 16.98 años después y finalmente, para el 2015, decae nuevamente hasta 17.37. Este comportamiento lo podemos visualizar en la Fig. 3.3.

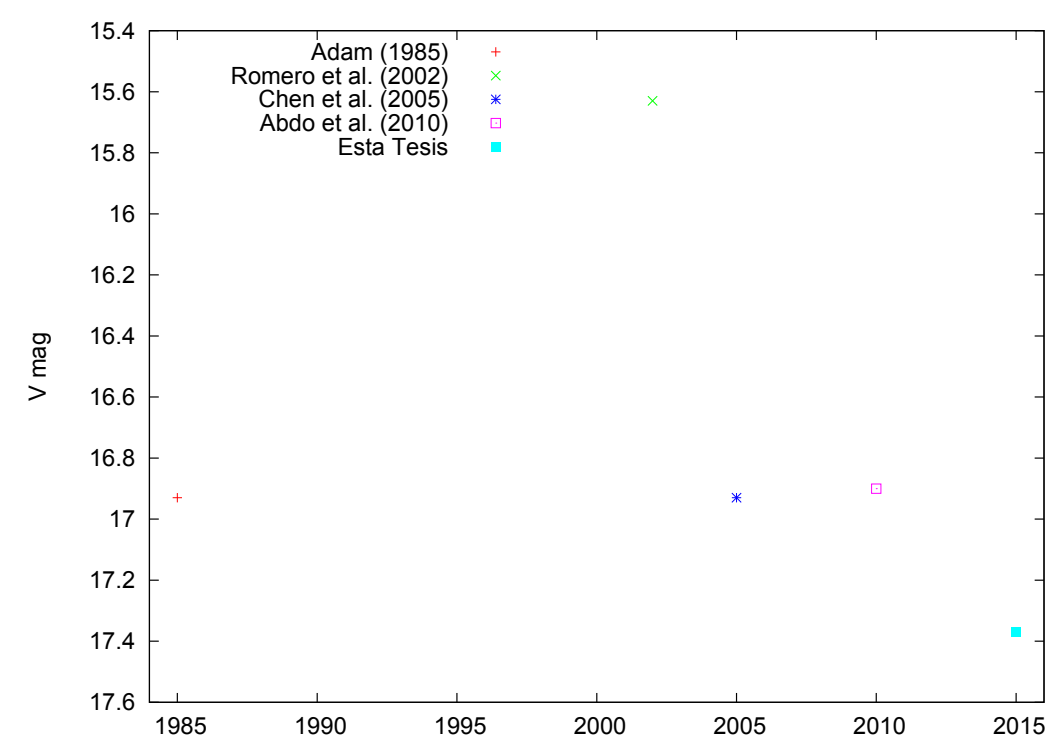

Figura 3.3. Valores comparativos para la magnitud estándar V del blazar [HB89] 0208-512.

Es decir, para las observaciones hechas en esta Tesis, el blazar estaría en un nivel de actividad bajo. Respecto a datos publicados para la magnitud en el filtro R, Chatterjee et al. (2013) estudiaron esta fuente en varias bandas del OIR (óptico/IR), incluyendo a R. Obtuvieron la curva de luz en la banda R para el período agosto 2008 hasta febrero 2012, en la cual se aprecian varios estallidos y variaciones. En la Fig. 3.4 mostramos la curva de luz obtenida por Chatterjee et al. (2013) en comparación con lo que nosotros obtuvimos. Nuestros valores en $\mathrm{R}$ resultan cercanos al valor medio que presentan los datos de Chatterjee et al. (2013).

Con los valores de las magnitudes estándares en $\mathrm{V}$ y $\mathrm{R}$, podemos obtener el índice de color $(\mathrm{V}-\mathrm{R})$ y calcular el índice espectral $\alpha_{V R}$. Para las 3 noches, este índice resultó $2.69 \pm 0.026$ en agosto, y $2.1 \pm 0.074$ y $2.1 \pm 0.026$ para las noches de septiembre, siendo el valor promedio de $\alpha_{V R}=2.33 \pm 0.065$. En la Fig. 3.5 se muestra cómo ha sido la variación en escala de meses del índice espectral. Se observa que de un mes a otro, el valor decrece, implicando que la distribución espectral se vuelve más blanda. Este decrecimiento en el valor está asociado a lo obtenido en las curvas de variación de la magnitud estándar en $\mathrm{V}$ y $\mathrm{R}$, donde se observa que la magnitud decrece (el objeto se vuelve más débil). Un espectro más blando, cuando el objeto cae en brillo, implica que se llega a energías más bajas.

Chatterjee et al. (2013) encuentran que $\alpha_{B J}$ varía de 0.8 a 1.7. En este caso, la 

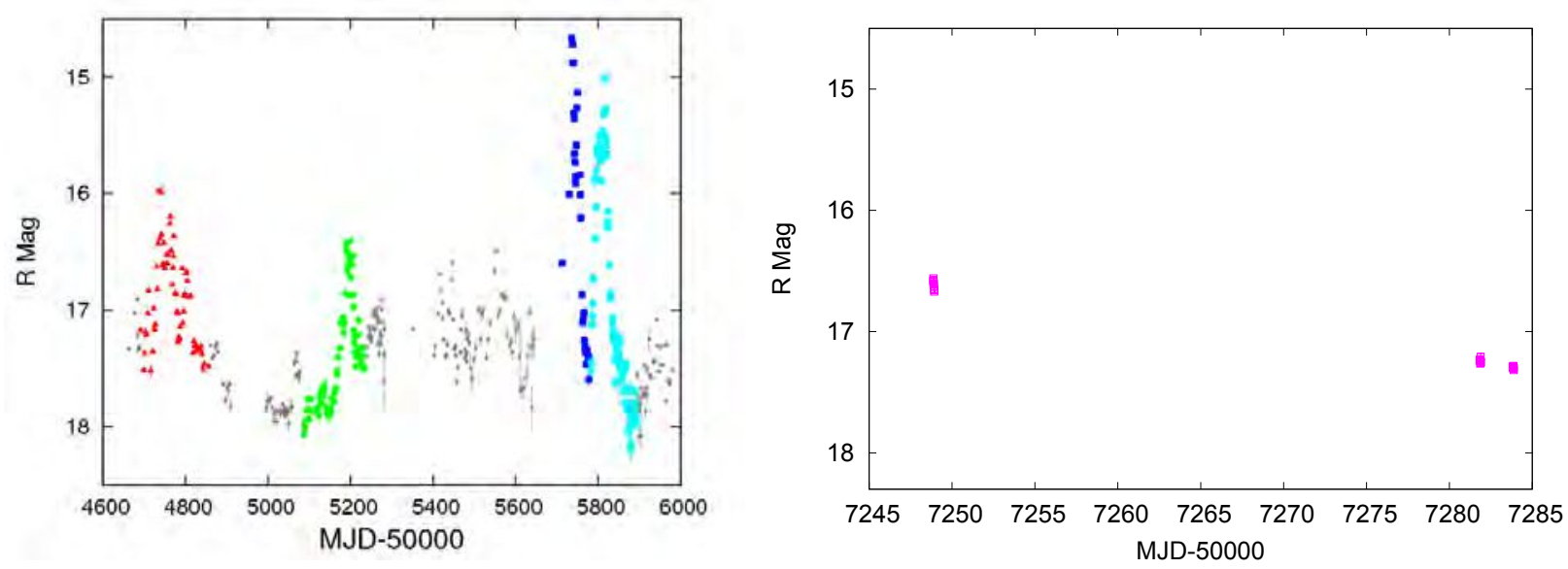

Figura 3.4. Curva de luz de R para 0208-512. A la izquierda corresponde al período agosto 2008 - febrero 2012 (Chatterjee et al. 2013), donde los puntos de color rojo, verde, azul y cian corresponden a los intervalos en los cuales se detectó un estallido intenso en las bandas óptico-NIR. A la derecha nuestros datos correspondientes al período agosto - septiembre 2015.

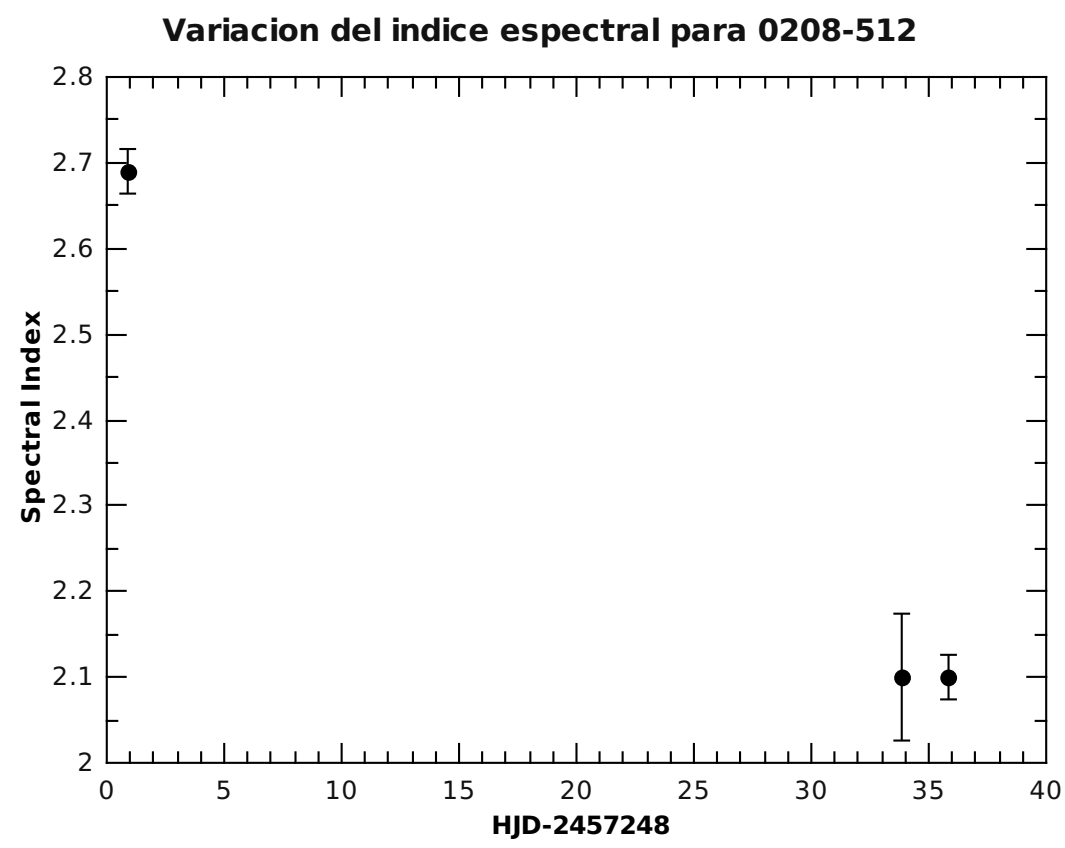

Figura 3.5. Variación del índice espectral para PKS0208-512.

fuente pasó de tener un espectro más blando a uno más endurecido, llegando a energías mayores. Junto a este comportamiento del índice espectral, más las variaciones que se detectaron, analizamos el diagrama magnitud-magnitud (ver Fig. 3.6), en el cual se puede apreciar que existe una correlación entre los datos en $\mathrm{V}$ y $\mathrm{R}$. El valor de la función DCF dio positivo, $\mathrm{DCF}=1.007 \pm 0.145$, indicando que existe correlación entre los datos. A su vez, se procedió a hacer un análisis de regresión lineal, el cual determinó una pendiente positiva, $\mathrm{A}=1.177 \pm 0.014$, corroborando la correlación hallada entre los 
datos (ver valores en la Tabla 3.4). Por último, se calculó el índice de correlación de Pearson $p=0.995$, confirmando que se tiene una fuerte correlación entre los datos. Estos resultados implican que ambas variaciones (a escalas de meses) se correlacionan, lo que se traduce a que las variaciones detectadas en ambos filtros provienen de la misma región, es decir, los fotones responsables de estas emisiones provienen de la misma zona y del mismo proceso físico.
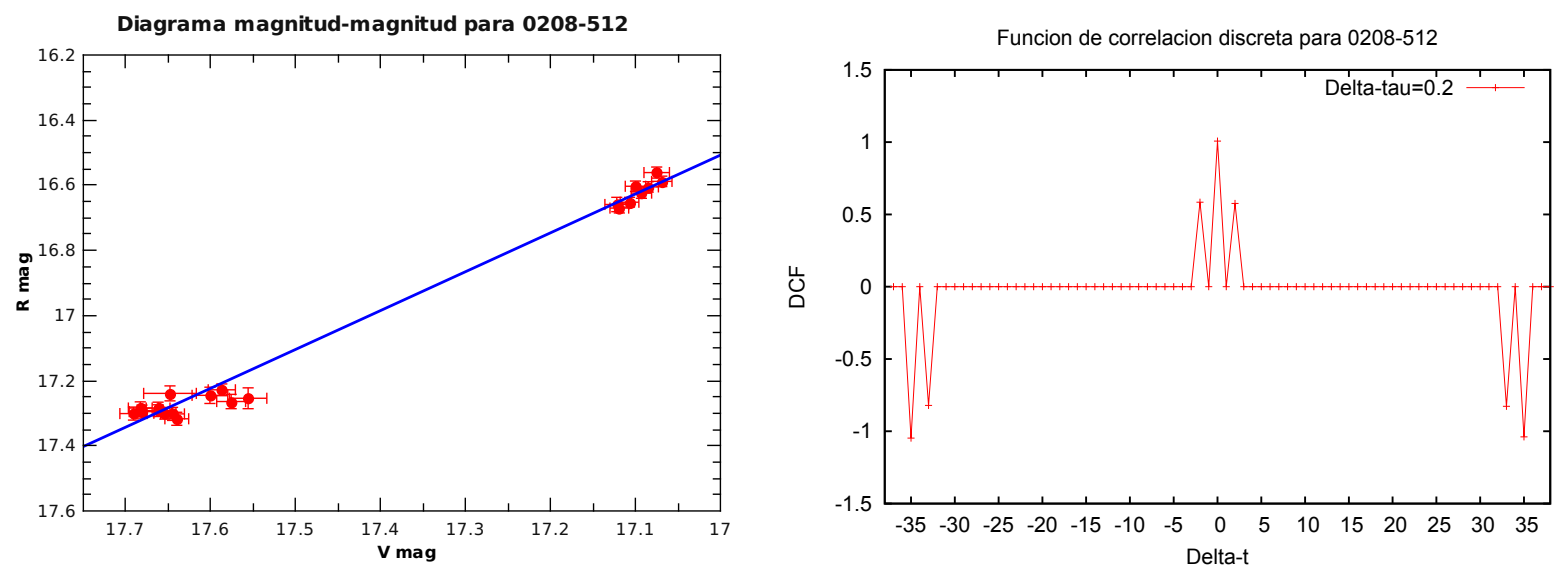

Figura 3.6. Diagrama magnitud-magnitud (izquierda) y función de correlación discreta (derecha) para 0208-512.

Finalmente, podemos analizar cuál sería el posible origen de las variaciones detectadas a partir del diagrama color-magnitud. En la Fig. 3.7, graficamos el comportamiento para la noche de agosto por un lado, y las noches de septiembre por el otro. Para los datos de agosto, se obtuvo un valor negativo del coeficiente de Pearson $(r=-0.690)$, resultando en una correlación negativa. Esto conlleva a que la fuente presenta una tendencia RWB. Para los datos de Septiembre, en cambio, se observa una tendencia BWB con valores en los parámetros del ajuste y del coeficiente de Pearson $(r=0.762)$ implicando una fuerte correlación. A escalas de meses, se tiene una fuerte correlación negativa $(r=-0.855)$ en los datos, lo que indicaría un comportamiento RWB para este blazar. Un comportamiento similar fue reportado por Chatterjee et al. (2013).

* [HB89] 0414+009: clasificado como BL Lac, se lo detectó a energías de TeV (H.E.S.S Colaboration, 2012). Posee un redshift de $z=0.287$ (Halpern et al. 1991). Su primera detección fue con el satélite HEAO 1 (Ulmer et al. 1980). Ulmer et al. (1983) encontraron que, a través de observaciones en radio, rayos $\mathrm{X}$ y óptico, este objeto estaba asociado a una fuente brillante en rayos X del tipo BL Lac. Por otro lado, con observaciones en rayos X, se logró determinar que esta fuente tiene la forma típica de un HBL en su SED (Walter et al. 1998; Beckmaann et al. 2002; actualmente HSP). Impey \& Tapia (1988) estudiaron su polarización confirmando que se trataba de un objeto tipo BL Lac. En cuanto al óptico, las primeras mediciones datan de la década del '80 (Pica et al., 1988; Hewitt et al. 1989). Ulmer et al. (1983), con datos en el óptico, calcularon su magnitud en el filtro V de 16.4 mag, además de observar un espectro sin líneas de emisión (característico de los BL Lacs). Falomo \& Tanzi (1991) analizaron las variaciones espectrales en el óptico y en NIR, encontrando variaciones grandes en 

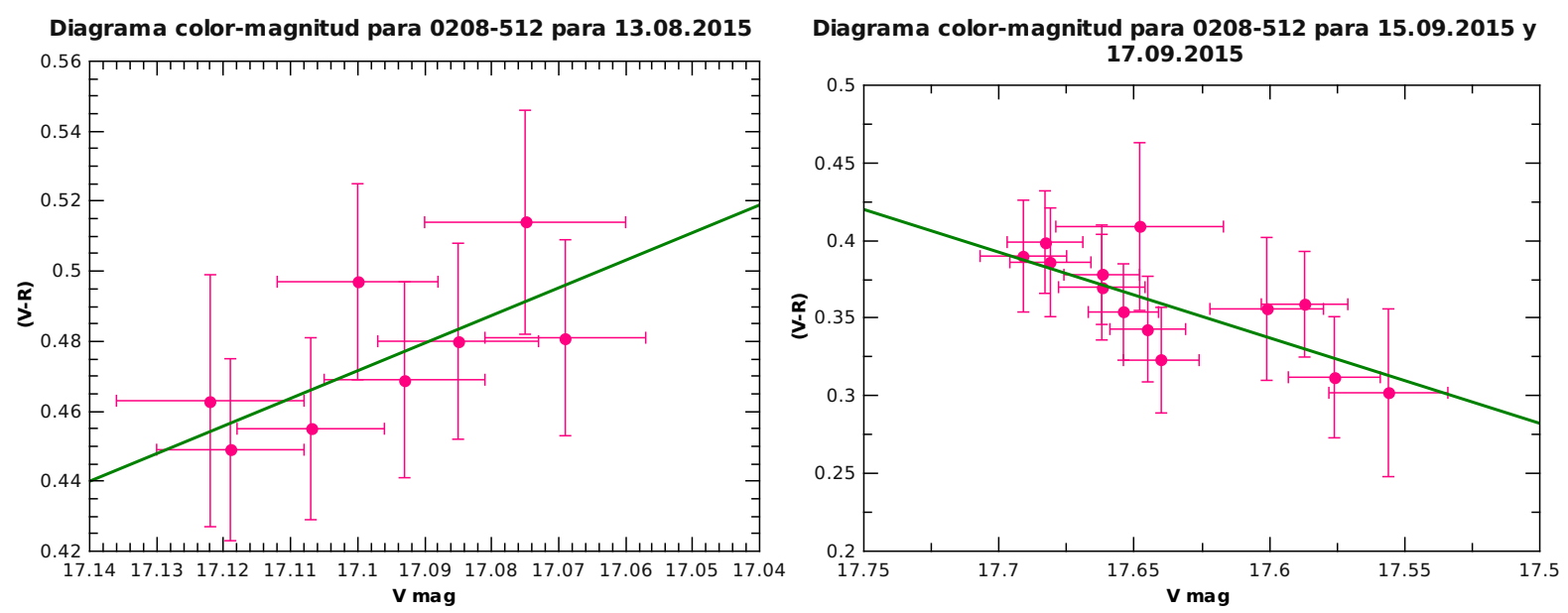

Figura 3.7. Diagrama color-magnitud para PKS 0208-512. A la izquierda se muestra el diagrama correspondiente a la noche del 13 agosto 2015, con un comportamiento BWB. A la derecha, el diagrama corresponde a las noches del 15 y 17 septiembre del 2015, donde se observa una tendencia RWB.

el óptico $(\Delta V=0.8)$ que no estaban asociadas a las pequeñas variaciones en el NIR $(\Delta K=0.1)$. Además, en el estado alto de la fuente, lograron ajustar un ley de potencia chata (con un índice espectral de 0.4) superpuesta al espectro de una galaxia elíptica. En el estado de baja actividad, en cambio, necesitaron de la presencia de componentes extras.

Este blazar fue observado durante 2 noches, 27 y 28 noviembre 2016. En la Tabla 3.3, se muestran los resultados del análisis de variabilidad para este objeto. Respecto a la variabilidad intra-noche, el objeto no presentó variaciones con ninguno de los dos parámetros y en ninguno de los dos filtros. En cuanto a las variaciones inter-noche, sólo el parámetro $\mathrm{F}$ detectó variaciones, en el filtro V. Las condiciones atmosféricas, sobretodo en la segunda noche, no han sido favorables, registrándose la presencia de nubosidad de moderada a alta. Con lo cual basándonos en lo analizado en el capítulo anterior, y observando que el criterio $\mathrm{C}$ no ha detectado variabilidad, no es considerada como una variación real la que se registra en el filtro $\mathrm{V}$ con el test $\mathrm{F}$. Por ende, tampoco se ha detectado variabilidad inter-noche.

En la Fig. 3.8 mostramos las curvas correspondientes a la magnitud estándar en el filtro V y R del blazar. La amplitud de estas curvas resultó de $\Delta m(V)=0.074$ y $\Delta m(R)=0.034$. Los valores promedios de las magnitudes estándares del blazar resultaron ser $16.197 \pm 0.016$ en $\mathrm{V}, \mathrm{y} 15.773 \pm 0.010$ en $\mathrm{R}$.

Diversos autores obtuvieron las magnitudes estándares en los filtros $\mathrm{V}$ y $\mathrm{R}$ a lo largo de varios años. En la Fig. 3.9, se muestra el comportamiento general para la magnitud V (panel superior) y para la magnitud R (panel inferior). Analizando la curva obtenida para la magnitud $\mathrm{R}$, observamos un incremento en el brillo de la fuente, donde McHardy et al. (1992) obtuvieron un valor de $\mathrm{R}=17.21$, mientras que Falomo \& Tanzi (1991) encontraron un valor para $R=16.6$. Raitieri et al. (1998), por su parte, hallaron al objeto en el estado más alto reportado hasta ese momento, observando un nuevo incremento desde 16.40 hasta 16.18. Luego, el blazar presenta variaciones 


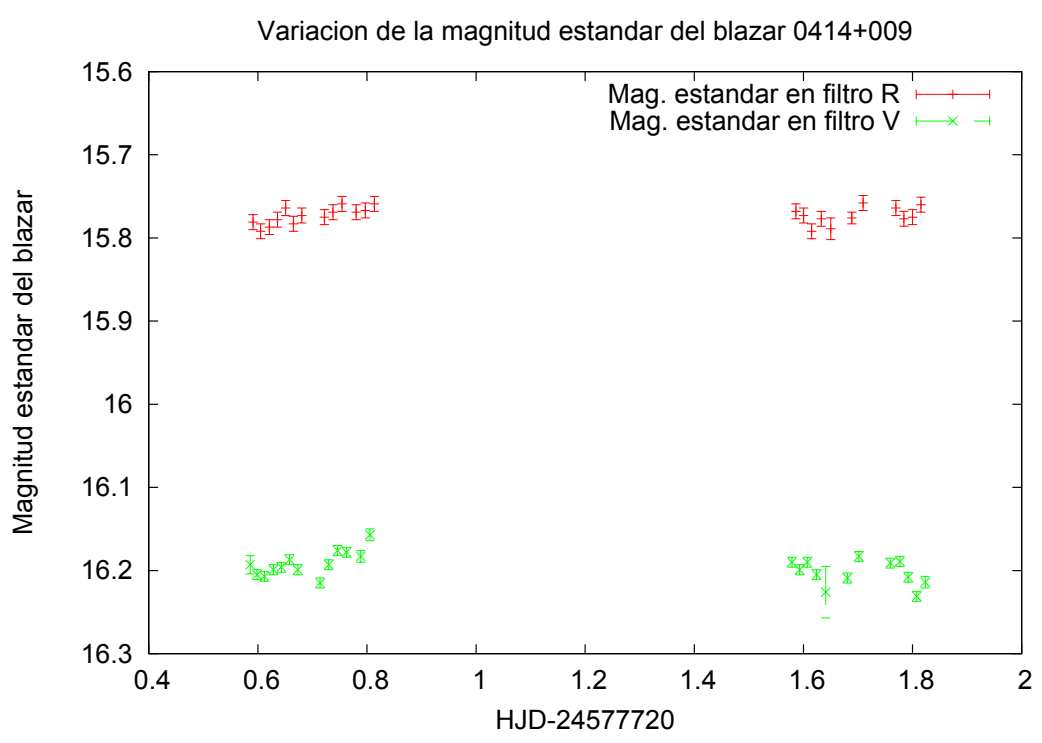

Figura 3.8. Curva de luz para la magnitud estándar de $0414+009$ en el filtro R (rojo) y en el V (verde).

a escalas largas, como lo muestra Kapanadze (2009), donde la magnitud en $\mathrm{R}$ oscila entre los valores 15.86 y 16.92. En esta Tesis, obtuvimos el valor más brillante del blazar reportado hasta el momento, con una magnitud $\mathrm{R}=15.77$. Vemos que a lo largo de 30 años aproximadamente, el blazar pasó por varios estados de actividad alta y baja, donde se ve reflejado en el comportamiento de la curva de la magnitud R. Un comportamiento análogo se observa para la magnitud V, donde Ulmer et al. (1993) encontró un valor de V de 16.38, mientras que Falomo \& Tanzi (1991) obtuvieron un incremento en la magnitud desde 17.5 a 16.8. McHardy et al. (1992) detectaron un valor más brillante para el blazar, 16.64 y luego Hewitt \& Burbidge (1993) encontraron que el blazar decayó en su brillo hasta 17.59. Nuevamente, Raitieri et al. (1998) encuentran al blazar con su brillo creciendo, llegando hasta 16.65. Por otra lado, en esta Tesis obtuvimos el valor más alto de la magnitud para el blazar, siendo éste de 16.19 mag.

Estudiamos el comportamiento en el diagrama magnitud-magnitud (ver Fig. 3.10) junto con el análisis de la DCF. Si bien se puede llegar a apreciar una correlación en los datos, el valor de la función de correlación resulta $\mathrm{DCF}=0.213 \pm 0.160$ con un valor de su incerteza del orden del valor de la función. Además, el coeficiente de Pearson para este ajuste fue $r=0.394$, indicando que se tiene una correlación débil entre los datos. En parte, esto puede deberse a los errores que presentan los datos (ver las barras de error del gráfico izquierdo de la Fig. 3.10). El ajuste lineal (ver la Tabla 3.4) posee un valor de $\chi^{2}$ reducido bajo $\left(\chi^{2}=0.127\right)$, confirmando que el ajuste presenta baja confiabilidad.

En base a los valores de las magnitudes estándares del objeto en $\mathrm{V}$ y $\mathrm{R}$, se calculó el índice espectral, $\alpha_{V R}$, cuyo valor promedio resultó ser $\alpha_{V R}=2.4 \pm 0.01$. Para cada una de las dos noches de observación, se obtuvieron $2.37 \pm 0.01$ y $2.43 \pm 0.02$ (ver Fig. 3.11). Asociado a este valor, se tiene el índice de color (V-R), cuyo valor promedio resultó $0.423 \pm 0.015$. Por otra parte, a partir de aquellos valores publicados en $\mathrm{V}$ y $\mathrm{R}$, podemos estimar el valor de $\alpha_{V R}$ y (V-R) para esos datos, con el objetivo de analizar el comportamiento a gran escala del índice espectral. Para los datos de Falomo \& 

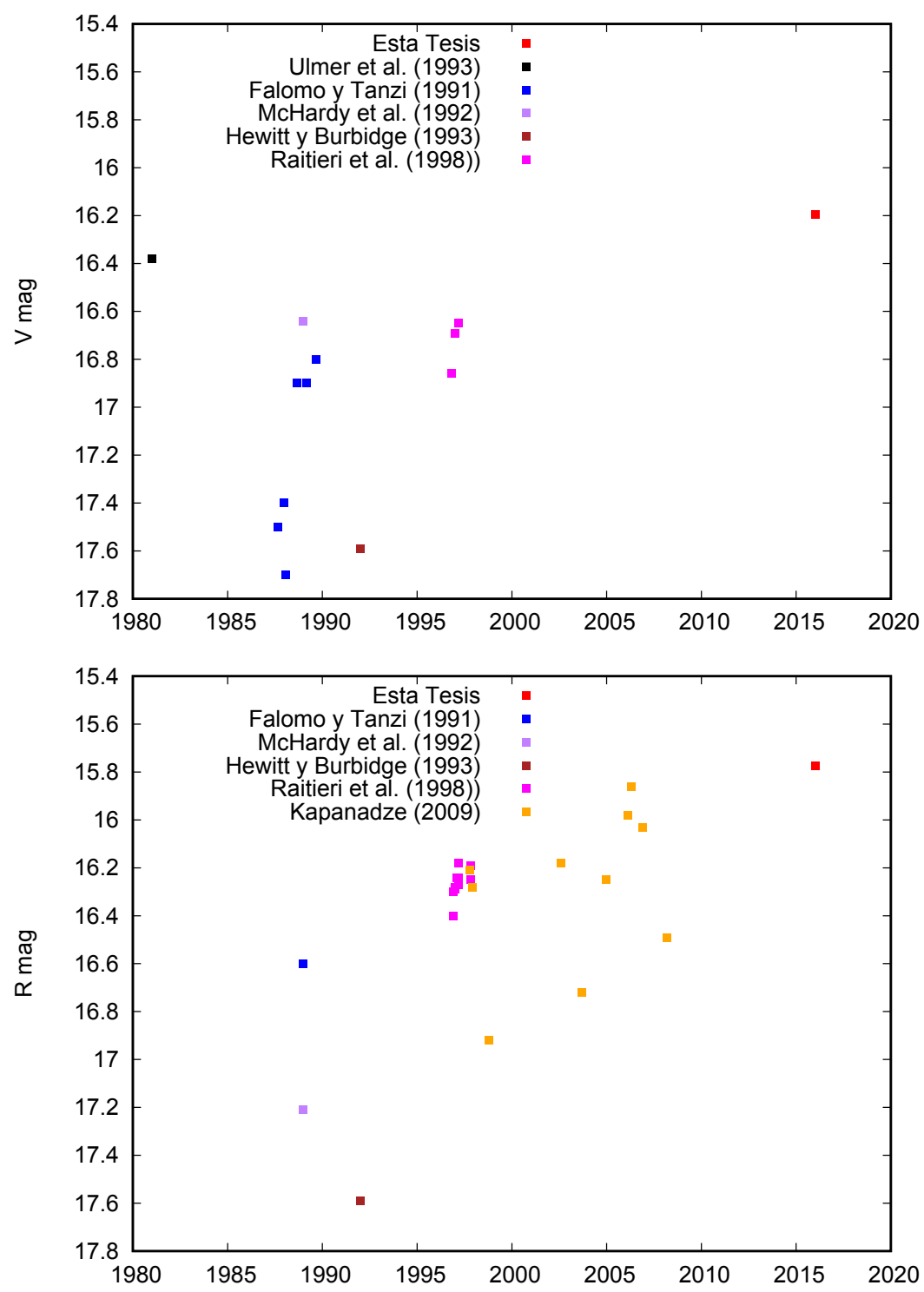

Figura 3.9. Comparación de la magnitud estándar en V (panel superior) y en $\mathrm{R}$ (panel inferior) para [HB89] 0414+009 a lo largo de los años.

Tanzi (1991), correspondientes a agosto de 1989, se obtuvo un valor de $(\mathrm{V}-\mathrm{R})=0.3$, resultando en $\alpha_{V R}=1.7$. En cuanto a los datos correspondientes a Raitieri et al. (1998), obtuvieron para datos correspondientes a 1997 un índice promedio de $(\mathrm{V}-\mathrm{R})=0.43 \mathrm{con}$ un $\alpha_{V R}=2.44$. De manera global, presentamos en la Fig. 3.12 el comportamiento de $\alpha_{V R}$ a lo largo de todos estos años.

Por último, analizamos el diagrama color-magnitud, que se muestra en la Fig. 3.13. Observamos que para nuestros datos, se tiene una correlación fuerte positiva (pues el coeficiente de Pearson resulta $\mathrm{r}=0.785$ ), lo que podría asociarse a un comportamiento del estilo BWB, tendencia esperable en objetos tipo BL Lac.

* [HB89] 0521-365: catalogado como BZU (blazar tipo incierto), fue primeramente clasificado como una galaxia N (Bolton et al. 1965) cuando la estudiaron como 

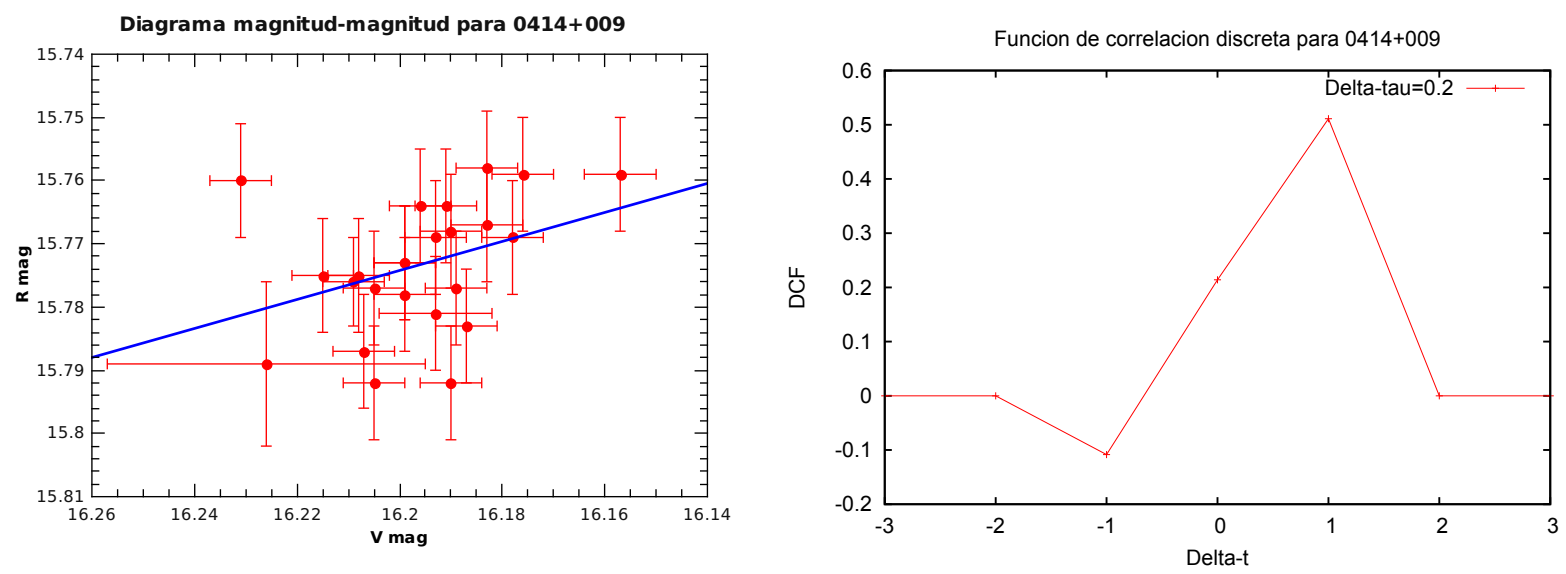

Figura 3.10. Diagrama magnitud-magnitud (izquierda) y función de correlación discreta (dercha) para 0414+009.

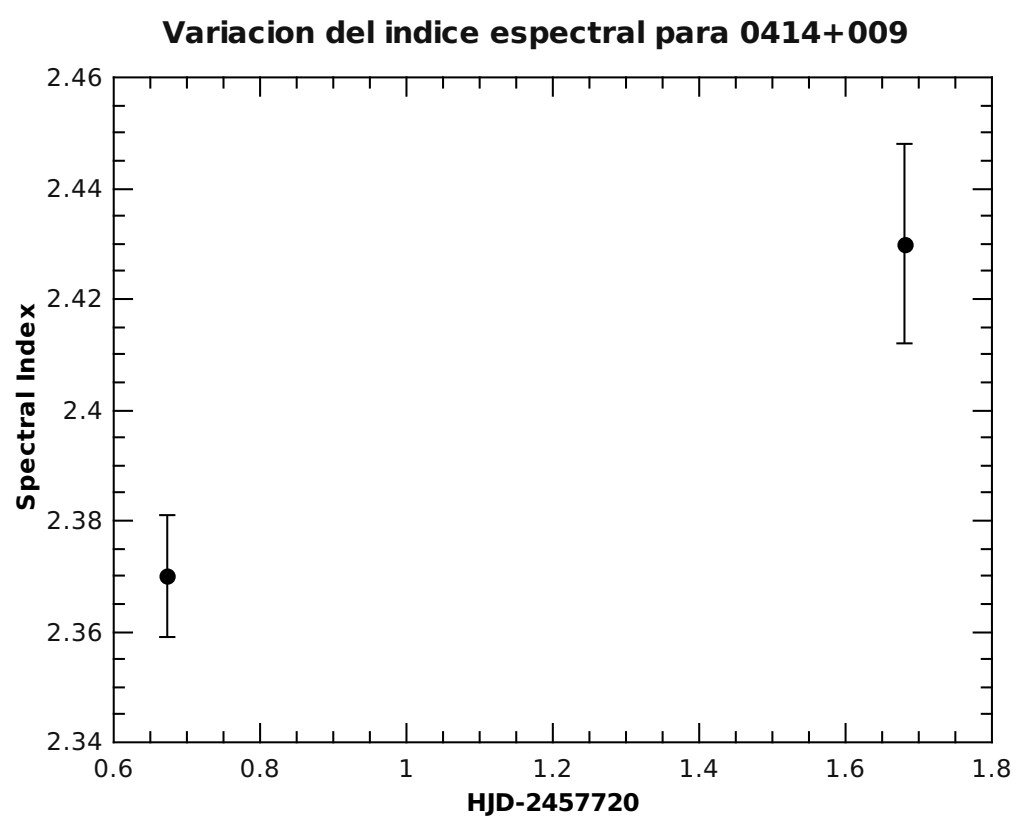

Figura 3.11. Variación del índice espectral para 0414+009.

contraparte óptica de una fuente de radio del catálogo de Parkes. Luego pasó a ser catalogado como un BL Lac (Danziger et al., 1979, Burbidge et al., 1987). Eggen (1970) muestra que la fuente varía más de una magnitud en el óptico en escalas temporales de meses, además de corroborar las primeras observaciones espectroscópicas de Westerlund \& Stokes (1966) y Searle \& Bolton (1968), las cuales muestran un continuo sin líneas de emisión, sugiriendo que la fuente se trata de un objeto tipo BL Lac. Se refuerza su naturaleza BL Lac a partir de la variabilidad de su continuo azul y a través de su polarización óptica reportada por Angel \& Stockman (1980). Se reportan las primeras observaciones en rayos X en el trabajo de Schwartz \& Ku (1983). En los trabajos de Danziger et al. $(1979,1983 b)$ notaron la presencia de un jet óptico y además presentaron espectros del núcleo variable tomados en el estado más alto de emisión del objeto. Este 


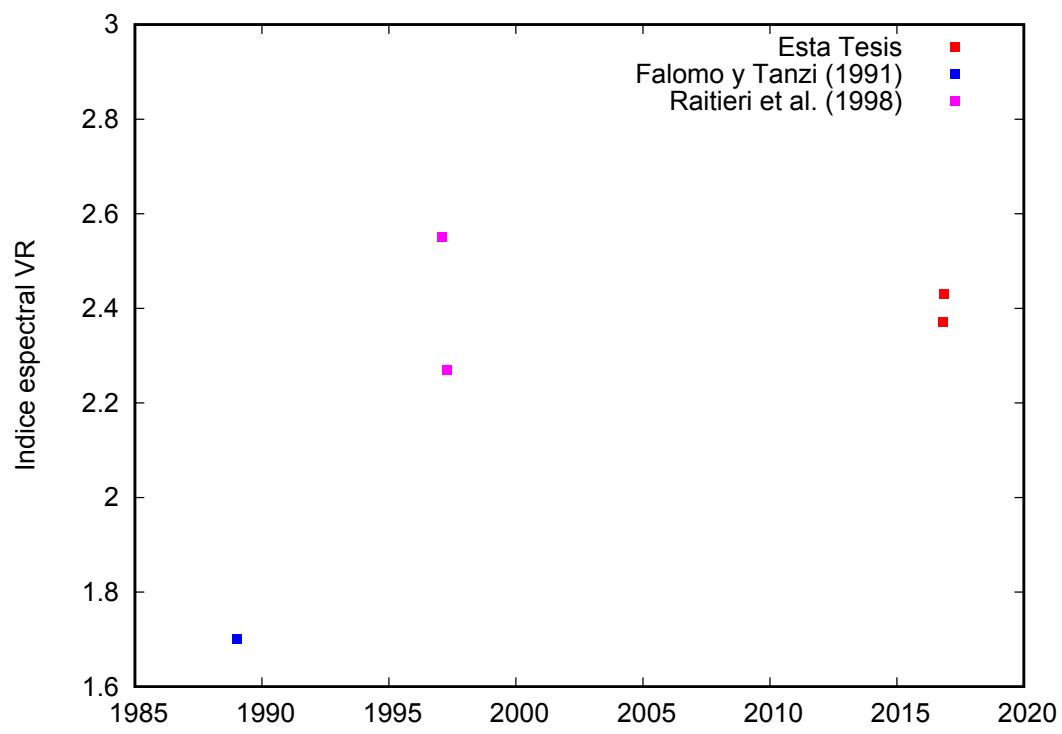

Figura 3.12. Comportamiento del índice espectral $\alpha_{V R}$ de $0414+009$ a partir de los datos obtenidos en esta Tesis y en comparación a los valores publicados.

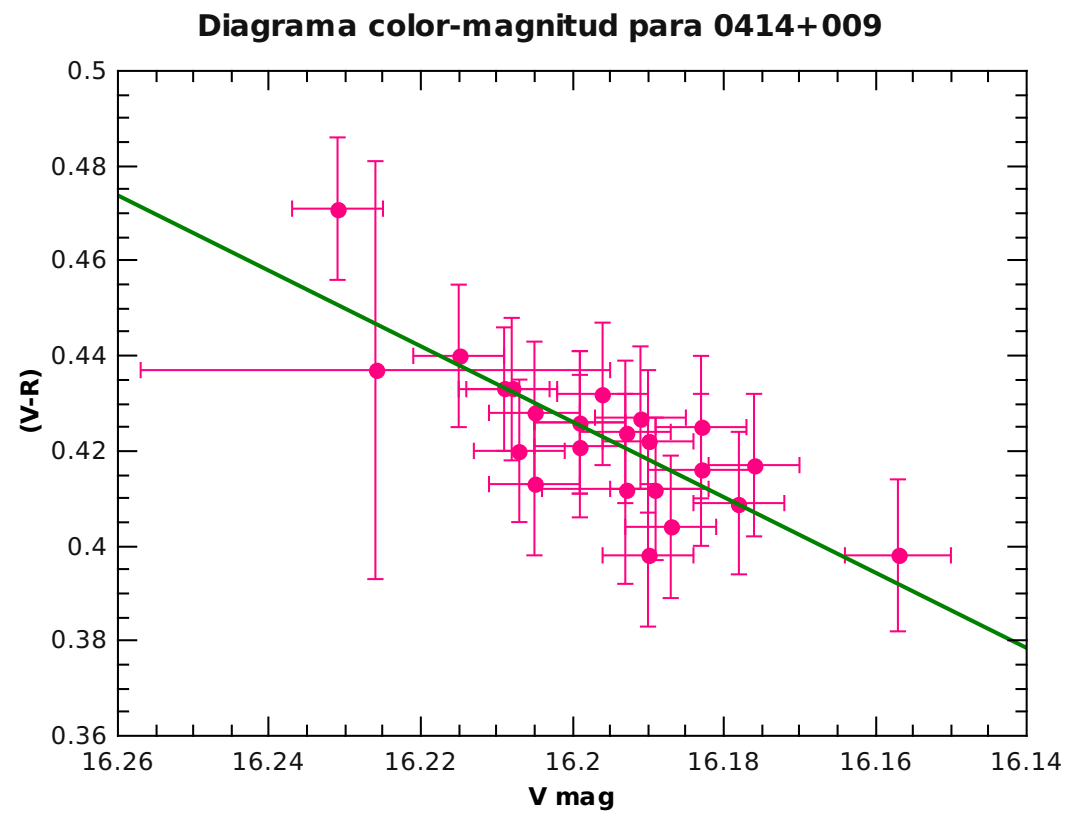

Figura 3.13. Diagrama color-magnitud para $0414+009$.

mismo jet se lo observó muy bien alineado con el jet en radio a escalas de kiloparsecs, con una clara correspondencia entre las estructuras en radio y óptico (Scarpa et al., 1999). El satélite Chandra detectó la contraparte en rayos $X$ de la parte más intensa del jet (Birkinshaw et al., 2002). En el rango de energía de rayos- $\gamma$, esta fuente fue detectada por el EGRET pero no fue incluida en el 3er catálogo del EGRET (Hartman et al., 1999). A partir de modelar la SED teniendo en cuenta las características en radio, Pian et al. (1996) derivaron un ángulo de visión de $30^{\circ}$ con un factor de Lorentz de 1.2. En cambio, para un factor de Lorentz de $\Gamma=5$, Giroletti et al. (2004) derivaron 
un rango angular entre $21^{\circ}-27^{\circ}$. Posee un valor de redshift de $z=0.0565$ (Jones, D.H. et al., 2009), aunque sus primeras mediciones datan del trabajo de Danzinger et al. (1979) en donde, a partir de algunas líneas débiles en el espectro, pudieron estimar $z$.

Esta fuente fue seguida durante una noche, 11 diciembre 2015, la cual presentó inestabilidades climáticas. Respecto a la variabilidad intra-noche, no se detecta variación en el filtro $\mathrm{V}$ ni en el filtro $\mathrm{R}$ con ningunos de los parámetros $\mathrm{C}$ y $\mathrm{F}$. Estos resultados están señalados en la Tabla 3.3.

Utilizando datos publicados de las magnitudes estándares de las estrellas de campo, pudimos estimar la magnitud estándar del blazar. Esto se debió a que no tenemos observaciones de campos estándares en la noche de observación. En el caso del filtro V, utilizamos los valores publicados en Andruchow (2006, PhD Thesis) y para el filtro $\mathrm{R}$, usamos los valores publicados en el catálogo de USNO-B1 (Monet et al., 2003). De esta manera, la magnitud estándar promedio para el blazar resultó ser 15.298 \pm 0.003 en $\mathrm{V}$ y $14.378 \pm 0.003$ en $\mathrm{R}$. La variación obtenida en cada filtro fue de $\Delta m(V)=0.044$ y $\Delta m(R)=0.062$ (ver Fig. 3.14).

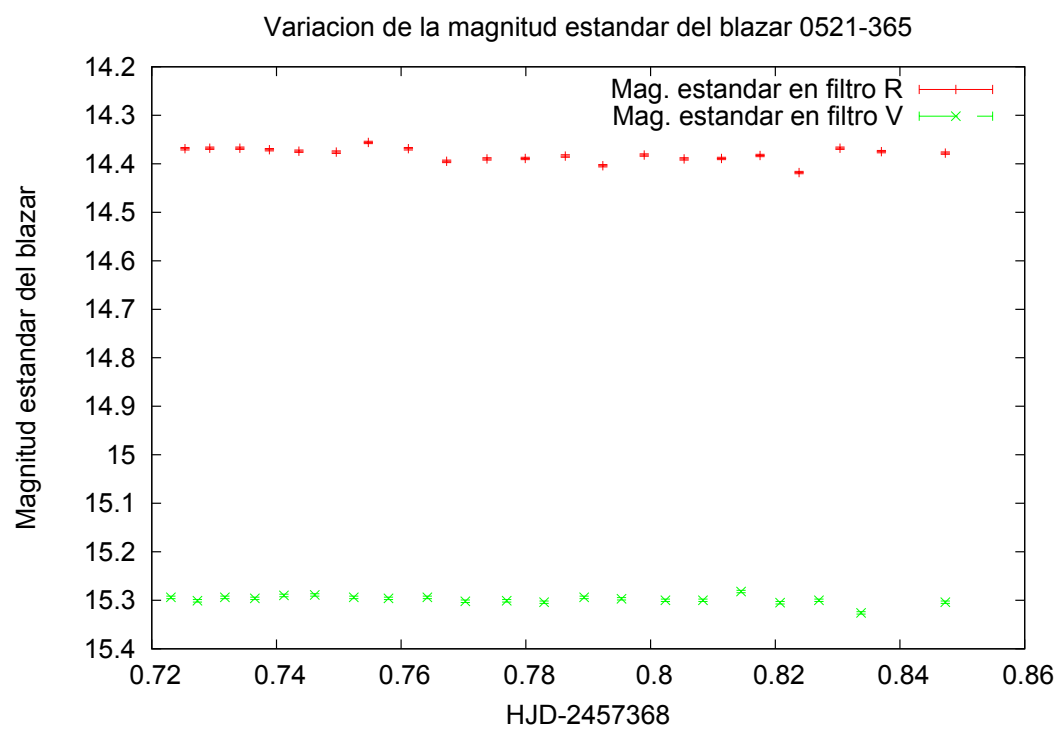

Figura 3.14. Curva de luz para la magnitud estándar de 0521-365 en el filtro R (rojo) y en el V (verde).

Comparando con los valores publicados de las magnitudes estándares $\mathrm{V}$ y $\mathrm{R}$, encontramos que a lo largo de casi 50 años el valor de la magnitud V se mantuvo estable. Westerlund \& Stokes (1966) obtuvieron un $\mathrm{V}=14.47 \pm 0.01$, mientras que Hamuy \& Maza (1987) observaron al blazar en 3 noches de febrero 1985, obteniendo valores en la magnitud de 15.02, 15.02 y 15.05. Por otra parte, Romero et al. (2002) encontraron que la magnitud promedio en las observaciones hechas en diciembre 1998 dieron un valor de $15.03 \pm 0.03$, con una variación en la magnitud de $\Delta m_{V}=0.063 \mathrm{mag}$. En nuestro caso, el valor obtenido es consistente con lo encontrado en las publicaciones, siendo éste $15.3 \pm$ 0.003. Para el caso de la magnitud R, Bergeron \& D'Odorico (1986) obtuvieron un valor de $14.8 \pm 0.3$ (agosto 1984). Por su parte, Hamuy \& Maza (1987) con las observaciones de febrero de 1985, obtuvieron valores de 14.44, 14.44 y 14.49. Un año más tarde, con datos obtenidos para septiembre de 1986, la magnitud del blazar decreció, 
llegando a 18.9 \pm 0.3 (Boissé \& Bergeron). Urry et al. (2000) utilizan observaciones del telescopio espacial Hubble, donde obtienen un valor de $\mathrm{R}=15.28 \pm 0.1$. Para nuestras observaciones, $\mathrm{R}=14.37 \pm 0.003$. Vemos que la magnitud $\mathrm{V}$ tiene un decrecimiento máximo en su brillo de $0.8 \mathrm{mag}$, al igual que la magnitud R. Las curvas de luz de las magnitudes estándares se muestran en la Fig. 3.15.
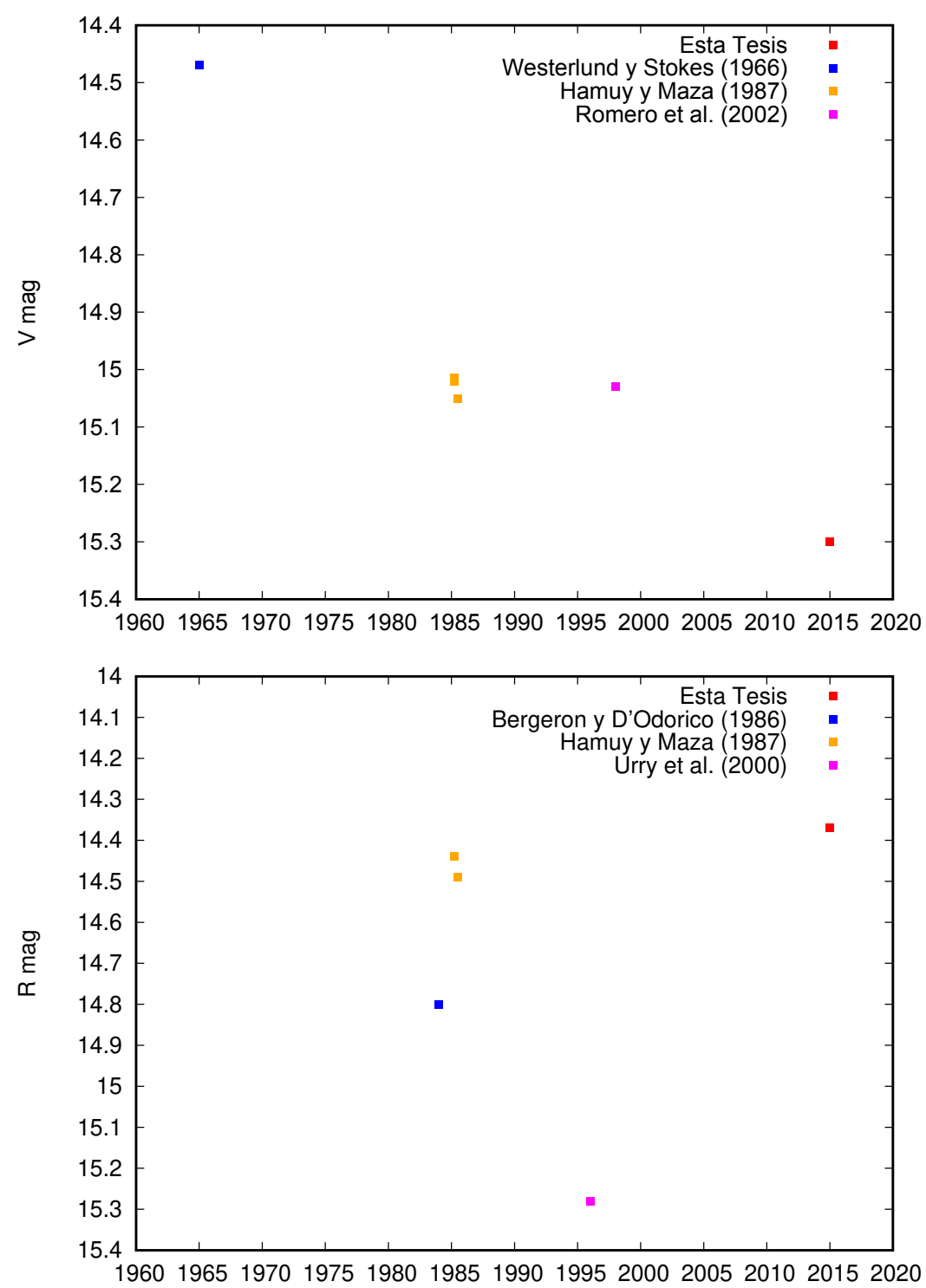

Figura 3.15. Comparación de la magnitud estándar en V (panel superior) y en $\mathrm{R}$ (panel inferior) para [HB89] 0521-365 a lo largo de los años.

$\mathrm{Al}$ analizar el diagrama magnitud-magnitud (Fig. 3.16), se observa una correlación entre los datos en $\mathrm{V}$ y $\mathrm{R}$, la cual es corroborada por los valores que se muestran en la Tabla 3.4 del ajuste lineal. Además, la función de correlación DCF también corrobora la correlación, con un valor de $\mathrm{DCF}=0.081 \pm 0.088$. Luego, la misma región física y el mismo proceso de radiación son los responsables de los fotones que emiten en estas dos bandas.

Con las magnitudes estándares obtenidas, calculamos el índice espectral, $\alpha_{V R}=5.20$ 

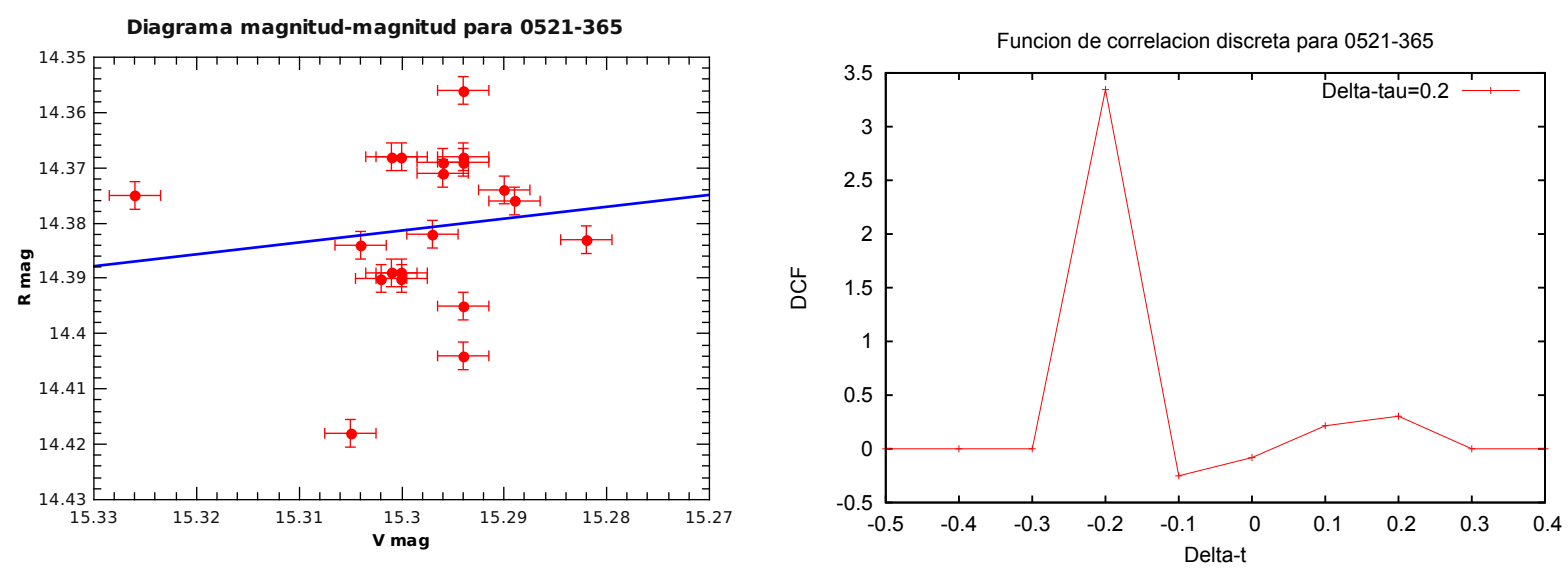

Figura 3.16. Diagrama magnitud-magnitud (izquierda) y función de correlación discreta (derecha) para 0521-365.

\pm 0.016 , el cual tienen asociado un índice de color de 0.93土 0.05. Hamuy \& Maza (1987) también obtuvieron los índices de color $(\mathrm{V}-\mathrm{R})$ para sus 3 noches de datos, resultando 0.58, 0.58 y 0.56. Aplicando el cálculo del índice espectral descripto previamente, se obtienen los valores de $\alpha_{V R}$, siendo $3.29,3.30$ y 3.18, y su valor promedio $\alpha_{V R}=3.30$. Entonces, entre 1985 y 2015, el índice espectral creció en 1.9. El espectro es ahora más duro de lo que era en 1985.

Por último, analizamos el diagrama color-magnitud que se muestra en la Fig. 3.17(izquierda). Los datos del diagrama muestran una tendencia negativa. Tanto el ajuste lineal como la función DCF y el coeficiente de Pearson $(r=0.424)$ indican que se trata de una correlación débil positiva. Esto implicaría que, para [HB89] 0521-365, se tendría una tendencia BWB, aunque el análisis no arroje resultados confiables. Si este mismo análisis lo aplicamos a los datos de Hamuy \& Maza (1987), se obtiene una tendencia contraria, esto es, un comportamiento tipo RWB (Fig. 3.17, derecha).
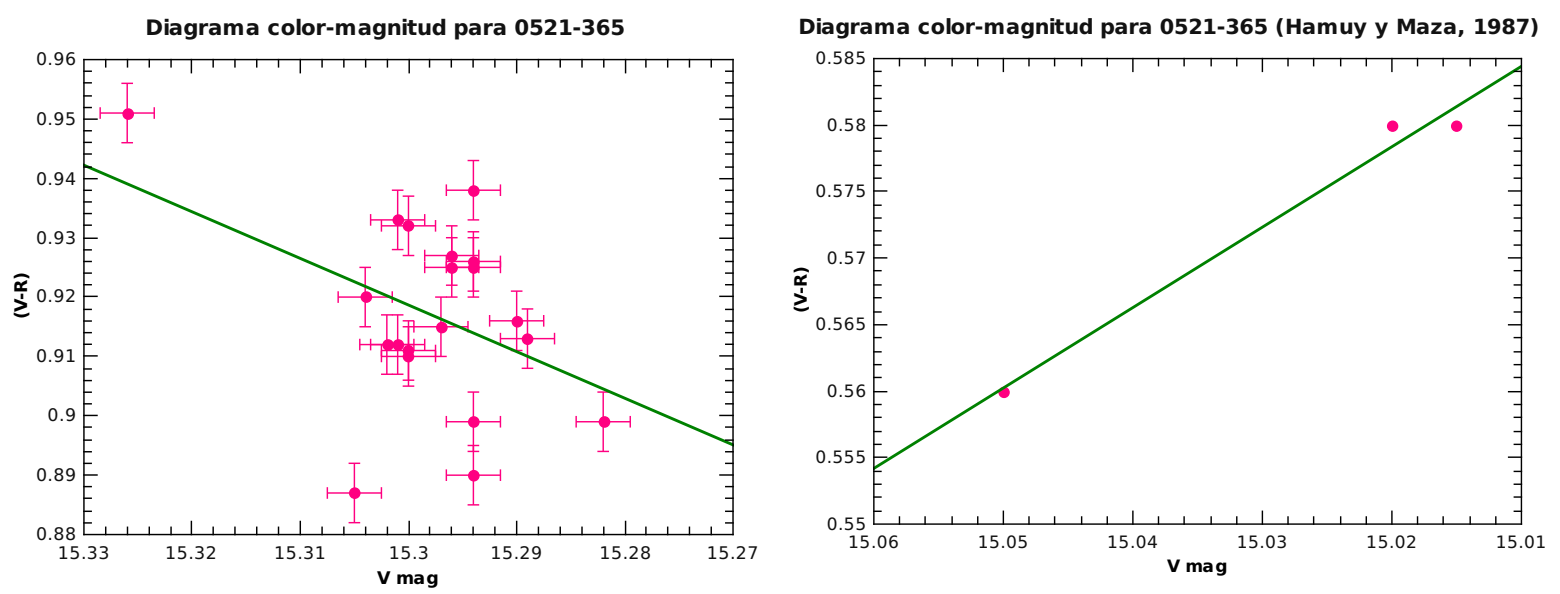

Figura 3.17. Diagrama color-magnitud para 0521-365, con nuestras observaciones (izquierda) y con los datos de Hamuy \& Maza (1987) (derecha).

Comparando en forma global los datos de Hamuy \& Maza (1987) y los nuestros, 
vemos que en un lapso de 30 años, la magnitud $V$ decrece en brillo pasando de $\sim 15.03$ a 15.30. En este mismo intervalo temporal, el índice espectral aumenta de 3.30 a 5.20, sugiriendo que el espectro se endureció. Y acompañando a este comportamiento, el blazar pasa de tener una tendencia BWB a RWB en estos 30 años.

* PMN J0847-2337: esta blazar está catalogado como un BZU y sólo lo han estudiado en sucesivos catálogos como el CRATES (Healey et al. 2007); los correspondientes al satélite Fermi (Abdo et al. 2010, Nolan et al. 2012, Ackermann et al. 2015); el de 2MASS (Huchra et al. 2012), conociéndose muy poco sobre su variabilidad. En cuanto a sus propiedades ópticas, Mahony et al. (2011) obtuvieron magnitudes de $\mathrm{B}=16.44 \mathrm{y}$ $\mathrm{R}=15.24$ pero sin poder calcular el valor de su redshift. Shaw et al. (2012) determinaron el valor de su redshift a partir de su espectro, $\mathrm{z}=0.059$ y su índice espectral $\alpha=-1.48$. Ghisellini et al. (2014) calcularon los parámetros del jet relativista, donde obtuvieron el valor de factor Doppler $\Gamma=5.0$, y el ángulo de visión $\theta_{V}=10$.

Este blazar fue seguido durante 6 noches en CASLEO, entre 22 y 27 abril 2014. En la Tabla 3.3 se muestran los resultados obtenidos de aplicar las herramientas $\mathrm{C}$ y $\mathrm{F}$. No se detecta variabilidad intra-noche en ninguno de los dos filtros coincidiendo en la detección de no variabilidad ambos parámetros $\mathrm{C}$ y $\mathrm{F}$, y en cuanto a las variaciones inter- noche, sólo se detecta variabilidad en ambos filtros con F. Por otra parte, en la Fig. 3.18 mostramos las curvas de las magnitudes estándares $\mathrm{V}$ (puntos verdes) y $\mathrm{R}$ (puntos rojos). Las magnitudes estándares promedio fueron $16.526 \pm 0.026$ para el filtro V y 16.093 \pm 0.019 para el filtro R. Dentro de la literatura, son escasos los trabajos que analizaron datos en el óptico, además de no haber registros sobre el estado de variabilidad. Mahony et al. (2011) obtuvieron un valor para la magnitud $\mathrm{R}=15.24$ mag. Comparando con lo que obtuvimos de nuestras observaciones $(\mathrm{R}=16.093 \pm 0.019 \mathrm{mag})$, encontramos al blazar con un brillo menor, cuya diferencia resulta $\Delta R=0.85$ mag en el transcurso de 7 años.

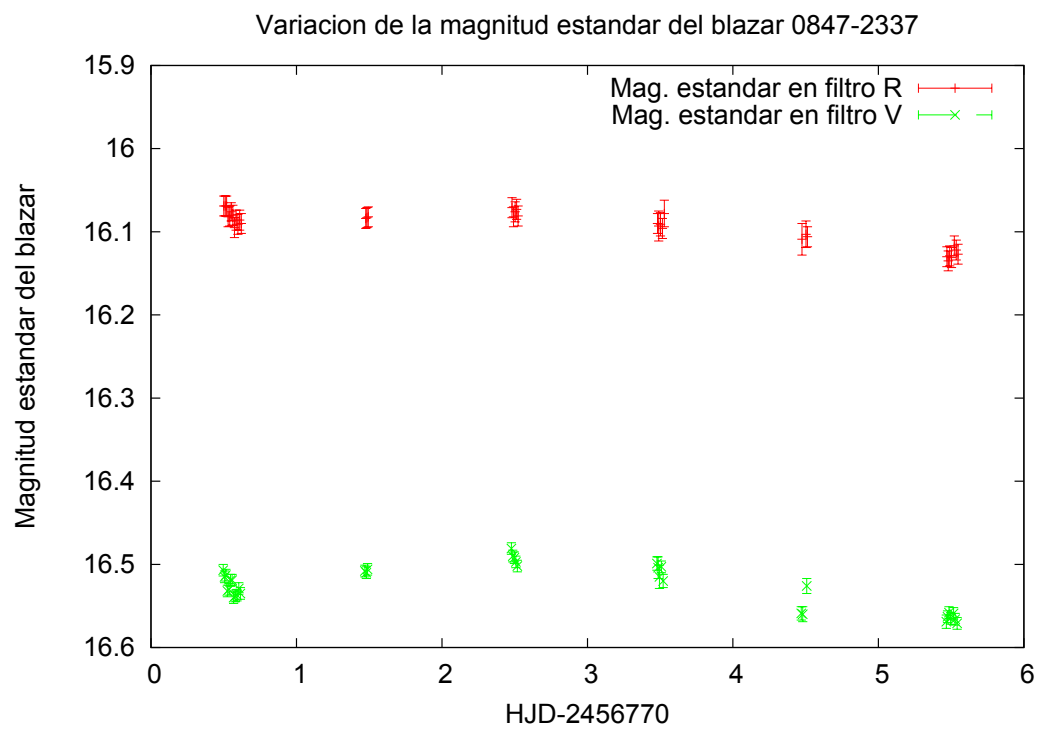

Figura 3.18. Curva de luz para la magnitud estándar de 0847-2337 en el filtro R (rojo) y en el V (verde). 
Por otro lado, a partir del diagrama magnitud-magnitud, podemos ver cómo están correlacionadas las magnitudes V y R. En la Fig. 3.19, se ve una fuerte correlación entre los datos. Tanto el ajuste lineal, el cual arroja una pendiente positiva (Tabla 3.4), como el valor de la función de correlación ( $\mathrm{DCF}=0.802 \pm 0.2)$, corroboran dicha correlación. Además, el coeficiente de Pearson también concluye en una correlación fuerte, siendo su valor $r=0.832$.
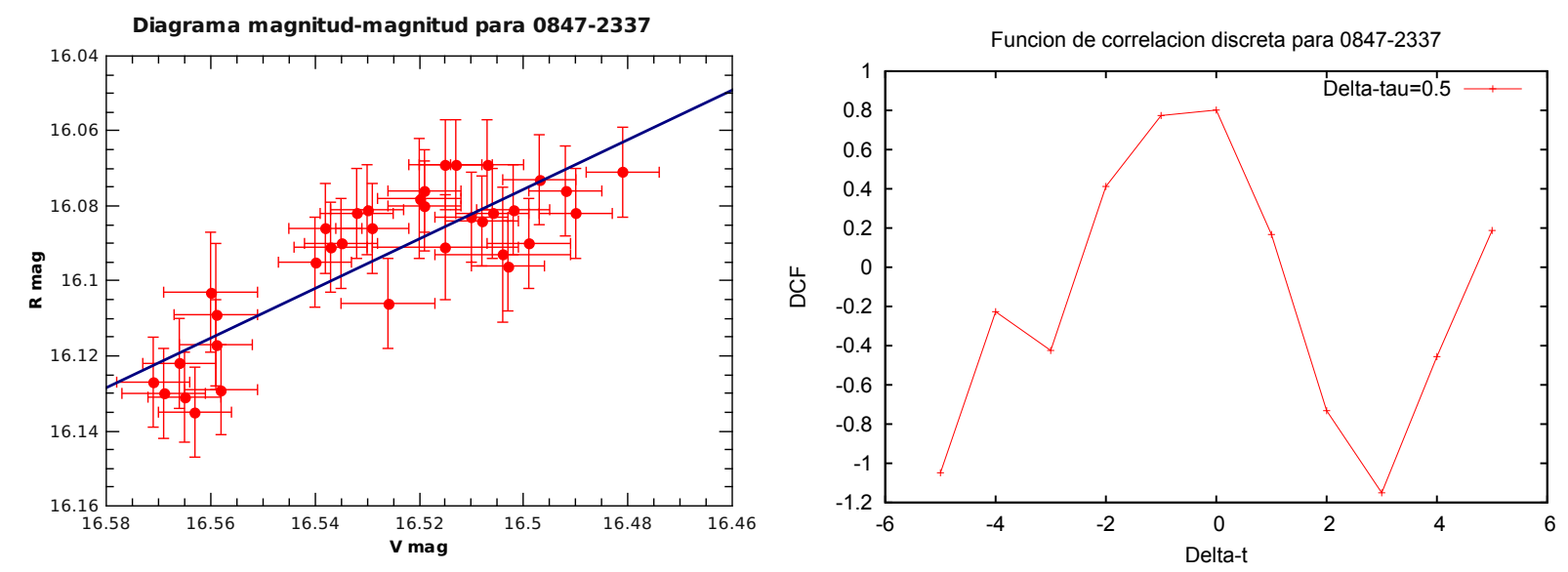

Figura 3.19. Diagrama magnitud-magnitud (izquierda) y función de correlación discreta (derecha) para 0847-2337.

Dado que se obtuvieron las magnitudes estándares en V y R, calculamos y analizamos el comportamiento del índice espectral $\alpha_{V R}$, presentado en la Fig. 3.20. En la primer noche su valor se encuentra en 2.53, para luego decaer hasta 2.36 en la tercer noche. Después el $\alpha_{V R}$ vuelve a crecer en su valor hasta llegar hasta 2.48 en la última noche. Su valor promedio fue de $\alpha_{V R}=2.43 \pm 0.019$.

Por último, a partir del índice de color (V-R), graficamos el diagrama color-magnitud, como se muestra en la Fig. 3.21. Tanto el ajuste lineal como el coeficiente de Pearson $(\mathrm{r}=0.644)$ concluyen que la correlación entre los datos es moderada y positiva. Con lo cual, la tendencia que se observa implicaría una tendencia BWB para PMN J0847-2337.

* [HB89] 1116-462: este blazar está clasificado como un FSRQ. Posee un redshift de $\mathrm{z}=0.713$ y el primer valor de la magnitud V fue de 17.0 (Tritton et al. 1971). Otros autores coinciden en el mismo valor detectado en la magnitud V (Adam 1985; Bozyan et al. 1990). Por otro lado, Hewitt \& Burbidge (1993) obtuvieron un valor de V=15.17. Ojha et al. (2009) estudiaron las bandas BVRI junto con los colores de más de 200 fuentes en distintas época, encontrando para [HB89] 1116-462 un valor de V=17.021 para el 2006 y $V=17.108, R=16.917$ y $(\mathrm{V}-\mathrm{R})=0.191$ para el 2007. Esta fuente aparece en catálogos en radio (Large et al., 1981, Griffith \& Wright 1993, Massardi et al., 2011), rayos X (Marshall et al., 2005) y rayos- $\gamma$ (Ackermann et al., 2015), pero es poco lo que se conoce sobre su estado de variabilidad.

Esta fuente fue observada durante dos noches, 13 y 14 abril 2015. En particular, el campo de este objeto es poblado y se tienen muchas estrellas con magnitudes que cumplen el criterio de Howell. Tanto el criterio C como el parámetro F han coincidido 


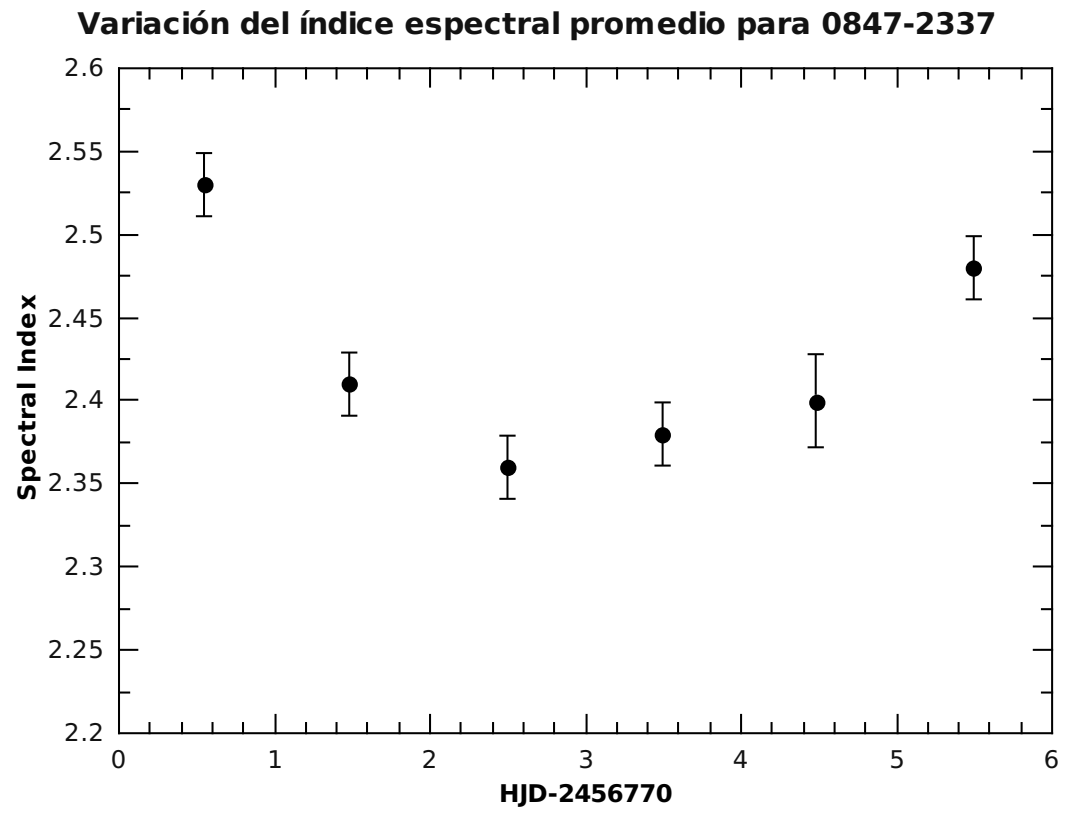

Figura 3.20. Variación del índice espectral para 0847-2337.

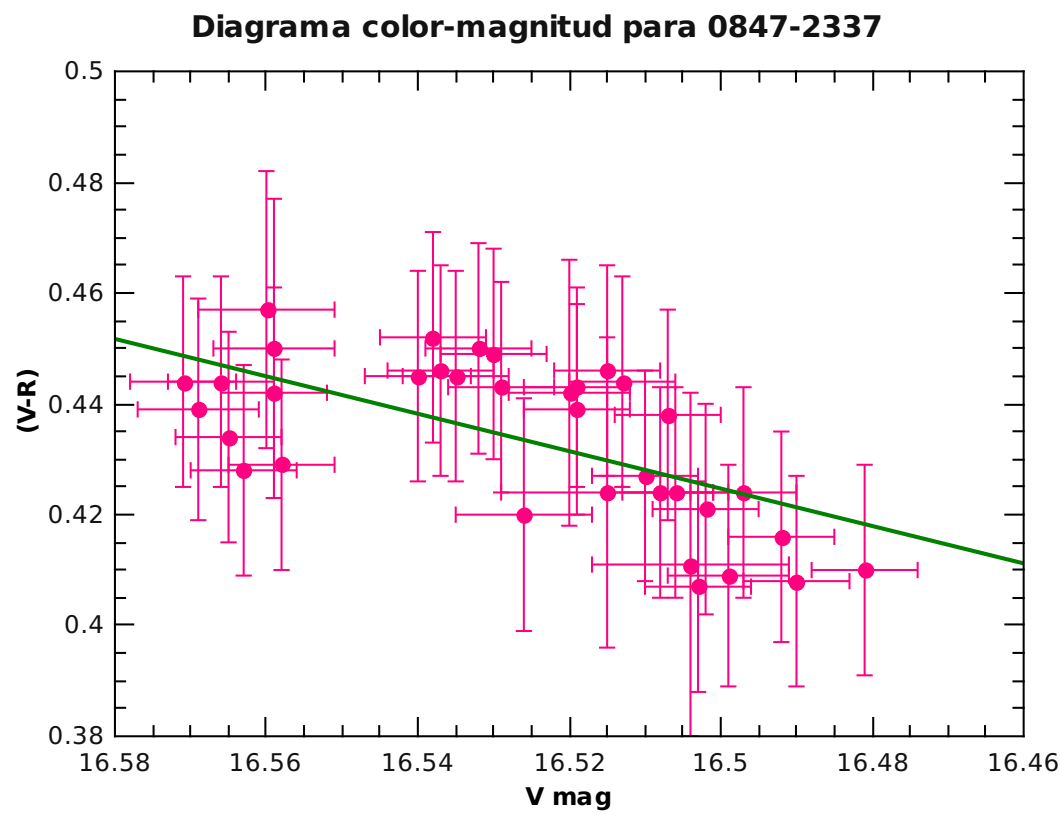

Figura 3.21. Diagrama color-magnitud para 0847-2337.

en los resultados de la variabilidad. En cuanto a la variabilidad intra-noche, se detectó sólo en el filtro $\mathrm{V}$ para la primer noche mientras que para la segunda se registraron variaciones en ambos filtros. Por otra parte, también se detectó variabilidad inter-noche.

Se calcularon las magnitudes estándares del blazar, siendo sus valores promedio $17.124 \pm 0.076$ para el filtro V, y $16.936 \pm 0.027$ para el filtro R. La curva de luz de estas magnitudes se muestra en la Fig. 3.22. La variación que se obtiene es $\Delta m=0.227$ en el filtro V y $\Delta m=0.100$ en el filtro R.

Respecto a la magnitud V, el valor que obtuvimos $(17.124 \pm 0.076)$ es comparable al 


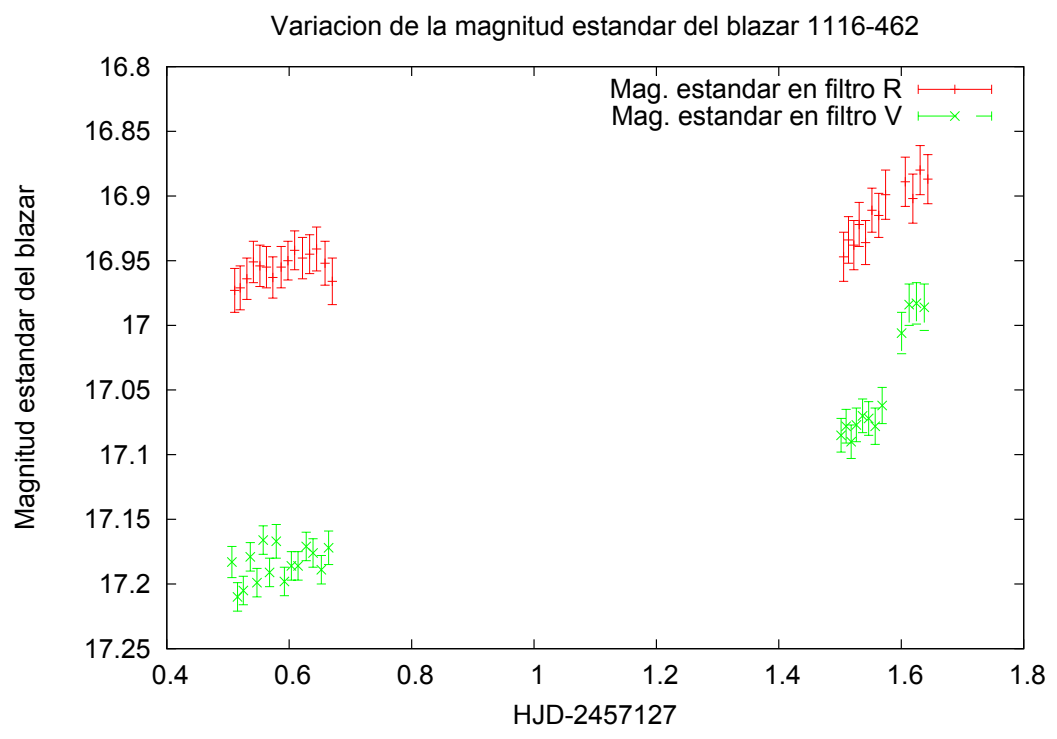

Figura 3.22. Curva de luz para la magnitud estándar de 1116-462 en el filtro R (rojo) y en el V (verde).

encontrado por Tritton et al. (1971), Adam (1985), Bozyan et al. (1990) y Ojha et al. (2009). En cuanto a la magnitud R, nuestro valor también es similar (16.936士0.027) al hallado por Ojha et al. (2009). En la Fig. 3.23 graficamos los distintos valores hallados en la literatura de la magnitud $\mathrm{V}$ junto con el obtenido en esta Tesis. Observamos que, a lo largo de $\sim 45$ años, la magnitud estándar del blazar disminuyó en 0.124 mag, encontrando en nuestro caso el valor más débil para esta magnitud.

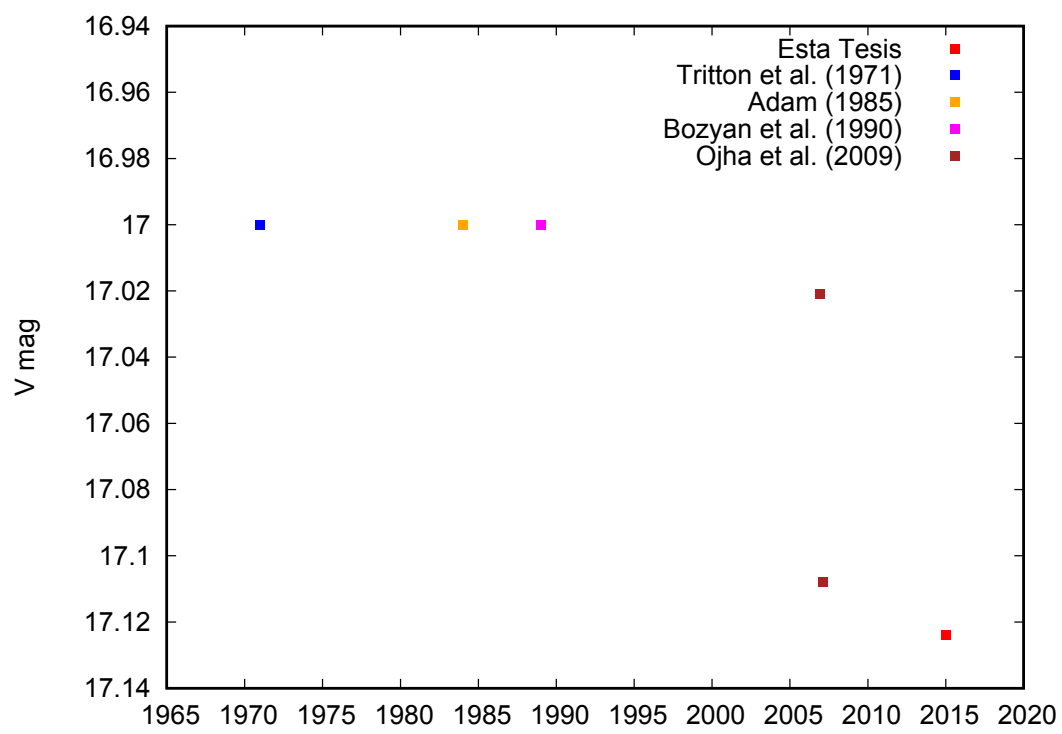

Figura 3.23. Comparación de la magnitud estándar en V para [HB89] 1116-462 a lo largo de los años.

A partir de las variaciones en las magnitudes estándares detectadas para nuestras observaciones, analizamos el diagrama magnitud-magnitud, mostrado en la Fig. 3.24. Se 
observa que se tiene una correlación entre los datos de ambos filtros $\mathrm{V}$ y $\mathrm{R}$, corroborada por el ajuste lineal realizado y la función DCF (ver valores en la Tabla 3.4). Además dicha correlación fuerte la confirma el valor del coeficiente de Pearson $r=0.911$.
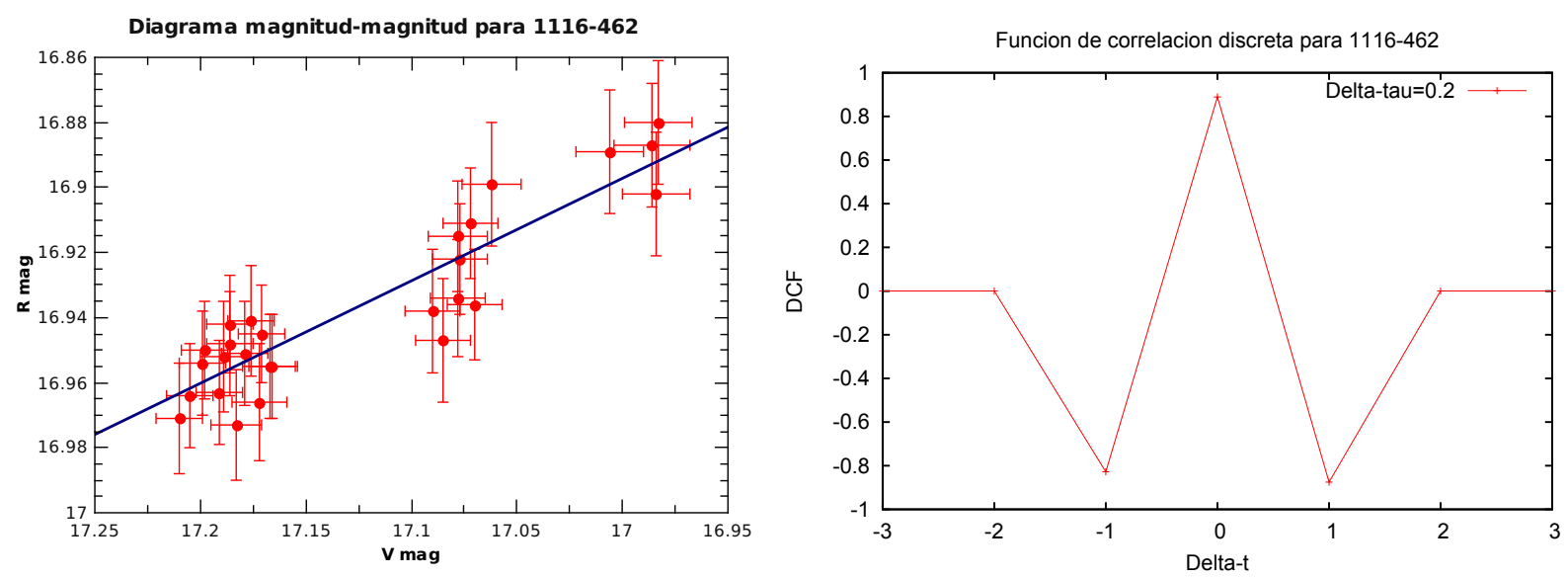

Figura 3.24. Diagrama magnitud-magnitud (izquierda) y función de correlación discreta (derecha) para [HB89] 1116-462.

Por otro lado, teniendo los valores de las magnitudes estándares, podemos analizar el índice espectral $\alpha_{V R}$ y ver cómo es su comportamiento. Calculando el índice de color (V-R), obtuvimos un valor promedio de $\alpha_{V R}=1.06 \pm 0.045$, el cual resultó de $1.30 \pm 0.014$ para la primer noche y de $0.760 \pm 0.027$ para la segunda. En la Fig. 3.25 se muestra cómo fue la variación de una noche a otra, donde el valor decrece en 0.54, indicando que la distribución espectral se vuelve más blanda. Para los datos publicados por Ojha et al. (2009), calculamos el respectivo $\alpha_{V R}$, resultando en un valor de $\alpha_{V R}=1.08$, comparable a lo que obtuvimos en promedio para el blazar.

Finalmente, para analizar el posible origen de las variaciones detectadas, estudiamos el diagrama color-magnitud, en la Fig.3.26. Para el conjunto de observaciones en el filtro V y R, se obtuvo una correlación significativamente fuerte y positiva $(r=0.978)$, como se observa en el gráfico. Este comportamiento indicaría una tendencia BWB en el blazar, esto es, más azul a medida que el brillo aumenta.

* [HB89] 1127-145: es un FSRQ, con un valor del redshift $\mathrm{z}=1.187$ (Burbidge \& Kinman 1966). Comenzó siendo detectado como una fuente en radio variable (Wehrle et al., 1992; Bondi et al. 1996). En la banda de rayos X, Siemiginowska et al. (2002) descubrieron la estructura de un jet con una longitud de $300 \mathrm{kpc}$, a partir de observaciones de Chandra. En cuanto a su núcleo, el satélite BeppoSAX detectó fuerte emisión en esta banda (Giommi et al. 2002) con un espectro que llega hasta los $300 \mathrm{keV}$. Respecto a la banda óptica, Bolton \& Kinman (1966), registraron un valor en la magnitud V de 16.9, al igual que Adam (1985). Por otro lado, Romero et al. (1999) reportaron variabilidad con una amplitud del $2.2 \%$ en $3.5 \mathrm{hr}$ en el filtro $\mathrm{V}$.

Observamos esta fuente dos noches, 10 y 12 abril 2015. No se detectaron variaciones intra-noche con ninguno de los filtros y con ninguno de los parámetros C y F.De la misma manera tampoco se han registrado variaciones inter-noche. 
Variación del índice espectral para 1116-462

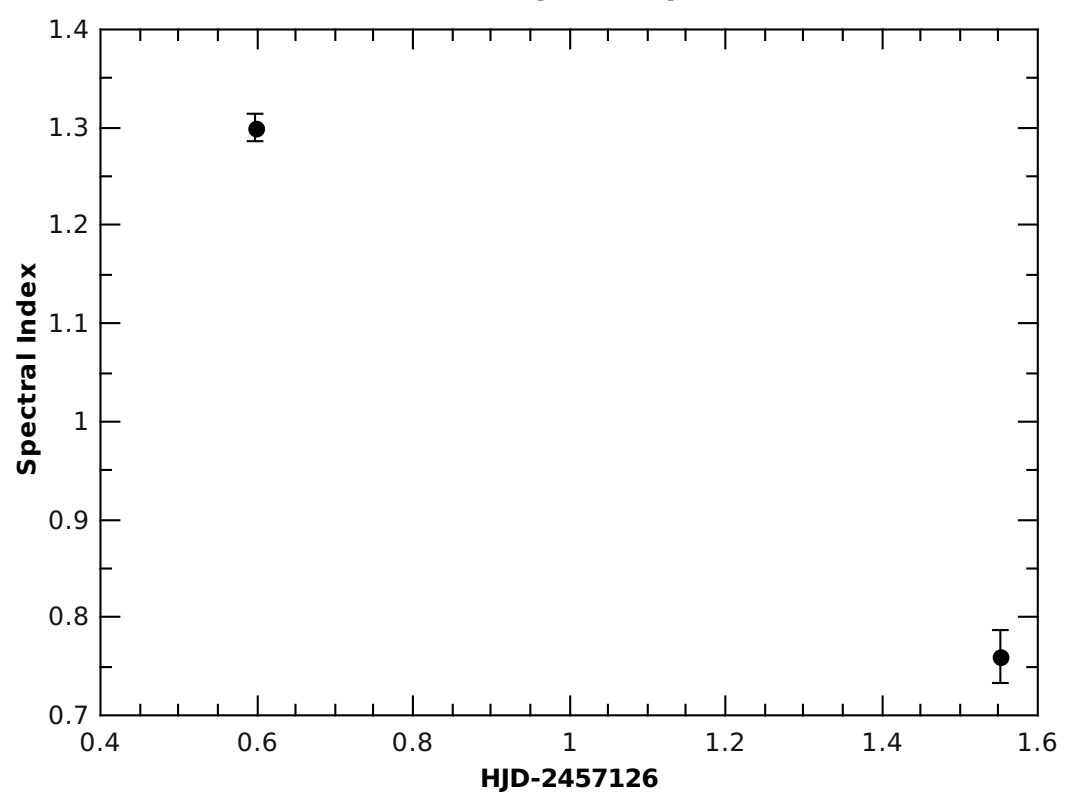

Figura 3.25. Variación del índice espectral para 1116-462.

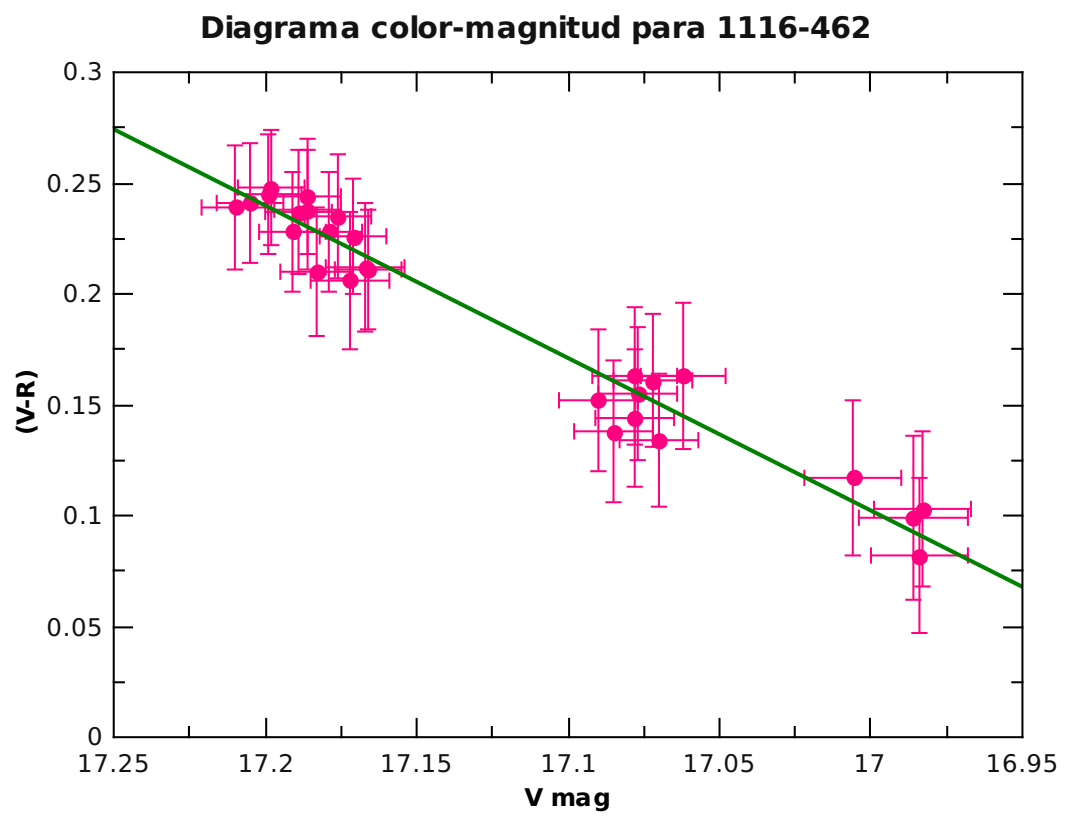

Figura 3.26. Diagrama color-magnitud para 1116-462.

En la Fig. 3.27, mostramos las curvas de luz obtenidas a partir de las magnitudes estándares $\mathrm{V}$ (puntos verdes) y $\mathrm{R}$ (puntos rojos). Las magnitudes estándares promedio resultaron $18.242 \pm 0.008$ para el filtro $\mathrm{V}$ y $17.419 \pm 0.008$ para el filtro $\mathrm{R}$.

Comparando con los valores publicados en la literatura, Bolton \& Kinman (1966) y Adam (1985) registraron un valor de $\mathrm{V}=16.9 \mathrm{mag}$, mientras que en esta Tesis obtuvimos un valor de $\mathrm{V}=17.4 \mathrm{mag}$, encontrando al blazar más débil que lo reportado en la literatura. 


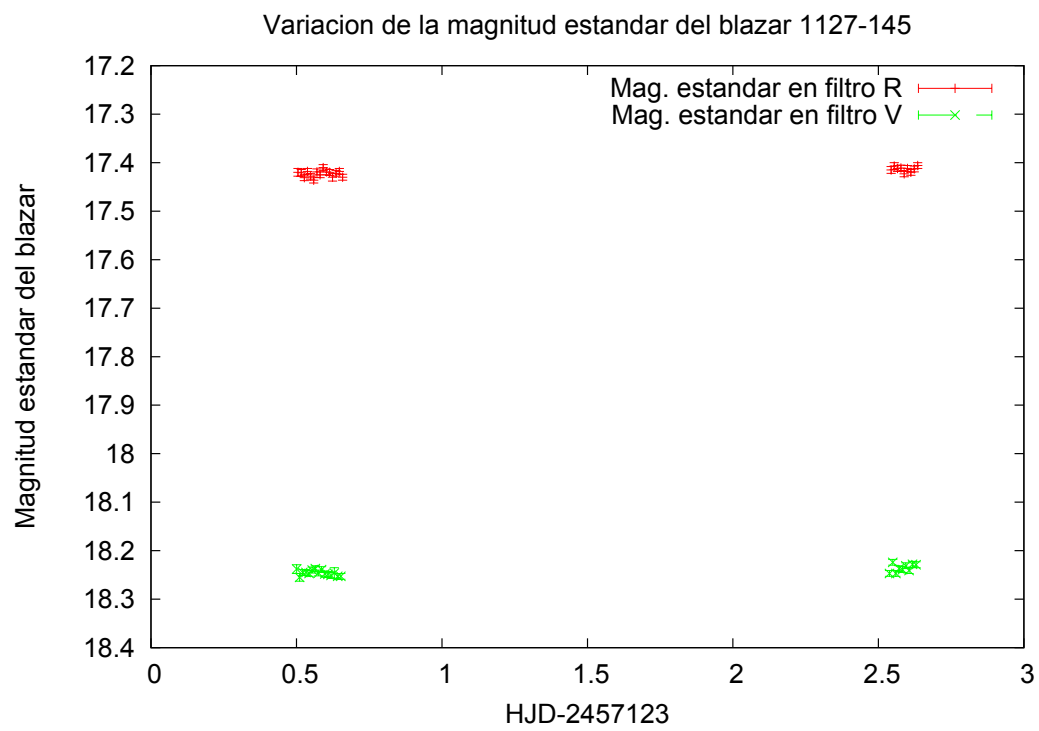

Figura 3.27. Curva de luz para la magnitud estándar de 1127-145 en el filtro R (rojo) y en el V (verde).

Analizamos también el diagrama magnitud-magnitud junto con la función de correlación discreta y el ajuste lineal (ver Fig. 3.28). Se puede observar que entre los datos en ambas bandas $\mathrm{V}$ y R, se aprecia una correlación, la cual es corroborada con el ajuste lineal (Tabla 3.4) y con el valor de la función, $\mathrm{DCF}=1.098 \pm 0.28$. Sin embargo, el coeficiente de Pearson arroja que dicha correlación es débil.
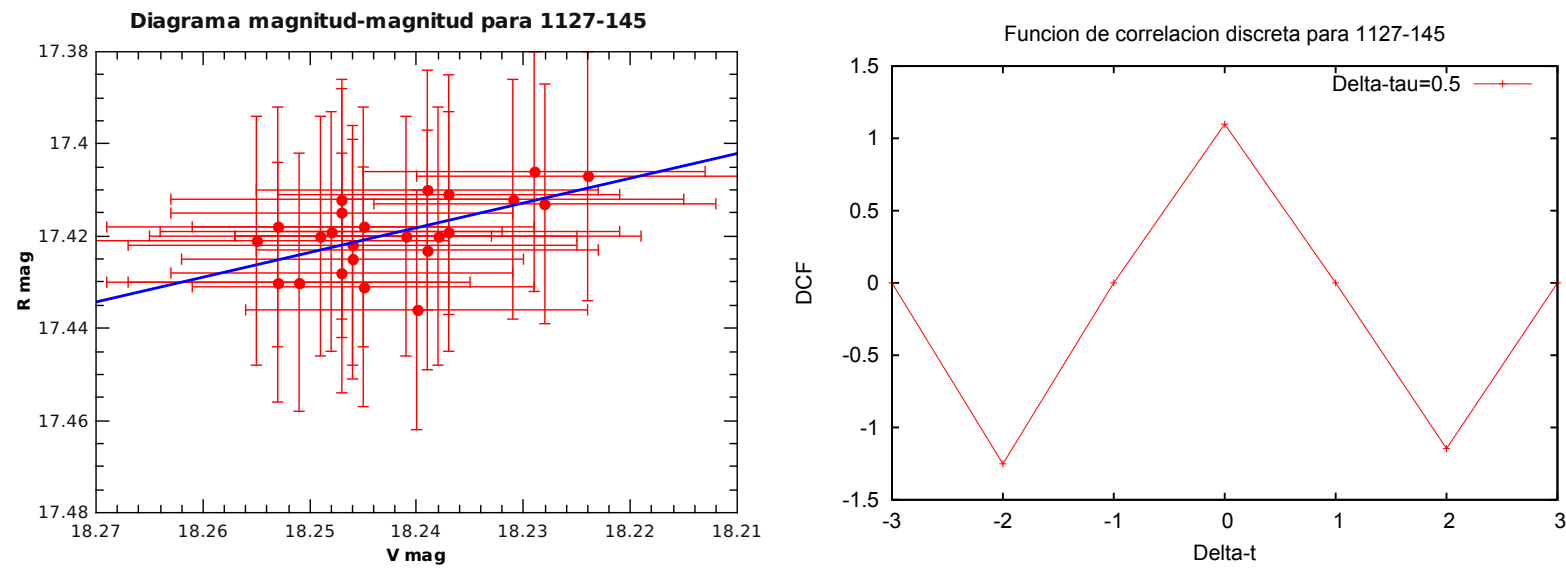

Figura 3.28. Diagrama magnitud-magnitud (izquierda) y función de correlación discreta (derecha) para 1127-145.

Por otro lado, teniendo las magnitudes estándares, calculamos el índice espectral, donde se obtuvo un valor de $4.67 \pm 0.008$ para ambas noches de observación, sin registrarse cambios en su comportamiento. Su valor promedio resultó $\alpha_{V R}=4.67 \pm 0.007$.

Finalmente, en cuanto al diagrama color-magnitud, presentado en la Fig. 3.30, tanto el ajuste lineal como el coeficiente de Pearson $(\mathrm{r}=0.511)$ coinciden en que la correlación positiva es entre moderada y débil. Con lo cual, no es del todo confiable asociarle a esta 


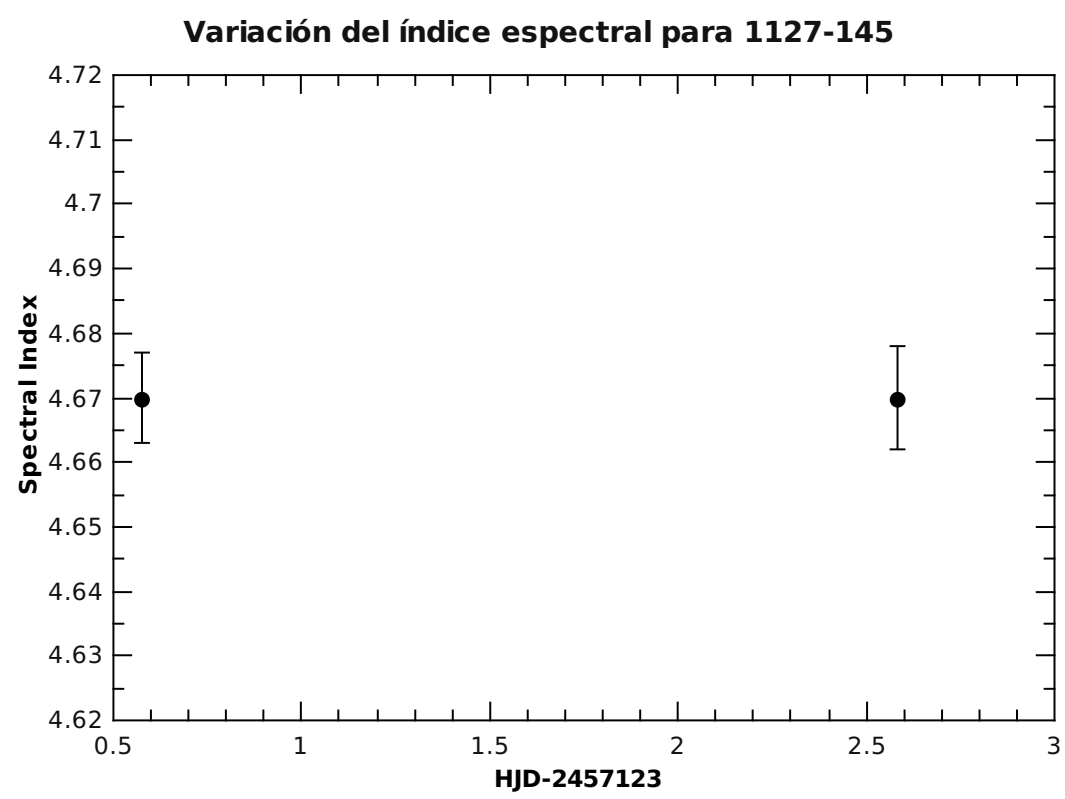

Figura 3.29. Variación del índice espectral para 1127-145.

fuente una tendencia BWB. Este resultado está condicionado por las grandes barras de error presentes.

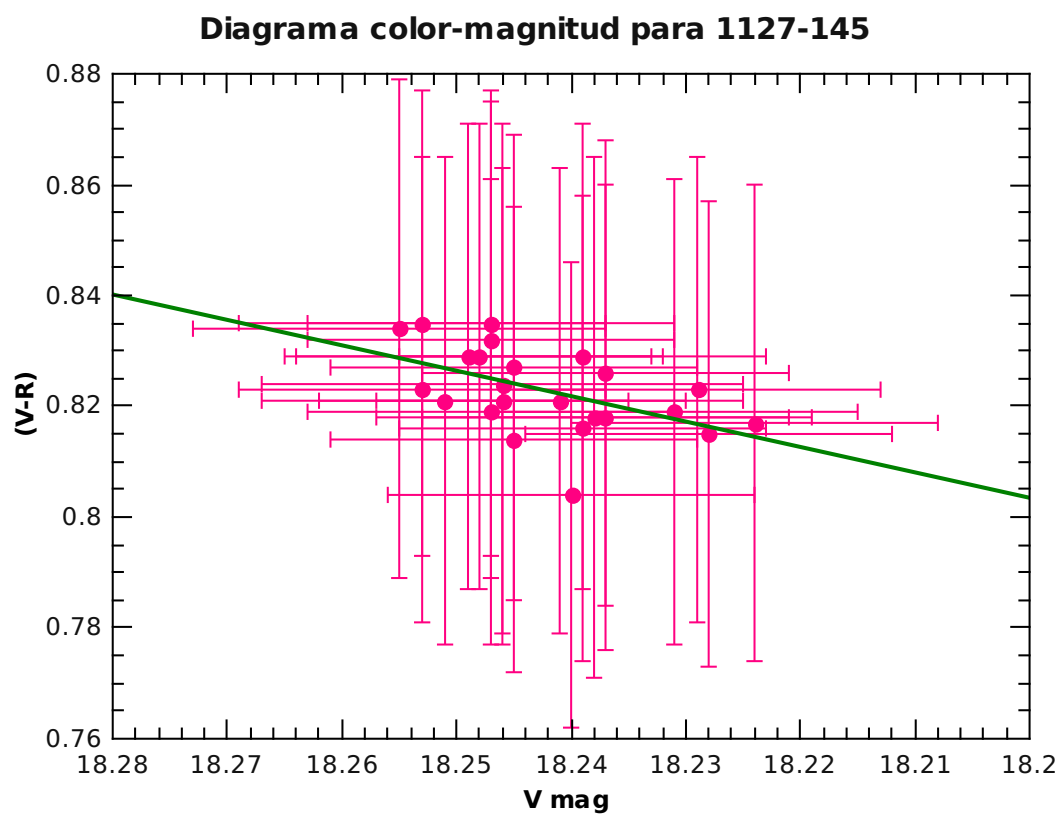

Figura 3.30. Diagrama color-magnitud para 1127-145.

* PKS 1229-02: este blazar fue catalogado como un FSRQ (LSP). Bolton \& Kinman (1966) obtuvieron un valor en la magnitud de V de 16.75 mag. Por otra parte, Kinman \& Burbidge (1967) calcularon su redshift, obteniendo éste $\mathrm{z}=1.038$. Pica et al. (1988) reportaron 34 observaciones correspondientes al sistema fotográfico, obteniendo una magnitud promedio de 16.76 mag entre mayo de 1971 y abril de 1987, registrando 
un mínimo de 16.28 y una variación en la magnitud de 0.94. Wilkes et al. (1994) encontraron un valor para la magnitud $V=16.75$, a partir del catálogo de Véron-Cetty \& Véron (1987). Villata et al. (1997) obtuvieron los valores de la magnitud en el filtro $\mathrm{R}$, con un rango desde $\mathrm{R}=16.39$ - 16.46. Por su parte, Romero et al. (2002) reportaron para el blazar una magnitud $\mathrm{V}=16.86$ mag para las dos noches en que observaron a este blazar, sin detectar variabilidad intra-noche. Con respecto a la detección de variaciones, existen trabajos donde han registrado variaciones de más de $1.2 \mathrm{mag}$, con una magnitud mínima de 16.9 (Adam 1978; Pica et al. 1980). En altas energías, este blazar es un fuerte emisor de rayos- $\gamma$ (Thompson et al. 1995; Cellotti \& Ghisellini 2008). Con observaciones del satélite Chandra, Tavecchio et al. (2007) y Russell et al. (2012) demostraron que este objeto posee una emisión extendida en rayos X. McLaughlin et al. (1996), en un estudio sobre la variabilidad de esta fuente en rayos- $\gamma$ con datos del satélite EGRET, no detectan variabilidad.

Este blazar fue observado durante una noche, 12 abril 2015, sin presentar variaciones en sus curvas de luz. Ambos parámetros C y F coinciden en la no detección de variabilidad en ninguno de los filtros $\mathrm{V}$ y $\mathrm{R}$.

En la Fig. 3.31, mostramos las curvas de luz de las magnitudes estándares en V y R, donde se obtuvieron los valores promedio de $16.79 \pm 0.006$ para el filtro $\mathrm{V}$ y $16.48 \pm 0.005$ para el filtro R. El comportamiento de la magnitud del blazar no refleja variación alguna en ningún filtro.

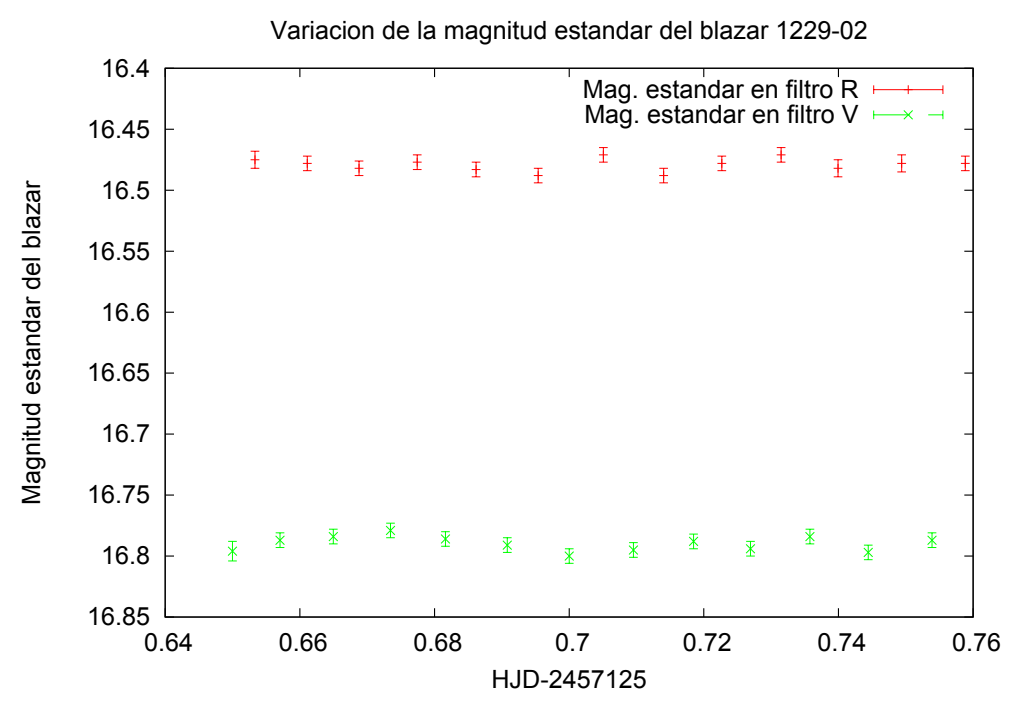

Figura 3.31. Curva de luz para la magnitud estándar de 1229-02 en el filtro R (rojo) y en el V (verde).

A partir de lo que mencionamos anteriormente, podemos comparar los valores obtenidos con lo publicado. En el filtro V, Bolton \& Kinman (1966) obtienen V=16.95, Pica et al. (1988) reportaron un valor promedio de $V=16.76$, Wilkes et al. (1994) reportaron un $\mathrm{V}=16.75$ y Romero et al. (2002) registraron un valor de $\mathrm{V}=16.86$. En nuestro caso, las observaciones arrojaron un valor de $\mathrm{V}=16.79$, acorde a lo publicado por otros autores. En lo que respecta al filtro R, Villata et al. (1997) obtuvieron valores entre $\mathrm{R}=16.39$ - 16.46 , mientras que nuestras observaciones dieron un valor de $\mathrm{R}=16.48$, también acorde a lo publicado. A lo largo de todos estos años, el brillo del 
objeto varió en $0.3 \mathrm{mag}$, y nuestras observaciones encuentran a la fuente en un estado bajo de actividad. Lo mismo sucede con el filtro R.

Con los valores de las magnitudes estándares, podemos construir el diagrama magnitudmagnitud. En este caso, obtuvimos un caso de anticorrelación entre los datos en V y $\mathrm{R}$, como lo indica el valor de la DCF y la pendiente negativa del ajuste lineal (Fig. 3.32). Sin embargo, el valor del $\chi^{2}$ reducido es extremadamente bajo (ver Tabla 3.4) y las incertezas son mayores a los valores propios de los parámetros. Además, el valor bajo del coeficiente de Pearson, $r=0.277$ indica que la correlación es débil, con lo cual no puede afirmarse que se trate de una anticorrelación.
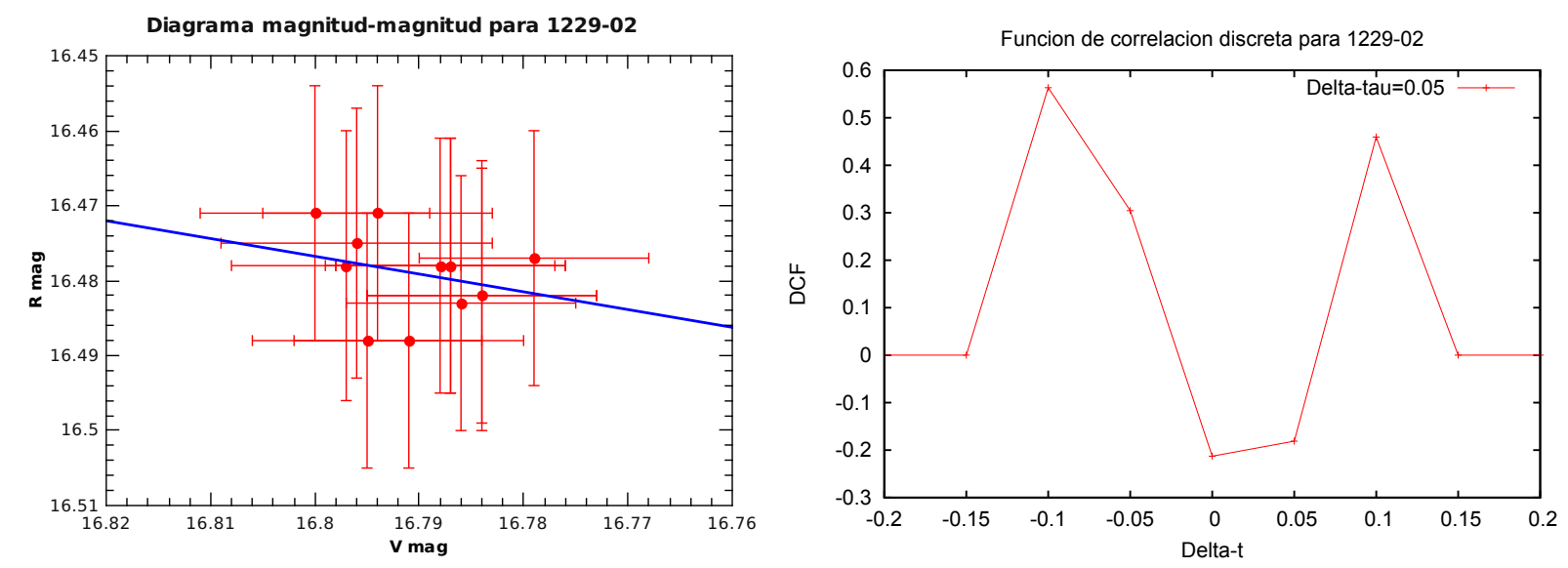

Figura 3.32. Diagrama magnitud-magnitud (izquierda) y función de correlación discreta (derecha) para 1229-02.

Finalmente, a partir del índice de color (V-R), se obtuvo el valor del índice espectral $\alpha_{V R}=1.76 \pm 0.009$. No se realizó un análisis del comportamiento de $\alpha_{V R}$ a lo largo de los años debido a la falta de valores publicados en $\mathrm{V}$ y $\mathrm{R}$ para un mismo conjunto de observaciones. En algunos trabajos, sólo estudiaron la magnitud V, mientras que en otros analizaron sólo a $\mathrm{R}$, sin tener ambas magnitudes estudiadas en un mismo trabajo. $\mathrm{Y}$ en cuanto al diagrama color-magnitud, se presenta una tendencia positiva fuerte (corroborada por el ajuste lineal y el coeficiente de Pearson, $r=0.829$ ) en los datos, lo que estaría indicando un comportamiento BWB (Fig. 3.33).

* PMN J1256-1146: esta fuente es clasificada como un BL Lac (HSP). Es muy poco lo que se conoce de esta fuente respecto del óptico y de su estado de variabilidad. Sólo se ha publicado en diversos catálogos como el CRATES (en la banda de radio, Healey et al. 2007); los catálogos de Fermi (en la banda de rayos- $\gamma$, Abdo et al. 2010, Nolan et al. 2012, Ackermann et al. 2015); y el RASS-6dFGS (en la banda de rayos X, Mahony et al. 2010), entre otros.

Sólo se lo observó durante una noche, 23 abril 2014, con muy pocas observaciones. Lo interesante de este caso es que el campo es poco poblado y el blazar es el objeto más brillante del campo, siendo la estrella siguiente en 3 mag más débil. No se ha registrado variaciones intra-noche (resultado coincidente entre los parámetros $\mathrm{C}$ y F) en ninguno de los dos filtros. 


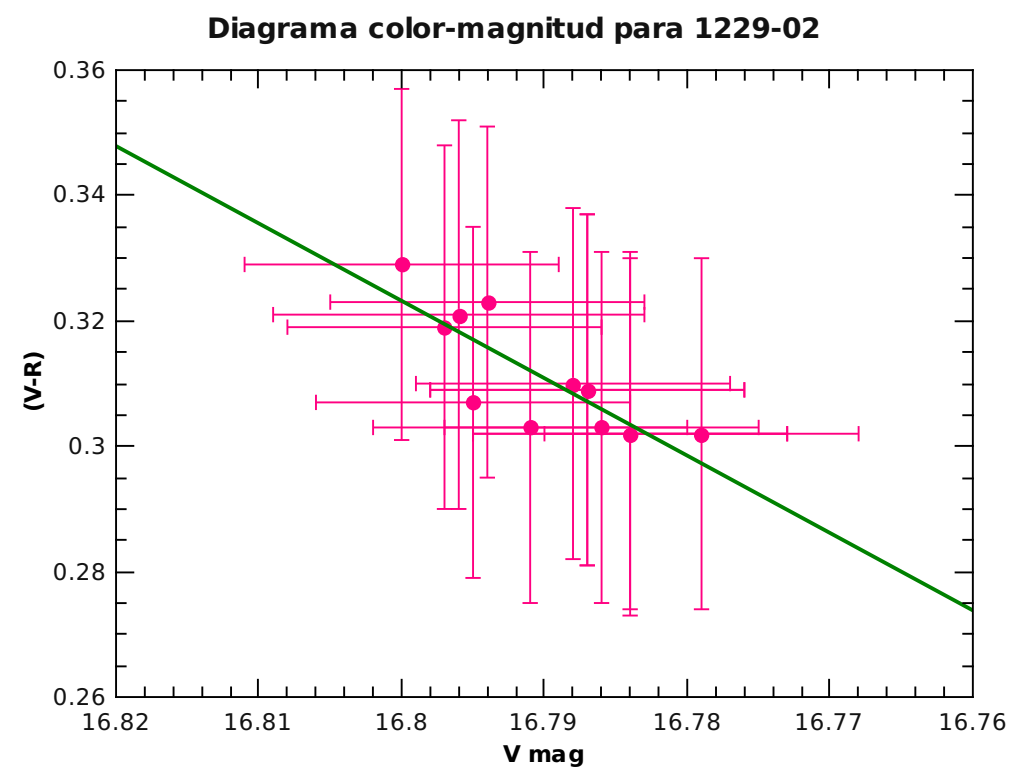

Figura 3.33. Diagrama color-magnitud para 1229-02.

Por otra parte, obtuvimos las magnitudes estándares, resultando en $12.055 \pm 0.005$ para el filtro V y $11.750 \pm 0.012$ para el filtro R, respectivamente. En la Fig. 3.34 presentamos las curvas de las magnitudes estándares para este objeto.

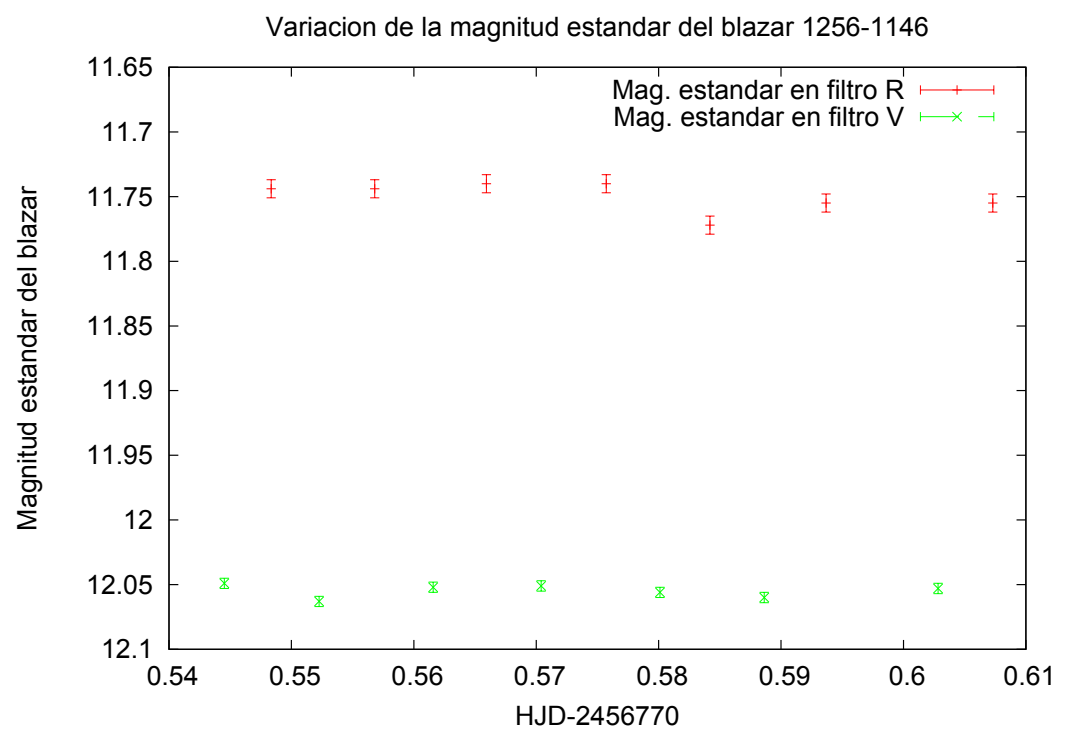

Figura 3.34. Curvas de luz de la magnitud estándar de 1256-1146 en el filtro R (rojo) y en el V (verde).

Estudiamos cómo es el diagrama magnitud-magnitud, obteniendo que en los filtros $\mathrm{V}$ y $\mathrm{R}$ los datos están correlacionados, dado que el ajuste lineal resultó con una pendiente positiva. Por otro lado, la DCF da un valor positivo ( $\mathrm{DCF}=0.097 \pm 0.34$ ), corroborando la correlación hallada. Sin embargo, los datos presentan barras de error grandes y el ajuste presenta una incerteza grande (Fig. 3.35). Esto lo corrobora el coeficiente de Pearson el cual concluye que la correlación que se observa es débil $(r=0.269)$. 

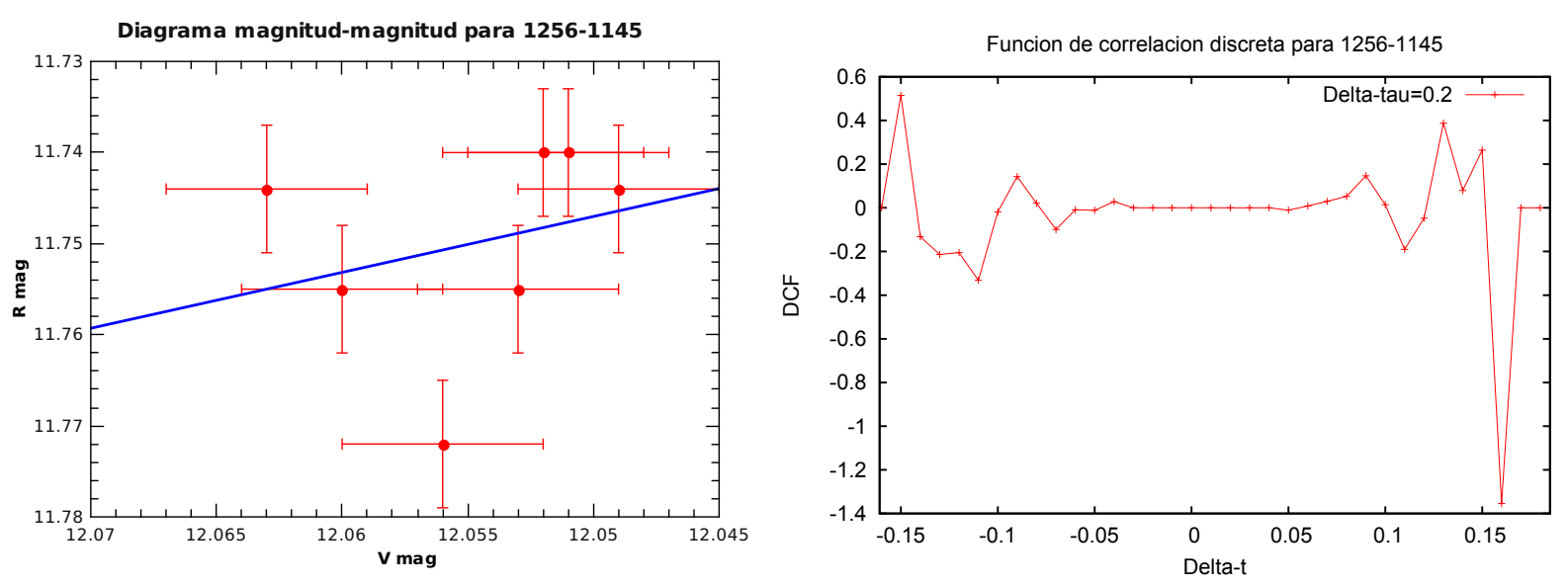

Figura 3.35. Diagrama magnitud-magnitud (izquierda) y función de correlación discreta (derecha) para 1256-1145.

A partir de los valores de las magnitudes $\mathrm{V}$ y $\mathrm{R}$ estándares, calculamos el índice espectral, el cual toma un valor promedio de $\alpha_{V R}=1.73 \pm 0.011$. Finalmente analizamos el diagrama color-magnitud, Fig. 3.36. Tanto el valor del $\chi^{2}$ del ajuste lineal (0.03) como el coeficiente de Pearson $(\mathrm{r}=0.173)$ indican una correlación positiva muy débil, lo que implicaría que no puede confirmarse el comportamiento BWB.

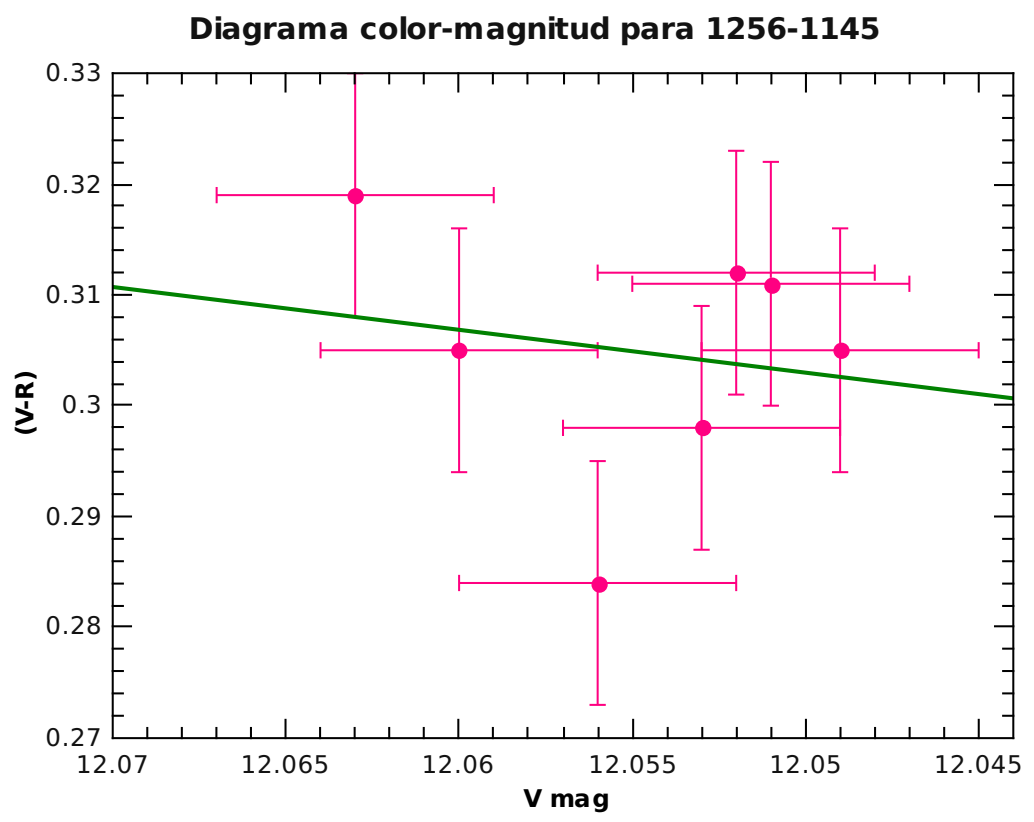

Figura 3.36. Diagrama color-magnitud para 1256-1145.

* [HB89] 1424-418: es un FSRQ, con un $z=1.522$ (White et al. 1988). Gilmore (1979) analizó la fuente encontrando variaciones en el flujo con amplitudes medias $(<0.5 \mathrm{mag})$ y rápidas $(<30 \mathrm{~d})$ en el visual (usando el método de $\chi^{2}$ para la detección de variabilidad). Por otra parte, Hewitt \& Burbidge (1993) reportaron un valor de 
$\mathrm{V}=17.7$, mientras que Romero et al. (2002) estudiaron la variabilidad en dos noche de 1999, sin encontrar variaciones intra-noche. Reportaron un valor de $\mathrm{V}=18.81 \pm 0.03$ para la primer noche y $V=18.85 \pm 0.02$ para la segunda. Mahony et al. (2011) obtuvieron un valor para la magnitud $\mathrm{R}$ de 16.03. Con respecto a las altas energías, esta fuente fue detectada por el satélite EGRET (Hartman et al. 1999) y forma parte de los catálogo de Fermi en rayos- $\gamma$ : 1FGL (Abdo et al. 2010) y 2FGL (Nolan et al. 2012).

Esta fuente fue observada durante 2 noches, 13 y 14 abril 2015. No se ha registrado variabilidad intra-noche en ninguna de las dos noches en ninguno de los dos filtros, ni tampoco variaciones inter-noche. Ambos parámetros $\mathrm{C}$ y $\mathrm{F}$ han coincidido en los resultados de variabilidad.

Los valores de las magnitudes estándares para el blazar son $17.634 \pm 0.037$ para el filtro V y $17.040 \pm 0.018$ para el filtro R. Las curvas de luz de las magnitudes estándares se muestran en la Fig. 3.37.

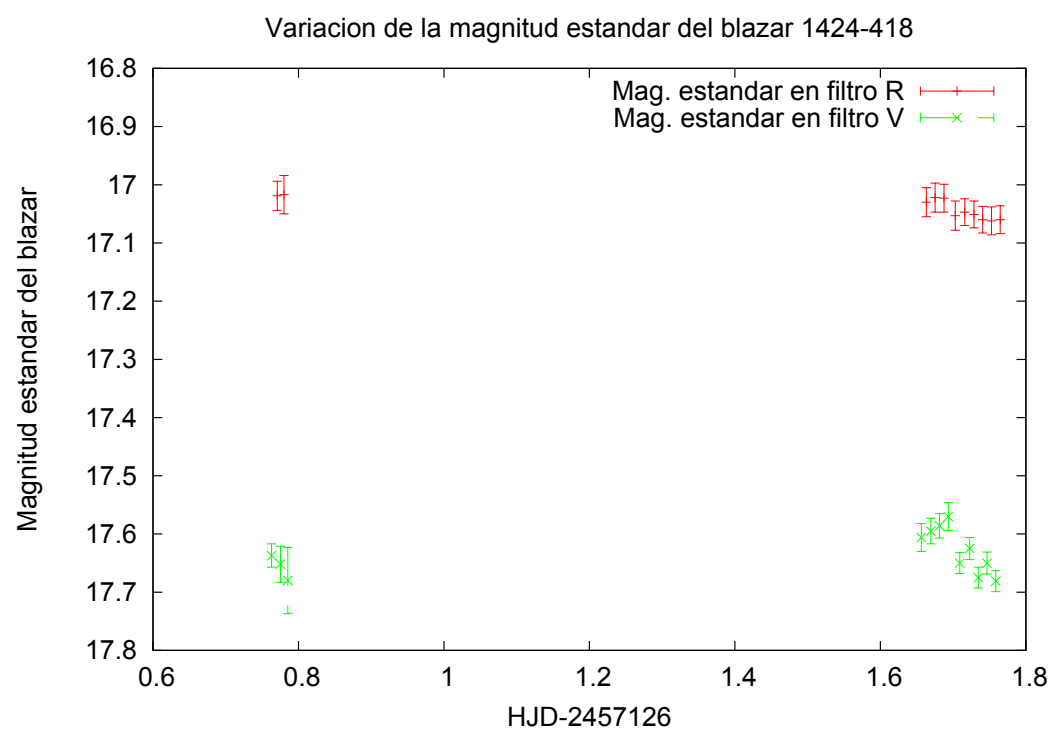

Figura 3.37. Curvas de luz de la magnitud estándar de 1424-418 en el filtro R (rojo) y en el V (verde).

Estos valores obtenidos los comparamos con lo publicado. En el caso de la magnitud V, Hewitt \& Burbidge (1993) obtuvieron $V=17.7$, similar a lo que obtuvimos en esta Tesis, $V=17.63$, mientras que Romero et al. (2002) reportaron un valor mayor, siendo su promedio $\mathrm{V}=18.83$. Por otra parte, Mahony et al. (2011) obtuvieron un $\mathrm{R}=16.03$, una magnitud más brillante a la obtenida aquí, $\mathrm{R}=17.04$. Esto es, 4 años después, encontramos a la fuente en un estado de baja actividad, siendo una magnitud más débil. En la Fig. 3.38, mostramos la curva de luz para la magnitud V. Entre 1993 y 2015, la fuente presentó un decaimiento en su brillo de casi 1 mag, aunque no se registran valores intermedios para poder estudiar con más precisión cuál fue el comportamiento de la variación en $\mathrm{V}$.

Analizamos el diagrama magnitud-magnitud, que se muestra en la Fig. 3.39. Los resultados de la función DCF indican que existe una anticorrelación en los datos, comportamiento que no se ve en el gráfico ni en el ajuste lineal realizado (ver valores en Tabla 3.4). Sin embargo, se puede observar del diagrama que las barras de error en 


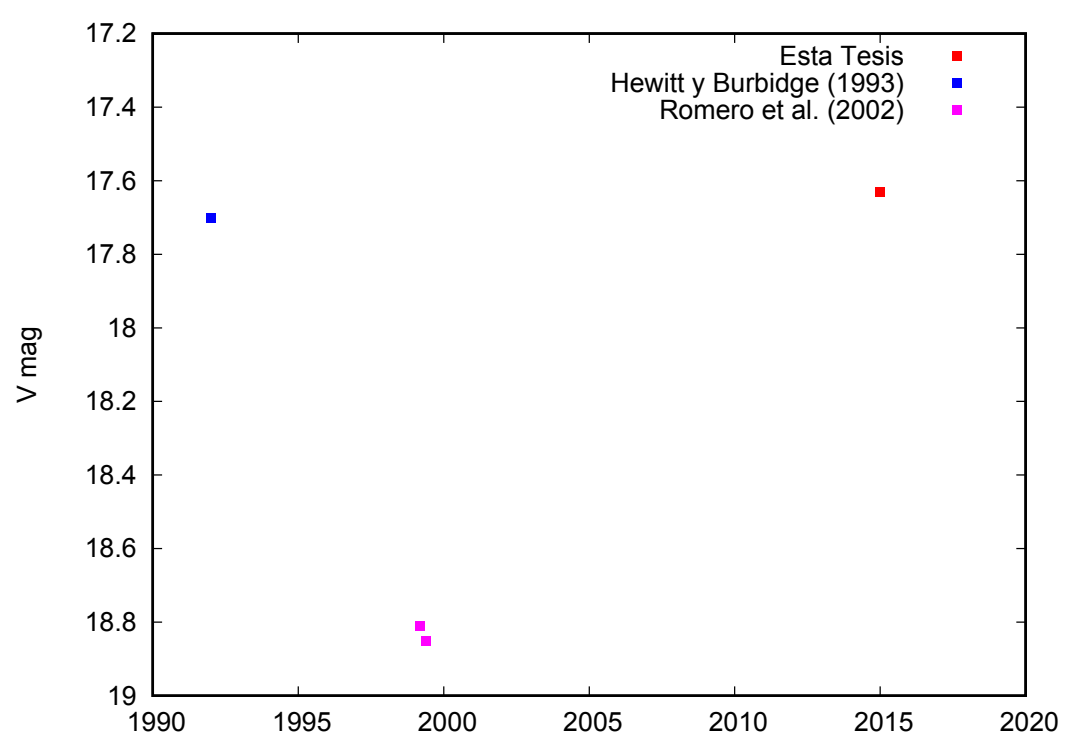

Figura 3.38. Curva de luz para la magnitud estándar en V para [HB89] 1424-418 a lo largo de los años.

los datos son grandes, haciendo que el ajuste presente una incerteza grande (el valor de $\chi^{2}$ reducido es de 0.022$)$. Además el valor del coeficiente de Pearson $(r=0.06)$ es extremadamente bajo, con lo cual no se puede concluir nada sobre la (anti)correlación entre los datos.
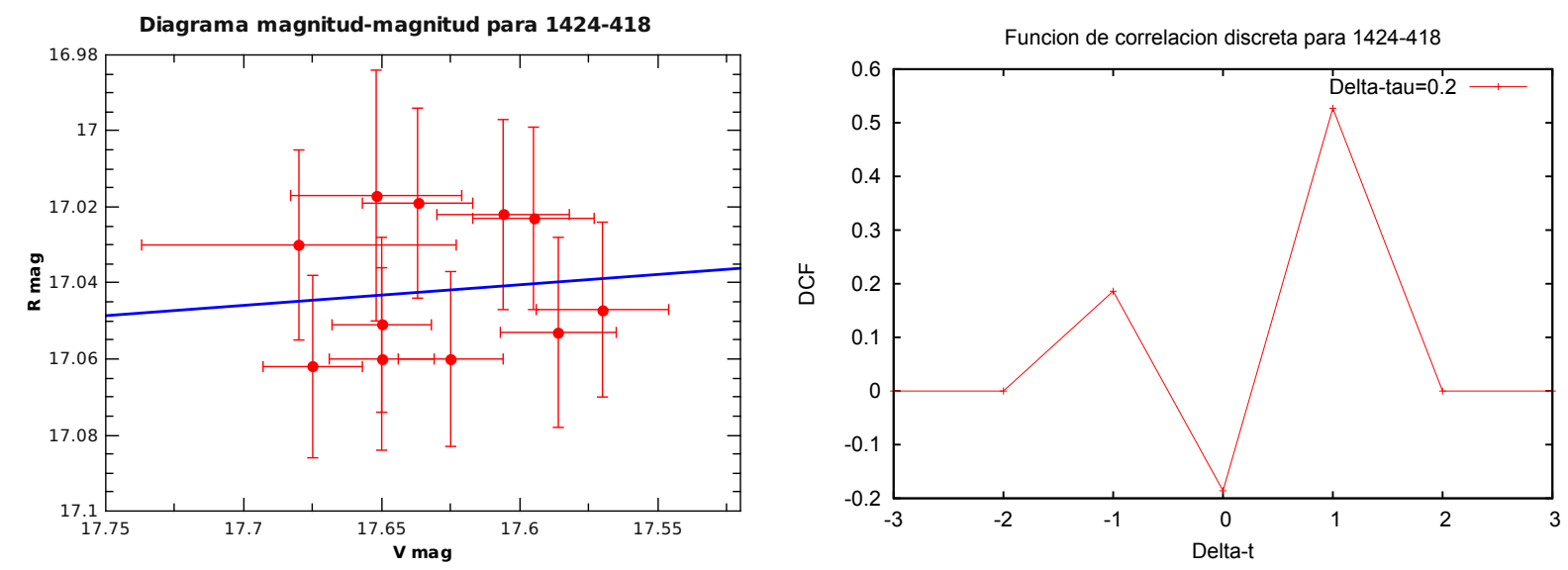

Figura 3.39. Diagrama magnitud-magnitud (izquierda) y función de correlación discreta (derecha) para 1424-418.

Respecto al índice espectral, para la primer noche se obtuvo un valor de $\alpha_{V R}=3.60$ \pm 0.016 , mientras que para la segunda noche resultó de 3.25 \pm 0.031 . En la Fig. 3.40 se muestran ambos valores, donde se tiene una variación de 0.35 entre una noche y otra, indicando que la distribución espectral se vuelve más blando. Su valor promedio fue de $\alpha_{V R}=3.34 \pm 0.040$.

Por último, analizando el diagrama color-magnitud (Fig.3.41), tanto el ajuste lineal como el coeficiente de Pearson $(\mathrm{r}=0.889)$ indican una fuerte correlación positiva. Esto 


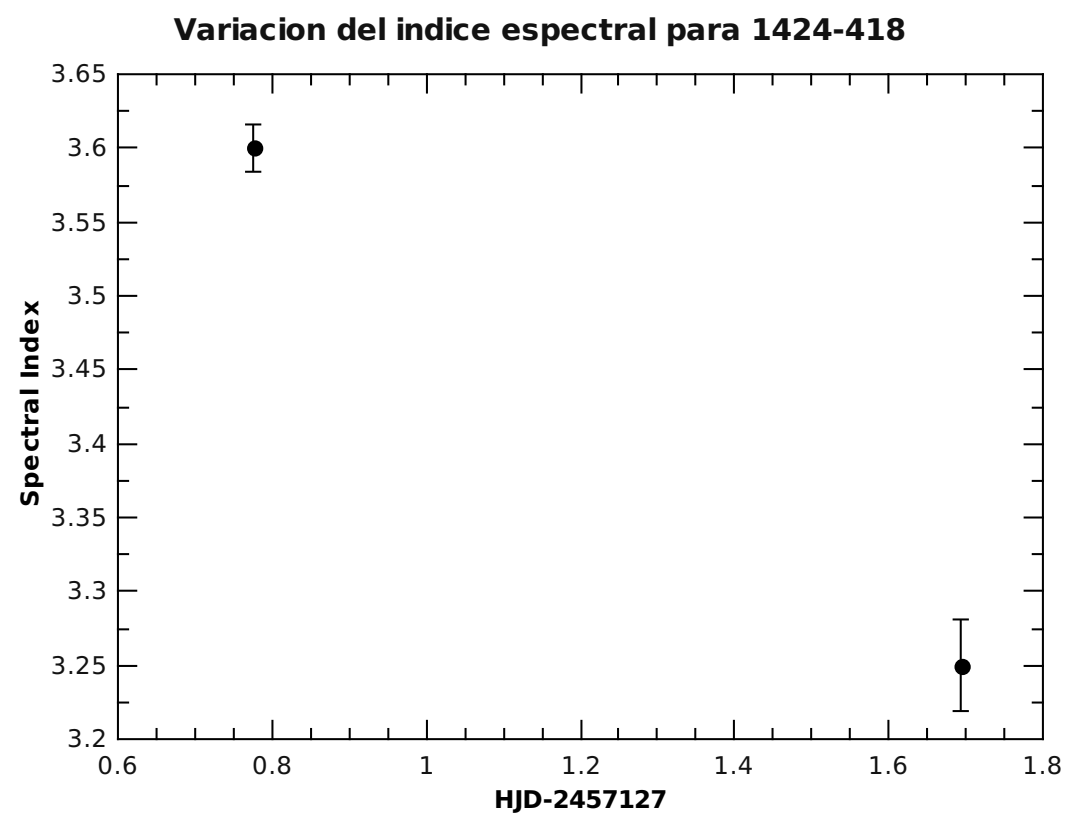

Figura 3.40. Variación del índice espectral para 1424-418.

indicaría que se tiene una tendencia BWB.

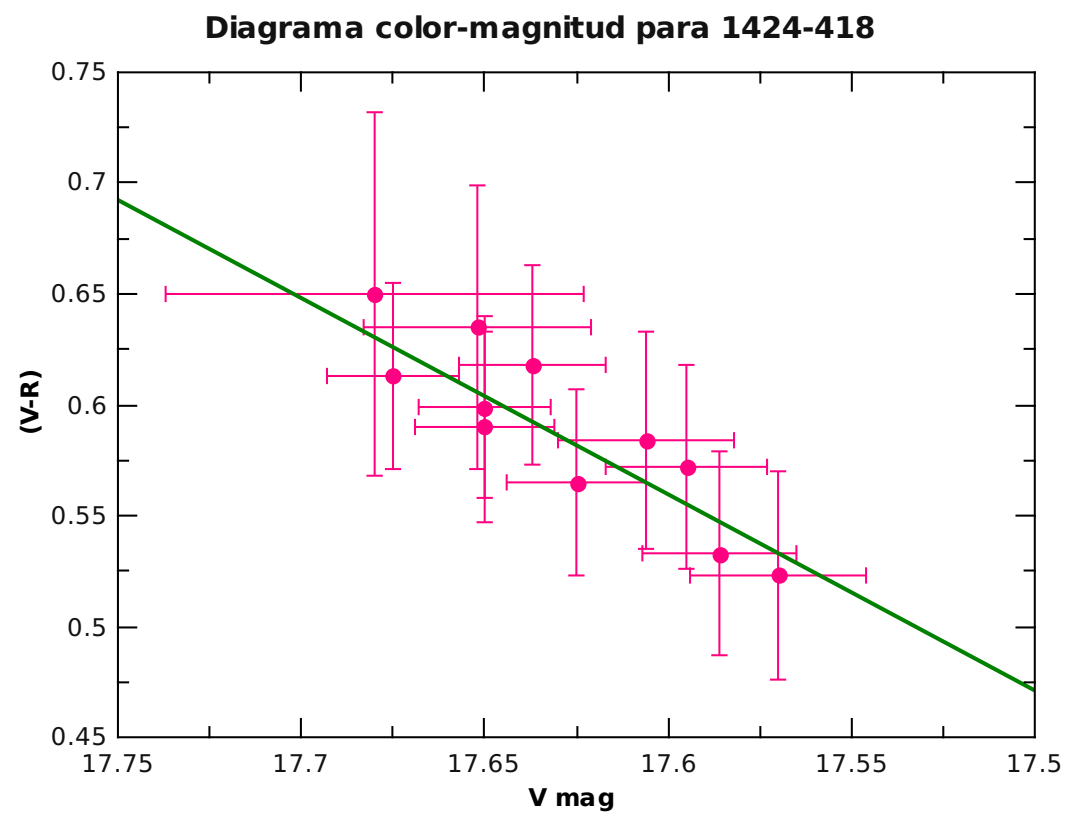

Figura 3.41. Diagrama color-magnitud para 1424-418.

* PKS J1443-3908: esta fuente está clasificada como un BL Lac (HSP), con un valor de $\mathrm{z}=0.065$. Como otras fuentes antes mencionadas, este blazar ha sido muy poco estudiado en el óptico y en lo que respecta a su variabilidad. En la literatura suele aparecer inmerso en los distintos catálogos disponibles, como por ejemplo, el RASS6dFGS de rayos X (Mahony et al. 2010), los de Fermi (Abdo et al. 2010, Nolan et 
al. 2012, Ackermann et al. 2015), el PARKES-MIT-NRAO (PMN, Griffith et al. 1993; Gregory et al. 1994), etc.

A este objeto lo seguimos durante 3 noches, 24, 25 y 27 abril 2014. En la Tabla 3.3, figuran los resultados del estado de variabilidad. En al primer noche se ha registrado variabilidad intra-noche sólo en el filtro R, con ambos parámetros estadísticos, mientras que en las restantes, se ha detectado variabilidad en ambos filtros $\mathrm{V}$ y $\mathrm{R}$. Respecto a la variabilidad inter-noche, si bien se ha detectado variación sólo en con el test $\mathrm{F}$, no se la considera como una variación real de la fuente. Aplicando lo analizado en el capítulo anterior, vemos que los valores de $\sigma_{2}$ son grandes en comparación a las otras noches, lo que implica DLC de control ruidosas. Hemos visto que el test F es sensible a clasificar como variable curvas ruidosas por lo que esta detección de variabilidad no será considerada.

Por otra parte se obtuvieron las magnitudes estándares para el blazar, resultando los valores promedios de $15.199 \pm 0.020$ para el filtro $\mathrm{V}$ y $14.796 \pm 0.024$ para el filtro $\mathrm{R}$, (Fig. 3.42),y con amplitudes de $\Delta m(V)=0.100$ y $\Delta m(R)=0.151$.

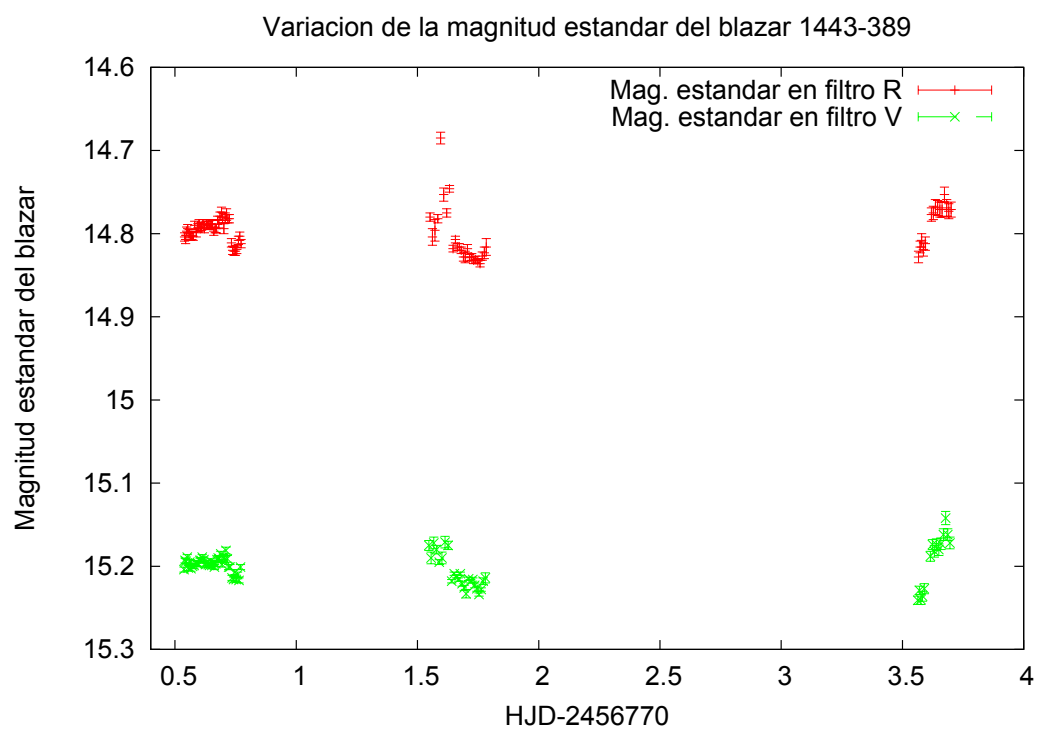

Figura 3.42. Curvas de luz de la magnitud estándar de 1443-3908 en el filtro R (rojo) y en el V (verde).

Por otra parte, analizamos el diagrama magnitud-magnitud, que se muestra en la Fig. 3.43, para poder explicar las variaciones detectadas. Se observa que presenta una correlación entre los datos en $\mathrm{V}$ y R, lo que se confirma con el ajuste lineal hecho (Tab. 3.4) y con el valor de la función $\mathrm{DCF}, \mathrm{DCF}=0.053 \pm 0.030$. Además el coeficiente de Pearson confirma que esta correlación es fuerte $(r=0.850)$.

Habiendo calculado las magnitudes estándares $\mathrm{V}$ y $\mathrm{R}$, podemos obtener el valor del índice espectral $\alpha_{V R}$. Para nuestras observaciones, obtuvimos un valor promedio de $\alpha_{V R}=2.29 \pm 0.016$, el cual oscila entre $2.28 \pm 0.016$ y $2.31 \pm 0.013$, sin presentar variaciones significativas (ver Fig. 3.44).

Para poder analizar el origen de las variaciones, estudiamos el diagrama colormagnitud, como se ve en la Fig. 3.45. A partir del ajuste lineal (con un valor del $\chi^{2}$ reducido muy bajo, 0.036) y del coeficiente de Pearson $\mathrm{r}=-0.148$, se tiene una correlación 

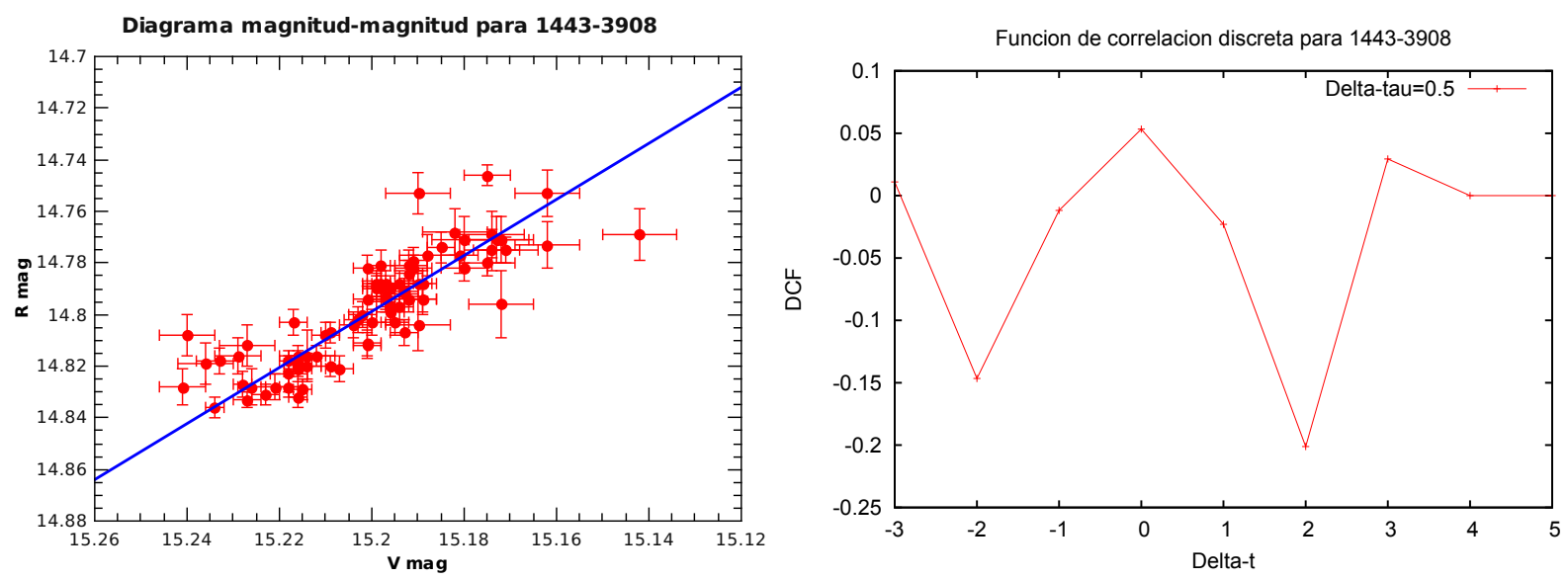

Figura 3.43. Diagrama magnitud-magnitud (izquierda) y función de correlación discreta (derecha) para 1443-3908.

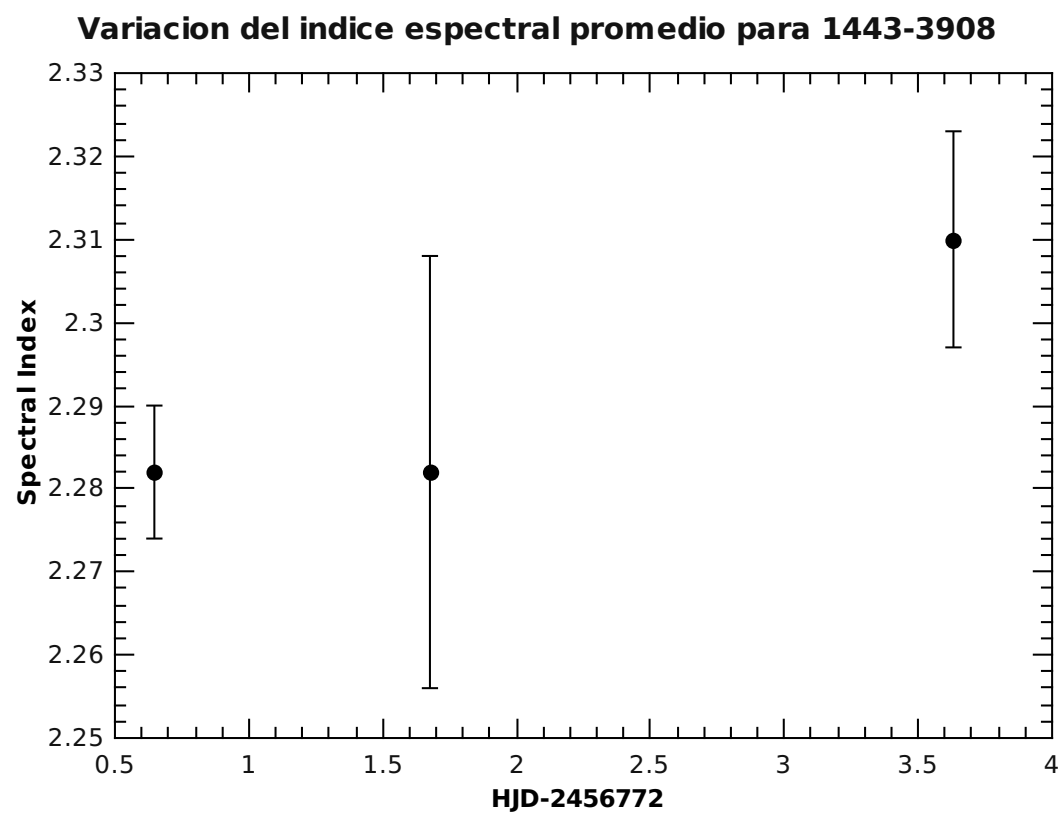

Figura 3.44. Variación del índice espectral para 1443-3908.

negativa muy débil. Con lo cual no se puede afirmar que se tiene un comportamiento RWB en la fuente. De hecho, el comportamiento de los puntos no pareciera ajustar una relación lineal, como se puede ver a simple vista.

* PKS 1510-089: Este blazar está clasificado como FSRQ $(z=0.361$, Burbidge \& Kinman, 1966; Thompson, Djorgovski \& de Carvalho 1990). La primera detección de variabilidad en el óptico data de la década de los 70 (Lu 1972). Por otra parte, Romero et al. (1999) estudiaron la microvariabilidad de esta fuente sin detectar variaciones, mientras que Sandrinelli et al. (2014) analizaron posibles variaciones tanto en escalas cortas como largas ( $~ 6$ años), midiendo amplitudes de $\Delta m=2.64$ mag y 3.43 mag en los filtros V y R, respectivamente. Gupta et al. (2016) también detectaron variaciones 


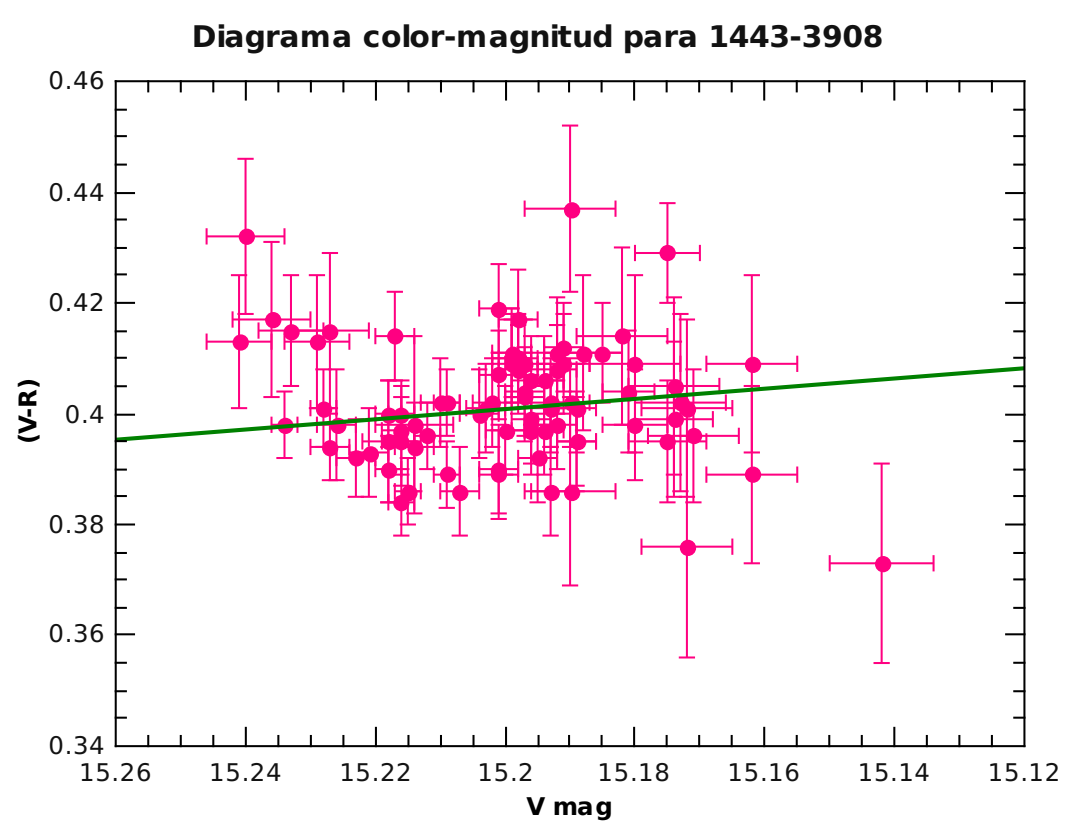

Figura 3.45. Diagrama color-magnitud para 1443-3908.

de amplitud $\Delta m=0.85 \mathrm{mag}$ en $\mathrm{V}$ y 0.75 en $\mathrm{R}$ en escala de meses. Una característica particular de esta fuente es la presencia de una loma pronunciada en el UV (UV bump) en el espectro. Ésta se cree que es debida a la emisión térmica del disco de acreción (Malkan \& Moore 1986, Pian \& Treves 1993, D'Ammando et al. 2009). En cuanto a las altas energías, Siebert et al. (1996) utilizaron datos del ROSAT para estudiar la parte blanda de la emisión en rayos X, al igual que Singh et al. (1997), quienes analizaron el espectro en esta banda, encontrando que era inusualmente duro, con un índice de fotones de $\Gamma=1.30 \pm 0.06$ y sugiriendo que la emisión está dominada por el jet relativista. Tavecchio et al. (2000) utilizaron datos del BeppoSAX para el análisis del espectro, el cual mostraba un exceso en la parte blanda de rayos X, además que la parte de bajas energías era más empinada que la de altas energías (ajustada con una ley de potencia). Por otra parte, en la banda de rayos- $\gamma$ esta fuente es un fuerte emisor, alcanzando energías de los GeV (detectado por los satélites EGRET, FermiLAT y AGILE). D'Ammando et al. (2009) reportaron la detección de un flare muy rápido con el AGILE, sumergido en una actividad muy alta con eventos de muy rápida variabilidad desde radio hasta rayos- $\gamma$. Los primeros trabajos multifrecuencia fueron reportados por Katakoa et al. (2008), con los satélites Swift y Suzaku, junto con datos en el óptico y radio. Además, Abdo et al. (2010) reportaron una alta actividad de la fuente en el transcurso de 2 años, con variabilidad detectadas en el óptico, UV, rayos $\mathrm{X}$ y rayos $-\gamma$ en escalas cortas.

Este objeto fue seguido durante tres noches consecutivas, del 12 al 14 abril de 2015 . Es interesante que dentro del campo observado, este blazar es uno de los objetos más brillantes. En la Tabla 3.3 se dan los resultados de aplicar los distintos test sobre las curvas de luz. Lo que observamos es que la fuente no presentó variabilidad (estos resultados coinciden con ambos parámetros $\mathrm{C}$ y F) en ninguno de los dos filtros durante la primera noche. Al contrario, tanto para la segunda como para la tercera noche se detectaron microvariabilidades en ambos filtros, tanto con el criterio $\mathrm{C}$ como con el test 
F. Y cuando se estudia el comportamiento sobre las tres noche consecutivas, la fuente resultó ser variable en ambos filtros con ambos parámetros.

En la Fig.3.46 se muestran las curvas del cambio de la magnitud estándar para PKS 1510-089 tanto para el filtro V (verde) como para el filtro R (rojo). Se detectó una amplitud promedio de $\Delta m=0.083$ en ambos filtros, siendo las amplitudes de variabilidad para la segunda y la tercera noche, donde se detecto microvariabilidad, de $\Delta m=0.08$ (filtro V) y $\Delta m=0.06$ (filtro R) en alrededor de 3 hs., y de $\Delta m=0.06$ (filtro $\mathrm{V}$ ) y $\Delta m=0.05$ (filtro R) en un lapso de $1.7 \mathrm{hr}$, respectivamente para cada de las noches mencionadas.

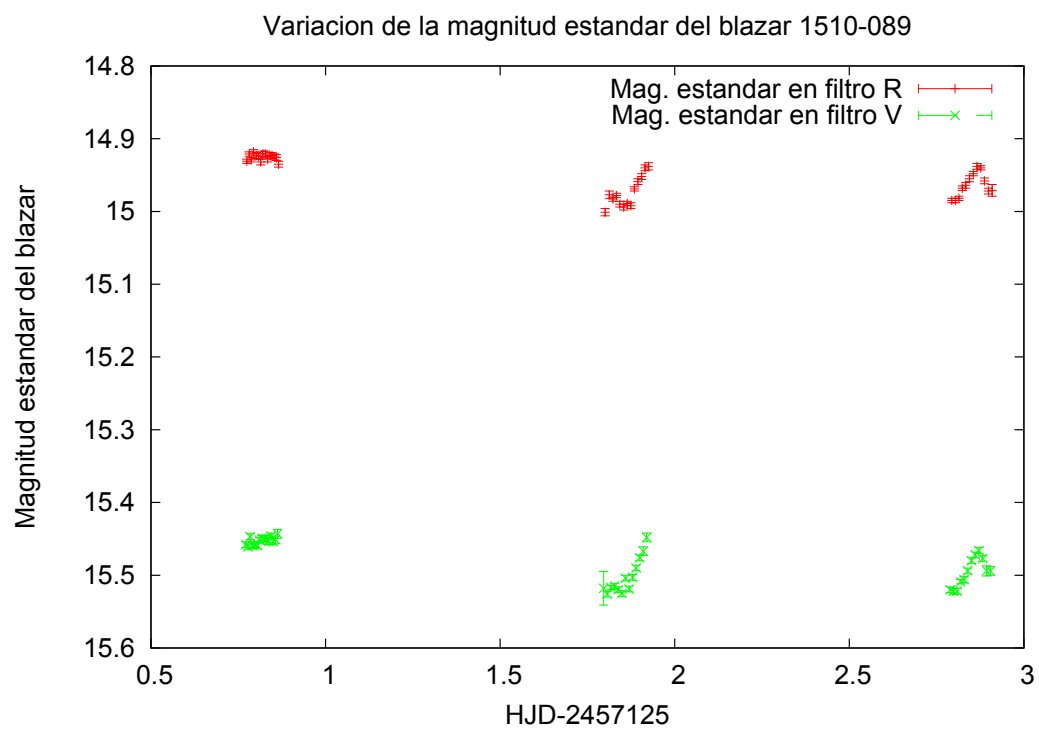

Figura 3.46. Curvas de luz de la magnitud estándar de PKS 1510-089 en el filtro $\mathrm{R}$ (rojo) y en el V (verde).

Recientemente, Gupta et al. (2016) reportaron el comportamiento del flujo óptico PKS 1510-089 sobre escalas temporales de 90 días a en un período que abarca de abril a agosto de 2014. En este trabajo encuentran variabilidad de días a meses con amplitudes $\Delta m=0.85$ en $\mathrm{V}$ y $\Delta m=0.75$ en $\mathrm{R}$. Entre estas observaciones y las nuestras transcurrió aproximadamente 1 año, en donde se observa que la magnitud en $\mathrm{V}$ decae en 0.768 y en 0.907 en R. Es decir, la fuente se vuelve más brillante de un año al otro. Por otro lado, Sandrinelli, Covino \& Treves (2014) también estudiaron la variabilidad de PKS 1510-089 a escalas largas y cortas. En un período de aproximadamente 6 años (enero 2006 - junio 2012), obtuvieron un $\Delta m=3.25$ en el filtro $\mathrm{V}$ (cuyo rango de magnitudes va desde $13.11 \mathrm{mag}$ a $16.36 \mathrm{mag}$ ), y $\Delta m=3.01$ en el filtro $\mathrm{R}$ (donde el rango va desde $13.27 \mathrm{mag}$ a $16.28 \mathrm{mag}$ ). En promedio, para la fuente se obtuvo una magnitud de 14.83 mag en V y 14.65 mag en R. En la Fig. 3.47 se muestra una comparación entre los valores obtenidos en esta Tesis y en los mencionados trabajos.

A partir de haber obtenido las magnitudes estándares del blazar, podemos calcular el valor promedio del índice espectral en la banda del óptico, $\alpha_{V R}$, tanto para cada noche como a lo largo de todas las noches. Los valores promedio obtenidos fueron: $2.99 \pm 0.009,3.00 \pm 0.010$ y $3.02 \pm 0.008$, respectivamente para cada una de las noches (ver Fig. 3.48), siendo el promedio general 3.00 \pm 0.009 . Este índice tiene asociado un 


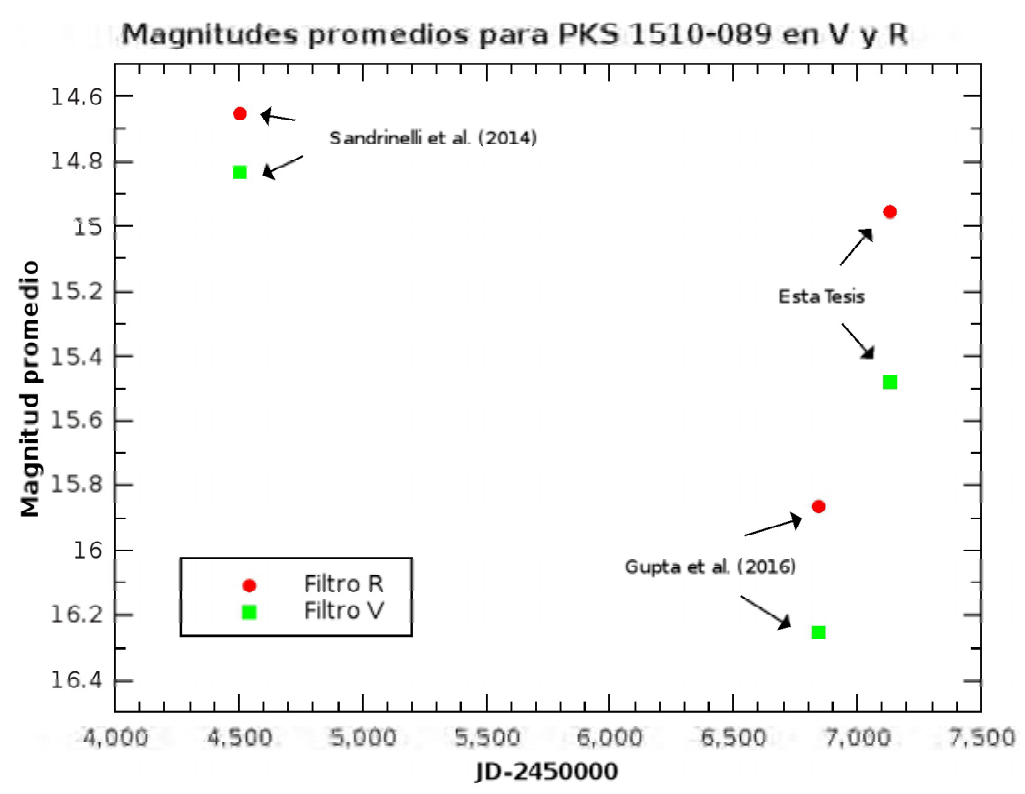

Figura 3.47. Comparación de las magnitudes promedio en los filtros V (verde) y R (rojo) para PKS 1510-089.

índice de color promedio para las 3 noches de $(\mathrm{V}-\mathrm{R})=0.5 \pm 0.008$, siendo comparable al encontrado por Gupta et al. (2016), cuyo valor promedio es $(\mathrm{V}-\mathrm{R})=0.4$. Tomando este valor y aplicamos el cálculo del índice espectral, resulta que en un valor de $\alpha_{V R}=2.27$ para sus datos.

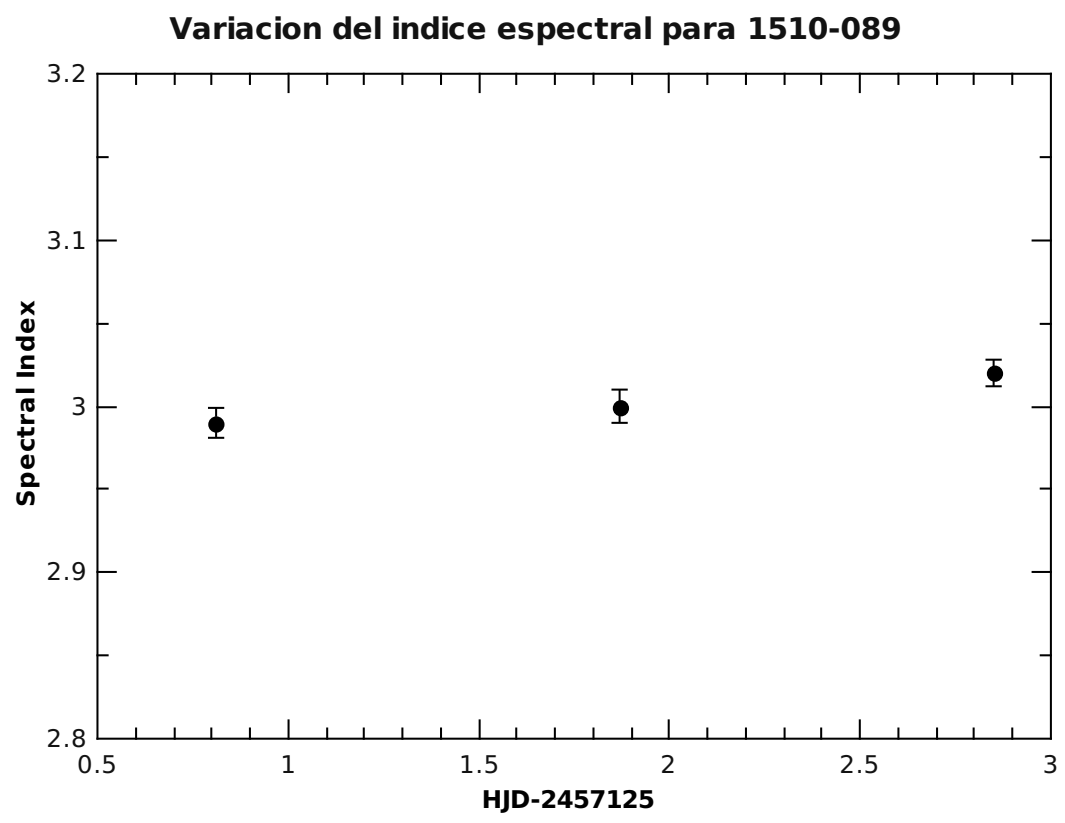

Figura 3.48. Variación del índice espectral a lo largo del turno.

A partir de los valores obtenidos en esta Tesis y agregando aquellos publicados por Gupta et al. (2016) y por Sandrinelli et al. (2014), podemos analizar el comportamiento a gran escala de $\alpha_{V R}$. Basados en la curva de variación de las magnitudes, y observando 
para qué valores del JD se daban los incrementos y decaimientos en el brillo de la fuente, tomamos 5 regiones en donde se ven picos de crecimiento en las magnitudes, y calculamos los respectivos valores de $\alpha_{V R}$ (ver Fig. 3.49). Para el caso de Gupta et al. (2016), se utilizaron los datos de los valores máximos y mínimos para las magnitudes con sus respectivos JD, y se procedió de igual manera en el cálculo del color y del índice espectral. El resultado final se muestra en el panel inferior de la Fig. 3.49. Cada vez que la fuente se vuelve más débil, el valor del índice $\alpha_{V R}$ decrece, lo que implica que la distribución espectral de energía en ese rango se vuelve más blanda. En cambio, cuando la fuente se vuelve más brillante, $\alpha_{V R}$ crece, lo que corresponde a un espectro más endurecido. De esta manera, podemos ver cómo los cambios en el brillo de PKS 1510-089 se ven reflejados en el comportamiento espectral.

Como vimos en las demás fuentes, analizamos qué sucede con el diagrama magnitudmagnitud, así podemos estudiar posibles correlaciones entre los datos en las bandas V y R. Dado el comportamiento encontrado para $\alpha_{V R}$, es esperable que exista una correlación. En la Fig. 3.50 (izquierda) se muestra el diagrama magnitud-magnitud, donde se observa una correlación positiva entre ambos conjuntos de datos. Dicho ajuste arroja un valor confiable de $\chi^{2}$ reducido de 0.91 (cercano a 1). Por otro lado, utilizamos la función de correlación discreta (DCF), como se muestra en la Fig. 3.50 (derecha). La función da como resultado un valor positivo de DCF para un lapso de tiempo positivo pero cercano a 0 (lapso temporal entre la toma de datos en $\mathrm{V}$ y $\mathrm{R}$ ), indicando una correlación positiva entre los datos (ver Tab. 3.4). Esto implica por un lado, y como es esperable, que los fotones en ambas bandas son emitidos por el mismo proceso físico y en la misma región física. Por otro lado, que este lapso positivo de tiempo muestra que las variaciones en el filtro $\mathrm{V}$ conducen a las variaciones en el filtro $\mathrm{R}$. Esta correlación también es corroborada por el coeficiente de Pearson $(r=0.95)$, el cual resulta en una correlación fuerte. Este comportamiento se lo suele interpretar como un efecto intrínseco de la opacidad de la fuente debido al proceso SSC (Kudryavtseva et al. 2011; Gupta et al. 2012).

Para estudiar el posible origen de las variaciones, analizamos el comportamiento del diagrama color-magnitud (Gupta et al. 2016, Agarwal et al. 2014). En la Fig. 3.51, se muestra el diagrama color-magnitud para PKS 1510-089. En la misma figura se graficó el ajuste lineal realizado, con un valor de $\chi^{2}$ reducido de 0.22 . Por otro lado, el coeficiente de Pearson indica una correlación positiva débil entre los datos $(\mathrm{r}=0.426)$. Con lo cual, los resultados de ambos ajustes no son robustos, lo que puede deberse a las grandes barras de error que poseen los datos. Por lo tanto, estaríamos observando que el objeto tendría un comportamiento RWB, tendencia contraria a la que enocntraron Gupta et al. (2016). Por su parte, Sasada et al. (2011), basados en observaciones realizados a lo largo de seis meses durante el 2009, reportan variaciones de esta fuente de, por ejemplo, 2.5 mag mas brillante en 7 días (flare), y otras de menor amplitud. Durante el período de crecimiento de la magnitud, la fuente siguió un comportamiento tipo BWB. Este flare se encuentra enmarcado dentro de un período mas largo de actividad, durante el cual, PKS 1510-089 mostró un comportamiento RWB. Además de estos cambios en la tendencia en el diagrama color-magnitud, se encontró que el flare que ocurrió en mayo de 2010 fue esencialmente acromático, a diferencia de otros flares (Bonning et al., 2012). Este comportamiento cambiante entre RWB, BWB o acromático presente en este objeto fue confirmado por Zhang et al., 


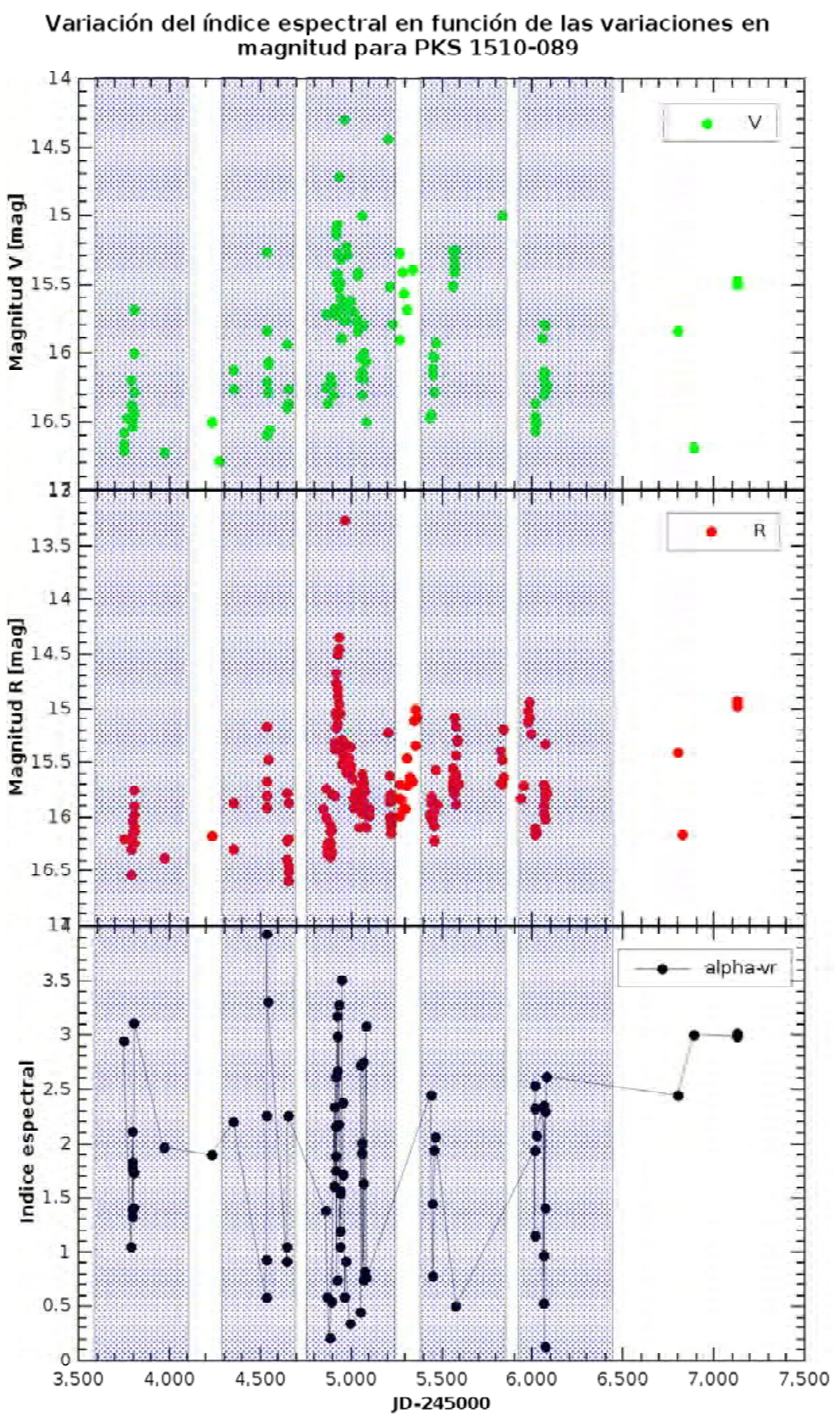

Figura 3.49. Panel Superior: comportamiento de la magnitud aparente en V (datos de Sandrinelli et al., 2014; Gupta et. al 2016, y la presente tesis). Panel medio: Idem para la magnitud aparente en R. Panel inferior: Curva de variación del índice espectral $\alpha_{V R}$ para PKS 1510-089.

2012. Todo esto favorece el escenario donde el origen de las distintas variaciones en el color pueden ser explicadas por dos componentes: una responsable de los long term flares, con un color relativamente rojo, y la otra relacionada con los short term flares, más azul. 

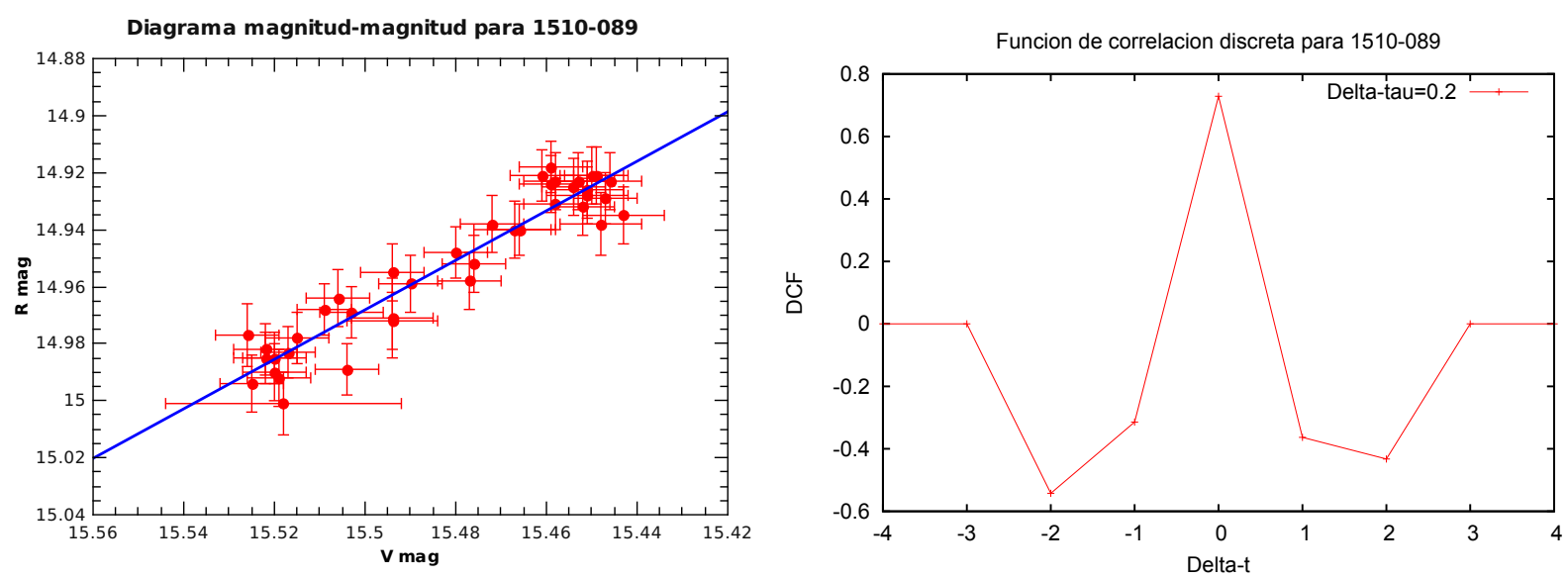

Figura 3.50. Diagrama magnitud-magnitud (izquierda) y función de correlación discreta para 1510-089 (derecha).

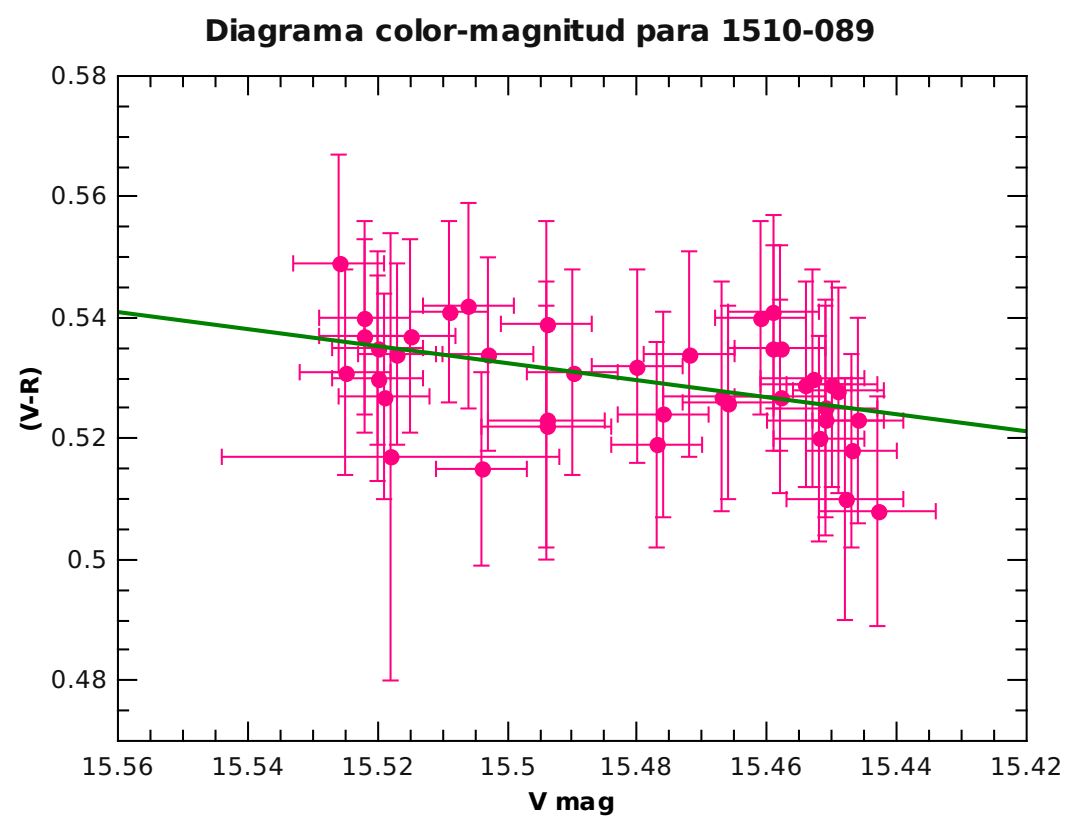

Figura 3.51. Diagrama color-magnitud para PKS 1510-089.

* PKS J1517-242: este BL Lac (ISP) posee un redshift de $\mathrm{z}=0.059$ (Disney et al. 1974; Jones et al. 2009). Este objeto fue uno de los primeros en ser clasificado como parte de los BL Lacs (Strittmatter et al. 1972). Bond (1971) reportó que la fuente en radio PKS 1517-2422 correspondía a la contraparte óptica de la fuente Ap Librae, la cual registraba una variabilidad óptica importante entre 15.0 y 16.1 mag (Ashbrook, 1942). Por su parte, Westerlund et al. (1982) estudiaron las propiedades de esta fuente a través de la fotometría UBV, encontrando valores para la magnitud V entre los años 1975 y 1980 (estos valores serán mostrados y analizados más adelante, Fig. 3.53). De la misma manera, Hamuy \& Maza (1987) reportaron no sólo valores para la magnitud V sino también para R. Fan \& Lin (2000) obtuvieron que la fuente presentaba variaciones en el filtro $\mathrm{V}$ de $\Delta m=1.45 \mathrm{mag}$ (con valores de la magnitud $\mathrm{V}$ entre 13.86 y 15.31), y 
en el filtro $\mathrm{R}$ de $\Delta m=1.03 \mathrm{mag}$ (con valores de la magnitud $\mathrm{R}$ entre 13.44 y 14.47 ), además de encontrar una anticorrelación entre el índice de color (B-U) y la magnitud V. Mahony et al (2011) reportaron un valor de 14.86 para el filtro R. En rayos X, este objeto fue descubierto por el satélite Einstein (Schartz \& Ku, 1983). En altas energías, la Colaboración H.E.S.S. en el años 2010 reportaron la detección de rayos- $\gamma$ a muy altas energías (VHE, Hofmann 2010), mientras que Kaufmann et al. (2011) reportaron la existencia de un jet en la banda de rayos $\mathrm{X}$ a partir de observaciones con el satélite Chandra.

Este blazar fue monitoreado durante 3 noches, 22, 23 y 26 abril 2014, y otras dos noches, 10 y 11 abril 2015. Para las observaciones del 2014, en cuanto a la variabilidad intra-noche, para cada una de las 3 noches no se detectan variaciones en ningún filtro ni con ninguno de los parámetros $\mathrm{C}$ y F. Considerando la variabilidad inter-noche, tampoco se registran variaciones en ningún filtro. Por otra parte, para los datos del 2015, no se registran variaciones intra-noche para ninguna de las dos noches de observación, al igual que para la variabilidad inter-noche. Ambos parámetros determinan la no variabilidad. Por último, en escalas de años, se obtuvo una variabilidad significativa en ambos filtros $\mathrm{V}$ y $\mathrm{R}$ y con ambos parámetros $\mathrm{C}$ y F.

En lo que respecta a la obtención de las magnitudes estándares, para los datos del 2014, los valores de las magnitudes promedio resultaron $14.905 \pm 0.061$ para el filtro V y $14.370 \pm 0.052$ para el filtro R. Para el 2015, se obtuvieron $14.995 \pm 0.020$ para el filtro V y $14.371 \pm 0.015$ para el filtro R. En la Fig. 3.52, se muestra la variación de la magnitud estándar del blazar en cada año y en el período 2014-2015. Las variaciones para el 2014-2015 fueron de $\Delta m(V)=0.299$ y $\Delta m(R)=0.170$.

De la Fig. 3.53, se puede observar que a lo largo de los años, el blazar crece en brillo, hasta llegar a su magnitud más brillante alrededor del año 2000, para luego decrecer. Es posible ver cómo cambia el estado de actividad de la fuente.

En la Fig. 3.54 se muestran los diagramas magnitud-magnitud para los 3 grupos de datos que se tienen. Para los tres conjuntos de datos, se obtuvo una correlación entre los datos en V y R. Si bien el ajuste lineal y el valor de la DCF corroboran en todos los casos la existencia de dicha correlación, en particular para los datos del 2015 es grande la incerteza que se tiene tanto en el ajuste, como en la función y en los datos (ver Tab. 3.4). Además, esto se ve reflejado en el diagrama del medio de la Fig. 3.54.

Por otra parte, en lo que respecta al índice espectral, como se muestra en la Fig. 3.55 , en cada año no se registran variaciones significativas en el valor del mismo. Su valor promedio es de $\alpha_{V R}=3.04 \pm 0.016$ para el 2014, y $\alpha_{V R}=3.54 \pm 0.022$ para el 2015. Si tomamos el período 2014-2015, la variación del índice es importante, como se muestra en el gráfico inferior de la Fig. 3.55. De un año a otro, $\alpha_{V R}$ crece, lo que implica que la distribución espectral se vuelve más dura. El valor promedio general para este período es de $\alpha_{V R}=3.21 \pm 0.046$. Nuestros valores los podemos comparar con los índices de otros autores. Estos valores los calculamos a partir de los valores de las magnitudes publicadas. Para el caso de Hamuy \& Maza (1987), encontramos un valor promedio de $\alpha_{V R}=3.18$, cercano al obtenido en esta Tesis. Para los datos de Fan \& Lin (2000), correspondería un valor de $\alpha_{V R}=2.5$, menor valor al nuestro. El comportamiento global de $\alpha_{V R}$ lo mostramos en la Fig. 3.56.

Se puede ver que nuestros datos son comparables con los obtenidos por Hamuy \& Maza (1987). Vemos que, alrededor del año 2000, donde la fuente presentaba su pico de 

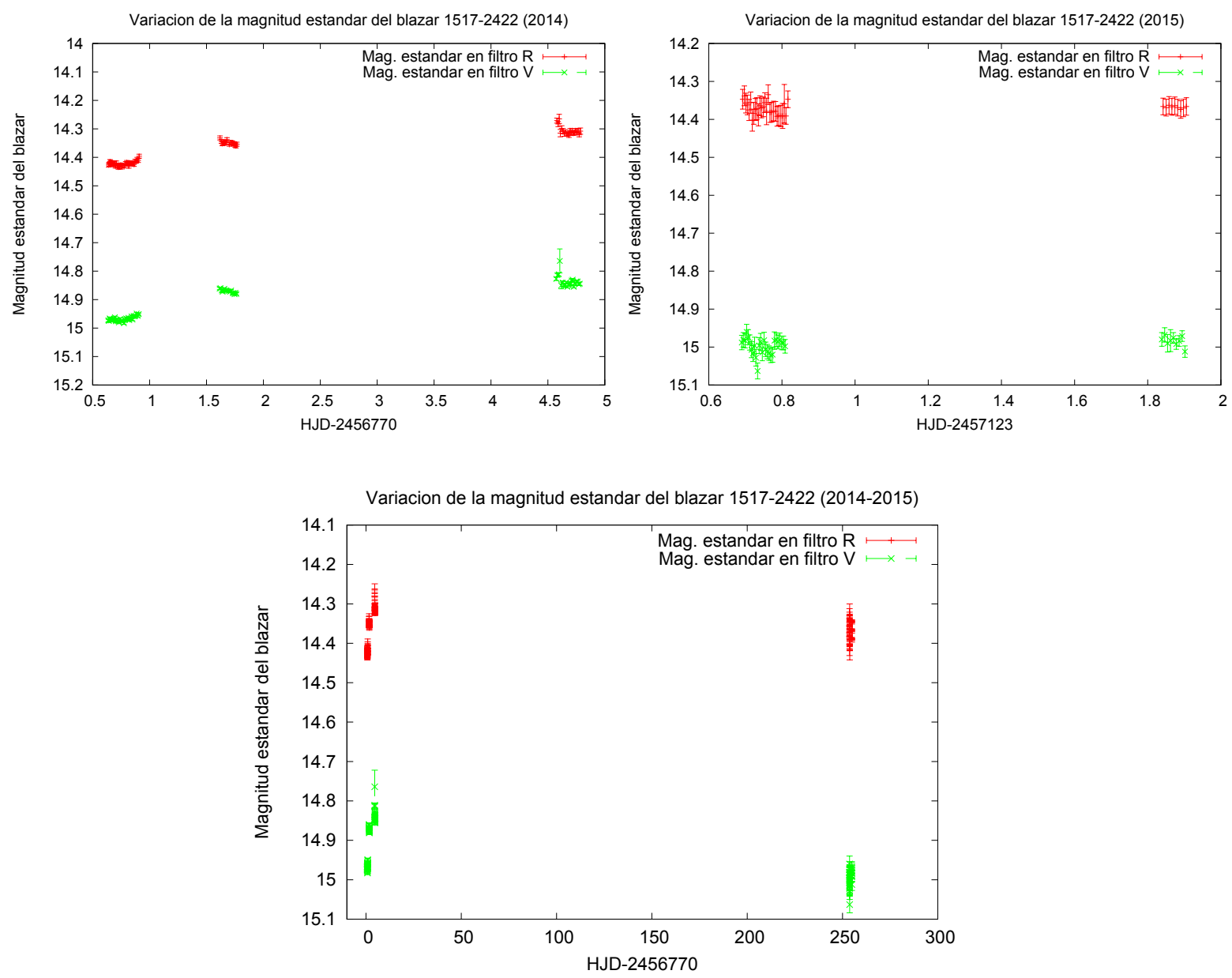

Figura 3.52. Curvas de luz de la magnitud estándar de 1517-2422 en el filtro R (rojo) y en el V (verde), en el panel superior izquierdo correspondiente al 2014, en el panel superior derecho, al 2015 y el último, al período 2014-2015.

máximo brillo, el valor de $\alpha_{V R}$ es el más bajo. Lo que estamos viendo es que, a medida que el blazar se vuelve más brillante, el valor de $\alpha_{V R}$ decrece, esto es, la distribución espectral se hace más blanda. Cuando el brillo comienza a decaer, el valor de $\alpha_{V R}$ aumenta, lo que implica que el espectro se endurece.

Finalmente, del análisis de los diagramas color-magnitud, presentados en la Fig. 3.57, vemos que tanto para los datos del $2014(\mathrm{r}=0.639)$ como del $2015(\mathrm{r}=0.749)$ y del período 2014-2015 ( $\mathrm{r}=0.747)$, se registra una tendencia positiva y moderada en los tres gráficos, indicando en cada caso que se tiene una tendencia BWB. Sin embargo, estas correlaciones no son fuertes, y visualmente puede apreciarse que las correlaciones no estarían siguiendo una tendencia lineal, sino parabólica. Por lo tanto, no resultaría del todo confiable asociar la tendencia BWB a esta fuente. 

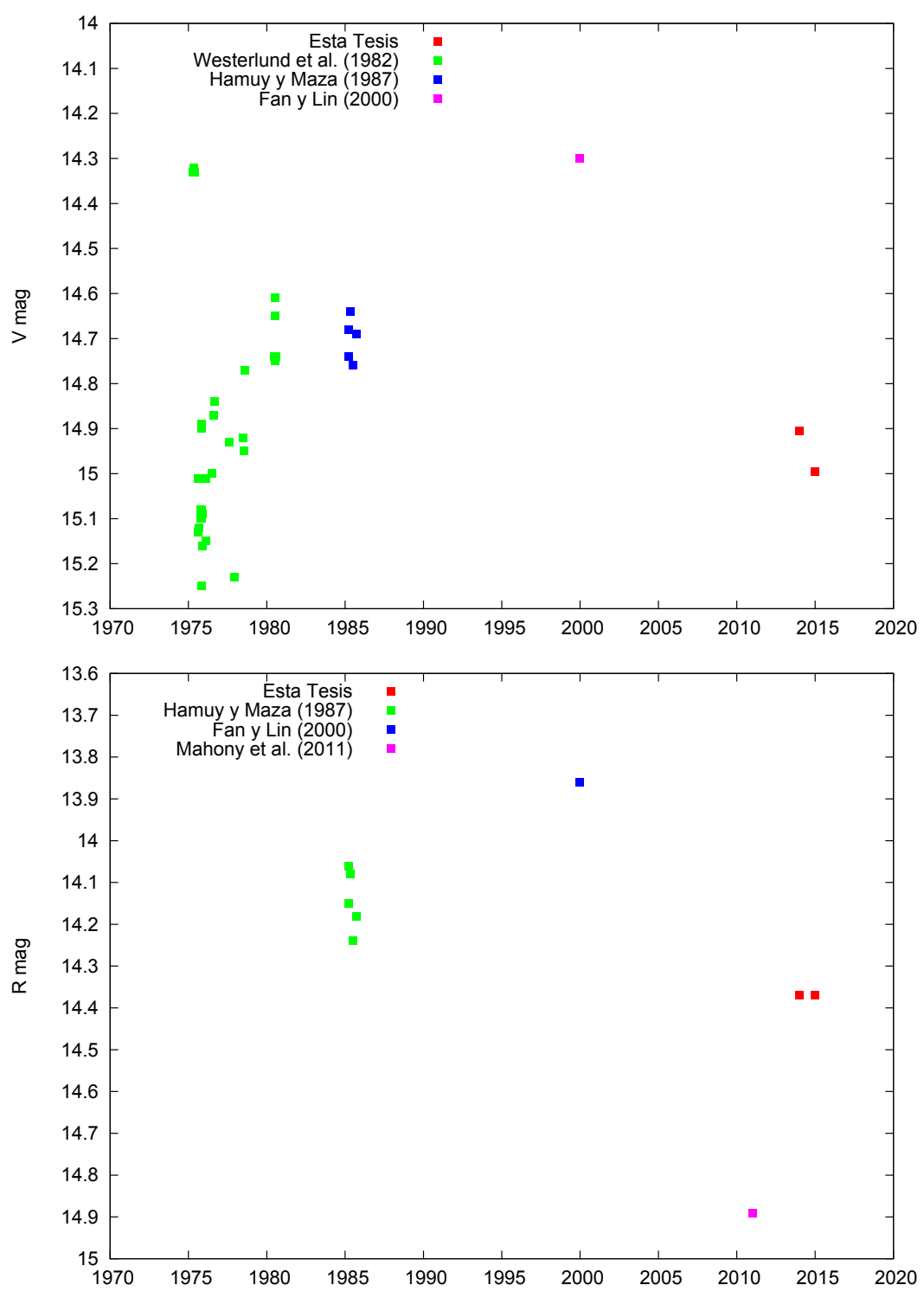

Figura 3.53. Valores comparativos para la magnitud estándar V (panel superior) y R (panel inferior) del blazar PKS 1517-2422. 

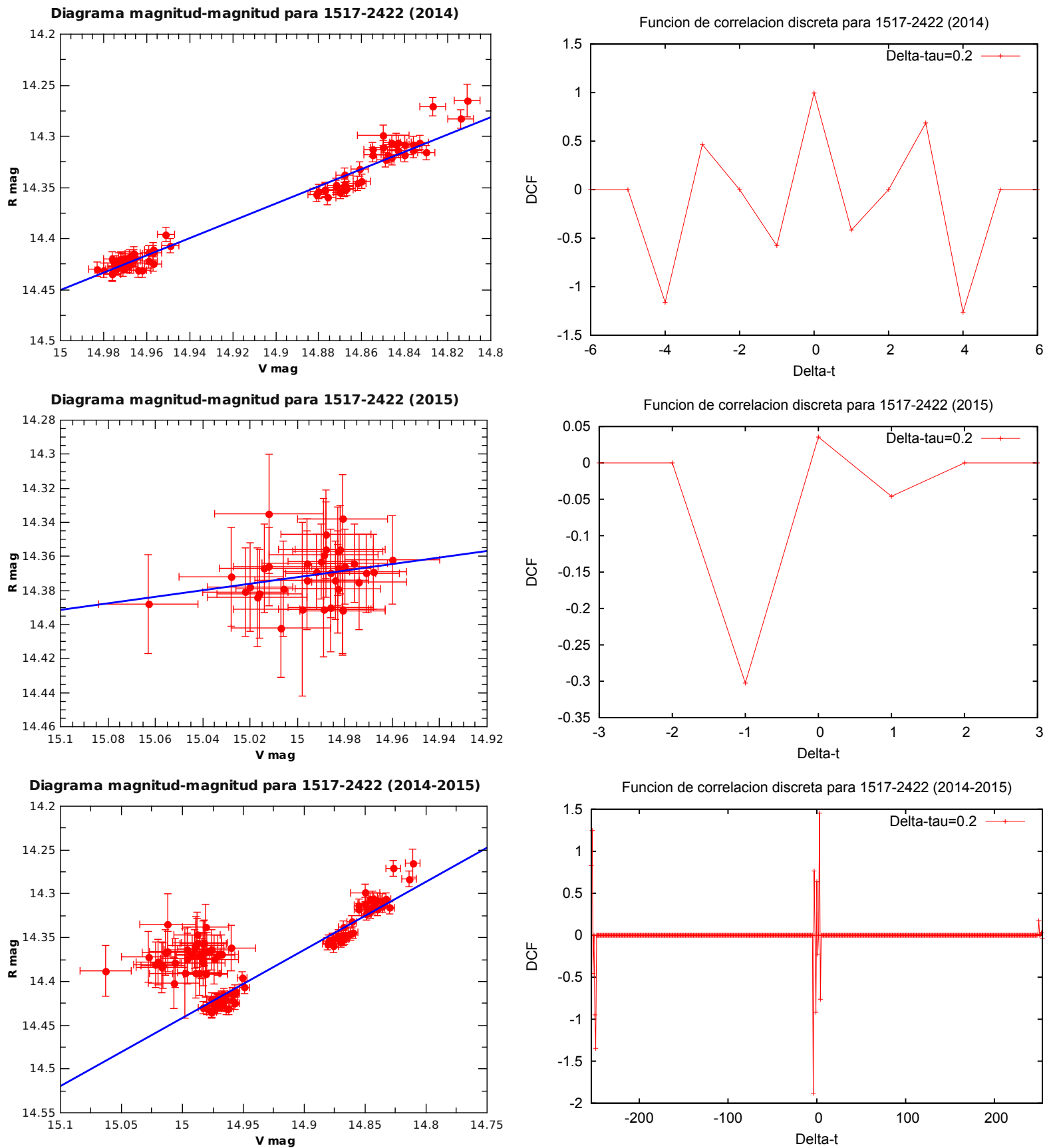

Figura 3.54. Diagrama magnitud-magnitud (izquierda) y función de correlación discreta para 1517-2422 (derecha). De arriba hacia abajo, se tienen los datos correspondientes al 2014, 2015 y el período 2014-2015. 

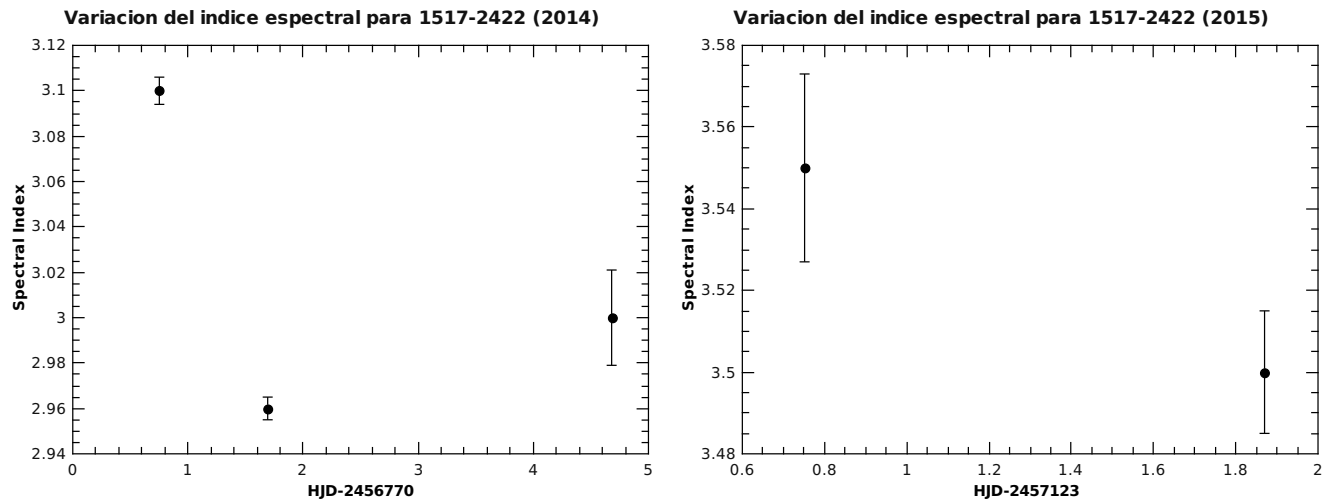

Variacion del indice espectral para 1517-2422 (2014-2015)

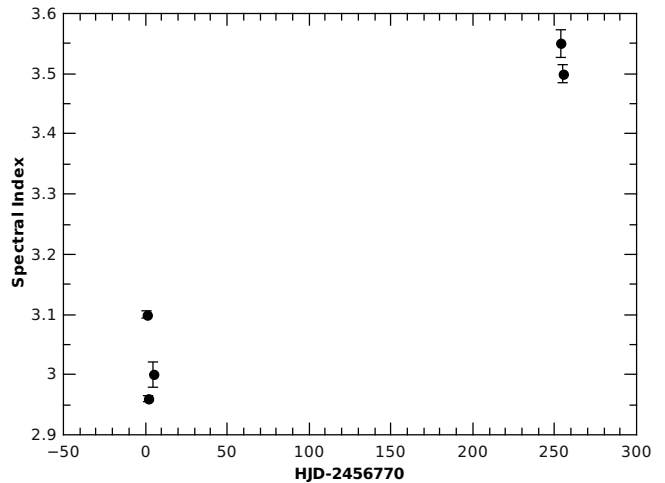

Figura 3.55. Variación del índice espectral para 1517-2422. En el panel superior izquierdo están los datos del 2014. En el panel superior derecho, los correspondientes al 2015 y en el panel inferior, los datos del período 2014-2015.

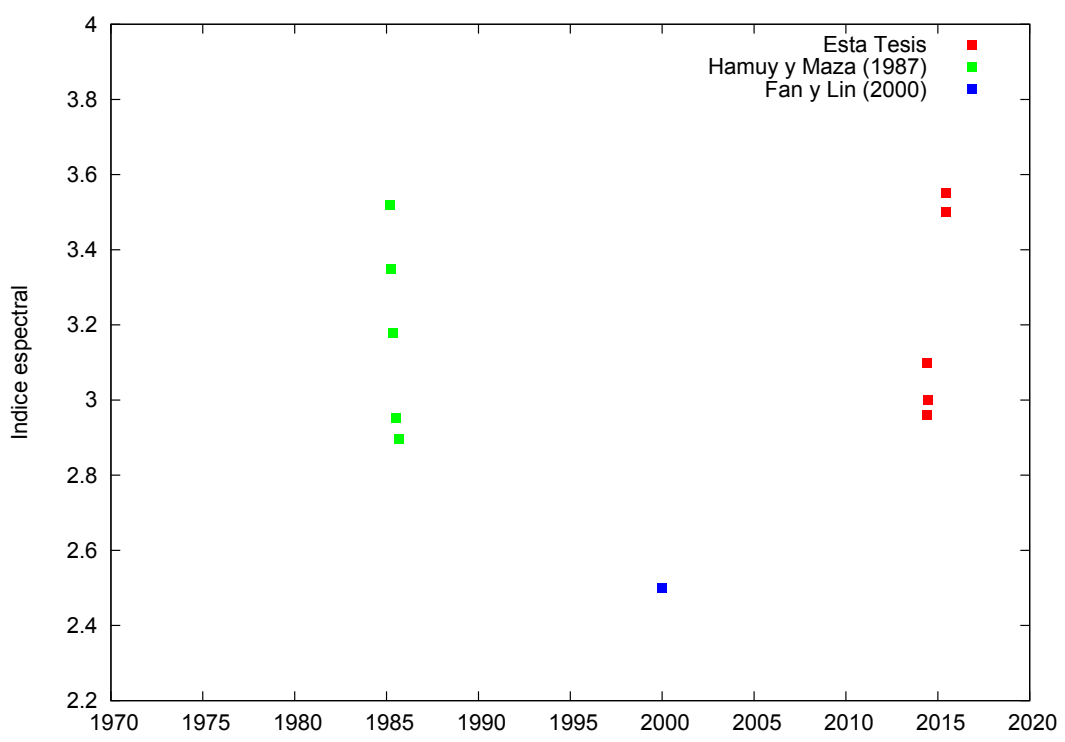

Figura 3.56. Comparación de los valores publicados de $\alpha_{V R}$ para 1517-2422. 

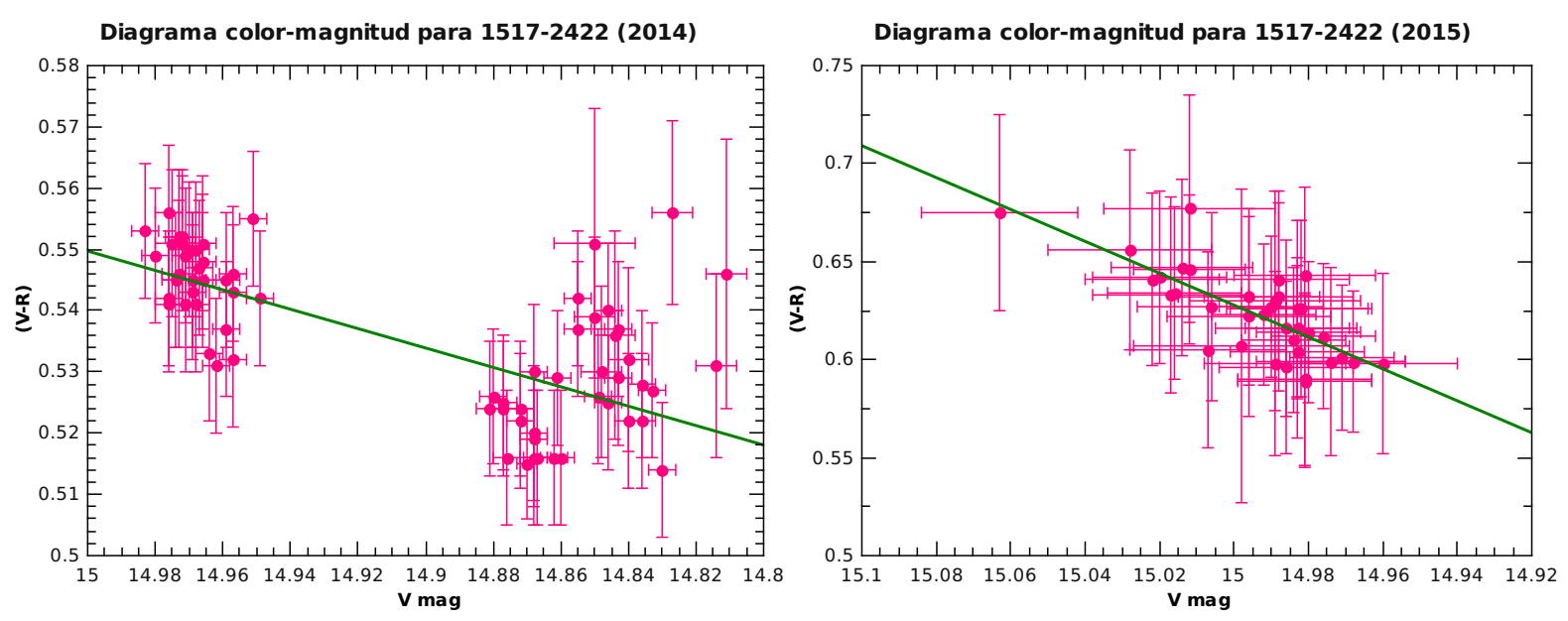

Diagrama color-magnitud para 1517-2422 (2014-2015)

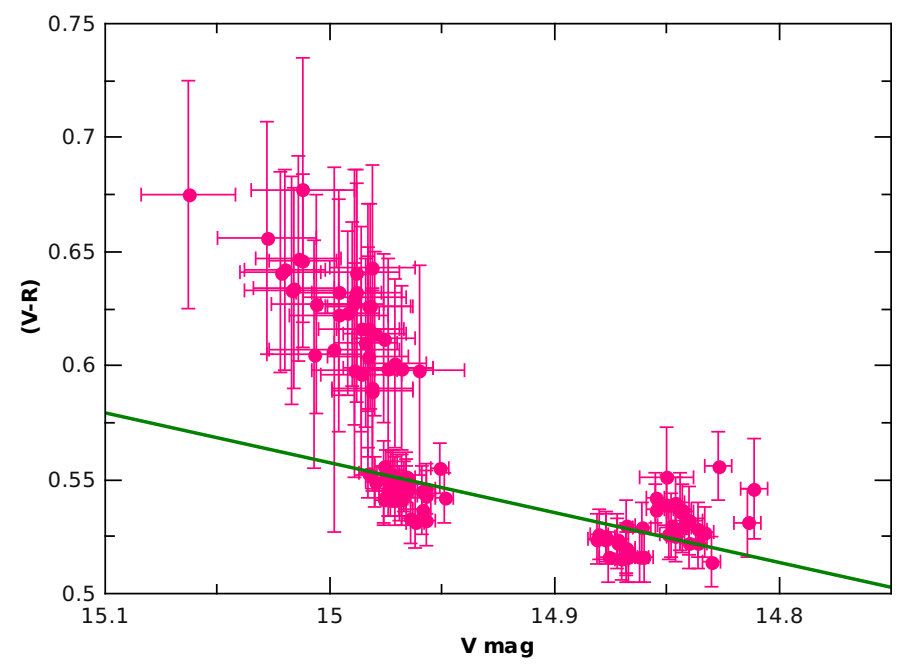

Figura 3.57. Diagrama color-magnitud para 1517-2422. En el panel superior izquierdo están los datos del 2014. En el panel superior derecho, los correspondientes al 2015 y en el panel inferior, los datos del período 2014-2015. 
* BZB J1917-192: catalogado como un BL Lac (HSP), este blazar posee un redshift de $\mathrm{z}=0.137 \pm 0.001$ (Carangelo et al. 2003; Sbarufatti et al 2005). Esta fuente forma parte de diversos catálogos en varias longitudes de onda, tales como los catálogos del satélite Fermi (en rayos- $\gamma$, Abdo et al. 2010, Nolan et al. 2012, Ackermann et al. 2015); en radio PARKES-MIT-NRAO (Griffith et al. 1994), VLBA (Kolalov et al. 2007): ROSAT (en rayos-X, Voges et al. 1999). Del catálogo de Véron-Cetty \& Véron (2001), este blazar registra una magnitud $V=18.5$.

Fue observado en dos noches, 25 y 26 abril 2014. Con respecto a la variabilidad intranoche, no se observan variaciones en ningún filtro en las dos noches. Esta detección de no-variabilidad se obtiene tanto con el criterio $\mathrm{C}$ como con el test F. Y en lo que respecta a la variabilidad inter-noche, sólo se registra variación para el filtro $\mathrm{R}$ y con el parámetro F. Sin embargo, las curvas resultan ruidosas, y a partir del análisis hecho en el capítulo 2, terminamos concluyendo que esta variación no es real, con lo cual no se tienen variaciones inter-noche.

Obtuvimos los valores de las magnitudes estándares del blazar, cuyos valores promedio son $15.542 \pm 0.023$ para el filtro V y $15.098 \pm 0.025$ para el filtro R. En la Fig. 3.58, mostramos las curvas de luz de las magnitudes.

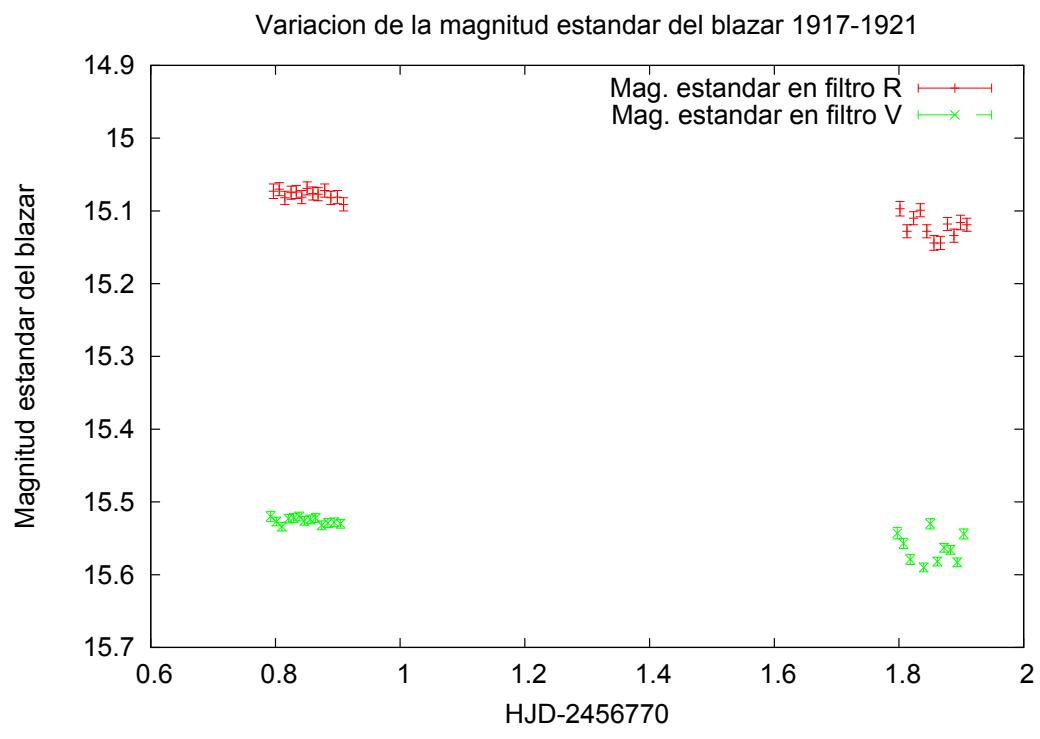

Figura 3.58. Curvas de luz de la magnitud estándar de 1917-1921 en el filtro R (rojo) y en el V (verde).

En cuanto al diagrama magnitud-magnitud, Fig. 3.59, se puede ver una correlación entre los datos, aunque los primeros valores graficados muestran barras de error grandes y están más dispersados, dificultando el ajuste en esa región (ver Tab. 3.4). Tanto el ajuste como el valor de la función $(\mathrm{DCF}=0.751 \pm 0.090)$ corroboran dicha correlación. Además, el coeficiente de Pearson indica que la correlación es fuerte.

En cuanto al comportamiento del índice espectral, se obtuvo un valor de $2.54 \pm 0.006$ para la primer noche y $2.51 \pm 0.025$ para la segunda, sin mostrar variación alguna (Fig. 3.60). Su valor promedio resultó ser $\alpha_{V R}=2.53 \pm 0.017$.

Por otra parte, analizando el diagrama color-magnitud, vemos que el comportamiento de los puntos implicaría que la fuente presenta una tendencia BWB, Fig. 3.61. 

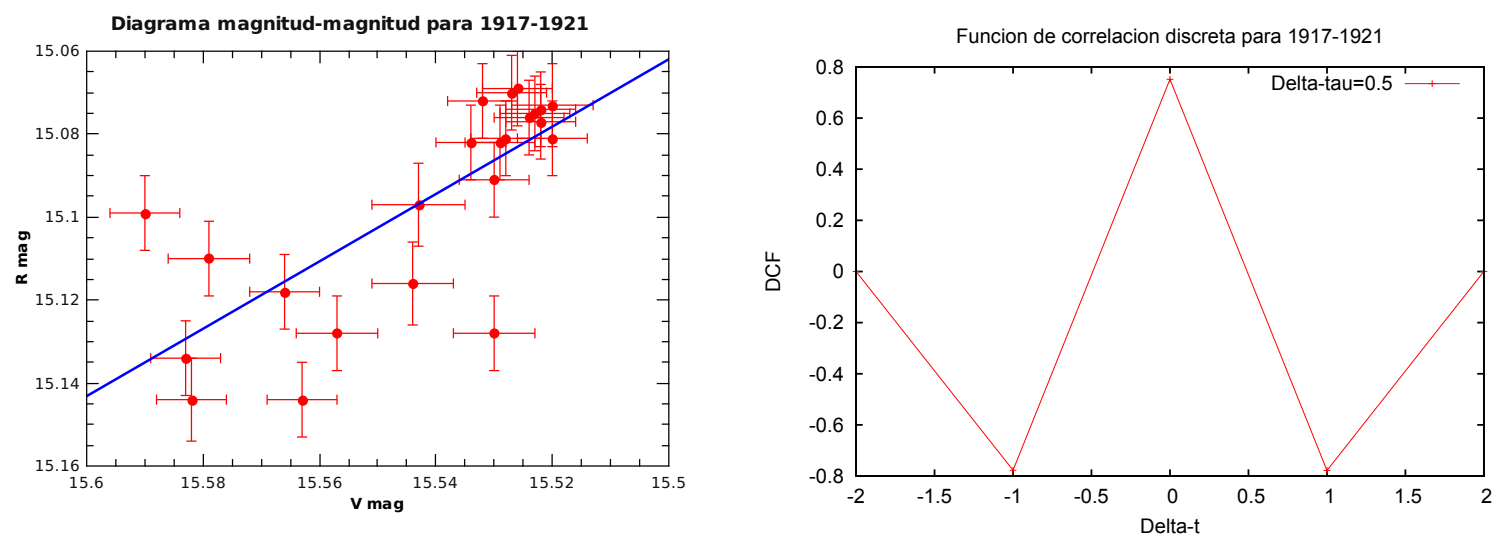

Figura 3.59. Diagrama magnitud-magnitud (izquierda) y función de correlación discreta para 1917-1921 (derecha).

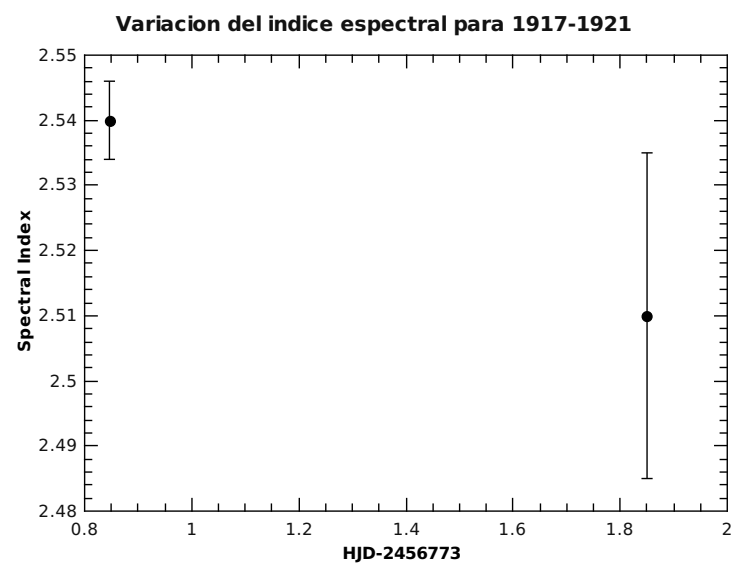

Figura 3.60. Variación del índice espectral para 1917-1921.

Sin embargo, tanto el ajuste lineal como el coeficiente de Pearson $(\mathrm{r}=0.238)$ coinciden en que la correlación es débil, con lo cual no se puede concluir sobre la tendencia de este blazar.

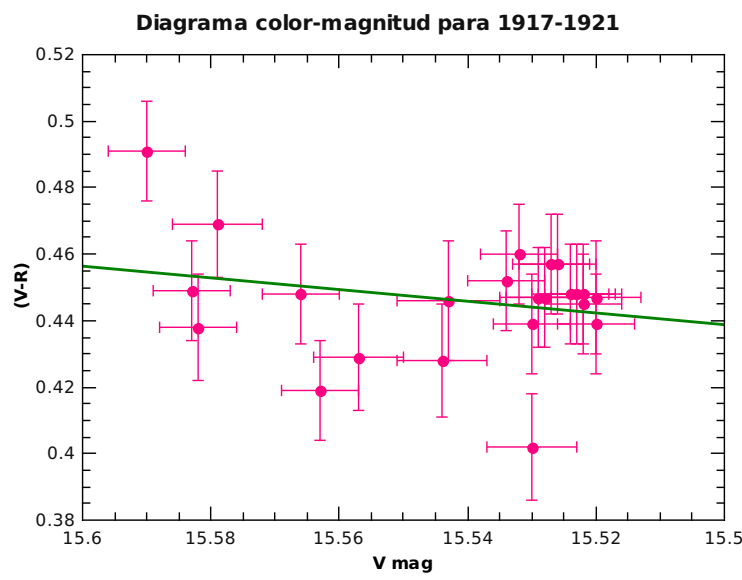

Figura 3.61. Diagrama color-magnitud para 1917-1921. 
* 3FGL J1958.2-3011: este blazar está catalogado como un BL Lac (HSP). Posee un $\mathrm{z}=0.119$ (Jones et al. 2009). Este objeto aparece en diversos catálogos dentro de la literatura, como el RBS-NVSS (en radio, Bauer et al. 2000), o los catálogos del satélite Fermi (en rayos- $\gamma$, Abdo et al. 2010, Ackermann et al. 2011, 2015; Acero et al. 2015).

Se lo observó en 2 noches, 23 y 24 de abril 2014. No presenta variaciones intra-noche en ninguno de los dos filtros. En cuanto a la variación inter-noche, tampoco se detecta variación alguna. Los resultados de los parámetros $\mathrm{C}$ y $\mathrm{F}$ coinciden en ambos casos. Ambos no detectan variabilidad.

En lo que respecta a las magnitudes estándares, se obtuvieron valores de 17.024 \pm 0.009 para el filtro V y $16.438 \pm 0.007$ para el filtro $\mathrm{R}$ y sus curvas de luz se muestran en la Fig. 3.62.

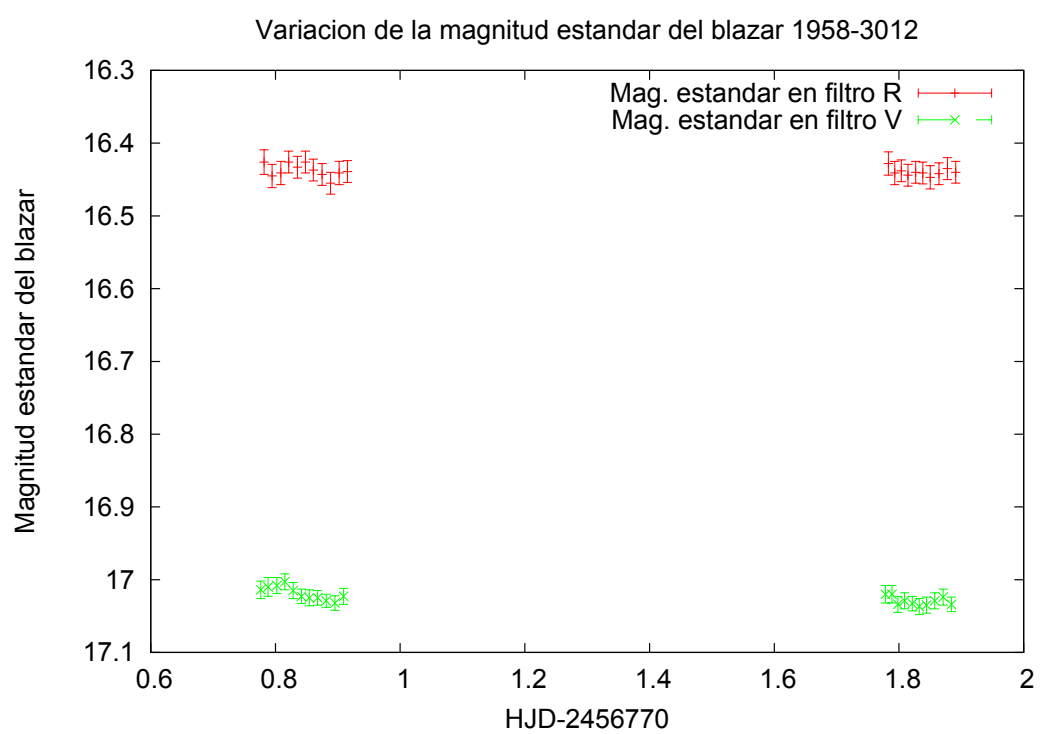

Figura 3.62. Curvas de luz de la magnitud estándar de 1958-3012 en el filtro R (rojo) y en el V (verde).

Analizando el diagrama magnitud-magnitud, que se muestra en la Fig. 3.63, se puede observar una correlación entre los datos de los filtros $\mathrm{V}$ y R. Esta correlación es corroborada por la función DCF, con un valor positivo, al igual que con el ajuste lineal, aunque éste último presenta algunas incertezas en sus valores (ver valores en la Tab. 3.4).

Obtuvimos el valor promedio del índice espectral, siendo éste $\alpha_{V R}=3.32 \pm 0.009$. Para la primer noche, registramos un valor de $3.30 \pm 0.009$, mientras que para la segunda, el valor fue de $3.34 \pm 0.005$. El comportamiento entre ambas noches se muestra en la Fig. 3.64.

Por último, estudiamos el diagrama color-magnitud (Fig. 3.65). Si bien los datos presentan barras de error grandes, tanto el ajuste lineal como el coeficiente de Pearson $(\mathrm{r}=0.681)$ indican una correlación moderada positiva de los mismos. Esto indicaría una tendencia BWB para este blazar. 

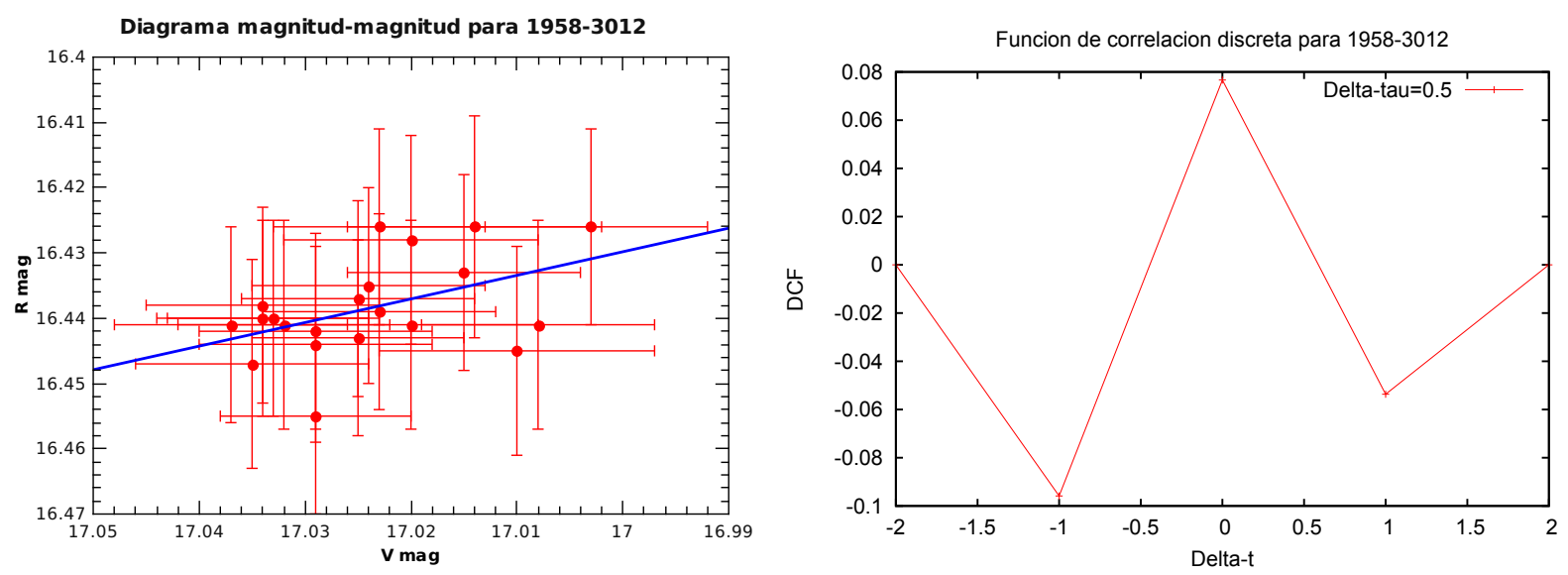

Figura 3.63. Diagrama magnitud-magnitud (izquierda) y función de correlación discreta para 1958-3012 (derecha).

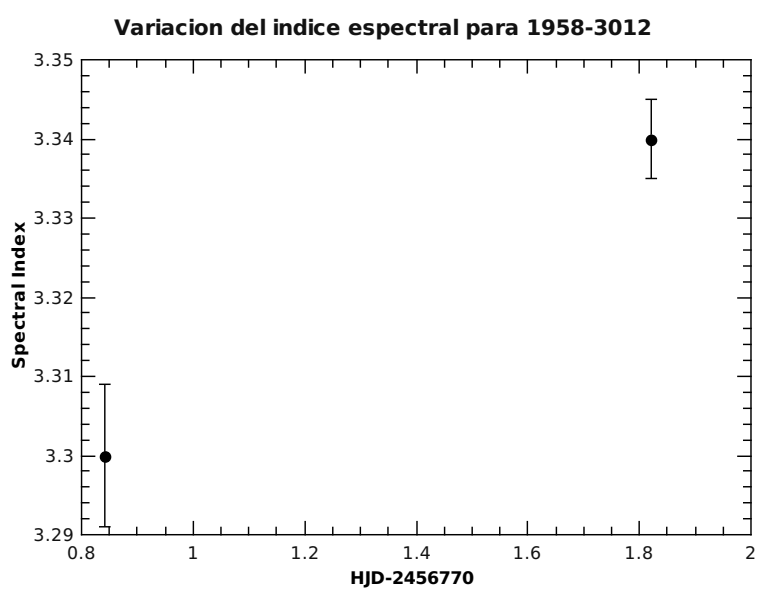

Figura 3.64. Variación del índice espectral para 1958-3012.

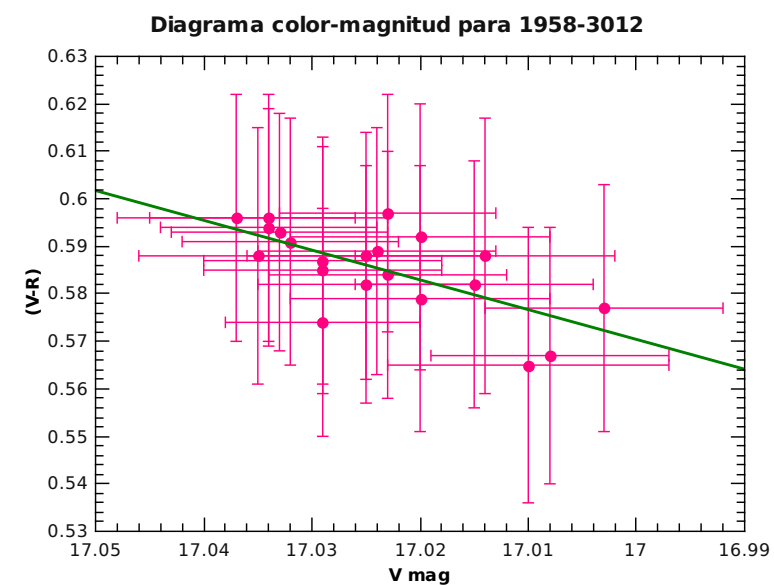

Figura 3.65. Diagrama color-magnitud para 1958-3012. 
* PKS 2005-489: es un BL Lac (HSP), con un valor de $\mathrm{z}=0.071$ (Falomo et al. 1987). En altas energías, fue detectado por el satélite EGRET en el rango de los 100 $\mathrm{MeV}$ (Lin et al. 1999). Se le detectaron dos flares potentes en rayos X a través del RXTE (Rossi X-Ray Timing Explorer, Remillard 1998; Perlman et al. 1999). Respecto a la banda óptica, Wall et al. (1986) reportaron variaciones a gran escala, con un $\Delta=$ 0.5 mag en la banda B, para dos conjuntos de datos espaciados en un año. En escalas temporales de días, Heidt \& Wagner (1996) mostraron amplitudes del $10 \%$ en la banda $\mathrm{R}$, con un valor promedio de 13.5. Resultados similares obtuvieron Rector \& Perlman (2003). Romero et al. (1999) no detectaron microvariabilidad para observaciones hechas en 1997. Dominici et al. (2004) presentaron por primera vez la curva de luz en el óptico a gran escala, desde 1996 hasta el 2000. Detectaron varios episodios de variabilidad con duraciones de aproximadamente 20 días. En escalas temporales de días, encontraron algunas correlaciones entre las curvas de luz en el óptico y las curvas en rayos X. Mahony et al. (2011) reportaron una magnitud $R=14.55$, mientras que Sandrinelli et al. (2014) estudiaron la variabilidad a gran escala, en búsqueda de quasi-periodicidades. Los valores en $\mathrm{V}$ y $\mathrm{R}$ serán mostrados más adelante.

Esta fuente fue observada durante una noche, 12 agosto 2015, en la cual se detectó variación en los datos. Para el filtro $\mathrm{V}$, se detectó variabilidad intra-noche para ambos parámetros $\mathrm{C}$ y $\mathrm{F}$, mientras que en el filtro $\mathrm{R}$, sólo se registró con el $\mathrm{F}$.

Por otra parte, obtuvimos los valores estándares para el blazar, en promedio resultaron $14.746 \pm 0.015$ para el filtro V y $14.383 \pm 0.021$ para el filtro $R$. Las variaciones en la magnitud estándar del blazar, como se observa en la Fig. 3.66, resultaron $\Delta m(V)=0.075$ y $\Delta m(R)=0.066$.

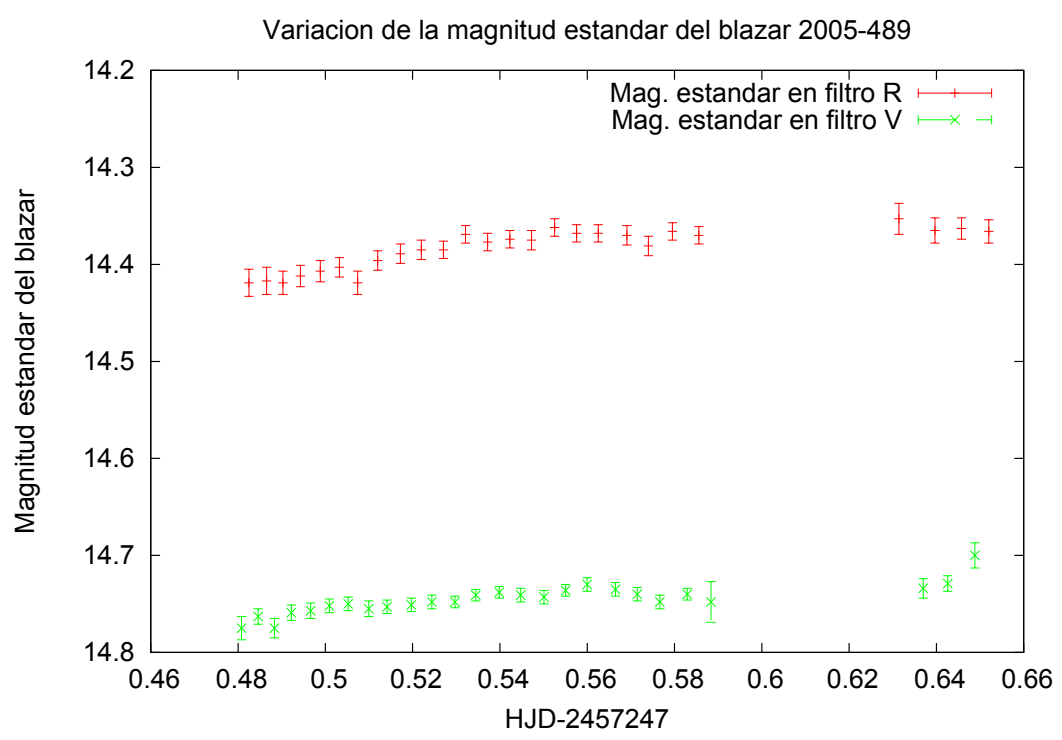

Figura 3.66. Curva de luz de la magnitud estándar de 2005-489 en el filtro R (rojo) y en el V (verde).

En la Fig. 3.67 comparamos nuestros resultados con lo que está publicado en la literatura. Vemos que la fuente presenta episodios en donde su brillo crece y luego decae. Y nuestros son comparables con los que halló Sandrinelli et al. (2014).

Analizando el diagrama magnitud-magnitud, Fig. 3.68, es clara la correlación que 

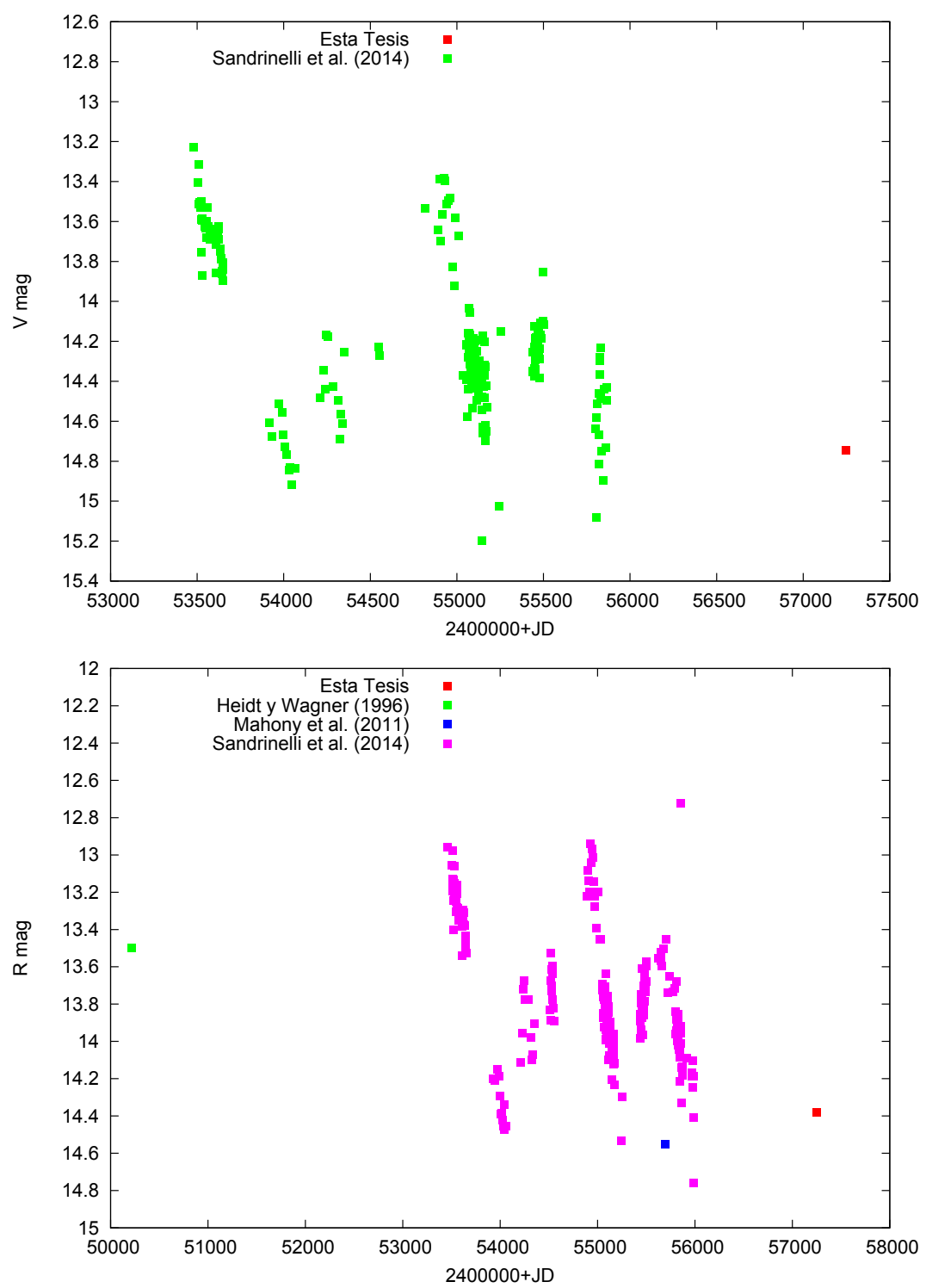

Figura 3.67. Valores comparativos para la magnitud estándar V (panel superior) y R (panel inferior) del blazar PKS 2005-489.

presentan los datos para este caso. Esto se corrobora con el ajuste lineal (Tab. 3.4) y el valor de la función $(\mathrm{DCF}=0.285 \pm 0.084)$.

El diagrama color-magnitud se presenta en la Fig. 3.69. Tanto el ajuste lineal como el coeficiente de Pearson $(\mathrm{r}=0.370)$ indican una correlación negativa débil. Por lo tanto, no podemos concluir que se tiene una tendencia RWB. Por otra parte, el índice espectral para esta fuente adquiere un valor promedio de $\alpha_{V R}=2.06 \pm 0.014$.

* [HB89] 2126-158: este blazar está clasificado como un FSRQ, con un z=3.269 (Jauncey et al. 1978) y es uno de los quasars más brillante, con $\mathrm{V}=17.3$ (Condon et al. 1977). Francis et al. (2000) reportaron valores para las magnitudes V y R de $16.92 \pm 0.05$ y $16.63 \pm 0.05$, respectivamente. Por otra parte, Ellison et al. (2005) obtuvieron un valor de $V=16.66 \pm 0.05$, mientras que Mahony et al. (2011) obtienen un valor para $R$ de 17.41. 

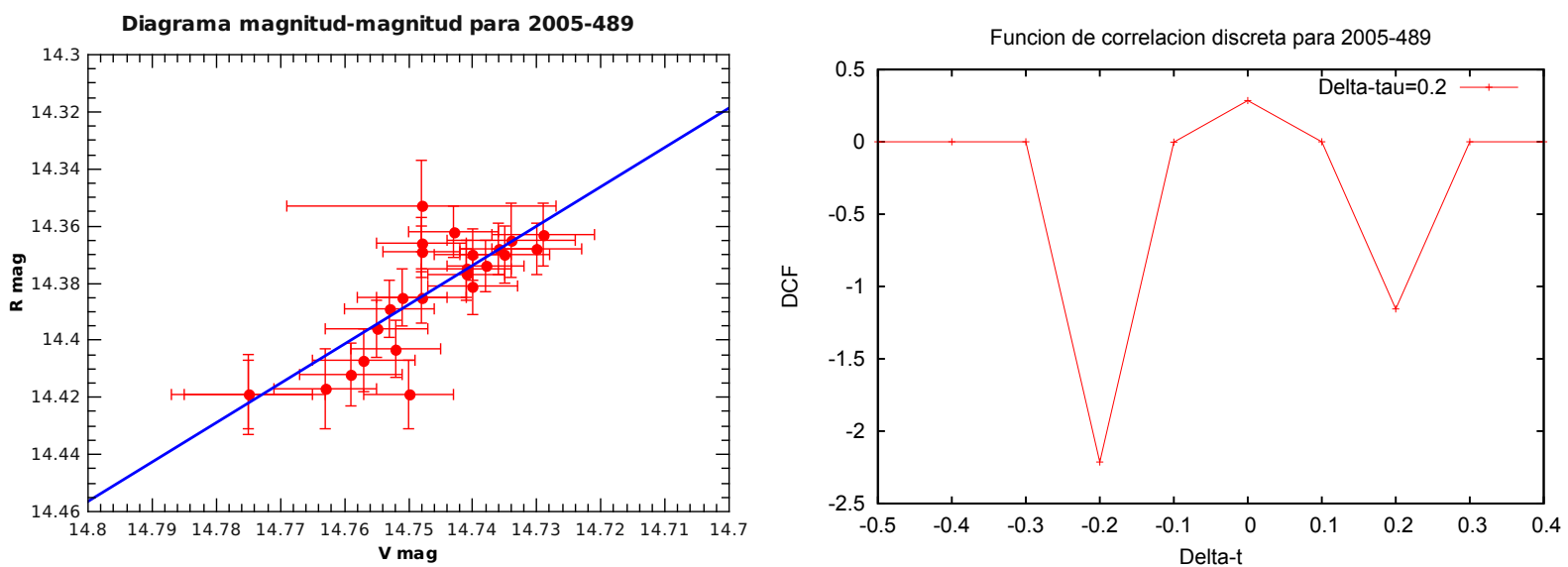

Figura 3.68. Diagrama magnitud-magnitud (izquierda) y función de correlación discreta para 2005-489 (derecha).

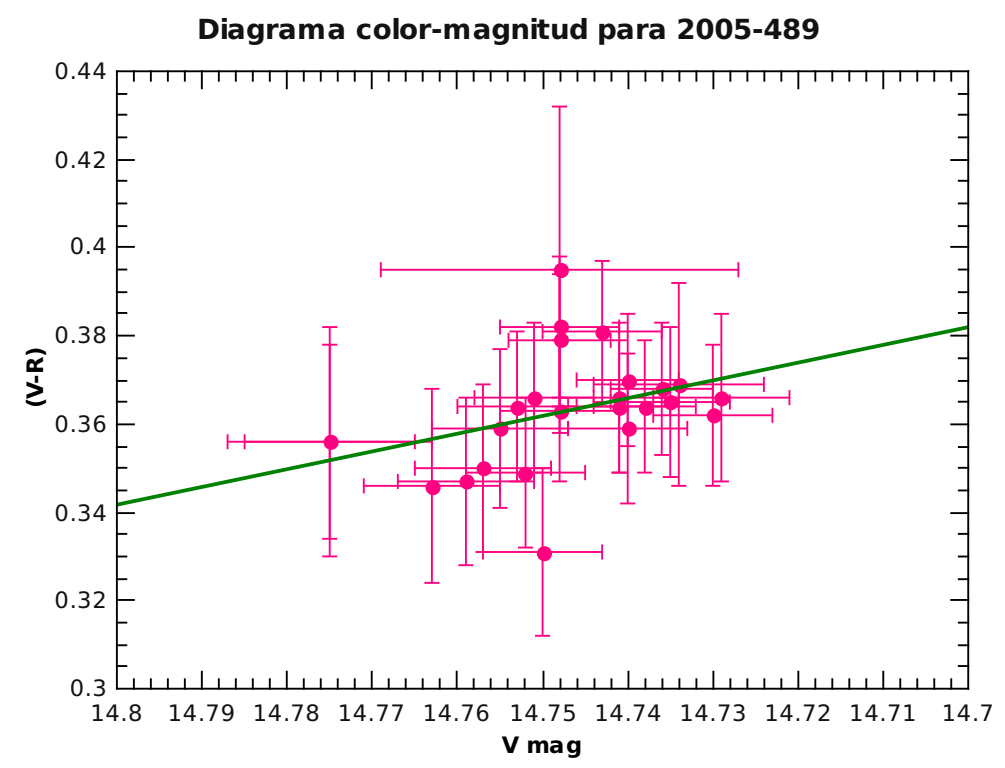

Figura 3.69. Diagrama color-magnitud para 2005-489.

Respecto a ésta última, Borgeest \& Schramm (1994) estudiaron la variabilidad en R, sin encontrar variación alguna en sus datos. En la banda de rayos X, este blazar es el más brillante con z $>3$ y es el segundo más brillante, después de PKS 2149-306, con z>2 (Fiore et al. 2003). Este el objeto de la muestra seleccionada con el valor de redshift más alto.

Esta fuente fue seguida durante dos noches, 15 y 17 septiembre 2015. En lo que respecta a variaciones intra-noche, en ninguna de las dos noches se detecta variabilidad, al igual que en el caso de variabilidad inter-noche. Tanto el criterio $\mathrm{C}$ como el test $\mathrm{F}$ coinciden en la no detección de variabilidad en ambas escalas.

Las magnitudes estándares que se obtuvieron para el blazar son 17.074 \pm 0.014 para el filtro V y 16.814 \pm 0.014 para el filtro R. Comparando nuestros valores con los publicados en la literatura, vemos que la fuente varió en su brillo, tanto en $\mathrm{V}$ como en $\mathrm{R}$. 
En la Fig. 3.71 mostramos este comportamiento.

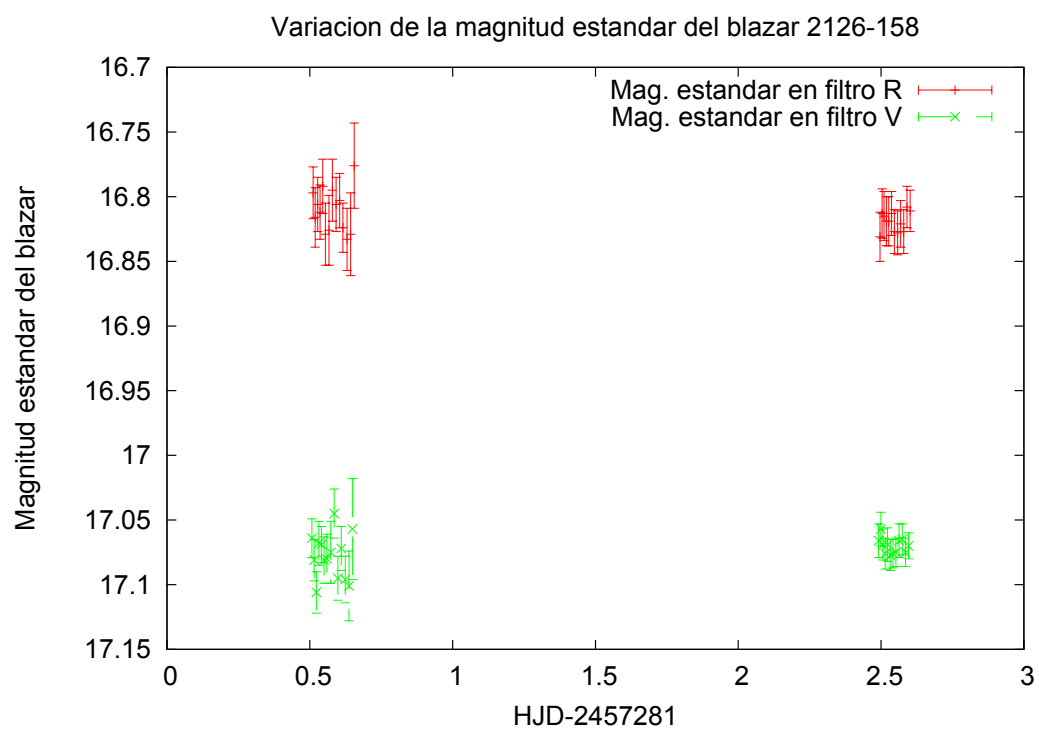

Figura 3.70. Curva de luz de la magnitud estándar de 2126-158 en el filtro R (rojo) y en el V (verde).

En cuanto al comportamiento del diagrama magnitud-magnitud, no hay coincidencia entre el ajuste lineal y la función de correlación. El diagrama, Fig. 3.72, muestra una correlación, aunque pequeña y los datos presentan errores grandes en ambas magnitudes. El ajuste da una recta de pendiente positiva pero con un valor del $\chi^{2}$ reducido cercano a 0 , con lo que el ajuste no resulta confiable. Por otra parte, la función toma un valor negativo, $\mathrm{DCF}=-0.215 \pm 0.087$, indicando una anticorrelación.

Con las magnitudes estándares, calculamos el índice espectral. Para la primer noche, obtuvimos un valor de $\alpha_{V R}=1.52 \pm 0.017$, mientras que para la segunda resultó $1.42 \pm 0.010$ (Fig. 3.73). Su valor promedio fue de $\alpha_{V R}=1.48 \pm 0.016$. Para los datos, Francis et al. (2000) obtenemos un valor de $\alpha_{V R}=1.65 \pm 0.016$, comparable a lo que obtuvimos.

Respecto al diagrama color-magnitud, el coeficiente de Pearson $(\mathrm{r}=0.701)$ al igual que el ajuste lineal coinciden en que se tiene una correlación positiva moderada, lo que indicaría que este FSRQ tiene una tendencia BWB.

* PKS 2149-306: está catalogado como FSRQ, con un valor de $\mathrm{z}=2.345$ (Wilkes 1986). En cuanto a los estudios en la banda óptica, no hay evidencia de variaciones en ninguna de las bandas, teniendo magnitudes en $\mathrm{V}=17.75 \pm 0.01$ y $\mathrm{R}=17.31 \pm 0.05$ (Francis et al. 2000). Por su parte, Mahony et al. (2011) reportaron una magnitud $\mathrm{R}=17.27$. En la banda de rayos $\mathrm{X}$, diversos autores lo han estudiado, encontrando que es un fuerte emisor en esta banda y además han registrado variaciones significativas (Siebert et al. 1996; Cappi et al. 1997; Ferrero \& Brinkman 2003; Sambruna et al. 2007; Bianchin et al. 2009; Soldi et al. 2014). Por otro lado, este objeto es uno de los 64 blazars con redshift alto $(\mathrm{z}>2)$ reportado por el catálogo de Fermi (3FGL, Acero et al. 2015).

Esta fuente fue observada durante dos noches, 12 agosto y 17 septiembre 2015. En 

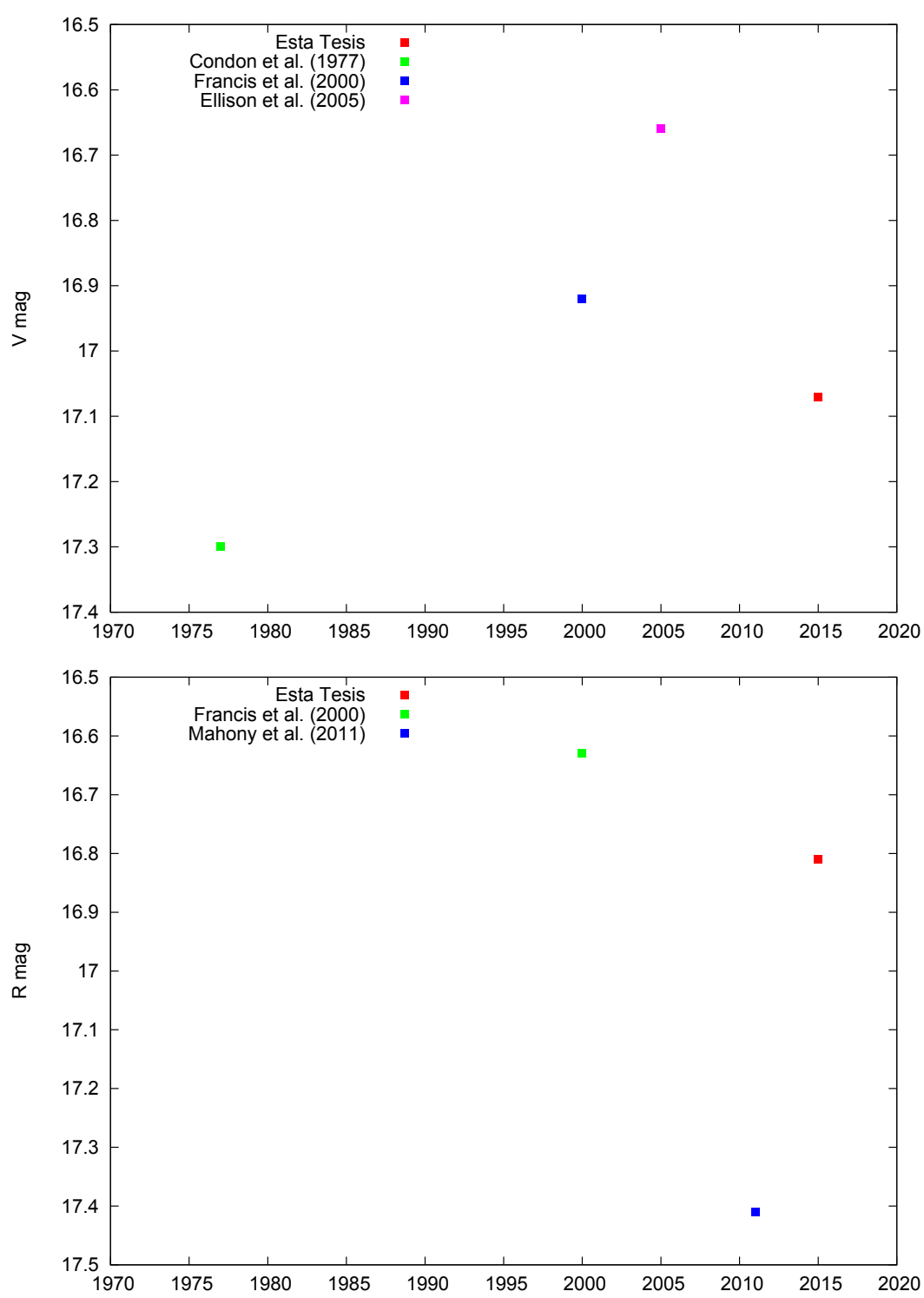

Figura 3.71. Valores comparativos para la magnitud estándar V (panel superior) y R (panel inferior) del blazar [HB89] 2126-158.

ambas noches se obtuvieron muy pocas observaciones. En ambas oportunidades el objetos presentó una magnitud muy débil, lo que llevó a mayores tiempos de integración. A esto se le sumó la interrupción de las observaciones durante agosto debido a malas condiciones climáticas (nevada). No se detectó variabilidad intra-noche para ninguna de las dos noche de observación. En cuanto a la variabilidad a escala de meses, tampoco se registran variaciones.

Se obtuvieron las magnitudes estándares para el blazar: $17.743 \pm 0.020$ para el filtro V y $17.535 \pm 0.024$ para el filtro $R$ (ver Fig. 3.75). obtuvieron $V=17.75 \pm 0.01$ y $\mathrm{R}=17.31 \pm 0.05$, mientras que Mahony et al. (2011) registraron $\mathrm{R}=17.27$. El valor que calculamos para la magnitud V es similar al de Francis et al. (2000), mientras que nuestro valor de $\mathrm{R}$ es mayor a los reportados por los otros autores, teniendo la magnitud más débil. 

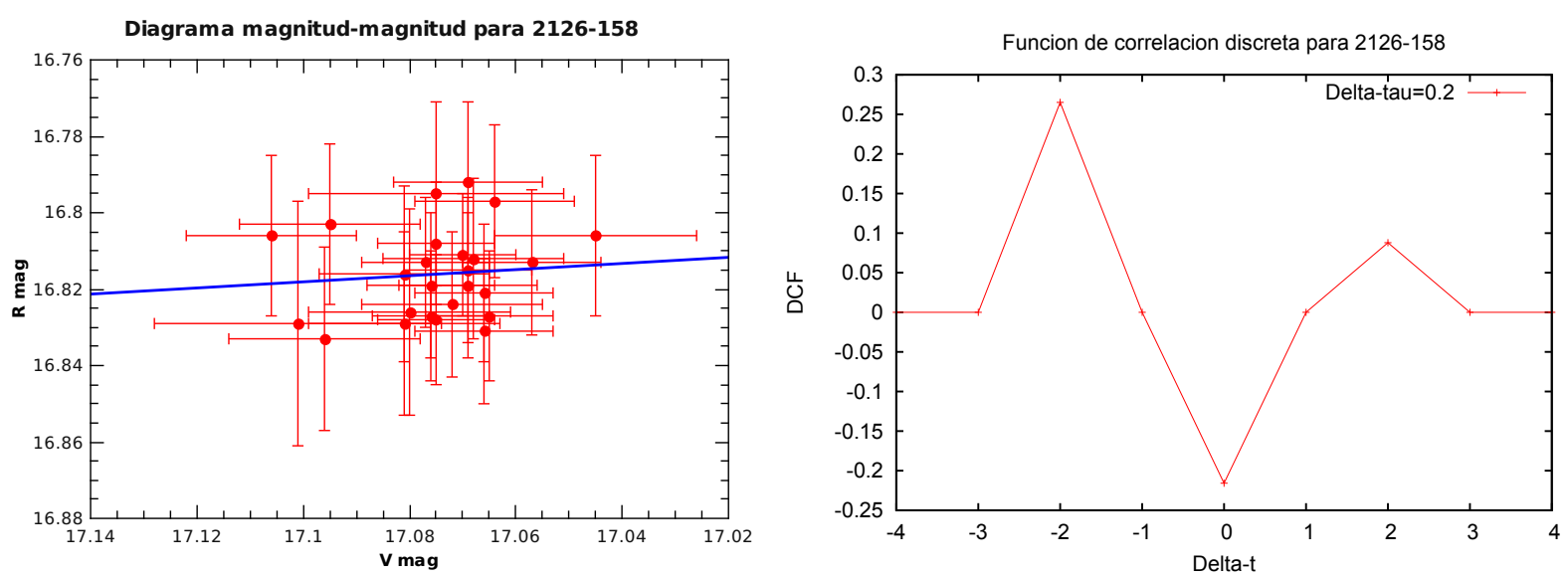

Figura 3.72. Diagrama magnitud-magnitud (derecha) y función de correlación discreta para 2126-158 (izquierda).

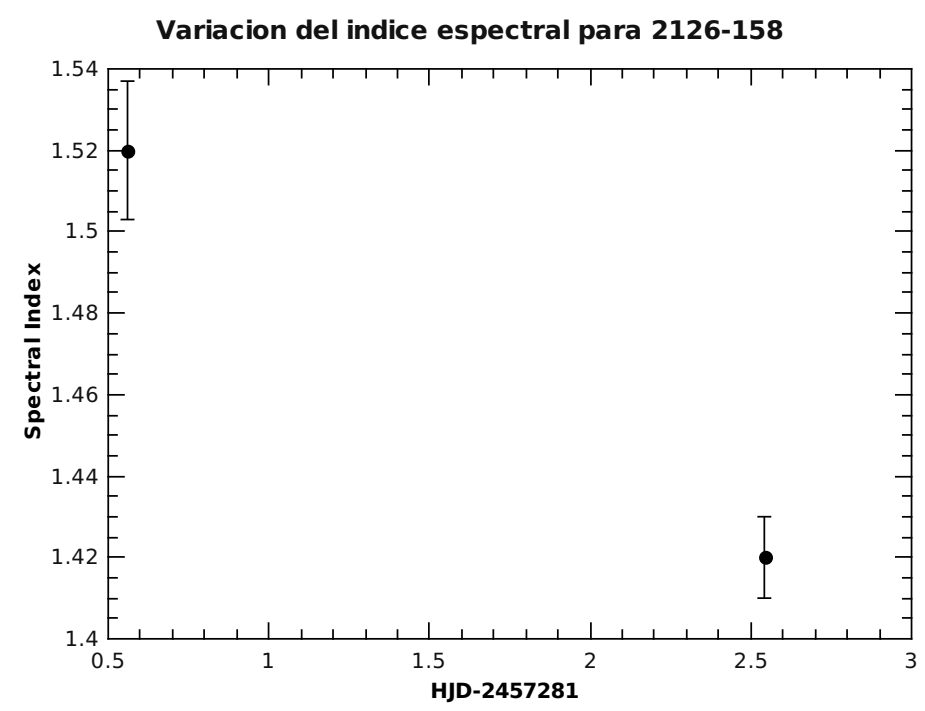

Figura 3.73. Variación del índice espectral para 2126-158.

Respecto al diagrama magnitud-magnitus, por una parte, se obtiene una recta de pendiente positiva pero con un error muy alto en su cálculo y un valor de $\chi^{2}$ reducido bajo (ver Tab. 3.4). Por la otra, el valor de la DCF resulta negativo ( $\mathrm{DCF}=-0.182 \pm 0.234$ ), con una incerteza mayor que el propio valor. La Fig. 3.76 refleja la no coincidencia poco definida.

En cuanto al índice espectral, no hay mucha variación en su valor, que va desde 1.10 a 1.23 (Fig. 3.77) y siendo su valor promedio $\alpha_{V R}=1.18 \pm 0.021$. De los datos publicado por Francis et al. (2000), podemos calcular el índice espectral a partir de sus valores publicado de $\mathrm{V}$ y R. El índice resultó $\alpha_{V R}=2.5 \pm 0.06$, lo que implica que, al momento de nuestras observaciones, la distribución espectral es más blanda respecto a los datos de este autor.

Por último, para el diagrama color-magnitud (Fig. 3.78) tanto el ajuste lineal como el coeficiente de Pearson $(\mathrm{r}=0.265)$ concuerdan con que se tiene una correlación posi- 


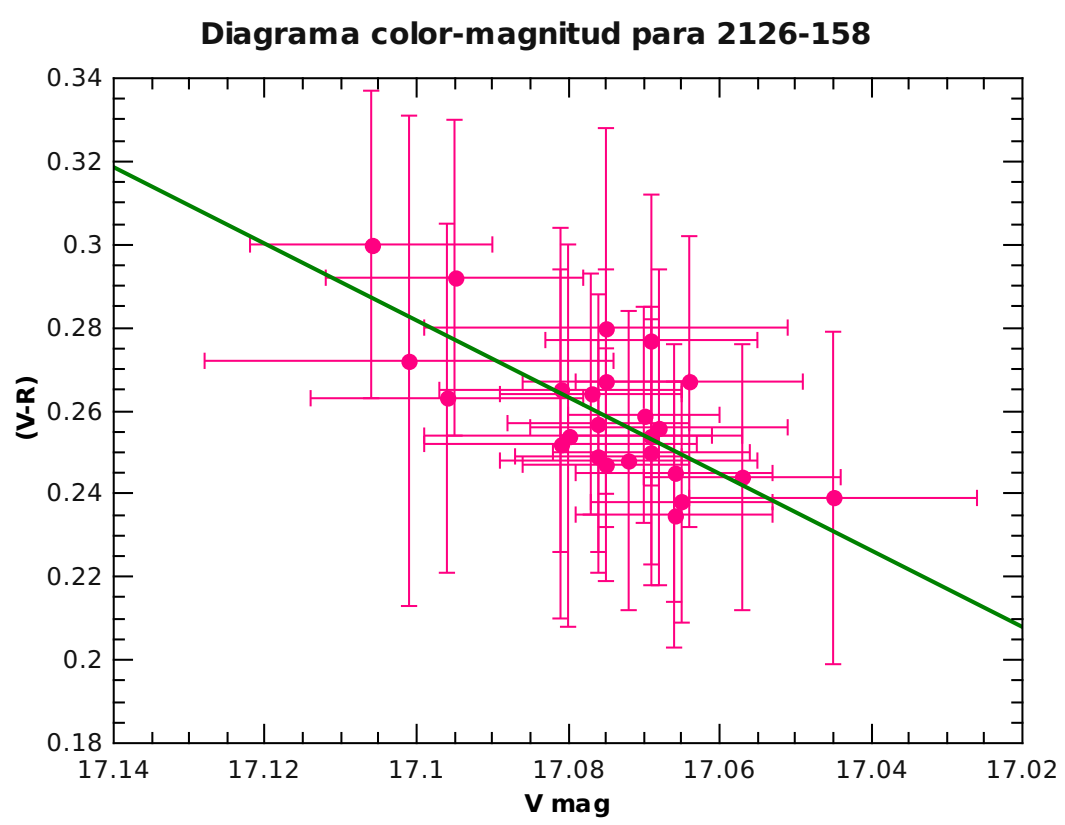

Figura 3.74. Diagrama color-magnitud para 2126-158.

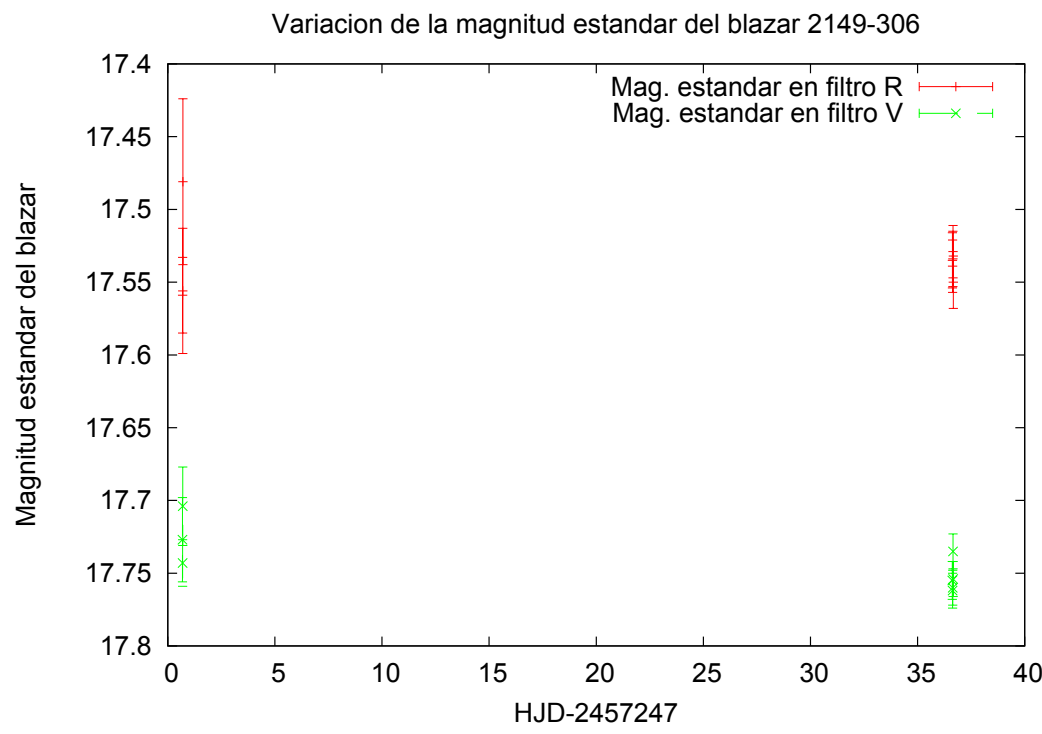

Figura 3.75. Curvas de luz de la magnitud estándar de 2149-306 en el filtro R (rojo) y en el V (verde).

tiva débil, lo que implicaría una tendencia BWB para este blazar.

* PKS 2155-304: está catalogado como BL Lac (HSP) y con un redshift de $z=$ 0.116 (Falomo, Pesce \& Treves, 1993). Es uno de los BL Lacs más brillantes en rayos X y EUV (Giommi et al. 1998). Fue clasificado como un TeV blazar con la detección de rayos- $\gamma$ de VHE (Chadwick et al. 1999) y luego confirmado por la colaboración H.E.S.S. con un nivel de significancia de $45 \sigma$ para energías mayores a $160 \mathrm{GeV}$ (Aharonian et al. 2005). Esta fuente ha sido observada en diversas escales temporales sobre un gran 

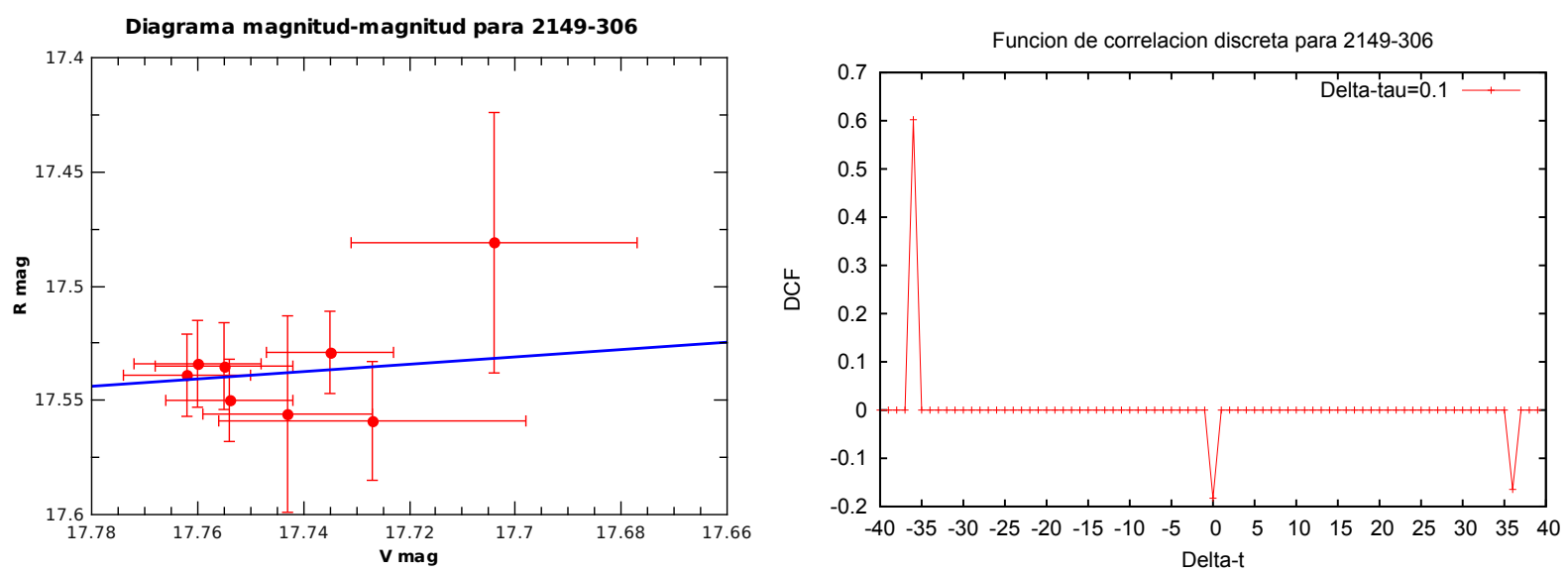

Figura 3.76. Diagrama magnitud-magnitud (izquierda) y función de correlación discreta para 2149-306 (derecha).

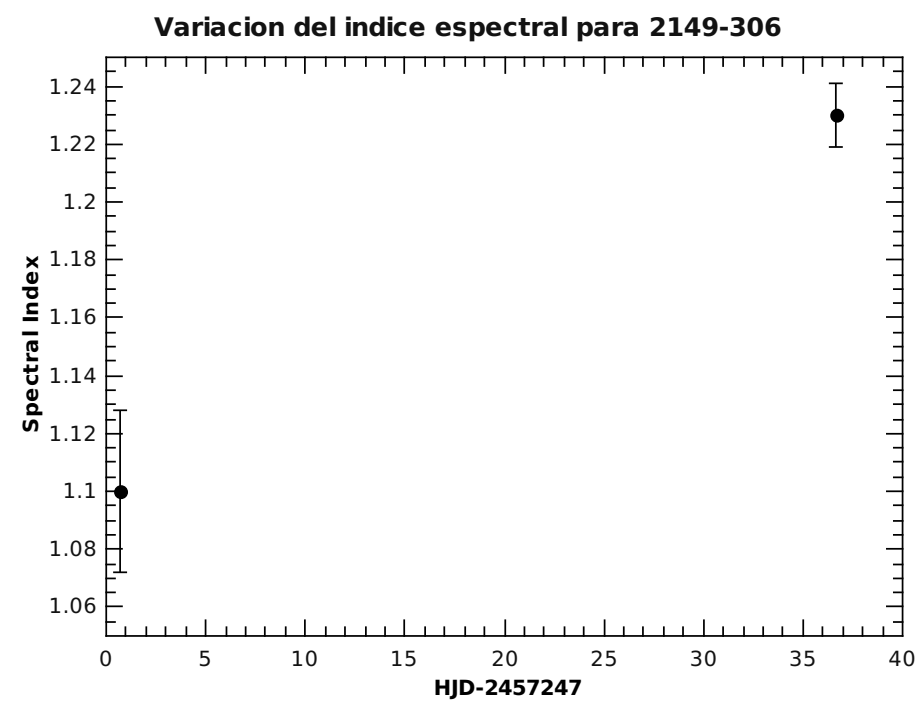

Figura 3.77. Variación del índice espectral para 2149-306.

rango de frecuencias desde radio hasta rayos- $\gamma$ VHE, y ha mostrado variabilidad fuerte y rápida (Dominici et al. 2006; Dolcini et al. 2007; Aharonian et al. 2007; Abramowski et al. 2010; Kastendieck et al. 2011; Abramowski et al. 2012; Aleksíc et al. 2012; Barkov et al. 2012). Son numerosos los trabajos que estudiaron a este blazar en cuanto a sus propiedades ópticas y su variabilidad. Tanto los valores de $\mathrm{V}$, como de $\mathrm{R}$ y $\mathrm{V}$ $\mathrm{R}$ encontrados en los siguientes trabajos, se muestran en la Fig. 3.80: Griffith et al. (1979) obtuvieron valores de la magnitud V en datos de 1978; Miller \& McAllister (1983) reportaron variabilidades rápidas $(\Delta m<0.08$ mag en dos días), como así también variaciones más largas ( $\Delta m \sim 0.75$ mag en 4 años); Hamuy \& Maza (1987) estudiaron a esta fuente en los filtros UBVRI, encontrando variaciones en $\mathrm{V}$ y $\mathrm{R}$ de $\Delta m=0.4$ mag (entre 12.9 y 13.3 ) y $\Delta m=0.5$ mag (entre 12.1 y 12.6), respectivamente; Carini \& Miller (1992) reportaron variabilidad óptica correspondiente a observaciones entre 1972 y 1990, a escalas temporales de varios minutos hasta años; Smith et al. (1992) 


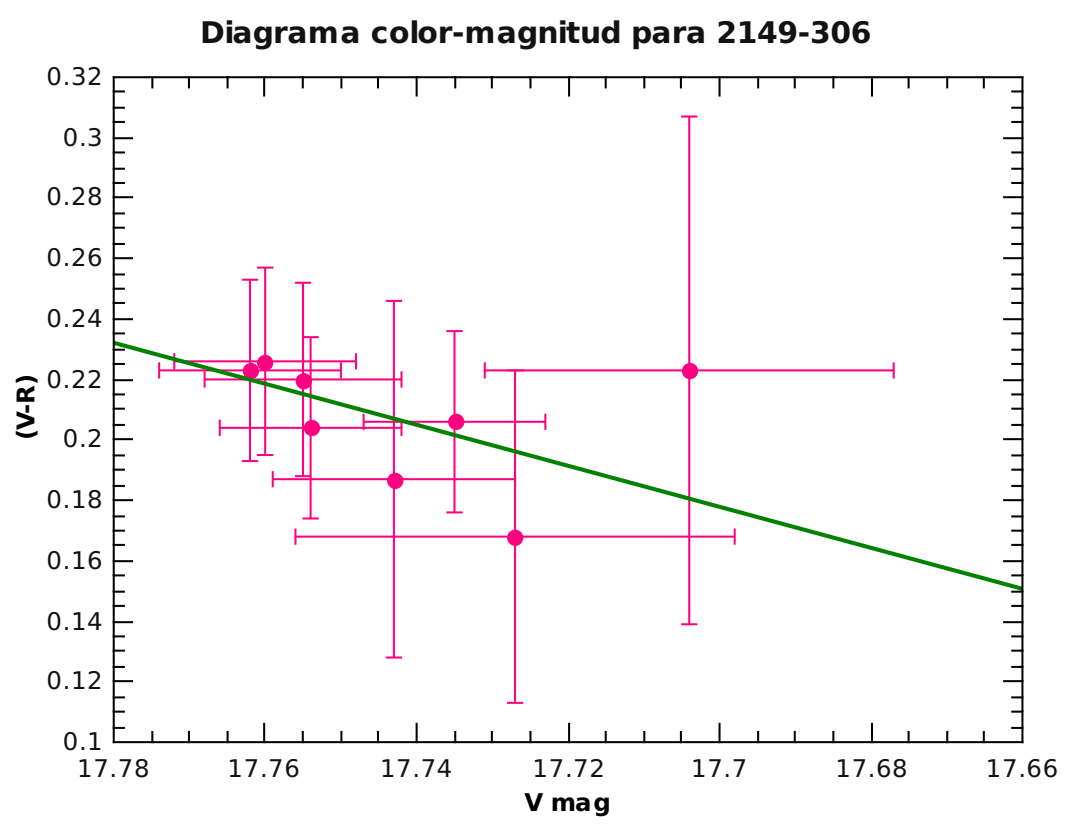

Figura 3.78. Diagrama color-magnitud para 2149-306.

y Jannuzi et al. (1993) estudiaron propiedades fotométricas y polarimétricas de esta fuente; Heidt et al. (1997) reportaron variaciones presentes en observaciones de 1990 y 1994, encontrando particularmente en Junio 1990 variaciones inter-noche; Paltani et al. (1997) encuentran escalas temporales asociadas a la variabilidad de este blazar de 15 min, y escalas mayores, entre 10 y 40 días; Romero et al. $(1999,2002)$ estudiaron las variaciones óptica en el filtro V, sin detectar microvariabilidad; Dominici et al. (2004) obtuvieron curvas de luz en el período 1996-2000 donde encontraron épocas de baja actividad en cuanto a la variabilidad, seguido de períodos de actividad alta, pero sin encontrar periodicidades. Respecto a esto último, son varios los trabajos que reportan periodicidades y quasi-periodicidades presentes en este blazar, que van desde 0.7 días hasta 315 días (Ulmer et al. 1993; Fan \& Lin 2000; Lachowicz et al. 2009; Zhang et al. 2014; Sandrinelli et al. 2014, 2016).

Este blazar fue observado durante dos noches, 13 agosto y 15 septiembre 2015. En cuanto a la variabilidad (intra-noche), se detectan variaciones en ambas noches (agosto y septiembre), tanto en el filtro $\mathrm{V}$ como en el filtro R. Considerando ahora escalas de meses, también se detecta variabilidad de gran amplitud entre los meses de agosto y septiembre, registrados en los dos filtros. Estos resultados de variabilidad fueron detectados por el criterio $\mathrm{C}$ y el test $\mathrm{F}$.

Obtuvimos las magnitudes estándares, con valores de $14.152 \pm 0.150$ para el filtro V y $14.010 \pm 0.150$ para el filtro R. La Fig. 3.79 muestra la variación significativa que se da en la magnitud del blazar, teniendo una importante amplitud de $\Delta m(V)=0.387 \mathrm{y}$ $\Delta m(R)=0.345$.

De la Fig. 3.80, vemos que existe un comportamiento similar entre ambas magnitudes, donde el brillo de la fuente crece en el período 1979-2000, para luego decaer entre 2005-2015. Además de este comportamiento a gran escala, se pueden observar fluctuaciones intermedias, donde el brillo de la fuente crece y decrece. Los valores obtenidos en la presente Tesis son comparables con los obtenidos por Sandrinelli et al. (2014). 


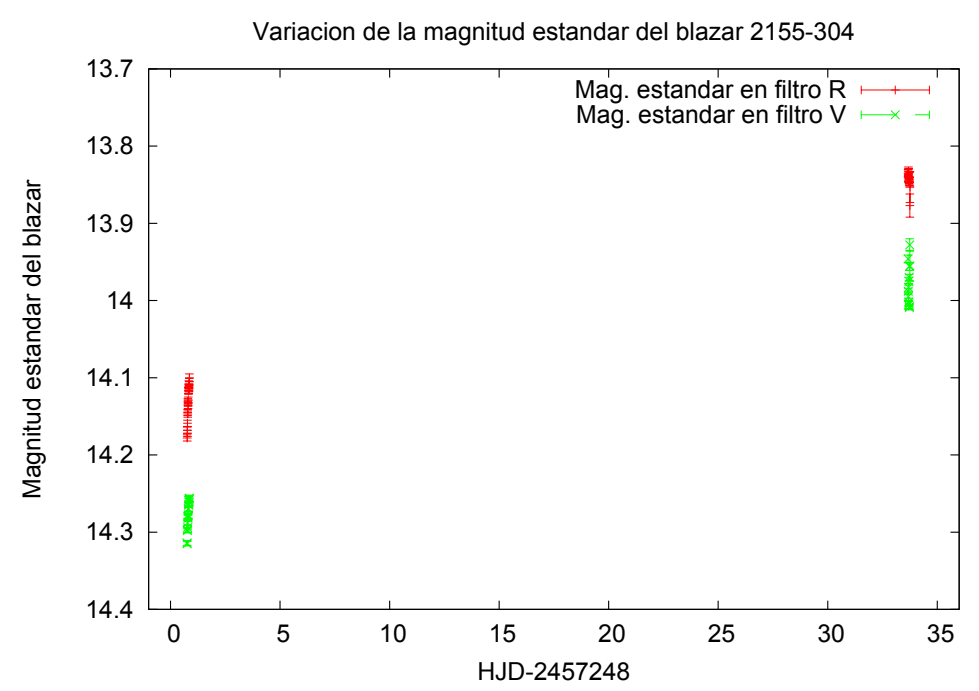

Figura 3.79. Curvas de luz de la magnitud estándar de PKS 2155-304 en el filtro $\mathrm{R}$ (rojo) y en el V (verde).

Por otra parte, se puede inferir que entre los años 1995 y 2005, el blazar presentó la época de mayor actividad en su variabilidad.

Analizamos el diagrama magnitud-magnitud, que se muestra en la Fig. 3.81. Vemos que existe una fuerte correlación entre los datos en V y R. El ajuste lineal (Tab. 3.4), con una pendiente positiva y un valor del $\chi^{2}$ reducido casi de 1 corroboran junto a los obtenido para la función DCF la correlación hallada. Además el coeficiente de Pearson indica que esta correlación es fuerte.

En cuanto al comportamiento del índice espectral, obtuvimos un valor promedio de $\alpha_{V R}=0.81 \pm 0.023$, con $0.83 \pm 0.008$ en la primer noche y $0.77 \pm 0.033$ en la segunda noche (Fig. 3.82, panel izquierdo).

A partir de los datos publicados en $\mathrm{V}$ y $\mathrm{R}$, podemos analizar el comportamiento de $\alpha_{V R}$ a lo largo de los años, como se muestra en la Fig. 3.82 (panel derecho). Comparando con las variaciones obtenidas para las magnitudes $\mathrm{V}$ y $\mathrm{R}$, vemos que $\alpha_{V R}$ crece (en el mismo período que crecen $\mathrm{V}$ y $\mathrm{R}$ ), y luego decrece (también coincidiendo con el período de decaimiento del brillo de la fuente). Esto implica que cada vez que la fuente se vuelve más débil, el valor del índice $\alpha_{V R}$ decrece, lo que implica que la distribución espectral de energía en ese rango se vuelve más blanda. En cambio, cuando la fuente se vuelve más brillante, $\alpha_{V R}$ crece, lo que corresponde a un espectro más endurecido.

Finalmente, estudiamos el diagrama color-magnitud, en la Fig. 3.83, para cada una de las noches de observación. En el caso de las observaciones de agosto 2015, se tiene que el coeficiente de Pearson $(\mathrm{r}=-0.526)$ indica una correlación moderada negativa, indicando que la fuente posee una tendencia RWB. Para los datos de septiembre 2015, el coeficiente de Pearson $(\mathrm{r}=0.465)$ implica una correlación débil positiva, asociada a una tendencia BWB. Con lo cual, de un mes a otro, el objeto estaría cambiando su comportamiento. En cuanto a su comportamiento global a escala de meses, el coeficiente arroja un valor de $\mathrm{r}=0.314$, indicando una correlación débil positiva, lo que implica que la fuente presentaría una tendencia BWB. 

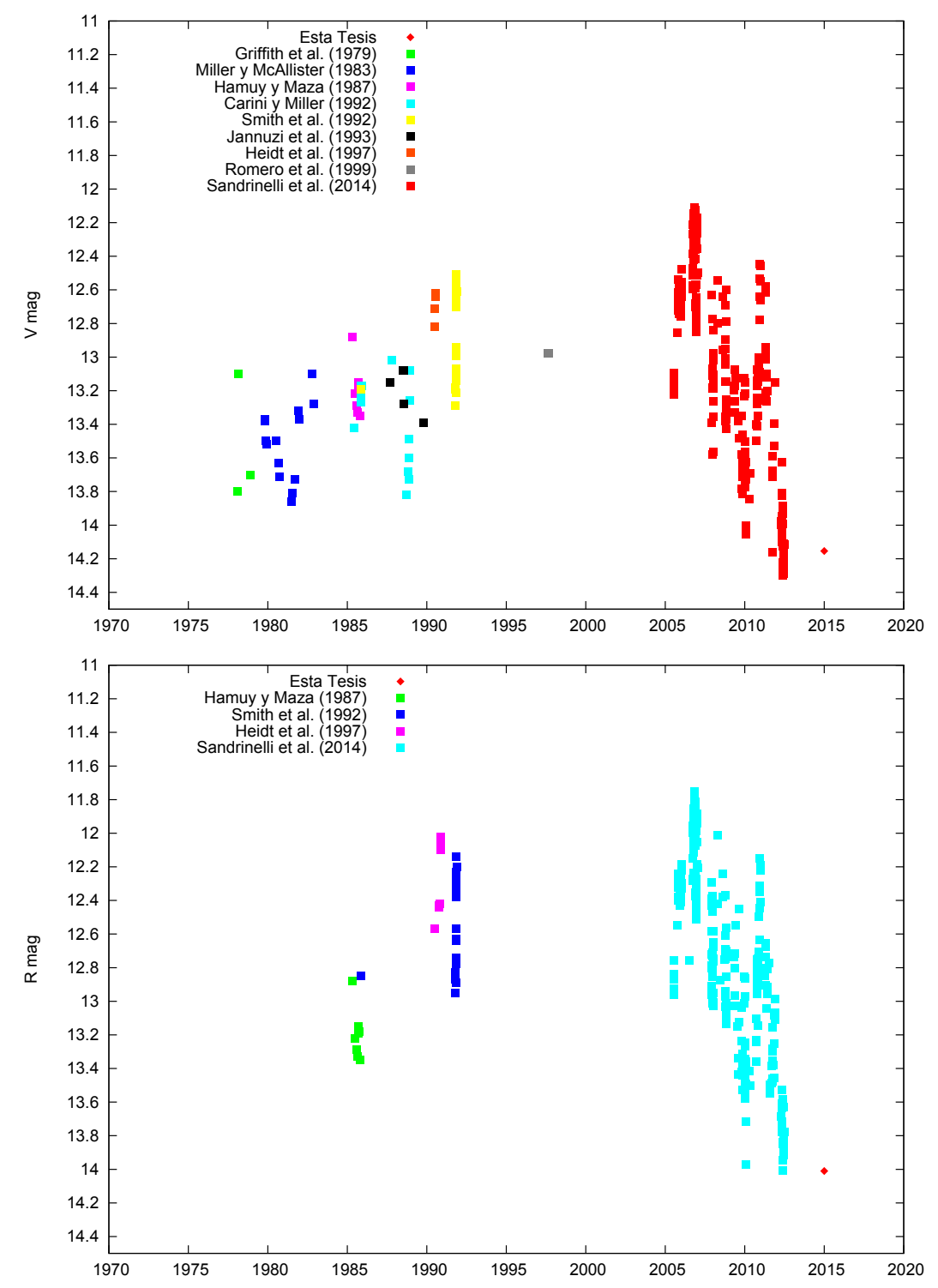

Figura 3.80. Valores comparativos para la magnitud estándar V (panel superior) y R (panel inferior) del blazar PKS 2155-304. 

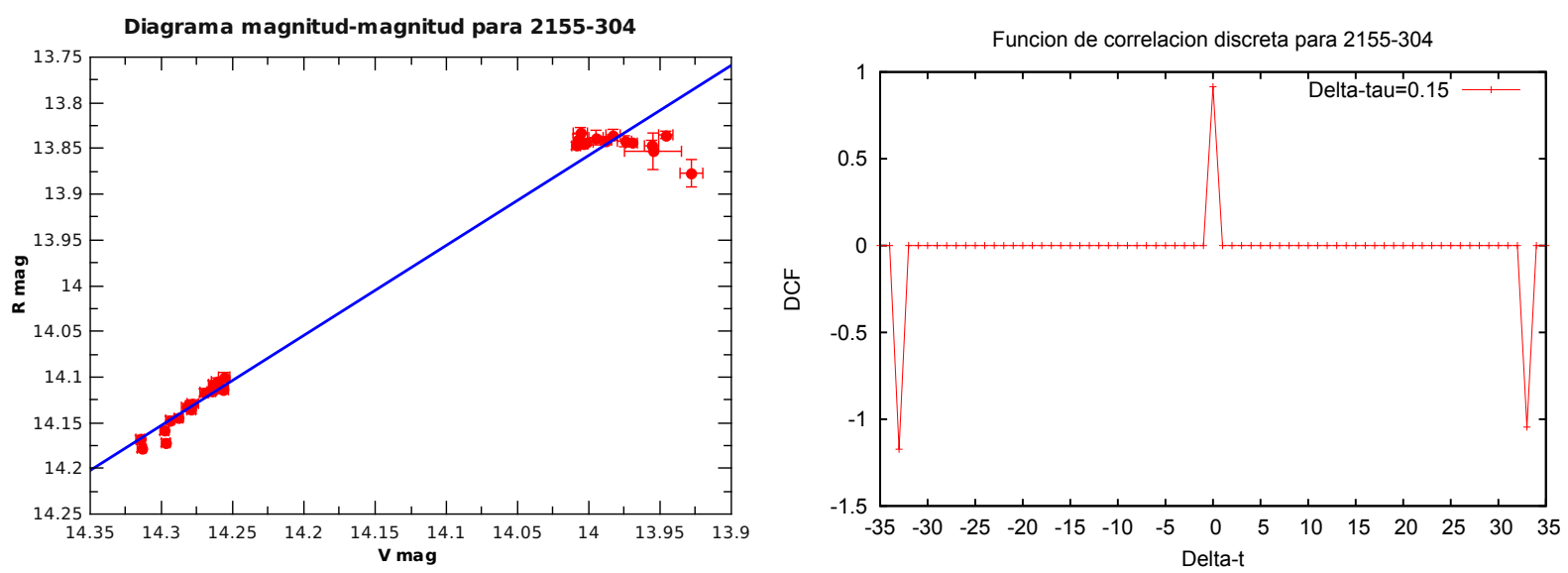

Figura 3.81. Diagrama magnitud-magnitud (izquierda) y función de correlación discreta para 2155-304 (derecha).
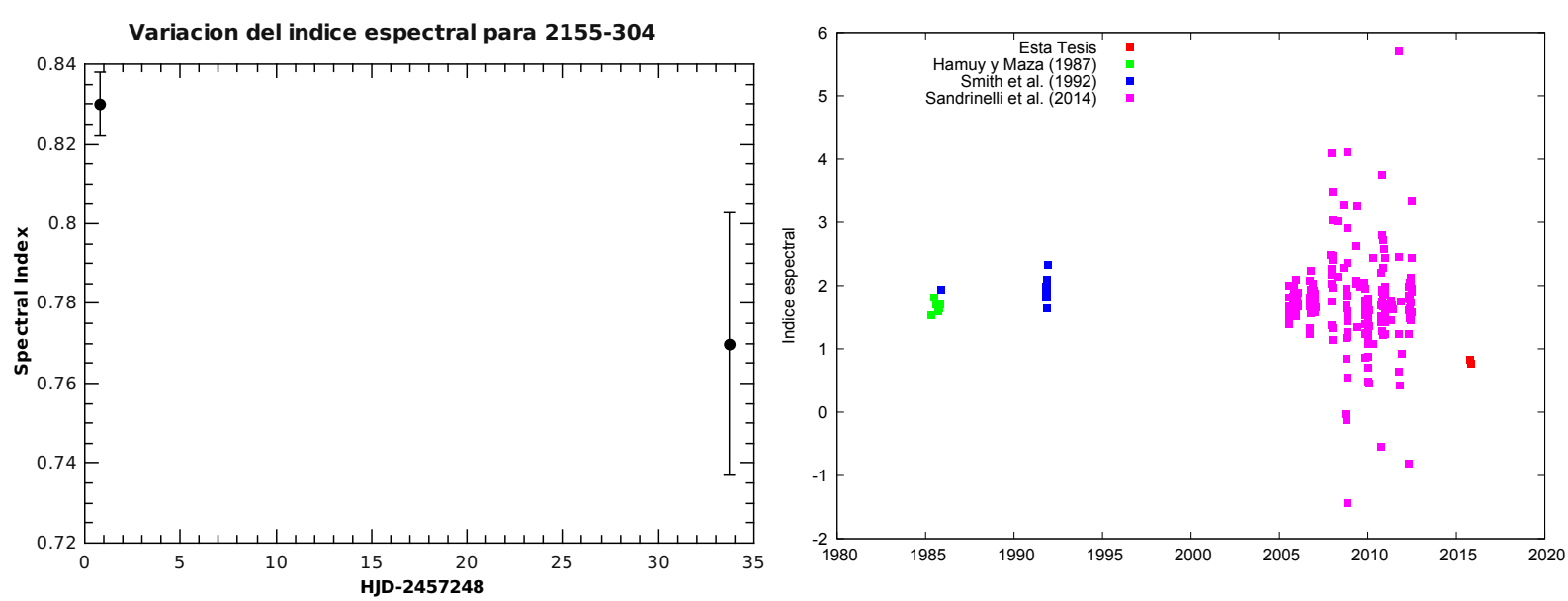

Figura 3.82. Índice espectral. Panel izquierdo: Variación del índice espectral para 2155-304. Panel derecho: Comparación del índice espectral para 2155-304 a lo largo de los años. 
Diagrama color-magnitud para 2155-304 para 13.08.15

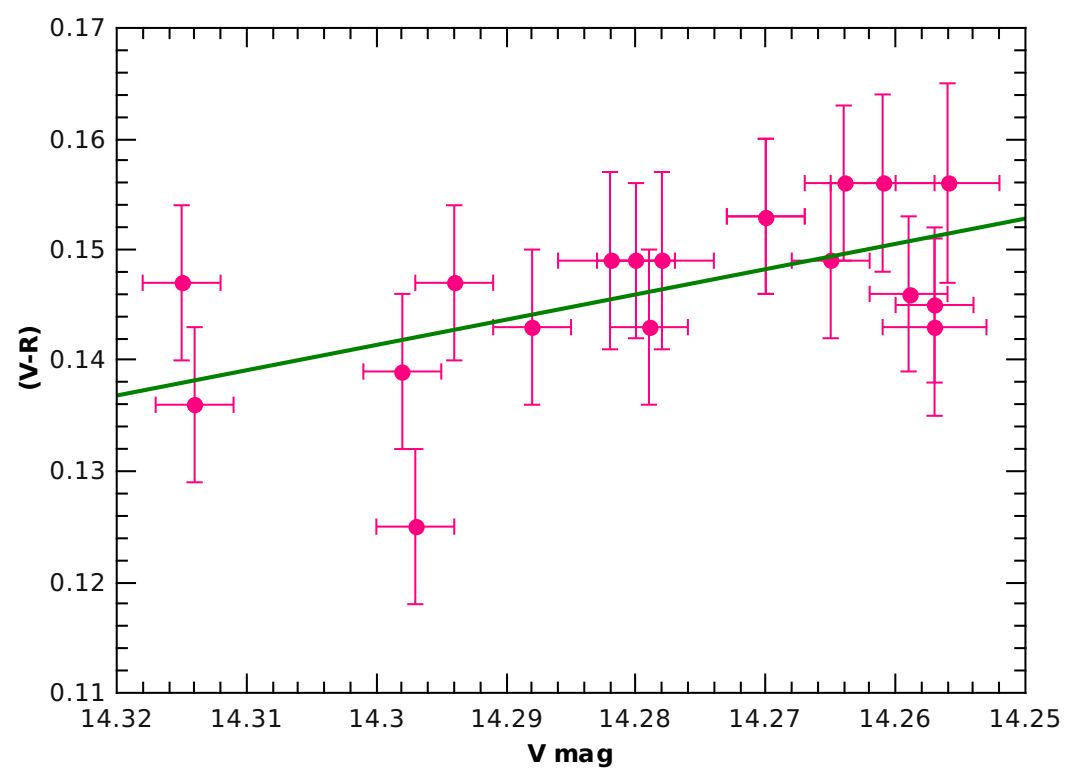

Diagrama color-magnitud para 2155-304 para 15.09.15

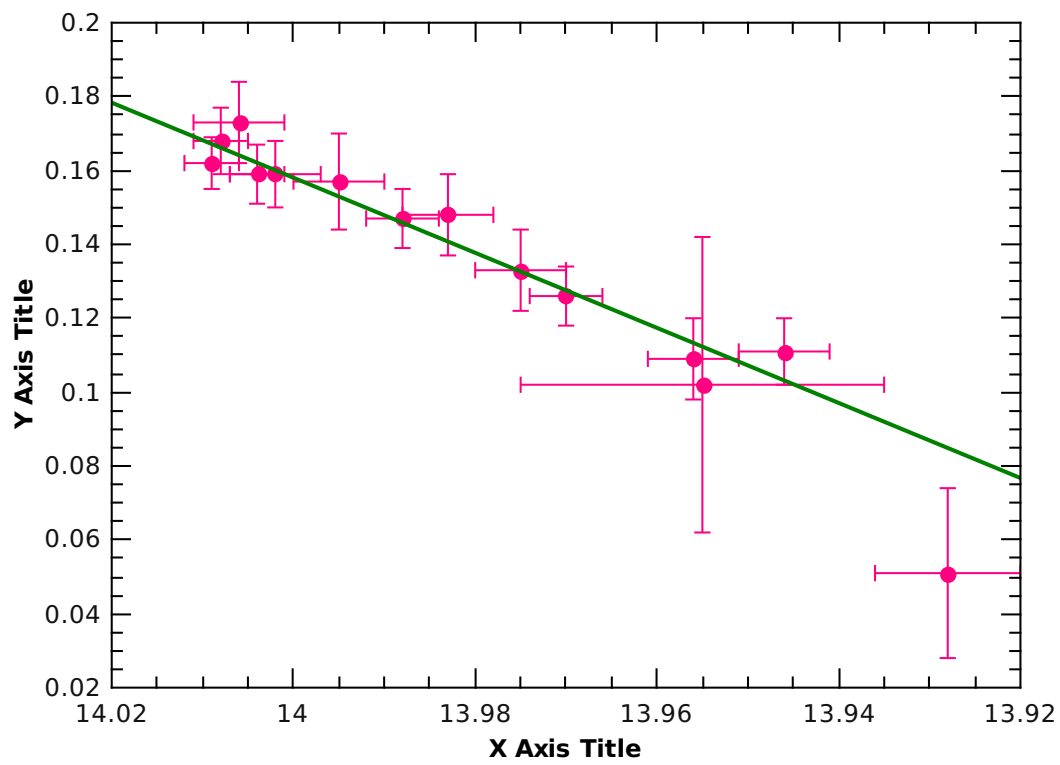

Figura 3.83. Diagrama color-magnitud para 2155-304. Panel superior: diagrama correspondiente a los datos de agosto 2015. Panel inferior: diagrama correspondiente a septiembre 2015 . 
* PMN J2310-4347: clasificado como BL Lac, esta fuente posee un valor de $\mathrm{z}=0.0886$ (Tucker et al. 1995). Caccianiga et al. (1997) y Worrall et al. (1999) reportaron emisión óptica muy débil, sin presencia de líneas de emisión (característico de objetos tipo BL Lac). Bliss et al. (2009) confirmaron con observaciones en el óptico, radio y rayos $\mathrm{X}$ que este blazar posee un continuo débil en el óptico, al igual que su emisión en radio, pero en cuanto a la banda de rayos $\mathrm{X}$, posee un continuo fuerte tipo ley de potencias.

Este blazar fue observado durante una noche, 17 septiembre 2015, sin registrar variabilidad intra-noche en ninguno de los filtros. Este resultado de no-variabilidad fue detectado por ambos parámetros $\mathrm{C}$ y F. Por otro lado, se obtuvieron las magnitudes estándares para el blazar, siendo $16.375 \pm 0.011$ para el filtro V y $15.681 \pm 0.005$ para el filtro R. Las curvas de luz de las magnitudes se muestran en la Fig. 3.84.

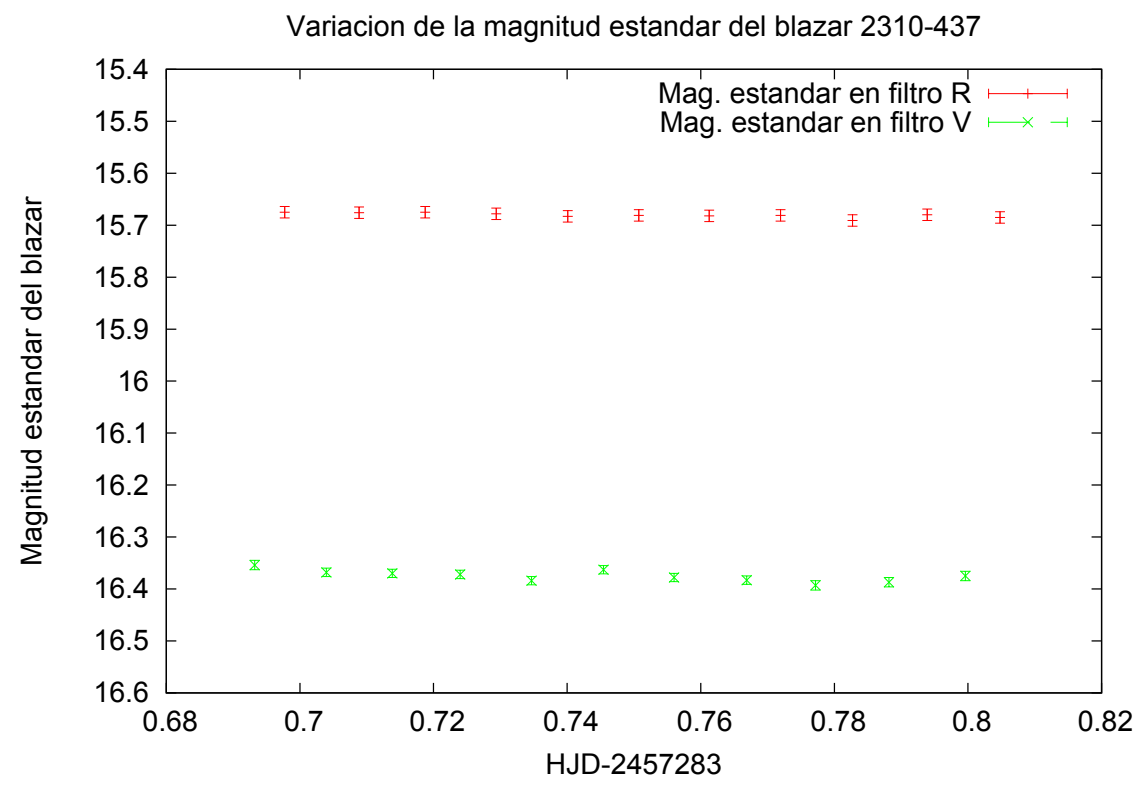

Figura 3.84. Curvas de luz para la magnitud estándar de 2310-437 en el filtro R (rojo) y en el V (verde).

Estudiando el diagrama magnitud-magnitud, presentado en la Fig. 3.85, observamos que se tiene una correlación entre los datos en $\mathrm{V}$ y $\mathrm{R}$, corroborada por el ajuste lineal y por el valor de la función, como puede verse en la Tabla 3.4.

En lo que respecta al índice espectral, se tiene un valor promedio para esta noche de observación de $\alpha_{V R}=3.95 \pm 0.008$.

Finalmente, analizando el diagrama color-magnitud (Fig. 3.86) el coeficiente de Pearson $(\mathrm{r}=0.923)$ indica que se tiene una correlación fuerte positiva entre los datos. Esto implicaría que el blazar presenta un comportamiento tipo BWB. 

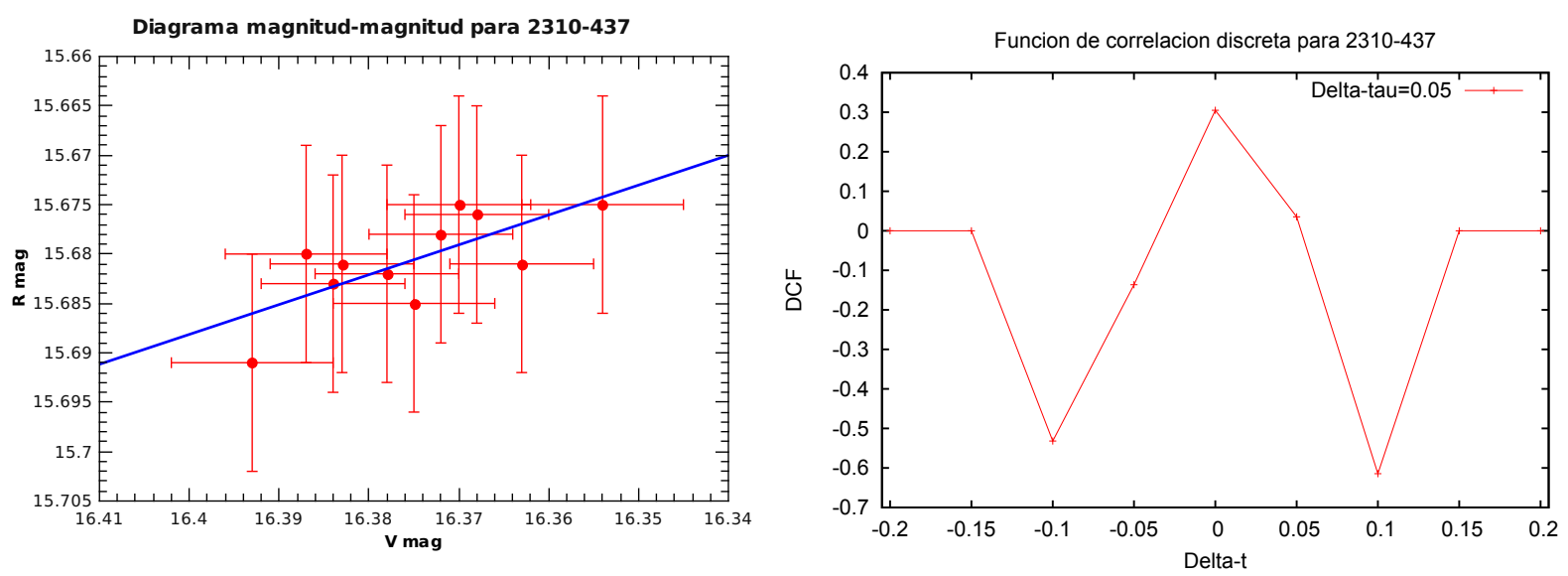

Figura 3.85. Diagrama magnitud-magnitud (izquierda) y función de correlación discreta para 2310-437 (derecha).

Diagrama color-magnitud para 2310-437

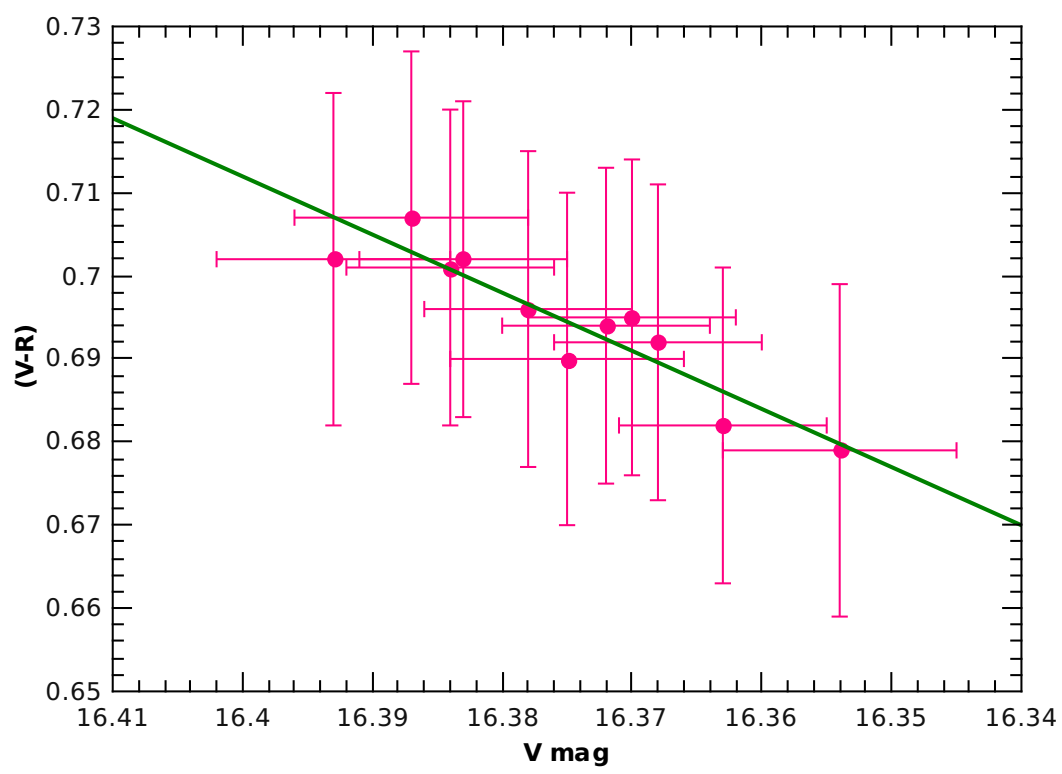

Figura 3.86. Diagrama color-magnitud para 2310-437. 


\subsubsection{Los blazares BZU}

Dentro de la muestra de blazares elegimos a 3 objetos que figuran como BZU en ROMA BZCAT, ya sea porque no se conoce su clasificación (si es BL Lac o FSRQ), o si se desconoce cuál es su clase espectral (HSP, ISP o LSP). Lo que podemos ver es que, si utilizando los resultados obtenidos en esta Tesis, podemos inferir, o contribuir a definir, qué clase de objetos son estos BZU. Para esto, a continuación presentamos un breve resumen de los resultados obtenidos para estas fuentes:

- PKS 0208-512: Se lo observó en agosto y septiembre de 2015. En cuanto a su variabilidad, sólo se detectó variabilidad intra-noche en el filtro R, correspondiente a los datos de agosto. Respecto a escalas mayores, esta fuente presenta una variabilidad significativa $(\Delta m \sim 0.7$, para ambos filtros) de un mes a otro. Esta variación resulta correlacionada en ambos filtros, V y R. Su índice espectral promedio fue de $\alpha_{V R}=2.33 \pm 0.065$, donde de un mes a otro este valor desciende, indicando que la distribución espectral se volvió menos dura. Finalmente, en el diagrama color-magnitud, encontramos una tendencia RWB, típica de objetos tipo FSRQs. El valor de su redshift es de $\mathrm{z}=1.003$. Recordando que tanto $\mathrm{BL}$ Lacs como FSRQs presentan distribuciones distintas con respecto a z, este valor podría asociarse a un objeto tipo FSRQs (Giommi et al. 2011).

- [HB89] 0521-365: En la única noche de observación en diciembre del 2015, no se registró variabilidad. A partir de los valores de las magnitudes estándares publicadas, obtuvimos una estimación de su magnitud estándar en $\mathrm{V}$ y $\mathrm{R}, \mathrm{y}$ de su índice de color, dando como resultado un valor del índice espectral de $\alpha_{V R}=5.20 \pm 0.016$. En cuanto a su diagrama color-magnitud, esta fuente muestra un comportamiento del tipo BWB (esperable en objetos tipo BL Lacs). Respecto al valor de su redshift, éste resulta un valor pequeño de $\mathrm{z}=0.055$, valor esperable para el caso de objetos de tipo BL Lac (debido su distribución respecto de los FSRQs).

- PMN J0847-2337: En las 6 noches en las que se observó a esta fuente en abril 2014, no se registró variabilidad intra-noche en ninguna de ellas. El valor de su índice espectral promedio resultó ser de $\alpha_{V R}=3.508 \pm 0.012$, aunque lo interesante es su comportamiento a lo largo de las 6 noches. En la primer noche su valor se encuentra en 3.56 y a la siguiente baja en 0.1, para quedarse estable en $\sim 3.45$ a los largo de 3 noche. Para las últimas 2, se incrementa en 0.06, llegando a $\sim 3.51$. El valor de su redshift es de $\mathrm{z}=0.059$, con lo cual tenemos una situación similar a la del objeto anterior. Respecto a su diagrama color-magnitud, la fuente presenta una tendencia BWB (esperable en objetos tipo BL Lacs).

Por otra parte, Hovatta et al. (2014) encuentran una relación entre el pico sincrotrón de la SED de los blazares en función de lo que se conoce como Índice de modulación intrínseca, $\bar{m}$. Esta relación resulta diferente según el tipo de objeto y es por eso que calculamos este índice para ver en qué lugar del diagrama caen los objetos de nuestra muestra. Este índice fue desarrollado por Richards et al. (2011) y se construye como el cociente entre la desviación estándar $\left(\sigma_{0}\right)$ de la densidad de flujo medido sobre el valor medio de dicha densidad $\left(S_{0}\right)$ : 


$$
\bar{m}=\frac{\sigma_{0}}{S_{0}} .
$$

Este índice se usa para estudiar el estado de variabilidad de la fuente. Considerando un nivel de significancia de $3 \sigma$, se puede calcular el valor crítico $\bar{m}_{3}$, a partir del cual se establece si la fuente presenta o no variabilidad. En el caso en que $\bar{m}$ caiga por fuera de la zona de los $3 \sigma$, se considera que una variabilidad estadísticamente significativa no puede ser establecida. En nuestro caso, calculamos $\bar{m}_{3}$ para toda la muestra observada incluyendo a los objetos clasificados como BZU. Se calculó el índice $\bar{m}$ para los flujos en el filtro $\mathrm{V}$, tomando una noche de observación (escala de la microvariabilidad) para cada fuente. Esto se hizo implementando un programa que desarrolló el Dr. Daniel Carpintero para realizar este cálculo en particular. Para la muestra total, obtuvimos como resultado que aproximadamente el $65 \%$ de los blazares resultó ser variable.

Aplicando este análisis a nuestros datos, podemos ver dónde se ubican los objetos BZU de nuestra muestra en esa relación. Los valores del pico sincrotrón para toda la muestra estudiada fueron recopilado usando el software SED Builder ${ }^{3}$. En la Fig. 3.87 se muestran los resultados, indicando en particular a las 3 fuentes BZU.

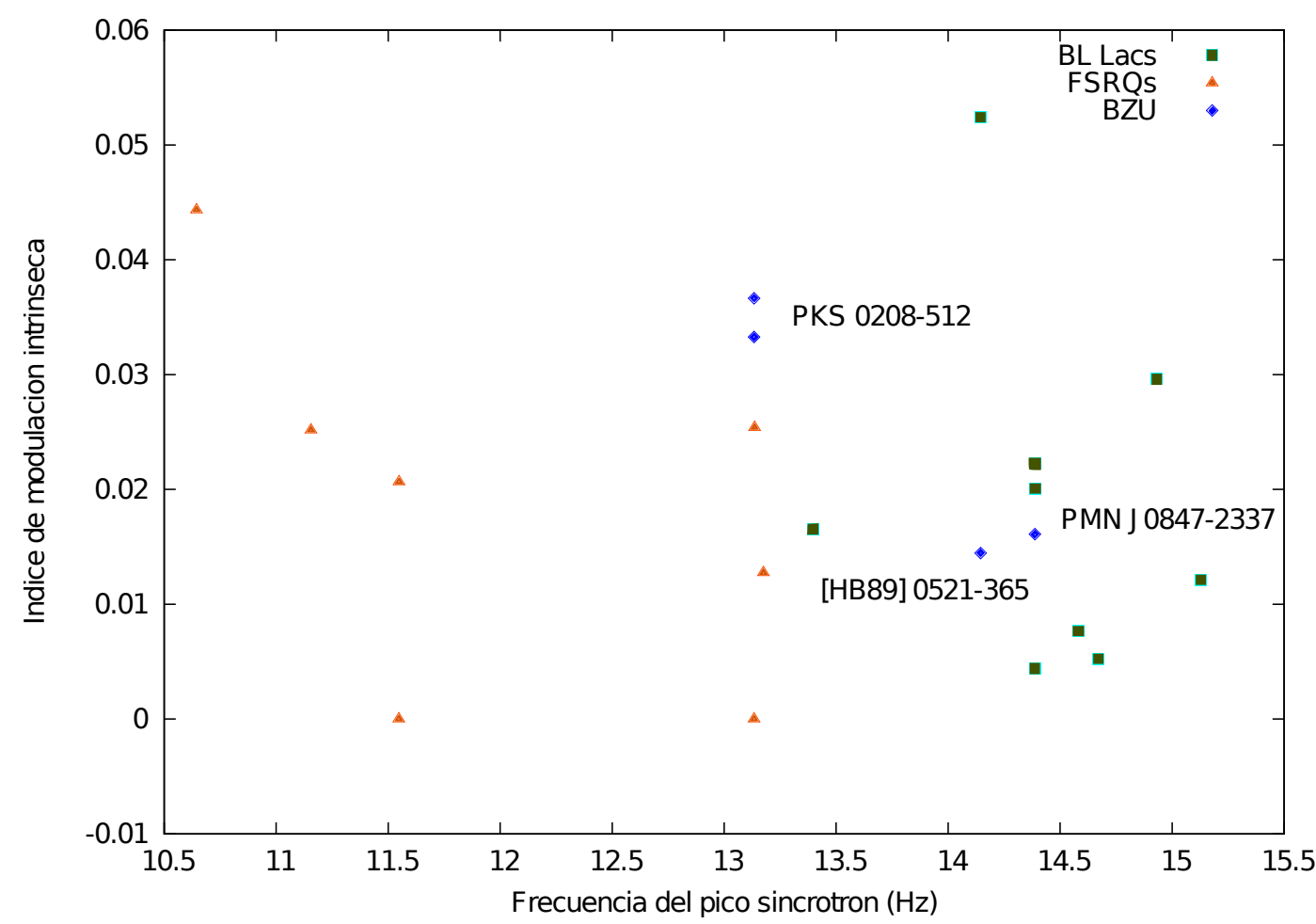

Figura 3.87. Índice de modulación intrínseca en función del pico sincrotrón de la SED del blazar (en log). Se muestran cuáles son los 3 objetos BZU, además de los BL Lacs y FSRQs conocidos.

Hovatta et al. (2014) también analizan la relación que hay entre el redshift del blazar y el índice de modulación intrínseca. En la Fig. 3.88 mostramos esta relación para nuestra muestra, en donde están señalizados los 3 objetos BZU. La distribución de puntos respecto al valor del redshift es similar a lo que obtienen Hovatta et al.

\footnotetext{
${ }^{3}$ https://tools.asdc.asi.it/SED/
} 
(2014), donde se refleja la distribución que tienen los BL Lacs y los FSRQs respecto al z (Giommi et al. 2011). En particular, en los gráficos presentados por Hovatta et al. (2014), se pueden observar que varios FSRQs tienen asociados valores mayores de $\bar{m}$ que los BL Lacs. Este mismo comportamiento se observa en el BZU de nuestra muestra, candidato a FSRQ. De este modo, vemos que los 3 blazares BZU se localizan en zonas bien definidas, siendo que [HB89] 0521-365 y PMN J0847-2337 están posicionados entre los BL lacs, mientras que PKS 0208-512 se encuentra en la región donde se ubican los FSRQs.

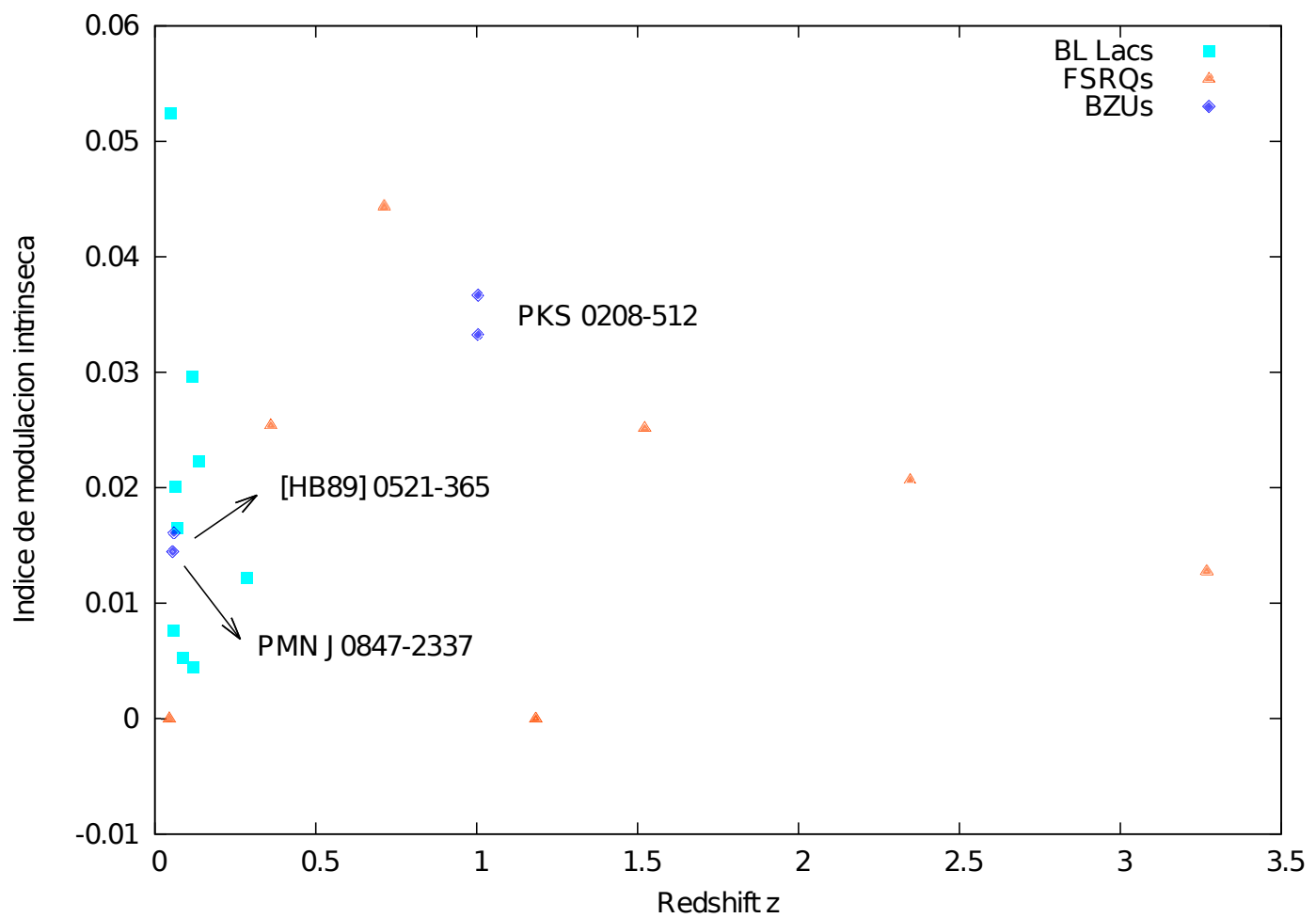

Figura 3.88. Índice de modulación intrínseca en función del redshift. Se indican a los 3 objetos BZU, además de los BL Lacs y FSRQs conocidos.

De los resultados obtenidos en el presente análisis, podemos confirmar la clasificación de estos tres objetos de la muestra: el blazar PKS 0208-512 se trataría de un FSRQ, coincidiendo con lo que sostienen la mayoría de los trabajos en la literatura. Por otro lado, en cuanto a [HB89] 0521-365 y PMN J0847-2337, serían objetos BL Lacs. 


\subsection{Discusión de los resultados obtenidos en el óp- tico de la muestra seleccionada}

A partir de todo el análisis llevado a cabo para las observaciones en la banda del óptico de los 19 blazares de la muestra, llegamos a los siguientes resultados globales:

- Del total de la muestra, el $54 \%$ son BL Lac y $46 \%$ son FSRQ.

- Considerando ambos índices de variabilidad, el $26 \%$ de los blazares registró variabilidad intra-noche para el filtro $\mathrm{V}$, mientras que el $32 \%$ resultó variable en el filtro $\mathrm{R}$ en la misma escala temporal.

- Una submuestra de 11 objetos pudo ser observada en más de una noche en un mismo turno. De estos objetos encontramos que se registró variabilidad internoche en el $26 \%$ de la muestra en ambos filtros V y R. Este porcentaje fue calculado considerando aquellos casos en los cuales ambos índices de variabilidad fueron coincidentes.

- El $84 \%$ de la muestra mostró un comportamiento BWB, mientras que el $16 \%$ restante presentó una tendencia RWB.

- Del total de BL Lacs, el $82 \%$ resultó BWB, mientras que el restante $18 \%$ RWB.

- Del total de FSRQs, el $87.5 \%$ resultó BWB y el 12.5\% RWB.

La microvariabilidad detectada en los blazares es una importante herramienta para el estudio tanto de los procesos físicos involucrados en la fuente como así también de la región emisora central. Dicha variabilidad puede ser explicada aplicando diferentes modelos que explican los mecanismos capaces de generar variaciones significativas en cortos plazos de tiempo. Por un lado, se tienen los modelos intrínsecos a la fuente, que incluyen a los modelos físicos (asociados a la emisión del blazar) y geométricos, tales como el modelos de shock-in-jet, Hot-spot y swinging jets. Y por el otro se tienen los modelos extrínsecos, como por ejemplo, el modelo de microlentes gravitacionales.

El comportamiento de los colores juega un papel importante en esta búsqueda del modelo que mejor explique lo observado. Como mencionamos anteriormente, variaciones en el flujo de la fuente inducen a variaciones en los colores y en el índice espectral. Luego, el estudio del diagrama color-magnitud permite analizar cómo las variaciones detectadas afectan a los colores y esto permite asociar un modelo en particular para explicar las observaciones. Diversos autores (Agarwal et al. 2015; Gupta et al. 2016) han encontrado que, entre los blazares, se tienen básicamente dos tipos de comportamientos entre el índice de color y la magnitud. La tendencia BWB (Bluer when brigther) suele ser observada en los BL Lacs, y puede ser explicada a través del modelos tipo shock-in-jet. Este comportamiento puede ser explicado invocando la presencia de dos componentes: una componente variable relativamente azul más una componente relativamente roja (Fiorucci et al. 2004). Por otro lado, la tendencia RWB (Redder when brigther) es más usual encontrarla en los FSRQs, y se la puede interpretar como la contribución de la emisión térmica del disco de acreción. Esta componente influye sobre la región más azul de la emisión cuando el jet es débil. Zhang et al. (2014, 2015) 
también estudian el comportamiento de una muestra de blazares en los diagramas color-magnitud. Sin embargo llegan a conclusiones totalmente opuestas a las halladas por Agarwal et al. (2015) y Gupta et al. (2016). Esto es debido a una interpretación errónea de los diagramas color-magnitud que estos autores analizan.

De la muestra de 19 blazares observados, reportamos un comportamiento BWB en 16 objetos, de los cuales 7 son FSRQs y 9 son Bl Lacs. Por otro lado, en 3 de ellos se reporta un comportamiento RWB, siendo un FSRQ y dos BL Lacs. En cuanto a los BL Lacs, encontramos que el $82 \%$ reportaron una tendencia BWB (como es esperable) y el resto resultaron RWB. Por otra parte, en cuanto a los FSRQs, casi el $88 \%$ posee una tendencia BWB, mientras que sólo el $12 \%$ mostró un comportamiento RWB, como se espera. En muchos de estos casos, los ajustes resultaron ser poco confiables (barras de error grandes y/o valores de $\chi^{2}$ reducido cercanos a 0), razón por la cual los BL Lacs reflejan en mayor medida el comportamiento esperable en los diagramas color-magnitud respecto de los FSRQs.

De los resultados obtenidos, podemos inferir que los comportamientos a escalas mayores (meses) pueden encontrarse también a escalas de horas a días. En particular, basados en la muestra observada en la presente Tesis, podemos ver que, en general, las tendencias esperadas para los distintos tipos de blazares se replican a menor escala.

Para poder obtener resultados concluyentes, es necesario ampliar la muestra observacional. Esto sería no solo en el número de fuentes observadas, sino también en el seguimiento de las mismas.

Cabe aclarar que, con el cálculo del índice de modulación intrínseca, como el mismo se calculó para toda la muestra en el filtro $\mathrm{V}$ y $\mathrm{R}$, obtuvimos que un mayor número de fuentes que resultaron ser variables con este índice, llegando a ser el $65 \%$ de la muestra. Del estudio previo realizado en el Cap. 2, de donde podemos desprender que, si una fuente resultó no variable tanto con el parámetro $\mathrm{C}$ como con el $\mathrm{F}$, existe una alta chance de que esta variabilidad ser real bajo cualquier circunstancia, nos resulta llamativo el valor encontrado con el índice de modulación intrínseca. Es por esto que sólo se usó a los fines de los diagramas utilizados, para estudiar la naturaleza de los BZUs. Es claro que se necesitan mayores estudios para este índice en particular. 


\subsection{Análisis en altas energías}

\subsubsection{Rayos X}

\section{Introducción}

La astronomía de rayos X está orientada al estudio de objetos cuya emisión está asociada a la presencia de plasmas a muy altas temperaturas (con $\mathrm{T}>10^{5}-10^{7} \mathrm{~K}$ ) o la interacción de partículas relativistas con campos o materia. La energía de los fotones de rayos $\mathrm{X}$ se mide en electrovoltios (eV), y se extiende desde $0.1-100 \mathrm{keV}$. Las radiaciones de alta frecuencia son producidas cuando los electrones son repentinamente desacelerados al interaccionar con el campo eléctrico o magnético de un núcleo. Esto se conoce como Radiación de Bremsstrahlung, o de frenado. También son producidos por desexcitación atómica cuando los electrones hacen transiciones a niveles de energía más bajos en los átomos. En particular, estudiar la emisión en rayos X de blazares permite conocer más en detalle las regiones más internas del núcleo activo, ya que esta emisión se origina muy cerca del SMBH central.

Debido a la absorción que sufren los rayos X a causa de la atmósfera terrestre, es necesario llevar a cabo las observaciones a través de telescopios orbitales. Dada la naturaleza de los rayos $\mathrm{X}$, cuando éstos inciden sobre un material determinado, son capaces de atravesarlo sin problemas. Sin embargo, si su ángulo de incidencia es menor a cierto ángulo crítico $\left(<1^{\mathrm{O}}\right)$, éstos pueden ser reflejados y por lo tanto enfocados en ciertas direcciones determinadas. Esto es lo que se conoce como Óptica de incidencia rasante. De aquí surge la geometría o la óptica WOLTER, la cual consta de dos superficies: una parabólica y otra hiperbólica. Este sistema de espejos es utilizados en satélites como XMM-Newton y Chandra. En la actualidad, además de estos últimos, existen otros satélites en funcionamiento tales como Integral, MAXI, NUSTAR, Suzaku y Swift.

\section{Características del satélite Chandra}

La nueva generación de observatorios orbitales como Chandra, el cual tiene una resolución espacial y espectral sin precedentes, permite realizar estudios en la banda de rayos $\mathrm{X}$ de fuentes relativamente débiles. Este satélite posee un espejo Wolter con geometría tipo 1 de alta resolución, dos detectores de imagen y dos sets de redes de transmisión. El sistema óptico consta de 4 espejos con superficie reflectora anidados. Además, tiene buena sensibilidad en la banda de energía de 0.1 - $10 \mathrm{keV}$ y el área colectora se encuentra entre los 6 y $7 \mathrm{keV}$. La combinación de la alta resolución, una gran área colectora y la sensibilidad a rayos $\mathrm{X}$ de altas energías permite el estudio de objetos extremadamente débiles, muchas veces fuertemente absorbidos, por el gas y polvo del medio interestelar.

Cuenta con dos instrumentos situados en el plano focal: High Resolution Camera (HRC) y Advanced CCD Imaging Spectometer (ACIS). El primero es un instrumento de microcanales de plata, que posee dos detectores: HRC-I para imagen, con un campo de visión de 30 arcmin $\times 30$ arcmin y HRC-S para espectroscopía, con un campo de 6 arcmin $\times 99$ arcmin. El segundo es una configuración de varios CCD, dispuestos en un arreglo bidimensional que permite hacer en forma simultánea imagen y espectroscopía: ACIS-I, el cual cuenta con un arreglo de $2 \times 2$ CCDs, cuyo campo de visión es de 16 


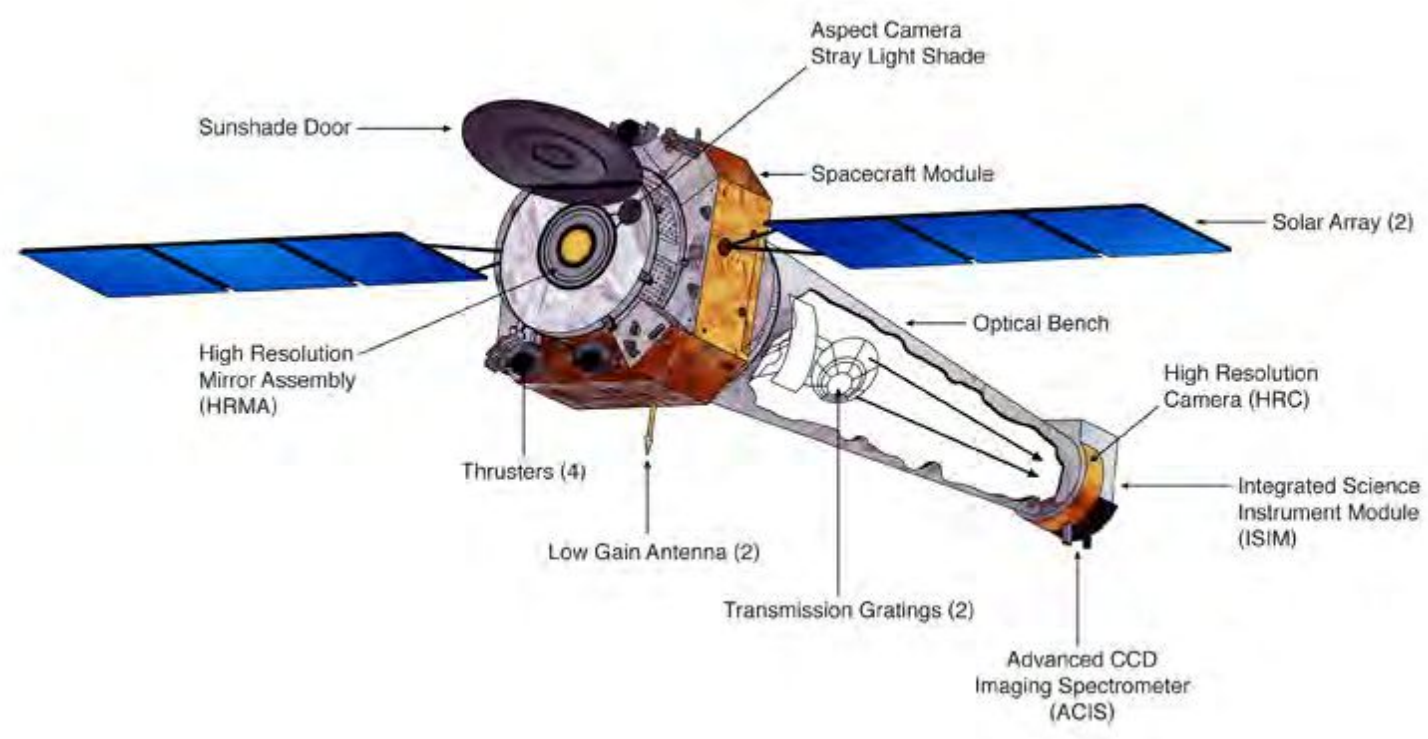

Figura 3.89. Esquema del satélite Chandra (NASA/CXC/SAO).

$\operatorname{arcmin} \times 16$ arcmin; y ACIS-S, el cual es un arreglo de redes 1x6 CCDs. La Fig. 3.89 muestra un esquema del satélite Chandra.

\section{Obtención y reducción de los datos}

Con el propósito de estudiar el comportamiento en la banda de los rayos X de la muestra de objetos observados en la banda óptica, se analizaron observaciones realizadas con el satélite Chandra, las cuales fueron extraídas del Chandra Data Archive (CDA). Estamos interesados en estudiar la variabilidad del flujo en rayos X. Para este tipo de análisis en los blazares, se necesitan observaciones que posean una gran cantidad de eventos y que haya sido observado por un rango de tiempo razonable. En total, 13 de los 19 blazares de la muestra de objetos estudiados en la banda óptica poseían observaciones realizadas con este satélite. Los datos adquiridos se procesan a través de tareas y scripts provistos por Chandra. El software $\mathrm{CIAO}^{4}$ (Chandra Interactive Analysis of Observations), permite procesar y analizar los datos en rayos X, y obtener los productos científicos de interés, tales como imágenes en distintos rangos de energía, curvas de luz, espectros, etc. Para la reducción de los datos, se implementa el script chandra_repro, para obtener los archivos de eventos que serán usados más adelante. Luego, se definen las regiones tanto de la fuente como del cielo para poder obtener las curvas de luz. Con el dmstat, se analiza en qué chip se encuentra la fuente y las regiones definidas y con la tarea dmextract, se procede a sustraer el fondo del cielo para obtener la curva de luz. Dentro de esta última tarea, se especifican tanto los GTIs (intervalos de tiempo bueno, del inglés Good Time Intervals), como el bineado temporal de los datos. El resultado final es la curva de luz que será analizada con las herramientas de variabilidad disponibles, tales como el glvary.

\footnotetext{
${ }^{4}$ http://cxc.harvard.edu/ciao/
} 
La información de los datos obtenidos con Chandra se muestran en la Tabla 3.6: la columna 1 indica el nombre de la fuente, la columna 2 su correspondiente número de identificación de la observación, la columna 3 la fecha de observación, la columna 4 el instrumento utilizado y finalmente la columna 5 el tiempo de exposición.

\section{Herramientas para el análisis de la variabilidad}

Para el análisis de los datos Chandra se usaron diversas tareas del software CIAO. Para la extracción de las curvas de luz de los blazares de cada una de las observaciones se uso la tarea dmextract, para la cual se eligió una región circular para la fuente y otra similar para extraer el fondo. El paso siguiente fue analizar la curva de luz para buscar posibles variaciones. Para el análisis de variabilidad en las curvas de luz obtenidas, aplicamos dos métodos: el algoritmo glvary de Chandra y un ajuste lineal (asociado con el test de $\chi^{2}$ ). La tarea glvary se basa en el algoritmo de Gregory-Loredo (Gregory \& Loredo, 1992). Este algoritmo fue desarrollado con el objetivo de poder detectar y medir una señal periódica en un conjunto de datos, aún si se desconocen sus características. Este método también puede expandirse a señales no periódicas. El algoritmo consiste en dividir los eventos en varios rangos de tiempo y buscar alguna posible desviación entre ellos. Los posibles valores que pueda tomar el índice de variabilidad junto con su significado se resumen en la Tabla 3.7. Aquí, Odd corresponde al cociente de las probabilidades, $P$ corresponde a la probabilidad de una señal variable, y $f 3$ y $f 5$ corresponden a las fracciones de la curva que se encuentran dentro de $3 \sigma$ y $5 \sigma$ respecto al promedio de la tasa de cuentas. La Fig. 3.90 muestra dos ejemplos de curvas de luz, en las que una no presenta variabilidad y la otra sí. Las dos principales ventajas de aplicar este método son: por un lado, que es insensible al tipo y forma de la variabilidad presente, y por otro lado, toma en cuenta las incertezas en la tasa de cuentas, de manera apropiada.

Por otro lado, realizamos un análisis de regresión lineal en las curvas, con el objetivo de ver si a cada curva de luz se le puede ajustar una recta. Este método se basa en el test de $\chi^{2}$, para determinar si las curvas presentan algún tipo de variación. En el caso en que la curva sea ajustada por una recta, se considera no variable. Caso contrario, la curva es considerada variable. La aplicación de esta herramienta fue tomada como un criterio independiente, para reforzar los resultados obtenidos con la herramienta glvary. En el caso de que la estadística de la fuente es baja, este tipo de test no resulta apropiado y por lo tanto no se ha tomado en cuenta. Por lo tanto, sólo el resultado de la tarea glvary es tomado como válido (ver Tabla 3.8 más adelante). 
Tabla 3.6. Información sobre los datos obtenidos en la banda de rayos X de la muestra. Las columnas son nombre de la fuente, la identificación de la observación, la fecha de observación, el instrumento utilizado y el tiempo de exposición.

\begin{tabular}{|c|c|c|c|c|}
\hline Objeto & ObsID & $\begin{array}{c}\text { Fecha } \\
\text { aaaa-mm-dd }\end{array}$ & Instrumento & $\begin{array}{l}\text { Exposición } \\
\text { kseg }\end{array}$ \\
\hline PKS 0208-512 & 4813 & $2005-02-24$ & ACIS-S & 48.67 \\
\hline \multirow[t]{2}{*}[HB89]{$0414+009$} & 2969 & 2002-08-01 & ACIS-S & 50.09 \\
\hline & 4284 & 2002-08-02 & ACIS-S & 36.44 \\
\hline \multirow[t]{3}{*}{$H$ B89]0521 - 365} & 846 & 1999-12-31 & ACIS-S & 9.12 \\
\hline & 4926 & 2004-06-17 & ACIS-I & 18.52 \\
\hline & 5837 & 2006-02-02 & ACIS-I & 27.68 \\
\hline PMN J0847-2337 & - & - & - & - \\
\hline$[H B 89] 1116-462$ & - & - & - & - \\
\hline \multirow[t]{2}{*}[HB89]{$1127-145$} & 866 & 2000-05-28 & ACIS-S & 27.36 \\
\hline & 5708 & $2005-04-25$ & ACIS-S & 105.51 \\
\hline \multirow[t]{3}{*}{ PKS 1229-02 } & 4841 & 2004-04-18 & ACIS-S & 18.31 \\
\hline & 11731 & 2010-04-26 & ACIS-S & 47.55 \\
\hline & 12205 & 2010-05-05 & ACIS-S & 51.97 \\
\hline PMN J1256-1146 & - & - & - & - \\
\hline$[H B 89] 1424-418$ & - & - & - & - \\
\hline PKS J1443-3908 & - & - & - & - \\
\hline \multirow[t]{2}{*}{ PKS 1510-089 } & 2141 & 2001-03-23 & ACIS-S & 9.24 \\
\hline & 11432 & 2010-04-05 & ACIS-S & 45.5 \\
\hline PKS J1517-242 & 3971 & 2003-07-04 & ACIS-S & 12.81 \\
\hline BZB J1917-192 & - & - & - & - \\
\hline \multirow[t]{2}{*}{ 3FGL J1958.2-3011 } & 12281 & 2010-08-22 & ACIS-I & 9.46 \\
\hline & 13136 & $2010-10-23$ & ACIS-I & 39.53 \\
\hline \multirow[t]{5}{*}{ PKS 2005-489 } & 842 & 2000-10-07 & ACIS-I & 5.85 \\
\hline & 12295 & 2010-08-13 & ACIS-S & 91.74 \\
\hline & 12296 & 2010-08-03 & ACIS-S & 47.25 \\
\hline & 13019 & 2010-08-05 & ACIS-S & 86.38 \\
\hline & 13022 & 2010-08-11 & ACIS-S & 56.82 \\
\hline$[H B 89] 2126-158$ & 376 & 1999-11-16 & ACIS-S & 34.13 \\
\hline \multirow[t]{2}{*}{ PKS 2149-306 } & 336 & 1999-11-18 & ACIS-S & 35.56 \\
\hline & 1481 & 1999-11-20 & ACIS-S & 54.07 \\
\hline
\end{tabular}


Tabla 3.6. Cont.

\begin{tabular}{|c|c|c|c|c|}
\hline Objeto & ObsID & $\begin{array}{c}\text { Fecha } \\
\text { aaaa-mm-dd }\end{array}$ & Instrumento & $\begin{array}{l}\text { Exposición } \\
\text { kseg }\end{array}$ \\
\hline \multirow[t]{37}{*}{ PKS 2155-304 } & 1014 & $2001-03-28$ & ACIS-S & 26.69 \\
\hline & 1015 & $2000-12-06$ & ACIS-S & 9.51 \\
\hline & 1790 & 2000-08-11 & ACIS-S & 20.89 \\
\hline & 1791 & 2000-08-11 & ACIS-S & 20.89 \\
\hline & 1792 & 2000-08-11 & ACIS-S & 20.89 \\
\hline & 1793 & 2000-08-11 & ACIS-S & 20.89 \\
\hline & 1794 & 2000-08-12 & ACIS-S & 20.89 \\
\hline & 1795 & 2000-08-07 & ACIS-S & 19.7 \\
\hline & 1796 & 2000-08-08 & ACIS-S & 19.51 \\
\hline & 1798 & 2000-08-08 & ACIS-S & 19.51 \\
\hline & 1799 & 2000-08-10 & ACIS-S & 19.89 \\
\hline & 2335 & $2000-12-06$ & ACIS-S & 29.07 \\
\hline & 337 & 1999-10-20 & ACIS-S & 38.66 \\
\hline & 1703 & 2000-05-31 & ACIS-S & 25.28 \\
\hline & 1705 & 2000-05-31 & ACIS-S & 25.5 \\
\hline & 6874 & 2006-07-29 & ACIS-S & 28.45 \\
\hline & 3167 & 2001-11-30 & ACIS-S & 29.65 \\
\hline & 3168 & 2001-11-30 & ACIS-S & 28.8 \\
\hline & 3169 & $2001-11-27$ & ACIS-I & 7.83 \\
\hline & 3170 & 2001-11-27 & ACIS-I & 7.66 \\
\hline & 3171 & $2001-11-28$ & ACIS-I & 8.05 \\
\hline & 3172 & $2001-11-28$ & ACIS-I & 7.66 \\
\hline & 3173 & 2001-11-29 & ACIS-I & 7.62 \\
\hline & 3174 & 2001-11-29 & ACIS-I & 7.69 \\
\hline & 3175 & 2002-04-01 & ACIS-I & 7.69 \\
\hline & 3176 & 2001-12-01 & ACIS-I & 7.83 \\
\hline & 3177 & 2001-12-01 & ACIS-I & 8.05 \\
\hline & 3667 & $2002-06-11$ & ACIS-S & 14.81 \\
\hline & 3668 & 2002-06-11 & ACIS-S & 13.47 \\
\hline & 3669 & 2002-06-11 & ACIS-S & 42.43 \\
\hline & 3706 & $2002-11-29$ & ACIS-S & 27.71 \\
\hline & 3707 & 2002-11-30 & ACIS-S & 26.93 \\
\hline & 3708 & 2002-11-29 & ACIS-S & 26.62 \\
\hline & 4416 & $2003-12-16$ & ACIS-S & 46.48 \\
\hline & 5173 & $2004-11-23$ & ACIS-S & 26.71 \\
\hline & 6090 & $2005-05-25$ & ACIS-S & 27.53 \\
\hline & 6091 & 2005-09-19 & ACIS-S & 29.24 \\
\hline PMN J2310-4347 & 2967 & $2002-07-26$ & ACIS-S & 26.75 \\
\hline
\end{tabular}



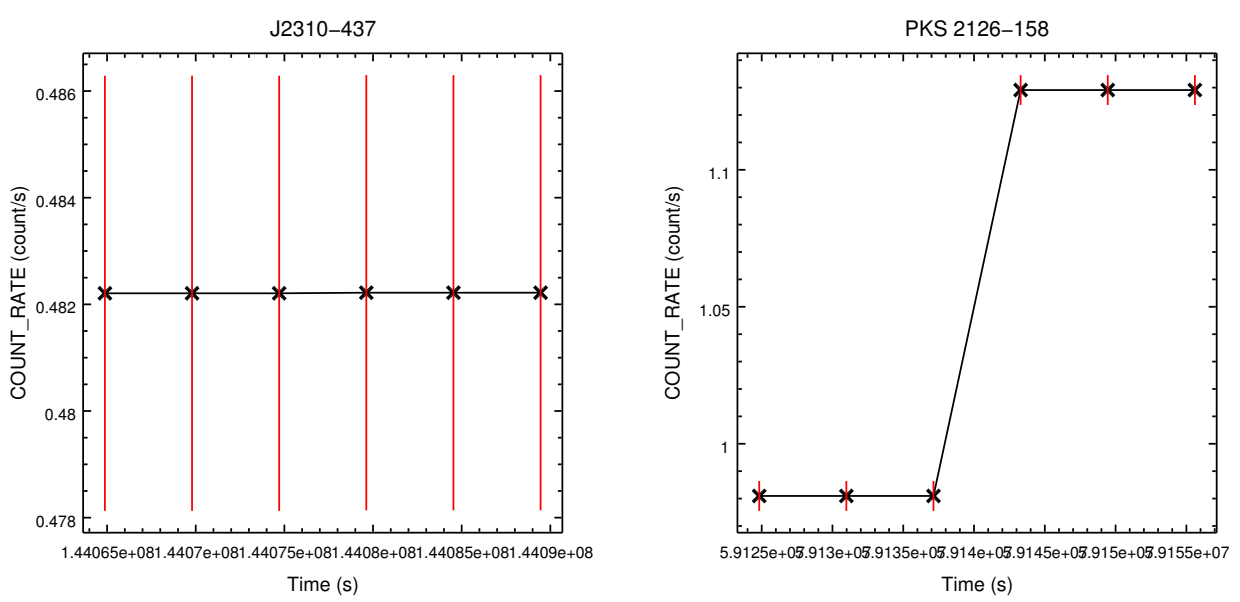

Figura 3.90. Ejemplos de dos curvas de luz que genera y utiliza la herramienta glvary para la detección de variabilidad. En el panel derecho, la herramienta no detecta variabilidad, mientras que la curva en el panel izquierdo es tomada como variable.

Tabla 3.7. Valores del algoritmo de glvary. (Tabla extraída de: http://cxc.cfa.harvard.edu/ciao/threads/variable/).

\begin{tabular}{ccc}
\hline \hline Índ. de variab. & Condición a cumplirse & Resultado \\
\hline 0 & $P \leq 0.5$ & Definit. no variable \\
1 & $0.5<P<2 / 3$ y $f 3>0.997$ y $f 5=1.0$ & Considerado no variable \\
2 & $2 / 3 \leq 0.9$ y $f 3>0.997$ y $f 5=1.0$ & Probablemente no variable \\
3 & $0.5 \leq P<0.6$ & Quizás variable \\
4 & $0.6 \leq P<2 / 3$ & Probablemente variable \\
5 & $2 / 3 \leq P<0.9$ & Considerado variable \\
6 & $0.9 \leq P$ y $O d d<2.0$ & Definit. variable \\
7 & $2.0 \leq O d d<4.0$ & Definit. variable \\
8 & $4.0 \leq O d d<10.0$ & Definit. variable \\
9 & $10.0 \leq O d d<30.0$ & Definit. variable \\
10 & $30.0 \leq O d d$ & Definit. variable \\
\hline
\end{tabular}




\section{Análisis de los resultados: Variabilidad}

La Tabla 3.8 muestra los resultados del análisis de la variabilidad, después de aplicar los dos métodos utilizados. En la columna 1 el objeto; en la columna 2 la identificación de la observación; en la columna 3 la fecha de observación; en la columna 4 el valor del algoritmo de glvary; en la columna 5, el valor de $\chi^{2}$ reducido; y finalmente en la columna 6 cómo resultó la clasificación de la curva de luz.

De los 13 blazares observados con Chandra, sólo a 5 se les detectó variabilidad intra-noche. Esto es, un $38 \%$ de la muestra analizada en rayos X resultó variable. De estas 5 fuentes, el $60 \%$ corresponden a blazares BL Lac (HSP), mientras que el restante $40 \%$ son del tipo FSRQs (LSP). A continuación, se detallan los resultados para cada uno de estos 5 blazares junto a sus curvas de luz.

[HB89] 1127-145: Este objeto fue observado en mayo del 2000 y abril del 2005 Para los datos correspondientes al 2000, no se ha detectado variación alguna en la curva (los resultados obtenidos con ambos métodos son concordantes). Para las observaciones del 2005, se registra variabilidad intra-noche. En este caso, la herramienta glvary arroja el índice de variabilidad más alto (10). Las curvas de luz obtenidas para ambos conjuntos de datos se muestran en la Fig. 3.91.

3FGL J1958.2-3011: A esta fuente, se la observó durante agosto y octubre del 2010. En el primer caso la fuente mostró un alto nivel de variabilidad. Con glvary, se obtuvo un índice máximo de variabilidad (10), mientras que el ajuste lineal corroboró lo hallado con un valor de $\chi^{2}$ reducido mucho mayor que 1 (26.678). En cuanto a los datos de octubre, no se registró variación significativa. Las curvas de luz de ambas observaciones se presentan en la Fig. 3.92.

PKS 2005-489: A este objeto se lo observó en octubre del 2000 y agosto del 2010. Para los datos del 2000, no se detectó variabilidad (consistente al aplicar ambos métodos), mientras que para los datos del 2010, sólo una de las observaciones (ObsID=13019) mostró variaciones, con un índice de 7 según el glvary. El ajuste lineal no fue tenido en cuenta dada la baja cantidad de cuentas de la fuente en dicha observación. Las curvas de luz obtenidas se muestran en la Fig. 3.93.

[HB89] 2126-158: Este blazar se lo observó en noviembre del 1999, en donde tanto el glvary (con un índice de variabilidad máximo de 10) como el ajuste lineal (con un valor del $\chi^{2}$ reducido de 3.729) muestra una clara variabilidad intra-noche. La curva se muestra en la Fig. 3.94.

PKS 2155-304: Este blazar fue observado por Chandra en varias oportunidades. Las observaciones utilizadas para esta fuente corresponden al período que va desde octubre del 1999 hasta julio del 2006, con un total de 37 ObsID. Estas observaciones se presentan en la Fig. 3.95. De estas observaciones, sólo 12 presentaron variabilidad y corresponden a los datos de agosto del 2000, noviembre del 2001, abril, junio y noviembre del 2002 y junio del 2006. Estos resultados se muestran en la Tabla 3.8 y las curvas, con sus respectivos ObsID se muestran en la Fig. 3.96. 
Tabla 3.8. Resultados del análisis de variabilidad en rayos X. Se muestra en la columna 1 el objeto; en la columna 2 la identificación de la observación; en la columna 3 la fecha de observación; en la columna 4 el valor del algoritmo de glvary; en la columna 5 , el valor de $\chi^{2}$ reducido; y finalmente en la columna 6 cómo resultó la clasificación de la curva de luz.

\begin{tabular}{cccccc}
\hline \hline Objeto & ObsID & Fecha & glvary & $\chi^{2}$ reducido & Variable? \\
\hline PKS 0208-512 & 4813 & $2005-02-24$ & 0 & 0.946005 & No \\
{$[$ HB89]0414+009 } & 2969 & $2002-08-01$ & 0 & 0.747326 & No \\
& 4284 & $2002-08-02$ & 0 & 0.847537 & No \\
{$[$ HB89]0521-365 } & 846 & $1999-12-31$ & 0 & 1.120160 & No \\
& 4926 & $2004-06-17$ & 0 & 1.002250 & No \\
[HB89]1127-145 & 5837 & $2006-02-02$ & 0 & 0.840983 & No \\
& 5706 & $2000-05-28$ & 0 & 0.898741 & No \\
PKS 1229-02 & 4841 & $2005-04-25$ & 10 & 1.329250 & Sí \\
& 11731 & $2004-04-18$ & 0 & 0.934654 & No \\
PKS 1510-089 & 12205 & $2010-05-05$ & 0 & 0.782025 & No \\
& 2141 & $2001-03-23$ & 0 & -612946 & No \\
PKS J1517-242 & 11432 & $2010-04-05$ & 0 & 0.840099 & No \\
3FGL J1958.2-3011 & 12281 & $2003-07-04$ & 0 & - & No \\
PKS 2005-489 & 13136 & $2010-10-23$ & 0 & 1.426190 & No \\
& 842 & $2000-10-07$ & 0 & 0.164835 & No \\
& 12295 & $2010-08-13$ & 0 & 0.816628 & No \\
& 12296 & $2010-08-03$ & 0 & 0.896487 & No \\
PKS 2149-306 & 13019 & $2010-08-05$ & 7 & - & Sí \\
& 13022 & $2010-08-11$ & 0 & 0.952798 & No \\
HB89]2126 - 158 & 376 & $1999-11-16$ & 10 & 3.729290 & Sí \\
& 1481 & $1999-11-18$ & 0 & 0.818381 & No \\
& & & 0 & 0.651177 & No \\
\hline
\end{tabular}


Tabla 3.8. Cont.

\begin{tabular}{|c|c|c|c|c|c|}
\hline Objeto & ObsID & Fecha & glvary & $\chi^{2}$ reducido & Variable? \\
\hline \multirow[t]{37}{*}{ PKS 2155-304 } & 1014 & 2001-03-28 & 0 & 1.018750 & No \\
\hline & 1015 & $2000-12-06$ & 0 & 0.997294 & $\mathrm{No}$ \\
\hline & 1790 & 2000-08-11 & 7 & 1.870230 & Sí \\
\hline & 1791 & 2000-08-11 & 0 & 1.328910 & No \\
\hline & 1792 & 2000-08-11 & 3 & 2.179170 & Sí \\
\hline & 1793 & 2000-08-11 & 9 & 2.963380 & Sí \\
\hline & 1794 & 2000-08-12 & 0 & 1.452320 & $\mathrm{No}$ \\
\hline & 1795 & 2000-08-07 & 9 & 9.627370 & Sí \\
\hline & 1796 & 2000-08-08 & 0 & 1.007300 & No \\
\hline & 1798 & 2000-08-08 & 1 & 1.159030 & No \\
\hline & 1799 & 2000-08-10 & 6 & 1.365970 & Sí \\
\hline & 2335 & 2000-12-06 & 0 & 0.853122 & No \\
\hline & 337 & 1999-10-20 & 0 & 1.186890 & No \\
\hline & 1703 & 2000-05-31 & 0 & 1.023280 & No \\
\hline & 1705 & 2000-05-31 & 0 & 0.916620 & No \\
\hline & 6874 & 2006-07-29 & 8 & 2.133370 & Sí \\
\hline & 3167 & 2001-11-30 & 0 & 0.937324 & No \\
\hline & 3168 & 2001-11-30 & 0 & 0.798052 & No \\
\hline & 3169 & $2001-11-27$ & 10 & 17.329800 & Sí \\
\hline & 3170 & $2001-11-27$ & 0 & 1.048760 & No \\
\hline & 3171 & $2001-11-28$ & 0 & 1.047850 & No \\
\hline & 3172 & 2001-11-28 & 10 & 9.742770 & Sí \\
\hline & 3173 & 2001-11-29 & 0 & 1.277170 & No \\
\hline & 3174 & 2001-11-29 & 0 & 1.418560 & No \\
\hline & 3175 & 2002-04-01 & 10 & - & Sí \\
\hline & 3176 & 2001-12-01 & 0 & - & No \\
\hline & 3177 & 2001-12-01 & 0 & 1.104720 & No \\
\hline & 3667 & 2002-06-11 & 10 & 5.410590 & Sí \\
\hline & 3668 & 2002-06-11 & 0 & 0.962367 & No \\
\hline & 3669 & 2002-06-11 & 8 & 4.445610 & Sí \\
\hline & 3706 & 2002-11-29 & 0 & 0.781266 & No \\
\hline & 3707 & 2002-11-30 & 7 & - & Sí \\
\hline & 3708 & 2002-11-29 & 0 & 1.138350 & No \\
\hline & 4416 & 2003-12-16 & 0 & 0.768056 & No \\
\hline & 5173 & $2004-11-23$ & 0 & 0.788472 & No \\
\hline & 6090 & $2005-05-25$ & 0 & 0.682120 & No \\
\hline & 6091 & 2005-09-19 & 0 & 0.809051 & No \\
\hline PMN J2310-4347 & 2967 & $2002-07-26$ & 0 & 0.800349 & No \\
\hline
\end{tabular}



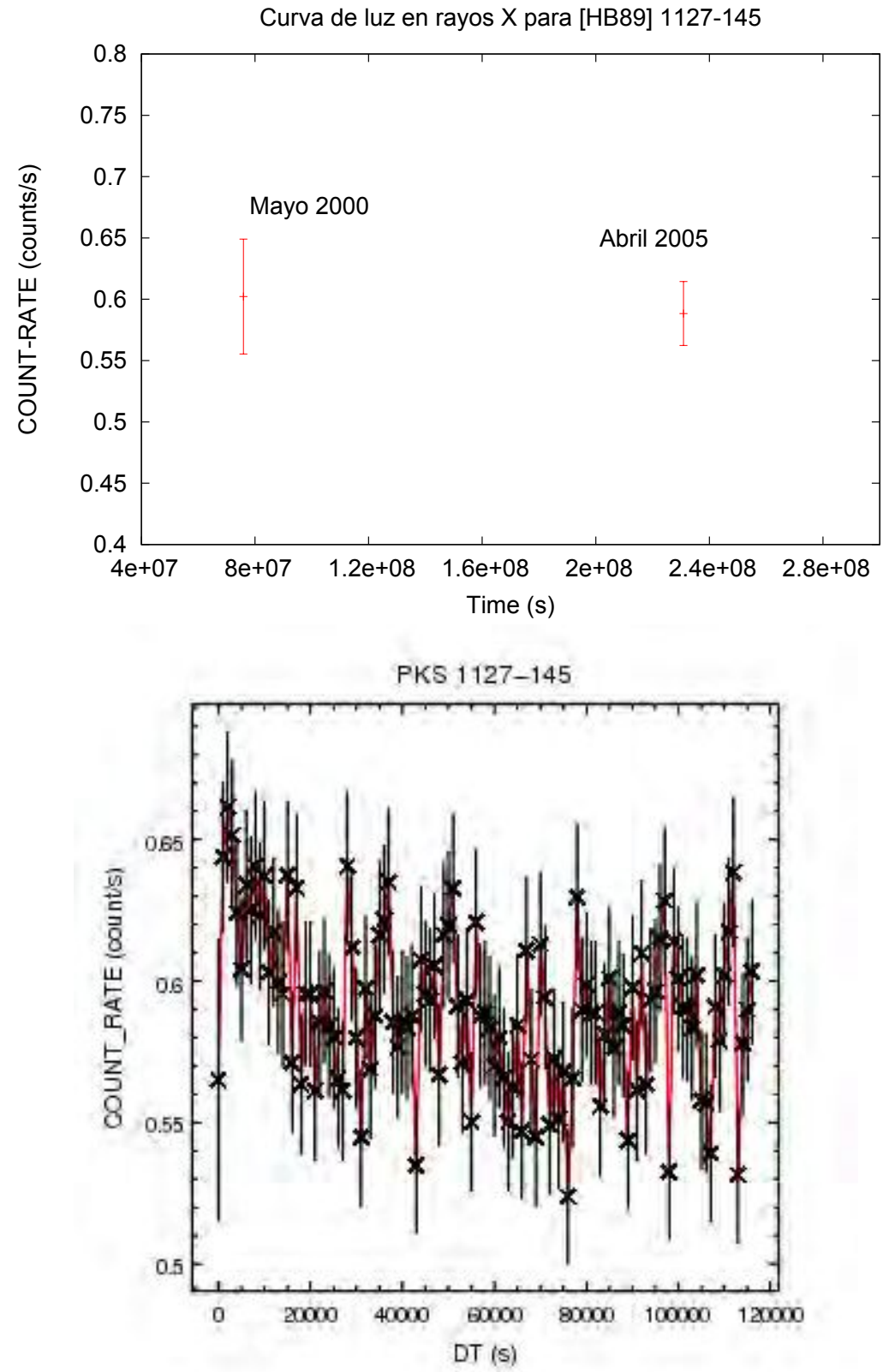

Figura 3.91. Curvas de luz para $[H B 89] 1127$ - 145. En el panel superior se encuentran todas las observaciones para esta fuente, representadas cada una de ellas por un punto. En el panel inferior, se muestra la curva de luz para la observación ObsID:5708, la cual presentó variabilidad. 

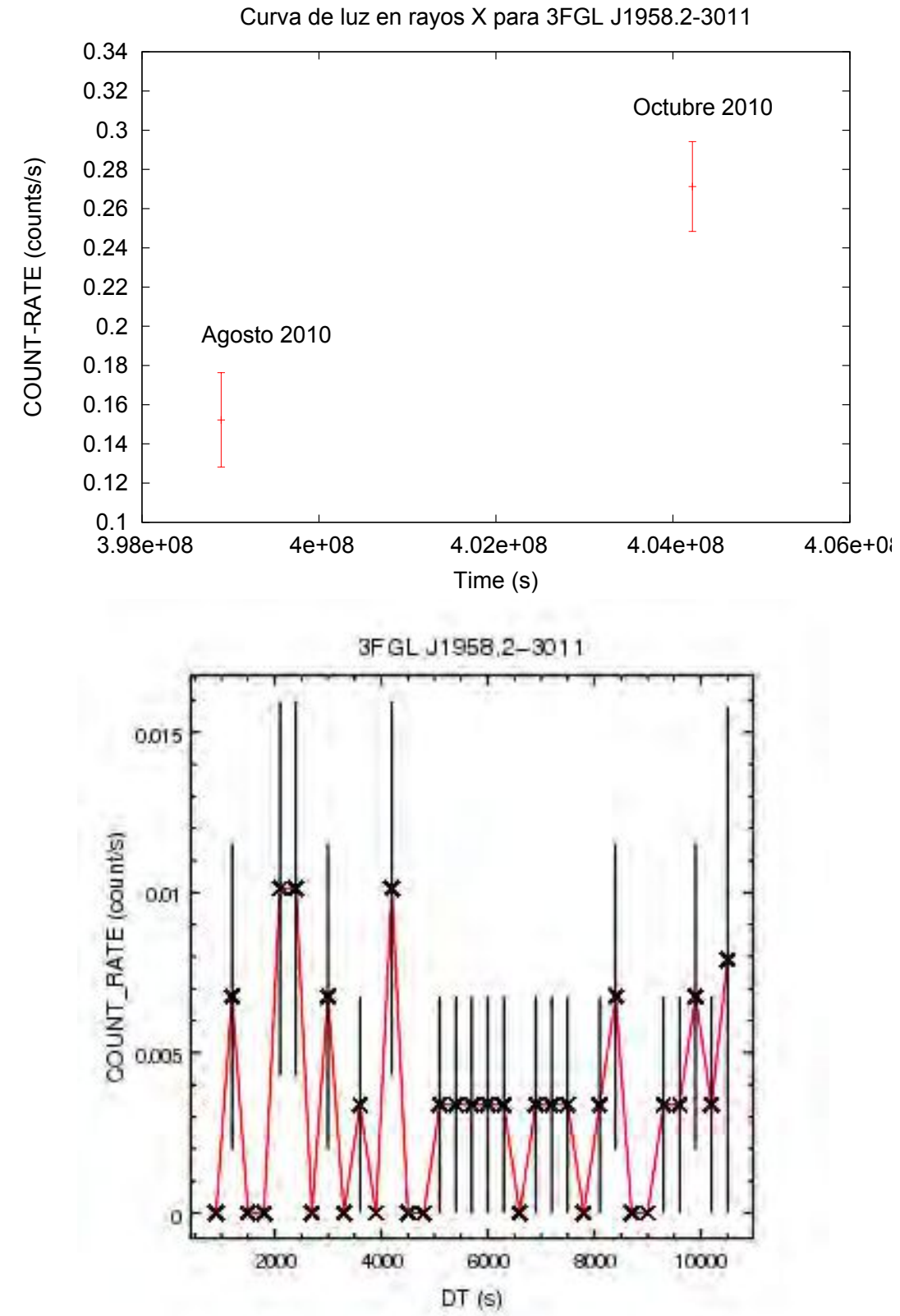

Figura 3.92. Curvas de luz para 3FGL J1958.2-3011. En el panel superior se encuentran todas las observaciones para esta fuente, representadas cada una de ellas por un punto. En el panel inferior, se muestra la curva de luz para la observación ObsID:12281, la cual presentó variabilidad. 

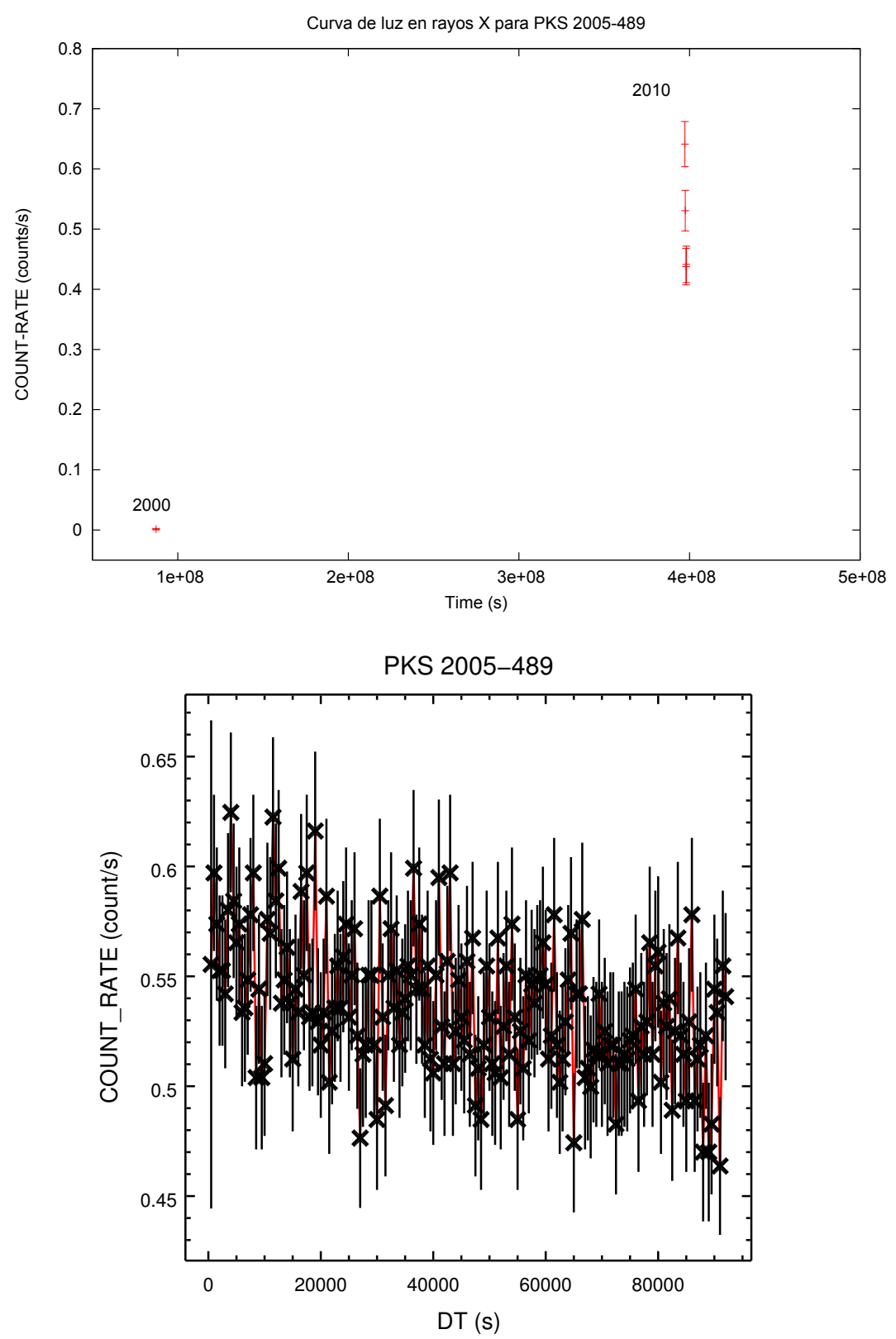

Figura 3.93. Curvas de luz para PKS 2005-489. En el panel superior se encuentran todas las observaciones para esta fuente, representadas cada una de ellas por un punto. En el panel inferior, se muestra la curva de luz para la observación ObsID:13019, la cual presentó variabilidad. 


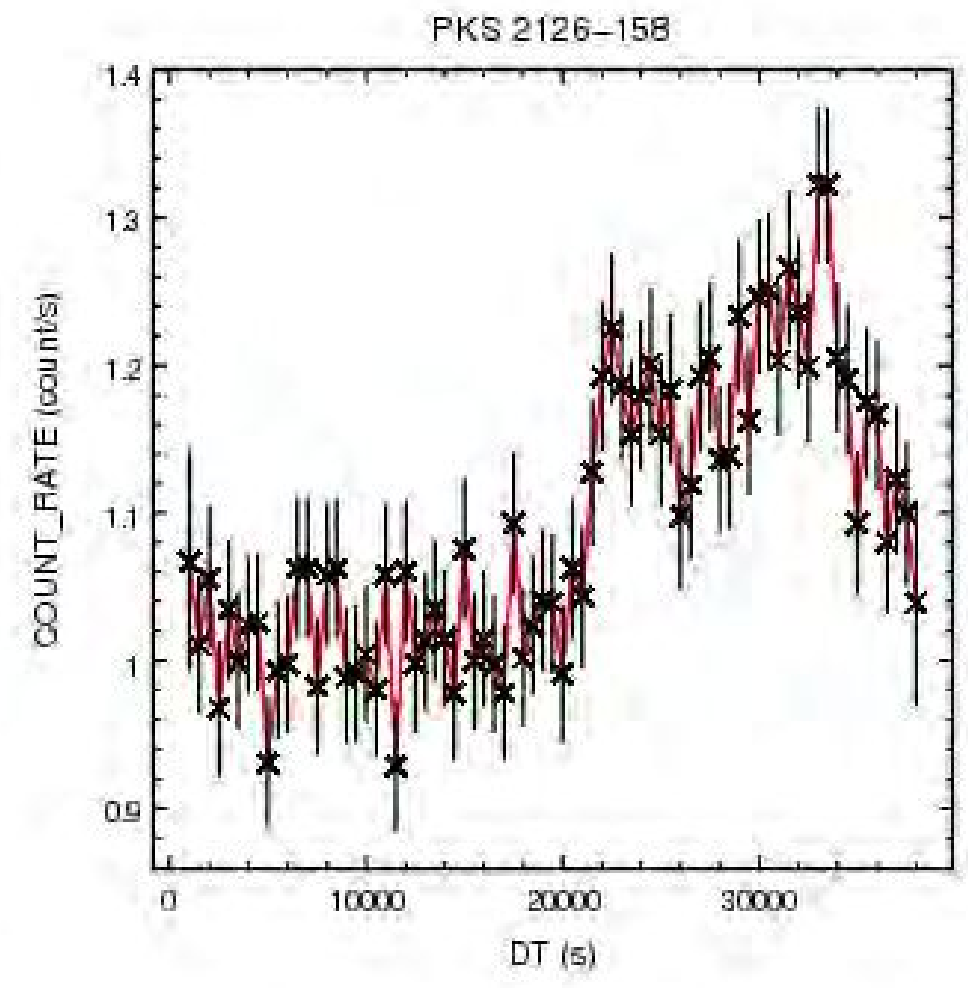

Figura 3.94. Curva de luz para $[H B 89] 2126$ - 158, correspondientes a las observaciones ObsID:376.

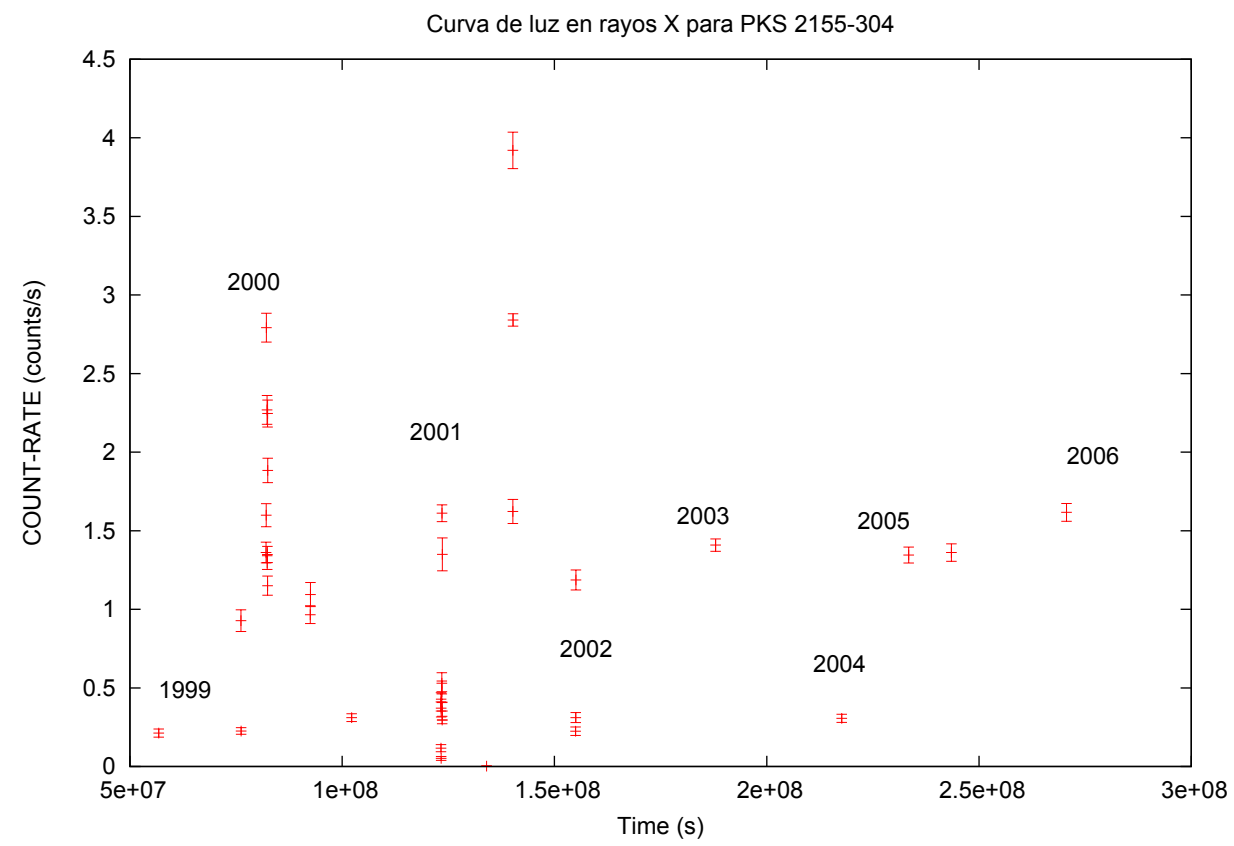

Figura 3.95. Curva de luz para PKS 2155-304. Se muestran todas las observaciones para esta fuente, representadas cada una de ellas por un punto. 

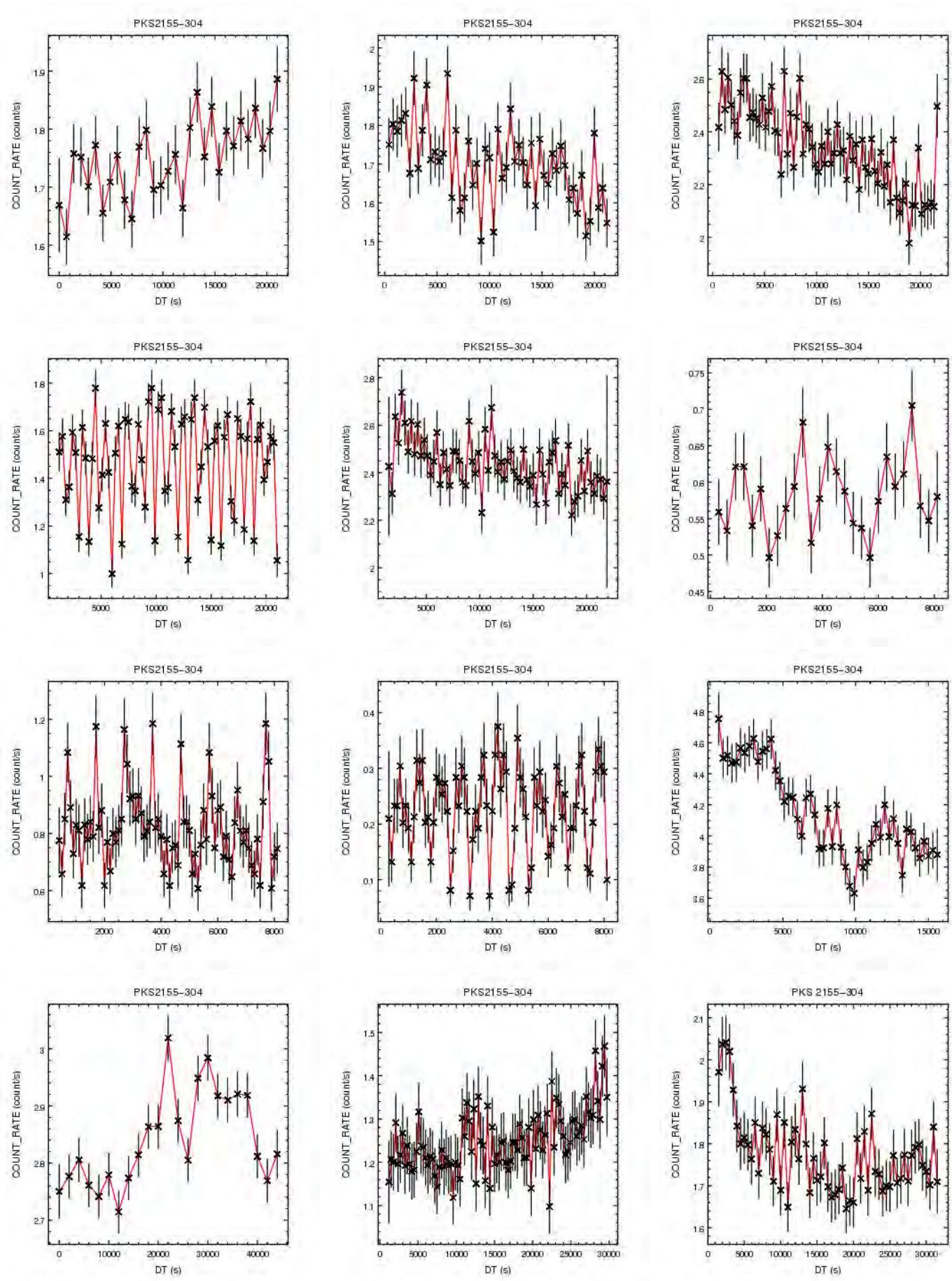

Figura 3.96. Curvas de luz para PKS 2155-304 que presentaron variabilidad, correspondientes a las observaciones ObsID:1790, ObsID:1792, ObsID:1793, ObsID:1795, ObsID:1799, ObsID:3169, ObsID:3172, ObsID:3175, ObsID:3667, ObsID:3669, ObsID:3707 y ObsID:6874. 


\subsubsection{Análisis multifrecuencia para 3FGL J1958.2-3011}

Como complemento a las observaciones realizadas en la banda óptica y de rayos X, se analizaron datos del satélite Fermi obtenidos en la banda de rayos- $\gamma$, con el objetivo de analizar el comportamiento a altas energías de la muestra de objetos estudiados. Se compararon las curvas de luz óptico $/ \gamma$ y de rayos $\mathrm{X} / \gamma$ de aquellos objetos que fueron observados simultáneamente a ambas frecuencias.

De toda la muestra, sólo 4 objetos cumplen con este tipo de observaciones simultáneas. Estos son: PKS 1229-02, PKS 1510-089, 3FGL J1958.2-3011 y PKS 2005-489. En el caso de estas fuentes, los datos ópticos fueron obtenidos entre el 2014 y 2015, mientras que los datos en rayos X corresponden al 2010. Dado que el telescopio Fermi se encuentra operativo a partir de 2008, el análisis de comparación entre las curvas de luz se realizó para el período 2008/2016. De esta manera, garantizamos la simultaneidad, por un lado, entre los datos en rayos $\mathrm{X}$ y rayos- $\gamma$, y por el otro, entre los datos en óptico y en rayos- $\gamma$. En particular, 2 de estos 4 blazares presentan variabilidad, 3FGL J1958.2-3011 y PKS 2005-489 (ver Tabla 3.8). Para 3FGL J1958.2-3011, tanto glvary como el ajuste lineal corroboran fuertemente este comportamiento, con un valor del índice del glvary de 10, y el valor de $\chi^{2}$ reducido 26.67. Para el otro blazar, sólo se tienen los resultados del glvary, dado que la estadística del $\chi^{2}$ reducido fue muy baja. Por lo tanto, para el análisis multifrecuencia, nos quedamos con 3FGL J1958.2-3011. En la Fig. 3.92 de la sección anterior se muestran las curvas de luz obtenidas, para cada ObsID. En particular, para el presente análisis, usamos las ObsID=12281, que corresponden al 2010.

\section{Rayos- $\gamma$}

Los rayos- $\gamma$ representan la radiación más energética del espectro electromagnético. Básicamente, existen tres procesos radiativos por los cuales se producen los rayos- $\gamma$ : Efecto Compton Inverso (la radicación $\gamma$ se produce cuando un fotón es dispersado por un electrón); Bremsstrahlung (se origina cuando un electrón relativista es acelerado en el campo magnetoestático de un núcleo o de otra partícula) y decaimiento de piones (la producción de piones se da en interacciones hadrónicas $p-p$. Una vez creado el pión neutro $\pi^{0}$, éste decae en un par de rayos- $\gamma$ ). Los rayos- $\gamma$ tienen la peculiaridad de que son muy difíciles de detectar a nivel terrestre, ya que son absorbidos fuertemente por la atmósfera (se desintegran al interactuar con las moléculas de aire). El fotón inicial crea un par electrón - positrón que interactúa a su vez con la materia y campos de la atmósfera y entregarán parte de su energía para crear fotones secundarios. Existen dos maneras de detectarlos: a través de telescopios terrestres (en forma indirecta), Telescopios Cherenkov, y otra a partir del uso de satélites espaciales. Con respecto a la primera, cuando un rayo- $\gamma$ llega a la atmósfera terrestre, inicia una cascada electromagnética; y si el rayo- $\gamma$ es muy energético, los leptones que hay en la atmósfera producen luz Cherenkov. Un problema para este tipo de observaciones es el ruido de fondo que generan los rayos cósmicos, dado que éstos también producen cascadas que terminan en emisión de luz Cherenkov. Por este motivo, es que se ha desarrollado la detección de los rayos- $\gamma$ desde el espacio, requiriendo distintas técnicas según el rango de energía involucrado. Debido a esto, el espectro $\gamma$ queda dividido en varias bandas: $<1 \mathrm{MeV}$, entre $1 \mathrm{MeV}$ y $30 \mathrm{MeV}$ y entre $30 \mathrm{MeV}$ y $300 \mathrm{GeV}$. 
Entre los satélites más conocidos, se encuentran: COMPTEL (telescopio Compton del Compton Gamma Ray Observatory); EGRET (Energetic Gamma Ray Experiment Telescope); GLAST; AGILE y Fermi LAT (Large Area Telescope).

\section{Características del satélite Fermi}

El satélite Fermi fue lanzado el 11 de junio 2008 y los datos de las observaciones hechas están disponibles a partir del 04 de agosto del 2008, para ser adquiridos y utilizados. Este satélite observa en la banda entre $10 \mathrm{keV}-300 \mathrm{GeV}$. Posee un telescopio de imagen en rayos- $\gamma$ y un instrumento que permite el estudio de los "estallidos $\gamma$ " (gamma-ray bursts). Su principal instrumento, el "Large Area Telescope (LAT)", tiene mayor área, resolución angular, campo de visión, y tiempo muerto, de modo que en conjunto proveen un factor de 30 o más en cuanto a la sensibilidad, y además provee la capacidad de estudiar los fenómenos transitorios. Por otro lado, el monitor de estallidos $\gamma$, Gamma-ray Burst Monitor (GBM), posee un campo de visión mucho mayor que el de LAT y provee una cobertura espectral de los estallido $\gamma$ que se extiende desde el límite inferior de LAT hasta $100 \mathrm{keV}$.

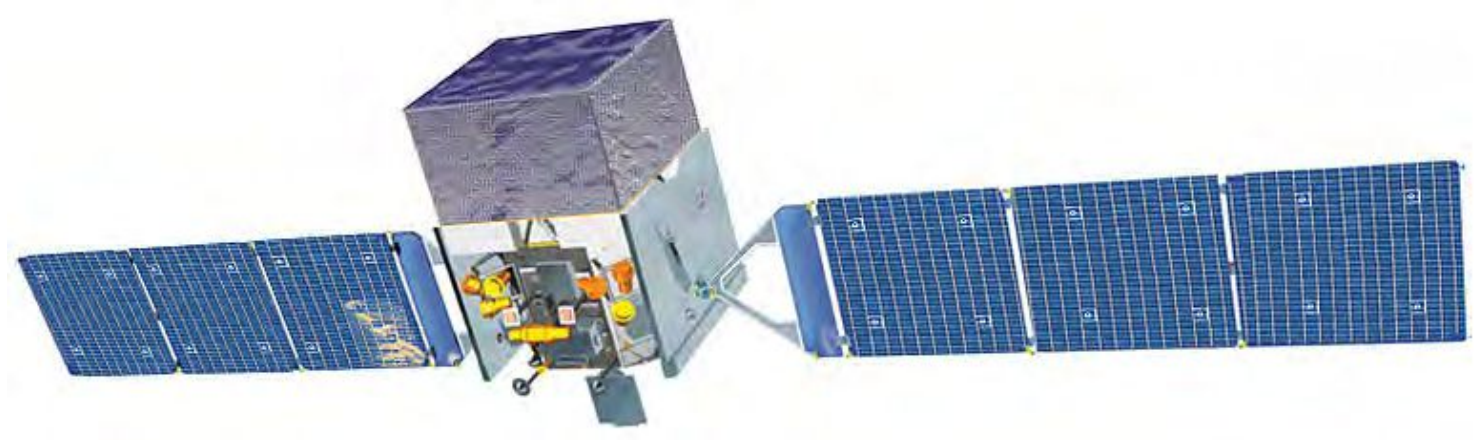

Figura 3.97. Esquema del satélite Fermi-LAT (NASA/Sonoma State University/Aurore Simonnet).

\section{Obtención y reducción de los datos}

Las observaciones de rayos- $\gamma$ del blazar 3FGL J1958.2-3011, obtenidos con el satélite Fermi-LAT, fueron extraídos de la base de datos Fermi $^{5}$, correspondientes al período 04/08/2008 hasta 02/06/2016.

$\mathrm{Al}$ igual que en rayos X, a través del software proporcionado por la misión Fermi, el Fermi Science Tools $^{6}$ se utilizaron las tareas apropiadas, tales como el gtselect, gtmktime, gtbin, para reducir y analizar los datos, de manera de obtener las curvas de luz y estudiar su comportamiento temporal en las mas altas energías. Los datos descargados se preparan a través de las tareas gtselect y gtbin. La primera es utilizada para hacer los cortes en el archivo de eventos, basados en columnas, como por ejemplo tiempo, energía, posición, ángulo cenital, coordenadas instrumentales, clase de evento y tipo de

\footnotetext{
${ }^{5}$ https://fermi.gsfc.nasa.gov/cgi-bin/ssc/LAT/LATDataQuery.cg

${ }^{6}$ https://fermi.gsfc.nasa.gov/ssc/data/analysis/
} 
evento. Adicionalmente, la segunda tarea hace cortes basados en el archivo del satélite y actualiza la extensión de los GTIs. Una vez que los datos están procesados, se recurre a la fotometría de apertura para generar las curvas de luz. Este método tiene como ventajas el proveer un modelo independiente para la medición del flujo, requerir de poco pasos para el análisis y no exige tanta demanda en tiempo de cómputo. Además, permite el uso de agrupamientos (o bineado) temporales cortos. Se genera la curva de luz a través de la tarea gtbin. Posteriormente, se utiliza la tarea gtexposure para determinar el tiempo de exposición de cada bin temporal establecido. En particular dentro de esta tarea, de ser posible, se necesita una estimación del índice espectral de fotones para la generación de la curva. Además, si se está interesado en la variabilidad a escalas cortas, se tiene que aplicar una corrección baricéntrica con la tarea gtbary. Finalmente, se obtiene la curva de luz en cuentas por bin temporal. Y con ftcalc se transforman las unidades de la curva en fotones $/ \mathrm{cm}^{2} / \mathrm{seg}$.

\section{Resultados obtenidos para 3FGL J1958.2-3011}

En la Fig.3.98 se muestran las imágenes en cada una de las bandas analizadas para el objeto: óptico (panel superior), rayos X (panel intermedio) y rayos- $\gamma$ (panel inferior). En el caso del óptico, la imagen corresponde a una de las observaciones hechas en CASLEO en abril del 2014, en el filtro V. Respecto a la imagen en rayos X, en color rojo se muestra la banda de energía de $0.2-1.5 \mathrm{keV}$, en verde se tiene el rango de energía entre $1.5-2.5 \mathrm{keV}$ y en color azul, entre $2.5-8.0 \mathrm{keV}$. En rayos- $\gamma$, se muestra el mapa de cuentas del campo de la fuente.

Como se ha mencionado en las secciones anteriores, este blazar es un BL Lac del tipo HSP. En el óptico, se lo siguió por dos noches y no se detectó variabilidad intranoche ni inter-noche, en ninguno de los dos filtros. Obtuvimos una magnitud estándar en el filtro $\mathrm{V}$ de $17.024 \pm 0.009$ y en el $\mathrm{R}$, de $16.438 \pm 0.007$. El flujo total en el filtro $\mathrm{R}$ fue de $F_{V}=5.644 \times 10^{-30} \pm 5.67 \times 10^{-32} \mathrm{mJy}$, mientras que en el filtro $\mathrm{R}$ se obtuvo un valor de flujo de $F_{R}=8.168 \times 10^{-30} \pm 1.16 \times 10^{-31} \mathrm{mJy}$. Analizando su diagrama colormagnitud (Fig. 3.65, Sec. 3.2.4), se observa una tendencia BWB en los datos. El valor promedio del índice espectral $\alpha_{V R}$ resultó de $3.32 \pm 0.009$, mientras que, comparando de una noche a otra, el valor aumenta desde 3.30 hasta 3.34. Es un crecimiento muy leve, pero estaría indicando que se tiene un espectro un poco más duro, llegando a más altas energías. En la banda de rayos X, se analizaron datos correspondientes a dos noches. De estas noches sólo se ha detectado variabilidad en la segunda. El análisis de variabilidad, usando la herramienta glvary arrojó un valor del índice de variabilidad igual a 10 (valor máximo), el cual resulta concordante con el valor del $\chi^{2}$ reducido usando el ajuste lineal. El valor del flujo para este objeto fue de $F_{0.5-7.0 \mathrm{keV}}=2.3 \times 10^{-12} \pm_{2.36 \times 10^{-12}}^{2.24 \times 12} \mathrm{erg}$ $\mathrm{cm}^{-2} \mathrm{seg}^{-1}$. La información de esta fuente en la banda de los rayos- $\gamma$ contenida en el 3er catálogo de Fermi (3FGL), indica que el flujo $\gamma$ es $F_{1-100 \mathrm{GeV}}=4.91 \times 10^{-10} \pm 1.17 \times 10^{-10}$ ph $\mathrm{cm}^{-2} \mathrm{seg}^{-1}$, su índice espectral es $\alpha=1.182 \pm 0.169$ y su índice de variabilidad es de 63.614. La Fig. 3.99 muestra la curva de luz en la banda de rayos- $\gamma$, del período 2008/2016, donde se tomó el bineado de 1 mes.

Debido a que no se cuenta con observaciones simultáneas en la banda del óptico y de rayos $\mathrm{X}$, se han utilizado datos en rayos- $\gamma$ como puente para unir ambas bandas, el cual puede ayudar a discernir la naturaleza del comportamiento variable detectado en 


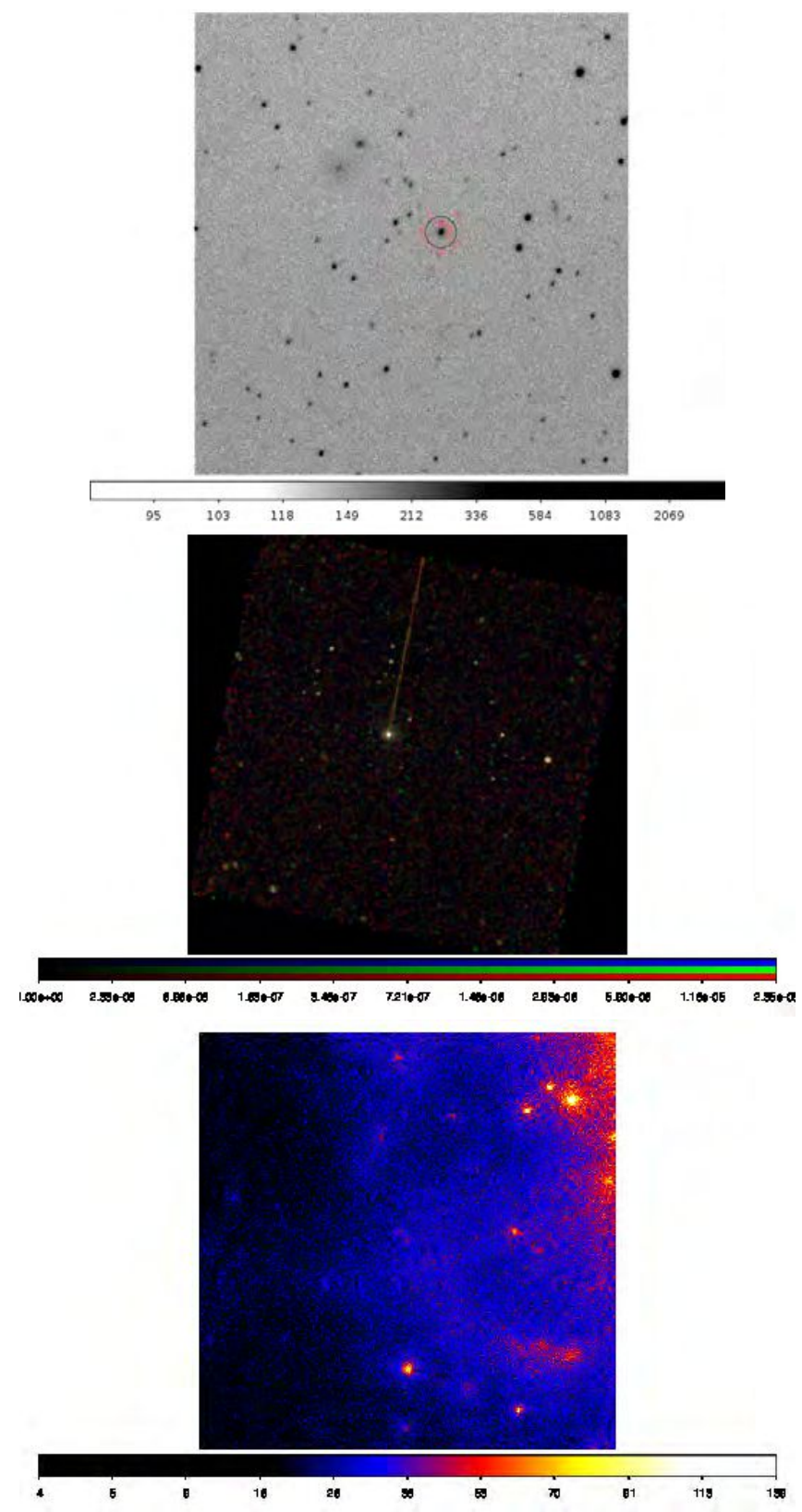

Figura 3.98. Imágenes de 3FGL J1958.2-3011 en las bandas del óptico (panel superior), rayos X (panel intermedio) y rayos- $\gamma$ (panel inferior).

el fuente. Presentamos a continuación las curvas de luz en la banda del óptico, rayos $\mathrm{X}$ y rayos- $\gamma$. En la Fig. 3.100, se muestran las curvas en el óptico (panel superior y medio), correspondientes a los días 24 y 25 abril 2014 y en rayos- $\gamma$ (panel inferior). La curva de luz en esta última banda posee un bineado de $1 \mathrm{hr}$. Se observa que, en el período correspondiente a la observación realizada en la banda óptica, la curva de luz en la banda de rayos- $\gamma$ no presenta variaciones significativas en su flujo. En la banda óptica, el blazar tampoco registra variaciones a escalas intra-noche ni inter-noche.

Por otro lado, en la Fig. 3.101, tenemos las curvas de luz de 3FGL J1958.2-3011 en las bandas de rayos X (panel superior), correspondientes a agosto y octubre 2010 y rayos- $\gamma$ (panel inferior), con un bineado temporal de $1 \mathrm{hr}$. En el caso de la curva de 


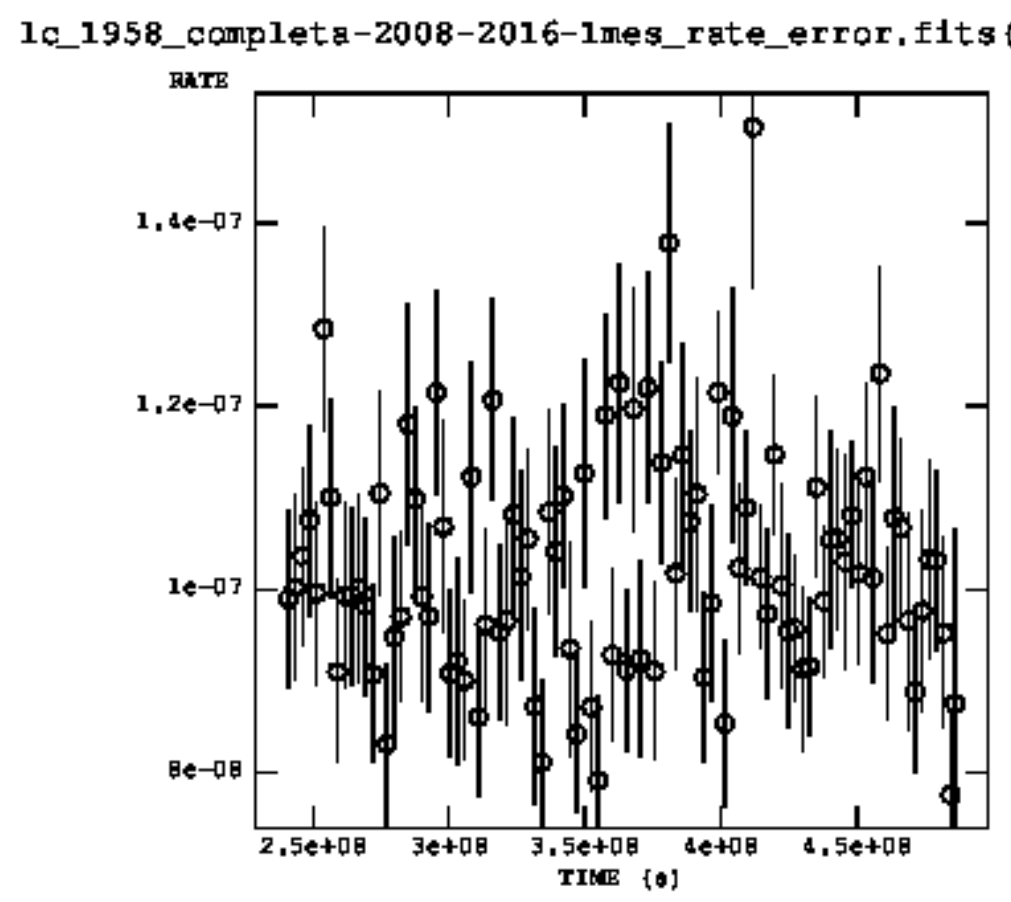

Figura 3.99. Curvas de luz de 3FGL J1958.2-3011 completa en la banda de rayos- $\gamma$, desde agosto de 2008 hasta junio de 2016. Se tomó un bineado de 1 mes.

luz en rayos- $\gamma$, nuevamente no se detectan variaciones significativas en su flujo para el período de tiempo considerado. En lo que respecta a la curva de luz en rayos X, se observa que sólo para la observación correspondiente a agosto 2010 se detecta variabilidad. Para este conjunto de datos en particular, no se detecta variabilidad en rayos- $\gamma$.

A partir de los que observamos en las curvas de luz en las distintas bandas, vemos que no se han detectado variaciones, salvo para la banda de rayos $\mathrm{X}$ en el período de agosto 2010, variación que no se refleja en la banda de rayos- $\gamma$. Esto no nos permite arribar a conclusiones acerca del comportamiento de la fuente en las distintas bandas estudiadas para el periodo temporal contemplado en cada análisis. Esto refleja la necesidad de incorporar más datos al estudio y poder analizar en más detalle el comportamiento del flujo en estas bandas. Observaciones simultáneas con telescopios ópticos y de rayos X-duros como NuStar ${ }^{7}$ pueden ser útiles para estudiar posibles correlaciones entre radiación no-térmica en ambas bandas (el gas térmico difícilmente llega a las temperaturas que mide este instrumento). Observaciones complementarias con telescopios Cherenkov pueden permitir revelar microvariaciones, como fue el caso para PKS 1510-089 en 2016 (Zacharias et al. 2017, arxiv:1708.00653).

\footnotetext{
${ }^{7}$ https://www.nustar.caltech.edu/
} 
Curvas de luz en optico y rayos gamma para 3FGL J1958.2-3011

Curva de luz en $\vee$ en la banda optica

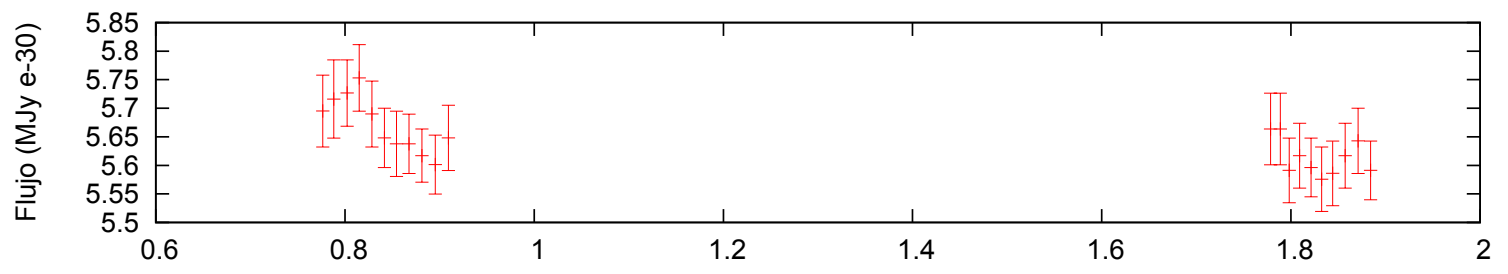

Curva de luz en $\mathrm{R}$ en la banda optica

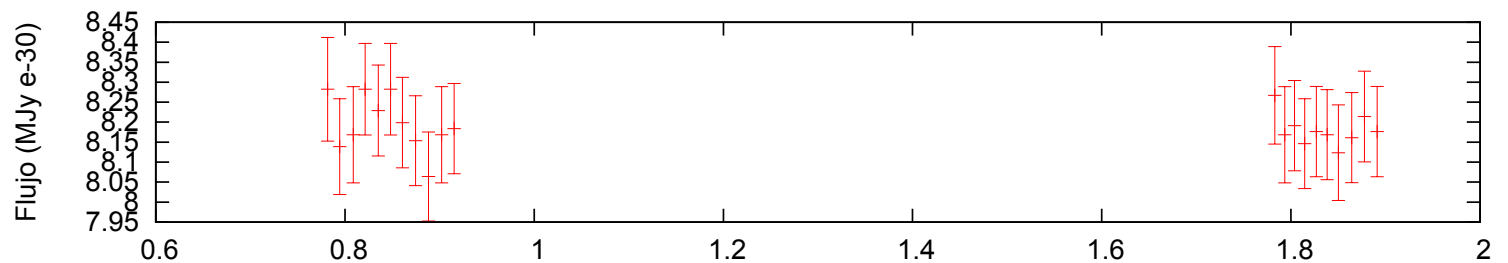

Curva de luz en la banda de rayos gamma

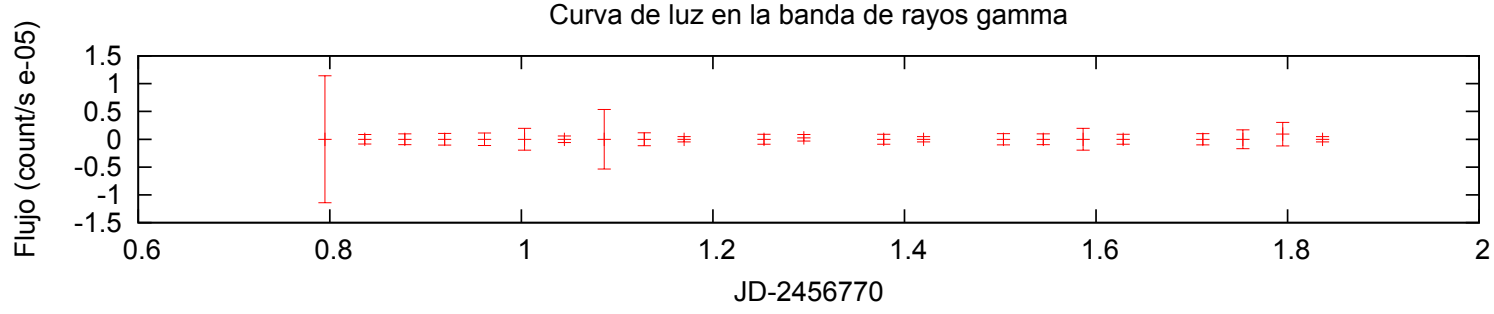

Figura 3.100. Curvas de luz de 3FGL J1958.2-3011 en la banda del óptico (panel superior y medio) en los filtros V y R, correspondientes a los días 24 y 25 abril 2014 y rayos- $\gamma$ (panel inferior). En esta última curva, se tomó un bineado temporal de 1 hora. 
Curvas de luz en rayos $X$ y rayos gamma para 3FGL J1958.2-3011

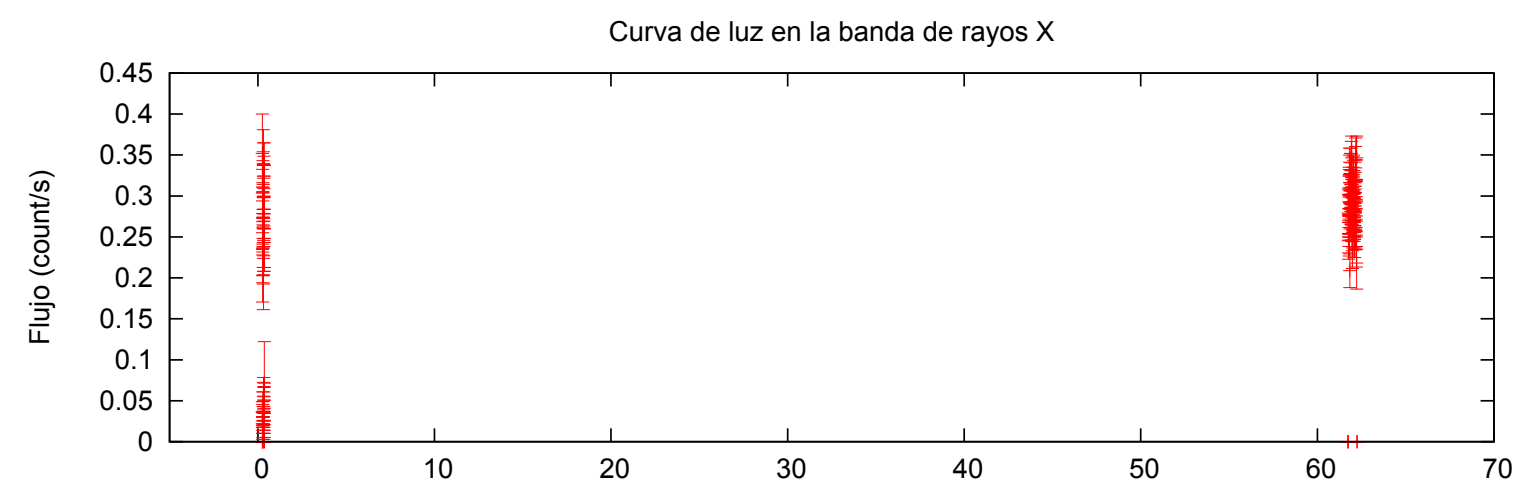

Curva de luz en la banda de rayos gamma

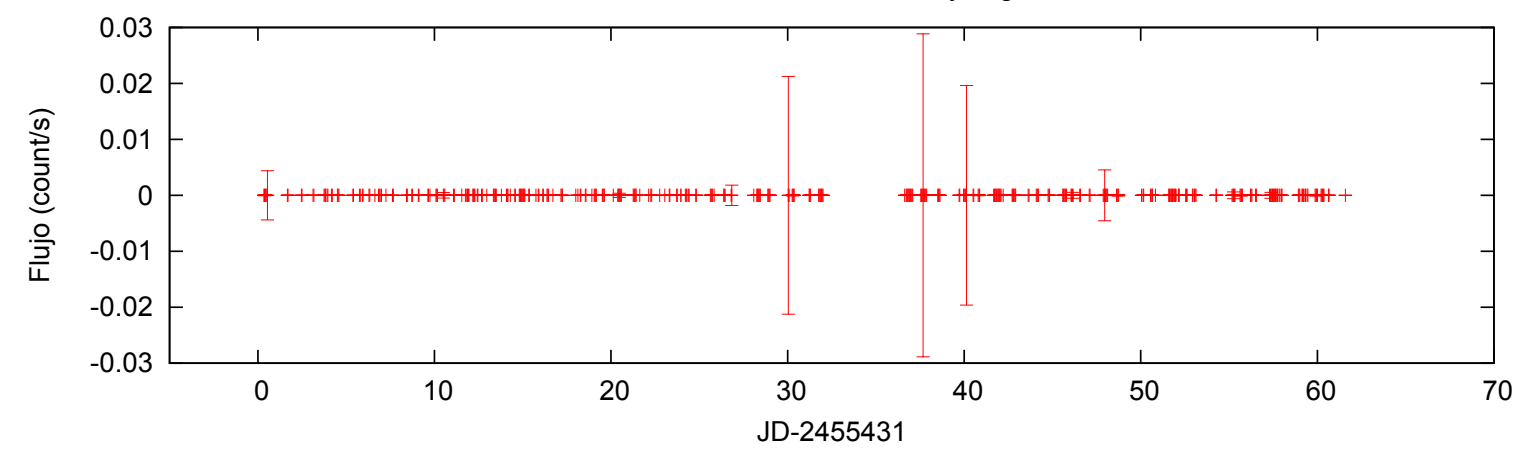

Figura 3.101. Curvas de luz de 3FGL J1958.2-3011 en las bandas de rayos X (panel superior), correspondientes a agosto y octubre 2010 y rayos- $\gamma$ (panel inferior). En esta última curva, se tomó un bineado temporal de 1 hora. 



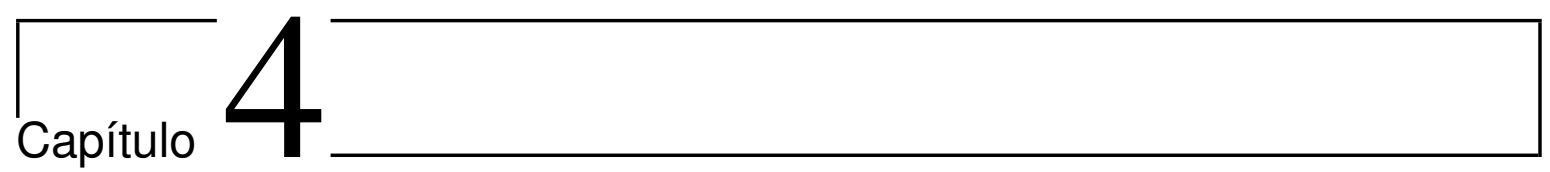

\section{Conclusiones}

En la presente Tesis, estudiamos el comportamiento del flujo óptico de los blazares.

Tener la certeza del estado de variabilidad en una curva de luz es de suma importancia para poder inferir sobre los mecanismos físicos que ocurren en la fuente y para poder explicar el comportamiento observado. Uno de los problemas que tiene emparentado el estudio de la microvariabilidad es el hecho de que las DLCs no están bien pobladas, siendo el valor máximo esperado en los seguimientos de los AGNs de alrededor de 40 puntos (en el mejor de los casos). La gran mayoría de los tests estadísticos necesitan un número entre cientos a miles de puntos en la curva de luz. Este hecho deriva en que es limitado el número de índices de variabilidad (y/o tests estadísticos) que pueden ser aplicados al estudio de estas curvas. Buscando un índice estadístico que nos devuelva el resultado más certero posible, es que planteamos una serie de simulaciones sobre las cuales poder aplicar dos herramientas en particular, el índice $\mathrm{C}$ y el test F. Ambos están basados en las desviaciones estándares de las curvas involucradas. Sokolovsky et al. (2017), encontraron que aquellos tests que involucran la dispersiones de una distribución, son más estables en relación al número de puntos en la curva (ver Fig. 5 del trabajo). Las simulaciones se realizaron generando una serie de imágenes sobre las cuales se incluyeron distintas condiciones atmosféricas: variaciones del seeing, distintas coberturas de nubes y diferentes estadíos de la presencia de la Luna. En función de estas imágenes, se construyeron las curvas de luz y de esta manera se pudieron obtener conjuntos de datos que involucraban casi por completo los distintos errores presentes en las observaciones reales.

Encontramos que se debe usar un factor de peso en los índices utilizados. En particular, analizamos el factor de peso de Howell (Howell et al. 1988), el cual tiene en consideración las diferencias entre las magnitudes del objeto estudiado y la estrellas de la fotometría diferencial.

Con el test $\mathrm{F}$ encontramos casos de falsos positivos. Esto se refleja en los resultados obtenidos en el estudio de los casos de AGNs planteados como no variables. El test $\mathrm{F}$ tiene tendencia a reconocer como variables curvas ruidosas. Sin embargo, podemos decir que este test es un buen parámetro para clasificar a las curvas como no variables.

Por el contrario, encontramos casos en los cuales el índice $\mathrm{C}$ arrojo falsos negativos. Esto ocurre cuando la amplitud de la variación es pequeña o cuando la variación planteada imita en cierta medida a los cambios en las condiciones atmosféricas. A pesar de esto, podemos afirmar que una fuente resultó variable si el índice $\mathrm{C}$ así lo indica. 
Determinados tipos de variabilidad son más factibles de ser afectados por los cambios en las condiciones atmosféricas, ya sea por la amplitud que presenta la variación originalmente o por el tipo de variabilidad. El caso ideal sería poder realizar estudios individuales sobre cada curva de luz, pudiendo ponderar las contribuciones espúreas que introducen factores como seeing atmosférico, puntos fotométricos malos, problemas instrumentales, etc. De esta manera, podríamos estimar estas contribuciones espúreas para intentar contrarrestar sus efectos sobre las DLCs. Esto dista de ser una tarea sencilla y realista de ser llevada a cabo. Es por eso que, a pesar de los problemas involucrados en los índices de variabilidad utilizados, éstos siguen siendo la mejor herramienta.

De la muestra de 19 blazares observados, encontramos que el $26 \%$ registró variabilidad intra-noche para el filtro $\mathrm{V}$, mientras que el $32 \%$ presentó variaciones en el filtro R. Estos resultados en la detección de variabilidad son producto de la aplicación de los parámetros $\mathrm{C}$ y $\mathrm{F}$ en conjunto con los resultados arrojados por las simulaciones, donde la confirmación de variabilidad detectada es corroborada por ambos parámetros. Por otro lado, reportamos que el $84 \%$ de los objetos mostró una tendencia BWB, mientras que el $16 \%$ de las fuentes resultaron RWB. En varios de estos objetos los ajustes resultaron ser poco confiables. Este resultado ocurre en menor medida para los BL Lacs que para los FSRQs. La tendencia BWB hallada se la puede asociar a modelos del tipo shock-in-jet, donde se tiene la presencia de dos componentes, una variable relativamente azul y otra relativamente roja, que están contribuyendo a la emisión general. Por su parte, una tendencia RWB se la puede relacionar con la contribución de la emisión térmica del disco de acreción, la cual influye sobre la región más azul del espectro, cuando se tiene emisión débil del jet. Basándonos en el gran porcentaje de blazares que resultaron tener una tendencia BWB, la naturaleza de la variabilidad encontrada podría ser mejor explicada por un modelo del tipo shock-in-jet.

Utilizando la posición en el diagrama índice de modulación intrínseca vs. la frecuencia del pico sincrotrón de la SED de la fuente, estudiamos la naturaleza de los 3 objetos BZU presentes en la muestra. Confirmamos la clasificación como FSRQ para PKS 0208-512, y [HB89] 0521-365 y PMN J0847-2337 se ubicaron en la región de los BL Lacs.

A una parte de la muestra le estudiamos el comportamiento en la banda de rayos $\mathrm{X}$, utilizando datos públicos tomados con el satélite Chandra. Estos datos no fueron coincidente en tiempo con los datos tomados en el óptico. De los 13 objetos estudiados, encontramos que 5 blazares presentan variaciones a escalas de tiempo cortas. Las características que presentan estas variaciones (rápidas y de baja amplitud) estarían asociadas a emisión térmica relacionada con la existencia de choques desplazándose dentro del plasma del jet. Para el caso del blazar, 3FGL J1958.2-3011, realizamos un estudio multifrecuencia con datos tomado por el satélite Fermi-LAT en la banda de rayos- $\gamma$, complementando las observaciones hechas en el óptico y los datos en rayos X. Estos datos en altas energías comparten simultaneidad con los datos obtenidos en la banda $\mathrm{X}$ en un período, y con las observaciones en la banda óptica obtenidas en esta Tesis en otro momento. Con esto buscamos relacionar el comportamiento en la banda $\mathrm{X}$ con los resultados obtenidos en el flujo óptico utilizando las curvas en rayos- $\gamma$ como vínculo. 
Obtuvimos que, al momento de las observaciones en el óptico, no se registraron variaciones en esta banda, mientras que para la curva de luz en rayos- $\gamma$ se detectan variaciones en el número de cuentas que se tiene. Por otro lado, en el período de observación de los datos en rayos $\mathrm{X}$, la fuente presenta variabilidad en rayos- $\gamma$ al igual que en rayos $\mathrm{X}$. Lo que estaría ocurriendo es que las regiones en donde la emisión es generada no son las mismas. Mientras que la emisión a altas energías se genera en la región cercana al núcleo, la emisión óptica está más alejada. Para observar la variación detectada en altas energías en la región del óptico, necesitamos escalas temporales mayores de días y horas, debido al retraso que se tiene entre la emisión en rayos X y rayos- $\gamma$ respecto de la emisión en el óptico.

A lo largo de los últimos años, han cobrado especial relevancia los estudios multifrecuencia, en particular los relacionados con los AGNs. La naturaleza de estos objetos es compleja, presentando variaciones a lo largo de todo el espectro electromagnético y pudiendo ser detectados desde radio hasta $\mathrm{TeV}$. Los mecanismos físicos que explican los comportamientos observados deben contemplar estas características. Son escasas las campañas que se pueden realizar simultáneamente en distintas bandas del espectro sobre una fuente en particular. Para poder caracterizar una clase en especial, necesitamos observaciones sobre una muestra de relevancia estadística de esa clase de objetos. Por el momento esto no es realizable. Es por esto que planteamos el enfoque presentado en esta Tesis para poder vincular los comportamientos reportados en las distintas bandas y analizar si los mismos pueden ser explicados invocando el mismo mecanismo físico. 



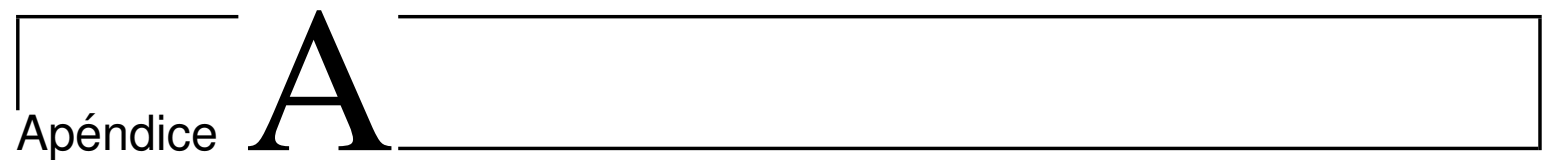

\section{Calibración de los objetos de la muestra al sistema estándar}

\section{A.1. Campos de estándares usados en la calibración}

En este apéndice presentamos los valores de las magnitudes estándares en los filtros V y R de las estrellas en cada uno de los campos de los blazares de la muestra, en donde están incluídas las estrellas de comparación y control elegidas. Para el cálculo de estos valores, se obtuvieron imágenes de campos de estándares del catálogo de Landolt (1992).

En cada semestre de los turnos asignados en CASLEO, se observaron los siguientes campos:

- CASLEO 2014A (Abril 2014): campo SA107.

- CASLEO 2015A (Abril 2015): campo SA104.

- CASLEO 2015B (Agosto-Sept-Dic 2015): campo Mark A.

- CASLEO 2016B (Noviembre 2016): campo SA95.

En la Fig. A.1 se muestran las secciones de los campos observados, juntos con las estrellas señaladas correspondiente al catálogo de Landolt.

Se ejecutaron las tareas de calibración de IRAF para obtener, en cada caso, los coeficientes y las ecuaciones de la transformación al sistema estándar. Los resultados obtenidos se presentan en la Tabla A.1. En la columna 1, se tiene el nombre del campo de Landolt; en la columna 2, las estrellas estándares presentes en ese campo; en las columnas 3 y 4, se muestran los valores de la magnitud en el $\mathrm{V}$ y el índice (V-R) publicados en el catálogo; y en las columnas 5 y 6 , mostramos el valor promedio obtenido en esta Tesis de la magnitud en $\mathrm{V}$ y del índice. 

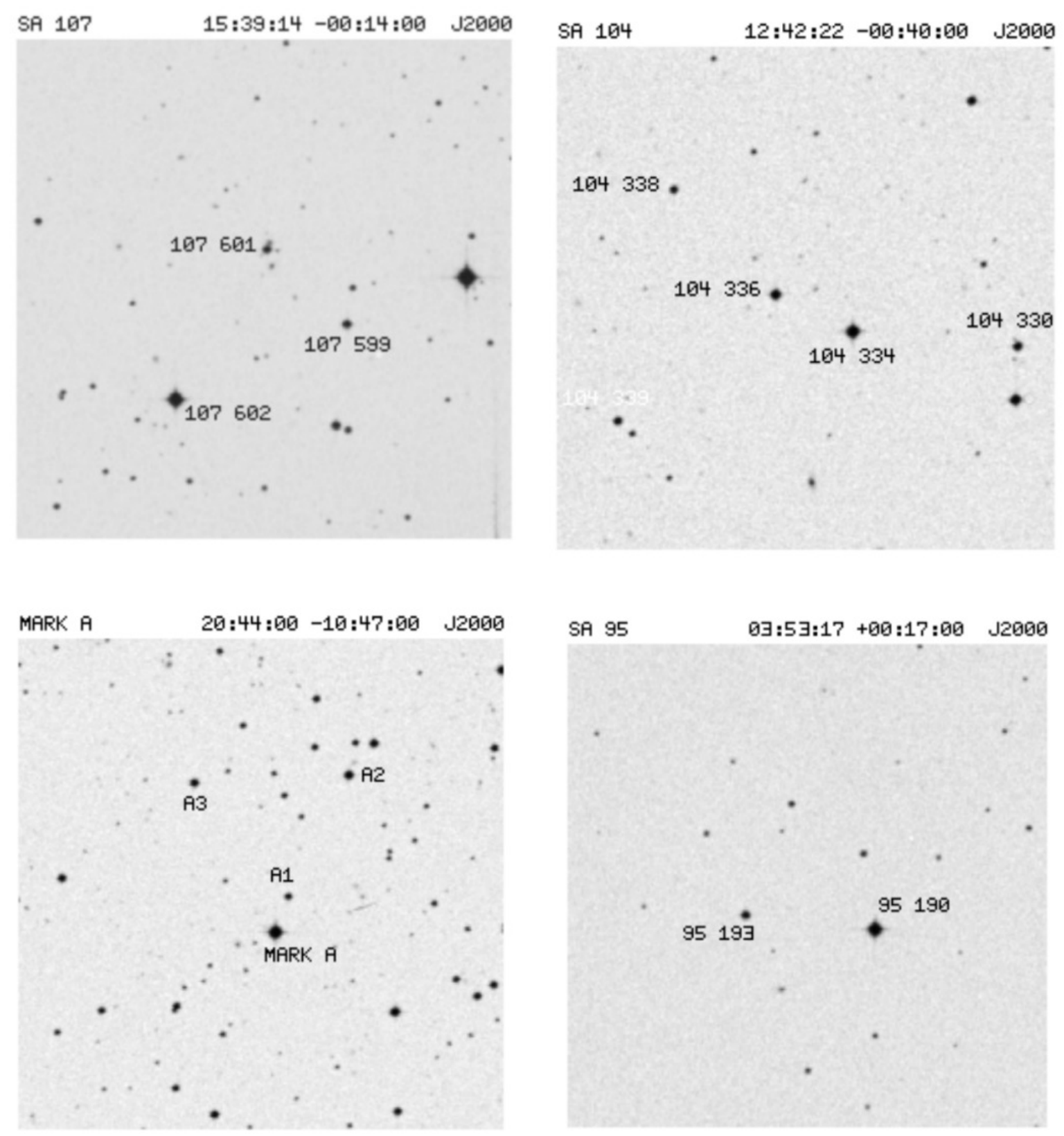

Figura A.1. Campos de estándares de Landolt usados. En el panel superior, izquierdo se encuentra el campo SA107 y en el derecho, SA104. En el panel inferior, izquierdo, Mark A y en el derecho, SA95. 
Tabla A.1. Valores de las magnitudes estándares de las estrellas de Landolt y los valores de dichas magnitudes calculadas con nuestras calibraciones. En la columna 1, se tiene el nombre del campo de Landolt; en la columna 2, las estrellas estándares presentes en ese campo; en las columnas 3 y 4 , se muestran los valores de la magnitud en el V y el índice (V-R) publicados en el catálogo; y en las columnas 5 y 6 , mostramos el valor promedio obtenido en esta Tesis de la magnitud en $\mathrm{V}$ y del índice.

\begin{tabular}{cccccc}
\hline \hline Campo & Estrella & \multicolumn{2}{c}{ Valores de Landolt } & \multicolumn{2}{c}{ Valores de esta Tesis } \\
& Núm. & V & V- R & $\bar{V}$ & $\bar{V}-R$ \\
\hline SA107 & 599 & 14.675 & 0.433 & 14.707 & 0.433 \\
& 601 & 14.646 & 0.923 & 14.655 & 0.923 \\
& 602 & 12.116 & 0.545 & 12.074 & 0.546 \\
\hline SA104b & 330 & 15.296 & 0.369 & 15.271 & 0.406 \\
& 334 & 13.484 & 0.323 & 13.481 & 0.321 \\
& 336 & 14.404 & 0.461 & 14.421 & 0.464 \\
& 338 & 16.059 & 0.348 & 16.076 & 0.363 \\
& 339 & 15.459 & 0.476 & 15.447 & 0.475 \\
\hline Mark & $\mathrm{A} 2$ & 14.540 & 0.379 & 14.537 & 0.383 \\
& $\mathrm{~A} 1$ & 15.911 & 0.367 & 15.913 & 0.369 \\
& $\mathrm{~A}$ & 13.258 & -0.115 & 13.258 & -0.117 \\
& $\mathrm{~A} 3$ & 14.818 & 0.587 & 14.819 & 0.582 \\
\hline SA95 & 190 & 12.627 & 0.195 & 12.627 & 0.195 \\
& 193 & 14.338 & 0.748 & 14.338 & 0.748 \\
\hline
\end{tabular}




\section{A.2. Resultados}

Presentamos cada uno de los campos de los blazares, juntos con las estrellas candidatas a ser comparación y control, en las Fig. A.2, A.3, A.4, A.5, A.6 y A.7.
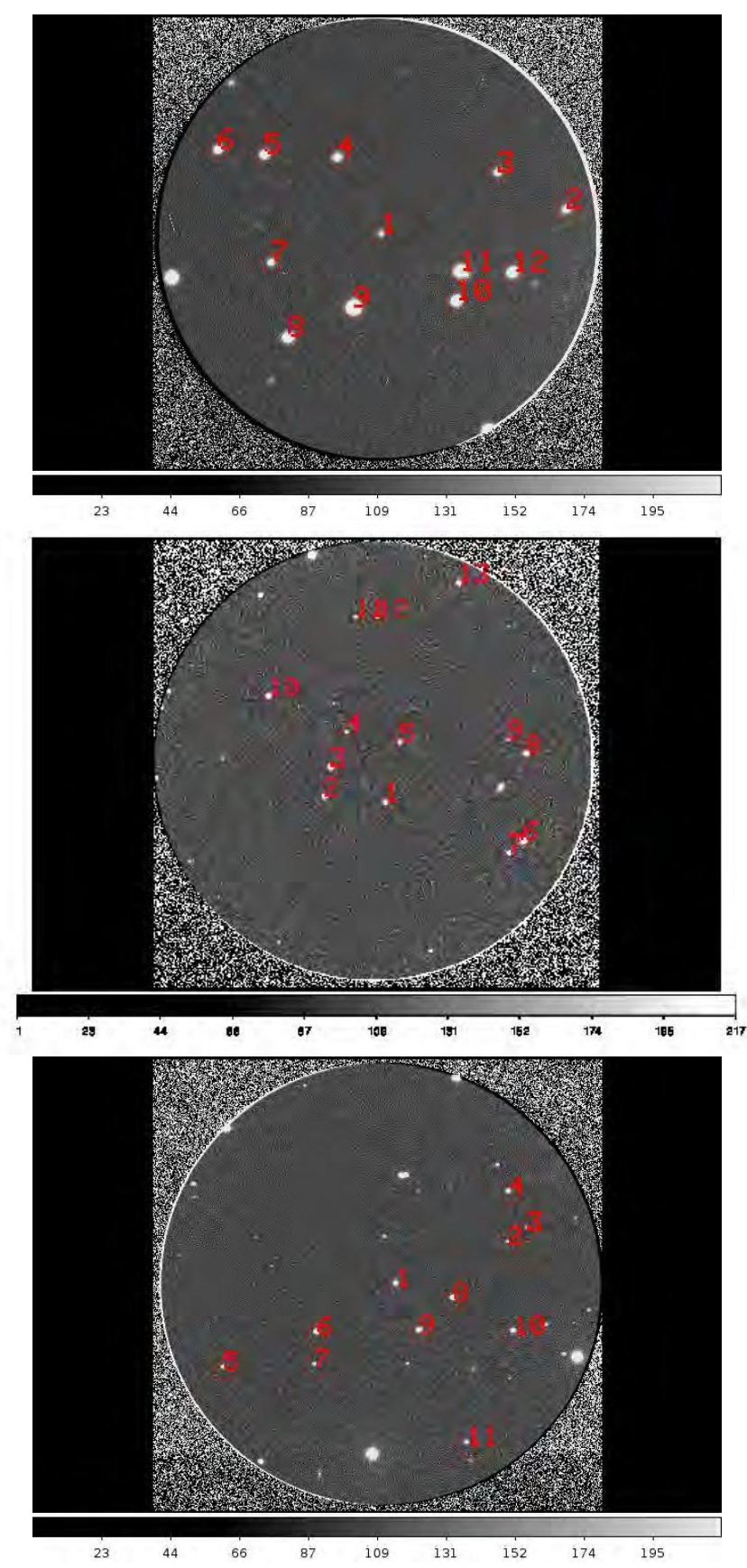

Figura A.2. Campos correspondientes a (en orden descendente): [HB89]0208-512, 1H 0414+009 y [HB89]0521-365. En todos los campos se indica con el número 1 al blazar. 

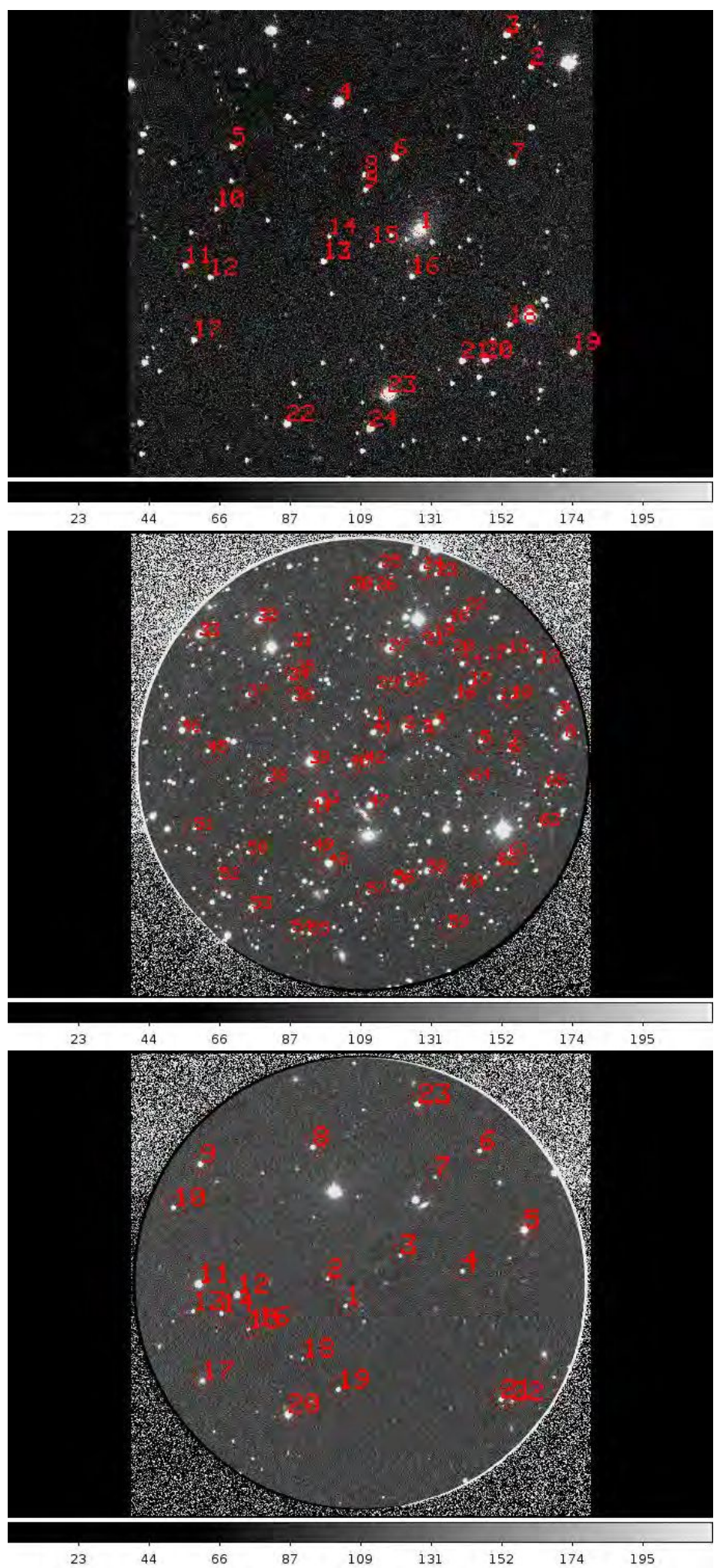

Figura A.3. Campos correspondientes a (en orden descendente): PMN J0847-2337, [HB89] 1116-462 y [HB89] 1127-145. En todos los campos se indica con el número 1 al blazar. 

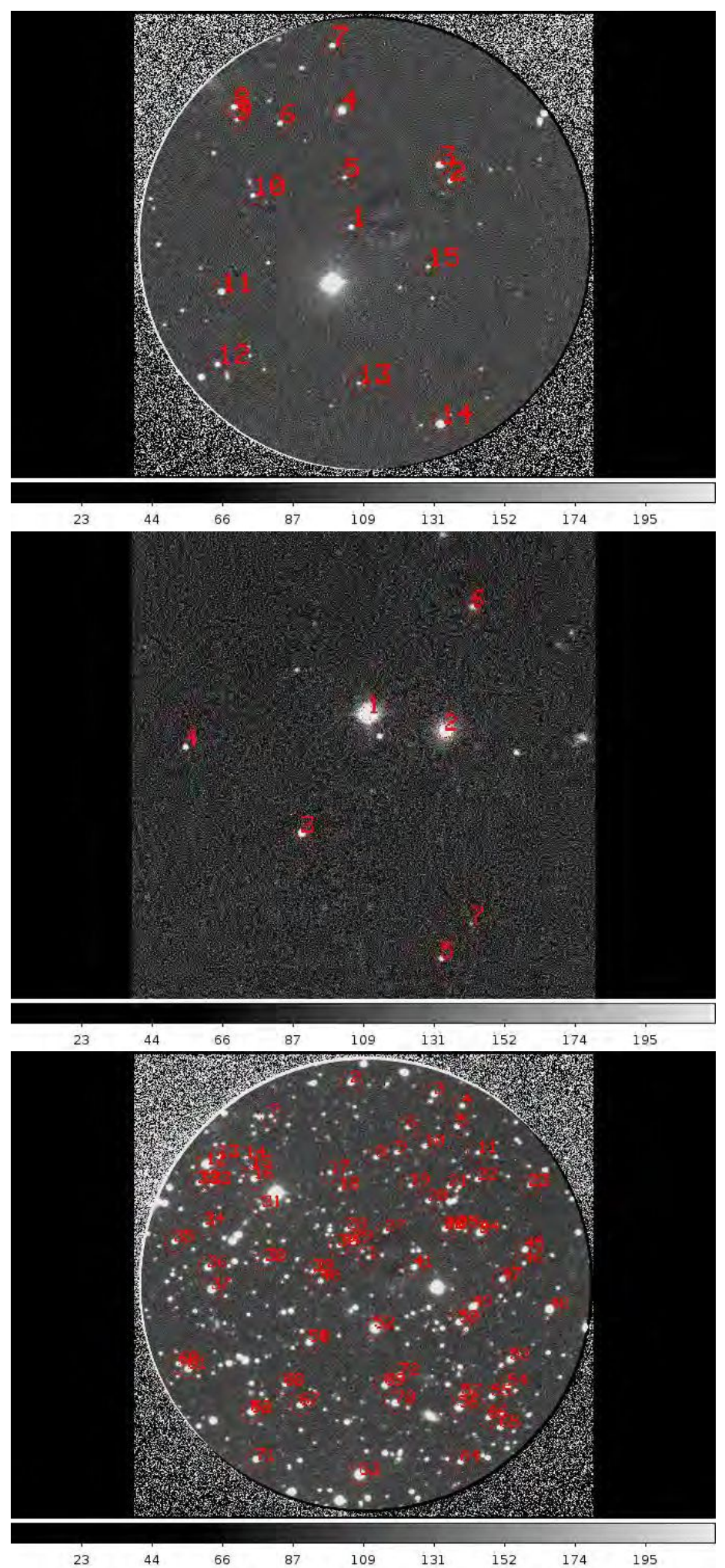

Figura A.4. Campos correspondientes a (en orden descendente): PKS 1229-02, PMN J1256-1146 y [HB89] 1424-418. En todos los campos se indica con el número 1 al blazar. 

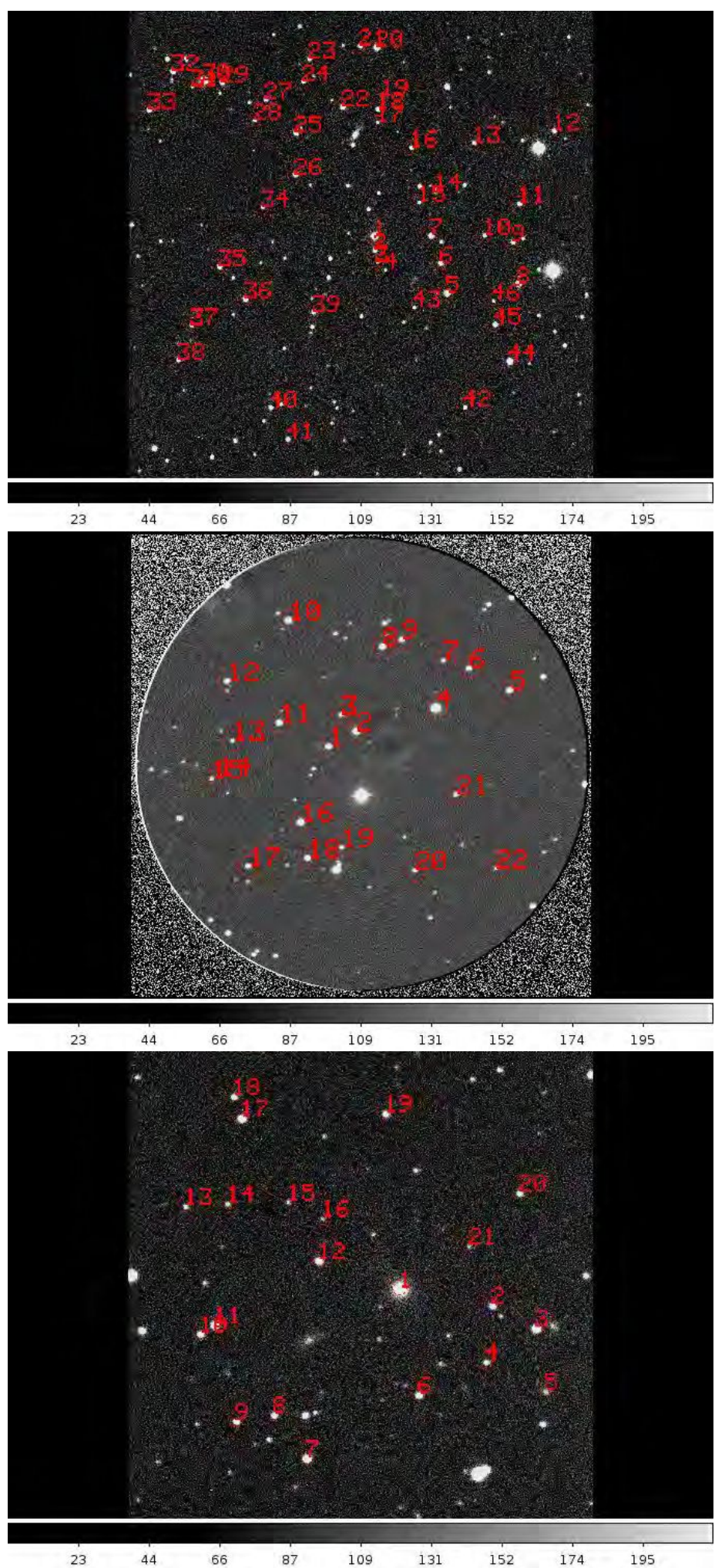

Figura A.5. Campos correspondientes a (en orden descendente): PKS 1443-389, PKS 1510-089 y PKS J1517-242. En todos los campos se indica con el número 1 al blazar. 

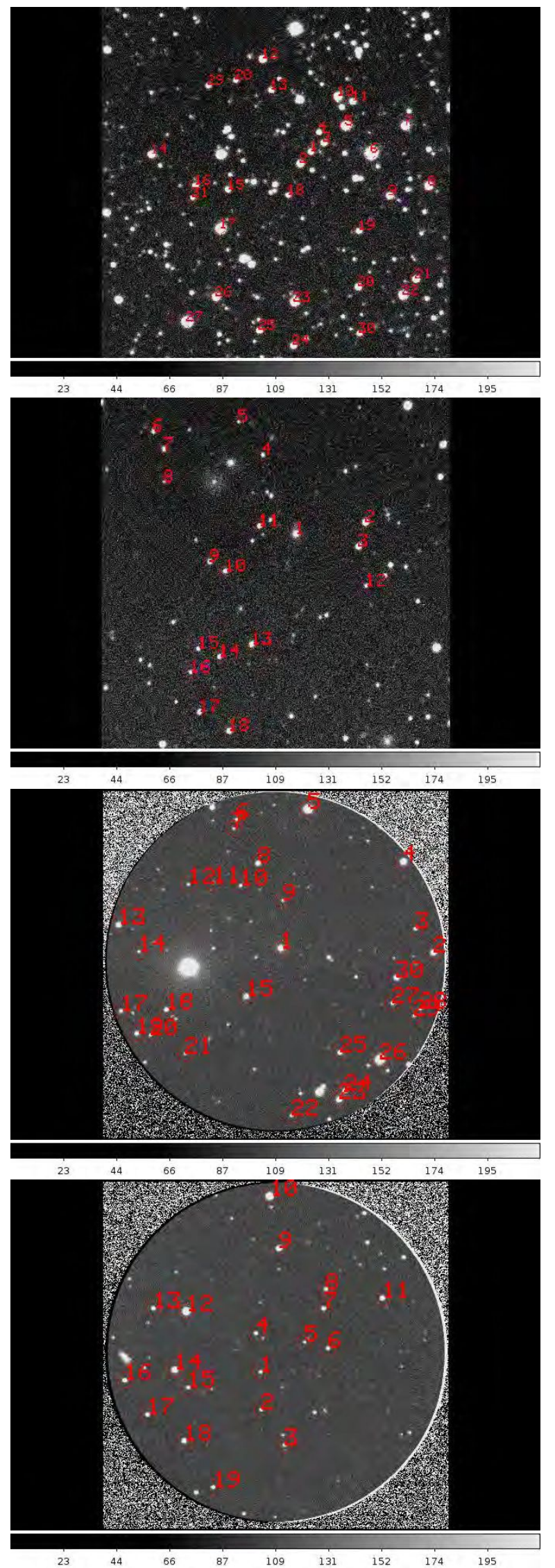

Figura A.6. Campos correspondientes a (en orden descendente): BZB J1917-192, 6dFJ1958148-301112, PKS 2005-489 y [HB89] 2126-158. En todos los campos se 202 indica con el nḿero 1 al blazar. 

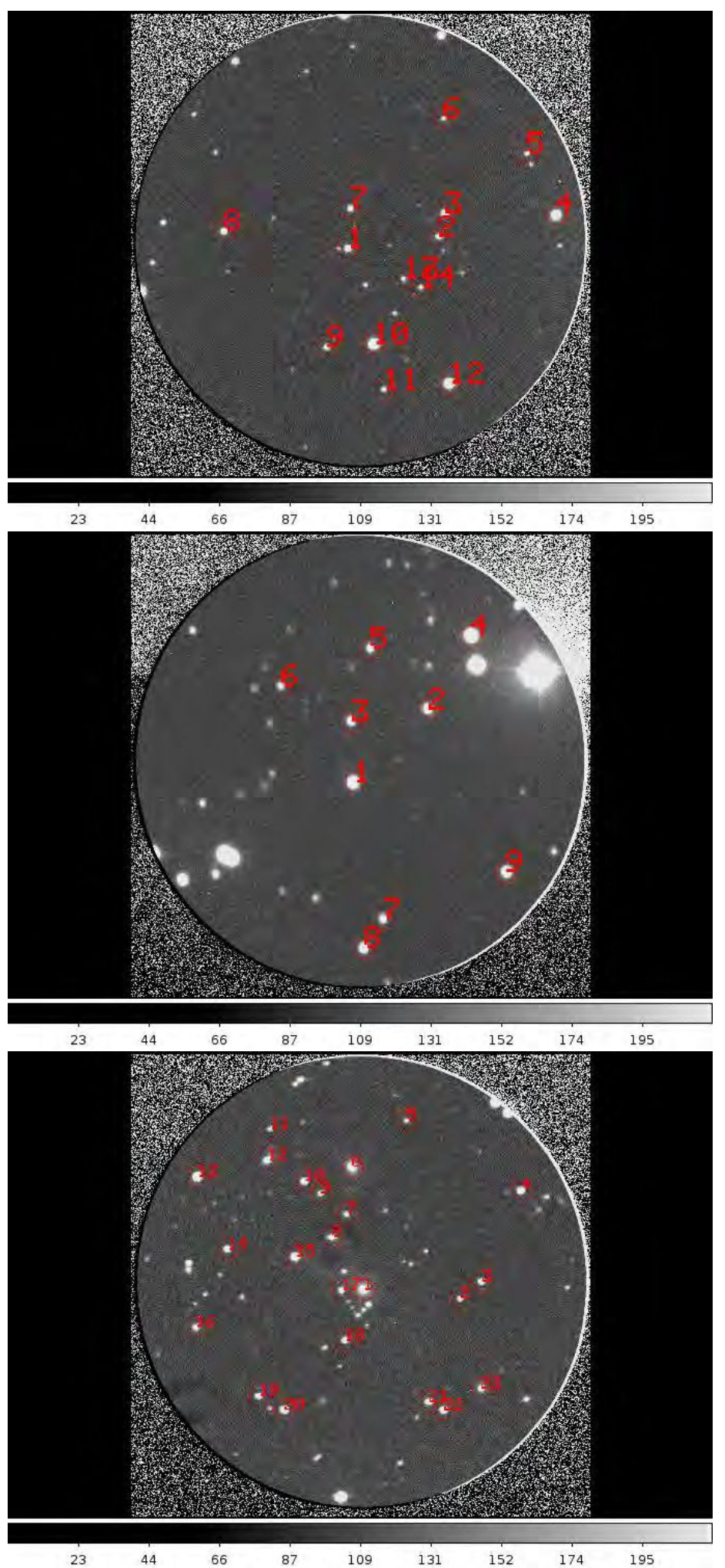

Figura A.7. Campos correspondientes a (en orden descendente): PKS 2149-306, PKS 2155-304 y PMN J2310-4374. En todos los campos se indica con el número 1 al blazar. 
En la Tabla A.2 presentamos los valores de las magnitudes estándares en el filtro $\mathrm{V}$ y del índice de color (V-R), con sus respectivos errores para estrellas de campo sobre la que se realizó la fotometría de apertura. En la columna 1 se presenta al blazar; en la columna 2, la identificación numérica de las estrellas de campo (igual a la mostrada en las figuras); en la columna 4, la magnitud estándar en el filtro V con su error; y en la columna 4, el índice de color con su respectivo error. En algunos casos, determinadas estrellas no aparecen, dado que los valores obtenidos de la calibración resultaron indefinidos. Esto suele suceder con algunas estrellas que caen muy cerca del borde de la imagen. Para cada objeto, se señala en color magenta la estrella que fue elegida como estrella de comparación y en color cian la estrella elegida como estrella de control utilizadas para la fotometría diferencial. En esta tabla aparecen todos los blazares correspondientes a los campos mostrados anteriormente, a excepción de [HB89] 0521-365. Para este objeto no se pudieron tomar campos de estándares en la noche de observación debido a las condiciones climáticas no ideales que se tuvieron.

Tabla A.2. Valores de las magnitudes estándares de las estrellas de campo para cada uno de los blazares de la muestra.

\begin{tabular}{cccc}
\hline \hline Objeto & Estrella & Magnitud V & Indice de color (V-R) \\
\hline [HB89]0208-512 & 2 & $16.300 \pm 0.004$ & $1.175 \pm 0.005$ \\
& 3 & $16.341 \pm 0.004$ & $0.876 \pm 0.006$ \\
4 & $15.917 \pm 0.003$ & $0.855 \pm 0.004$ \\
& 5 & $15.529 \pm 0.003$ & $0.979 \pm 0.004$ \\
6 & $15.746 \pm 0.003$ & $0.880 \pm 0.004$ \\
& 7 & $16.436 \pm 0.004$ & $0.871 \pm 0.006$ \\
8 & $14.739 \pm 0.002$ & $1.000 \pm 0.002$ \\
9 & $13.305 \pm 0.001$ & $0.750 \pm 0.001$ \\
10 & $14.837 \pm 0.002$ & $0.849 \pm 0.003$ \\
& 11 & $14.234 \pm 0.001$ & $0.812 \pm 0.001$ \\
& 12 & $14.871 \pm 0.002$ & $0.861 \pm 0.003$ \\
\hline 2 & $15.318 \pm 0.002$ & $0.635 \pm 0.003$ \\
& 3 & $14.040 \pm 0.001$ & $0.452 \pm 0.002$ \\
4 & $16.920 \pm 0.005$ & $0.638 \pm 0.007$ \\
& 5 & $17.559 \pm 0.009$ & $0.569 \pm 0.011$ \\
6 & $13.399 \pm 0.001$ & $0.432 \pm 0.002$ \\
& 7 & $16.868 \pm 0.005$ & $0.483 \pm 0.007$ \\
8 & $15.228 \pm 0.002$ & $0.419 \pm 0.003$ \\
9 & $17.035 \pm 0.005$ & $0.916 \pm 0.006$ \\
& 10 & $15.404 \pm 0.002$ & $0.485 \pm 0.003$ \\
11 & $16.366 \pm 0.003$ & $1.011 \pm 0.004$ \\
12 & $17.925 \pm 0.011$ & $0.955 \pm 0.013$ \\
\hline
\end{tabular}


Tabla A.2. Cont.

\begin{tabular}{cccc}
\hline \hline Objeto & Estrella & Magnitud V & Indice de color (V-R) \\
\hline PMN J0847-2337 & 2 & $17.572 \pm 0.014$ & $0.222 \pm 0.021$ \\
3 & $15.978 \pm 0.004$ & $0.305 \pm 0.005$ \\
4 & $14.102 \pm 0.001$ & $0.128 \pm 0.002$ \\
5 & $16.570 \pm 0.006$ & $0.105 \pm 0.009$ \\
6 & $15.537 \pm 0.003$ & $0.547 \pm 0.003$ \\
7 & $15.844 \pm 0.003$ & $0.194 \pm 0.006$ \\
8 & $17.157 \pm 0.010$ & $0.141 \pm 0.016$ \\
9 & $17.709 \pm 0.018$ & $0.299 \pm 0.024$ \\
10 & $18.329 \pm 0.025$ & $0.489 \pm 0.034$ \\
11 & $17.011 \pm 0.008$ & $0.581 \pm 0.010$ \\
12 & $17.272 \pm 0.008$ & $0.234 \pm 0.016$ \\
13 & $16.842 \pm 0.007$ & $0.278 \pm 0.011$ \\
14 & $18.565 \pm 0.032$ & $0.425 \pm 0.044$ \\
15 & $18.148 \pm 0.023$ & $0.284 \pm 0.034$ \\
16 & $17.037 \pm 0.010$ & $0.213 \pm 0.015$ \\
17 & $16.733 \pm 0.006$ & $0.318 \pm 0.009$ \\
18 & $17.165 \pm 0.011$ & $0.214 \pm 0.017$ \\
19 & $16.198 \pm 0.005$ & $0.100 \pm 0.007$ \\
20 & $15.742 \pm 0.003$ & $0.355 \pm 0.004$ \\
21 & $16.090 \pm 0.004$ & $0.121 \pm 0.006$ \\
22 & $15.644 \pm 0.003$ & $0.229 \pm 0.004$ \\
23 & $12.601 \pm 0.001$ & $-0.026 \pm 0.002$ \\
24 & $15.194 \pm 0.002$ & $0.113 \pm 0.003$ \\
& &
\end{tabular}


Tabla A.2. Cont.

\begin{tabular}{|c|c|c|c|}
\hline Objeto & Estrella & Magnitud V & Indice de color (V-R) \\
\hline \multirow[t]{43}{*}{ [HB89]1116-462 } & 2 & $14.830 \pm 0.001$ & $0.392 \pm 0.002$ \\
\hline & 3 & $18.301 \pm 0.015$ & $0.725 \pm 0.016$ \\
\hline & 4 & $14.436 \pm 0.001$ & $0.606 \pm 0.002$ \\
\hline & 5 & $15.795 \pm 0.003$ & $0.468 \pm 0.003$ \\
\hline & 6 & $17.135 \pm 0.006$ & $0.433 \pm 0.008$ \\
\hline & 7 & $17.970 \pm 0.013$ & $0.568 \pm 0.014$ \\
\hline & 8 & $13.776 \pm 0.001$ & $0.481 \pm 0.002$ \\
\hline & 9 & $14.543 \pm 0.001$ & $0.591 \pm 0.002$ \\
\hline & 10 & $17.386 \pm 0.008$ & $0.458 \pm 0.008$ \\
\hline & 11 & $15.954 \pm 0.003$ & $0.367 \pm 0.003$ \\
\hline & 12 & $15.591 \pm 0.003$ & $0.453 \pm 0.003$ \\
\hline & 13 & $16.913 \pm 0.005$ & $0.517 \pm 0.005$ \\
\hline & 14 & $15.973 \pm 0.003$ & $0.416 \pm 0.003$ \\
\hline & 15 & $17.224 \pm 0.006$ & $0.434 \pm 0.008$ \\
\hline & 16 & $17.862 \pm 0.010$ & $0.659 \pm 0.011$ \\
\hline & 17 & $18.250 \pm 0.014$ & $0.600 \pm 0.015$ \\
\hline & 18 & $15.941 \pm 0.003$ & $0.457 \pm 0.003$ \\
\hline & 19 & $17.105 \pm 0.006$ & $0.395 \pm 0.008$ \\
\hline & 20 & $17.755 \pm 0.009$ & $0.569 \pm 0.010$ \\
\hline & 21 & $17.677 \pm 0.010$ & $0.372 \pm 0.012$ \\
\hline & 22 & $15.808 \pm 0.003$ & $0.542 \pm 0.003$ \\
\hline & 23 & $16.739 \pm 0.005$ & $0.477 \pm 0.005$ \\
\hline & 24 & $13.954 \pm 0.001$ & $0.441 \pm 0.002$ \\
\hline & 25 & $15.388 \pm 0.003$ & $0.594 \pm 0.002$ \\
\hline & 26 & $16.274 \pm 0.004$ & $0.480 \pm 0.004$ \\
\hline & 27 & $13.432 \pm 0.001$ & $0.352 \pm 0.002$ \\
\hline & 28 & $15.868 \pm 0.003$ & $0.415 \pm 0.003$ \\
\hline & 29 & $17.306 \pm 0.008$ & $0.449 \pm 0.008$ \\
\hline & 30 & $17.272 \pm 0.006$ & $0.568 \pm 0.007$ \\
\hline & 31 & $16.003 \pm 0.003$ & $0.410 \pm 0.003$ \\
\hline & 32 & $13.609 \pm 0.001$ & $0.308 \pm 0.002$ \\
\hline & 33 & $13.285 \pm 0.001$ & $0.219 \pm 0.002$ \\
\hline & 34 & $16.455 \pm 0.004$ & $0.415 \pm 0.005$ \\
\hline & 35 & $17.205 \pm 0.006$ & $0.637 \pm 0.007$ \\
\hline & 36 & $15.236 \pm 0.003$ & $0.506 \pm 0.002$ \\
\hline & 37 & $15.582 \pm 0.003$ & $0.380 \pm 0.003$ \\
\hline & 38 & $15.187 \pm 0.001$ & $0.397 \pm 0.002$ \\
\hline & 39 & $13.026 \pm 0.001$ & $0.282 \pm 0.002$ \\
\hline & 40 & $15.854 \pm 0.003$ & $0.566 \pm 0.003$ \\
\hline & 41 & $15.028 \pm 0.001$ & $0.551 \pm 0.002$ \\
\hline & 42 & $15.999 \pm 0.003$ & $0.327 \pm 0.003$ \\
\hline & 43 & $14.057 \pm 0.001$ & $0.396 \pm 0.002$ \\
\hline & 44 & $15.938 \pm 0.003$ & $0.451 \pm 0.003$ \\
\hline
\end{tabular}


Tabla A.2. Cont.

\begin{tabular}{|c|c|c|c|}
\hline Objeto & Estrella & Magnitud V & Indice de color (V-R) \\
\hline [HB89]1116-462 & 45 & $16.808 \pm 0.005$ & $0.395 \pm 0.006$ \\
\hline \multirow[t]{20}{*}{ Cont. } & 46 & $14.704 \pm 0.001$ & $0.436 \pm 0.002$ \\
\hline & 47 & $14.547 \pm 0.001$ & $0.362 \pm 0.002$ \\
\hline & 48 & $13.858 \pm 0.001$ & $0.443 \pm 0.002$ \\
\hline & 49 & $16.962 \pm 0.005$ & $0.558 \pm 0.006$ \\
\hline & 50 & $16.951 \pm 0.005$ & $0.444 \pm 0.006$ \\
\hline & 51 & $17.003 \pm 0.005$ & $0.483 \pm 0.006$ \\
\hline & 52 & $18.045 \pm 0.011$ & $0.963 \pm 0.012$ \\
\hline & 53 & $15.221 \pm 0.003$ & $0.426 \pm 0.002$ \\
\hline & 54 & $16.108 \pm 0.004$ & $0.430 \pm 0.004$ \\
\hline & 55 & $16.386 \pm 0.004$ & $0.403 \pm 0.005$ \\
\hline & 56 & $15.086 \pm 0.001$ & $0.455 \pm 0.002$ \\
\hline & 57 & $16.705 \pm 0.005$ & $0.340 \pm 0.005$ \\
\hline & 58 & $14.627 \pm 0.001$ & $0.358 \pm 0.002$ \\
\hline & 59 & $16.562 \pm 0.004$ & $0.613 \pm 0.005$ \\
\hline & 60 & $15.297 \pm 0.003$ & $0.550 \pm 0.002$ \\
\hline & 61 & $15.743 \pm 0.003$ & $0.467 \pm 0.003$ \\
\hline & 62 & $16.849 \pm 0.005$ & $0.447 \pm 0.006$ \\
\hline & 63 & $16.645 \pm 0.005$ & $0.532 \pm 0.005$ \\
\hline & 64 & $17.618 \pm 0.008$ & $0.652 \pm 0.008$ \\
\hline & 65 & $16.169 \pm 0.004$ & $0.444 \pm 0.004$ \\
\hline \multirow[t]{22}{*}{ HB89]1127-145 } & 2 & $18.347 \pm 0.015$ & $0.803 \pm 0.016$ \\
\hline & 3 & $18.378 \pm 0.015$ & $0.906 \pm 0.016$ \\
\hline & 4 & $17.348 \pm 0.006$ & $0.845 \pm 0.007$ \\
\hline & 5 & $14.425 \pm 0.001$ & $0.840 \pm 0.002$ \\
\hline & 6 & $17.267 \pm 0.006$ & $0.934 \pm 0.006$ \\
\hline & 7 & $18.871 \pm 0.023$ & $0.893 \pm 0.023$ \\
\hline & 8 & $16.395 \pm 0.004$ & $0.919 \pm 0.004$ \\
\hline & 9 & $15.965 \pm 0.003$ & $0.862 \pm 0.003$ \\
\hline & 10 & $17.018 \pm 0.005$ & $0.972 \pm 0.005$ \\
\hline & 11 & $14.224 \pm 0.001$ & $0.881 \pm 0.002$ \\
\hline & 12 & $15.269 \pm 0.001$ & $0.920 \pm 0.002$ \\
\hline & 13 & $17.935 \pm 0.010$ & $1.218 \pm 0.010$ \\
\hline & 14 & $17.199 \pm 0.006$ & $0.943 \pm 0.006$ \\
\hline & 15 & $18.727 \pm 0.020$ & $1.059 \pm 0.020$ \\
\hline & 16 & $19.895 \pm 0.052$ & $1.516 \pm 0.048$ \\
\hline & 17 & $18.144 \pm 0.013$ & $1.098 \pm 0.012$ \\
\hline & 18 & $19.492 \pm 0.039$ & $0.997 \pm 0.039$ \\
\hline & 19 & $16.977 \pm 0.005$ & $1.323 \pm 0.005$ \\
\hline & 20 & $14.599 \pm 0.001$ & $0.781 \pm 0.002$ \\
\hline & 21 & $16.825 \pm 0.005$ & $1.111 \pm 0.005$ \\
\hline & 22 & $19.069 \pm 0.028$ & $1.051 \pm 0.028$ \\
\hline & 23 & $16.088 \pm 0.003$ & $0.856 \pm 0.003$ \\
\hline
\end{tabular}


Tabla A.2. Cont.

\begin{tabular}{|c|c|c|c|}
\hline Objeto & Estrella & Magnitud V & Indice de color (V-R) \\
\hline \multirow[t]{14}{*}{ PKS 1229-02 } & 2 & $17.340 \pm 0.009$ & $0.885 \pm 0.009$ \\
\hline & 3 & $15.235 \pm 0.003$ & $0.388 \pm 0.003$ \\
\hline & 4 & $16.166 \pm 0.004$ & $0.575 \pm 0.005$ \\
\hline & 5 & $17.902 \pm 0.013$ & $0.848 \pm 0.013$ \\
\hline & 6 & $17.075 \pm 0.008$ & $0.960 \pm 0.008$ \\
\hline & 7 & $17.108 \pm 0.008$ & $0.697 \pm 0.008$ \\
\hline & 8 & $16.227 \pm 0.005$ & $0.409 \pm 0.005$ \\
\hline & 9 & $18.106 \pm 0.018$ & $0.482 \pm 0.020$ \\
\hline & 10 & $17.280 \pm 0.009$ & $0.354 \pm 0.011$ \\
\hline & 11 & $15.743 \pm 0.004$ & $0.414 \pm 0.004$ \\
\hline & 12 & $16.483 \pm 0.005$ & $0.528 \pm 0.006$ \\
\hline & 13 & $18.027 \pm 0.017$ & $0.309 \pm 0.020$ \\
\hline & 14 & $14.143 \pm 0.001$ & $0.514 \pm 0.002$ \\
\hline & 15 & $17.846 \pm 0.014$ & $0.386 \pm 0.017$ \\
\hline \multirow[t]{6}{*}{ PMN J1256-1146 } & 2 & $15.640 \pm 0.002$ & $0.580 \pm 0.003$ \\
\hline & 3 & $16.811 \pm 0.004$ & $0.302 \pm 0.007$ \\
\hline & 4 & $18.106 \pm 0.013$ & $0.713 \pm 0.018$ \\
\hline & 5 & $18.503 \pm 0.020$ & $0.805 \pm 0.026$ \\
\hline & 6 & $18.172 \pm 0.014$ & $0.706 \pm 0.020$ \\
\hline & 7 & $19.592 \pm 0.053$ & $0.970 \pm 0.067$ \\
\hline \multirow[t]{22}{*}{ [HB89] 1424-418 } & 2 & $15.900 \pm 0.003$ & $0.593 \pm 0.003$ \\
\hline & 3 & $15.846 \pm 0.003$ & $0.450 \pm 0.003$ \\
\hline & 4 & $16.782 \pm 0.005$ & $0.498 \pm 0.006$ \\
\hline & 5 & $16.553 \pm 0.005$ & $0.470 \pm 0.005$ \\
\hline & 6 & $17.538 \pm 0.009$ & $0.916 \pm 0.009$ \\
\hline & 7 & $17.586 \pm 0.010$ & $0.429 \pm 0.012$ \\
\hline & 8 & $16.883 \pm 0.005$ & $0.580 \pm 0.006$ \\
\hline & 9 & $16.692 \pm 0.005$ & $0.468 \pm 0.006$ \\
\hline & 10 & $16.470 \pm 0.004$ & $0.470 \pm 0.005$ \\
\hline & 11 & $17.605 \pm 0.009$ & $0.448 \pm 0.011$ \\
\hline & 12 & $13.776 \pm 0.001$ & $0.376 \pm 0.002$ \\
\hline & 13 & $17.412 \pm 0.009$ & $0.547 \pm 0.011$ \\
\hline & 14 & $17.783 \pm 0.011$ & $0.444 \pm 0.013$ \\
\hline & 15 & $17.797 \pm 0.014$ & $0.436 \pm 0.016$ \\
\hline & 16 & $15.677 \pm 0.003$ & $0.619 \pm 0.003$ \\
\hline & 17 & $17.480 \pm 0.009$ & $0.451 \pm 0.010$ \\
\hline & 18 & $17.634 \pm 0.009$ & $0.522 \pm 0.010$ \\
\hline & 19 & $17.492 \pm 0.008$ & $0.984 \pm 0.008$ \\
\hline & 20 & $16.625 \pm 0.005$ & $0.474 \pm 0.006$ \\
\hline & 21 & $17.191 \pm 0.008$ & $0.739 \pm 0.008$ \\
\hline & 22 & $16.568 \pm 0.005$ & $0.428 \pm 0.005$ \\
\hline & 23 & $17.602 \pm 0.009$ & $0.551 \pm 0.010$ \\
\hline
\end{tabular}


Tabla A.2. Cont.

\begin{tabular}{|c|c|c|c|}
\hline Objeto & Estrella & Magnitud V & Indice de color (V-R) \\
\hline [HB89] 1424-418 & 24 & $14.884 \pm 0.001$ & $0.584 \pm 0.002$ \\
\hline \multirow[t]{42}{*}{ Cont. } & 25 & $15.588 \pm 0.003$ & $0.396 \pm 0.003$ \\
\hline & 26 & $16.024 \pm 0.004$ & $0.474 \pm 0.004$ \\
\hline & 27 & $16.560 \pm 0.005$ & $0.512 \pm 0.005$ \\
\hline & 28 & $16.921 \pm 0.006$ & $0.442 \pm 0.008$ \\
\hline & 29 & $16.008 \pm 0.004$ & $0.450 \pm 0.005$ \\
\hline & 30 & $17.490 \pm 0.010$ & $0.700 \pm 0.011$ \\
\hline & 31 & $16.544 \pm 0.006$ & $0.446 \pm 0.007$ \\
\hline & 32 & $16.407 \pm 0.004$ & $0.496 \pm 0.005$ \\
\hline & 33 & $17.321 \pm 0.008$ & $0.556 \pm 0.009$ \\
\hline & 34 & $16.454 \pm 0.004$ & $0.507 \pm 0.005$ \\
\hline & 35 & $16.208 \pm 0.004$ & $0.587 \pm 0.004$ \\
\hline & 36 & $15.331 \pm 0.003$ & $0.642 \pm 0.002$ \\
\hline & 37 & $14.463 \pm 0.001$ & $0.549 \pm 0.002$ \\
\hline & 38 & $16.139 \pm 0.004$ & $0.544 \pm 0.004$ \\
\hline & 39 & $16.922 \pm 0.006$ & $0.485 \pm 0.007$ \\
\hline & 40 & $16.541 \pm 0.005$ & $0.531 \pm 0.005$ \\
\hline & 41 & $16.035 \pm 0.004$ & $0.444 \pm 0.004$ \\
\hline & 42 & $16.024 \pm 0.004$ & $0.474 \pm 0.004$ \\
\hline & 43 & $15.588 \pm 0.003$ & $0.396 \pm 0.003$ \\
\hline & 44 & $14.884 \pm 0.001$ & $0.584 \pm 0.002$ \\
\hline & 45 & $16.062 \pm 0.004$ & $0.558 \pm 0.004$ \\
\hline & 46 & $17.781 \pm 0.010$ & $0.444 \pm 0.012$ \\
\hline & 47 & $16.104 \pm 0.004$ & $0.435 \pm 0.005$ \\
\hline & 48 & $14.357 \pm 0.001$ & $0.481 \pm 0.002$ \\
\hline & 49 & $14.860 \pm 0.001$ & $0.615 \pm 0.002$ \\
\hline & 50 & $15.567 \pm 0.003$ & $0.478 \pm 0.003$ \\
\hline & 51 & $15.058 \pm 0.003$ & $0.413 \pm 0.002$ \\
\hline & 52 & $13.346 \pm 0.001$ & $0.575 \pm 0.002$ \\
\hline & 53 & $14.978 \pm 0.001$ & $0.444 \pm 0.002$ \\
\hline & 54 & $16.253 \pm 0.004$ & $0.618 \pm 0.005$ \\
\hline & 55 & $16.127 \pm 0.004$ & $0.600 \pm 0.004$ \\
\hline & 56 & $14.442 \pm 0.001$ & $0.660 \pm 0.002$ \\
\hline & 57 & $16.324 \pm 0.004$ & $0.629 \pm 0.005$ \\
\hline & 58 & $15.058 \pm 0.003$ & $0.413 \pm 0.002$ \\
\hline & 59 & $15.125 \pm 0.003$ & $0.411 \pm 0.003$ \\
\hline & 60 & $16.525 \pm 0.005$ & $0.555 \pm 0.006$ \\
\hline & 61 & $14.952 \pm 0.003$ & $0.432 \pm 0.002$ \\
\hline & 62 & $15.125 \pm 0.003$ & $0.411 \pm 0.003$ \\
\hline & 63 & $13.226 \pm 0.001$ & $0.703 \pm 0.002$ \\
\hline & 64 & $16.220 \pm 0.004$ & $0.451 \pm 0.005$ \\
\hline & 65 & $15.969 \pm 0.004$ & $0.382 \pm 0.005$ \\
\hline & 66 & $16.556 \pm 0.005$ & $0.455 \pm 0.005$ \\
\hline
\end{tabular}


Tabla A.2. Cont.

\begin{tabular}{|c|c|c|c|}
\hline Objeto & Estrella & Magnitud V & Indice de color (V-R) \\
\hline [HB89] 1424-418 & 67 & $15.462 \pm 0.003$ & $0.417 \pm 0.003$ \\
\hline \multirow[t]{5}{*}{ Cont. } & 68 & $17.146 \pm 0.006$ & $0.607 \pm 0.008$ \\
\hline & 69 & $15.208 \pm 0.003$ & $0.383 \pm 0.003$ \\
\hline & 70 & $15.114 \pm 0.003$ & $0.594 \pm 0.002$ \\
\hline & 71 & $15.381 \pm 0.003$ & $0.465 \pm 0.003$ \\
\hline & 72 & $17.252 \pm 0.008$ & $0.545 \pm 0.008$ \\
\hline \multirow[t]{36}{*}{ PKS 1443-389 } & 2 & $16.916 \pm 0.008$ & $0.372 \pm 0.012$ \\
\hline & 3 & $18.537 \pm 0.031$ & $0.347 \pm 0.049$ \\
\hline & 4 & $18.947 \pm 0.040$ & $0.390 \pm 0.061$ \\
\hline & 5 & $15.460 \pm 0.002$ & $0.590 \pm 0.003$ \\
\hline & 6 & $16.672 \pm 0.005$ & $0.811 \pm 0.007$ \\
\hline & 7 & $15.837 \pm 0.003$ & $0.391 \pm 0.005$ \\
\hline & 8 & $15.505 \pm 0.002$ & $0.573 \pm 0.003$ \\
\hline & 9 & $16.750 \pm 0.006$ & $0.446 \pm 0.009$ \\
\hline & 10 & $17.679 \pm 0.012$ & $0.616 \pm 0.018$ \\
\hline & 11 & $16.966 \pm 0.007$ & $0.579 \pm 0.010$ \\
\hline & 12 & $16.899 \pm 0.008$ & $0.621 \pm 0.012$ \\
\hline & 13 & $17.418 \pm 0.010$ & $0.495 \pm 0.015$ \\
\hline & 14 & $16.554 \pm 0.005$ & $0.507 \pm 0.008$ \\
\hline & 15 & $18.260 \pm 0.024$ & $0.452 \pm 0.035$ \\
\hline & 16 & $17.362 \pm 0.009$ & $0.464 \pm 0.014$ \\
\hline & 17 & $18.653 \pm 0.033$ & $0.592 \pm 0.046$ \\
\hline & 18 & $16.979 \pm 0.007$ & $0.385 \pm 0.012$ \\
\hline & 19 & $18.368 \pm 0.024$ & $0.794 \pm 0.032$ \\
\hline & 20 & $15.367 \pm 0.002$ & $0.449 \pm 0.003$ \\
\hline & 21 & $17.151 \pm 0.008$ & $0.512 \pm 0.012$ \\
\hline & 22 & $16.145 \pm 0.003$ & $0.452 \pm 0.005$ \\
\hline & 23 & $17.561 \pm 0.011$ & $0.378 \pm 0.018$ \\
\hline & 24 & $17.632 \pm 0.012$ & $0.395 \pm 0.018$ \\
\hline & 25 & $16.411 \pm 0.004$ & $0.380 \pm 0.006$ \\
\hline & 26 & $16.692 \pm 0.005$ & $0.406 \pm 0.008$ \\
\hline & 27 & $17.539 \pm 0.011$ & $0.429 \pm 0.017$ \\
\hline & 28 & $18.015 \pm 0.016$ & $0.491 \pm 0.025$ \\
\hline & 29 & $16.756 \pm 0.006$ & $0.410 \pm 0.009$ \\
\hline & 30 & $14.319 \pm 0.001$ & $0.300 \pm 0.002$ \\
\hline & 31 & $18.092 \pm 0.020$ & $0.718 \pm 0.026$ \\
\hline & 32 & $16.488 \pm 0.005$ & $0.492 \pm 0.008$ \\
\hline & 33 & $16.435 \pm 0.004$ & $0.490 \pm 0.00$ \\
\hline & 34 & $17.815 \pm 0.013$ & $0.397 \pm 0.021$ \\
\hline & 35 & $16.729 \pm 0.005$ & $1.078 \pm 0.006$ \\
\hline & 36 & $16.430 \pm 0.004$ & $0.533 \pm 0.006$ \\
\hline & 37 & $17.746 \pm 0.013$ & $0.490 \pm 0.020$ \\
\hline
\end{tabular}


Tabla A.2. Cont.

\begin{tabular}{|c|c|c|c|}
\hline Objeto & Estrella & Magnitud V & Indice de color (V-R) \\
\hline PKS 1443-389 & 38 & $17.398 \pm 0.010$ & $0.445 \pm 0.015$ \\
\hline \multirow[t]{8}{*}{ Cont. } & 39 & $17.138 \pm 0.008$ & $0.388 \pm 0.013$ \\
\hline & 40 & $17.065 \pm 0.008$ & $0.448 \pm 0.012$ \\
\hline & 41 & $16.951 \pm 0.006$ & $0.454 \pm 0.009$ \\
\hline & 42 & $17.427 \pm 0.010$ & $0.509 \pm 0.015$ \\
\hline & 43 & $18.160 \pm 0.019$ & $0.641 \pm 0.026$ \\
\hline & 44 & $15.383 \pm 0.002$ & $0.427 \pm 0.003$ \\
\hline & 45 & $16.734 \pm 0.005$ & $0.559 \pm 0.008$ \\
\hline & 46 & $18.580 \pm 0.028$ & $0.420 \pm 0.042$ \\
\hline \multirow[t]{21}{*}{ PKS 1510-089 } & 2 & $14.710 \pm 0.003$ & $0.434 \pm 0.003$ \\
\hline & 3 & $16.724 \pm 0.009$ & $0.453 \pm 0.010$ \\
\hline & 4 & $13.309 \pm 0.001$ & $0.377 \pm 0.002$ \\
\hline & 5 & $15.825 \pm 0.005$ & $0.539 \pm 0.005$ \\
\hline & 6 & $16.459 \pm 0.006$ & $0.457 \pm 0.008$ \\
\hline & 7 & $17.473 \pm 0.015$ & $0.472 \pm 0.017$ \\
\hline & 8 & $15.520 \pm 0.004$ & $0.496 \pm 0.005$ \\
\hline & 9 & $16.283 \pm 0.006$ & $0.561 \pm 0.007$ \\
\hline & 10 & $14.905 \pm 0.003$ & $0.493 \pm 0.003$ \\
\hline & 11 & $15.317 \pm 0.004$ & $0.625 \pm 0.004$ \\
\hline & 12 & $16.076 \pm 0.005$ & $0.865 \pm 0.005$ \\
\hline & 13 & $17.459 \pm 0.014$ & $0.762 \pm 0.015$ \\
\hline & 14 & $17.667 \pm 0.018$ & $0.846 \pm 0.018$ \\
\hline & 15 & $17.305 \pm 0.013$ & $0.597 \pm 0.014$ \\
\hline & 16 & $14.471 \pm 0.003$ & $0.475 \pm 0.002$ \\
\hline & 17 & $16.304 \pm 0.006$ & $0.560 \pm 0.007$ \\
\hline & 18 & $15.582 \pm 0.004$ & $0.862 \pm 0.004$ \\
\hline & 19 & $16.997 \pm 0.010$ & $0.492 \pm 0.012$ \\
\hline & 20 & $16.943 \pm 0.010$ & $0.485 \pm 0.012$ \\
\hline & 21 & $16.530 \pm 0.008$ & $0.428 \pm 0.008$ \\
\hline & 22 & $17.766 \pm 0.018$ & $0.699 \pm 0.019$ \\
\hline \multirow{12}{*}{$\begin{array}{l}\text { PKS J1517-242 } \\
\quad \text { (Año 2014) }\end{array}$} & 2 & $17.102 \pm 0.007$ & $0.528 \pm 0.010$ \\
\hline & 3 & $15.776 \pm 0.002$ & $0.393 \pm 0.003$ \\
\hline & 4 & $17.626 \pm 0.010$ & $0.357 \pm 0.016$ \\
\hline & 5 & $18.139 \pm 0.017$ & $0.696 \pm 0.023$ \\
\hline & 6 & $16.925 \pm 0.006$ & $0.418 \pm 0.009$ \\
\hline & 7 & $15.893 \pm 0.002$ & $0.389 \pm 0.003$ \\
\hline & 8 & $17.213 \pm 0.007$ & $0.476 \pm 0.011$ \\
\hline & 9 & $17.164 \pm 0.007$ & $0.436 \pm 0.011$ \\
\hline & 10 & $16.946 \pm 0.006$ & $0.481 \pm 0.009$ \\
\hline & 11 & $15.928 \pm 0.002$ & $0.524 \pm 0.003$ \\
\hline & 12 & $16.630 \pm 0.004$ & $0.290 \pm 0.007$ \\
\hline & 13 & $18.083 \pm 0.015$ & $0.298 \pm 0.024$ \\
\hline
\end{tabular}


Tabla A.2. Cont.

\begin{tabular}{|c|c|c|c|}
\hline Objeto & Estrella & Magnitud V & Indice de color (V-R) \\
\hline PKS J1517-242 & 14 & $18.172 \pm 0.017$ & $0.442 \pm 0.025$ \\
\hline \multirow[t]{7}{*}{ Cont. } & 15 & $18.739 \pm 0.028$ & $1.021 \pm 0.034$ \\
\hline & 16 & $19.077 \pm 0.038$ & $1.226 \pm 0.045$ \\
\hline & 17 & $16.124 \pm 0.003$ & $0.464 \pm 0.005$ \\
\hline & 18 & $17.420 \pm 0.008$ & $0.655 \pm 0.012$ \\
\hline & 19 & $17.173 \pm 0.007$ & $1.150 \pm 0.009$ \\
\hline & 20 & $17.406 \pm 0.009$ & $0.294 \pm 0.014$ \\
\hline & 21 & $18.744 \pm 0.029$ & $1.085 \pm 0.035$ \\
\hline \multirow{16}{*}{$\begin{array}{c}\text { PKS J1517-242 } \\
\text { (Año 2015) }\end{array}$} & 2 & $16.842 \pm 0.014$ & $0.351 \pm 0.017$ \\
\hline & 3 & $14.450 \pm 0.003$ & $0.873 \pm 0.002$ \\
\hline & 4 & $17.004 \pm 0.014$ & $0.525 \pm 0.017$ \\
\hline & 5 & $15.108 \pm 0.004$ & $0.649 \pm 0.005$ \\
\hline & 6 & $16.195 \pm 0.008$ & $0.476 \pm 0.009$ \\
\hline & 7 & $16.031 \pm 0.008$ & $0.450 \pm 0.009$ \\
\hline & 8 & $15.656 \pm 0.005$ & $0.693 \pm 0.005$ \\
\hline & 9 & $17.283 \pm 0.019$ & $0.473 \pm 0.022$ \\
\hline & 10 & $17.668 \pm 0.026$ & $0.472 \pm 0.031$ \\
\hline & 11 & $16.505 \pm 0.010$ & $0.568 \pm 0.012$ \\
\hline & 12 & $17.531 \pm 0.020$ & $1.170 \pm 0.020$ \\
\hline & 13 & $15.921 \pm 0.006$ & $0.518 \pm 0.008$ \\
\hline & 14 & $16.323 \pm 0.009$ & $0.507 \pm 0.010$ \\
\hline & 15 & $16.030 \pm 0.006$ & $0.678 \pm 0.007$ \\
\hline & 16 & $13.861 \pm 0.001$ & $0.480 \pm 0.002$ \\
\hline & 17 & $15.367 \pm 0.004$ & $0.441 \pm 0.005$ \\
\hline \multirow[t]{19}{*}{ BZB J1917-1921 } & 2 & $15.315 \pm 0.001$ & $0.380 \pm 0.002$ \\
\hline & 3 & $15.902 \pm 0.003$ & $0.417 \pm 0.005$ \\
\hline & 4 & $16.521 \pm 0.004$ & $0.452 \pm 0.006$ \\
\hline & 5 & $14.068 \pm 0.001$ & $0.327 \pm 0.002$ \\
\hline & 6 & $12.504 \pm 0.001$ & $0.387 \pm 0.002$ \\
\hline & 7 & $14.535 \pm 0.001$ & $0.497 \pm 0.002$ \\
\hline & 8 & $14.752 \pm 0.001$ & $0.576 \pm 0.002$ \\
\hline & 9 & $15.646 \pm 0.002$ & $0.603 \pm 0.003$ \\
\hline & 10 & $14.656 \pm 0.001$ & $0.643 \pm 0.002$ \\
\hline & 11 & $15.913 \pm 0.002$ & $0.444 \pm 0.003$ \\
\hline & 12 & $15.257 \pm 0.001$ & $0.394 \pm 0.002$ \\
\hline & 13 & $16.409 \pm 0.003$ & $0.424 \pm 0.005$ \\
\hline & 14 & $15.491 \pm 0.002$ & $0.553 \pm 0.002$ \\
\hline & 15 & $16.686 \pm 0.004$ & $0.453 \pm 0.006$ \\
\hline & 16 & $16.253 \pm 0.003$ & $0.398 \pm 0.005$ \\
\hline & 17 & $13.601 \pm 0.001$ & $0.348 \pm 0.002$ \\
\hline & 18 & $16.868 \pm 0.005$ & $0.445 \pm 0.008$ \\
\hline & 19 & $16.370 \pm 0.003$ & $0.442 \pm 0.005$ \\
\hline & 20 & $15.669 \pm 0.002$ & $0.542 \pm 0.003$ \\
\hline
\end{tabular}


Tabla A.2. Cont.

\begin{tabular}{|c|c|c|c|}
\hline Objeto & Estrella & Magnitud V & Indice de color $(\mathrm{V}-\mathrm{R})$ \\
\hline \multirow{10}{*}{$\begin{array}{l}\text { BZB J1917-1921 } \\
\text { Cont. }\end{array}$} & 21 & $15.487 \pm 0.002$ & $0.403 \pm 0.003$ \\
\hline & 22 & $14.120 \pm 0.001$ & $0.543 \pm 0.002$ \\
\hline & 23 & $14.538 \pm 0.001$ & $0.555 \pm 0.002$ \\
\hline & 25 & $16.117 \pm 0.003$ & $0.463 \pm 0.005$ \\
\hline & 26 & $14.811 \pm 0.001$ & $0.588 \pm 0.002$ \\
\hline & 27 & $13.241 \pm 0.001$ & $0.729 \pm 0.002$ \\
\hline & 28 & $16.888 \pm 0.005$ & $0.480 \pm 0.007$ \\
\hline & 29 & $16.736 \pm 0.004$ & $0.625 \pm 0.005$ \\
\hline & 30 & $16.752 \pm 0.005$ & $0.550 \pm 0.007$ \\
\hline & 31 & $17.204 \pm 0.007$ & $0.411 \pm 0.010$ \\
\hline \multirow[t]{15}{*}{ 6dFJ1958148-301112 } & 2 & $16.109 \pm 0.002$ & $0.398 \pm 0.003$ \\
\hline & 3 & $16.316 \pm 0.003$ & $0.481 \pm 0.005$ \\
\hline & 4 & $17.892 \pm 0.010$ & $0.510 \pm 0.015$ \\
\hline & 5 & $18.546 \pm 0.020$ & $0.950 \pm 0.025$ \\
\hline & 6 & $17.726 \pm 0.009$ & $0.490 \pm 0.014$ \\
\hline & 7 & $17.512 \pm 0.007$ & $0.388 \pm 0.012$ \\
\hline & 8 & $19.003 \pm 0.028$ & $1.127 \pm 0.034$ \\
\hline & 9 & $17.306 \pm 0.006$ & $0.385 \pm 0.010$ \\
\hline & 10 & $17.529 \pm 0.007$ & $0.481 \pm 0.011$ \\
\hline & 11 & $17.468 \pm 0.008$ & $0.491 \pm 0.012$ \\
\hline & 12 & $18.038 \pm 0.012$ & $0.446 \pm 0.018$ \\
\hline & 13 & $17.034 \pm 0.005$ & $1.074 \pm 0.006$ \\
\hline & 14 & $17.563 \pm 0.008$ & $0.464 \pm 0.012$ \\
\hline & 15 & $17.930 \pm 0.011$ & $0.431 \pm 0.017$ \\
\hline & 16 & $18.422 \pm 0.017$ & $0.364 \pm 0.028$ \\
\hline \multirow[t]{17}{*}{ PKS 2005-489 } & 2 & $14.262 \pm 0.002$ & $0.437 \pm 0.003$ \\
\hline & 3 & $16.302 \pm 0.005$ & $0.340 \pm 0.007$ \\
\hline & 4 & $13.667 \pm 0.001$ & $0.506 \pm 0.001$ \\
\hline & 5 & $12.238 \pm 0.001$ & $0.312 \pm 0.001$ \\
\hline & 6 & $16.665 \pm 0.008$ & $0.415 \pm 0.010$ \\
\hline & 7 & $17.007 \pm 0.009$ & $0.493 \pm 0.011$ \\
\hline & 8 & $15.010 \pm 0.003$ & $0.516 \pm 0.004$ \\
\hline & 9 & $16.697 \pm 0.008$ & $0.331 \pm 0.010$ \\
\hline & 10 & $16.575 \pm 0.006$ & $0.321 \pm 0.009$ \\
\hline & 11 & $17.155 \pm 0.010$ & $0.753 \pm 0.012$ \\
\hline & 12 & $16.802 \pm 0.008$ & $0.377 \pm 0.010$ \\
\hline & 14 & $17.761 \pm 0.066$ & $0.596 \pm 0.091$ \\
\hline & 15 & $14.998 \pm 0.003$ & $0.320 \pm 0.004$ \\
\hline & 16 & $15.523 \pm 0.004$ & $0.346 \pm 0.005$ \\
\hline & 18 & $15.525 \pm 0.004$ & $0.348 \pm 0.005$ \\
\hline & 20 & $18.496 \pm 0.249$ & $1.680 \pm 0.259$ \\
\hline & 21 & $16.886 \pm 0.009$ & $0.406 \pm 0.011$ \\
\hline
\end{tabular}


Tabla A.2. Cont.

\begin{tabular}{|c|c|c|c|}
\hline Objeto & Estrella & Magnitud V & Indice de color $(\mathrm{V}-\mathrm{R})$ \\
\hline PKS 2005-489 & 22 & $16.294 \pm 0.005$ & $0.391 \pm 0.007$ \\
\hline \multirow[t]{8}{*}{ Cont. } & 23 & $14.065 \pm 0.002$ & $0.388 \pm 0.003$ \\
\hline & 24 & $14.900 \pm 0.003$ & $0.416 \pm 0.004$ \\
\hline & 25 & $15.197 \pm 0.003$ & $0.355 \pm 0.004$ \\
\hline & 26 & $11.980 \pm 0.001$ & $0.576 \pm 0.001$ \\
\hline & 27 & $16.004 \pm 0.005$ & $0.397 \pm 0.007$ \\
\hline & 28 & $16.448 \pm 0.006$ & $0.737 \pm 0.008$ \\
\hline & 29 & $16.885 \pm 0.009$ & $0.460 \pm 0.011$ \\
\hline & 30 & $14.497 \pm 0.002$ & $0.51 \pm 0.003$ \\
\hline \multirow[t]{18}{*}{ [HB89] 2126-158 } & 2 & $17.082 \pm 0.005$ & $0.439 \pm 0.007$ \\
\hline & 3 & $17.156 \pm 0.005$ & $0.535 \pm 0.007$ \\
\hline & 4 & $16.556 \pm 0.004$ & $0.421 \pm 0.005$ \\
\hline & 5 & $17.708 \pm 0.009$ & $0.408 \pm 0.011$ \\
\hline & 6 & $16.589 \pm 0.004$ & $0.573 \pm 0.005$ \\
\hline & 7 & $16.146 \pm 0.003$ & $0.451 \pm 0.004$ \\
\hline & 8 & $16.695 \pm 0.004$ & $0.690 \pm 0.005$ \\
\hline & 9 & $14.853 \pm 0.001$ & $0.376 \pm 0.001$ \\
\hline & 10 & $13.184 \pm 0.001$ & $0.436 \pm 0.001$ \\
\hline & 11 & $15.816 \pm 0.002$ & $0.595 \pm 0.003$ \\
\hline & 12 & $12.827 \pm 0.001$ & $0.524 \pm 0.001$ \\
\hline & 13 & $16.386 \pm 0.003$ & $0.538 \pm 0.004$ \\
\hline & 14 & $13.720 \pm 0.001$ & $0.254 \pm 0.001$ \\
\hline & 15 & $16.688 \pm 0.004$ & $0.416 \pm 0.006$ \\
\hline & 16 & $16.083 \pm 0.003$ & $0.481 \pm 0.004$ \\
\hline & 17 & $16.154 \pm 0.003$ & $0.586 \pm 0.004$ \\
\hline & 18 & $15.395 \pm 0.002$ & $0.410 \pm 0.003$ \\
\hline & 19 & $16.585 \pm 0.004$ & $0.422 \pm 0.005$ \\
\hline \multirow[t]{13}{*}{ PKS 2149-306 } & 2 & $15.678 \pm 0.002$ & $0.444 \pm 0.003$ \\
\hline & 3 & $15.191 \pm 0.002$ & $0.392 \pm 0.003$ \\
\hline & 4 & $13.469 \pm 0.001$ & $0.399 \pm 0.001$ \\
\hline & 5 & $16.595 \pm 0.004$ & $0.393 \pm 0.006$ \\
\hline & 6 & $16.982 \pm 0.005$ & $0.528 \pm 0.007$ \\
\hline & 7 & $15.888 \pm 0.003$ & $0.694 \pm 0.004$ \\
\hline & 8 & $15.272 \pm 0.002$ & $0.395 \pm 0.003$ \\
\hline & 9 & $15.691 \pm 0.002$ & $0.392 \pm 0.003$ \\
\hline & 10 & $12.991 \pm 0.001$ & $0.516 \pm 0.001$ \\
\hline & 11 & $16.428 \pm 0.003$ & $0.618 \pm 0.004$ \\
\hline & 12 & $13.148 \pm 0.001$ & $0.416 \pm 0.001$ \\
\hline & 13 & $16.474 \pm 0.004$ & $0.462 \pm 0.005$ \\
\hline & 14 & $16.737 \pm 0.004$ & $0.709 \pm 0.005$ \\
\hline
\end{tabular}


Tabla A.2. Cont.

\begin{tabular}{|c|c|c|c|}
\hline Objeto & Estrella & Magnitud V & Indice de color (V-R) \\
\hline \multirow[t]{8}{*}{ PKS 2155-304 } & 2 & $15.331 \pm 0.004$ & $0.266 \pm 0.005$ \\
\hline & 3 & $15.862 \pm 0.005$ & $0.120 \pm 0.007$ \\
\hline & 4 & $13.376 \pm 0.001$ & $0.339 \pm 0.001$ \\
\hline & 5 & $16.277 \pm 0.006$ & $0.304 \pm 0.009$ \\
\hline & 6 & $16.781 \pm 0.009$ & $0.500 \pm 0.011$ \\
\hline & 7 & $16.271 \pm 0.006$ & $0.645 \pm 0.008$ \\
\hline & 8 & $15.342 \pm 0.004$ & $0.249 \pm 0.005$ \\
\hline & 9 & $14.702 \pm 0.003$ & $0.115 \pm 0.004$ \\
\hline \multirow[t]{22}{*}{ PMN J2310-4374 } & 2 & $17.196 \pm 0.006$ & $0.444 \pm 0.008$ \\
\hline & 3 & $16.395 \pm 0.003$ & $0.391 \pm 0.004$ \\
\hline & 4 & $16.039 \pm 0.003$ & $0.609 \pm 0.004$ \\
\hline & 5 & $18.210 \pm 0.014$ & $0.806 \pm 0.017$ \\
\hline & 6 & $16.692 \pm 0.004$ & $0.732 \pm 0.005$ \\
\hline & 7 & $17.851 \pm 0.010$ & $0.684 \pm 0.012$ \\
\hline & 8 & $17.159 \pm 0.006$ & $0.650 \pm 0.008$ \\
\hline & 9 & $17.805 \pm 0.010$ & $0.685 \pm 0.012$ \\
\hline & 10 & $15.945 \pm 0.003$ & $0.494 \pm 0.004$ \\
\hline & 11 & $18.130 \pm 0.012$ & $1.126 \pm 0.013$ \\
\hline & 12 & $16.852 \pm 0.005$ & $0.686 \pm 0.007$ \\
\hline & 13 & $14.647 \pm 0.001$ & $0.476 \pm 0.001$ \\
\hline & 14 & $16.316 \pm 0.003$ & $0.757 \pm 0.004$ \\
\hline & 15 & $14.800 \pm 0.001$ & $0.445 \pm 0.001$ \\
\hline & 16 & $17.538 \pm 0.008$ & $0.670 \pm 0.010$ \\
\hline & 17 & $17.414 \pm 0.008$ & $0.669 \pm 0.010$ \\
\hline & 18 & $16.828 \pm 0.004$ & $1.304 \pm 0.005$ \\
\hline & 19 & $16.511 \pm 0.004$ & $0.393 \pm 0.005$ \\
\hline & 20 & $14.975 \pm 0.002$ & $0.359 \pm 0.002$ \\
\hline & 21 & $16.138 \pm 0.003$ & $0.908 \pm 0.004$ \\
\hline & 22 & $15.459 \pm 0.002$ & $0.611 \pm 0.003$ \\
\hline & 23 & $17.324 \pm 0.006$ & $0.701 \pm 0.008$ \\
\hline
\end{tabular}




\section{Bibliografía}

[1] Abdo, A. A, Ackermann, M., Ajello, M. et al., 2009, ApJ, 700, 597

[2] Abdo, A. A., Ackermann, M., Ajello M., et al., 2010a, ApJ, 715, 429

[3] Abdo, A. A., Ackermann, M., Agudo I., et al., 2010b, ApJ, 716, 30

[4] Abdo, A. A, Ackermann, M., Ajello M. et al., 2010c, ApJS, 188, 405

[5] Abdo, A. A, Ackermann, M., Ajello M. et al., 2010d, ApJ, 721, 1425

[6] Abdo, A. A, Ackermann, M., Ajello M. et al., 2010e, ApJ, 722, 520

[7] Abramowski, A., Acero, F., Aharonian F., et al., 2010, A\&A, 520, 83

[8] Abramowski, A., Acero, F., Aharonian F., et al., 2012, A\&A, 539, 149

[9] Ackermann, M., Ajello, M.; Allafort, A, et al., 2011, ApJ, 743, 171

[10] Ackermann, M., Ajello, M.; Allafort, A, et al., 2013, ApJS, 209, 34

[11] Ackermann, M., Ajello, M., Atwood, W. B., et al., 2015, ApJ, 810, 14

[12] Acero, F., Ackermann, M., Ajello, M. et al., 2015, ApJS, 218, 23

[13] Adam, 1978, A\&A, 531, 151

[14] Adam, 1985, A\&A, 61, 225

[15] Aharonian, F., Akhperjanian, A. G., Aye, K. M., et al., 2005, A\&A, 430, 865

[16] Aharonian, F., Akhperjanian, A. G., Bazer-Bachi, A. R., et al., 2007, ApJL, 664, L71

[17] Aleksić, J., Alvarez, E. A., Antonelli, L. A., et al., 2012, A\&A, 544, 75

[18] Andruchow, I., Cellone, S. A., Romero, G. E., et al., 2003, A\&A, 409, 857

[19] Andruchow, I., Romero, G. E. \& Cellone, S. A., 2005, A\&A, 442, 97

[20] Andruchow, I., Cellone, S. A.; Romero, G. E., 2008, MNRAS, 388, 1766 
[21] Angel \& Stockman, 1980, Ann. Rev. Astron. Astrophys., 18, 321

[22] Antonucci, R., 1993, ARA\&A, 31, 473

[23] Ashbrook, M.D, 1942, Harvard Ann, 109, No. 7

[24] Barkov, M. V., Aharonian, F. A., Bogovalov, S. V., et al., 2012, ApJ, 749, 119

[25] Bauer, Franz E.; Condon, J. J.; Thuan, Trinh X.; Broderick, J. J., ApJS, 129, 547

[26] Beckmann, V.; Wolter, A.; Celotti, A., et al., A\&A, 383, 410

[27] Bergeron, J.; Dodorico, S., 1986, MNRAS, 220, 833

[28] Bertsch, D. L.; Dingus, B. L.; Fichtel, C. E., et al., 1993, ApJ, 405, 21

[29] Bessell, M.S., 1979, PASP, 91, 589

[30] Bessell, M.S., Castelli, F. \& Plez, B., 1998, A\&A, 333, 231

[31] Bhattacharya,Debbijoy; Misra, Ranjeev; Rao, A. R.; Sreekumar, P. 2013, MNRAS, 431, 1618

[32] Bianchin, V.; Foschini, L.; Ghisellini, G. et al. 2009, A\&A, 469, 423

[33] Birkinshaw, M.; Worrall, D. M.; Hardcastle, M. J., 2002, MNras, 355, 142

[34] Blandford, R. D., Rees, M. J., 1978, Phys. Scr., 17, 265

[35] Blandford, R. D., Königl, A., 1979, ApJ, 232, 34

[36] Bliss, A. F.; Worrall, D. M.; Birkinshaw, et al., 2009, ApJ, 698, 1061

[37] Blom, J. J.; Bennett, K.; Bloemen, H. et al., 1995, A\&A, 298, 33

[38] Boisse, P.; Bergeron, J., A\&A, 192, 1

[39] Bolton, J. G, .Gardner, F. F.; Mackey, M. B., 1964, Aust. J. Phys., 17, 340

[40] Bolton, J. G., Clarke, M. E. \& Ekers, R. D., 1965, Aust. J. Phys., 18, 627

[41] Bolton, J. G., Kinman, T. D., 1966, ApJ, 145, 195

[42] Bond, Howard E., 1971, aPj, 167, 79

[43] Bondi, M.; Padrielli, L.; Fanti, R. et al., 1996, A\&A, 308415

[44] Bonning, E. W.; Bailyn, C.; Urry, C. M. et al., 2009, ApJ, 697, 81

[45] Bonning, E.; Urry, C. M.; Bailyn, C. et al, 2012, ApJ, 756, 13

[46] Böttcher, M., 2007, Ap\&SS, 309, 95

[47] Böttcher, M., Principe, D., ApJ, 692, 1374 
[48] Bozyan, Elizabeth P.; Hemenway, Paul D.; Argue, A. Noel, 1990, AJ, 991421

[49] Brinkmann, W.; Siebert, J.; Boller, Th., 1994, A\&A, 281, 355

[50] Burbidge, E.M., Kinman, T. D., 1987, AJ, 93, 1

[51] Burbidge, G.; Hewitt, A., 1987, AJ, 93, 1

[52] Caccianiga, Alessandro; Maccacaro, Tommaso, 1997, AJ, 114, 2350

[53] Cappi, M.; Matsuoka, M.; Comastri, A. et al, 1997, ApJ, 478, 492

[54] Carini, M. T., Miller, H. R. \& Goodrich, B. D., 1990, AJ, 100, 347

[55] Carini, M. T., Miller, H. R., Noble, J. C. \& Sadun, A. C., 1991, AJ, 101, 1196

[56] Carini, M. T., Miller, H. R., ApJ, 385, 146

[57] Carini, M. T., Noble, J. C., Taylor, R. \& Culler R., 2007, AJ, 133, 303

[58] Cellone, S. A., Romero, G. E. \& Combi, J. A., 2000, AJ, 119, 1534

[59] Cellone, S. A., Romero, G. E. \& Araudo, A. T., 2007, MNRAS, 374, 357

[60] Celotti, A.; Ghisellini, G., MNRAS, 2008, 385, 283

[61] Chadwick, P. M., Lyons, K., McComb, T. J. L. et al., 1999, ApJ, 513, 161

[62] Chatterjee, R., Jorstad, Svetlana G.; Marscher, Alan P., et al., 2008, ApJ, 689, 79

[63] Chatterjee, Ritaban; Bailyn, C. D.; Bonning, E. W. et al., 2012, ApJ, 749, 191

[64] Chatterjee, R., Fossati, G.; Urry, C. M. et al., 2013, ApJ, 763, 11

[65] Chen, P. S.; Fu, H. W.; Gao, Y. F., 2005, NewA, 11, 27

[66] Ciardi, D. R., von Braun, K., Bryden, G., et al., 2011, AJ, 141, 108

[67] Condon, J. J.; Hicks, P. D.; Jauncey, D. L., 1977, AJ, 82, 692

[68] Curtis, H. D., 1917, PASP, 29, 91

[69] Curtis, H. D., 1920, Modern theories of the spatial nebulae. J. R: Astron. Soc. Can., 14, 317

[70] D’Ammando, F.; Pucella, G.; Raiteri, C. M., 2009, A\&A, 508, 181

[71] Danziger, I. J.; Fosbury, R. A. E.; Goss, W. M.; Ekers, R. D., 1979, MNRAS, 188, 415

[72] Danziger, I. J.; Bergeron, J.; Fosbury, R. A. E., et al., 1983, MNRAS, 203, 565

[73] de Diego, J. A., 2010, AJ, 139, 1269

[74] de Diego, J. A., 2014, AJ, 148, 93 
[75] de Diego, J. A., Dultzin-Hacyan, D., Ramirez A., Benitez, E., 1998, ApJ, 501, 69

[76] de Diego, J. A., Polednikova, J., Bongiovanni, A., Pérez García, A. M., De Leo, M. A., Verdugo, T., Cepa, J., 2015, AJ, 150, 44

[77] Disney, M. J., 1974, ApJ, 193, 103

[78] Dolcini, A., Farfanelli, F., Ciprini, S., et al., 2007, A\&A, 469, 503

[79] Dominici, T. P.,Abraham, Z.; Teixeira, R. et al., 2004, AJ, 128, 47

[80] Dominici, T. P., Abraham, Z. \& Galo, A. L., 2006, A\&A, 460, 665

[81] Donato, D.; Sambruna, R. M.; Gliozzi, M., 2005, A\&A, 433, 1163

[82] Edelson, J.A. \& Krolik, J.H., 1988, ApJ, 333, 646

[83] Ellison, Sara L.; Hall, Patrick B.; Lira, Paulina, 2005, AJ, 130, 1345

[84] Fath, E.A., 1909, Lick Observatory Bulletin, 5, 71

[85] Falomo, R.; Maraschi, L.; Tanzi, E. G.; Treves, A., 1987, ApJ, 318, 39

[86] Falomo, R., Tanzi, E. G., 1991, AJ, 102, 1294

[87] Falomo, R., Pesce, J. R. \& Treves, A., 1993, ApJ, 411, 63

[88] Falomo, R.; Pian, E.; Treves, A., 2014, A\&ARv., 22, 73

[89] Fan, J. H. \& Lin, R. G., 2000, A\&A, 355, 880

[90] Fan, J.H; Cheng, K. S.; Zhang, Li, 2002, PASJ, 54, 533

[91] Fanaroff \& Riley, 1974, MNRAS, 167, 31

[92] Ferrero, E.; Brinkmann, W., 2003, A\&A, 402, 465

[93] Fiore, F.; Elvis, M.; Maiolino, R., 2003, A\&A, 409, 57

[94] Fiorucci, M.; Ciprini, S.; Tosti, G., 2004, A\&A, 419, 25

[95] Fossati, G.; Maraschi, L.; Celotti, A. et al., 1998, MNRAS, 299, 433

[96] Francis, Paul J.; Whiting, Matthew T.; Webster, Rachel L., 2000, PASA, 17, 56

[97] Gambill, J. K.; Sambruna, R. M.; Chartas, G., 2003, A\&A, 401, 505

[98] Gaur, Haritma; Gupta, Alok C.; Strigachev, A., 2012, MNRAS, 425, 3002

[99] Ghisellini G.; Tavecchio, F.; Foschini, L.; Ghirlanda, G., 2011, MNRAS, 414, 2674

[100] Gilmore, G., 1979, MNRAS, 187, 389

[101] Giommi, P., Fiore, F., Guainazzi, M., et al., 1998, A\&A, 333, L5 
[102] Giommi, P.; Capalbi, M.; Fiocchi, M., 2002, babs.conf, 63

[103] Giommi, P.; Capalbi, M.; Cavazzuti, E. et al, 2007, A\&A, 468571

[104] Giommi, P., 2011, xau5.confE, 25

[105] Gopal-Krishna \& Subramanian, K., 1991, Nature, 349, 766

[106] Gopal-Krishna \& Wiita, P. J., 1992, A\&A, 259, 109

[107] Gopal-Krishna; Sagar, Ram; Wiita, Paul J., 1995, MNRAS, 274, 701

[108] Goyal, A.; Gopal-Krishna; Wiita, P. J. et al., 2012, A\&A, 544, 37

[109] Gregory, P. C.; Loredo, Thomas J., 1992, ApJ, 3998, 146

[110] Gregory, P. C.; Vavasour, J. D.; Scott, W. K.; Condon, J. J, 1994, ApJS, 90, 173

[111] Griffith, M. et al., 1979, ApJ, 238, 810

[112] Griffith, Mark R.; Wright, Alan E., 1993, AJ, 105, 1666

[113] Griffith, Mark R.; Wright, Alan E.; Burke, B. F.; Ekers, R. D., 1994, ApJS, 90, 179

[114] Gu, M. F.; Lee, C.-U.; Pak, Soojong, et al., 2006, A\&A, 450, 39

[115] Gupta, Alok C.; Fan, J. H.; Bai, J. M.; Wagner, S. J., 2008, AJ, 135, 1348

[116] Gupta, Alok C.; Krichbaum, T. P.; Wiita, P. J. et al., 2012, MNRAS, 425, 1375

[117] Gupta, Alok C.; Agarwal, A.; Bhagwan, J. et al., 2016, MNRAS, 458, 1127

[118] Hamuy, M.; Maza, J., 1987, A\&AS, 68, 383

[119] Halpern, Jules P.; Chen, Vera S.; Madejski, Greg M.; Chanan, Gary A., 1991, AJ, 101, 818

[120] Hartman, R. C.; Bertsch, D. L.; Bloom, S. D. et al., 1999, ApJS, 123, 79

[121] Healey, Stephen E.; Romani, Roger W.; Cotter, Garret et al. 2008, ApJS, 175, 97

[122] Heidt, J.; Wagner, S. J., 1996, A\&A, 305, 42

[123] Heidt, J.; Wagner, S. J.; Wilhelm-Erkens, U., 1997, A\&A, 325, 27

[124] H.E.S.S. Colaboration, 2012, A\&A, 538, 103

[125] Hewitt, A.; Burbidge, G., 1989, ApJS, 171, 61

[126] Hewitt, A.; Burbidge, G., 1993, ApJS, 87, 451

[127] Hofmann, W., 2010, ATel, 2743, 1 
[128] Howell, S. B., 2008, Astronomische Nachrichten, 329, 259

[129] Howell, S. B. \& Jacoby G. H., 1986, PASP, 98, 802

[130] Howell, S. B., Mitchell, K. J. \& Warnock III A., 1988, AJ, 95, 247

[131] Hoyle, F. \& Fowler, W. A., 1963, MNRAS, 125, 169

[132] Ikejiri, Yuki; Uemura, Makoto; Sasada, Mahito et al., 2011, PASJ, 63, 639

[133] Impey, C. D.; Neugebauer, G., 1988, AJ, 95, 307

[134] Impey, C. D.; Tapia, S., 1988, ApJ, 333, 666

[135] Jang, M. \& Miller, H. R., 1995, ApJ, 452, 582

[136] Jang, M. \& Miller, H. R., 1997, AJ, 114, 565

[137] Jannuzi, Buell T.; Smith, Paul S.; Elston, Richard, 1993, ApJS, 85, 265

[138] Jauncey, D. L.; Wright, A. E.; Peterson, B. A.; Condon, J. J., 1978, ApJ, 219, 1

[139] Jones, D.H. et al., 2009, MNRAS, 399, 683

[140] Joshi, U. C.; Baliyan, K. S.; Ganesh, S., 2007, BASI, 35, 261

[141] Joshi, R., Chand, H., Gupta, A. C., Wiita, P. J., 2011, MNRAS, 412, 2717

[142] Kapanadze, Bidzina Z., 2009, MNRAS, 398, 832

[143] Kastendieck, M. A., Ashley, M. C. B \& Horns, D., 2011, A\&A, 531, A123

[144] Kataoka, J.; Madejski, G.; Sikora, M., 2008, ApJ, 672, 787

[145] Kaufmann, Sarah, 2011, ICRC, 8, 201

[146] Kendall, M. G. \& Stuart, A., 1969, The Advanced Theory of Statistics (Vol.1, Third Edition). Charles Griffin \& Co. Ltd.

[147] Kesteven, M. J. L., Bridle, A. H., Brandie, G. W., 1976, AJ, 81, 919

[148] Kidger, M.; Takalo, L.; Sillanpaa, A., 1992, A\&A, 264, 32

[149] Kinman, T. D.; Burbidge, E. M., 1976, ApJ, 148, 59

[150] Kotilainen, J. K., Falomo, R. \& Scarpa, R., 1998, A\&A, 332, 503

[151] Kovalov et al. 2007, AJ, 133, 1236

[152] Kreikenbohm, A.; Dorner, D.; Kadler, M. et al., 2017, xru.cof, 119

[153] Ku, W. H.-M., 1980, HiA, 5, 677

[154] Kudryavtseva, N. A.; Gabuzda, D. C.; Aller, M. F.; Aller, H. D., 2011, MNRAS, 415,1631 
[155] Kumar, P. \& Gopal-Krishna, Hum C., 2015, MNRAS, 448, 1463

[156] Lachowicz, P.; Gupta, A. C.; Gaur, H.; Wiita, P. J., 2009, A\&A, 506, 17

[157] Landolt, A. U., 1992, AJ, 104, 340

[158] Large, M.I., Mills, B.Y., Little, A.G. et al., 1981, MNRAS, 194, 693

[159] Larsson, S.; Fermi LAT Collaboration; many multiwavelength collaborators Gamma-ray variability and correlation properties of blazars observed with Fermi LAT, 2012, JPhCS, 355, 2029

[160] Liller, M. H.; Liller, W., 1975, ApJ, 199, 133

[161] Liu, B. F.; Yuan, W.; Meyer, F., 1999, ApJ, 527, 17

[162] Lu, Phillip K., 1972, AJ, 77, 829

[163] Mahony, Elizabeth K.; Croom, Scott M.; Boyle, Brian J., 2010, MNRAS, 401, 1151

[164] Mahony, Elizabeth K.; Sadler, Elaine M.; Croom, Scott M. et al., 2011, MNRAS, 401, 115

[165] Malkan, M. A.; Moore, R. L., 1986, ApJ, 300, 216

[166] Mangalam, Arun V.; Wiita, Paul J., 1993, ApJ, 406, 420

[167] Mao, L. S., 2011, NewA, 16, 503

[168] Marshall et al., 2005, ApJS, 156, 13

[169] Marscher, A. P.; Gear, W. K., 1985, ApJ, 298, 114

[170] Marscher, A. P., Jorstad S. G., D’Acangelo F. D. et al., 2008, Natur, 452, 966

[171] Massardi, M., Bonaldi, A., Bonavera, L. et al., 2011, MNRAS, 415, 1597

[172] Massaro, E., Giommi, P., Leto, C. et al., 2009, A\&A, 495, 691

[173] Massaro, F.; Masetti, N.; D’Abrusco, R.; Paggi, A.; Funk, S., 2014, AJ, 148, 66

[174] McHardy, I. M.; Luppino, G. A.; George, I. M.; Abraham, R. G.; Cooke, B. A., 1992, MNRAS, 256, 655

[175] McLaughlin, M. A.; Mattox, J. R.; Cordes, J. M.; Thompson, D. J., 1996, ApJ, 473, 763

[176] Miller, H. R.\& McAlister, H. A., 1983, ApJ, 272, 26

[177] Miller, H. R., Carini, M. T. \& Goodrich, B. D., 1989, Nat, 337, 627

[178] Miller, H. R. \& Noble, J. C., 1996, ASPC, 110, 17 
[179] Mortlock et al., 2011, Nature, 474, 616

[180] Mücke, A., Protheroe, R. J., Engel, R. et al., 2003, Astropart. Phys., 18, 593

[181] Myers, S.T., Jackson, N.J., Browne, I.W.A et al., 2008, MNRAS, 341, 1

[182] Noble, John C.; Carini, Michael T.; Miller, H. Richard; Goodrich, Bret, 1997, AJ, 113, 1995

[183] Nolan, P. L.; Tompkins, W. F.; Grenier, I. A.; Michelson, P. F., 2003, ApJ, 597, 615

[184] Nolan, P. L.; Abdo, A. A.; Ackermann, M. et al., 2012, ApJS, 199, 31

[185] Ojha, Roopesh., et al., 2009, AJ, 138, 845

[186] Padovani, P. \& Giommi, P., 1995, ApJ, 444, 567

[187] Padovani, P., Alexander, D.M., Assef, R.J. et al., 2017, Astron Astrophys Rev 25,2

[188] Paltani, S.; Courvoisier, T. J.-L.; Blecha, A.; Bratschi, P., 1997, A\&A, 327, 539

[189] Perlman, Eric S.; Madejski, Greg; Stocke, John T.; Rector, Travis A., 1999, ApJ, 523,11

[190] Perlman, ric S.; Georganopoulos, Markos; Marshall, Herman L. et al., 2011, ApJ, 739,65

[191] Peterson B. A.; Jauncey, D. J.; Wright, A. E.; Condon, J. J., 1976, ApJ, 207, 5

[192] Peterson B. A.; Wright, A. E.; Jauncey, D. L.; Condon, J. J., 1979, ApJ, 232, 400

[193] Pian, E.; Treves, A., 1993, ApJ, 416, 130

[194] Pian, E.; Falomo, R.; Ghisellini, G. et al., 1996, ApJ, 459, 169

[195] Pian, E.; Amati, L.; Antonelli, L. A., 1999, A\&AS, 138, 463

[196] Pica, A. J.; Pollock, J. T.; Smith, A. G., 1980, AJ, 85, 1442

[197] Pica, Andrew J.; Smith, Alex G.; Webb, James R. et al., 1988, AJ, 96, 1215

[198] Pohl, M.; Hartman, R. C.; Jones, B. B.; Sreekumar, P., 1997, A\&A, 326, 51

[199] Racine, 1970, ApJ, 159, 99

[200] Raiteri, C. M., Villata, M., Lanteri, L., Cavallone, M., Sobrito, G., 1998, A\&AS, 130,495

[201] Raiteri, C. M.; Villata, M.; de Francesco, G. et al., 1998, A\&AS, 132, 361

[202] Raiteri, C. M.; Villata, M.; Aller, H. D. et al., 2001,A\&A, 377, 396 
[203] Ramírez, A., de Diego, J. A., Dultzin, D., González-Pérez, J.-N., 2009, AJ, 138, 991

[204] Ramírez, A., de Diego, J. A., Dultzin-Hacyan D., González-Pérez, J. N., 2004, A\&A, 421, 83

[205] Rani, Bindu; Wiita, Paul J.; Gupta, Alok C., 2009, ApJ, 696, 2170

[206] Rani, Bindu; Gupta, Alok C.; Strigachev, A. et al., 2010, MNRAS, 404, 1992

[207] Rector, Travis A.; Perlman, Eric S., 2003, AJ, 126, 47

[208] Richards, J. L., Max-Moerbeck, W., Pavlidou, V., King, O. G., Pearson, T. J., Readhead, A. C. S., Reeves, R., Shepherd, M. C., Stevenson, M. A., Weintraub, L. C., Fuhrmann, L., Angelakis, E., Zensus, J. A., Healey, S. E., Romani, R. W., 2011, ApJs, 194, 29

[209] Romero, G. E.; Benaglia, P.; Combi, J. A., 1995, A\&A, 301, 33

[210] Romero, G. E., Cellone, S. A. \& Combi, J. A., 1999, A\&AS, 135, 477

[211] Romero, G. E., Cellone, S. A. \& Combi, J. A., 2000a, A\&A, 360, L47

[212] Romero, G. E., Cellone, S. A. \& Combi, J. A., 2000b, AJ, 120, 1192

[213] Romero, G. E., Cellone, S. A., Combi, J. A., Andruchow, I., 2002, A\&A, 390, 431

[214] Romero, G. E., Combi J. A. \& Colomb, F. R., 1994, A\&A, 288, 731

[215] Russell, H. R.; Fabian, A. C.; Taylor, G. B. et al., 2012, MNRAS, 422, 590

[216] Sagar, Ram; Stalin, C. S.; Gopal-Krishna; Wiita, Paul J., 2004, MNRAS, 348, 176

[217] Saito, S.; Stawarz, T.; Tanaka, Y. T., 2013, ApJ, 766, 11

[218] Salpeter, E. E., 1964, Accretion of interestellar matter by massive objects. Astrophys. J., 140, 796-800

[219] Sambruna, Rita M.; Donato, Davide; Tavecchio, F. et al., 2007, ApJ, 670, 74

[220] Sandrinelli, A.; Covino, S.; Treves, A., 2014, A\&A, 562, 79

[221] Sandrinelli, A.; Covino, S.; Dotti, M.; Treves, A., 2016, AJ, 151, 54

[222] Savage, A.; Bolton, J. G., 1979, MNRAS, 188, 599

[223] Sbarufatti, B.; Treves, A.; Falomo, R., 2005, ApJ, 635, 173

[224] Scarpa,R. \& Falomo, R., 1997, A\&A, 325, 109

[225] Scarpa, Riccardo; Urry, C. Megan; Falomo, Renato; Treves, Aldo., 1999, ApJ, 526,643 
[226] Schwartz \& Ku, 1983, AJ, 266, 459

[227] Schwartz, D. A.; Marshall, H. L.; Lovell, J. E. J. et al., 2006, ApJ, 640, 592

[228] Searle \& Bolton, J. G., 1968, Astrophys.J., 154, 101

[229] Sefako, R. R.; Glass, I. S.; Kilkenny, D.; de Jager, O. C. et al., 1999, MNRAS, 309, 1043

[230] Seyfert, C. K., 1943,Nuclear emission in spiral nebulae. Astrophys. J., 97, 28

[231] Siebert, J.; Brinkmann, W.; Morganti, R., 1996, MNRAS, 279, 1331

[232] Siemiginowska, Aneta; Bechtold, Jill; Aldcroft, Thomas L. et al., 2002, 570, 543

[233] Sillanpaa, A.; Haarala, S.; Valtonen, M. J.; Sundelius, B.; Byrd, G. G., 1988, ApJ, 325, 628

[234] Singh, K. P.; Shrader, C. R.; George, I. M., 1997, ApJ, 491, 515

[235] Slipher, V. M., 1913, The radial velocity of the Andromeda Nebula. Lowell Obs. Bull., 2, 56-57

[236] Smith, Paul S.; Hall, Patrick B.; Allen, Richard G.; Sitko, Michael L., 1992, ApJ, 400,115

[237] Sokolovsky, K. V.; Gavras, P.; Karampelas, A. et al., 2017, MNRAS, 464, 274

[238] Soldi, S.; Beckmann, V.; Baumgartner, W. H. et al., 2014, A\&A, 563, 57

[239] Stalin, C. S.; Gopal-Krishna; Sagar, Ram; Wiita, Paul J., 2004, MNRAS, 350, 175

[240] Stocke, John T.; Perlman, Eric S.; Wurtz, Ron; Morris, Simon L., 1991, ASPC, 21,218

[241] Strittmatter, P. A.; Serkowski, K.; Carswell, R. et al., 1972, 175, 7

[242] Taris, F.; Andrei, A.; Klotz, A. et al., 2013, A\&A, 552, 98

[243] Tavecchio, F.; Maraschi, L.; Ghisellini, G. et al., 2000, ApJ, 543, 535

[244] Tavecchio, F.; Maraschi, L.; Wolter, A. et al., 2007, ApJ, 662, 900

[245] Thompson, D. J.; Djorgovski, S.; de Carvalho, R., PASP, 102, 1235

[246] Thompson, D. J.; Bertsch, D. L.; Dingus, B. L. et al., 1995, ApJS, 101, 259

[247] Tingay S. J.; Edwards, P. G.; Costa, M. E. et al., 1996, ApJ, 464, 170

[248] Tritton, K. P., 1971, MNRAS, 155, 1

[249] Tucker, W. H.; Tananbaum, H.; Remillard, R. A., 1995, ApJ, 444, 532 
[250] Ulmer, M. P.; Shulman, S.; Evans, W. D. et al., 1980, ApJ, 235, 351

[251] Ulmer, M. P.; Brown, R. L.; Schwartz, D. A. et al., 1983, ApJ, 270, 1

[252] Ulmer, A.; Fenimore, E. E.; Epstein, R. I. et al., 1993, ApJ, 418, 395

[253] Ulrich, M. H., Maraschi, L \& Urry, C. M., 1997, ARA\&A, 35, 445

[254] Urry, C. M. \& Padovani, P., 1991, ApJ, 371, 60

[255] Urry, C. M. \& Padovani, P., 1995, PASP, 107, 803

[256] Vagnetti, Fausto; Trevese, Dario; Nesci, Roberto, 2003, ApJ, 590, 123

[257] Valtonen, M. J.; Lehto, H. J.; Sillanpaa, A. et al., 2006, ApJ, 646, 36

[258] Vercellone, S.; Soldi, S.; Chen, A. W.; Tavani, M., 2004, MNRAS, 353, 890

[259] Véron-Cetty, M.-P. \& Véron, P., 1987, S\&T, 74, 613

[260] Véron-Cetty, M.-P. \& Véron, P., 2001, A\&A, 374, 92

[261] Véron-Cetty, M.-P. \& Véron, P., 2010, A\&A, 518, A10

[262] Villata, M.; Raiteri, C. M.; Ghisellini, G. et al., 1997, A\&A, 121, 119

[263] Villata, M.; Raiteri, C. M.; Balonek, T. J. et al., 2006, A\&A, 453, 817

[264] Voges, W.et al., 1998, A\&A, 349, 389

[265] von Montigny, C.; Bertsch, D. L.; Chiang, J.et al., 1995, ApJ, 440, 525

[266] Wagner, S. J. \& Witzel, A., 1995, ARA\&A, 33, 163

[267] Wall, J. V.; Danziger, I. J.; Pettini, M. et al., 1986, MNRAS 219, 23

[268] Wall, J. V. \& Jenkins, C. R., 2012, Practical Statistics for Astronomers. Cambridge, UK: Cambridge University Press

[269] Walter, R.; Aubord, A.; Bartholdi, P. et al., 1998, ASPC, 145, 1458

[270] Wehrle, Ann E.; Cohen, Marshall H.; Unwin, Stephen C. et al., 1992, ApJ, 391, 589

[271] Wehrle, A. E.; Pian, E.; Urry, C. M. et al., 1998, ApJ, 497, 178

[272] Westerlund \& Stokes, 1966,, Astrophys.J., 145, 354

[273] Westerlund, B. E.; Wlerick, G.; Garnier, R., 1982, A\&A, 105, 284

[274] Wierzcholska, A., Ostrowski, M., Stawarz, L., Wagner, S., Hauser, M., 2015, A\&A, 573, AA69

[275] White, Graeme L.; Jauncey, David L.; Savage, Ann et al., 1988, ApJ, 327, 561 
[276] Wilkes, B. J., 1986, MNRAS, 218, 331

[277] Wilkes, Belinda J.; Tananbaum, Harvey; Worrall, D. M. et al., 1994, ApJS, 92, 53

[278] Woltjer, L., 1959, Emission nuclei in galaxies. Astrophys. J., 130, 38

[279] Worral et al., 1999, ApJ, 516, 1163

[280] Wright, A. E., 1984, PASAu, 5, 510

[281] Zel'Dovich, Y. B. \& Novikov, I. D., 1964, The radiation of gravity waves by bodies moving in the field of a collapsing star. Sov. Phys. Dokl., 9, 246

[282] Zhang, B-K. et al., 2014, RAA, 14, 933

[283] Zhang, B-K. et al., 2015, RAA, 15, 1784

[284] Zibecchi, L., Andruchow, I., Cellone, S. A., Romero, G. E., Combi, J. A., 2011, BAAA, 54, 325

[285] Zibecchi, L., Andruchow, I., Cellone, S. A., Carpintero, D. D., Romero, G. E., Combi, J. A., 2017, MNRAS, 467, 340 